\title{
Versuche zur Erzeugung des Pentacyclopropylcyclopentadienyl-Kations, und Synthese von cyclopropylierten Oligoenen, Carbo- und Heterocyclen
}

\author{
Dissertation \\ zur Erlangung des Doktorgrades \\ der Mathematisch-Naturwissenschaftlichen Fakultäten \\ der Georg-August-Universität zu Göttingen
}

vorgelegt von

Stefan Redlich

aus Göttingen

Göttingen 2004 
D7

Referent: Prof. Dr. A. de Meijere Korreferent: Prof. Dr. L. F. Tietze

Tag der mündlichen Prüfung: 02. Nov. 2004 
Die vorliegende Arbeit wurde in der Zeit von November 2001 bis September 2004 am Institut für Organische und Biomolekulare Chemie der Georg-August-Universität Göttingen unter der Leitung von Herrn Prof. Dr. A. de Meijere und in der Abteilung Organische Chemie I der Universität Ulm unter der Leitung von Herrn Prof. Dr. H.-U. Siehl angefertigt.

Meinem Lehrer, Herrn Prof. Dr. A. de Meijere, danke ich herzlich für die interessante Aufgabenstellung, die hilfreichen Diskussionen, sowie für die während der Arbeit erwiesene Unterstützung.

Herrn Prof. Dr. H.-U. Siehl danke ich besonders für die freundliche Aufnahme, sowie für die Unterstützung und stete Diskussionsbereitschaft während des Forschungsaufenthalts in Ulm. 
Für meine Eltern, meine Schwester und Jasmin 
"Wie im gewöhnlichen Leben die Denkweise und Gemütsbeschaffenheit eines Menschen leichter sich verrät, wenn er in Leidenschaft geraten ist, so enthüllen sich auch die Verborgenheiten der Natur besser unter den Eingriffen der Kunst, als wenn man sie in ihrem Gang ungestört lässt.“

Sir Francis Bacon, 1561 - 1626, Naturforscher und Philosoph 


\section{Inhaltsverzeichnis}

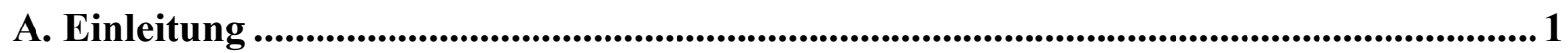

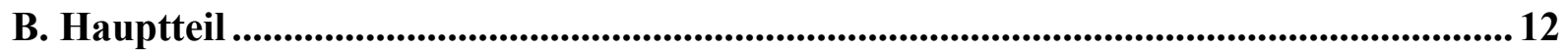

1. Bisherige Untersuchungen von Cyclopentadienyl-Kationen........................................ 12

2. Elektronische Struktur, Geometrie und quantenmechanische Berechnungen von

Cyclopropyl-substituierten Cyclopentadienyl-Kationen ............................................ 14

3. Synthese geeigneter Vorstufen für Cyclopropyl-substituierte, kationische

Cyclopentadienyl-Spezies und deren spektroskopische Untersuchung....

3.1. Retrosynthetische Überlegungen und generelle Aspekte zur Präparation von

Carbokationen .24

3.2. Synthetische Zugänge zu Cyclopropyl-substituierten Cyclopentadienen .25

3.3. Synthese von 1,2,3,4,5-Pentacyclopropylcyclopenta-1,3-dien (15) 29

3.4. Potentielle Vorstufen für Cyclopropyl-substituierte, kationische

Cyclopentadienyl-Spezies

3.5. Chlorpentacyclopropylcyclopentadien (36) als potentielle Vorstufe für das

Pentacyclopropylcyclopentadienyl-Kation (12)

3.6. Versuche zur Erzeugung und Charakterisierung des

Pentacyclopropylcyclopentadienyl-Kations (12) 40

3.7. Interpretation der experimentellen Daten im Hinblick auf die berechneten

Parameter von $c \operatorname{Pr}_{5} \mathrm{Cp}^{+}(\mathbf{1 2})$ 64

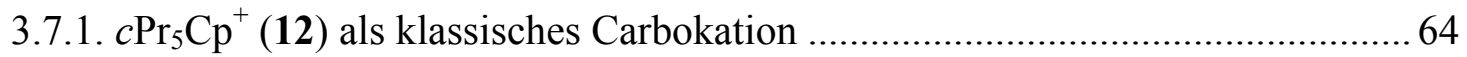

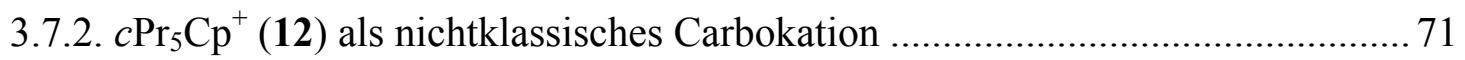

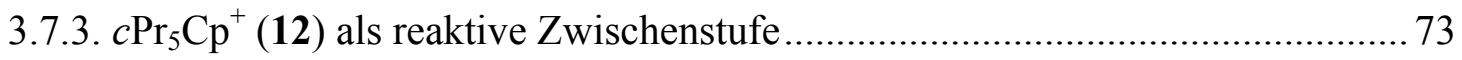

4. Tetra- und Pentacyclopropylcyclopentadien 14 und 15 als Bausteine für planarchirale Heteroferrocen-Liganden

4.1. Versuche zur Darstellung eines neuartigen N,P-Heteroferrocen-Liganden (16)..... 84

4.2. Versuche zur Darstellung eines gemischten Tetra- oder Pentayclopropylsubstituierten Ferrocens (89) 
4.3. Versuche zur Darstellung von Eisentricarbonyl-Cyclopentadien-Komplexen 88

5. Oligocyclopropylierte Diene als Substrate in DIELS-ALDER-Reaktionen. 90

5.1. DIELS-ALDER-Reaktionen mit Dimethylacetylendicarboxylat (97) und $N$-Phenylmaleimid (98) 91

5.2. Versuche zum Aufbau eines Heptacyclopropylnorbornadienyl-Gerüsts 97

5.3. Versuche zum Aufbau eines oligocyclopropylierten Tropylium-Grundgerüsts .... 100

6. Neue percyclopropylierte, fünfgliedrige Heterocyclen 105

6.1. Cyclopropylierte fünfgliedrige Heterocyclen mit einem Heteroatom ................... 108

6.2. Cyclopropylierte fünfgliedrige Heterocyclen mit zwei Heteroatomen................... 114

7. Neuartige Cyclopropyl-substituierte cyclische und acyclische Oligoene

7.1. Synthese eines Octacyclopropyl-substituierten Tricyclo[4.2.0.0 $\left.0^{2,5}\right]$ octadiens

(165) als Vorstufe für Octacyclopropylcuban (169).

7.2. Versuch der Darstellung von 1,2,3,4,5,6,7,8-Octacyclopropylcyclooctatetraen (176)

7.3. Cyclopropyl-substituierte 1,4-Dihalogenbutadiene als Kupplungskomponenten für oligocyclopropylierte acyclische Triene und Tetraene

8. Versuche zur Darstellung des 6,6-Dicyclopropyl-substituiertem Fulven 24, und die präparativ interessante Bildung von Allylidencyclobutenen

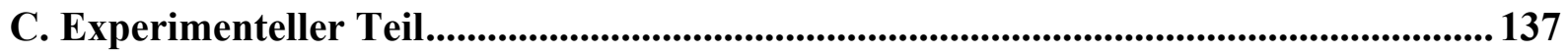

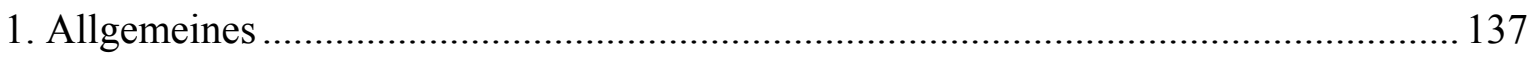

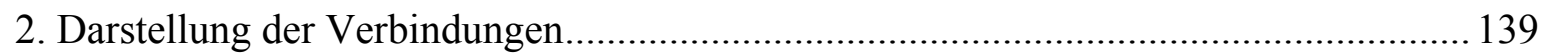

2.1. Synthese und Reinigung der verwendeten Reagenzien ...................................... 139

2.2. Carbokationen-Präparation und Tieftemperatur-NMR-Messungen ...................... 140

2.3. Darstellung der Verbindungen aus Teil B Kapitel 3 ........................................... 143

2.4. Darstellung der Verbindungen aus Teil B Kapitel 4.......................................... 151

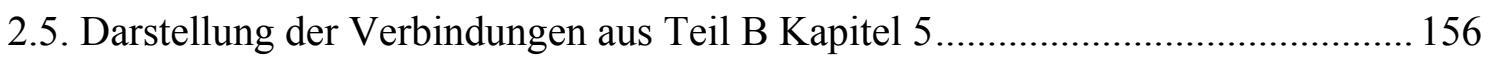

2.6. Darstellung der Verbindungen aus Teil B Kapitel 6........................................... 168

2.7. Darstellung der Verbindungen aus Teil B Kapitel 7 ............................................ 176

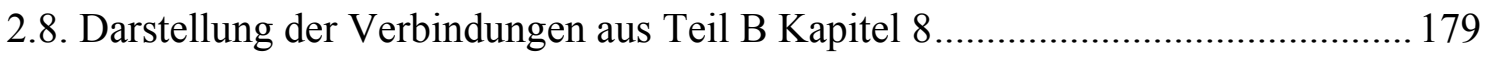




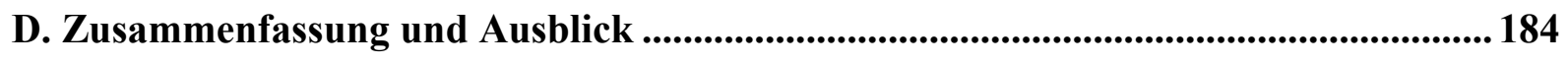

E. Literatur und Anmerkungen ...................................................................................................... 190

F. Spektrenanhang ….................................................................................................................. 205

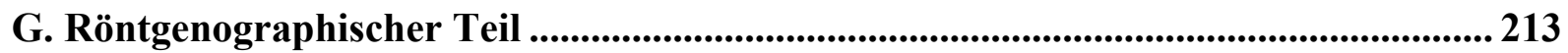




\section{A. Einleitung}

Die Chemie der Carbokationen kann heute - Dank der Wissenschaftler, die sie vorantrieben - auf eine Geschichte von über einem Jahrhundert zurückblicken. Eine der originellsten und kühnsten Ideen in der Organischen Chemie war seinerzeit die Annahme, dass positiv geladene Kohlenstoffverbindungen als Intermediate bei der Reaktion von neutralen Ausgangsmaterialien zu kovalent gebundenen Produkten auftreten. ${ }^{[1]}$ Obwohl die Beziehung zwischen dem kationischen Charakter von Triarylmethyl-Salzen und deren intensiver Farbe schon seit 1901 durch die Pionierarbeiten von NORRIS, KEHRMANN und VON BAEYER (Nobelpreis 1905) bekannt war, $^{[2]}$ wurde die generelle Bedeutung von Carbokationen längere Zeit nicht erkannt. Erst 1922 interpretierte MEERWEIN die WAGNERUmlagerung von Camphenhydrochlorid zum Isobornylchlorid mit dem Auftreten von ionischen Zwischenstufen, wodurch das moderne Konzept der Carbokationen geboren war. ${ }^{[3]}$ In den folgenden Jahrzehnten wurden die von Hughes, INGOLD und WiTMORE vorgeschlagenen Mechanismen der nucleophilen Substitution und der Eliminierung maßgebend weiterentwickelt und etabliert. ${ }^{[4]}$ Ihnen dienten kinetische und stereochemische Kriterien zum Nachweis der Beteiligung von Carbokationen an chemischen Reaktionen. Trotzdem wurden Carbokationen weiterhin als prinzipiell instabile und kurzlebige Spezies angesehen. Einen neuen Aufschwung erhielt die Carbokationen-Chemie in den 1960er Jahren durch die fundamentalen Arbeiten von OLAH (Nobelpreis 1994), der Carbokationen in wenig nucleophilen, supersauren Medien persistent machte und mit Hilfe von spektroskopischen Verfahren direkt nachwies. ${ }^{[5,6]}$ Er führte auch die Einteilung der Carbokationen in trivalente, „klassische“ Carbeniumionen und hypervalente, „nichtklassische“ Carboniumionen ein. Vor kurzem aber befand OLAH selbst diese Klassifizierung als nicht mehr zeitgemäß, da die Begriffe „klassisch“ und „nichtklassisch“ nur die Grenzen eines Spektrums definieren, in die sich alle Carbokationen einordnen. ${ }^{[7]}$

Es ist sicherlich kein Zufall, dass sowohl das erste Carbeniumion als auch das kurz zuvor entdeckte erste stabile Kohlenstoff-Radikal (GOMBERG-Radikal) beides Derivate des 
Triphenylmethans waren. ${ }^{[8]}$ Die bemerkenswerte Stabilität dieser Spezies konnte rasch auf das Vorhandensein der Phenyl-Substituenten in Nachbarschaft zum Elektronenmangel-Zentrum zurückgeführt werden. Alsbald wurden auch Effekte von Alkyl- und HeteroatomSubstituenten intensiv studiert. Unter den Alkyl-Substituenten fiel - allerdings erst sehr viel später - speziell die Cyclopropylgruppe auf, der aufgrund ihrer ausgeprägten Fähigkeit zur Wechselwirkung mit benachbarten p-Elektronenzentren, besondere Beachtung zuteil wurde. ${ }^{[9]}$

Die Gründe für ihre besonderen Eigenschaften sind in den außergewöhnlichen Bindungsverhältnissen des Cyclopropanringes zu finden. ${ }^{[10]}$ Die Bindungen im Cyclopropanring stehen der C,C-Doppelbindung eines Olefins näher als den C,C-Einfachbindungen eines Cyclobutanrings. Im gleichseitigen Dreieck, das die geometrische Grundstruktur des Cyclopropanringes darstellt, beträgt der Winkel $60^{\circ}$. Gerade diese extreme Abweichung vom tetraedrischen Bindungswinkel zwingt die Kohlenstoffatome, einen einzigartigen Bindungstyp auszubilden. Es existieren zwei äquivalente Bindungs-Modelle zur Beschreibung der ungewöhnlichen Eigenschaften und Reaktivitäten von Cyclopropanderivaten.

Aus der Sichtweise des WALSH-Modells ist jedes Kohlenstoffatom sp $^{2}$-hybridisiert. ${ }^{[11]}$ Die C,C-Bindungen kommen demnach durch Überlappung je eines $\mathrm{sp}^{2}$-Hybridorbitals jedes Kohlenstoffatoms im Zentrum des Dreiringes ( $\sigma$-Anteil der Bindung) und durch die exocyclische Überlappung der atomaren p-Orbitale ( $\pi$-Anteil der Bindung) zustande. Das aus der Linearkombination der drei $\mathrm{sp}^{2}$-Orbitale resultierende Molekülorbital (MO) wird von zwei Elektronen besetzt, die aus zwei Linearkombinationen von atomaren p-Orbitalen gebildeten zwei entarteten MOs beherbergen vier Elektronen. Jedes der beiden verbleibenden $\mathrm{sp}^{2}$-Hybridorbitale an den Kohlenstoffatomen ist dann für die Ausbildung der C,H-Bindungen verantwortlich (Schema 1). Neuere Ergebnisse besagen jedoch, dass die WALSH-Orbitale die Zustände im Cyclopropan nur unpräzise beschreiben. ${ }^{[12]}$ 

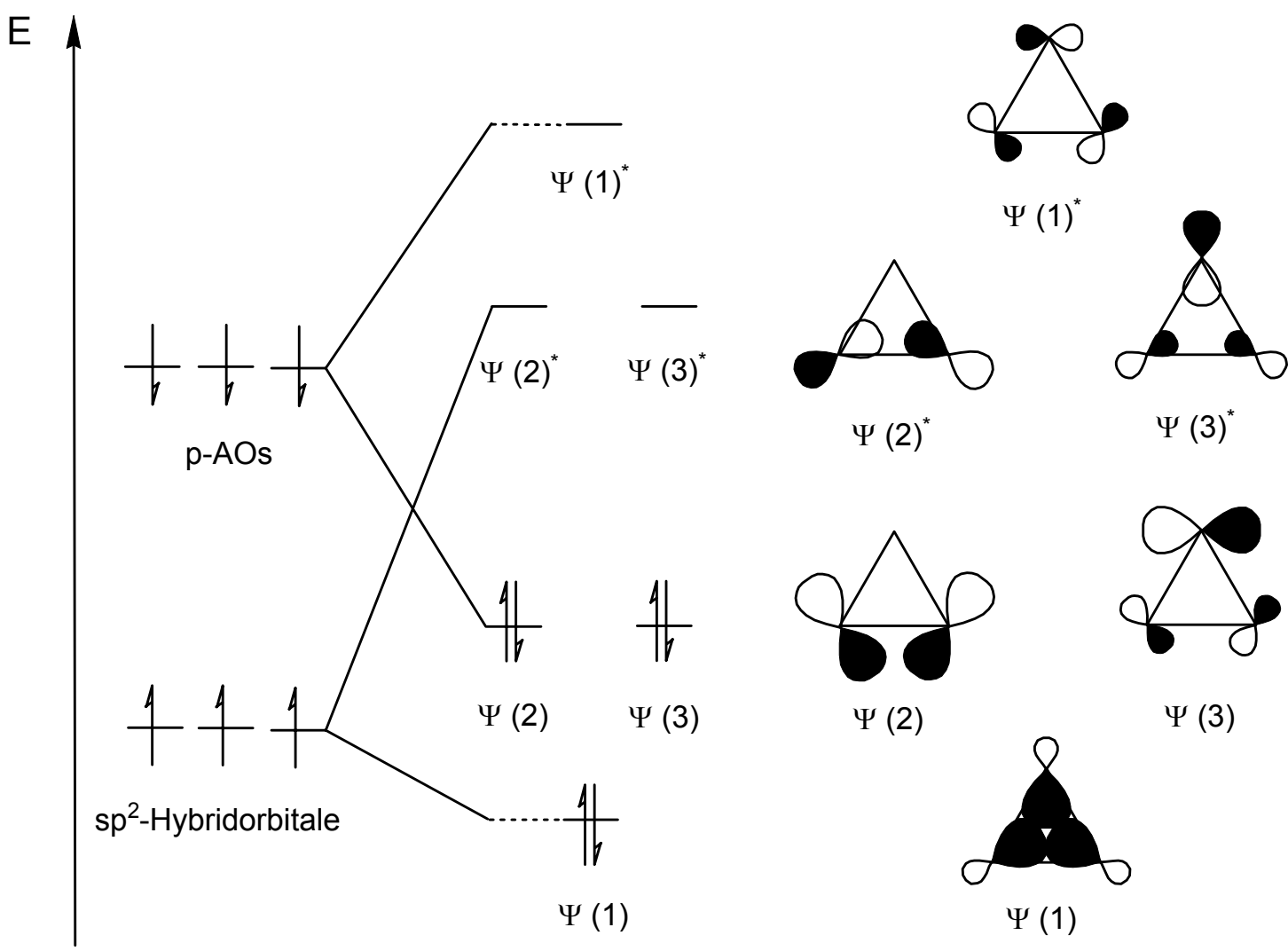

$\Psi(1)$

Schema 1. Molekülorbital-Schema des Cyclopropans nach WALSH.

Ein detaillierteres Modell wurde von FöRSTER, COULSON und MOFFIT auf Basis des Valenz-Bindungs-Modells erstellt. ${ }^{[13]}$ Sie erklären den Aufbau der carbocyclischen Bindungen durch Überlappung von $\mathrm{sp}^{5}$-Hybridorbitalen jedes Kohlenstoffatoms, die ebenfalls nicht entlang der Kern-Kern-Verbindungsachse, sondern nach außen hin orientiert sind („,bent bonds" ${ }^{\text {(14] }}{ }^{[1]}$ Der Winkel, der die Abweichung der Orbitalrichtung von der direkten Verbindungslinie angibt, wurde zu $20.5^{\circ}$ berechnet. Wie im WALSH-Modell werden auch hier die C,H-Bindungen durch $\mathrm{sp}^{2}$-Hybridorbitale an den Kohlenstoffatomen gebildet (Abbildung 1).

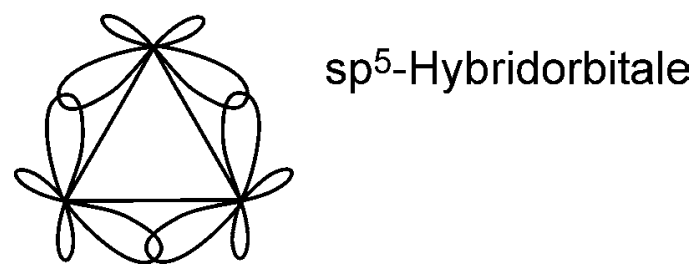

Abbildung 1. Bindungsmodell des Cyclopropans nach FÖRSTER, COULSON und MOFFIT. 
Die Fähigkeit von Cyclopropylgruppen, mit benachbarten $\pi$ - und p-Elektronenzentren in Konjugation zu treten, soll hier anhand von Konformationsanalysen des Vinylcyclopropans und des Cyclopropylmethyl-Kations hervorgehoben werden. So ist die Wechselwirkung zwischen Cyclopropanring und der olefinischen Doppelbindung im Vinylcyclopropan dann maximal, wenn die p-Orbitalachsen der Doppelbindung parallel zur Dreiringebene angeordnet sind. In der antiperiplanaren Konformation mit einem Diederwinkel von $180^{\circ}$ ist Vinylcyclopropan um ca. $4 \mathrm{~kJ} / \mathrm{mol}$ stabiler als das entsprechende synclinale Konformer mit $\varphi=65^{\circ}$ (Abbildung 2). ${ }^{[10]}$
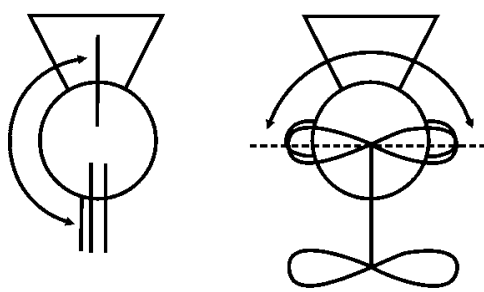

$\varphi=180^{\circ}$

antiperiplanar (s-trans)
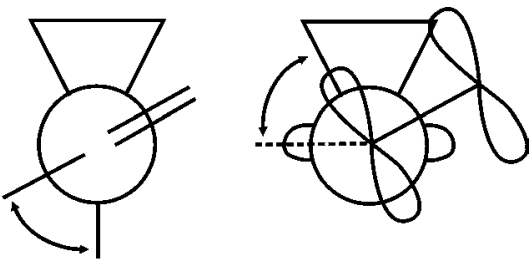

$\varphi=65^{\circ}$

synclinal (gauche)

Abbildung 2. Konformationsanalyse des Vinylcyclopropans.

Wesentlich größer dagegen ist die Energiedifferenz zwischen günstigerer bisektierter und instabilerer senkrechter Konformation beim Cyclopropylmethyl-Kation mit ca. 40-60 kJ/mol (Abbildung 3). ${ }^{[15,16]}$ Größenordnungsmäßig entspricht dies der Stabilisierungsenergie eines Allyl- oder Benzyl-Kations gegenüber einem nicht zur Konjugation fähigen primären Carbeniumion. ${ }^{[17]}$
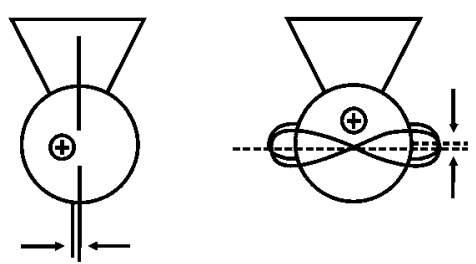

$\varphi=0^{\circ}$

bisektiert (max. Wechselwirkung)
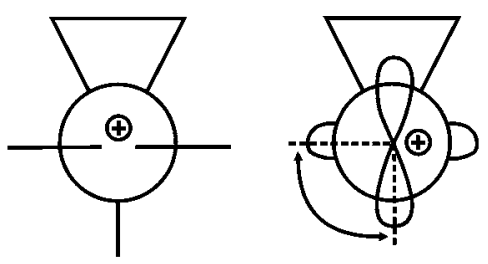

$\varphi=90^{\circ}$ senkrecht (min. Wechselwirkung)

Abbildung 3. Zur Konformation des Cyclopropylmethyl-Kations. 
Die Stabilisierung durch $\beta$-C,C-Hyperkonjugation läßt sich durch Wechselwirkung des besetzten antisymmetrischen WALSH-Orbitals $\psi(3)$ mit dem formal vakanten $p_{z}$-Orbital an $\mathrm{C}(\alpha)^{+}$beschreiben. Es resultiert ein bindendes MO vom $\pi$-Typ $(\psi(3)+\lambda p)$, welches über das Molekül delokalisiert ist (Schema 2). $\Psi(2)$ wird nicht mit dem p-Orbital linearkombiniert und bildet ein Orbital vom $\sigma$-Typ. Die Folge dieser Wechselwirkung ist die Verlagerung von Elektronendichte aus dem Cyclopropanring hin $\mathrm{zu} \mathrm{C}(\alpha)^{+}$. Dadurch wird die bindende Wechselwirkung der Orbitale der proximalen $\mathrm{C}_{\beta}, \mathrm{C}_{\gamma}$-Bindungen geschwächt - die Bindungen werden verlängert. Gleichzeitig wird die antibindende Wechselwirkung der $\mathrm{C}_{\gamma}, \mathrm{C}_{\gamma}$-Bindungsorbitale geschwächt, was zu einer Stärkung der distalen Bindung und damit zu einer Verkürzung der $\mathrm{C}_{\gamma}, \mathrm{C}_{\gamma}$-Bindungslänge führt.

$\mathrm{E}$

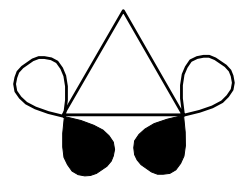

$\Psi(2)$

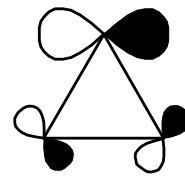

$\Psi(3)$

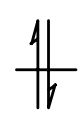

$\Psi(2)$ $p-\lambda \Psi(3)$

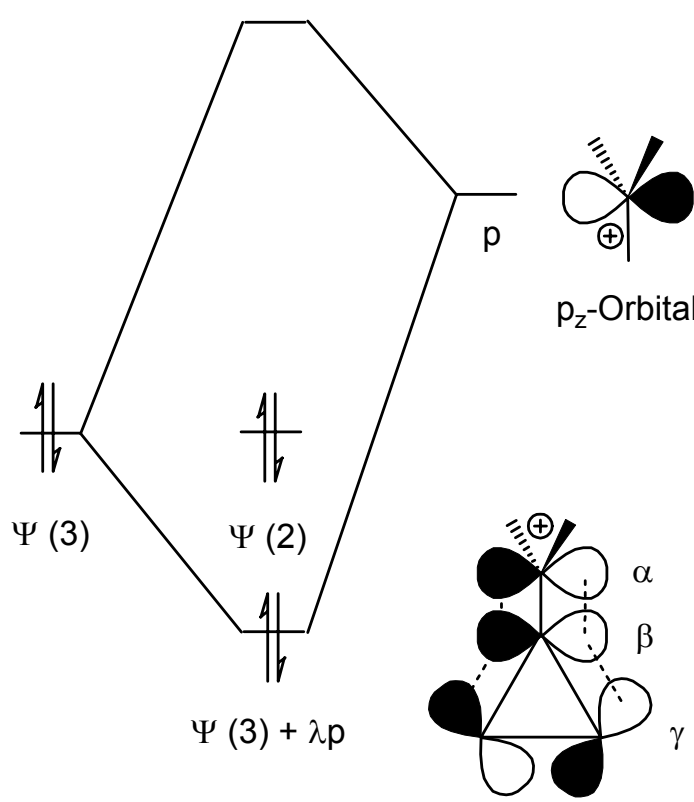

Schema 2. MO-Schema der $\beta$-C,C-Hyperkonjugation in Cyclopropylmethyl-Kationen. ${ }^{[18]}$

Das unsubstituierte Cyclopropenyl-Kation (1) ist der kleinste aromatische Carbocyclus, der die HÜCKEL-Regel befolgt. Auch mit dessen $\pi$-Elektronensystem kann der CyclopropylSubstituent vorteilhaft wechselwirken. Dadurch ist das Tricyclopropylcyclopropenyl-Kation (4) unter den rein Alkyl-substituierten Vertretern $\mathbf{2}$ bis $\mathbf{5}$ das stabilste (Abbildung 4). 


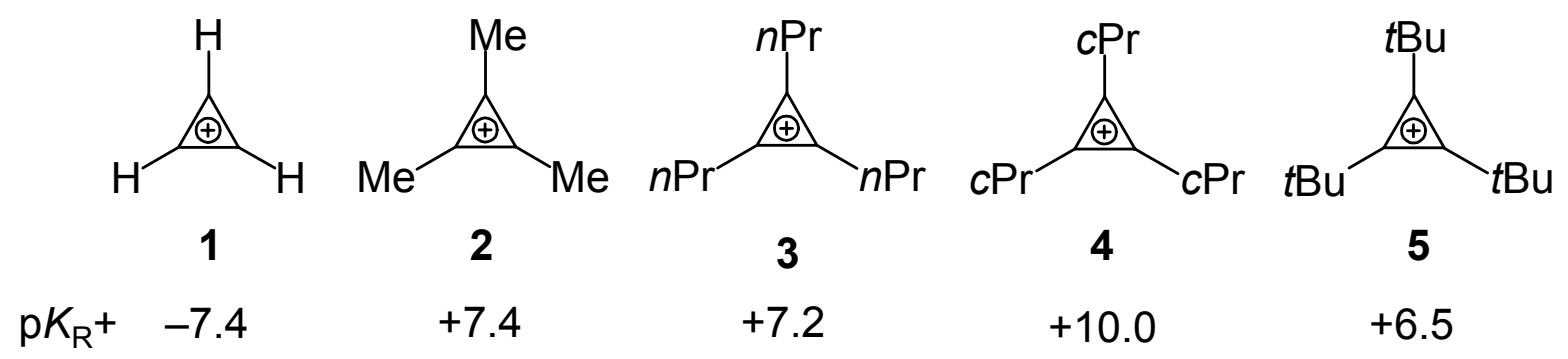

Abbildung 4. $\mathrm{p} K_{\mathrm{R}}{ }^{+}$-Werte ausgewählter Alkyl-substituierter Cyclopropenyl-Kationen. ${ }^{[19]}$

Die relative Stabilität eines Carbokations wird am besten durch seinen $\mathrm{p} K_{\mathrm{R}}{ }^{+}$-Wert wiedergegeben. In verdünnter wässriger Lösung $\left(H_{\mathrm{R}}\right.$ ist die Aciditätsfunktion des Reaktionsmediums; in Wasser: $\left.\mathrm{p} H \approx H_{\mathrm{R}}\right)$ ist der $\mathrm{p} K_{\mathrm{R}}{ }^{+}$-Wert gleichbedeutend mit dem $\mathrm{pH}$-Wert, bei dem das Carbokation und der korrespondierende Alkohol in gleicher Konzentration vorliegen (Schema 3). ${ }^{[6 \mathrm{~d}, 20]}$ Positive Werte zeigen dabei ein durch elektronenliefernde Substituenten stabilisiertes Kation an und umgekehrt.

$$
\begin{gathered}
\mathrm{R}-\mathrm{OH}+\mathrm{H}^{+} \rightleftharpoons \mathrm{R}^{+}+\mathrm{H}_{2} \mathrm{O} \\
\mathrm{p} K_{\mathrm{R}^{+}}=\lg \frac{\left[\mathrm{R}^{+}\right]}{[\mathrm{R}-\mathrm{OH}]}+H_{\mathrm{R}}
\end{gathered}
$$

Schema 3. Ableitung und Bedeutung des $\mathrm{p} K_{\mathrm{R}}{ }^{+}$-Werts für Carbokationen.

Die Bezeichnung Aromatizität ist Teileines ein Konzepts, für das die meisten Chemiker ein vermeintlich intuitives Verständnis besitzen, das aber vergleichsweise schwierig zu definieren ist. ${ }^{[21]}$ Ungleich diffiziler ist folglich eine adäquate Beschreibung von Antiaromatizität. $^{[22]}$ Chemische Reaktivität war ein frühes Kriterium für Aromatizität und es war eines der ersten, das auf die Untersuchung der Antiaromatizität angewandt wurde. Auffällig war bespielsweise die Schwierigkeit, antiaromatische Spezies - wie das prototypische Cyclopentadienyl-Kation (6a) - aus entsprechenden Edukten frei $\mathrm{zu}$ setzen. Diese Beobachtung wies auf deren extreme Instabilität hin. So konnten BRESLOW et al. durch Reaktion von Iodcyclopentadien (7) mit Silberperchlorat in Propionsäure bei $-15{ }^{\circ} \mathrm{C}$ nicht das antiaromatische Cyclopentadienyl-Kation erhalten, sondern identifizierten stattdessen ein 
DiELS-AlDER-Dimer von 7. ${ }^{[23]}$ Erst durch den Einsatz von Antimonpentafluorid als starke LEWIS-Säure gelang BRESLOW, SAUNDERS und WASSERMAN die Erzeugung von 6a aus Bromcyclopentadien (8) in einer Di- $n$-butylphthalat-Matrix (Schema 4). ${ }^{[24]}$

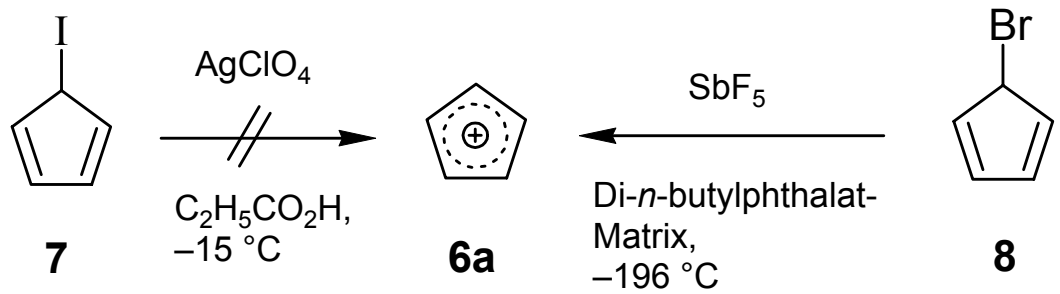

Schema 4. Versuche zur Erzeugung des unsubstituierten Cyclopentadienyl-Kations (6).

Im Hinblick auf die Aromatizität von Carbocyclen ist die HÜCKEL-Regel - zusammen mit der DEWAR-Resonanzenergie - als heuristisches Prinzip nach wie vor unübertroffen. ${ }^{[25]}$ Als Bezugssystem für die Bestimmung der Resonanzenergie nach DEWAR dienen die offenkettigen Polyene mit gleicher Zahl der $\pi$-Bindungen. Die Einführung des Konzepts der Antiaromatizität geht auf BRESLOW zurück, der konjugierte, carbocyclische Verbindungen mit (4n)- $\pi$-Elektronen und einer negativen DEWAR-Resonanzenergie (also eine Destabilisierung aufweisen) als Antiaromat bezeichnete. ${ }^{[26]}$ Er schlug erstmalig auch magnetische, spektroskopische und thermodynamische Kriterien vor, die in einer gegensätzlichen Beziehung zu denen für aromatische Verbindungen stehen. ${ }^{[27]}$ So werden NMR-Signale von Protonen an Antiaromaten, nicht wie solche an Aromaten ins Tieffeld, sondern zu höherem Feld verschoben. Weiterhin ist der HOMO-LUMO-Abstand bei aromatischen größer als bei antiaromatischen Verbindungen. Während Bindungslängen-Ausgleich charakteristisch für aromatische Systeme ist, weist Bindungslängen-Alternanz eher auf das Vorliegen von Antiaromatizität hin. Eine zusätzliche Charakteristik von antiaromatischen Systemen ist das Auftreten einer Strukturisomerie. Dies wird durch einen JAHN-TELLER-Effekt hervorgerufen, der eine geometrische Verzerrung verursacht, um ungleiche Besetzung von degenerierten Orbitalen zu verhindern. ${ }^{[28]}$ Diese verzerrten Spezies besitzen eine niedrigere Symmetrie als die voll konjugierten symmetrischen Strukturen, und etwaige Energieunterschiede können gering ausfallen. 


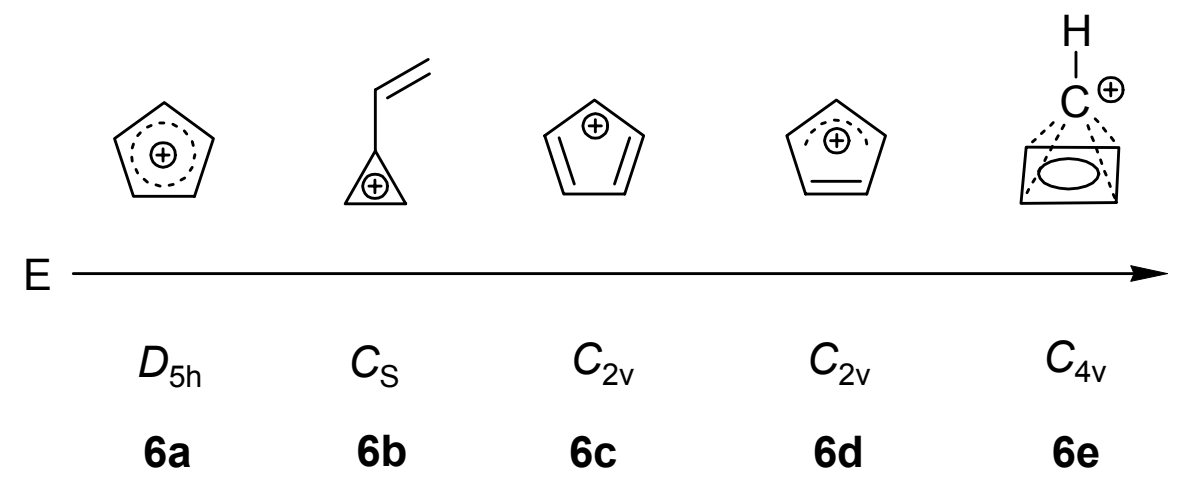

Abbildung 5. Strukturisomere des unsubstituierten Cyclopentadienyl-Kations (6).

Das ESR-Spektrum des unsubstituierten Cyclopentadienyl-Kations (6) wies dieses als eine Triplett-Spezies im Grundzustand aus. ${ }^{[24]}$ Ab initio Rechnungen auf hohem Niveau bestätigten die um $8 \mathrm{kcal} / \mathrm{mol}$ (MP4-Niveau) niedrigere Energie des $D_{5 \mathrm{~h}}$-Tripletts $6 \mathbf{a}$ relativ zu den $C_{2 \mathrm{v}}$-Singulett-Spezies $\mathbf{6 c}$ und $\mathbf{6 d}$, weswegen man auch vermutet hatte, dass $\mathbf{6 a}$ einer Pseudorotation mit 6c und 6d als Zwischenstufen unterliegt.[29] WiLliams, STOHRER und $\operatorname{HOFFMAN}^{[30]}$ formulierten eine dreidimensionale aromatische, quadratisch-pyramidale Struktur 6e mit $C_{4 \mathrm{v}}$-Symmetrie, die aber energetisch um $12 \mathrm{kcal} / \mathrm{mol}$ (MP4-Niveau) höher als 6a anzusiedeln ist. Das Vinylcyclopropenyl-Kation $\mathbf{6 b}$ - bislang als das stabilste in der Familie der $\mathrm{C}_{5} \mathrm{R}_{5}{ }^{+}$-Kationen angesehen - ist neueren Rechnungen zufolge mit 6a nahezu isoenergetisch (Abbildung 5). ${ }^{[29]}$

Eine Abschätzung der relativen Stabilität (oder besser Destabilisierung) des Cyclopentadienyl-Kations (6a) gelang auf elektrochemischem Wege, wobei ein $\mathrm{p} K_{\mathrm{R}}{ }^{+}$-Wert für 6a von -40 durch Messung der Halbstufen-Potentiale des $\mathrm{C}_{5} \mathrm{H}_{5}$-Anions und $\mathrm{C}_{5} \mathrm{H}_{5}$-Radikals berechnet werden konnte. ${ }^{[31]}$ Dieser Wert liegt um 20 Einheiten unter dem Wert von repräsentativen konjugierten Kationen, z. B. dem Allyl-Kation: $\mathrm{p}{K_{\mathrm{R}}}^{+} \approx-20$.

Nach den ersten Berichten von BRESLOW über das Cyclopentadienyl-Kation (6a) Ende der 60er Jahre sind eine Reihe von Aryl- und Heteroatom-substituierten CyclopentadienylKationen mit Hilfe von Matrixisolations-Techniken dargestellt und mit spektrokopischen Methoden charakterisiert worden. ${ }^{[23,24,31,32]}$ Allerdings war unter diesen lange Zeit kein Alkyl- 
substituiertes Cyclopentadienyl-Kation - bis SITZMANN 1993 von dem ungewöhnlich stabilen Pentaisopropylcyclopentadienyl-Kation (9) berichtete. ${ }^{[33]}$ Versuche von LAMBERT et al. ein Pentamethyl-substituiertes Cyclopentadienyl-Kation $\mathbf{1 0}$ zu erzeugen, resultierten in der Isolierung des wohlbekannten Pentamethylcyclopentenyl-Kations. ${ }^{[34]}$ Hinweise auf ein Bis(homoadamantyl)-anneliertes Cyclopentadienyl-Kations des Typs 11 konnten KoMATSU et al. bisher nur unter solvolytischen Bedingungen gewinnen. ${ }^{[35]}$ Allerdings war das Intermediat derart instabil, dass es Umlagerungen des Homoadamantyl-Gerüsts unterlag (Abbildung 6).<smiles></smiles>

9<smiles></smiles>

10

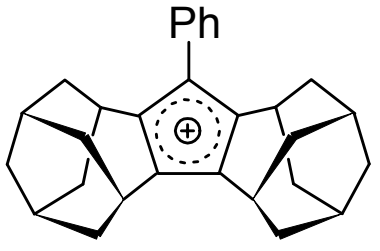

11

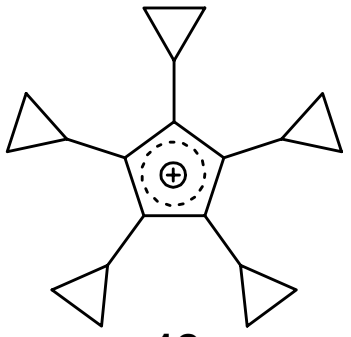

12

Abbildung 6. Untersuchte Alkyl-substituierte Cyclopentadienyl-Kationen 9 bis 11 und das angestrebte Pentacyclopropylcyclopentadienyl-Kation 12.

Trotz vielfältiger präparativer Untersuchungen und umfangreicher theoretischer Analysen konnte der Ursprung der Antiaromatizität in cyclischen konjugierten (4n)- $\pi$-Systemen bisher nicht vollständig aufgedeckt werden. Die Synthese eines Pentacyclopropyl-substituierten Cyclopentadienyl-Kations (12) ist daher ein lohnenswertes Ziel - zumal die unter den Alkylsubstituenten einzigartigen Fähigkeiten der Cyclopropylgruppe für eine Stabilisierung des antiaromatischen $4 \pi$-Elektronensystems sorgen könnten. Das Pentacyclopropylcyclopentadienyl-Kation (12) könnte so als weiterer Mosaikbaustein dienen, um das Bild der Antiaromatizität zu vervollständigen (Abbildung 6).

Das Grundgerüst des Pentacyclopropylcyclopentadienyl-Kations (12) konnte jüngst durch eine einstufige Synthese aus dem Dicyclopropylethin (13) in Form des percyclo- 
propylierten Cyclopentadiens $\mathbf{1 5}$ in recht guter Ausbeute zugänglich gemacht werden (Schema 5). ${ }^{[36]}$

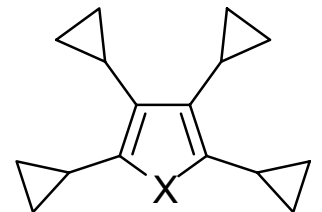

$18(X=N R)$

$19(X=O)$

$20(X=S)$

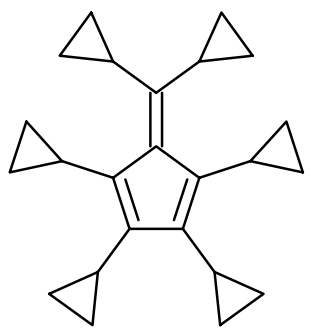

24

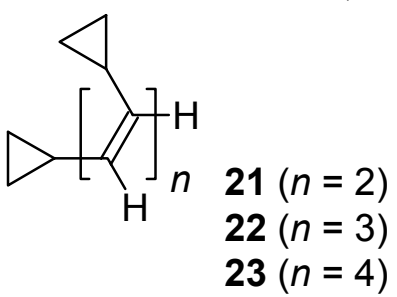

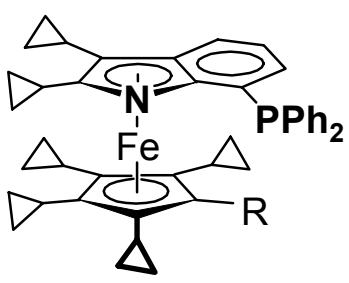

$16 \mathbf{a}(\mathrm{R}=\mathrm{H})$

$16 \mathrm{~b}(\mathrm{R}=c \mathrm{Pr})$

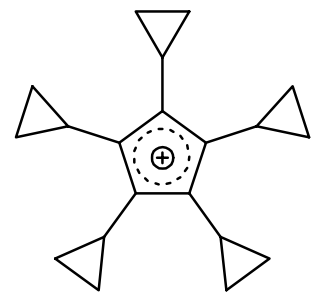

12

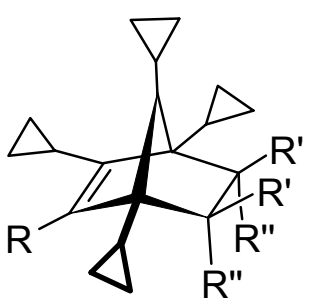

17

Schema 5. Produktpalette an cyclopropylierten Oligoenen, Carbo- und Heterocyclen ausgehend von Dicyclopropylethin (13).

Sowohl das Penta- als auch das Tetracyclopropylcyclopentadien 15 bzw. 14 wurden kürzlich zur Synthese von Oligocyclopropyl-substituierten Metallocen-Komplexen des Eisens und des Titans eingesetzt. ${ }^{[36,37]}$ An diesen Erfolg anknüpfend, sollte im Rahmen dieser Arbeit auch der Aufbau von planar-chiralen N,P-Liganden ${ }^{[38]}$ wie 16 aus den Cyclopentadienen 14 und 15 untersucht werden. In ihrer Eigenschaft als cyclisches Dien könnten diese Cyclopentadiene durch DIELS-ALDER-Reaktionen mit diversen Dienophilen auch als Edukte für cyclopropylierte Bicyclo[2.2.1]heptene des Typs 17 dienen. Außerdem sollten die Möglichkeiten der Darstellung von percyclopropylierten fünfgliedrigen Heterocyclen wie den Pyrrolen des Typs 18, dem Furan 19 oder dem Thiophen 20 $^{[39]}$ aus Dicyclopropylethin (13) 
beleuchtet werden. Letzteres könnte auch als monomerer Baustein für Cyclopropylsubstituierte, acyclische Oligoene wie 21, 22 oder 23 fungieren, die durch einen dirigierenden Effekt der Cyclopropylgruppen in eine helicale Konformation gezwungen werden könnten. Prinzipiell könnte das Acetylen 13 durch eine suksessive Carbopalladierung zweier seiner Moleküle in Tetracyclopropyl-substituierte Fulvene wie $\mathbf{2 4}$ transformiert werden. In Anbetracht der weit gefächerten Produktpalette (Schema 5), ${ }^{[40]}$ die durch die Transformation des Dicyclopropylethins (13) zugänglich wäre, stellt die Auslotung dieser Möglichkeiten neben der Erzeugung des Pentacyclopropylcyclopentadienyl-Kations 12, ein weiteres lohnendes Ziel dieser Arbeit dar. 


\section{B. Hauptteil}

\section{Bisherige Untersuchungen von Cyclopentadienyl-Kationen}

Das unsubstituierte Cyclopentadienyl-Kation, dessen cyclisch konjugiertes und planares $\mathrm{C}_{5}$-Kohlenstoffgerüst $4 \pi$-Elektronen an ausschließlich $\mathrm{sp}^{2}$-hybridisierten Kohlenstoffzentren beherbergt, repräsentiert - im Einklang mit den Kriterien von HüCKELs Regel ${ }^{[25]}$ - die prototypisch antiaromatische Cyclopentadienylspezies und wird daher als prinzipiell destabilisiertes Carbokation angesehen. Diese bindungstheoretischen Überlegungen fördern und fordern demnach begründet das Interesse, Cyclopentadienyl-Kationen so zu präparieren, dass sie mittels spektroskopischer Verfahren untersucht werden können.

Seit Beginn der sechziger Jahre sind sowohl die Stammverbindung 6a als auch die substituierten Cyclopentadienyl-Kationen, wie das Pentachlor- (25), die Pentaaryl- (27) oder das jüngst charakterisierte Pentaisopropylcyclopentadienyl-Kation (9) Gegenstand zahlreicher experimenteller Studien gewesen (Abbildung 7).<smiles>c1ccccc1</smiles>

$6 a$<smiles></smiles>

25<smiles></smiles>

26<smiles></smiles>

27<smiles></smiles>

9

$$
\begin{array}{ll}
\mathrm{R}^{\prime}=\mathrm{Ph}, \mathrm{CN} & \mathrm{Ar}=\mathrm{Ph}, p-\mathrm{C}_{6} \mathrm{H}_{4} \mathrm{X} \\
\mathrm{R}^{\prime \prime}=\mathrm{NMe}_{2}, \mathrm{H} & \mathrm{X}=\mathrm{Hal}, \mathrm{OMe}, \mathrm{OH}
\end{array}
$$

Abbildung 7. Bisher charakterisierte Cyclopentadienyl-Kationen.

Durch ESR-Spektroskopie in einer Antimonpentafluorid-Dibutylphthalat-Matrix bei $-196{ }^{\circ} \mathrm{C}$ konnten BRESLOW et al. den Triplett-Charakter der unsubstituierten Spezies 6a im Grundzustand nachweisen. ${ }^{[41]}$ Das Gleiche trifft für das Pentachlorcyclopentadienyl-Kation (25) zu, dessen Symmetrie $\left(D_{5 \mathrm{~h}}\right)$ zusätzlich von NovAK et al. durch IR-Spektroskopie an einer 
Tieftemperatur-SbF 5 -Matrix bestimmt werden konnte. ${ }^{[42]}$ Die von GOMPPER et al. als stabile, kristalline Materialien charakterisierten Heteroatom-substituierten Cyclopentadienyl-Kationen 26 mit Amino- und Cyano-Gruppen liegen in einem Singulett-Grundzustand vor (Abbildung 7). Sie müssten aber eher als die korrespondierenden Azafulvenderivate angesehen werden. ${ }^{[43]}$ Pentaarylcyclopentadienyl-Kationen als Lösungen in Dichlormethan oder Chloroform mit Bortrifluorid als LEWIS-Säure, besonders solche mit Donorsubstituierten Arylgruppen, wurden von BRESLOW, BROSER und KURRECK untersucht und sind bis $\mathrm{zu}-40{ }^{\circ} \mathrm{C}$ persistent. Sie liegen aufgrund einer geringen Übergangsenergie sowohl im Triplett- als auch im Singulett-Zustand nebeneinander vor. ${ }^{[44]}$ Das erste Alkyl-substituierte Pentaisopropylcyclopentadienyl-Kation (9), von SITZMANN et al. erforscht, zeigte sich in tiefgekühlten Dichlormethanlösungen bei $-90^{\circ} \mathrm{C}$ mit $\mathrm{SbF}_{5}$ als LEWIS-Säure stabil und wies einen Triplett-Grundzustand auf. ${ }^{[45]}$

Erst kürzlich versuchten LAMBERT et al. durch Hydridabstraktion aus einfachem Pentamethylcyclopentadien $\mathbf{2 8}$ mit einem Tritylsalz das Pentamethylcyclopentadienyl-Kation (10) darzustellen (Schema 6) und formulierten einen Singulett-Grundzustand mit zwei olefinischen, aber durch einen JAHN-TELLER-Effekt pyramidalisierten Kohlenstoffzentren. ${ }^{[46]}$<smiles></smiles>

10

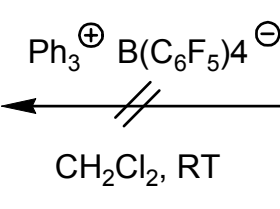

$\mathrm{CH}_{2} \mathrm{Cl}_{2}, \mathrm{RT}$

Schema 6. Versuchte Erzeugung des Pentamethylcyclopentadienyl-Kations (10).

Nach gründlicher Durchsicht der röntgenographischen und NMR-spektroskopischen Daten stellte sich dies allerdings als das wohlbekannte Pentamethylcyclopentenyl-Kation (29) heraus, das hier aufgrund des verwendeten extrem wenig nucleophilen bzw. LEWIS-basischen Tetrakis(pentafluorphenyl)boratanions als Gegenion in Form eines kristallinen, luftstabilen und langlebigen Carbokations erhalten wurde. 
Die potentielle $\sigma$-Stabilisierung eines Cyclopentadienyl-Kations durch Methylgruppen bleibt demnach fraglich, obwohl mit störungstheoretischen Rechnungen auf hohem Niveau eine um $63 \mathrm{kcal} / \mathrm{mol}$ geringere Bildungswärme für das Pentamethylcyclopentadienyl-Kation im Triplettzustand im Vergleich zum experimentellen Wert für $\mathrm{C}_{5} \mathrm{H}_{5}{ }^{+}$vorausgesagt wurde. ${ }^{[47]}$ JUTZI et al. lieferten einen chemischen Hinweis auf das Pentamethylcyclopentadienyl-Kation als reaktive Zwischenstufe, indem sie es in Solvolyse-Experimenten mit verschiedensten Nucleophilen abfingen. ${ }^{[48]}$

\section{Elektronische Struktur, Geometrie und quantenmechanische Berechnungen von Cyclopropyl-substituierten Cyclopentadienyl-Kationen}

Wie eingangs erläutert genießt die Cyclopropylgruppe mit ihren elektronischen und sterischen Besonderheiten unter den Alkyl-Substituenten eine herausgehobene Stellung. Durch ihre einzigartigen Donoreigenschaften ist sie sehr gut zur Stabilisierung eines benachbarten Elektronenmangelzentrums befähigt. ${ }^{[10]}$

Eine planare Fünfringstruktur mit $D_{5 \mathrm{~h}}$-Symmetrie vorausgesetzt, sollten alle Cyclopropylgruppen des Pentacyclopropylcyclopentadienyl-Kations (12) eine bisektierte Konformation einnehmen, um eine maximale Stabilisierung hervorzurufen.

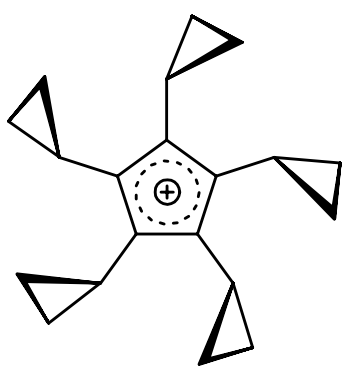

12

Abbildung 8. All-bisektiertes Pentacyclopropylcyclopentadienyl-Kation (12).

Entgegen dieser Annahme ergaben erste semiempirische Rechnungen auf AM1-Niveau eine Struktur, bei der zwei Cyclopropylreste eine optimale bisektierte, eine eine senkrechte 
und die beiden anderen eine gauche-Anordnung einnehmen (Abbildung 9). Dieses Konformer mit $C_{\mathrm{S}}$-Symmetrie enthielte demnach zwei Substituenten mit stark, zwei weitere mit schwach stabilisierender sowie einem mit destabilisierender Wirkung auf das Cyclopentadienyl-Kation 12.

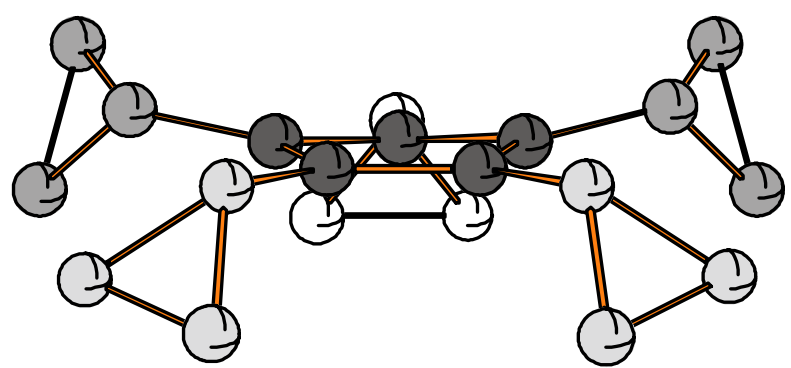

Abbildung 9. AM1-Struktur des Pentacyclopropylcyclopentadienyl-Kations (12).

Eine eingehendere Betrachtung der Molekülorbitale (MO) kann diesen Sachverhalt erklären. Die relative energetische Abfolge der MOs des Cyclopentadienyl-Kations im Grundzustand kann in erster Näherung in der Form eines FrOST-MusULIN-Diagramms behandelt werden (Schema 7). ${ }^{[49]}$

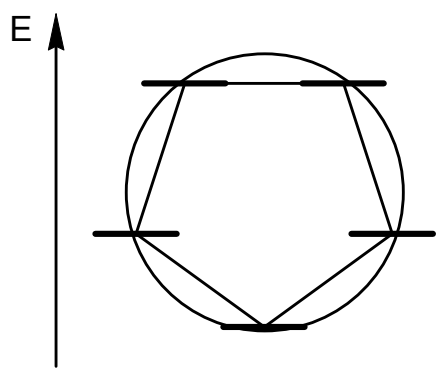

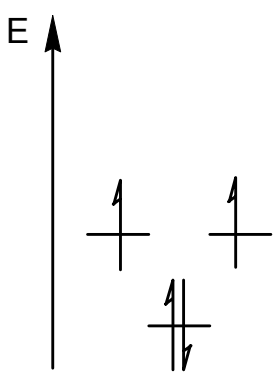

Triplett Grundzustand

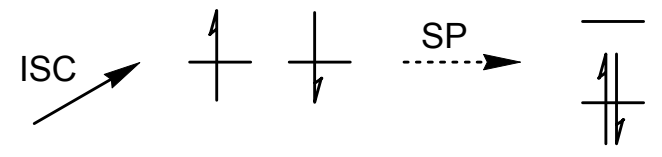

Singulett (open shell)
Singulett (closed shell)

Schema 7. Frost-MUSULIN-Diagramm und Energiediagramm für CyclopentadienylKationen.

Der Triplett-Grundzustand ist durch volle Besetzung des energieärmsten Orbitals durch ein Elektronenpaar und jeweilige Halbbesetzung der energetisch nächst höher liegenden, entarteten Orbitale mit einem Elektron charakterisiert. Durch Energiezufuhr kann dieser 
Triplettzustand ein intersystem crossing (ISC) vollziehen und so in einen open shell-SingulettZustand mit entgegengesetztem Spin der beiden Elektronen transformiert werden. Eine sich anschließende Spinpaarung (SP), die gleichzeitig die Entartung der Orbitale aufhebt, führt zu einem energetisch niedriger liegenden, und daher günstigeren closed shell-Singulett-Zustand (Schema 7).

Die Fähigkeit des Cyclopropyl-Substituenten, als Elektronendonor zu fungieren, beruht auf der Wechselwirkung seines höchst besetzten Molekülorbitals $\left(\mathrm{HOMO}^{[50]}\right)$ mit dem kationischen Cyclopentadienyl-Gerüst (Schema 8). Liegt dieses im Triplett-Grundzustand vor, erfolgt die Wechselwirkung mit einem der entarteten einfach besetzten Molekülorbitale $\left(\mathrm{SOMO}^{[51]}\right)$, wobei ein Elektron auf ein energetisch höheres, antibindendes Niveau angehoben würde. Hier könnte nur der Beitrag von einem der beiden Elektronen im bindenden MO zum Gesamtenergiegewinn beitragen. Im Singulett-Grundzustand erfolgt die Wechselwirkung mit dem energetisch niedrigsten unbesetzten Molekülorbital $\left(\mathrm{LUMO}^{[52]}\right)$ des CyclopentadienylKations. In diesem Falle kann eine positive Energiebilanz leicht realisiert werden, wenn die Besetzung des bindenden MO durch die Elektronen aus dem Cyclopropan-HOMO mehr Energie freisetzt als das intersystem crossing zum Cyclopentadienyl-LUMO kostete.
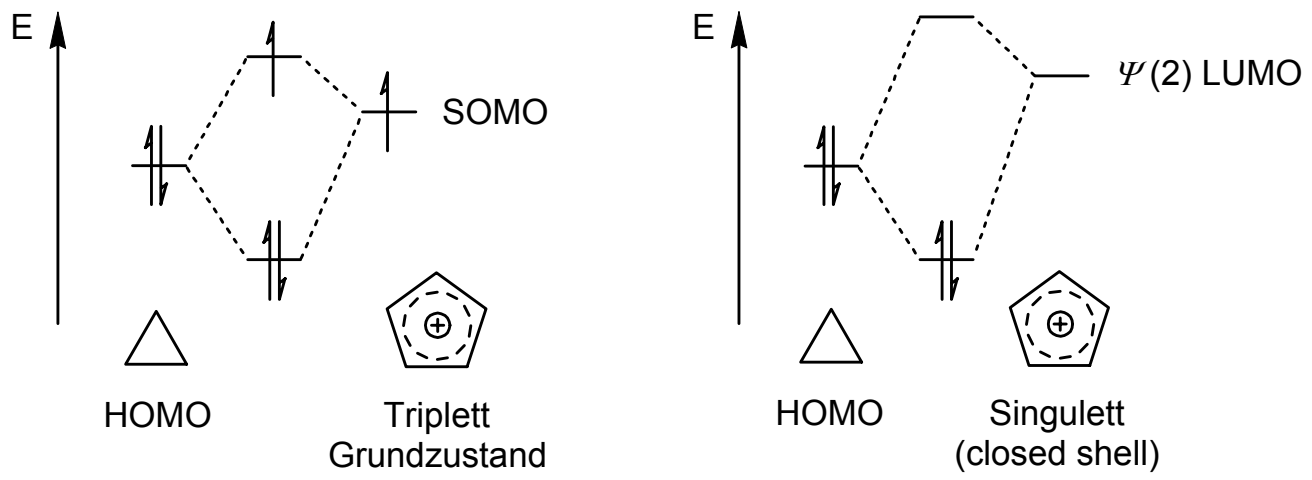

Schema 8. MO-Schema der Wechselwirkungen des $c$ Pr-Substituenten mit dem kationischen Cyclopentadienyl-Gerüst. 

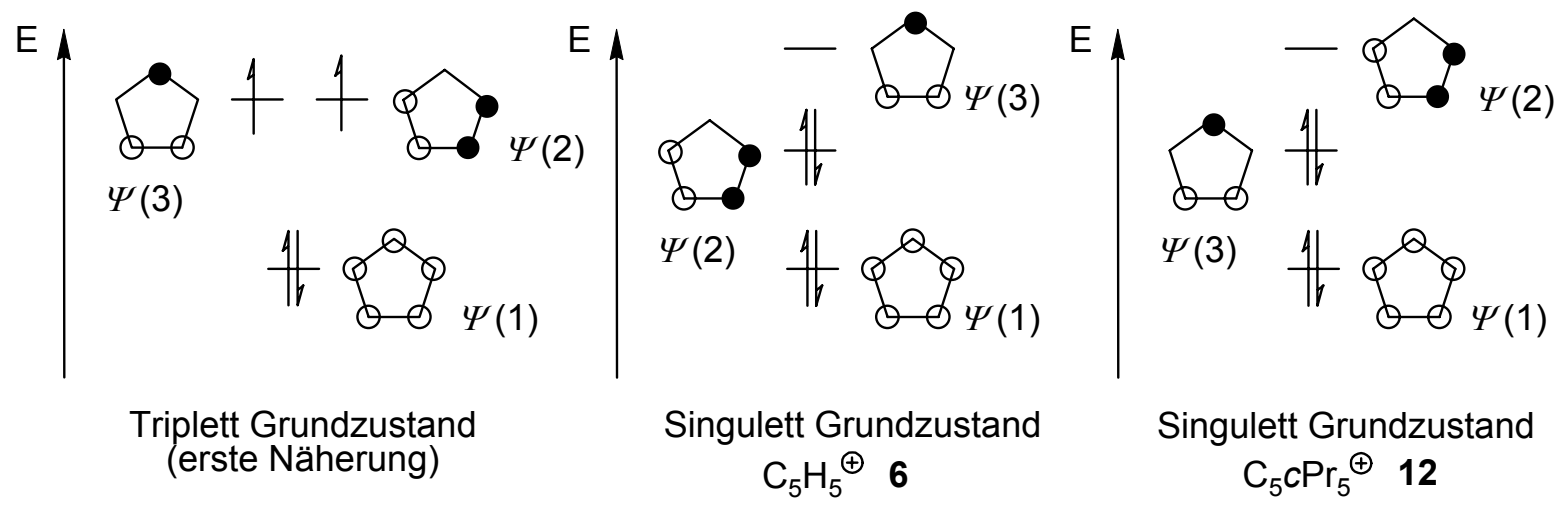

Singulett Grundzustand

$$
\mathrm{C}_{5} \mathrm{CPr}_{5}{ }^{\oplus} 12
$$

Schema 9. Energieabfolge der MOs von Cyclopentadienyl-Kationen im Grundzustand.

Existieren Cyclopentadienyl-Kationen in einem Triplett-Grundzustand, so sind deren Molekülorbitale, die durch die Wellenfunktionen $\psi(2)$ und $\psi(3)$ beschrieben werden, annähernd energetisch entartet. Nach dem JAHN-TELLER-Theorem ist aber ein nichtlineares Molekül mit nicht voll besetzten entarteten Orbitalen nicht stabil. Dies führt im SingulettKation daher zu einer Aufhebung der Entartung der SOMOs und unter Spinpaarung zur vollständigen Besetzung der energieärmeren Orbitale. Für die unsubstituierte Stammverbindung können kaum bzw. keine Effekte der Wasserstoffsubstituenten angenommen werden - dies führt zu der erwarteten Reihenfolge der MOs $\psi(2)$ und $\psi(3)$ des Cyclopentadienyl-Kations im Singulett-Grundzustand. Berechnungen für das Pentacyclopropylcyclopentadienyl-Kation im Singulett-Grundzustand zeigten dagegen, dass in der Energieabfolge der Orbitale das $\psi(3)$ - energetisch tiefer zu liegen kommt als das $\psi(2)-\mathrm{MO}$ (Schema 9). ${ }^{[53]}$

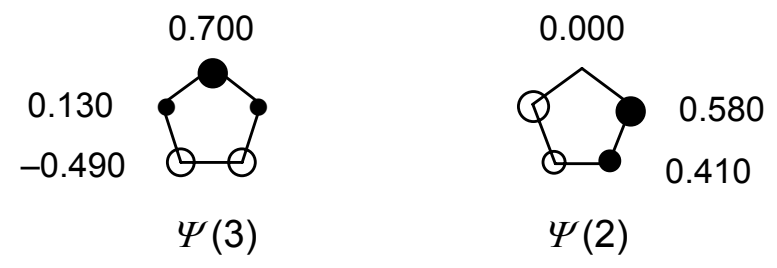

Abbildung 10. Für das Pentacyclopropylcyclopentadienyl-Kation (12) berechnete Atomorbitalkoeffizienten. 
Die fünf Atomorbitalkoeffizienten des $\psi(2)$-LUMO in $c \operatorname{Pr}_{5} \mathrm{C}_{5}{ }^{+}$weisen nicht alle den Gleichen Wert auf (Abbildung 10). Dies erklärt, warum nicht alle Cyclopropyl-Substituenten im gleichen Maße mit dem Cyclopentadienyl-System interagieren. Die Cyclopropylgruppen an den Fünfring-Kohlenstoffatomen mit dem größten Orbitalkoeffizienten $(+0.580)$ befinden sich fast in einer bisektierten Konformation, genau wie es in der AM1-Struktur vorhergesagt wurde (siehe Abbildung 9). Die Cyclopropan-Substituenten an den Kohlenstoffatomen mit den kleineren Orbitalkoeffizienten von +0.410 weichen auch deutlich von der bisektierten Anordnung in der AM1-Struktur ab. Ebenfalls in Übereinstimmung mit der AM1-Rechnung ist der Cyclopropanring an dem Kohlenstoffatom mit einem Orbitalkoeffizienten von Null senkrecht zum $\pi$-System des Cyclopentadienyl-Kations ausgerichtet (d. h. die WALSHOrbitale stehen senkrecht zum benachbarten und die positive Ladung tragenden $\pi$-Orbital) und weist damit keinen stabilisierenden, hyperkonjugativen Effekt auf.

Um eine relative Abschätzung der Stabilität von Cyclopentadienyl-Kationen untereinander $\mathrm{zu}$ erreichen, wurden homodesmotische Dichtefunktional-Rechnungen auf B3LYP/6-311G*-Niveau herangezogen. Hier wurde vorhergesagt, dass die Bildungswärme des Pentacyclopropylcyclopentadienyl-Kations (12) im Singulett-Zustand $19.4 \mathrm{kcal} / \mathrm{mol}$ geringer wäre als die des Pentaisopropylcyclopentadienyl-Kations und $29.3 \mathrm{kcal} / \mathrm{mol}$ geringer als die des Pentamethylcyclopentadienyl-Kations (Schema 10). ${ }^{[53]}$
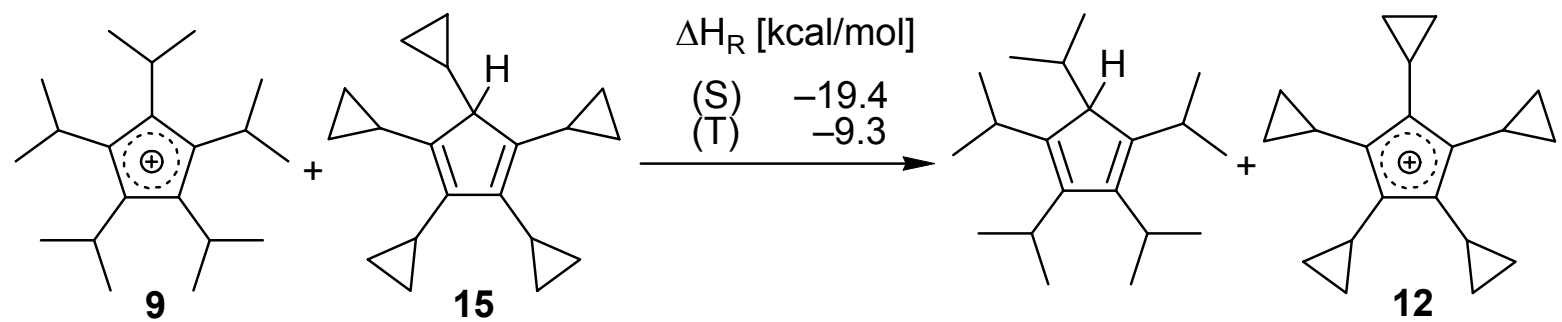

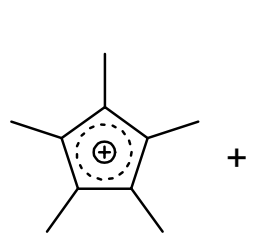

10

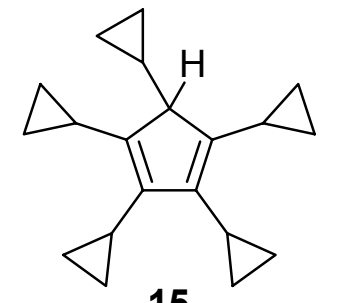

15
$\Delta \mathrm{H}_{\mathrm{R}}[\mathrm{kcal} / \mathrm{mol}]$

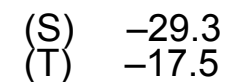

(T) $\quad-17.5$

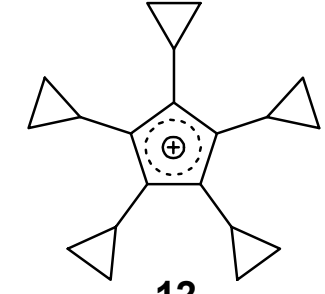

12

Schema 10. Relative Bildungswärmen aus DFT-Rechnungen für homodesmotische Reaktionen zum $c \operatorname{Pr}_{5} \mathrm{C}_{5}^{+}$aus $i \operatorname{Pr}_{5} \mathrm{C}_{5}^{+}$und $\mathrm{Me}_{5} \mathrm{C}_{5}^{+}$. 
Für eine bessere quantitative Abschätzung des Beitrags der Cyclopropyl-Substituenten zur Stabilisierung wurde die von SCHLEYER et al. vorgestellte Berechnung des nucleusindependent $c$ hemical shift Wertes angewandt. ${ }^{[54]}$

NICS-Werte definieren die berechnete, masselose, absolute magnetische Abschirmung im Zentrum von cyclischen Molekülen in enger Analogie zur Konvention für die chemische Verschiebung. Demnach drückt sich magnetische Abschirmung in negativen NICS-Werten aus, die für aromatische $(4 n+2) \pi$-Elektronen-Systeme beobachtet werden. Magnetische Entschirmung wird dagegen durch einen positiven NICS-Wert angezeigt, der in antiaromatischen $(4 n) \pi$-Elektronen-Systemen beobachtet wird.

In einer detaillierten Studie von Cyclopropyl-substituierten Cyclopentadienyl-Kationen durch SCHREINER wurden alle möglichen Substitutionsmuster und Geometrien neben der unsubstituierten Stammverbindung und im Anschluss daran die adiabatischen Singulett/Triplett-Übergangsenergien auf B3LYP/6-311G*-Level berechnet, um zu bestimmen, welche Cyclopentadienyl-Kationen eher in einem Triplett- als in einem SingulettZustand existieren. Für eine Aussage über die relative Aromatizität bzw. Antiaromatizität, wurden ebenfalls sämtliche NICS-Werte berechnet (Abbildung 11a,b,c).

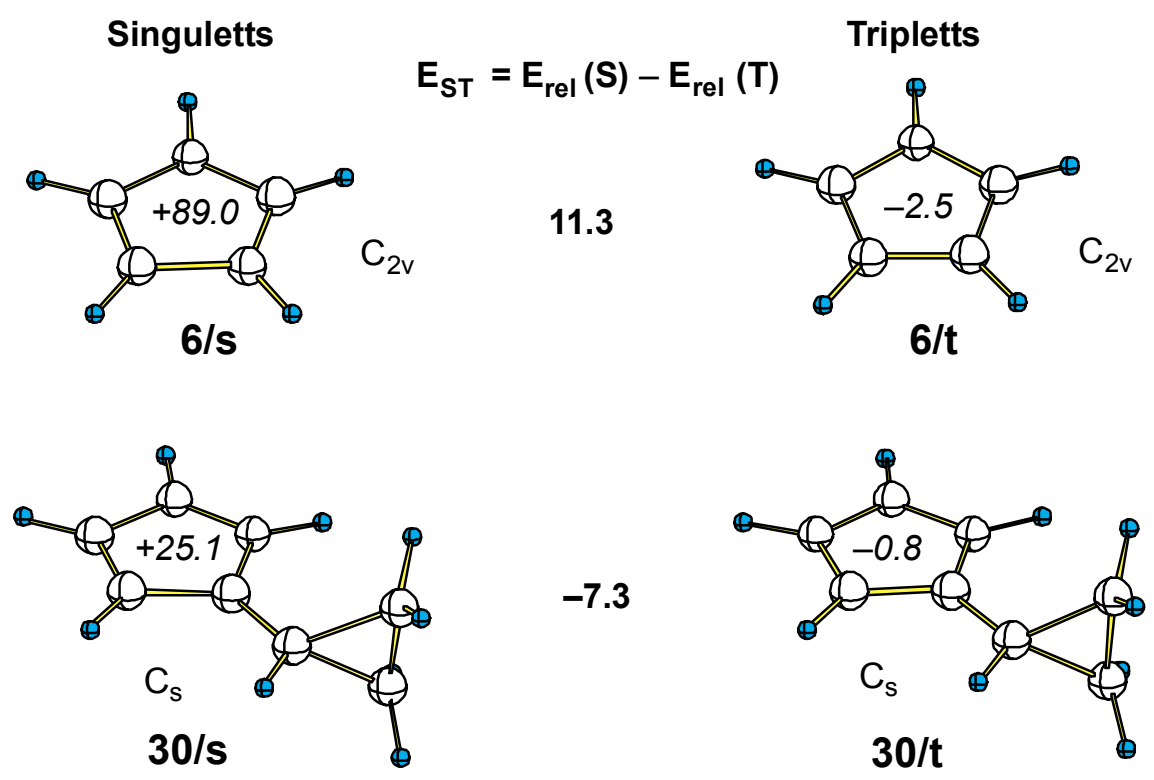

Abbildung 11a. Geometrien, NICS-Werte im Zentrum und relative Energien [kcal/mol] Cyclopropyl-substituierter Cyclopentadienyl-Kationen (B3LYP/6-311G*//B3LYP/6-31G*). 


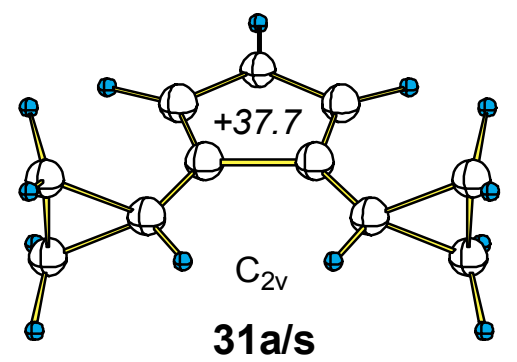

1.4
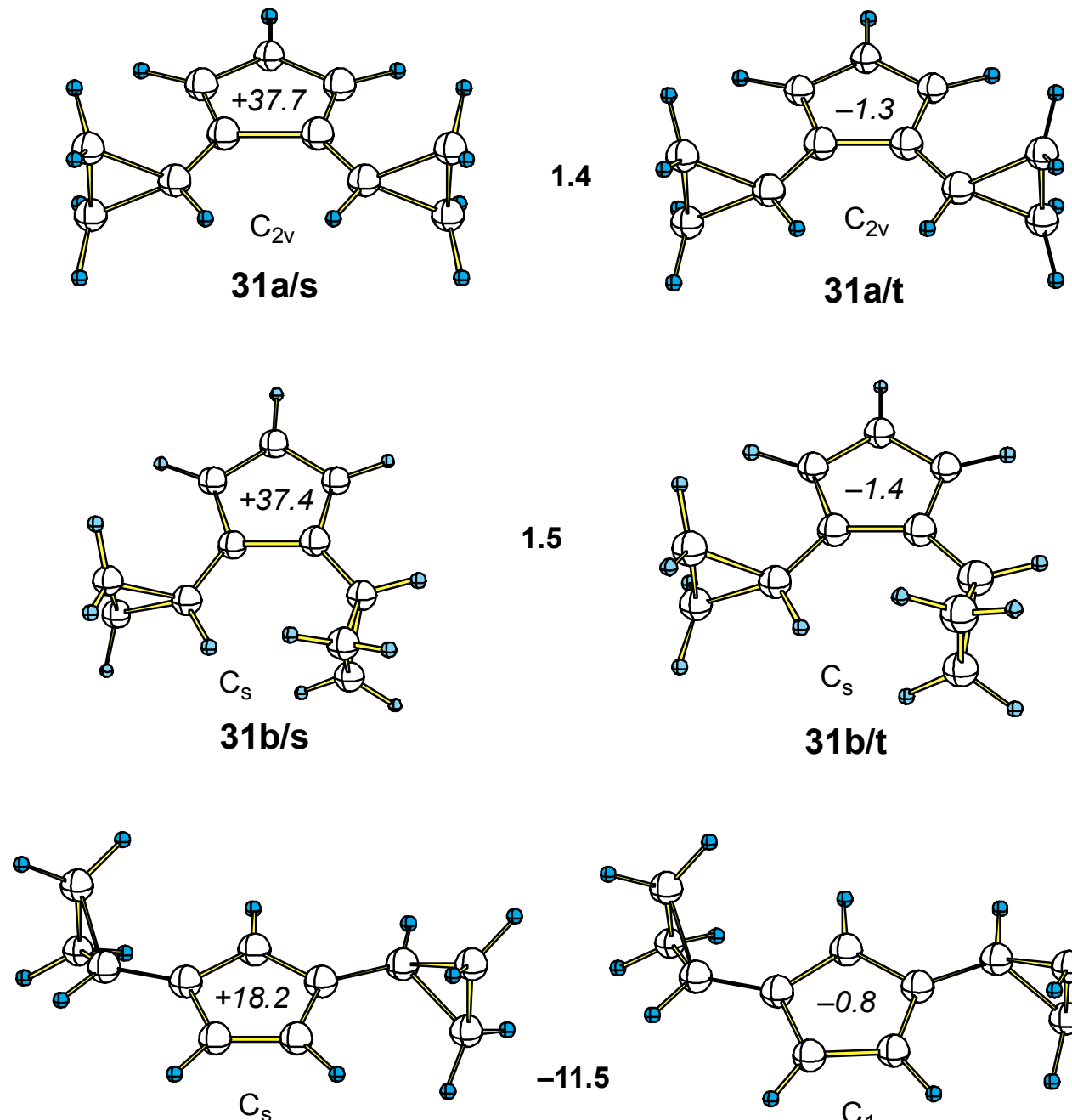

$32 \mathrm{c} / \mathrm{s}$

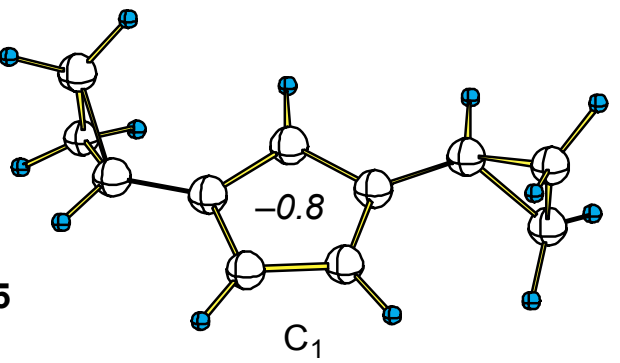

$32 \mathrm{c} / \mathrm{t}$
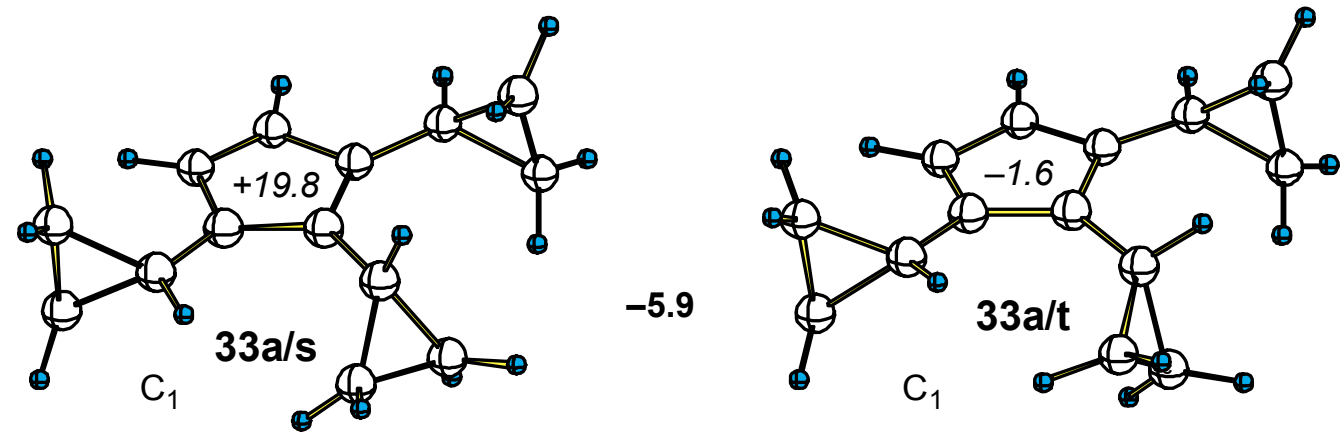

Abbildung 11b. Geometrien, NICS-Werte im Zentrum und relative Energien [kcal/mol] Cyclopropyl-substituierter Cyclopentadienyl-Kationen (B3LYP/6-311G*//B3LYP/6-31G*). 


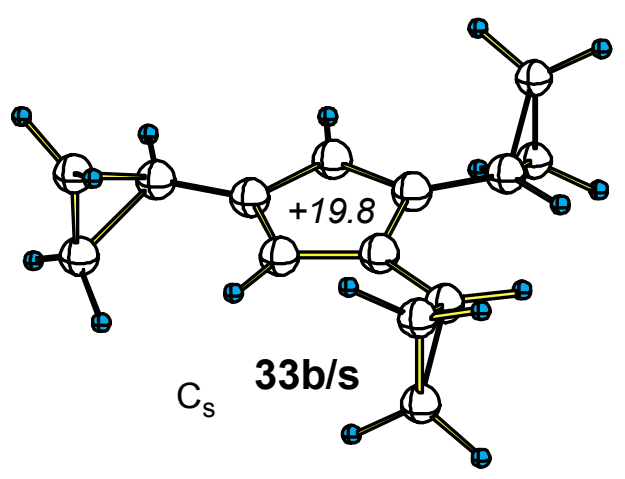

$-8.1$
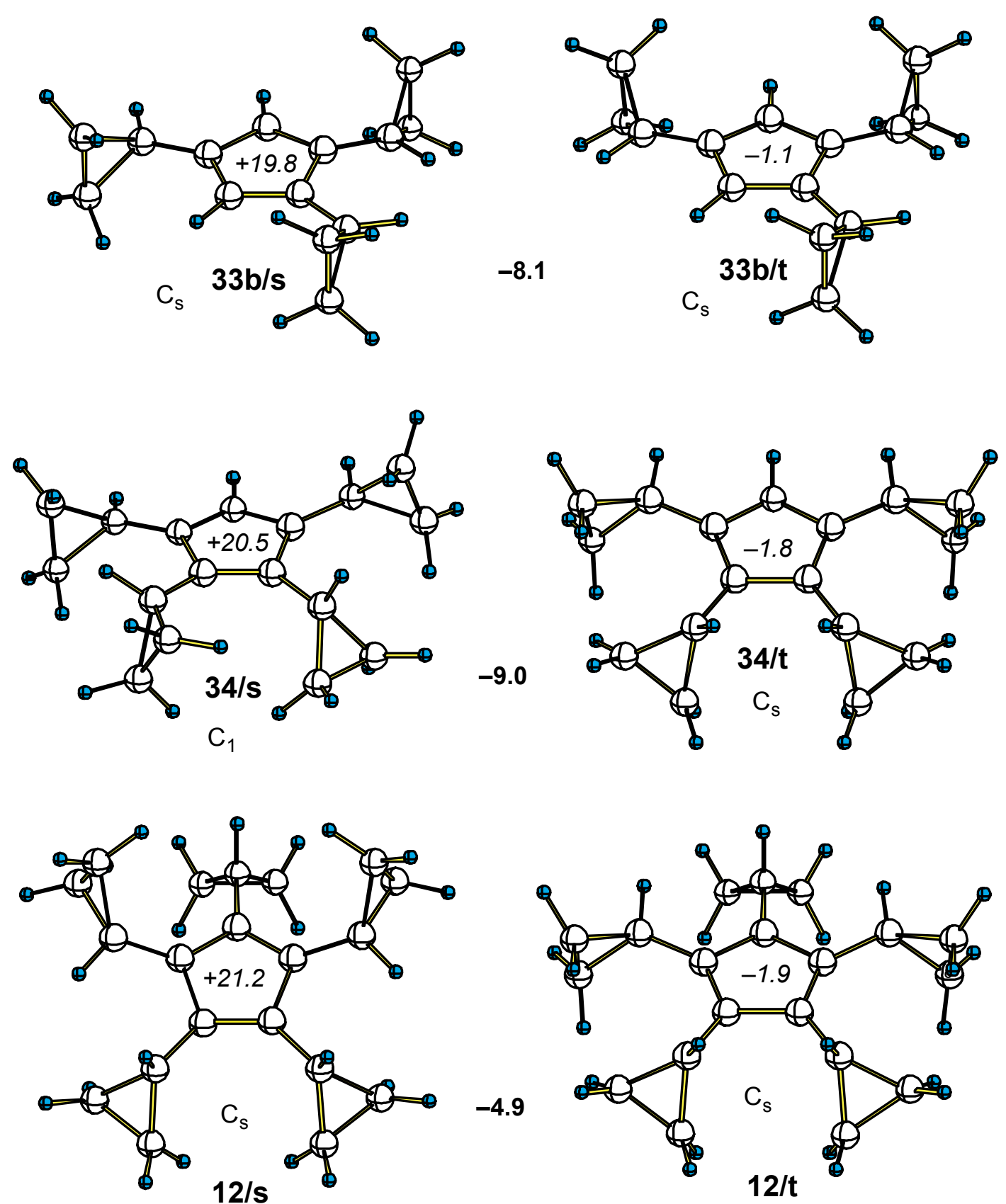

Abbildung 11c. Geometrien, NICS-Werte im Zentrum und relative Energien [kcal/mol] Cyclopropyl-substituierter Cyclopentadienyl-Kationen (B3LYP/6-311G*//B3LYP/6-31G*).

Die NICS-Werte der Cyclopropyl-substituierten Kationen 6/t bis 34/t im TriplettZustand sind durchweg negativ und zeigen den aromatischen Charakter dieser Spezies an (Abbildung 11a,b,c). Untersuchungen von BAIRD ${ }^{[55]}$ führten $\mathrm{zu}$ seinem Postulat, dass die niedrigsten Triplett-Zustände von $\mathrm{C}_{5} \mathrm{R}_{5}{ }^{+}$-Kationen eher als aromatisch eingestuft werden können. Weitere Studien auf der Basis umfassender ab initio Rechnungen, stützen diese 
Vermutung. ${ }^{[56]}$ Offenbar haben weder die Zahl der Cyclopropylgruppen noch das Substitutionsmuster am Cyclopentadienylkation einen signifikanten Einfluss auf die berechneten NICS-Werte $(-2.5$ bis -0.8$)$. Eine höhere Zahl an Cyclopropylgruppen kann somit auch keinen höheren Beitrag zur Stabilisierung der Cyclopentadienyl-Kationen im Triplett-Zustand liefern. Im Falle des eingangs vorgestellten PentachlorcyclopentadienylKations (25) konnten NOVAK et al. durch die Abwesenheit von hypsochrom verschobenen Schwingungsfrequenzen für die C-Cl-Bindungen bei IR-spektroskopischen Untersuchungen ebenfalls keinen stabilisierenden $\alpha$-Halogeneffekt der Chloratome (durch freie Elektronenpaare ausgeübter + M-Effekt) im Triplett-Zustand des Kations feststellen. ${ }^{[42 b]}$

Auf der anderen Seite weisen die NICS-Werte der Cyclopropyl-substituierten Kationen 6/s bis 34/s im Singulett-Zustand durchweg einen negativen Betrag auf, was auf den antiaromatischen Charakter dieser Spezies hinweist (Abbildung 11a,b,c). Weiterhin haben sowohl die Anzahl der Cyclopropylgruppen als auch das Substitutionsmuster am zentralen Ring in diesem Fall sehr wohl einen deutlichen Einfluss in diesen Cyclopentadienyl-Kationen. Schon ein einzelner Cyclopropyl-Substituent in bisektierter Position erniedrigt den NICSWert von +89.0 für das stark destabilisierte antiaromatische Cyclopentadienyl-Kation (6) auf +25.1 für das Monocyclopropylcyclopentadienyl-Kation (30/s). Zwei CyclopropylSubstituenten in 1- und 2-Position führen zu zwei Konformeren 31a/s und 31b/s mit fast gleich großen NICS-Werten $(+37.7$ und +37.4$)$, die bemerkenswerterweise größer sind als der für das monosubstituierte Kation (30/s). Dies liegt sicherlich an der gegenseitigen sterischen Beeinflussung, die die Cyclopropanringe davon abhält, sich optimal bisektiert zu positionieren. Im Gegensatz dazu lieferten die Rechnungen für das 1,3-disubstituierte Kation 32/s einen sehr viel kleineren NICS-Wert von +18 .2. In diesem Kation befinden sich die Cyclopropyl-Substituenten genau an den Kohlenstoffatomen mit den größten Atomorbitalkoeffizienten im $\psi(2)$-LUMO und können nun ungehindert die für eine maximale Wechselwirkung mit dem kationischen Cyclopentadienyl-Gerüst nötige Konformation einnehmen. Die Einführung eines dritten oder vierten Dreiring-Substituenten wie in 33a/s, 33b/s oder in 34/s bewirkt kaum eine Veränderung in den berechneten NICS-Werten, wie 
man sie durch einfache Extrapolation vermutet hätte $(\mathbf{3 3 a} / \mathbf{s}:+19.8, \mathbf{3 3 b} / \mathbf{s}:+19.8, \mathbf{3 4} / \mathbf{s}:+20.5)$. Auch hier können nicht alle Cyclopropylgruppen eine bisektierte Stellung einnehmen. Das Gleiche trifft für das Pentacyclopropylcyclopentadienyl-Kation (12/s) zu, in dem der fünfte Substituent am Kohlenstoffatom mit dem Atomorbitalkoeffizienten von Null senkrecht zum Cyclopentadienylsystem ausgerichtet ist. Dieser kann daher nicht zu einer Delokalisierung der positiven Ladung in 12/s beitragen und übt in dieser Anordnung sogar einen -I-Effekt aus. Er zwingt die benachbarten Cyclopropylgruppen noch dazu aus ihrer optimalen bisektierten Konformation, was zu einem geringfügig höheren NICS-Wert von +21.2 für 12/s führt (Abbildung 11a,b,c).

Eine Analyse der Energiedifferenzen für den adiabatischen Singulett-/Triplett-Übergang zeigt, dass das unsubstituierte $\mathrm{C}_{5} \mathrm{H}_{5}{ }^{+} \mathbf{6}$ mit einem positiven $\Delta \mathrm{E}_{\mathrm{ST}}$ Wert von $+11.3 \mathrm{kcal} / \mathrm{mol} \mathrm{im}$ Triplett-Zustand vorliegt. Die Einführung des einzelnen $c$ Pr-Substituenten im Kation 30/s lässt die Energiedifferenz zwischen Singulett- und Triplett-Niveau $\Delta \mathrm{E}_{\mathrm{ST}}=-7.3 \mathrm{kcal} / \mathrm{mol}$ negativ werden, d. h. das Kation sollte den Singulett-Zustand bevorzugen. Bis auf die Kationen 31a/s und 31b/s, gilt dieses auch für alle anderen Cyclopropyl-substituierten Cyclopentadienyl-Kationen (Abbildung 11a,b,c).

Diese vielversprechenden Vorhersagen lassen es sehr lohnend erscheinen, das Pentacyclopropylcyclopentadienyl-Kation präparativ zugänglich zu machen und einen spektroskopischen Hinweis auf die potentielle Stabilisierung des antiaromatischen Cyclopentadienyl-Kations durch Cyclopropyl-Substituenten zu erhalten. 
3. Synthese geeigneter Vorstufen für Cyclopropyl-substituierte, kationische Cyclopentadienyl-Spezies und deren spektroskopische Untersuchung

\subsection{Retrosynthetische Überlegungen und generelle Aspekte zur Präparation von Carbo- kationen}

Ideale Vorläufermoleküle, die sich zur Erzeugung von Carbokationen eignen, sind mit dem entsprechenden, interessierenden Kohlenstoff-Grundgerüst sowie einer funktionellen Gruppe ausgestattet, durch deren Behandlung mittels Brönsted- und/oder LEWIS-Säuren das zu untersuchende Kation freigesetzt werden kann. Außer durch Protonierung von C,CDoppel- oder Dreifachbindungen, ${ }^{[57,20]}$ werden Carbokationen überwiegend durch Heterolyse von $\mathrm{C}-\mathrm{OH}$ - oder $\mathrm{C}-\mathrm{Halogen}-\mathrm{Bindungen} \mathrm{erzeugt.} \mathrm{In} \mathrm{seltenen} \mathrm{Fällen} \mathrm{werden} \mathrm{unter} \mathrm{anderem}$ auch Ether, Chlorsulfonsäureester oder Kohlenwasserstoffe eingesetzt. Erstere können durch ionisierende Fragmentierung ${ }^{[58]}$ in Carbokationen überführt werden, letztere durch Hydridabstraktion. ${ }^{[59,82]}$

Im Allgemeinen sind Halogenide verglichen mit Hydroxy-Gruppen die bevorzugten Abgangsgruppen. Die exotherme Ionisation von z. B. Alkylhalogeniden kann mit einer um bis zu $10 \mathrm{kcal} / \mathrm{mol}$ geringeren Wärmetönung und damit schonender als die Protonierung und Dehydratisierung entsprechender Alkohole verlaufen. Diese setzen pro Carbokation auch je ein Molekül Wasser frei, das durch Bindung an z. B. LEWIS-Säuren inaktiviert werden muss. ${ }^{[60]}$

Die Untersuchungsmethode beeinflusst ebenfalls die Wahl der Abgangsgruppe. So werden Leitfähigkeits- oder kryoskopische Messungen bevorzugt in protischen Säuren wie Schwefelsäure durchgeführt. ${ }^{[61]}$ Dabei bieten sich Alkohole als Kationenvorläufer an. Neuere Methoden kombinieren die IR-Spektroskopie mit Matrix-Kondensations-Techniken. Dies ermöglicht die Untersuchung der Bildung und auch Umlagerung von reaktiven Kationen in kryogenischer Antimon(V)fluorid-Matrix. ${ }^{[62]}$ 
Nach diesen Überlegungen könnten das Fulven 35 und das Cyclopentadien 15 sowie sein korrespondierendes Halogenderivate wie $\mathbf{3 6}$ potentielle Vorstufen für das Pentacyclopropylcyclopentadienyl-Kation (12) sein (Schema 11).

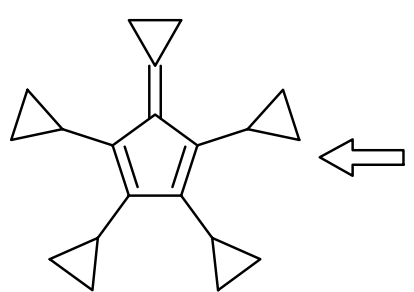

35

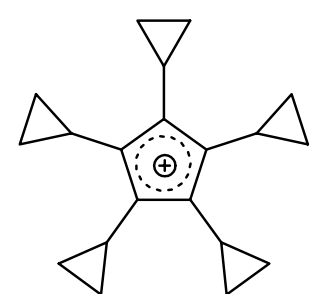

12

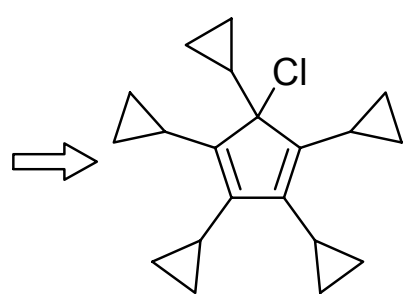

36

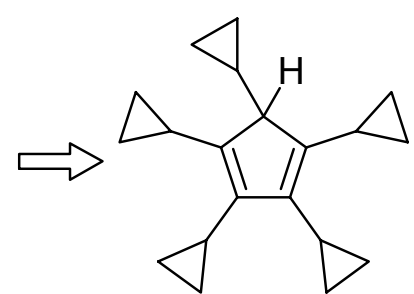

15

Schema 11. Retrosynthetische Überlegungen zum Pentacyclopropylcyclopentadienyl-Kation (12).

\subsection{Synthetische Zugänge zu Cyclopropyl-substituierten Cyclopentadienen}

Cyclopentadien und dessen substituierte Derivate gehören $\mathrm{zu}$ den am häufigsten verwendeten Liganden für Metallkomplexe. ${ }^{[63,37]}$ Allerdings ist nur wenig über Cyclopropylsubstituierte Cyclopentadiene bekannt, obwohl die Cyclopropylgruppe nicht nur wegen ihrer einzigartigen elektronischen Eigenschaften heraussticht. Dabei kommt sie sterisch einer Ethylgruppe näher als einer Isopropylgruppe. ${ }^{[64]}$ Unterschiedliche Methoden können zum Aufbau eines Cyclopentadien-Grundgerüsts herangezogen werden. Übergangsmetallvermittelte Reaktionen mit FISCHER-Carben-Chromkomplexen, ${ }^{[65]}$ die PAUSON-KHANDReaktion $^{[66]}$ oder die NAZAROV-Cyclisierung ${ }^{[67]}$ seien hier beispielhaft genannt.

So konnte EMME durch Reaktion des $\alpha, \beta$-ungesättigten FISCHER-Carben-Komplexes 37 mit Mono- oder Dicyclopropylethin die Di- bzw. Tricyclopropyl-substituierten Cyclopentenone 38 und 39 in über 60\% Ausbeute herstellen (Schema 12). ${ }^{[37]}$ 


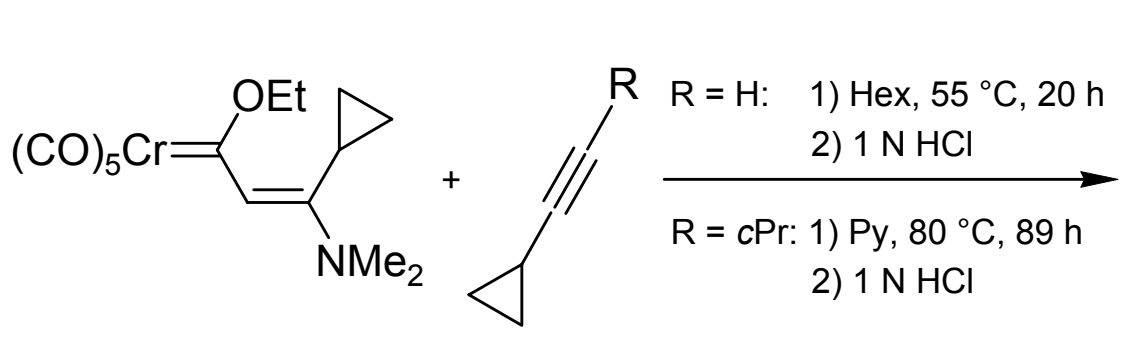

37

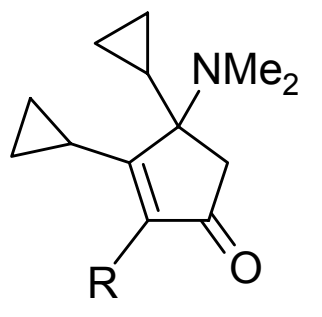

38: $\mathrm{R}=\mathrm{H}, 88 \%$

39: $\mathrm{R}=c \mathrm{Pr}, 62 \%$

Schema 12. Darstellung Cyclopropyl-substituierter Cyclopentenone mittels FISCHER-CarbenChrom-Komplexen.

Allerdings konnten weder $\mathbf{3 8}$ noch $\mathbf{3 9}$ in nachfolgenden Versuchen erfolgreich in die korrespondierenden 1,2-Di- bzw. 1,2,3,4-Tetracyclopropyl-substituierten Cyclopentadiene transformiert werden. Im ersteren Fall führte die angestrebte Eliminierung von Dimethylamin zur Öffnung des vicinalen Dreirings und im letzteren Fall fand bei der gewünschten 1,2-Addition von Cyclopropylmagnesiumbromid an das Enon eine Eliminierung der Dimethylaminofunktion statt, an die sich eine Dimerisierung des Tricyclopropylcyclopentadienons im Sinne einer DIELS-ALDER-Reaktion anschloss.

Der Versuch, das Tetracyclopropyl-substituierte Cyclopentenon 40 durch eine klassische PAUSON-KHAND-Reaktion des aus Dicobaltoctacarbonyl und Dicyclopropylethin (13) gebildeten Komplexes mit 1,2-Dicyclopropylethen darzustellen, gelang nicht. ${ }^{[37]}$

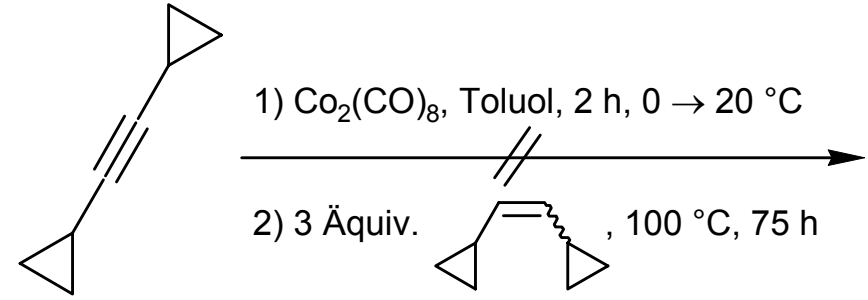

13

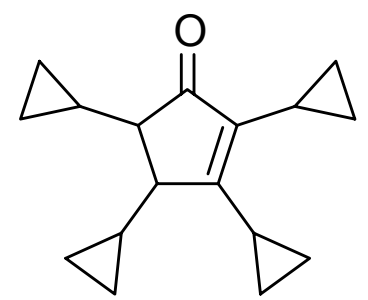

40

Schema 13. Tetracyclopropylcyclopentenon 40 durch PAUSON-KHAND-Reaktion zugänglich?

Der erstmals von VICTOR und SAREL dargestellte Komplex $\mathbf{4 1}^{[68]}$ erschien EMME ${ }^{[37]}$ als aussichtsreiches Edukt zur Herstellung des percyclopropylierten CyclopentadienylGrundgerüstes. Aber auch hier führte die Behandlung mit Trimethylamin- $N$-oxid zwar zur 
Dekomplexierung des Tetracyclopropylcyclopentadienons, doch konnte ausschließlich dessen DIELS-ALDER-Dimer isoliert werden. Zwar gelang in diesem Fall die Umsetzung von 41 zum Pentacyclopropyl-substituierten Cyclopentadienyl-Eisen-Komplex 42. Jedoch erwies sich dieser für weitere notwendige Transformationen als zu instabil.

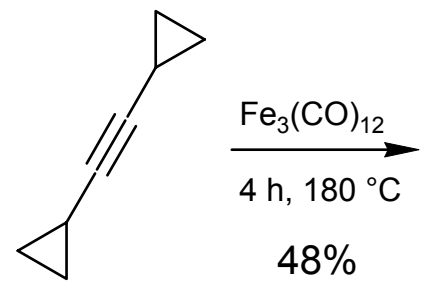

13

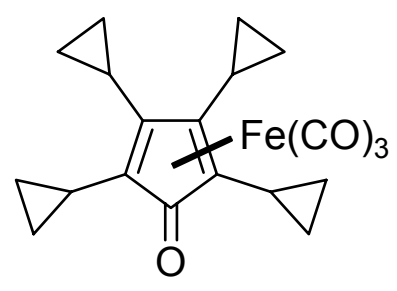

41

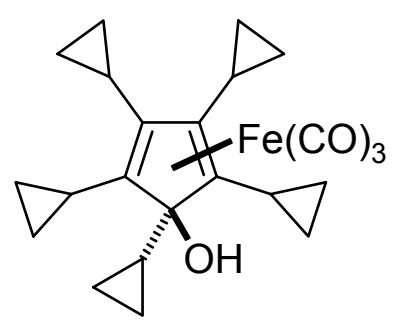

42

Schema 14. Erzeugung und weitere Umsetzung des Tricarbonyleisen-komplexierten Cyclopentadienons 41.

Nach NAZAROV werden Divinylketone bei Einwirkung von Protonen- oder LEWISSäuren in Cyclopentenone umgewandelt. Die dabei maßgebliche Cyclisierung ist eine thermisch erlaubte, konrotatorische $4 \pi$-Elektrocyclisierung eines Pentadienyl zu einem Cyclopentenyl-Kation. ${ }^{[67,69]} \mathrm{Um}$ mit dieser Synthesestrategie vierfach und auch fünffach Cyclopropyl-substituierte Cyclopentadiene 14 oder 15 zugänglich zu machen, versuchte EMME, aus Dicyclopropylethin nach Bildung einer geeigneten metallorganischen Verbindung und deren Addition an Ameisensäure- $n$-butylester, den entsprechenden Tetracyclopropylsubstituierten Bisallylalkohol $\mathbf{4 3}$ herzustellen. ${ }^{[37]}$ Anschließende Oxidation der Hydroxygruppe zu 44 und dessen NAZAROV-Cyclisierung sollte das Cyclopentenon 40 ergeben. Die Reduktion der Carbonylgruppe würde zu einem sekundären, die Addition einer Cyclopropylorganometall-Spezies zu einem tertiären Alkohol des Typs 45 führen, dessen anschließende Dehydratisierung die Cyclopentadiene 14 oder 15 liefern würde (Schema 15). 


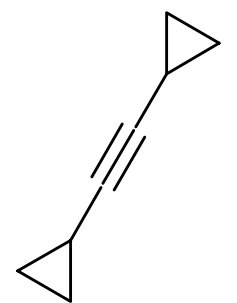

13

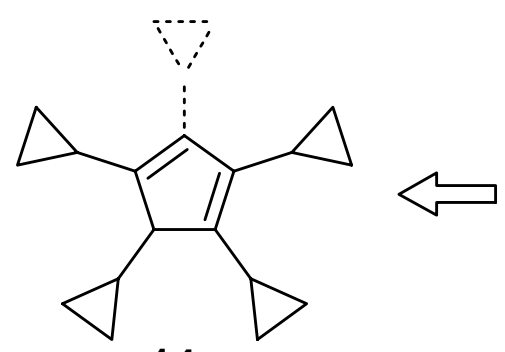

14

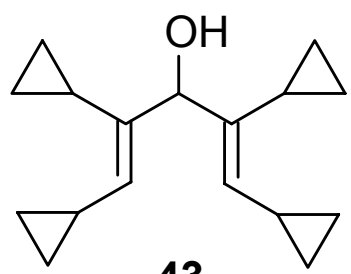

43

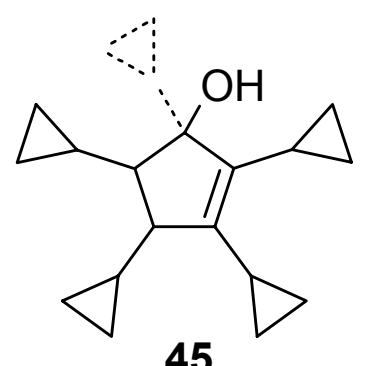

45

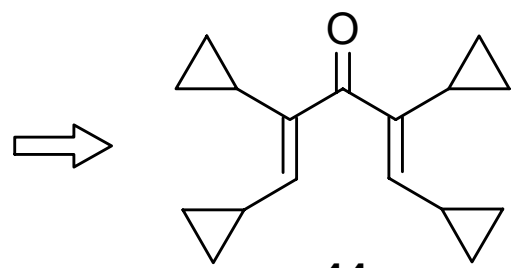

44<smiles>[CH]1C=C1</smiles>

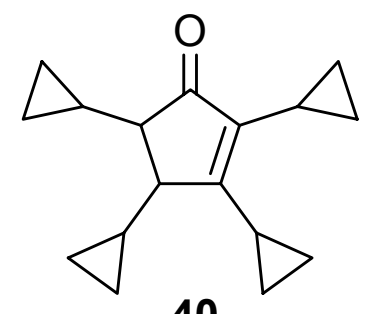

40

Schema 15. Möglicher Zugang zu Tetra- (14) und Pentacyclopropylcyclopentadien (15) durch NAZAROV-Cyclisierung.

Zur Überführung des Dicyclopropylethins in eine reaktive metallorganische Verbindung wurde die von $\mathrm{SATO}^{[70]}$ etablierte Methode der Hydromagnesierung von Alkinen mit einer katalytischen Menge Biscyclopentadienyltitandichlorid verwendet. Die Aktivierung des $\mathrm{Cp}_{2} \mathrm{TiCl}_{2}$ erfolgt dabei durch Reaktion mit zwei Äquivalenten Isobutylmagnesiumbromid unter Bildung einer niedervalenten $\mathrm{Cp}_{2} \mathrm{Ti}(i \mathrm{Bu})_{2}$-Spezies. Durch anschließende $\beta$-Hydrideliminierung entsteht Isobuten und ein Hydridotitan-Intermediat, welches sich an die Dreifachbindung des Alkins 13 addieren kann. Nach Ummetallierung der so gebildeten VinylTitanspezies entsteht das 1,2-Dicyclopropylethenylmagnesiumbromid (46). Unter diesen Bedingungen bildet sich 46 aus Dicyclopropylethin in einer exothermen Reaktion unter starker Gasentwicklung und intensiver Grünfärbung der Lösung. Wurde THF anstelle von Diethylether als Lösungsmittel verwendet, fand keine Bildung des GRIGNARD-Reagenzes statt. Die Vinyl-GRIGNARD-Verbindung 46 sollte anschließend durch zweifache Addition an Ameisensäure- $n$-butylester zum Diallylalkokohol 43 umgesetzt werden. 


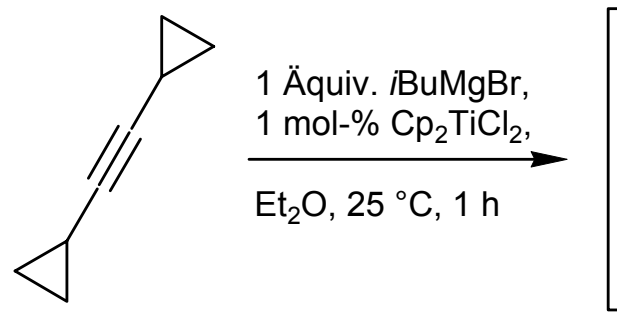

13

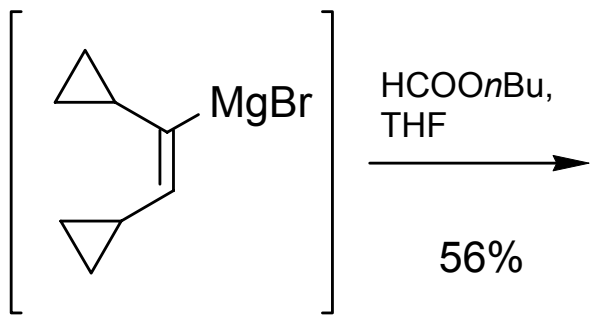

46

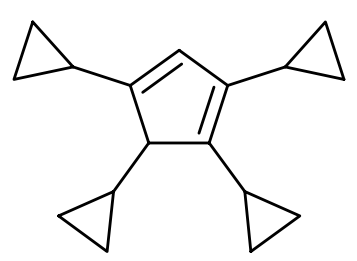

14

Schema 16. Einstufige Synthese von 1,2,4,5-Tetracyclopropylcyclopenta-1,3-dien nach EMME. $^{[37]}$

Überraschenderweise konnte schon bei dieser Reaktion das eigentlich gewünschte Tetracyclopropylcyclopentadien 14 als Hauptprodukt in einer recht guten Ausbeute von 56\% isoliert werden, so dass die weiteren Umsetzungen des eigentlich erwarteten Diallylalkokohols entfielen. Ausgehend von einfach herzustellendem Dicyclopropylethin steht hiermit eine einstufige - anstatt der ursprünglich vorgesehenen fünfstufigen - Synthese dieses neuartigen Cyclopentadienyl-Liganden zur Verfügung (Schema 16). ${ }^{[36 \mathrm{~b}]}$

\subsection{Synthese von 1,2,3,4,5-Pentacyclopropylcyclopenta-1,3-dien (15)}

Um einen Zugang zum entsprechenden Pentacyclopropylcyclopentadiens $\mathbf{1 5}$ zu schaffen, war der offensichtlich nächste Schritt, analog dem Protokoll zur Herstellung des Tetracyclopropylcyclopentadiens, eine etherische Lösung des GRIGNARD-Reagenzes 46 zu Cyclopropylcarbonsäuremethylester in THF zu geben.

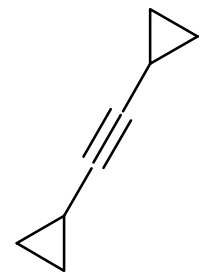

13

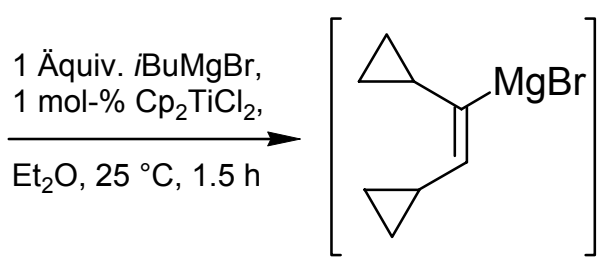

46

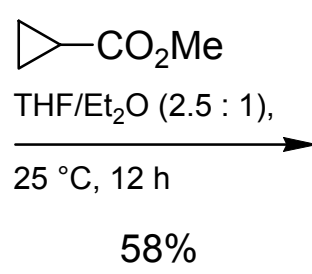

$58 \%$

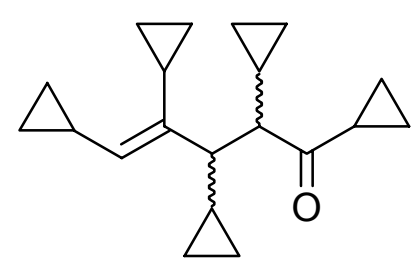

47

Schema 17. Versuchte Darstellung von Pentacyclopropylcyclopentadien und Bildung eines MiCHAEL-Addukts an (Dicyclopropylvinyl)cyclopropylketon. 
Nach der Reaktion konnte ein Gemisch zweier Diastereomere des 1,2,3,4,5-Pentacyclopropylpent-4-en-2-ons 47 als Hauptprodukt isoliert werden. ${ }^{[37]}$ Letzteres $^{2}$ musste sich aus zwei Molekülen des GRIGNARD-Reagenzes 46 durch 1,2-Addition an Cyclopropancarbonsäuremethylester und darauffolgende 1,4-Addition an das Enon gebildet haben (Schema 17).

Die in einigen Fällen kaum sicher vorhersagbare Chemoselektivität bei der Addition von GRIGNARD-Reagenzien an $\alpha, \beta$-ungesättigte Carbonylverbindungen ist wohl bekannt. So treten Enolisierung, Reduktion, Kondensation und auch konjugierte Addition als häufige Nebenreaktionen auf. Diese Problematik lässt sich durch den Einsatz der entsprechend weniger basischen Organocer(III)-Verbindungen umgehen.<smiles>O=C(/C=C/c1ccccc1)c1ccccc1</smiles>

48
A: 1.5 Äq. $\mathrm{PhMgBr}$, THF, $0{ }^{\circ} \mathrm{C}, 1 \mathrm{~h}$

B: 1.5 Äq. $\mathrm{PhMgBr} \cdot \mathrm{CeCl}_{3}$, THF, $0{ }^{\circ} \mathrm{C}, 1 \mathrm{~h}$

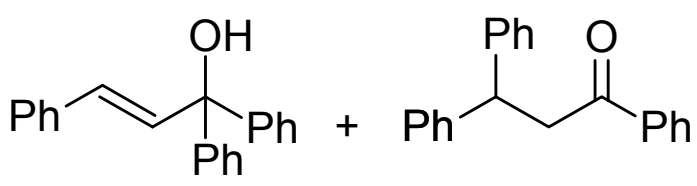

49
A: $5 \%$
$81 \%$
B: $\quad 86 \%$
$8 \%$

Schema 18. Steuerbare 1,2- oder 1,4-GRIGNARD-Addition durch Einsatz von Cer(III)chlorid.

So konnten IмАмОто et al. bei ihren Untersuchungen zum Einsatz von Organolanthanoid-Verbindungen in der organischen Synthese zeigen, dass Cer-vermittelte Additionen von Organolithium- und auch GRIGNARD-Verbindungen an Carbonylverbindungen unter milden Bedingungen die gewünschten Alkohole lieferten, selbst wenn die Carbonylverbindungen stark zur Enolisierung neigten oder eine konjugierte Doppelbindung enthielten. ${ }^{[71]}$ So entsteht aus 1,3-Diphenylpropenon (48) mit 
Phenylmagnesiumbromid als Hauptprodukt einer 1,4-Addition 1,3,3-Triphenyl-propan-1-on (50). Führt man die gleiche Reaktion Cer-vermittelt durch, so ist 1,1,3-Triphenyl-prop-2-en1-ol (49) hoch chemoselektiv das Hauptprodukt (siehe Schema 18).

Die ursprünglich ins Auge gefasste Synthesestrategie für das Pentacyclopropylcyclopentadien wurde dementsprechend modifiziert, um den beschriebenen unerwünschten Reaktionsweg zu unterdrücken. Demzufolge wurde zu einer Mischung aus einer etherischen Lösung des GRIGNARD-Reagenzes 46 und die Mischung in THF suspendiertem, wasserfreien $\mathrm{CeCl}_{3}$ der Cyclopropancarbonsäuremethylester gegeben und eine Stunde bei Raumtemperatur gerührt. Nach Hinzufügen von 15prozentiger Essigsäure und säulenchromatographischer Trennung des erhaltenen Rohprodukts, konnte das gewünschte Pentacyclopropylcyclopentadien 15 mit einer reproduzierbaren Ausbeute von über 60\% isoliert werden (Schema 19).

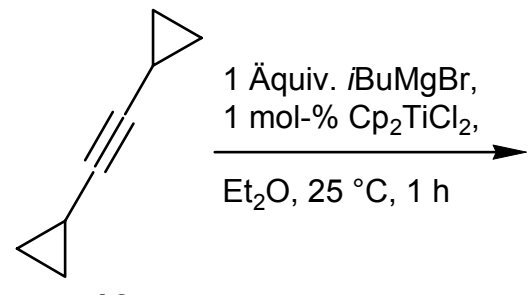

13

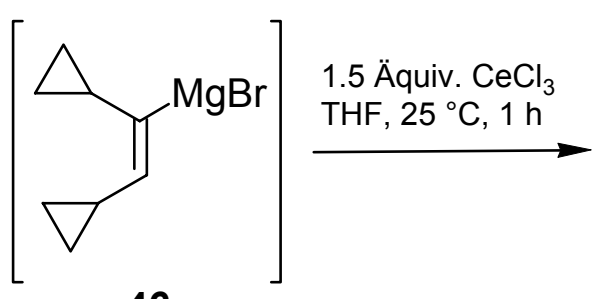

46

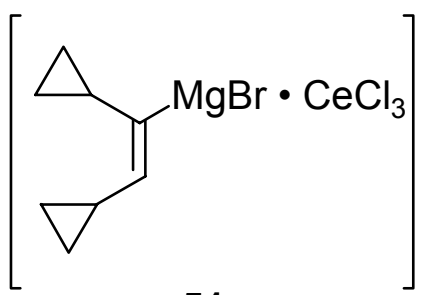

51

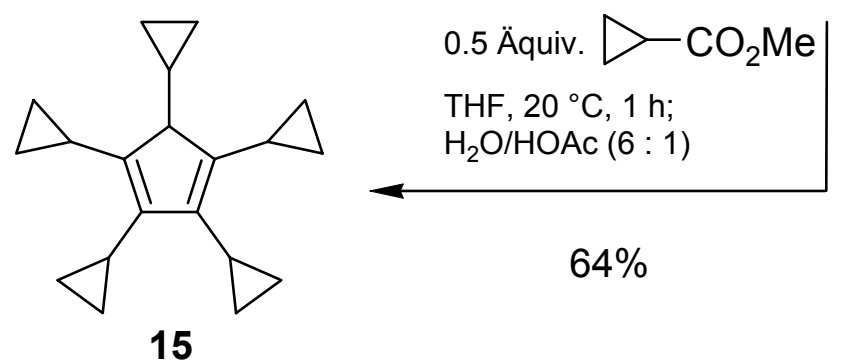

Schema 19. Optimierung der Cer-vermittelten einstufigen Synthese von Pentacyclopropylcyclopentadien 15 nach EMME.

Genauso wie bei der Bildung des Tetracyclopropylcyclopentadiens 14 entsteht das Pentacyclopropylcyclopentadien $\mathbf{1 5}$ hier vermutlich ebenfalls durch eine wohl bekannte, konrotatorisch verlaufende $4 \pi$-Elektrocyclisierung des Pentadienyl-Kations $\mathbf{5 3}$ zum 
Cyclopentenyl-Kation 54 und anschließende Eliminierung eines Protons. Die PentadienylKationen könnten hier wiederum durch Mitwirkung des LEWIS-aciden Magnesiumhalogenids aus den Pentadienolaten entstanden sein (Schema 20).

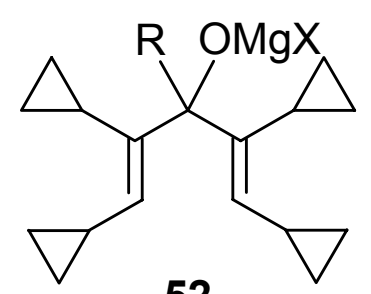

52

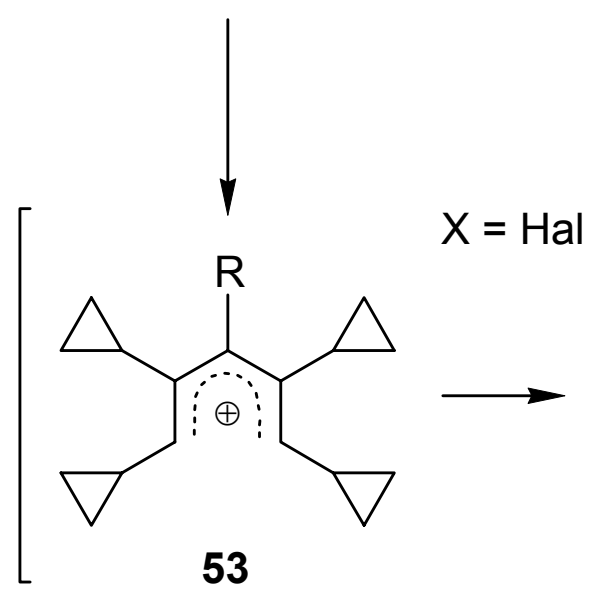

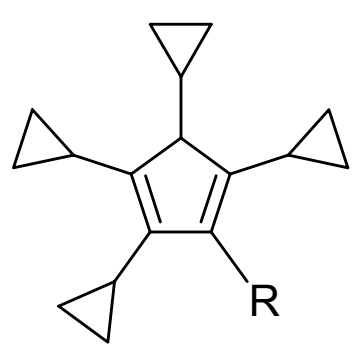

$14(\mathrm{R}=\mathrm{H})$

$15(\mathrm{R}=\mathrm{cPr})$

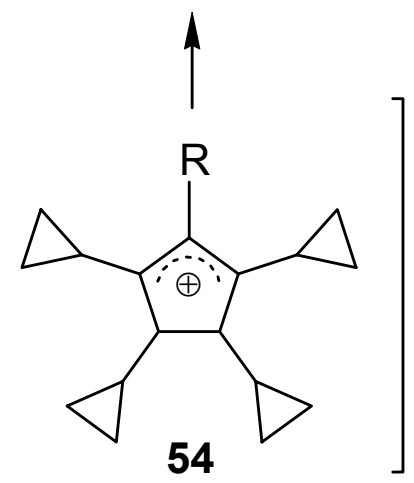

Schema 20. Bildung der Cyclopentadiene 14 und 15 durch intramolekulare $4 \pi$-Elektrocyclisierung intermediärer Pentadienyl-Kationen.

Bemerkenswert ist jedoch, dass im Gegensatz zur Bildung des Tetracyclopropylcyclopentadiens, das sich schon vor dem Aufarbeiten durch Dünnschichtchromatographie (DC) nachweisen lässt, das Pentacyclopropylcyclopentadien 15 erst nach dem Zusatz von verdünnter Essigsäure durch DC detektiert werden kann. Dies spricht dafür, dass zunächst der tertiäre Alkohol $\mathbf{5 2}$ gebildet wird. Aus diesem müsste dann durch Einwirkung der schwachen BRØNSTED-Säure Essigsäure das Pentadienyl-Kation 53 entstehen, welches umgehend zum Cyclopentenyl-Kation 54 cyclisiert (Schema 20). Verwendet man verdünnte Salzsäure oder nur Wasser, so erhält man große Mengen polymeren Materials und kein Cyclopentadien 15. Auch die Lösungsmittelmenge und das Verhältnis von THF zu Et $\mathrm{Z}_{2} \mathrm{O}$ sind einflussnehmende Faktoren. So erfolgt die Hydromagnesierung des 1,2-Dicyclopropylethin ausschließlich in 
$\mathrm{Et}_{2} \mathrm{O}$ und nicht in THF. Die Reaktion mit dem Cyclopropancarbonsäuremethylester scheint dagegen besser in einem größeren Volumen THF abzulaufen, wobei sich ein Verhältnis von $\mathrm{THF} / \mathrm{Et}_{2} \mathrm{O}$ von 6:1 bisher als optimal erwiesen hat.

Mit dieser Modifizierung stand nun auch eine einstufige Synthese für das Pentacyclopropylcyclopentadien $\mathbf{1 5}$ mit befriedigender Ausbeute zur Verfügung. Dieser neu erschlossene Zugang zum Cyclopentadien-Liganden 15 bildete die Grundlage für die Untersuchungen des Cyclopropyl-substituierten Cyclopentadienyl-Kations 12.

\subsection{Potentielle Vorstufen für Cyclopropyl-substituierte, kationische Cyclopentadienyl-Spezies}

Rein formal betrachtet könnte - wie eingangs erwähnt - der korrespondierende Kohlenwasserstoff selbst als Vorläufermolekül des interessierenden Cyclopentadienyl-Kations dienen, welches durch die Entfernung eines Hydridions von C-5 freigesetzt werden könnte. Eine starke LEWIS-Säure mit hinreichender Hydrid-Affinität könnte solch einen Vorgang bewerkstelligen und Tritylsalze, selbst Carbokationen, wären mögliche Kandidaten dafür.

LAMBERT et al. versuchten eine Hydridabstraktion von Pentamethylcyclopentadien (28) mit Hilfe von Trityl(tetrakispentafluorophenyl)borat als Hydridakzeptor und konnten ein kristallines, luftstabiles Material isolieren (siehe Schema 6). ${ }^{[46 a]}$ Die darüber gewonnenen experimentellen und spektroskopischen Daten wurden dahingehend interpretiert, dass der Theorie folgend das Pentamethylcyclopentadienyl-Kation 10 erhalten worden sein müsste, obwohl deutliche Unstimmigkeiten erkennbar gewesen wären. Anhand weitergehender Untersuchungen durch BERTRAND ${ }^{[46 b]}$ und MüLLER ${ }^{[46 c]}$ et al. konnten die Kristalle als das wohlbekannte Pentamethylcyclopentenyl-Kation 29 identifiziert werden, welches durch einen unerwarteten und bisher nicht eindeutig erklärbaren Protonentransfer entstanden sein musste. ${ }^{[46 \mathrm{~d}, 72,73]}$ 
Voraussetzung für einen solchen Hydridtranfer wäre, dass das entstehende Cyclopentadienyl-Kation thermodynamisch stabiler ist als das zur Hydridabstraktion eingesetzte Tritylsalz. Die relative Stabilität eines Carbeniumions wird am besten mit seinem $\mathrm{p} K_{\mathrm{R}}{ }^{+}$-Wert bewertet. In verdünnter wässriger Lösung $\left(H_{\mathrm{R}}\right.$ ist die Aciditätsfunktion des Reaktionsmediums; in Wasser: $\mathrm{p} H \approx H_{\mathrm{R}}$ ) ist der $\mathrm{p} K_{\mathrm{R}}{ }^{+}$-Wert gleichbedeutend mit dem $\mathrm{pH}$-Wert, bei dem Carbeniumion und der korrespondierende Alkohol in gleicher Konzentration vorliegen. ${ }^{[6 \mathrm{~d}, 20 \mathrm{~b}]}$

$$
\begin{gathered}
\mathrm{R}-\mathrm{OH}+\mathrm{H}^{+} \rightleftharpoons \mathrm{R}^{+}+\mathrm{H}_{2} \mathrm{O} \\
\mathrm{pK} \mathrm{R}^{+}=\lg \frac{\left[\mathrm{R}^{+}\right]}{[\mathrm{R}-\mathrm{OH}]}+H_{\mathrm{R}}
\end{gathered}
$$

Schema 21. Ableitung und Bedeutung des $\mathrm{p} K_{\mathrm{R}}{ }^{+}-$Werts für Carbokationen.

Auf diese Weise ist es allerdings nur möglich, den $\mathrm{p} K_{\mathrm{R}}{ }^{+}$-Wert von relativ stabilen Kationen zu bestimmen. Dabei zeigen positivere Werte ein durch elektronenliefernde Substituenten stabileres Kation an und umgekehrt.<smiles>c1ccccc1</smiles>

6<smiles></smiles>

27<smiles></smiles>

55<smiles>C=CC=O</smiles>

56<smiles>Pc1ccccc1</smiles>

57

$$
\begin{array}{llllll}
\mathrm{pK} K_{\mathrm{R}+} & -40 & -18.9 & -10.8 & -20 & -6.63
\end{array}
$$

Schema 22. Ausgewählte $\mathrm{p} K_{\mathrm{R}}{ }^{+}$-Werte für einige Cyclopentadienyl-Kationen und (konjugierte) Vergleichsverbindungen.

BRESLOW et al. konnten von den Kohlenwasserstoffen ausgehend auf elektrochemischem Wege über Messung der Halbstufenpotentiale des korrespondierenden Cp-Anions und des Cp-Radikals den $\mathrm{p} K_{\mathrm{R}}{ }^{+}$-Wert verschiedener Cyclopentadienyl-Kationen 
bestimmen, unter anderen auch den des unsubstituierten Stammsystems 6, der mit -40 um 20 Einheiten niedriger liegt als beispielsweise der des Allyl-Kations mit ca. -20 oder der des Trityl-Kations mit -6.63 (Schema 22). ${ }^{[41 \mathrm{c}]}$

Nahc diesen Betrachtungen ist es prinzipiell unwahrscheinlich, dass man mit einem Tritylsalz aus einem - wenn auch Donor-substituierten - Cyclopentadien das entsprechende Kation erzeugen kann. Trotzdem konnten hier nur Experimente letztliche Klarheit schaffen. Deshalb wurde analog zu den von LAMBERT publizierten Protokollen das Pentacyclopropylcyclopentadien (15) in Lösungen von deuteriertem Dichlormethan und Benzol jeweils mit den Tritylsalzen 58 oder 59 bei unterschiedlichen Temperaturen umgesetzt und unmittelbar danach NMR-spektroskopisch untersucht (Schema 23).

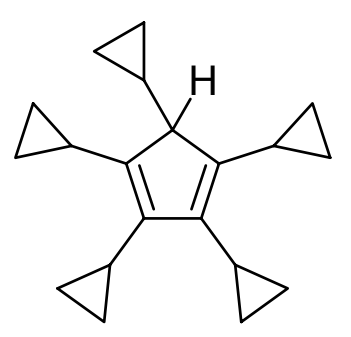

15

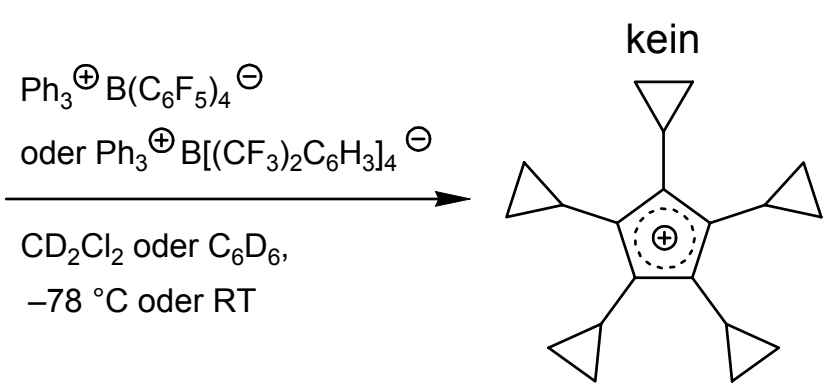

12

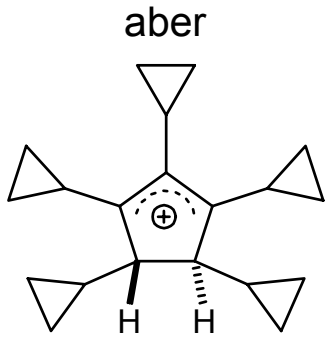

60

Schema 23. Versuchte Erzeugung von 12 durch Hydridabstraktion aus dem Pentacyclopropylcyclopentadien (15) mit Tritylsalzen.

Neben vielen Signalen sowohl im ${ }^{1} \mathrm{H}$ - als auch im ${ }^{13} \mathrm{C}-\mathrm{NMR}-$ Spektrum, die nicht zugeordnet werden konnten, waren charakteristische Signale erkennbar, die auf die Bildung des Pentacyclopropylcyclopentenyl-Kations (60) schließen ließen. Dieses CyclopentenylKation 60 konnte auch schon auf anderem Wege erzeugt und NMR-spektroskopisch vollständig charakterisiert werden. Es entsteht durch Protonierung des Cyclopentadiens 15 mit Tetrafluoroborsäure-Diethylether-Komplex in Deuterodichlormethan als tiefrote Lösung, die bei Raumtemperatur mehrere Minuten stabil ist (Schema 24). 


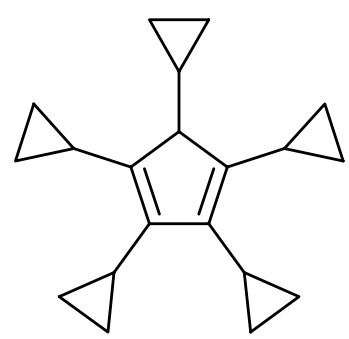

15

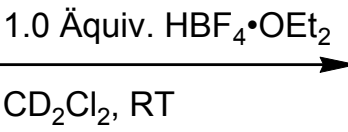

quant.

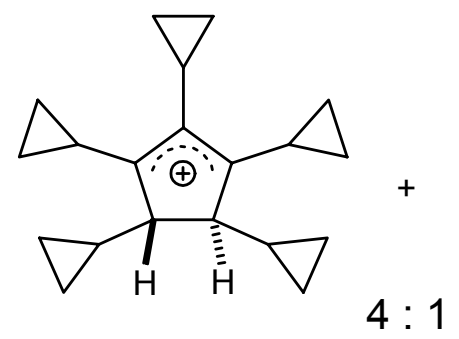

trans-60

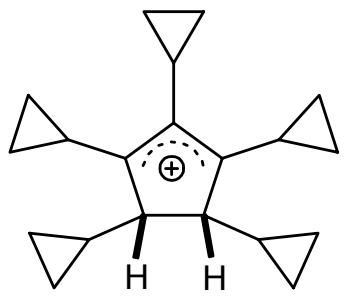

cis-60

Schema 24. Erzeugung des bei Raumtemperatur stabilen Cyclopentenyl-Kations 60 in Form seiner cis/trans-Isomere im Verhältnis 1:4.

Genauso wie bei den von LAMBERT beschriebenen Experimenten konnte hier also auch im Falle des Pentacyclopropylcyclopentadiens 15 lediglich das entsprechende CyclopentenylKation 60 erhalten werden. Eine theoretisch denkbare Erzeugung von 12 durch Hydridabstraktion mittels eines Tritylsalzes war demnach nicht weiter erfolgversprechend.

Als mögliche Kationen-Vorstufe wurde auch das Fulven 35 - durch Hydridabstraktion aus dem Lithiumpentacyclopropylcyclopentadienid erhältlich - in Betracht gezogen, das notwendige elektronische Verhältnisse angenommen - durch Protonierung das gewünschte Pentacyclopropylcyclopentadienyl-Kation ergeben würde (Schema 25).

In früheren Arbeiten konnte jedoch gezeigt werden, dass das 6,6-ethanoverbrückte Tetracyclopropylfulven 35 bei Zugabe von Tetrafluoroborsäure-Diethylether-Komplex nicht an C-6, sondern aufgrund des großen Atomorbitalkoeffizienten des HOMO an C-2 protoniert wird (Schema 25). Das dadurch entstehende Fulvenyl-Kation 61 konnte als intensiv gelbe Lösung in Deuterodichlormethan bei $-50{ }^{\circ} \mathrm{C}$ NMR-spektroskopisch charakterisiert werden und war bei dieser Temperatur über mehrere Stunden hinweg stabil. ${ }^{[36 a, 40]}$ 


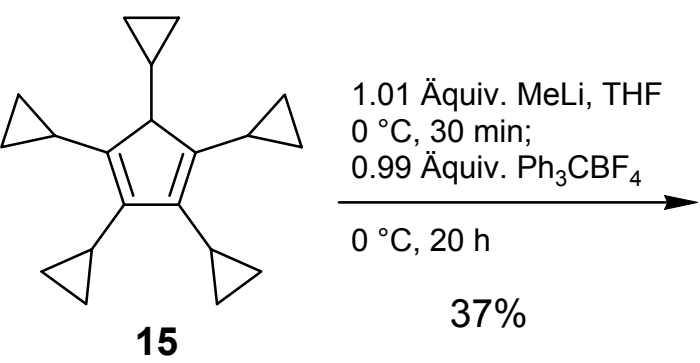

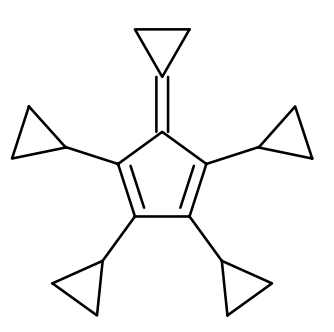

35

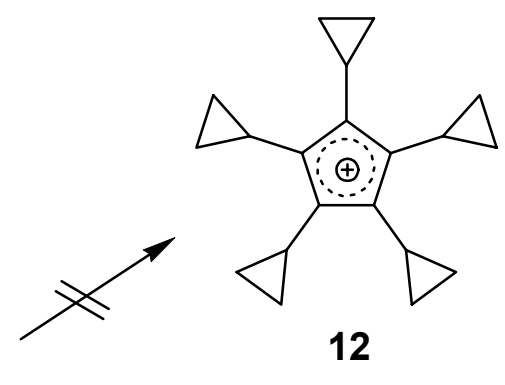

1.0 Äquiv. $\mathrm{HBF}_{4} \cdot \mathrm{Et}_{2} \mathrm{O}$

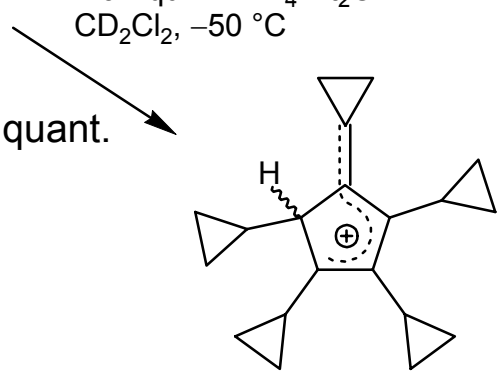

61

Schema 25. Erzeugung des 6,6-Ethano-2,3,4,5-tetracyclopropylfulvenyl-Kations (61) in $\mathrm{CD}_{2} \mathrm{Cl}_{2}$-Lösung bei $-50{ }^{\circ} \mathrm{C}$.

3.5. Chlorpentacyclopropylcyclopentadien (36) als potentielle Vorstufe für das Pentacyclopropylcyclopentadienyl-Kation (12)

An das jetzt leicht zugängliche fünffach Cyclopropyl-substituierte CyclopentadienylGrundgerüst musste offenbar eine geeignete Abgangsgruppe angebracht werden, die einerseits labil genug, aber die Vorstufe noch praktisch handhabbar lassen sollte. Ein Halogenid erschien hier als die beste Abgangsgruppe. Die Kohlenstoff-Fluor-Bindung ist mit ca. $108 \mathrm{kcal} / \mathrm{mol}$ die energiereichste unter den C-Hal-Bindungen und damit relativ schwer zu brechen. JUTZI und SITZMANN verwendeten bei ihren Untersuchungen zum Pentamethyl- bzw. Pentaisopropylcyclopentadienyl-Kation die entsprechenden Bromide, die als recht labile Verbindungen beschrieben wurden. ${ }^{[45,48]}$ Um ein ausgewogenes Verhältnis von Stabilität und Reaktivität der Vorstufe zu erzielen, wurde das Chloridion als Abgangsgruppe angestrebt, dessen Einführung schon bei der von EMME durchgeführten Synthese von Chlortetracyclopropylcyclopentadien erfolgreich verlief. ${ }^{[36 a, 37]}$ Er verwendete $N$-Chlorsuccinimid als Chlor-Quelle, um das Tetracyclopropylcyclopentadienylanion in THF zu chlorieren. 
Mit dieser Methode konnte auch das Chlorpentacyclopropylcyclopentadien als luftempfindliches Öl erfolgreich hergestellt werden (Schema 26).

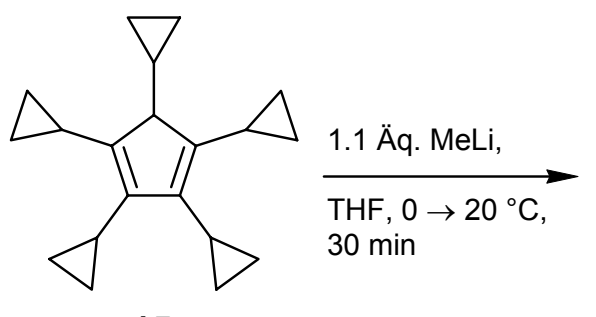

15

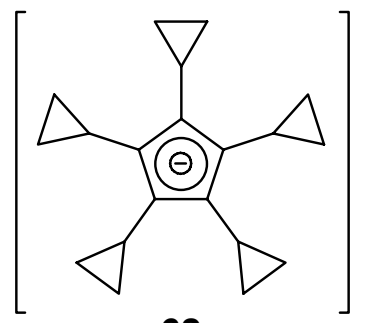

62

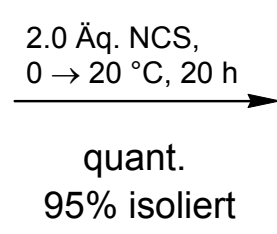

$95 \%$ isoliert

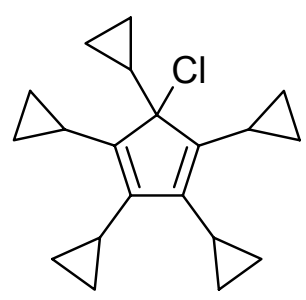

36

Schema 26. Synthese des 5-Chlor-1,2,3,4,5-pentacyclopropylcyclopenta-1,3-diens (36).

Allerdings mussten die Reaktionsbedingungen und die Aufarbeitung der Reaktivität des Cyclopentadienids 62 und der Empfindlichkeit des Chlorids 36 optimal angepasst werden. So gelang die quantitative Synthese von $\mathbf{3 6}$ nur bei Verwendung von Methyllithium als Base und durch Einsatz von zwei Äquivalenten NCS. Nach 20 Stunden wurde das THF im Vakuum entfernt und das Rohprodukt in Pentan suspendiert, wobei das Lithiumsalz des Succinimids und nicht umgesetztes NCS ausfielen. Anschließende Filtration in einer Argonatmosphäre und Entfernen des Lösungsmittels im Vakuum lieferte ein orangegelbes Öl, welches seinen NMRSpektren nach sauberes 36 ohne etwaige Dreiringöffnungsprodukte war. So konnte reines 5-Chlor-1,2,3,4,5-pentacyclopropylcyclopenta-1,3-dien (36) in 95\% Ausbeute isoliert werden (Schema 26, Tabelle 1, Eintrag 10).

Das Chlorid 36 erwies sich in Lösung als nicht sehr stabil. So konnte bei einer Lösung von 36 in $\mathrm{CH}_{2} \mathrm{Cl}_{2}$ nach $5 \mathrm{~h}$ bei $20^{\circ} \mathrm{C}$ das Fulven 64 nachgewiesen werden. Dieses kann sich nur durch eine Cyclopropylcarbinyl-Homoallyl-Umlagerung ${ }^{[74]}$ aus 12 gebildet haben (Schema 27). 
Tabelle 1. Optimierung der Reaktion von 15 mit $N$-Chlorsuccinimid (NCS) in THF.

\begin{tabular}{cccccccc}
\hline Eintrag & $\begin{array}{c}n \text {-BuLi } \\
\text { Äq. }\end{array}$ & Äq. & Äq. & $\begin{array}{c}\text { Nemp. } \\
{\left[{ }^{\circ} \mathrm{C}\right]}\end{array}$ & $\begin{array}{c}\text { Zeit } \\
{[\mathrm{h}]}\end{array}$ & $\begin{array}{c}\text { Umsatz } \\
(\%)\end{array}$ & $\begin{array}{c}\text { Ausbeute } \\
(\%)\end{array}$ \\
\hline 1 & 1.0 & & 1.0 & -29 & 1 & 70 & \\
2 & 1.1 & & 1.1 & -29 & 1 & 75 & \\
3 & 1.1 & & 1.2 & -29 & 2 & 80 & \\
4 & 1.1 & & 1.1 & $-29 \rightarrow 0$ & 2 & 80 & \\
5 & 1.1 & & 1.1 & $-29 \rightarrow 20$ & 20 & 85 & \\
6 & 1.5 & & 2.0 & $-29 \rightarrow 20$ & 20 & 90 & \\
7 & 1.5 & & 3.0 & $-29 \rightarrow 20$ & 20 & 80 & \\
8 & 1.1 & & 1.5 & $-29 \rightarrow 20$ & 40 & 85 & \\
\hline 9 & & 1.1 & 1.1 & $-29 \rightarrow 20$ & 15 & 60 & \\
\hline 10 & & 1.1 & 2.0 & $0 \rightarrow 20$ & 20 & 100 & 95 \\
\hline
\end{tabular}
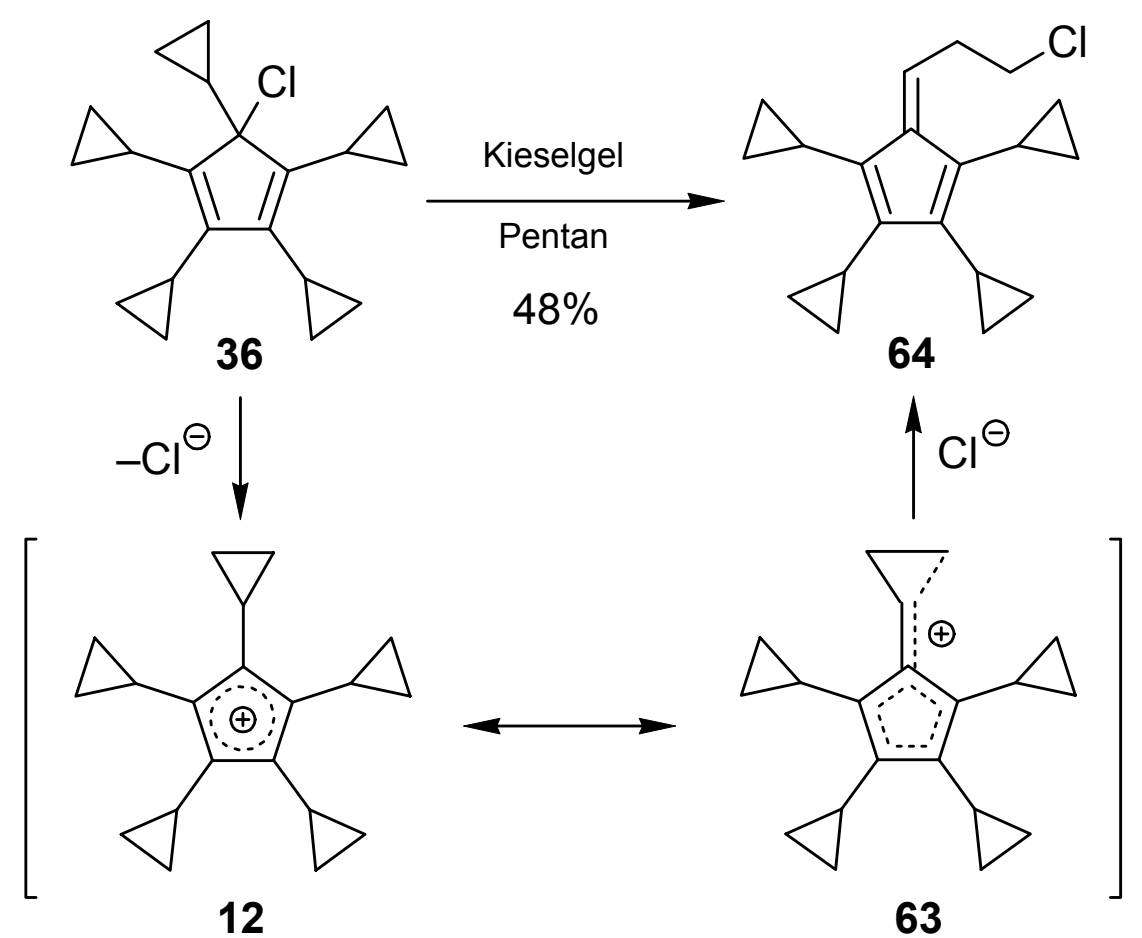

Schema 27. Umlagerung des Pentacyclopropylcyclopentadienylchlorids (36) zum Fulven 64. 
Dabei unterstützt $\mathrm{CH}_{2} \mathrm{Cl}_{2}$ als recht polares Lösungsmittel wahrscheinlich die Heterolyse der C-Cl-Bindung, wobei zwischenzeitlich das Pentacyclopropylcyclopentadienyl-Kation 12 zumindest als Kontaktionenpaar mit Chlorid enstanden sein muss. Eine kationische Cyclopropylcarbinyl-Homoallyl-Umlagerung und nachfolgender Abfang der Zwischenstufe 63 durch das Chloridion führt zum 1-(3'-Chlorpropyliden)-2,3,4,5-tetracyclopropylcyclopenta-2,4-dien (64), das sich präparativ auch durch Filtration des Chlorids 36 über Kieselgel mit Pentan als Eluent in $48 \%$ Ausbeute erhalten lässt. ${ }^{[75]}$

3.6. Versuche zur Erzeugung und Charakterisierung des PentacyclopropylcyclopentadienylKations (12)

Mit dem Cyclopentadienylchlorid 36 als direkte Kationenvorstufe in Händen wurden zunächst Solvolyse-Abfangexperimente mit Nucleophilen durchgeführt, um das intermediär auftretende Pentacyclopropylcyclopentadienyl-Kation in Form seines Substitutionsproduktes von 36 nachzuweisen.

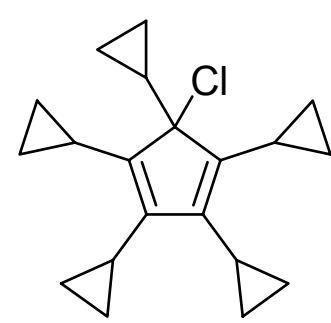

36
1) 2 Äquiv. $\mathrm{AgBF}_{4}$

$\mathrm{CH}_{2} \mathrm{Cl}_{2} / \mathrm{MeOH}(1: 1)$

Pentan, 5 min

2) wässriges $\mathrm{K}_{2} \mathrm{CO}_{3}$

$\mathrm{T}=-78{ }^{\circ} \mathrm{C}, 75 \%$

$\mathrm{T}=0{ }^{\circ} \mathrm{C}, 72 \%$

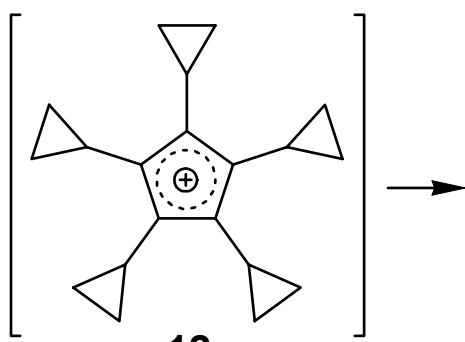

12

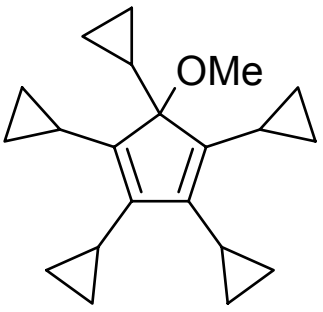

65

Schema 28. Silbertetrafluoroborat-assistierte Methanolyse des Pentacyclopropylcyclopentadienylchlorids (36).

In der Tat konnte 12 als Zwischenstufe dieser $\mathrm{S}_{\mathrm{N}} 1$-Reaktion indirekt nachgewiesen werden, als man bei $-78^{\circ} \mathrm{C}$ eine Lösung von 36 in Pentan zu einer Suspension von Silbertetrafluoroborat in Dichlormethan/Methanol (1:1) tropfte (Schema 28). Nach fünf Minuten wurde mit Kaliumcarbonat-Lösung aufgearbeitet, und nach säulenchromatographischer Reinigung des Rohproduktes erhielt man mit über 70\% Ausbeute den erwarteten Methylether 
65 mit völlig intaktem Kohlenstoffgerüst. Auch bei $0{ }^{\circ} \mathrm{C}$ durchgeführt ergab diese Solvolyse das Methoxycyclopentadien 65 in über 70\% Ausbeute.

Das alleinige Auftreten des Methoxycyclopentadiens 65 als Abfangprodukt ohne weitere Umlagerungsprodukte selbst bei $0{ }^{\circ} \mathrm{C}$ lässt darauf schließen, dass das Pentacyclopropylcyclopentadienyl-Kation unter diesen Bedingungen hinreichend stabil ist, um vom Methanol im Sinne einer $\mathrm{S}_{\mathrm{N}} 1$-Reaktion nucleophil abgefangen $\mathrm{zu}$ werden. Das Abfangen des Kations verläuft offenbar rascher als die damit prinzipiell in Konkurrenz stehende Cyclopropylcarbinyl-Homoallyl-Umlagerung. Dies würde bedeuten, dass das Cyclopentadienyl-Kation durch die fünf Cyclopropyl-Substituenten am Cyclopentadien-Ring in ausreichendem Maße stabilisiert wird. Der Methylether 65 kann dagegen nicht durch eine $\mathrm{S}_{\mathrm{N}} 2$-Reaktion entstanden sein, bei der nur das Methanol den Chlor-Substituenten durch einen Rückseitenangriff verdrängt haben müsste. Ein solcher $\mathrm{S}_{\mathrm{N}} 2$-artiger Angriff wäre an eben diesem quartären $\mathrm{sp}^{3}$-Kohlenstoffzentrum nicht bevorzugt. Eine Assistenz der LEWIS-sauren Silberionen muss demnach im ersten Schritt der Solvolyse involviert gewesen sein.

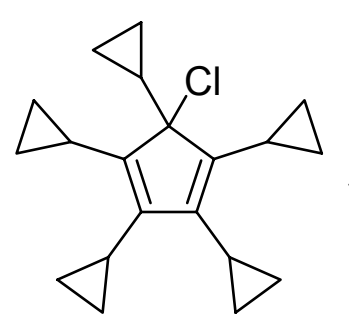

36
1) 2 Äquiv. $\mathrm{AgBF}_{4}$ $\mathrm{CH}_{2} \mathrm{Cl}_{2}$, Pentan $-78^{\circ} \mathrm{C}, 1 \mathrm{~min}$

2) $\mathrm{MeOH}$ wässriges $\mathrm{K}_{2} \mathrm{CO}_{3}$

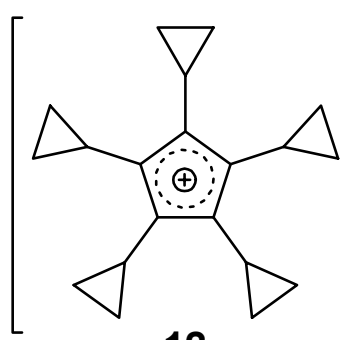

12

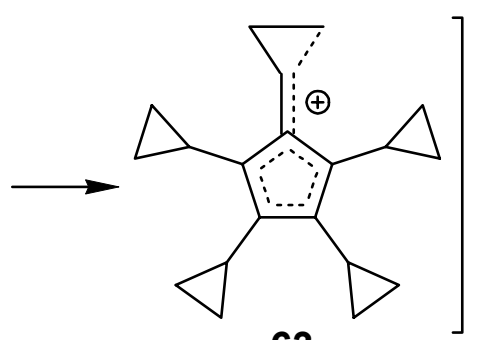

63<smiles>ClCCC=C1C(C2CC2)=C(C2CC2)C(C2CC2)=C1C1CC1</smiles>

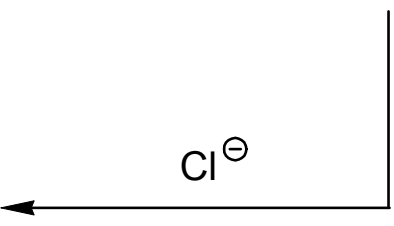

$18 \%$

64

Schema 29. Silbertetrafluoroborat-assistierte Solvolyse des Pentacyclopropylcyclopentadienylchlorids (36) mit Abfang des intermediären Kations durch Nucleophile. 
Die Behandlung des Chlorcyclopentadiens 36 mit Silbertetrafluoroborat in Dichlormethan/Pentan bei $-78{ }^{\circ} \mathrm{C}$ und erst darauffolgende Aufarbeitung mit Methanol und Kaliumcarbonat ergab neben einer großen Menge nicht identifizierten Materials in 18\% Ausbeute das schon bekannte Fulven 64 - das Produkt einer Cyclopropylcarbinyl-HomoallylUmlagerung (Schema 29). In Abwesenheit von Methanol als Lösungsmittel und Nucleophil wird das Pentacyclopropylcyclopentadienyl-Kation demnach zunächst ebenfalls gebildet, kann sich dann aber umlagern, wenn es nicht unmittelbar abgefangen wird.

Die am häufigsten verwendeten Reagenzien für die Erzeugung von Carbokationen sind klassischerweise die Brönsted-Säuren Fluorsulfonsäure $\left(\mathrm{FSO}_{3} \mathrm{H}\right)$ und Fluorwasserstoff $(\mathrm{HF})$, die LEWIS-Säure Antimon(V)fluorid $\left(\mathrm{SbF}_{5}\right)$ oder deren Mischungen, sogenannte magische Säuren. Für die Darstellung von stabilen Carbokationen in Lösung ist nicht allein die Säurestärke der ionisierenden Reagenzien entscheidend, sondern auch eine möglichst geringe Basizität und Nucleophilie der aus den eingesetzten Protonen- oder LEWIS-Säuren stammenden Gegenionen und des verwendeten Lösungsmittels. Letzteres sollte eine möglichst hohe Dielektrizitätskonstante aufweisen. Vor allem Sulfurylchloridfluorid $\mathrm{SO}_{2} \mathrm{ClF}$ (Sdp. $7{ }^{\circ} \mathrm{C}$, Schmp. $-125^{\circ} \mathrm{C}$ ) oder Sulfuryldifluorid $\mathrm{SO}_{2} \mathrm{~F}_{2}\left(\mathrm{Sdp} .-55^{\circ} \mathrm{C}\right.$, Schmp. $-136{ }^{\circ} \mathrm{C}$ ), Schwefeldioxid $\mathrm{SO}_{2}$ und auch das praktisch einfacher handhabbare Dichlormethan (meist deuteriert, $\mathrm{CD}_{2} \mathrm{Cl}_{2}$ ) werden häufig verwendet. In diesen Lösungsmitteln ist bei Verwendung von $\mathrm{SbF}_{5}$ als LEWIS-Säure die Bildung von oligomeren Anionen des Typs $\left[\mathrm{Sb}_{\mathrm{x}} \mathrm{F}_{5 \mathrm{x}} \mathrm{Cl}\right]^{-}$ begünstigt (Schema 30; im Gegensatz zu SbCl 5 , das in Dichlormethan stabile, lagerfähige Lösungen bildet, kann $\mathrm{SbF}_{5}$ nicht zusammen mit $\mathrm{CH}_{2} \mathrm{Cl}_{2}$ verwendet werden, da sich dabei sehr schnell das Chlormethyl-Kation bildet, das oberhalb von $-50{ }^{\circ} \mathrm{C}$ zu einer Zersetzung der Lösung führt). ${ }^{[20]}$

$$
\mathrm{R}-\mathrm{Cl}+\left(\mathrm{SbF}_{5}\right)_{\mathrm{x}} \longrightarrow \mathrm{R}^{\oplus}\left[\mathrm{Sb}_{\mathrm{x}} \mathrm{F}_{5 \mathrm{x}} \mathrm{Cl}\right]^{\ominus}
$$

Schema 30. Bildung oligomerer Antimonhalogenid-Anionen. 
Je nach Stabilität des Carbokations muss zu dessen vollständiger Bildung $\mathrm{SbF}_{5}$ in bis zu zehnfachem Überschuss eingesetzt werden. Geringere Konzentrationen können die Bildung von Chloronium-Ionen begünstigen. ${ }^{[76]}$

Ein optimal geeignetes, schwach koordinierendes Gegenion für reaktive Kationen sollte idealerweise eine möglichst geringe Nucleophilie und Basizität aufweisen. ${ }^{[77]}$ Anfang der 70er Jahre veröffentlichten RosenTHAL et al. Struktur- und spektroskopische Daten aus denen hervorging, dass Anionen wie Perchlorat $\left(\mathrm{ClO}_{4}^{-}\right)$, Nitrat $\left(\mathrm{NO}_{3}{ }^{-}\right)$oder Tetrafluoroborat $\left(\mathrm{BF}_{4}^{-}\right)$ in wässriger Lösung nicht koordinierend sind, aber bei Ausschluss von Wasser deutliche Wechselwirkungen mit Kationen eingehen können. ${ }^{[78]}$ Weiterhin wäre es von Vorteil, wenn das Anion geeignete spektroskopisch erfassbare Charakteristika besitzen und dem reaktiven Kation gute Löslichkeits- und Kristallisationseigenschaften verleihen würde. In diesem Zusammenhang haben aufgrund ihrer Größe, der Abwesenheit freier Elektronenpaare und ihrer extremen chemischen Inertheit fluorierte Tetraphenylborate und Carborane große Aufmerksamkeit gewonnen. Sie waren für viele Anwendungen Mittel der Wahl. So konnten die Katalysekraft LEWIS-saurer Lithiumionen gesteigert, ${ }^{[79]}$ neuartige „starke und zugleich milde“ Supersäuren entwickelt, ${ }^{[80]}$ Polymerisations-Katalysatoren für Olefine kommerziell zugänglich gemacht ${ }^{[77 \mathrm{a}]}$ und ungewöhnlich reaktive Kationen wie $\mathrm{C}_{60}{ }^{\bullet+}, \mathrm{Bu}_{3} \mathrm{Sn}^{+}$oder $\mathrm{Cu}(\mathrm{CO})_{4}{ }^{+}$isoliert werden. ${ }^{[81]}$

Erst kürzlich konnten REED et al. mit Hilfe der extrem inerten Carboran-Anionen $\left[\mathrm{CB}_{11} \mathrm{H}_{6} \mathrm{Br}_{6}\right]^{-}$bzw. $\left[\mathrm{CHB}_{11} \mathrm{Me}_{5} \mathrm{Br}_{6}\right]^{-[82]}$ das Silyl-substituierte Vinyl-Kation 66 und diverse tertiäre Alkyl-Kationen, wie beispielsweise 67, kristallin gewinnen und durch Röntgenstrukturanalysen charakterisieren (Schema 31).

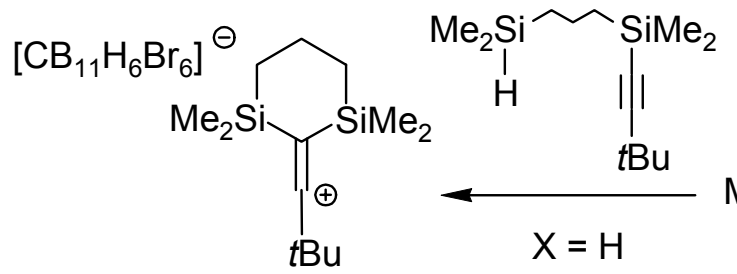

66<smiles>CC(C)[Mg]Br</smiles>

67

Schema 31. Kristalline Carbokationen mit Carboran-Anionen. 
In Zusammenarbeit mit der Arbeitsgruppe von REED wurde versucht das Pentacyclopropylcyclopentadienylchlorid (36) mit dem Silbersalz des Carborans $\mathrm{Ag}^{+}\left[\mathrm{CHB}_{11} \mathrm{Me}_{5} \mathrm{Br}_{6}\right]^{-}$in verschiedenen Lösungsmitteln umzusetzen. Dabei konnte das Pentacyclopropylcyclopentadienyl-Kation aber nicht isoliert werden.

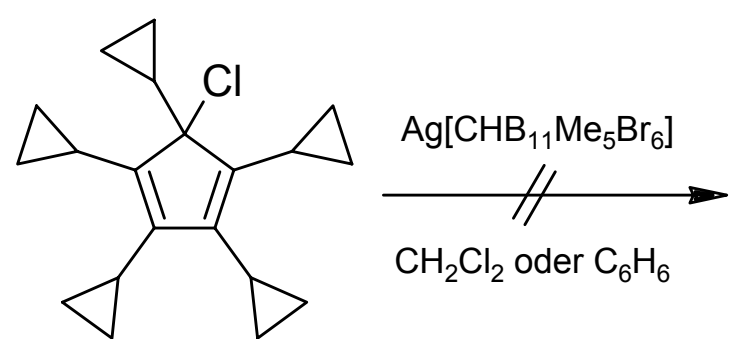

36

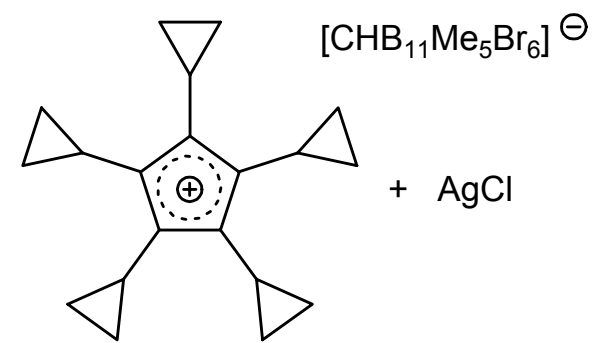

12

Schema 32. Versuch zur Darstellung des Pentacyclopropylcyclopentadienyl-Kations 12 mit Carboran-Gegenion.

Sowohl die eingangs aufgeführten theoretischen Betrachtungen als auch die durchgeführten Abfangexperimente gaben begründete Hinweise darauf, dass das Pentacyclopropylcyclopentadienyl-Kation (12) für eine Darstellung und spektroskopische Charakterisierung ausreichend stabil sein müsste. Andererseits wiesen die Erfahrungen der ersten Versuceh zur Präparation und zum spektroskopischen Nachweis darauf hin, dass dies wohl nicht auf Anhieb bei normalen Temperaturen und unter Schlenk-Bedingungen gelingen würde.

Um einen adäquaten spektroskopischen Hinweis auf die potentielle Stabilisierung des antiaromatischen Cyclopentadienyl-Kations durch Cyclopropyl-Substituenten zu erhalten, wurde in Zusammenarbeit mit der Arbeitsgruppe SIEHL ${ }^{[83]}$ an der Universität Ulm versucht, 12 unter klassischen „stable cation conditions“ herzustellen und NMR-spektroskopisch zu charakterisieren. ${ }^{[84]}$ 


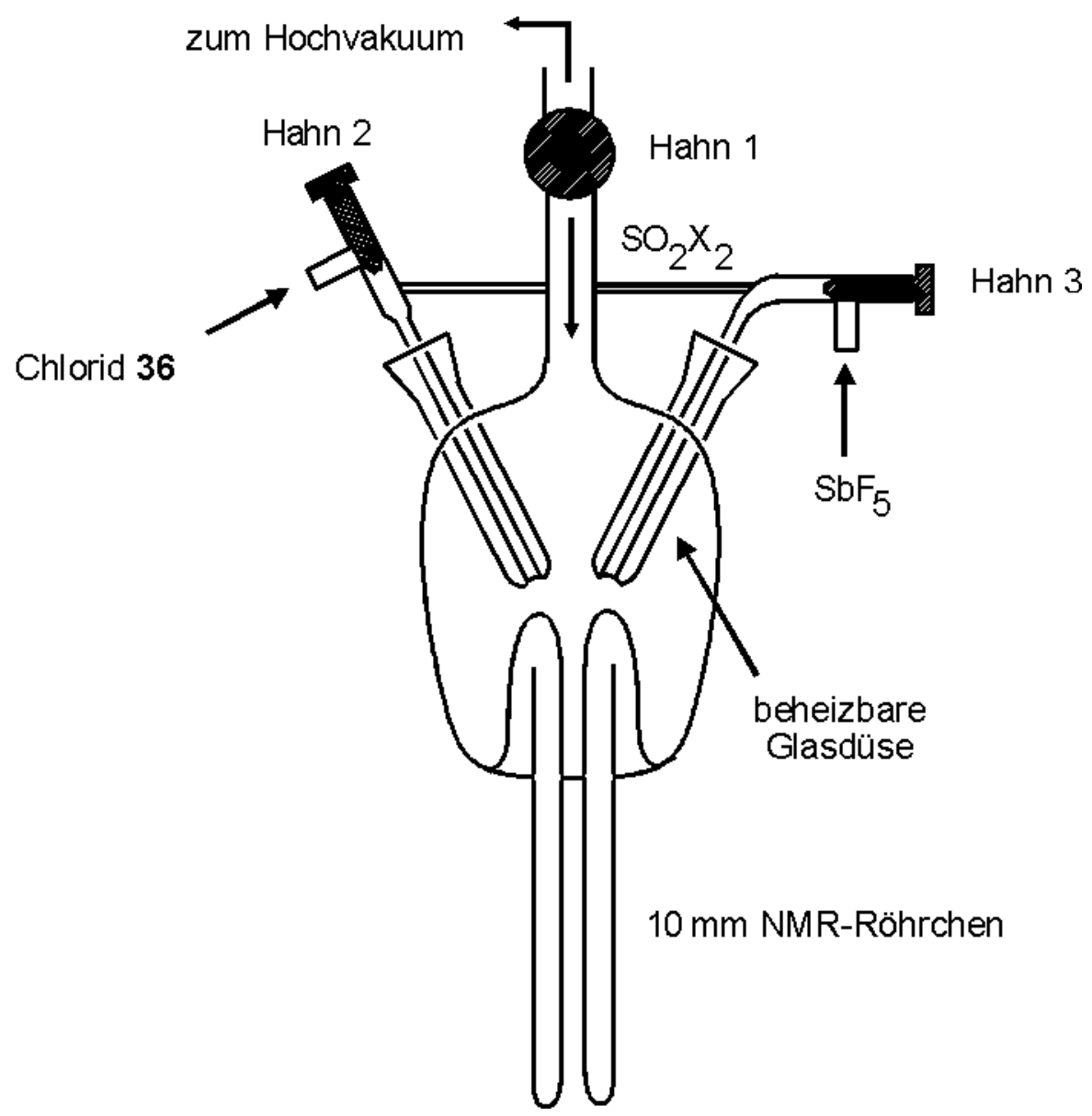

Abbildung 12. Apparat zur Darstellung von Carbokationen unter ,stable cation conditions“.

Dazu wurde eine Modifikation der Hochvakuum-Matrix-Cokondensationstechnik ${ }^{[85]}$ verwendet, bei der die Kationenvorstufe 36 auf dem Boden des Glasreaktors (Abbildung 12) vorgelegt wurde und im Hochvakuum durch dosiertes Einkondensieren von abwechselnd Lösungsmittel und LEWIS-Säure eine Matrix bestehend aus Schichten des Chlorids 36 (zuunterst), $\mathrm{SbF}_{5}$ und einem Gemisch aus $\mathrm{SO}_{2} \mathrm{ClF}$ und $\mathrm{SO}_{2} \mathrm{~F}_{2}$ im Verhältnis 2:1 bei $-196{ }^{\circ} \mathrm{C}$ erzeugt wurde. Eine solche Mischung bildet ein bei $-142{ }^{\circ} \mathrm{C}$ schmelzendes Eutektikum, das eine Untersuchung der Kationenprobe unterhalb der Schmelztemperaturen von $\mathrm{SO}_{2} \mathrm{ClF}$ $\left(-125{ }^{\circ} \mathrm{C}\right)$ und $\mathrm{SO}_{2} \mathrm{~F}_{2}\left(-136{ }^{\circ} \mathrm{C}\right)$ ermöglicht. Durch Homogenisierung dieser Matrix bei $-125{ }^{\circ} \mathrm{C}$ wurde eine tiefrote viskose Kationenlösung erhalten, die sofort in ein an den Glasreaktor angeschmolzenes und vorgekühltes $10 \mathrm{~mm}$-NMR-Rohr überführt wurde. Mit seinem größeren Durchmesser hat das NMR-Rohrs den Vorteil einer größeren Wärmekapazität, wodurch es bei den entsprechenden Arbeitsschritten weniger schnell auftaut. 
Die Menge der Reagenzien wurde so gewählt, dass die Konzentration der Kationenvorstufe und demnach auch des Carbokations - in der erhaltenen Lösung typischerweise ca. $0.15 \mathrm{~mol} / \mathrm{L}$ betrug. Nach Einfrieren der NMR-Probe im Hochvakuum wurde das Röhrchen vom Reaktor abgeschmolzen und weiter in flüssigem Stickstoff gelagert.

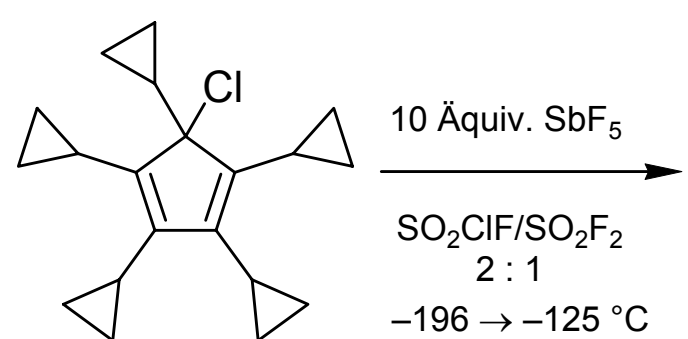

36

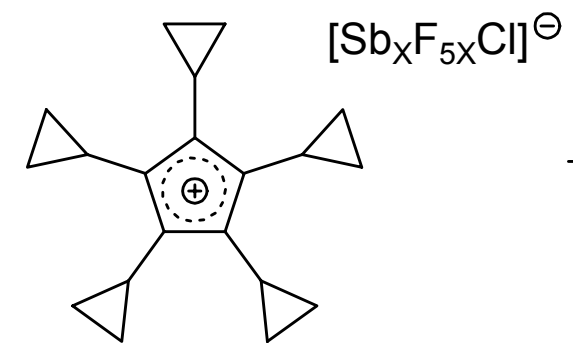

12

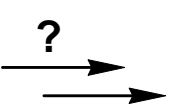

$$
\begin{gathered}
\text { tiefrote, } \\
\text { viskose Lösung }
\end{gathered}
$$

Schema 33. Erzeugung des Pentacyclopropylcyclopentadienyl-Kations 12 unter ,stable cation conditions“.

Für die NMR-spektroskopischen Experimente wurden die Kationenproben jeweils im tiefgefrorenen Zustand in das vorher temperierte NMR-Gerät (Bruker DRX $400 \mathrm{MHz}$ ) eingebracht und in einem Temperaturbereich von -140 bis $-110^{\circ} \mathrm{C}$ untersucht. Zur Bestimmung der Temperatur im NMR-Gerät wurde zuvor eine Kalibrierkurve mit Hilfe der Temperaturabhängigkeit der chemischen Verschiebung der Kohenstoffatome C-3 und C-4 von 2-Chlorbutan ermittelt, das in einer Kapillare in $\mathrm{SO}_{2} \mathrm{ClF}$ verwendet wurde. Der ${ }^{19} \mathrm{~F}$-LockKanal des NMR-Geräts wurde auf die Frequenz von $\mathrm{SO}_{2} \mathrm{ClF}$ abgestimmt. 


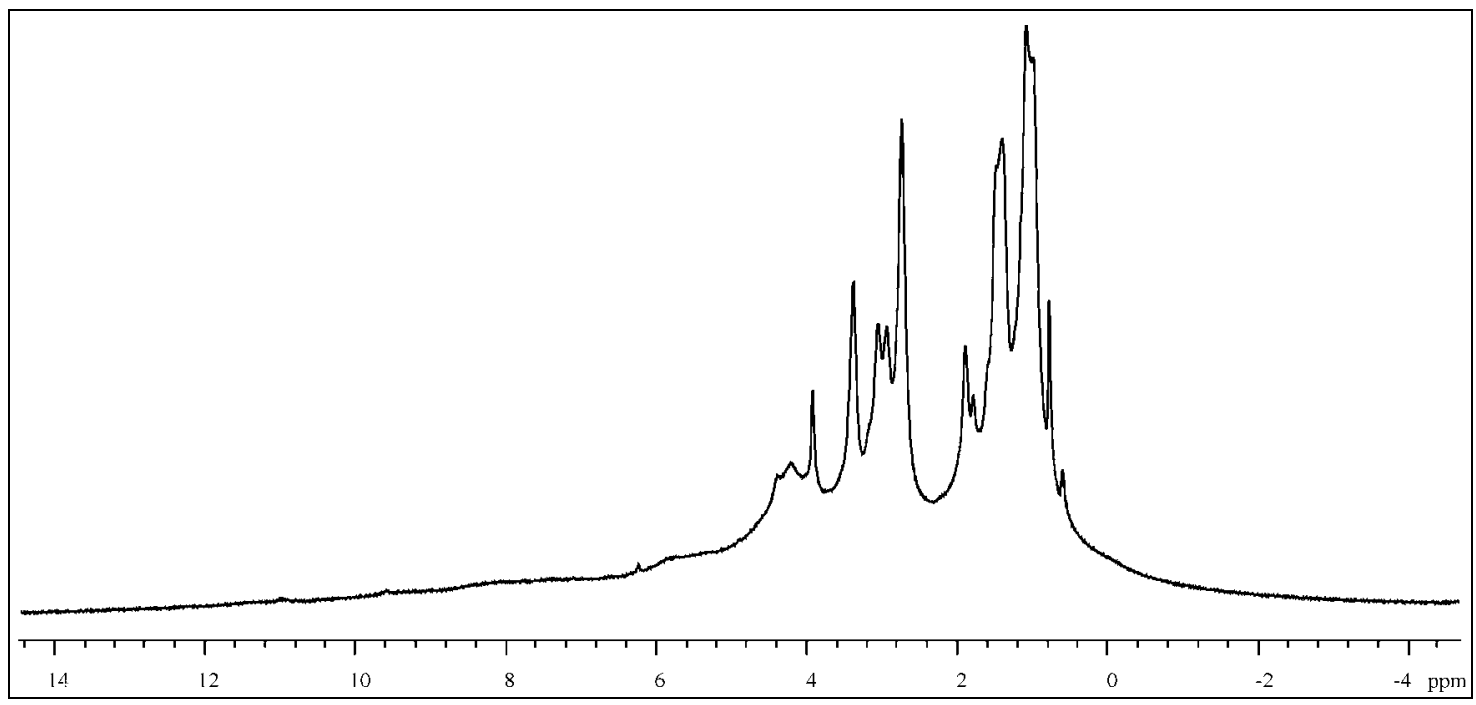

Abbildung 13. ${ }^{1} \mathrm{H}-$ Spektrum (400 MHz) einer typischen Kationenprobe bei $-110^{\circ} \mathrm{C}$.

Eine so hergestellte Kationenprobe wies bei $-140{ }^{\circ} \mathrm{C}$ ein ${ }^{1} \mathrm{H}-\mathrm{NMR}-\mathrm{Spektrum}$ mit breiten Multiplettsignalen auf, die beim Aufwärmen des NMR-Röhrchens auf $-110{ }^{\circ} \mathrm{C}$ in Schritten von $10 \mathrm{~K}$ zunehmend schärfer wurden und teilweise in diskrete Linien übergingen (Abbildung 13). Diese Linienverbreiterung bei niedrigerer Temperatur lässt sich auf die hohe Viskosität der Lösung zurückführen. Die erste Gruppe hochfeldverschobener Signale liegt zwischen 0.1 und 1.9 ppm und die zweite tieffeldverschobene Gruppe liegt zwischen 2.1 und 4.2 ppm. Diese Signale sollten ausschließlich von Cyclopropyl-Wasserstoffatomen stammen. Die leicht erhöhte Basislinie kann durch geringe Verunreinigungen erklärt werden, die durch Zersetzung des Kations auftreten könnten. Möglicherweise entstehen diese schon während der Präparation oder auch später während der NMR-Messungen. Das Vorhandensein von Ringöffnungsprodukten, wie dem schon bekannten Fulven 64, kann dagegen ausgeschlossen werden, da die zu erwartenden entsprechenden Signale nicht detektiert werden konnten. 


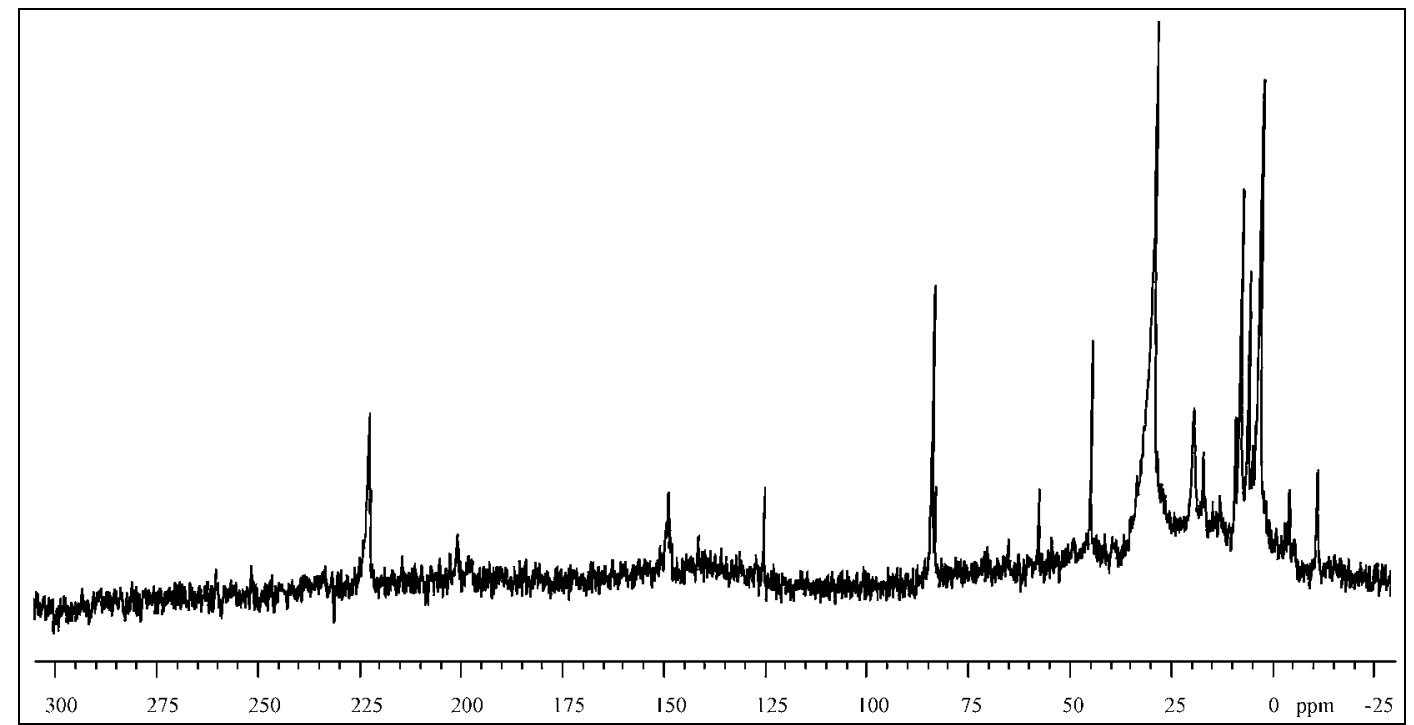

Abbildung 14. ${ }^{13} \mathrm{C}-\mathrm{NMR}-$ Spektrum $(100 \mathrm{MHz})$ einer typischen Kationenprobe bei $-115{ }^{\circ} \mathrm{C}$.

Auf den ersten Blick zeigte das ${ }^{13} \mathrm{C}$-NMR-Spektrum bei $-115{ }^{\circ} \mathrm{C}$ im wesentlichen zwölf Signale, die eine signifikante Linienbreite von durchschnittlich $100 \mathrm{~Hz}$ aufwiesen (Abbildung 14). Bei diesen konnte zwischen hochfeldverschobenen Linien bei 2.9, 5.5, 7.5, 16.5, 19.0 und $29.2 \mathrm{ppm}$ und tieffeldverschobenen Peaks bei 82.3, 83.2, 148.3, 149.2, 221.9 und 222.6 ppm unterschieden werden. Letztere traten jeweils in Form eines intensiven Signals mit einer kleineren Schulter als Paar auf. Einige wenige zusätzliche Signale rührten wahrscheinlich von den schon genannten möglichen Verunreinigungen her. Die Zuordnung der Signale konnte mit Hilfe von DEPT135 (distortionsless enhancement by polarization transfer, Pulswinkel $135^{\circ}$ ) und durch gekoppelte ${ }^{13} \mathrm{C}$-NMR-Spektren vorgenommen werden (Abbildungen 15 und 16). Die tieffeldverschobenen Peaks wiesen im gekoppelten ${ }^{13} \mathrm{C}-\mathrm{NMR}$ Spektrum keine Multiplettaufspaltung auf und im entsprechenden DEPT135-Spektrum, beide von derselben Probe aufgenommen, waren sie nicht zu detektieren. Somit wären sämtliche Peaks von 82 bis $222 \mathrm{ppm}$ auf quartäre Kohlenstoffatome im Cyclopentadienyl-Gerüst zurückzuführen. Da diese daher auch zur selben funktionellen Einheit gehören, war es unter Annahme ähnlicher Relaxationszeiten dieser quartären C-Atome legitim, eine Integration durchzuführen. Diese lieferte ein ungefähres Verhältnis von 2 C-Atomen für die Signale bei 222 ppm, 1 C-Atom für die bei 149 ppm und 2 C-Atome für die bei 83 ppm - oder ein entsprechendes Vielfaches davon. In den DEPT135-Spektren konnte auch gezeigt werden, 
dass die Signale bei 2.9 und 7.5 ppm den $\mathrm{CH}_{2}$-Gruppen und die Linien bei 5.5, 16.5, 19.0 und 29.2 ppm den CH-Gruppen der Cyclopropanringe zugeordnet werden können. Diese Signale zeigen jeweils sowohl die charakteristische Triplett- als auch Dublett-Signalaufspaltung im gekoppelten ${ }^{13} \mathrm{C}$-NMR-Spektrum.

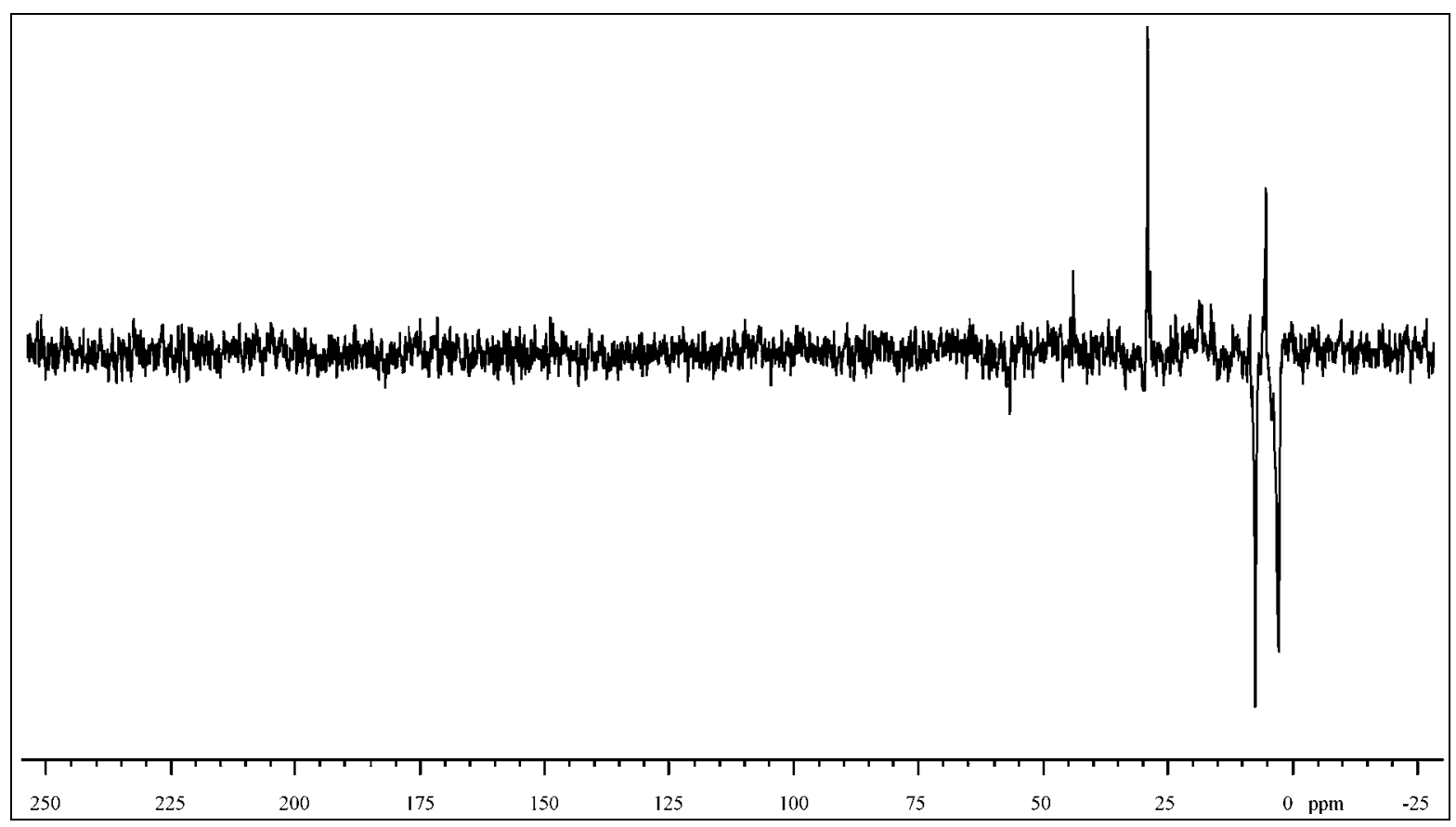

Abbildung 15. DEPT135-Spektrum (100 MHz) bei $-115{ }^{\circ} \mathrm{C}$.

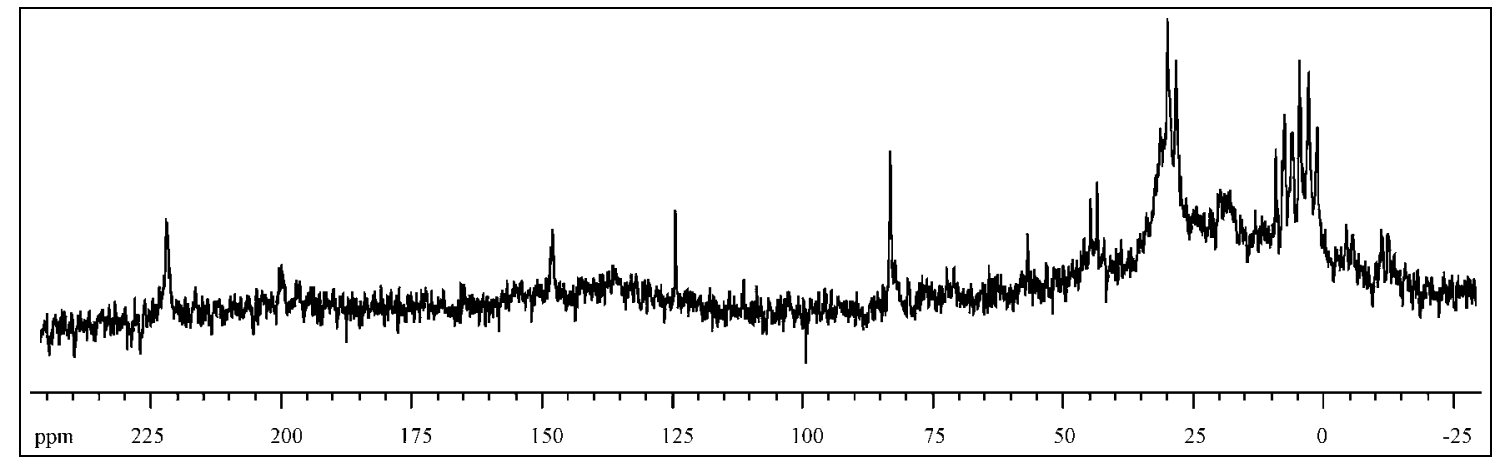

Abbildung 16. Gekoppeltes ${ }^{13} \mathrm{C}-\mathrm{NMR}$-Spektrum $(100 \mathrm{MHz})$ bei $-115{ }^{\circ} \mathrm{C}$.

Diese Beobachtungen ließen erste Überlegungen zur Struktur des Kations zu. Das Cyclopentadien-Gerüst müsste hier erhalten geblieben sein, da ein Zerfall des Fünfringes zu linearen Strukturen führen würde, an deren Enden zusätzlich tertiäre C-Zentren auftreten müssten. Solche Fragmente wiesen auch eine niedrigere Symmetrie auf. Zum einen muss aber 
das hier beobachtete Kation mindestens eine Spiegelebene $\sigma_{\mathrm{v}}$ besitzen, die durch ein C-Atom des Fünfring-Gerüstes verläuft, d. h. das Kation muss $C_{\mathrm{S}}$-Symmetrie aufweisen. Die Peaks bei 222 ppm könnten dabei zu den C-Atomen gehören, die vorwiegend die positive Ladung tragen und dadurch stark entschirmt würden. Charakteristisch ist auch die chemische Verschiebung des Signals bei 29 ppm, die in dem typischen Bereich für solche CyclopropylSubstituenten liegt, die eine positive Ladung in ihrer Nachbarschaft hyperkonjugativ stabilisieren und dadurch selbst zum tieferen Feld verschoben werden.

In einem aufgenommenen $\mathrm{C}, \mathrm{H}-$ Korrelationsspektrum (HSQC) konnten Kreuzpeaks detektiert werden, die eine Kopplung zwischen den Signalen der ${ }^{13} \mathrm{C}-\mathrm{CH}-$ Gruppen bei 5.5, 16.5, 19.0 und $29.2 \mathrm{ppm}$ und den ${ }^{1} \mathrm{H}$-Signalen bei 1.4, 1.6 und $2.9 \mathrm{ppm}$ aufzeigen. Zwischen den ${ }^{13} \mathrm{C}_{-} \mathrm{CH}_{2}$-Gruppen bei 2.9 und 7.5 ppm und den ${ }^{1} \mathrm{H}$-Signalen bei 0.6 und $1.3 \mathrm{ppm}$ wurden ebenfalls Kopplungen gemessen, was darauf hinweist, dass es sich im ${ }^{1} \mathrm{H}-\mathrm{NMR}-\mathrm{Spektrum}$ tatsächlich um cyclopropylische Protonensignale handeln musste.

Um auch eine direkte Beziehung zwischen den beobachteten ${ }^{1} \mathrm{H}$-Signalen der cyclopropylischen Wasserstoffatome untereinander $\mathrm{zu}$ bestimmen, wurde versucht, $\mathrm{H}, \mathrm{H}-$ Korrelationsspektren aufzunehmen. Jedoch führte keine der angewendeten Aufnahmetechniken zum Erfolg. Immerhin wies dies darauf hin, dass das System von Wasserstoffatomen sehr schnell relaxieren muss, was eine Bestimmung der H,H-Kopplungen verhinderte.

Nach jedem NMR-Experiment wurde ein ${ }^{1} \mathrm{H}$-Spektrum aufgenommen, um die Stabilität und Qualität der Kationenprobe während der Messungen zu verfolgen. Nach 3 Stunden konnten aber nur geringe Veränderungen festgestellt werden. Nach bis zu 6 Stunden Messzeit, je nach Konzentration der Probe und untersuchtem Temperaturbereich, waren die ursprünglich aufgezeichneten Signale fast vollständig verschwunden, so dass keine weiteren zuverlässigen Messungen möglich waren. Aus dieser Beobachtung ließ sich eine 
Halbwertszeit von ungefähr vier Stunden bei $-125{ }^{\circ} \mathrm{C}$ für das untersuchte Carbokation abschätzen.

Einige der vermessenen NMR-Röhrchen wurden repräsentativ für die durchgeführten NMR-Experimente zu Abfangexperimenten herangezogen. Dazu wurden die tiefgefrorenen 10 mm-Röhrchen jeweils am oberen Ende aufgebrochen und der langsam schmelzende Inhalt in einer Schutzgasatmosphäre bei $-90{ }^{\circ} \mathrm{C}$ in eine intensiv gerührte Suspension von Kaliumcarbonat in Methanol getropft. Nach wässriger Aufarbeitung konnte anhand von ${ }^{1} \mathrm{H}-$ und ${ }^{13} \mathrm{C}-\mathrm{NMR}-$ Spektren (Abbildungen 17 und 18) das schon bekannte Methoxycyclopentadien 65 identifiziert werden, und es war in dem erhaltenen Rohprodukt zu ca. 23\% enthalten (Schema 34).

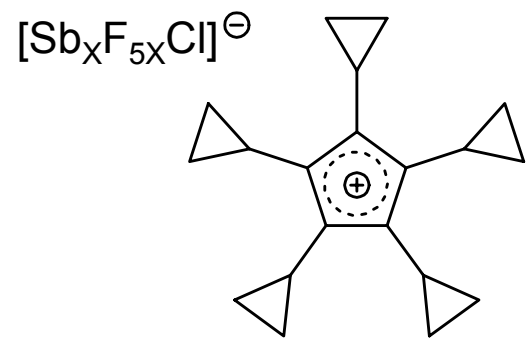

12

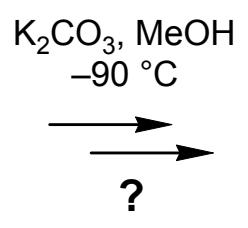

tiefrote, viskose Lösung

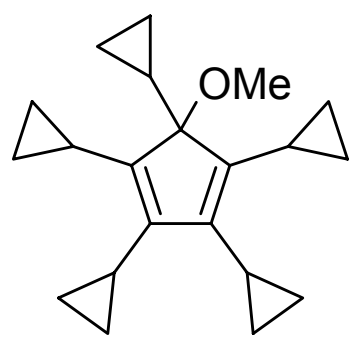

65

Schema 34. Abfangexperiment einer repräsentativen NMR-Probe mit $\mathrm{K}_{2} \mathrm{CO}_{3} / \mathrm{Methanol}$.

Dieser Methylether 65 kann prinzipiell auf drei Arten entstanden sein. Denkbar wäre erstens eine Reaktion mit noch verhandenem, unvollständig ionisiertem Pentacyclopropylcyclopentadienylchlorid 36. Wie aber in Kapitel 4.6 erläutert, kann die Reaktion nicht nach einem $\mathrm{S}_{\mathrm{N}} 2$-Mechanismus stattfinden (tertiäres Reaktionszentrum), sondern nur nach $\mathrm{S}_{\mathrm{N}} 1$. Außerdem wurde bei der Bildung der Kationenlösung auf eine Homogenisierung der Mischung geachtet, so dass keine Partikel ungelöst und damit unumgesetzt im Glasreaktor verblieben. In den aufgenommenen NMR-Spektren konnten auch keine charakteristischen Linien für eventuell noch vorhandenes Chlorid 36 erkannt werden. Weiterhin wäre eine $\mathrm{S}_{\mathrm{N}} 1$ - 
Reaktion genau gleichbedeutend mit der Herstellung der carbokationischen Zwischenstufe mit Hilfe einer starken LEWIS-Säure als erstem (geschwindigkeitsbestimmenden) Schritt der Reaktion und Abfang des Kations durch Methanol im zweiten raschen Schritt - eben die beschriebene Präparation unter „stable cation conditions“, die NMR-spektroskopische Untersuchung der so „gefangenen“ oder ,isolierten“ Kationen-Zwischenstufe und deren nachfolgender chemischer Nachweis durch diese Abfangexperimente. Das Abfangprodukt kann also zweitens direkt aus der Reaktion des Pentacyclopropylcyclopentadienyl-Kations (12) mit Methanol hervorgegangen sein. Als dritte Möglichkeit kommt eine Umwandlung des ursprünglich entstandenen Kations in eine weitere Zwischenstufe in Frage (bespielsweise ein Dimer), die in einer dynamischen Beziehung mit dem PentacyclopropylcyclopentadienylKation steht und bei Reaktion mit Methanol das monomere Abfangprodukt 65 freisetzt.

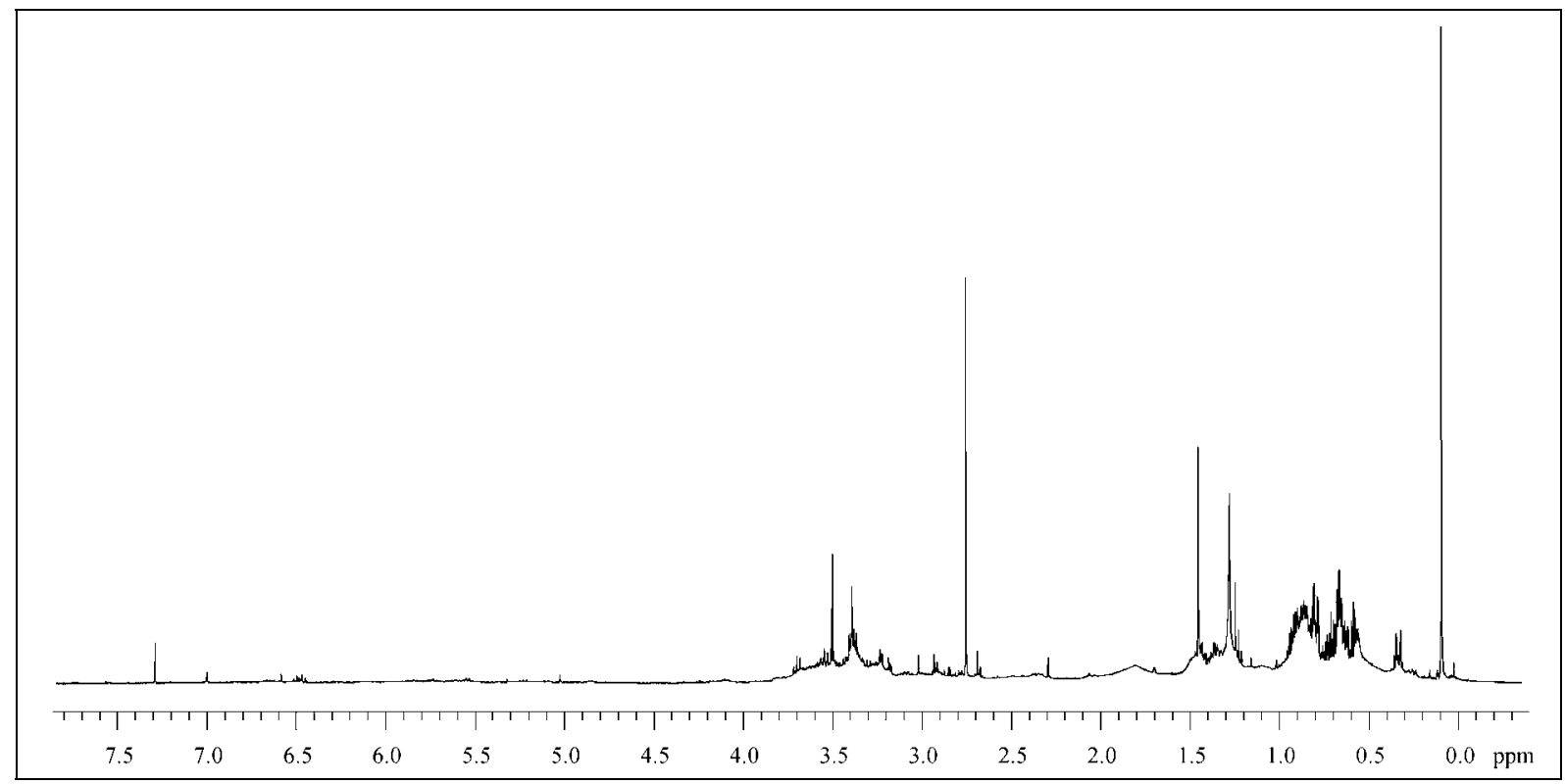

Abbildung 17. ${ }^{1} \mathrm{H}-\mathrm{NMR}-\mathrm{Spektrum} \quad\left(400 \mathrm{MHz}, \quad \mathrm{CDCl}_{3}\right)$ des rohen Abfangprodukts (hauptsächlich 65). 


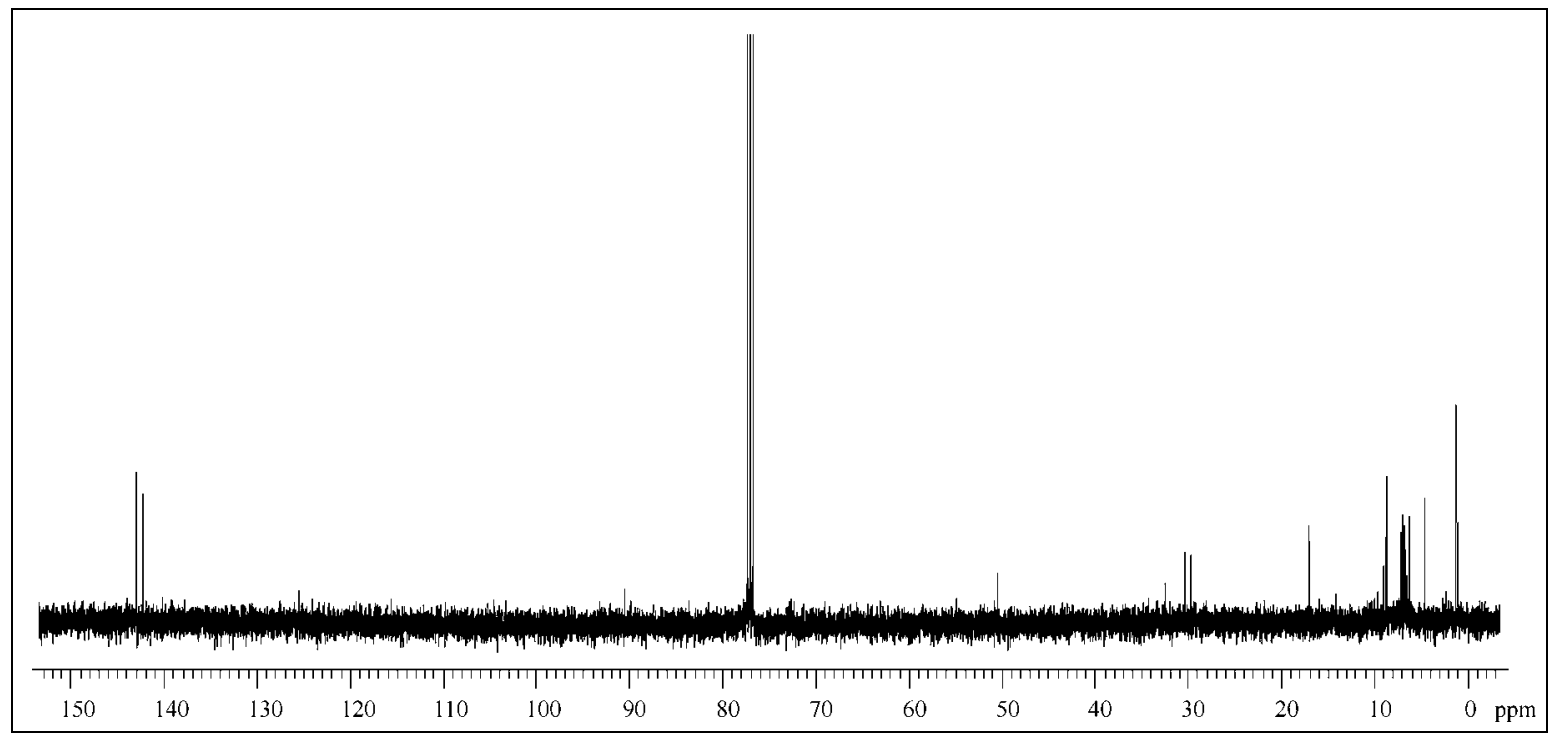

Abbildung 18. ${ }^{13} \mathrm{C}-\mathrm{NMR}-$ Spektrum $\quad\left(100 \mathrm{MHz}, \quad \mathrm{CDCl}_{3}\right)$ des rohen Abfangprodukts (hauptsächlich 65).

Dass hier immerhin ca. 23\% des Methylethers 65 detektiert werden konnten ist insofern bemerkenswert, als dass beim Eintragen der NMR-Lösung in das Methanol natürlich auch die Lösungsmittel selbst, nämlich $\mathrm{SO}_{2} \mathrm{ClF}$ und $\mathrm{SO}_{2} \mathrm{~F}_{2}$, zersetzt werden, aus denen in Folge dessen große Mengen starker Protonensäuren, wie $\mathrm{HF}, \mathrm{HCl}$ und $\mathrm{H}_{2} \mathrm{SO}_{4}$ freigesetzt werden, die nicht sofort vollständig vom vorhandenen Carbonat neutralisiert werden können. Somit ist sicherlich ein signifikanter Teil des säureempfindlichen cyclopropylierten CyclopentadienylGerüstes protoniert und anschließend in polymeres Material umgewandelt worden. In der Tat sind in dem NMR-Spektrum breite, nicht identifizierte Signale sichtbar. Eine massenspektrometrische Analyse des rohen Abfangproduktes bestätigte die Molmasse $\left(\mathrm{M}^{+}=\right.$ 296) für 65.

In diesem Stadium konnte ausgeschlossen werden, dass die erhaltenen NMR-Spektren von dem Pentacyclopropylcyclopentenyl-Kation 60 oder dem Fulvenyl-Kation 61 herrühren. Diese wurden, wie in Kapitel 4.4 ausgeführt, auf anderem Wege dargestellt und charakterisiert; sie weisen deutlich andere chemische Verschiebungen auf (Tabelle 2). 
Tabelle 2. ${ }^{13} \mathrm{C}-$ Chemische Verschiebungen $[\mathrm{ppm}]$ der kationischen, Cyclopropylsubstituierten Cyclopentadienyl-Spezies 60, 61 (je in $\mathrm{CD}_{2} \mathrm{Cl}_{2}$ ) und 12 (in $\mathrm{SO}_{2} \mathrm{ClF} / \mathrm{SO}_{2} \mathrm{~F}_{2}$ ).

$\begin{array}{ccc}\text { Pentacyclopropyl- } & \text { Ethanotetracyclopropyl } & \text { Pentacyclopropyl- } \\ \text { cyclopentenyl-Kation } & \text { fulvenyl-Kation } 61 & \text { cyclopentadienyl-Kation }\end{array}$

$6012(?)$

$\begin{array}{cccc}\text { Cyclopentadienyl } & \text { trans: } & & \\ \text { Gerüst } & \text { 229.2, } 154.7,57.1 ; & 216.0,206.5,155.5, & 222.6,(221.9),(149.2 \\ & \text { cis: } & 151.1,136.6,54.7 & 148.3,83.2,(82.3) \\ & 231.5,152.7,59.5 & & \\ \text { Cyclopropan- } & \text { trans: } 25.2 \text { bis } 5.4 ; & 23.3 \text { bis } 5.6 & 29.2 \text { bis } 2.9 \\ \text { ringe } & \text { cis: } 25.8 \text { bis } 5.4 & & \end{array}$

Daraus kann man weiterhin schließen, dass ein ähnlich dem bei LAMBERTs Versuchen zur Darstellung des Pentamethylcyclopentadienyl-Kations (10) beobachteter ungewöhnlicher Protonentransfer ausblieb.

Aus den obigen Ausführungen über die bis dahin gewonnenen Daten ergeben sich allerdings weitere Fragestellungen: 1) So wäre zum einen zu klären, warum die beobachteten ${ }^{13} \mathrm{C}$-Signale eine bemerkenswerte Halbwertsbreite von durchschnittlich $100 \mathrm{~Hz}$ aufweisen obwohl die Linie des tert-Butyl-Kations, das ebenfalls in der Probe zur Referenzierung vorhanden war, nur eine durchschnittliche Halbwertsbreite von $20 \mathrm{~Hz}$ aufwies. Demnach können die auffällig breiten Linien des neu beobachteten Carbokations nicht nur mit einer erhöhten Viskosität der Lösung oder paramagnetischen Verunreinigungen erklärt werden, die eine Spin-Gitter (bzw. Spin-Spin)-Relaxation des Spinsystems verlangsamt. Im Gegenteil wurde bei Versuchen, H,H-Kopplungen zu detektieren, ein schnell relaxierendes System gefunden. 2) Die breiten Linien weisen vielmehr auf ein dynamisches Phänomen hin, dem das Pentacyclopropylcyclopentadienyl-Kation unterliegt. Unterstützt wird diese Vermutung durch das Auftreten von Signalpaaren im Tieffeldbereich des ${ }^{13} \mathrm{C}-\mathrm{NMR}$-Spektrums, was für das 
Vorliegen eines Hauptisomers (222.6, 148.3 und 83.2 ppm) und eines Mindermengenisomers (221.9, 149.2 und 82.3 ppm) spricht. 3) Handelt es sich hier um Konformere, bei denen die Rotation der Cyclopropyl-Substituenten bei den tiefen Temperaturen eingefroren ist oder liegen hier Strukturisomere in einer dynamischen Beziehung nebeneinander vor? 4) Die chemische Verschiebung des Signalpaars bei 83 ppm wäre recht ungewöhnlich für ein quartäres und gleichzeitig $\mathrm{sp}^{2}$-hybridisiertes Kohlenstoffatom. 5) Könnte sich aus dem zunächst entstandenen Pentacyclopropylcyclopentadienyl-Kation ein weiteres reaktives Intermediat bilden, das ebenfalls Elemente für eine hohe Gesamtsymmetrie aufweist und aus dem bei Reaktion mit Methanol der Methylether 65 wieder freigesetzt wird? 6) Zusätzlich zu dem chemischen Hinweis werden detailliertere spektroskopische Informationen darüber benötigt, ob sowohl das Cyclopentadienyl-System als auch die Cyclopropanringe an diesem in Lösung intakt sind.

Antworten auf diese Fragen sollten einerseits theoretische Berechnungen der NMRVerschiebungen des Pentacyclopropylcyclopentadienyl-Kations 12 und andererseits eine ${ }^{13} \mathrm{C}$ Markierung der Kationenvorstufe 36. Durch die damit zu gewinnende Signalverstärkung könnte sich die Messzeit verkürzen (was einer länger gleichbleibenden Qualität der NMRProbe entspräche). Durch verschiedene NMR-Experimente könnten so wertvolle Informationen über die Cyclopentadienyl- und die Cyclopropyl-Region gewonnen werden.

In Anbetracht der zur Verfügung stehenden Reaktionen zum Aufbau des Pentacyclopropylcyclopentadiens $\mathbf{1 5}$ und nachfolgend des Chlorids $\mathbf{3 6}$ bat sich eine ${ }^{13} \mathrm{C}$ Markierung entweder des Dicyclopropylethins, von dem 2 Äquivalente eingesetzt werden müssten, oder des Cyclopropancarbonsäuremethylesters an, von dem nur 1 Äquivalent benötigt würde. Die zum Chlorid 36 führende Reaktion verläuft über das Pentacyclopropylcyclopentadienylanion, in welchem alle C-Atome des Cyclopentadienylrings chemisch äquivalent sind. Mit der Markierung von nur einem C-Atom erhält man deshalb eine vollständige Verteilung über den Cyclopentadienring in Form von drei Isotopomeren. Das Gleiche würde für die entsprechenden Positionen im Cyclopropanring zutreffen. ${ }^{[86]}$ 
Das Dicyclopropylethin (13) kann ausgehend von Cyclopropylacetylen oder Acetylen und 1-Brom-3-chlorpropan in zwei bzw. drei Stufen aufgebaut werden. Man könnte also ausgehen von kommerziell erhältlichem ${ }^{13} \mathrm{C}$-markiertem Acetylen (68), und/oder von ${ }^{13} \mathrm{C}$ markiertem Bromchlorpropan, das je nach Markierungsposition ausgehend von einem $\mathrm{C}_{1}$-Baustein schrittweise aufgebaut werden müsste. In Anbetracht dessen und der Tatsache, dass zwei Äquivalente des Dicyclopropylethins für die Synthese des Cyclopentadiens 15 nötig wären, wurde zunächst eine Synthese für den markierten Cyclopropancarbonsäuremethylester 71 entworfen.<smiles>C#C</smiles>

68

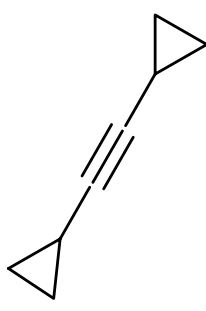

13

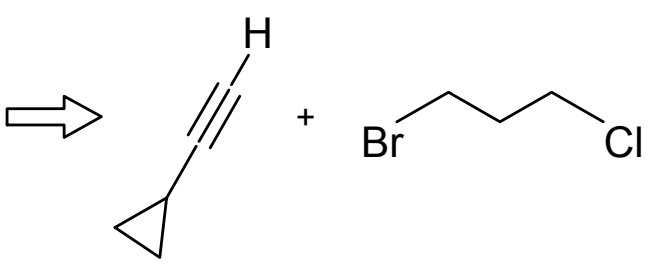

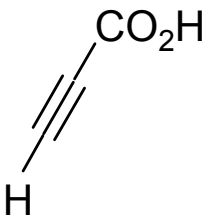

69

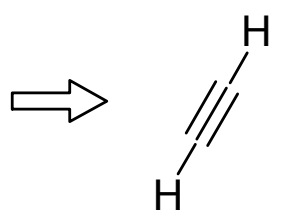

68

Schema 35. Retrosynthese des Dicyclopropylethins und des Cyclopropancarbonsäuremethylesters (71).

Einfach markierter 1-[ $\left[{ }^{13} \mathrm{C}\right]$-Cyclopropancarbonsäuremethylester (71a) wurde durch Carboxylierung von Cyclopropylmagnesiumbromid mit ${ }^{13} \mathrm{CO}_{2}$ in $\mathrm{THF}$ bei $-78{ }^{\circ} \mathrm{C}$ und anschließende Veresterung mit Diazomethan in Diethylether in insgesamt 45\% Ausbeute hergestellt (Schema 36). Zwei- und dreifach markierter $11^{\prime}, 2^{\prime}-{ }^{13} \mathrm{C}$ - bzw. 1,1',2'- ${ }^{13} \mathrm{C}$ Cyclopropancarbonsäuremethylester (71b,c) wurde jeweils ausgehend von doppelt markiertem Acetylen (Cambridge Isotopes Inc., Eurisotop France) hergestellt (Schema 36). Dieses wurde bei $-196{ }^{\circ} \mathrm{C}$ in einen Schlenk-Kolben kondensiert und bei dieser Temperatur mit einer Lösung von Methylmagnesiumchlorid in THF versetzt. Die Deprotonierung setzte 
beim Auftauen der Reaktionslösung auf Raumtemperatur ein. Die dabei gebildete EthinylGRIGNARD-Verbindung wurde nachfolgend bei $-78^{\circ} \mathrm{C}$ entweder mit unmarkiertem Kohlendioxid umgesetzt oder zur Erzielung einer Dreifachmarkierung mit ${ }^{13} \mathrm{CO}_{2}$.

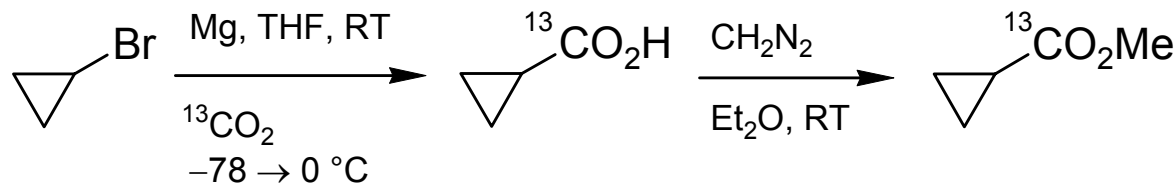

71a: $45 \%$ (gesamt)

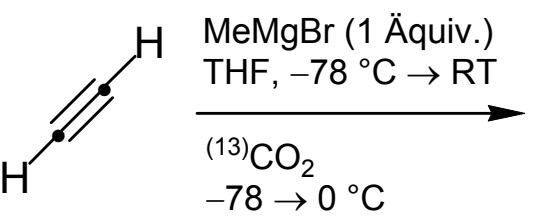

$-78 \rightarrow 0{ }^{\circ} \mathrm{C}$

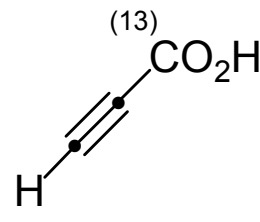

$69 a, b$

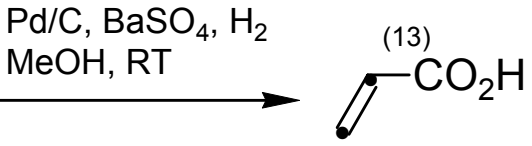

$70 a, b$

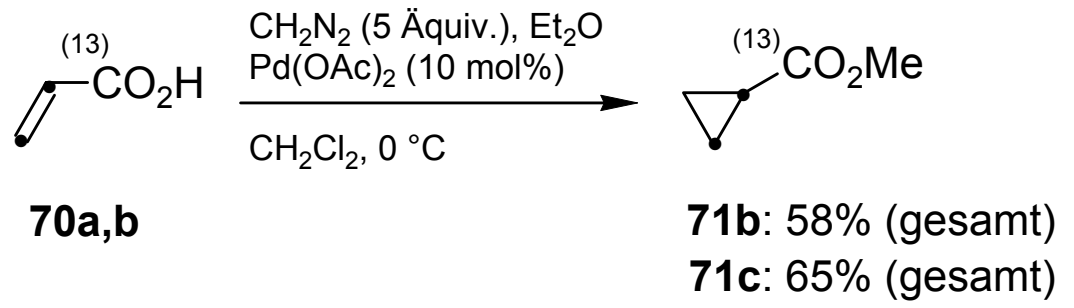

Schema 36. Optimierte Synthesen des einfach, zweifach bzw. dreifach ${ }^{13} \mathrm{C}$-markierten Cyclopropancarbonsäuremethylesters 71a,b,c.

Die so erhaltene zwei- bzw. dreifach ${ }^{13} \mathrm{C}$-markierte Propiolsäure $(\mathbf{6 9 a}, \mathbf{b})$ wurde in Methanol mit Wasserstoff in Gegenwart von LINDLAR-Katalysator bei Raumtemperatur zu der entsprechenden Acrylsäure (69a,b) reduziert. Die vorherige Veresterung der Propiolsäure mit Diazomethan bot keine Vorteile, da die nachfolgende Hydrierung zur vollständigen Zersetzung führte und nach Aufarbeitung der Reaktionsmischung kein Acrylsäuremethylester nachgewiesen werden konnte. Sowohl die Veresterung der Acrylsäure (70) als auch deren in situ Cyclopropylierung gelang bei $0^{\circ} \mathrm{C}$ in einer 3:1 Mischung von Diethylether und Dichlormethan mit Diazomethan und zeitversetzter Zugabe von 10 mol-\% Palladiumacetat zur Reaktionslösung. 1',2'-[ $\left.{ }^{13} \mathrm{C}\right]-$ bzw. 1,1',2'-[ $\left.{ }^{13} \mathrm{C}\right]-$ Cyclopropancarbonsäuremethylester (71b,c) konnten in einer Gesamtausbeute von 58 bzw. 65\% gewonnen werden (Schema 36). 
Wurde diese Reaktion nur in Diethylether oder Dichlormethan alleine oder in einem anderen Verhältnis als dem angegeben durchgeführt, so fand kein quantitativer Umsatz statt.

Diese Cyclopropancarbonsäuremethylester wurden dann jeweils auf dem in Kapitel 4.2 und Kapitel 4.3 beschriebenen Wege zu den ${ }^{13} \mathrm{C}$-markierten Cyclopentadienen 15a,b,c und diese nachfolgend $\mathrm{zu}$ den ${ }^{13} \mathrm{C}$-markierten Chloriden 36a,b,c umgesetzt, die in einer Gesamtausbeute von 26\% für das einfach, $28 \%$ für das zweifach und $34 \%$ für das dreifach markierte Chlorid isoliert wurden. Die ${ }^{13} \mathrm{C}-\mathrm{NMR}-\mathrm{Spektren}$ der markierten Pentacyclopropylcyclopentadiene wiesen eine zunächst nicht erwartete Randomisierung der ${ }^{13} \mathrm{C}$-Markierung aus, indem bei der einfach markierten Verbindung alle Signale des Cyclopentadienringes und bei dem zweifach markierten Cyclopentadien alle Cyclopropansignale deutlich verstärkt auftraten, (bei der dreifach markierten Verbindung dementsprechend alle). Offensichtlich findet im Pentacyclopropylcyclopentadien eine rasche $1,5-H$-Verschiebung statt, die die ${ }^{13} \mathrm{C}$ Markierung rein formal über alle Fünfring-Kohlenstoffatome und die daran geknüpften Cyclopropylringe verteilt, was zu einem Gemisch von drei Isotopomeren führt. Eine 1,5- $H$ Verschiebung bei einem Cyclopentadien ist an sich keine ungewöhnliche Beobachtung. Es ist aber auffällig, dass eine solche Protonenwanderung beim Tetracyclopropylcyclopentadien 14 nicht auftritt. Dieses müsste dann nämlich das thermodynamisch stabilere 1,2,3,4Tetracyclopropylcyclopenta-1,3-dien und nicht das gefundene 1,2,4,5-Tetracyclopropylcyclopenta-1,3-dien bilden. Letzteres erhält man aber erst bei wässriger Aufarbeitung des Tetracyclopropylcyclopentadienylanions. ${ }^{[37]}$

Aus den so ${ }^{13} \mathrm{C}$-markierten Kationenvorstufen wurden unter den anfangs für das unmarkierte Chlorid 36 beschriebenen Bedingungen ebenfalls NMR-Proben hergestellt. 


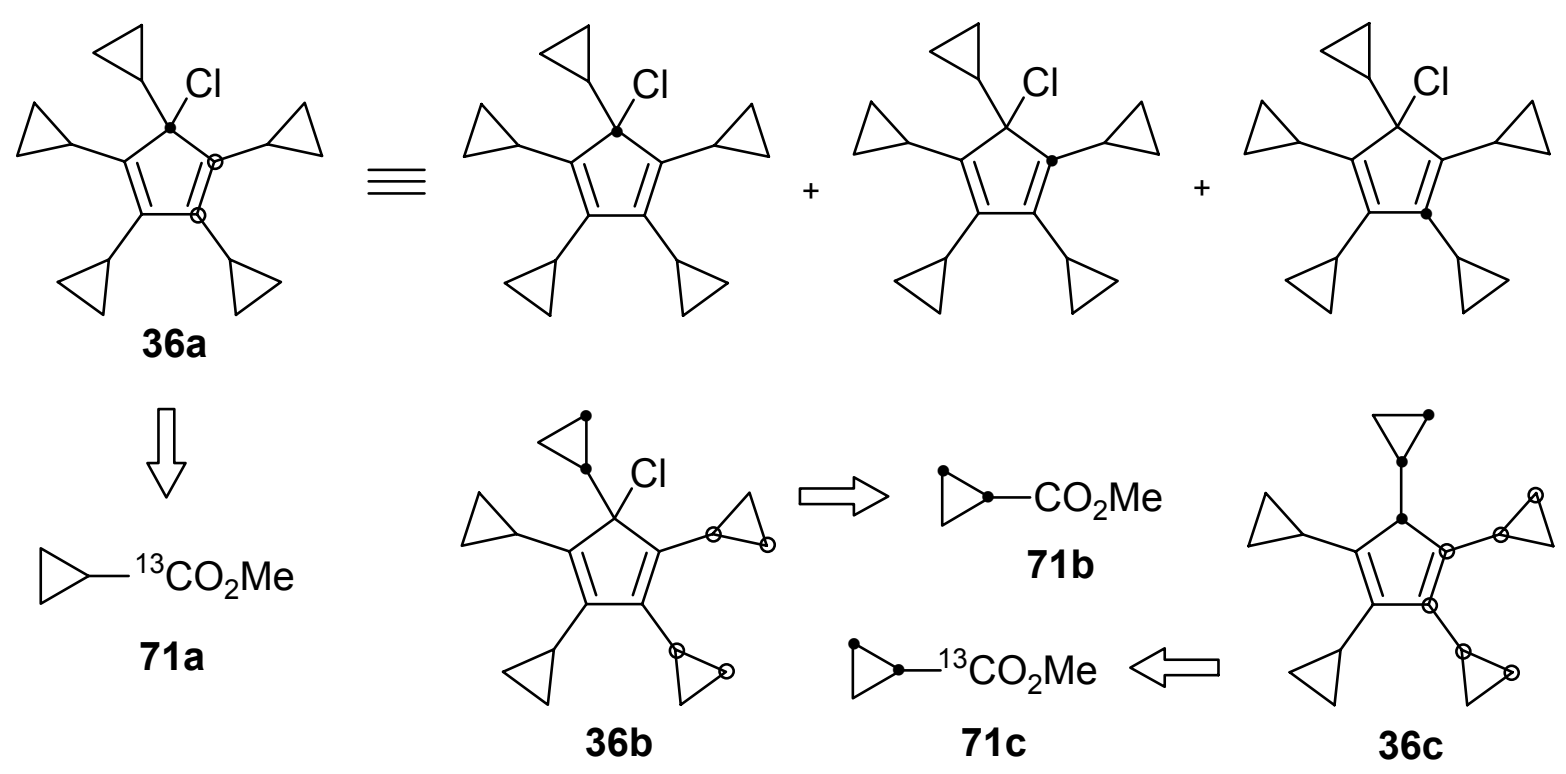

Schema 37. Isolierte Isotopomere der Chloride 36a,b,c.

Dabei sollte die einfach markierte Kationenvorstufe 36a einen gezielten Einblick in das Cyclopentadienyl-Gerüst ermöglichen und das zweifach markierte Chlorid $\mathbf{3 6 b}$ nähere Informationen über die Cyclopropyl-Kohlenstoffatome liefern können. Durch die Dreifachmarkierung in 36c würden alle Signale insgesamt verstärkt werden, was auf den ersten Blick wenig Nutzen hätte. Das ${ }^{13} \mathrm{C}$-NMR-Spektrum sähe genauso aus wie das des unmarkierten Kations, aber die C,C-Kopplungen sollten nun leichter messbar werden. Außerdem könnten dann auch C,C-Korrelationstechniken angewandt werden, die Aufschluss über die Konnektivitäten im Kohlenstoffgerüst des beobachteten Kations geben könnten.

Das ${ }^{13} \mathrm{C}$-NMR-Spektrum des doppelt markierten Carbokations zeigte eindeutig die verstärkten Cyclopropyl-Signale bei 2.9, 5.5, 7.5, 16.5, 19.0 und 29.2 ppm. Unglücklicherweise konnten aber die ${ }^{1} J_{\mathrm{C}, \mathrm{C}}$-Kopplungen nicht wie erwartet abgelesen werden. Für Cyclopropanringe werden diese größenordnungsmäßig im Bereich von $10 \mathrm{~Hz}$ erwartet. Leider waren diese kleinen ${ }^{1} J_{\mathrm{C}, \mathrm{C}}$-Kopplungen in den sehr breiten Signale nicht aufgelöst.

Mit Hilfe von DEPT90-Spektren (distortionless $\boldsymbol{e}$ nhancement by polarization transfer, Pulswinkel $90^{\circ}$ ), die allein $\mathrm{CH}-$ Gruppen anzeigen, konnte die ursprünglich getroffene 
Zuordnung von $\mathrm{CH}$ und $\mathrm{CH}_{2}$-Signalen bestätigt werden. Die aufgenommenen DEPT90Spektren wiesen nur die verstärkten Signale bei 5.5, 16.5, 19.0 und 29.2 ppm auf.

Der Einsatz des dreifach markierten Chlorids 36c bei der Kationenerzeugung ermöglichte die Anwendung der sogenannten INADEQUATE-Aufnahmetechnik (incredible natural abundance double quantum transfer experiment). ${ }^{[87]}$

Diese NMR-Methode wird verwendet, um die Signale von benachbarten Kohlenstoffatomen zu identifizieren, indem sie die starken Signale nicht koppelnder Kerne unterdrückt und so nur die Linien der Satelliten hervorbringt. Wie der Name der Methode schon andeutet, liegt ein großes Hindernis der Methode in der mangelnden Sensitivität, die wiederum eine Folge des geringen Vorkommens des NMR-aktiven ${ }^{13} \mathrm{C}$-Kerns von ca. $1 \%$ ist. Die Wahrscheinlichkeit, zwei ${ }^{13} \mathrm{C}$-Kerne in einem Molekül miteinander verbunden anzutreffen, liegt damit bei 1:10000. Praktisch erfordert diese Methode daher große Mengen an Substanz (bis zu $800 \mathrm{mg}$ ) und sehr lange Messzeiten (unter Umständen Tage), wenn sie bei natürlicher ${ }^{13} \mathrm{C}$-Häufigkeit durchgeführt wird. Sie ist deswegen häufig die letzte, aber auch sehr hilfreiche Wahl bei schwierigen Strukturproblemen. Ein 2D-INADEQUATE-Spektrum erscheint nicht wie ein COSY-Spektrum. Es gibt keine Diagonalpeaks. Stattdessen wird die chemische Verschiebung in die $F_{2}$-Dimension projiziert und die Doppelquantenfrequenz in die $F_{1}$-Dimension, die Summe der beiden Korrelationsfrequenzen. Eine Diagonale lässt sich dagegen durch die Mitte der jeweils korrelierenden C-Atome legen. 


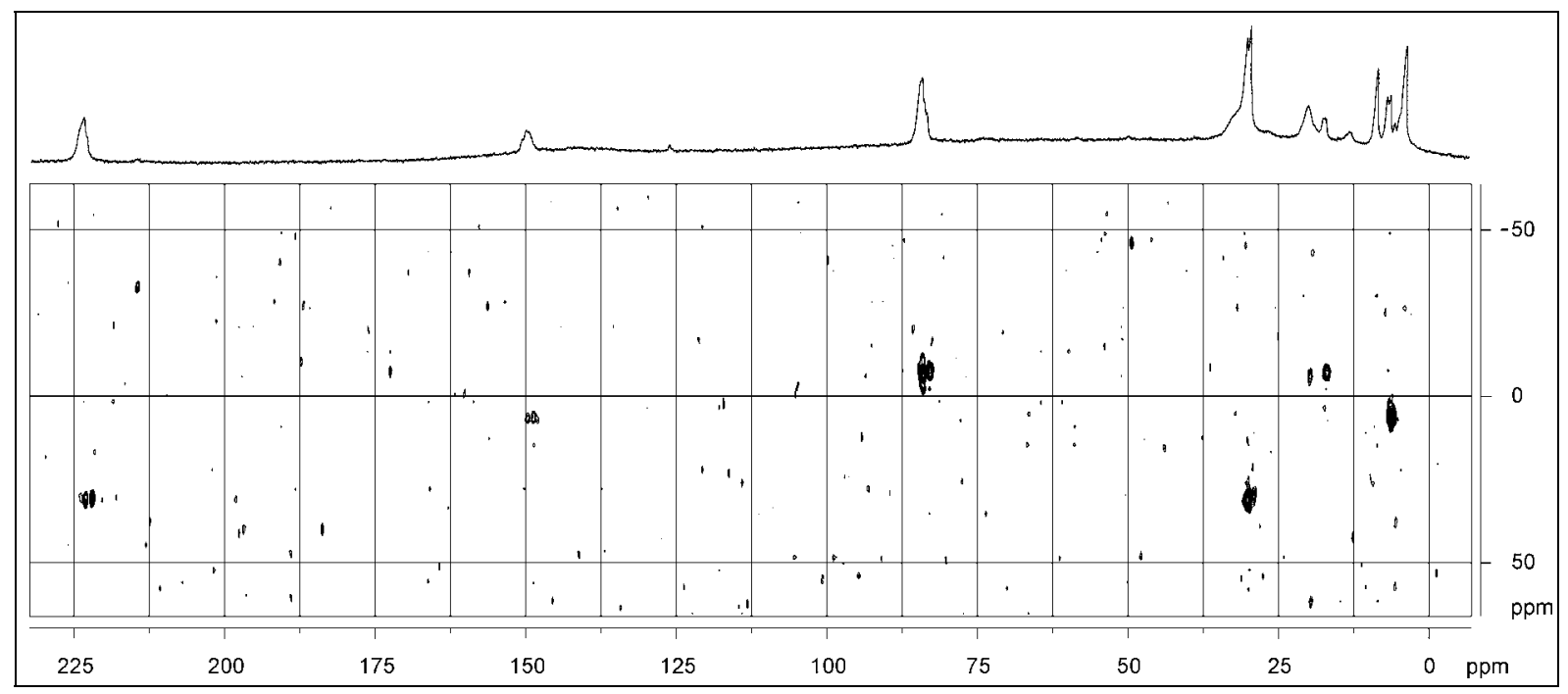

Abbildung 19. 2D-INADEQUATE-Spektrum $\quad(100 \mathrm{MHz})$ der dreifach ${ }^{13} \mathrm{C}$-markierten Kation-Probe bei $-125^{\circ} \mathrm{C}$.

Mit der nun zur Verfügung stehenden 100prozentig dreifach markierten Vorstufe konnten beide Punkte überwunden werden. Das aufgezeichnete 2D-INADEQUATESpektrum wies drei deutliche Korrelationen auf (Abbildung 19). Es zeigte eindeutig die Bindung zwischen den quartären Kohlenstoffen des Signalpaares bei 222 ppm und denen der CH-Signale bei 29.2 ppm an, die Verknüpfung der quartären Kohlenstoffe des Signalpaares bei 148 ppm und denen der CH-Signalen bei 5.5 ppm und die Nachbarschaft der quartären Kohlenstoffe des Signalpaares bei 83 ppm und denen der CH-Signalen bei 16.5 und 19.0 ppm. Damit bestätigte sich die Vermutung, dass die tieffeldverschobenen CH-Signale bei 29.2 ppm von Cyclopropylgruppen stammen, die mit den am stärksten positivierten quartären Cyclopentadien-C-Atomen verknüpft sind. Die Linienabstände in den wie „Dubletts“ aussehenden Korrelationspeaks der quartären C-Atome repräsentieren in diesem Fall allerdings nicht die ${ }^{1} J_{\mathrm{C}, \mathrm{C}}$-Kopplungskonstanten, die normalerweise aus einem 2D-INADEQUATE abgelesen werden können. Diese stammen von ihren als Paar auftretenden Signalen. In diesem Experiment konnten nur die Korrelationen bestimmt werden, die einen recht großen Betrag als Kopplungskonstante besaßen (der Wert $\Delta$ t, der für die Pausen zwischen den Anregungspulsen steht, sollte für die jeweils zu beobachtende Kopplung $J$ auf $1 /(4 J)$ gesetzt werden; mit $J$ in Hz). Die Korrelationen der Cyclopropyl- 
Kohlenstoffatome untereinander konnte auch in weiteren Experimenten nicht erhalten werden, was wahrscheinlich an der großen Signalbreite der beobachteten Peaks lag. Es war aber möglich, die ${ }^{1} J_{\mathrm{C}, \mathrm{H}}$-Kopplungskonstanten der tertiären und sekundären Kohlenstoffatome mit Hilfe von nicht entkoppelten ${ }^{13} \mathrm{C}-\mathrm{NMR}$ - und DEPT135-Spektren (Abbildung 20a) zu bestimmen.

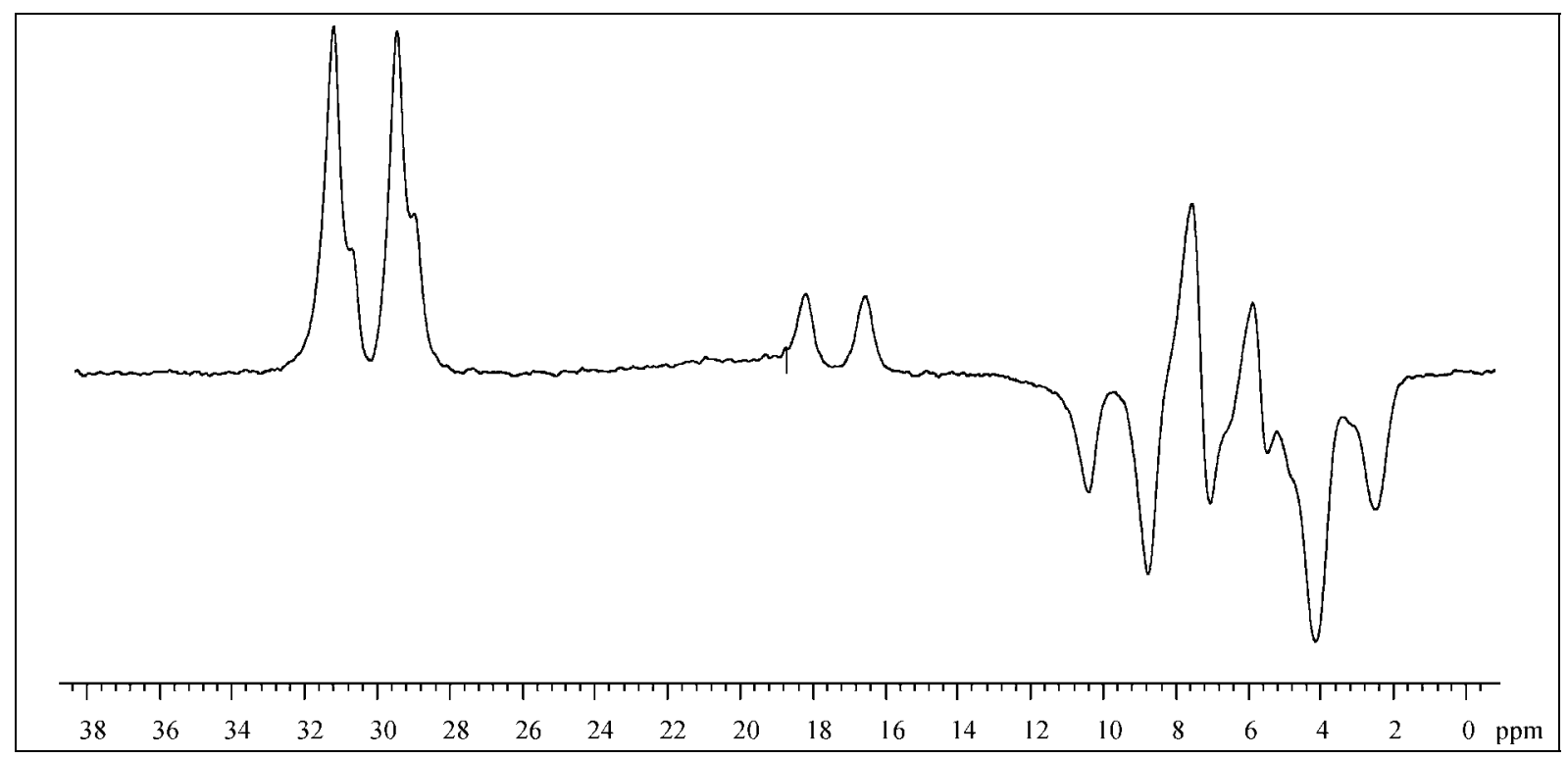

Abbildung 20a. Gekoppeltes DEPT135-Spektrum (100 MHz) der dreifach ${ }^{13} \mathrm{C}$-markierten Probe der Kation-Spezies bei $-125^{\circ} \mathrm{C}$.

Aus diesen konnten drei verschiedene Werte für die $\mathrm{CH}$-Signale erhalten werden, nämlich 172, 166 und $164 \mathrm{~Hz}$, was ebenfalls auf die schon erwartete $C_{\mathrm{S}}$-Symmetrie hinwies. Die Kopplungskonstante der sekundären C-Atome betrug ${ }^{1} J_{\mathrm{C}, \mathrm{H}}=164 \mathrm{~Hz}$. Die beobachteten ${ }^{1} J_{\mathrm{C}, \mathrm{H}}$-Kopplungskonstanten liegen damit alle in einem für hochgespannte Cyclopropanderivate, typischen Bereich. Durch das 2D-INADEQUATE und die gekoppelten ${ }^{13} \mathrm{C}$ - und DEPT135-Spektren war auf jeden Fall klar, dass die $\mathrm{CH}$ - und $\mathrm{CH}_{2}$-Gruppen tatsächlich von intakten Cyclopropanringen stammten und dass diese mit einem kationischen Cyclopentadienyl-Grundgerüst verknüpft waren.

Ein genauer Blick auf die ${ }^{13} \mathrm{C}-\mathrm{NMR}-\mathrm{Spektren}$ des einfach markierten Kations bei unterschiedlichen Temperaturen zeigte nicht nur die erwartete Intensivierung der Cyclopenta- 
dienyl-Signale an. Durch stufenweises Erwärmen der NMR-Proben beginnend bei $-140{ }^{\circ} \mathrm{C}$ in Schritten von $10 \mathrm{~K}$ bis hin $\mathrm{zu}-110^{\circ} \mathrm{C}$ ließ sich eine Abnahme der Signalbreite der beobachteten Paare bei 222, 148 und 83 ppm feststellen. Zusätzlich wurde aber auch eine Abnahme des chemischen Verschiebungsunterschiedes der jeweiligen Signalpaare beim Erwärmen gemessen. Die Signale des ebenfalls in der Lösung enthaltenen tert-Butyl-Kations (zur Referenzierung der Spektren) veränderten sich in dem Temperaturbereich dagegen nur minimal (Abbildung 20b).

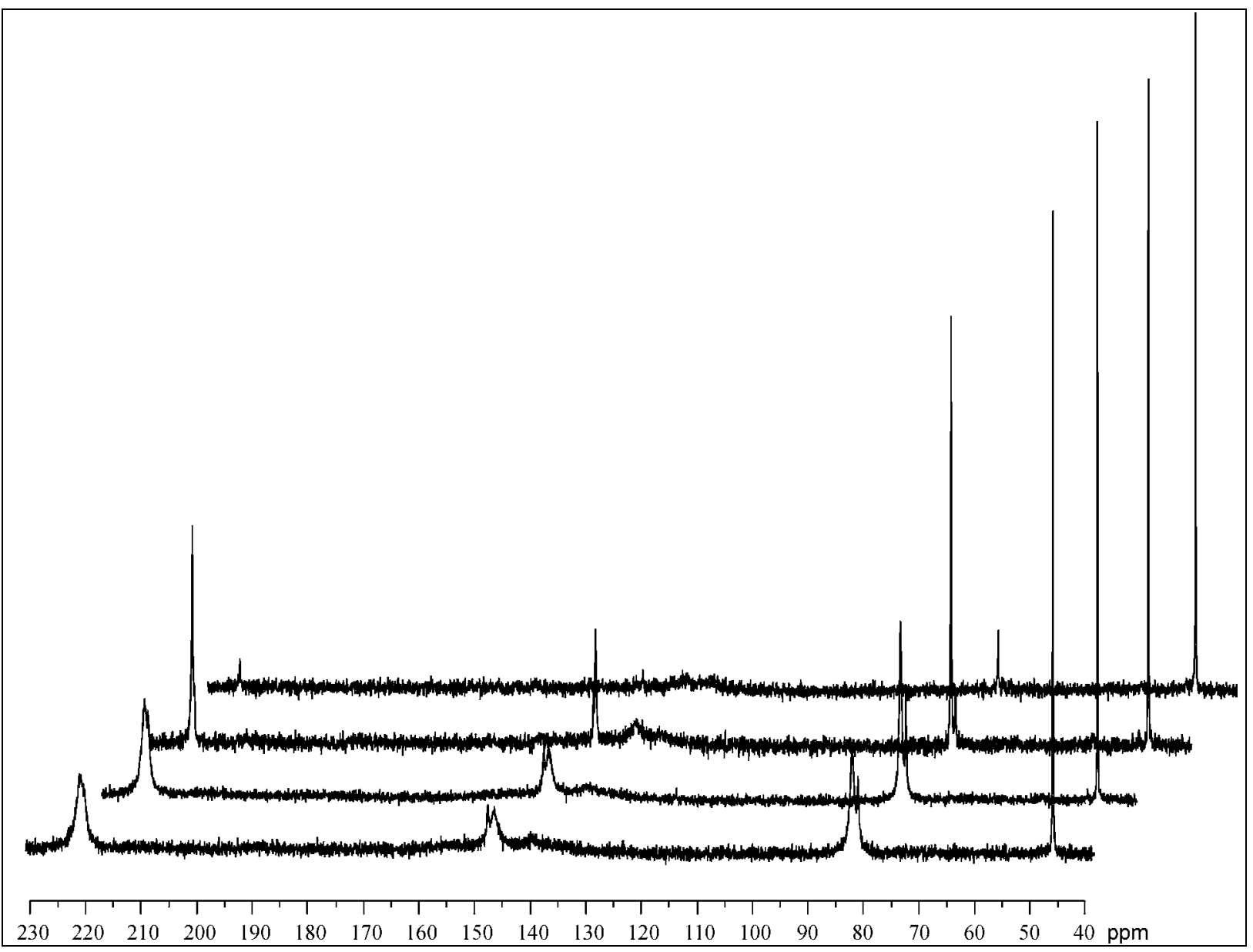

Abbildung 20b. ${ }^{13} \mathrm{C}-\mathrm{NMR}-$ Spektren $(100 \mathrm{MHz})$ der einfach markierten Probe bei -140 bis $-110^{\circ} \mathrm{C}$ (von vorne in Schritten von $\left.10 \mathrm{~K}\right)$.

Beide Phänomene können durch die Überlagerung der Signale nur schwer quantifiziert werden. Doch ließ sich ein signifikanter Trend ablesen. Die erste Beobachtung ist zunächst nicht ungewöhnlich, da bei höheren Temperaturen eine schnellere Relaxation naturgemäß zu 
schmaleren Signalen führt. Die zweite aber legt die Vermutung einer bevorstehenden Koaleszenz nahe. Beide Phänomene zusammen kann man aber auf den ersten Blick nicht mit einem einfachen Koaleszenz-Modell erklären, denn schmaler werdende Siganle treten im Bereich oberhalb einer Koaleszenztemperatur auf. Konvergieren würden die Signale dagegen nur unterhalb einer Koaleszenztemperatur.

3.7. Interpretation der experimentellen Daten im Hinblick auf die berechneten Parameter von $c \operatorname{Pr}_{5} \mathrm{Cp}^{+}$(12)

\subsection{1. $\mathrm{CPr}_{5} \mathrm{Cp}^{+}$(12) als klassisches Carbokation}

An dieser Stelle ist es notwendig, die gewonnenen Erkenntnisse mit dem Vorliegen des Pentacyclopropylcyclopentadienyl-Kations $\mathbf{1 2}$ in Einklang zu bringen. Dabei würden die spektroskopischen Daten auf einen Singulett-Grundzustand mit $C_{\mathrm{S}}-$ Symmetrie hindeuten. Diese Annahme würde auch mit dem Bild der für das PentacyclopropylcyclopentadienylKation 12 berechneten Orbitalkoeffizienten im Singulett-Zustand harmonieren (Abbildung 21).
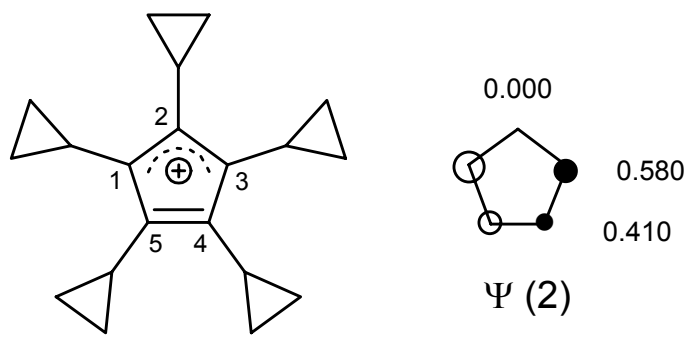

$\Psi(2)$

\section{$12 / s$}

Abbildung 21. Versuch zur Interpretation der experimentellen Daten für das vermeindliche Pentacyclopropylcyclopentadienyl-Kation.

Das quartäre Signalpaar bei 222 ppm wäre den Cyclopentadienyl-Positionen C-1 und C-3, das bei 148 ppm C-2 und das bei 83 ppm C-4 und C-5 zuzuordnen. Dies würde ebenfalls 
mit dem erhaltenen Intensitätsverhältnis von 2:1:2 übereinstimmen. Dadurch waren auch die Spiegelebene und die zweizählige Drehachse bestätigt. Die am stärksten mit dem kationischen Cyclopentadien-Gerüst durch Hyperkonjugation wechselwirkenden Cyclopropylgruppen befänden sich, wie auch die Rechnungen voraussagen, in 1,3-Position, was die Tieffeldverschiebung von $222 \mathrm{ppm}$ für $\mathrm{C}-1,3$ und von $29 \mathrm{ppm}$ für die benachbarten Cyclopropyl-Substituenten erklären würde. Wie könnte nun das paarweise Auftreten der quartären Cyclopentadienyl-C-Atome erklärt werden? Denkbar wären nebeneinander existierende Konformere, die durch eine eingeschränkte Drehbarkeit der Cyclopropylgruppen an den Positionen C-1 und C-3 entstünden. Dabei hätten die Dreiringe jeweils zwei Möglichkeiten, bisektiert $\mathrm{zu}$ stehen und dadurch die benachbarte positive Ladung $\mathrm{zu}$ delokalisieren. Typische Rotationsbarrieren für Cyclopropylcarbinyl-Kationen liegen in der Größenordnung von ca. $+15 \mathrm{kcal} / \mathrm{mol}$, was bei Temperaturen um $-100{ }^{\circ} \mathrm{C}$ durchaus zum Einfrieren der Rotation des Cyclopropyl-Substituenten um die Bindung zum kationischen Zentrum führen und anhand von zusätzlichen Signalen im ${ }^{13} \mathrm{C}-\mathrm{NMR}-$ Spektrum beobachtet werden kann. ${ }^{[88]}$ Die Cyclopropylgruppen an den Positionen C-2, C-4 und C-5, die kaum oder gar nicht an der Hyperkonjugation beteiligt wären, würden sich weiterhin ungehindert drehen können. SORENSEN et al. konnten genau solche Phänomene bei Untersuchungen an Alkyl- und Aryl-substituierten Dicyclopropylcarbinyl-Kationen feststellen. ${ }^{[88]}$

Da beide Cyclopropylgruppen an C-1,3 eine bisektierte Position einnehmen können, sind insgesamt vier Konformere denkbar, die sich durch die unterschiedliche Stellung der Cyclopropylgruppen relativ zueinander unterscheiden. Bezeichnet man die bisektierten Positionen zur leichteren Unterscheidung in den Projektionen mit „hoch“ und „runter“ stehen in dem Konformer A beide Dreiringe hoch, in B' und B', steht jeweils einer hoch und der andere runter und in $\mathbf{C}$ stehen beide Cyclopropylgruppen runter (Schema 38). 


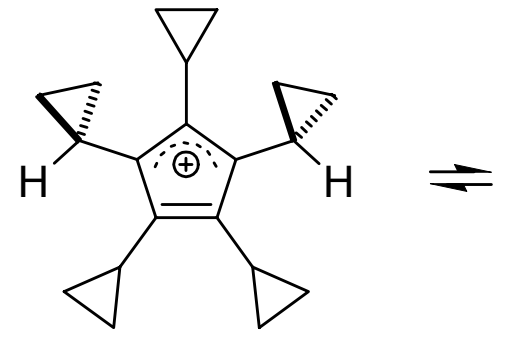

A

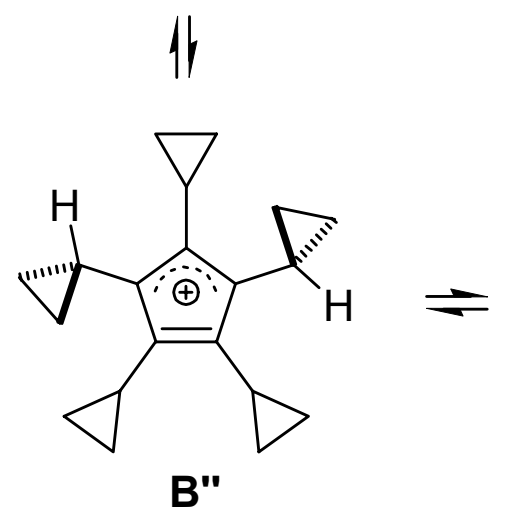

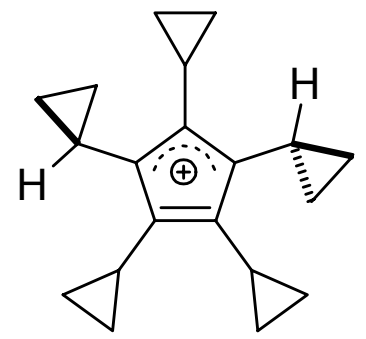

B'

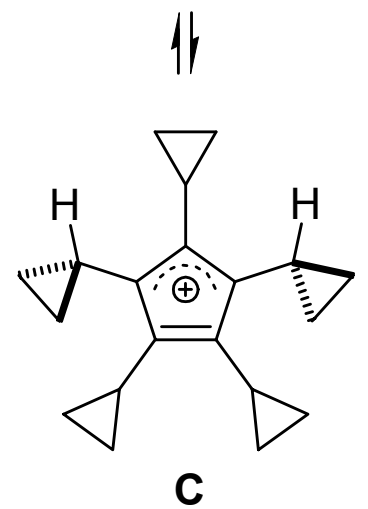

Schema 38. Erste Näherung zur Struktur der möglichen Konformere von 12.

Eine Abschätzung der relativen Energien solcher Konformere ließe sich aus einer Betrachtung der Bindungsverhältnisse und der Raumerfüllung der Substituenten ableiten. Betrachtet man das $C_{\mathrm{S}}$-symmetrische Cyclopentadienyl-Kation-Gerüst im Singulett-Zustand, so kann man annehmen, dass die Einfachbindungen zwischen C-1 und C-5 und C-3 und C-4 vergleichsweise lang und die Doppelbindungen zwischen C-4 und C-5 eher kurz wären. Die Bindungslängen zwischen C-1 und C-2 und C-2 und C-3 wären aufgrund der Konjugation und des damit anteiligen Doppelbindungscharakters sicherlich kürzer als eine C,C-Einfachbindung. Wollte die Cyclopropylgruppe einer stärkeren sterischen Abstoßung ausweichen, wäre es am günstigsten, wenn sich wie im Bild des Konformers C beide Dreiringe in Richtung der längeren Bindungen und damit also der weniger sterisch befrachteten Raumes (C-1/C-5 und C-3/C-4) drehen. Das Konformer A wäre durch seine beiden in den sterisch stärker belasteten Raum gedrehten Cylopropyl-Substituenten am energiereichsten. Die Konformere B' und B', wären demzufolge auf einem Energieniveau einzuordnen und isoenergetisch (Schema 39). 


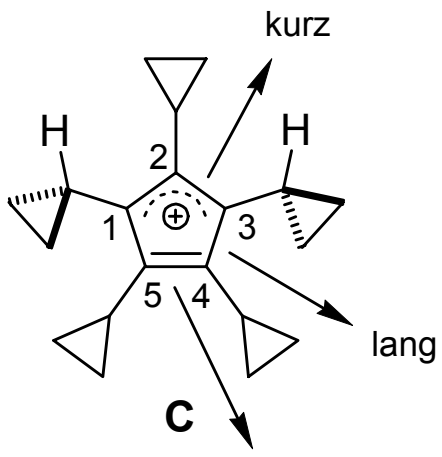

kurz
E

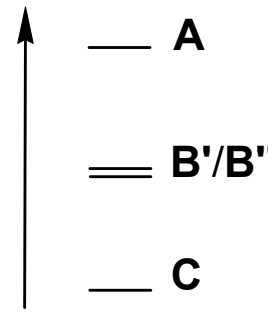

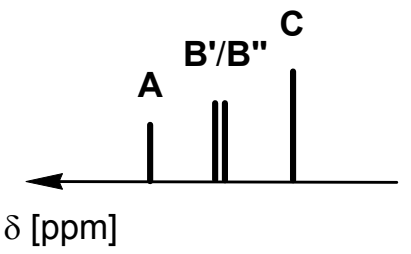

Schema 39. Abschätzung der Energieabfolge der Konformere A, B'/B', und $\mathbf{C}$ von $\mathbf{1 2}$ und aus deren bevorzugter Population resultierende NMR-Peaks.

Bei einer gegebenen, tiefen Temperatur müssten diese Energiezustände unterschiedlich stark besetzt sein, d. h. das Konformer C müsste vorherschen. Von den Konformeren B' und B' läge weniger vor, und A würde vermutlich kaum existieren. Daraus ergäben sich entsprechend jeweils drei NMR-Signale unterschiedlicher Intensität für die CyclopentadienylPositionen C-1,3, C-2 und C-45, wobei C das intensivste Signal erzeugen sollte. Eine Erhöhung der Temperatur würde nun unter Ausbildung zweier neuer Peaks zu einer ersten Koaleszenz der drei Signale führen, nämlich einem großen und einem kleineren (Schema 40). Dies könnte die im ${ }^{13} \mathrm{C}$-Spektrum tatsächlich beobachteten Signalpaare bei 222, 148 und 83 ppm erklären.

\section{Koaleszenz?}

1. Koaleszenz

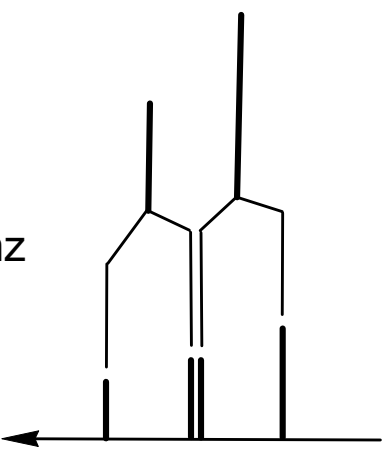

A B'/B" C
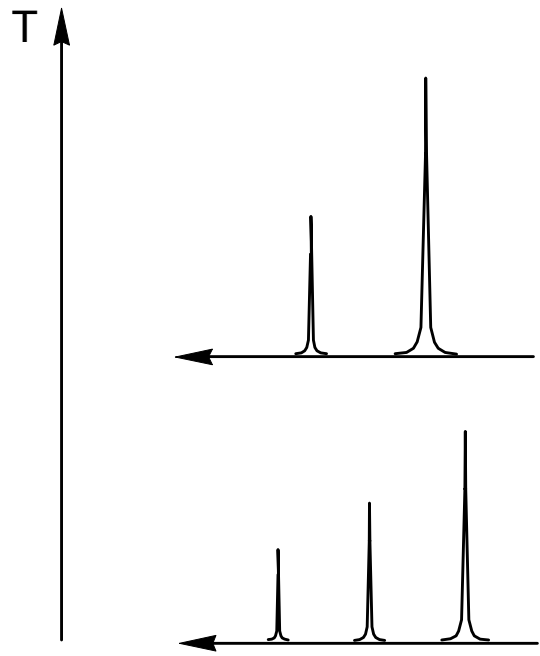

A $B^{\prime} / B^{\prime \prime} \quad C$

Schema 40. Erklärungsansatz für das Auftreten von Signalpaaren durch Koaleszenz der Signale der Konformere von 12. 
Unter diesen Annahmen wären die zwischen -140 und $-110^{\circ} \mathrm{C}$ aufgenommenen Spektren also Momentaufnahmen nach einer 1. Koaleszenz bei niedrigerer Temperatur, wonach die Signale noch eine gewisse Breite aufwiesen, die dann bei stufenweiser Erwärmung, wie beobachtet, schmaler würden. Die zweite Beobachtung des bei Erwärmen weiteren Zusammenrückens dieser Peaks legt die Folgerung nahe, dass sich die Konformere einer nächsten, zweiten Koaleszenz nähern, was durch die deutliche Abnahme des Signals für das Mindermengenkonformer bis hin $\mathrm{zu}$ seinem Verschwinden bei $-110{ }^{\circ} \mathrm{C}$ untermauert würde. Die freie Aktivierungsenthalpie $\Delta G^{\neq}$dieser Umwandlung lässt sich aus der Koaleszenztemperatur $\left(T_{\mathrm{c}}\right.$ in $\left.\mathrm{K}\right)$ und der Differenz der chemischen Verschiebung der konvergierenden Signale ( $\Delta v$ in $\mathrm{Hz})$ näherungsweise berechnen. Bei der Koaleszenztemperatur gilt für die Geschwindigkeitskonstante näherungsweise $k_{\mathrm{Tc}} \approx 2.22 \Delta \mathrm{v}$. Setzt man diese Beziehung in die Eyring-Gleichung ein, so erhält man nach Umformen $\Delta G^{\neq}=0.0191 T_{\mathrm{c}}$ $\left(9.91+\lg T_{\mathrm{c}}-\lg \Delta v\right) .{ }^{[89]}$ Mit $T_{\mathrm{c}}=163.15 \mathrm{~K}$ (entspricht $\left.-110^{\circ} \mathrm{C}\right)$ und $\Delta v=110 \mathrm{~Hz}$ (größte beobachtete Differenz der chemischen Verschiebung der Signalpaare) erhält man eine freie Aktivierungsenthalpie von ca. $\Delta G^{ \pm} \approx+7 \mathrm{kcal} / \mathrm{mol}$. Dieser Wert entspräche auch ungefähr der Rotationsbarriere des Dreirings am Cylopentadienyl-Kation und läge in einer dafür typischerweise beobachteten Größenordnung. ${ }^{[88]}$

$\mathrm{Zu}$ diskutieren wären jetzt noch die beobachteten chemischen Verschiebungen. Obwohl auch im ${ }^{1}$ H-NMR-Spektrum die Signale relativ breit waren, konnten doch die meisten von ihnen mittels C,H-Korrelationsspektren eindeutig auf cyclopropylische Protonen zurückgeführt werden. Sowohl die Cyclopropyl-Region als auch die CyclopentadienC-Atome im ${ }^{13} \mathrm{C}$-NMR-Spektrum konnten anhand von ${ }^{13} \mathrm{C}$-Markierungen und gekoppelten DEPT- und INADEQUATE-Spektren identifiziert werden. Allerdings fällt unter den chemischen Verschiebungen der Cyclopentadien-Kohlenstoffe das Signalpaar bei 83 ppm deutlich heraus. Diese chemische Verschiebung wäre für ein quartäres C-Atom, das im Cyclopentadienyl-Kation-System eingebunden $\mathrm{sp}^{2}$-hybridisiert sein sollte, höchst ungewöhnlich. Signale von olefinischen C-Atomen werden normalerweise jenseits von 100 ppm erwartet. Um diese Diskrepanz näher zu beleuchten, wurden von SCHREINER 
ab initio NMR-Rechnungen für das Pentacyclopropylcyclopentadienyl-Kation im Triplettund im Singulett-Zustand bei verschiedenen Geometrien durchgeführt. Die GIAORechnungen wurden auf dem B3LYP/6+311G* Niveau der Theorie durchgeführt und ergaben für die Triplett-Kationen sowohl von $C_{1^{-}}$als auch von $C_{\mathrm{S}^{-}}$Symmetrie jeweils eine Bindungsäquilibrierung des Cyclopentadien-Systems $\left(C_{1}: \quad 1.436-1.446 \AA, \quad C_{\mathrm{S}}\right.$ : 1.438-1.456 $\AA$ ) und geringe Unterschiede in den ${ }^{13} \mathrm{C}-\mathrm{NMR}-$ Verschiebungen der C-Atome 1-5 ( $\left.C_{1}: 174.7-181.7 \mathrm{ppm}, C_{\mathrm{S}}: 179.1-184.5 \mathrm{ppm}\right)$. Die Signale der Cyclopropylgruppen wurden durch die Rechnungen im Bereich von 18-28 ppm vorhergesagt. Das Pentacyclopropylcyclopentadienyl-Kation 12 im Triplett-Zustand mit $C_{\mathrm{S}}$-Symmetrie war dabei um $+4.6 \mathrm{kcal} / \mathrm{mol}$ energiereicher als $12 \mathrm{im}$ Triplett-Zustand mit $C_{1}$-Symmetrie. Für die Singulett-Kationen wurde ein durch Bindungsalternanz charakterisiertes CyclopentadienylSystem berechnet $\left(C_{1}: \quad 1.370-1.564 \AA, \quad C_{\mathrm{S}}: \quad 1.349-1.529 \AA\right), \quad$ dessen $\quad{ }^{13} \mathrm{C}-\mathrm{NMR}-$ Verschiebungen sehr stark von der Symmetrie des Kations abhingen $\left(C_{1}\right.$ : 140.6-196.3 ppm, $C_{\mathrm{S}}:$ 131.4-233.1 ppm). Die Signale der Cyclopropylgruppen wurden für $\mathbf{1 2} / \mathbf{s}$ mit $C_{1}$-Symmetrie im Bereich von $8-47 \mathrm{ppm}$ erwartet und die für 12/s mit $C_{\mathrm{S}}$-Symmetrie bei $5-11 \mathrm{ppm}$.

Tabelle 3. Vergleich der experimentellen und berechneten ${ }^{13} \mathrm{C}-\mathrm{NMR}-$ Verschiebungen des kationischen Cyclopentadienyl-Systems von 12.

\begin{tabular}{cccccc}
\hline & C-1 & C-2 & C-3 & C-4 & C-5 \\
\hline$C_{1}$, Singulett & 140.6 & 244.3 & 143.2 & 190.9 & 196.3 \\
$C_{\mathrm{S}}$, Singulett & 233.1 & 131.4 & 233.1 & 154.2 & 154.2 \\
\hline experimentell & $222.6,221.9$ & $149.2,148.3$ & $222.6,221.9$ & $83.2,82.3$ & $83.2,82.3$ \\
\hline$C_{1}$, Triplett & 176.0 & 178.7 & 176.0 & 174.7 & 181.7 \\
$C_{\mathrm{S}}$, Triplett & 179.7 & 184.5 & 179.7 & 180.3 & 180.3 \\
\hline
\end{tabular}


Vergleicht man die experimentellen und die berechneten ${ }^{13} \mathrm{C}$-Verschiebungen miteinander, so lässt sich klar erkennen, dass das hier möglicherweise beobachtete Cyclopentadienyl-Kation 12 aufgrund der gravierenden Abweichungen sicher nicht im Triplett-Zustand (weder mit $C_{1}$ - noch mit $C_{\mathrm{S}}$-Symmetrie) vorliegt. Dies wurde ja auch schon anhand von Berechnungen aller Cyclopropyl-substituierten Cyclopentadienyl-Kationen (Abbildung 11) und von homodesmotischen Reaktionen (Schema 10) vorhergesagt. Ein Blick auf die berechneten ${ }^{13} \mathrm{C}$-Verschiebungen des Cyclopentadienyl-Kations $\mathbf{1 2}$ als SingulettSpezies zeigt, dass das hier erhaltene Kation sicher ebenso wenig das berechnete $C_{1}$-symmetrische Cyclopentadienyl-Kation ist. In bester Übereinstimmung stehen die aufgezeichneten NMR-Signale immerhin mit den für die als $C_{\mathrm{S}^{-}}$-symmetrisch berechnete kationische Singulett-Spezies. Allerdings tritt hier für die cyclopentadienylischen Kohlenstoffatome C-4 und C-5 eine enorme Diskrepanz von 70 ppm zwischen den experimentellen und den berechneten Werten auf, die weit außerhalb der Fehlerwahrscheinlichkeit von bis $\mathrm{zu} \pm 20 \mathrm{ppm}$ für die verwendeten theoretischen Methoden liegt. Der rechnerisch vorhergesagte Wert von 154.2 ppm für C-4,5 bestätigt die Annahme, dass die Signale dieser quartären und $\mathrm{sp}^{2}$-hybridisierten $\mathrm{C}$-Atome eine chemische Verschiebung von mehr als 100 ppm haben sollten.

Offensichtlich bewirkt hier ein bisher außer Acht gelassener Effekt (oder ein Zusammenspiel mehrerer) eine für das Cyclopentadienyl-Kation 12 unerwartete Abschirmung der C-Atome 4 und 5. Geht man weiter von dem Vorliegen des Cyclopentadienyl-Kations 12 aus, so gäbe es mehrere denkbare Wechselwirkungen, die die Elektronendichte an C-4,5 beeinflussen könnten. So könnte eine Koordination durch das Anion $\left[\mathrm{Sb}_{\mathrm{x}} \mathrm{F}_{5 \mathrm{x}} \mathrm{Cl}\right]^{-}$oder durch Lösungsmittelmoleküle die Elektronendichte des Cyclopentadienyl-Systems erhöhen. Dies ist aber sehr unwahrscheinlich, da diese Reagenzien eben wegen ihrer inherenten chemischen Inertheit zur Darstellung von reaktiven Intermediaten eingesetzt werden. Weiterhin wäre zumindest rein formal - eine Aggregation zweier oder mehrer Cyclopentadienyl-Kationen selbst vorstellbar, wobei das substrukturelle Allyl-Kation mit der formalen Doppelbindung eines nächsten Cyclopentadienyl-Kations koordinative Bindungen ausbilden könnte. Selbst 
wenn der sterische Anspruch des Pentacyclopropyl-substituierten Kations diesen Vorgang erlauben könnte, würde dieser Prozess wie auch alle anderen möglichen Wechselwirkungen mit der Doppelbindung zwischen C-4 und C-5 aber einen Abzug von Elektronendichte bewirken und in der Folge eine Entschirmung dieser C-Atome. Es würde also genau der gegenteilige Fall eintreten - eben eine Tieffeldverschiebung statt einer festgestellten Hochfeldverschiebung.

Noch kann keine sichere Aussage darüber getroffen werden, ob wirklich das Pentacyclopropylcyclopentadienyl-Kation $\mathbf{1 2}$ vorliegt oder ein weiteres evtl. daraus hervorgehendes ebenfalls $C_{\mathrm{S}}$-symmetrisches kationisches Intermediat. Dagegen liegt die Folgerung nahe, dass an den Positionen 4 und 5 des angenommenen Kations 12 nicht olefinische Kohlenstoffatome mit $\mathrm{sp}^{2}$-Hybridisierung vorhanden sind, sondern - zwar ebenfalls quartäre - aber vielleicht eher $\mathrm{sp}$ - oder $\mathrm{sp}^{3}$-hybridiserte C-Atome. Eine sp-Hybridisierung kann wohl ausgeschlossen werden, da dies einem Cyclopentin-Grundgerüst entspräche, das unter den gegebenen Bedingungen nicht existenzfähig wäre.

\subsection{2. $c \mathrm{Pr}_{5} \mathrm{Cp}^{+}$(12) als nichtklassisches Carbokation}

Das Pentacyclopropylcyclopentadienyl-Kation 12 kann prinzipiell auch als ein nichtklassisches, also hypervalentes Carbokation betrachtet werden. Diesen Vorschlag machten WILLIAMS et al. ersmals und formulierten eine quadratisch-pyramidale Struktur für das unsubstituierte Cyclopentadienyl-Kation basierend auf der isoelektronischen Struktur von Pentaboran. ${ }^{[90]}$ STOHRER und HOFFMANN konnten diese Struktur durch theoretische Berechnungen bestätigen. Es kann als ein mit einem $\mathrm{CH}^{+}$-Fragment komplexiertes Cyclobutadien angesehen werden. Je nach theoretischen Niveau der bis heute angewandten Methoden sagen solche Rechnungen in guter Übereinstimmung voraus, dass das pyramidale Kation 6e weniger stabil als das isomere Singulett-Kation 6d sei. Letzteres sollte wiederrum weniger stabil als das isomere Triplett-Kation 6a sein. ${ }^{[47]}$ 


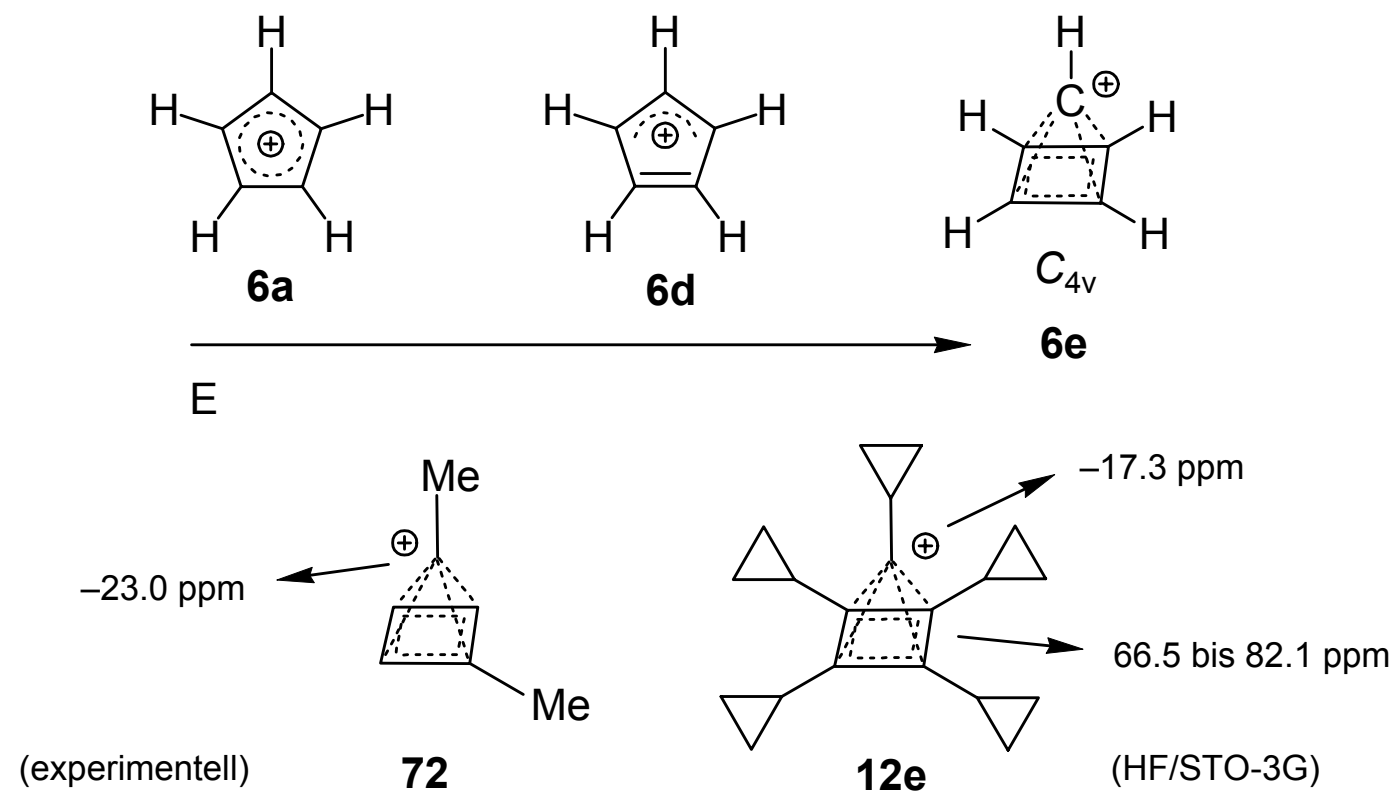

Schema 41. Das Pentacyclopropylcyclopentadienyl-Kation als nichtklassisches Kation 12e mit quadratisch-pyramidaler Struktur?

Obwohl das unsubstituierte pyramidale Cyclopentadienyl-Kation 6e noch nicht erzeugt worden ist, konnten Masamune et al. von Dimethyltricyclo[2.1.0.0 $\left.0^{2,5}\right]$ pentanolen ausgehend das Dimethyl-Derivat $\mathbf{7 2}$ unter stable cation conditions präparieren und NMRspektroskopisch charakterisieren (Schema 41). Bemerkenswert ist die starke Abschirmung des apikalen Kohlenstoffatoms von $-23.0 \mathrm{ppm}$ in 72. Angesichts dessen besäße ein hypothetisches Pentacyclopropyl-substituiertes Cyclopentadienyl-Kation des Typs 12e sicher auch eine hohe Symmetrie und würde dementsprechend drei quartäre ${ }^{13} \mathrm{C}$-Signale aufweisen. Bei eingeschränkter Rotation der apikalen Cyclopropylgruppe könnte auch das beobachtete dynamische Phänomen erklärt werden und als Abfangprodukt wäre auch der Methylether 65 zu erwarten. Allerdings würde wohl wie im Falle von $\mathbf{7 2}$ eine erhebliche Hochfeldverschiebung des apikalen Kohlenstoffatoms auch im Kation 12e auftreten. NMRBerechnungen auf einfachem ab initio Niveau (HF/STO-3G) bestätigen diese Tendenz. Dies steht aber in deutlichem Widerspruch zu den aufgenommenen ${ }^{13} \mathrm{C}-\mathrm{NMR}$-Spektren, so dass das Vorliegen eines quadratisch-pyramidalen Pentacyclopropylcyclopentadienyl-Kations 12e ausgeschlossen werden kann. Eine nur leicht verzerrte Struktur des Isomers 12f in der Art 
eines Briefumschlages erwies sich rechnerisch nicht als Minimum, sondern kollabierte je nach Grad der Faltung zum pyramidalen 12e oder planaren Kation 12d (Schema 42).

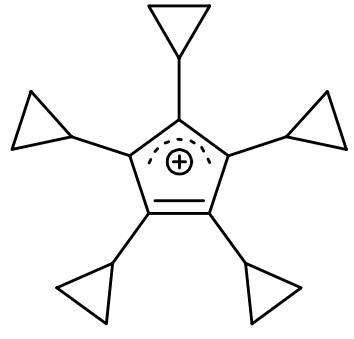

12d

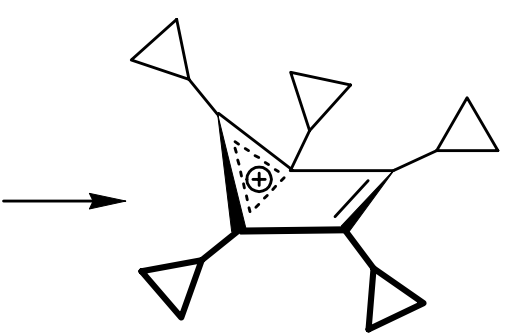

$12 f$

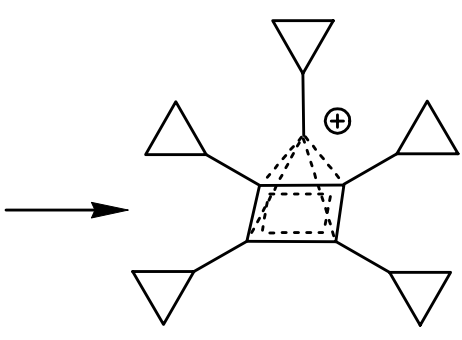

$12 \mathrm{e}$

Schema 42. Isomerisierung des planaren Kations 12d zum pyramidalen Kation 12e über das „Briefumschlag“-Konformer $12 f$.

\subsection{3. $c \operatorname{Pr}_{5} C p^{+}$(12) als reaktive Zwischenstufe}

Führt man sich die chemischen Eigenschaften des kleinsten neutralen, $4 \pi$-Elektronen enthaltenden und damit antiaromatischen Cyclobutadiens vor Augen, so ist es durch extreme Instabilität und hohe Reaktivität, sofern es nicht durch sperrige Substituenten ${ }^{[91]}$ oder durch Komplexierung mit Metallen $^{[92]}$ stabilisiert ist, charakterisiert. ${ }^{[93]}$ Freies Cyclobutadien reagiert zur Stabilisierung sehr rasch auch bei tiefen Temperaturen mit einem weiteren Molekül im Sinne einer [2+2]-Cycloaddition oder einer DIELS-ALDER-Reaktion zum anti- und syn-Tricyclo[4.2.0.0 2,5$]$ octa-3,7-dien. Postuliert man eine solche [2+2]-Dimerisierung auch für ein intermediär gebildetes Pentacyclopropylcyclopentadienyl-Kation (12), so würde das zu einem Decacyclopropyltricyclo[5.3.0.0 $\left.0^{2,6}\right]$ deca-4,8-dien-3,10-diyldikation führen, das wiederum als anti- und syn-Diastereomer anfallen könnte. 


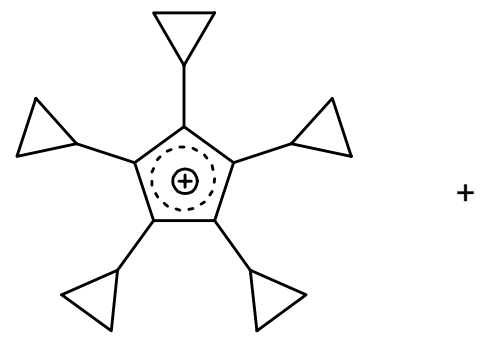

12

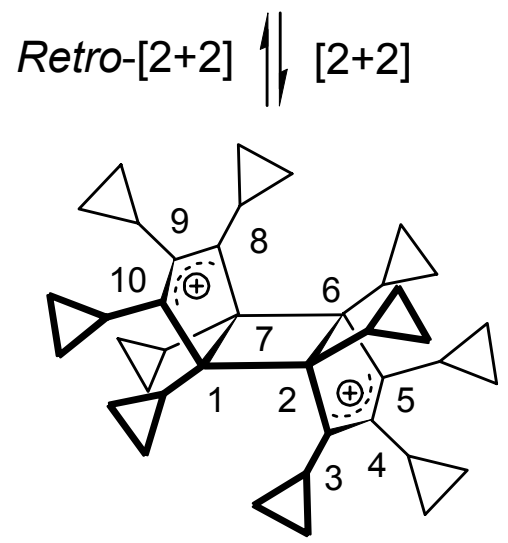

anti-73

$\mathrm{C}_{2 \mathrm{~h}}$

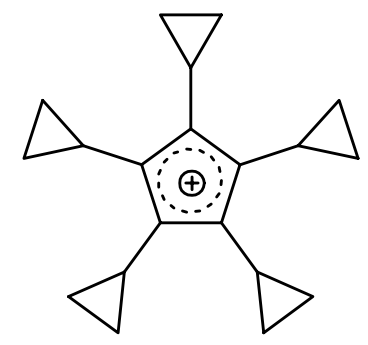

12

Retro-[2+2] $\|$ [2+2]

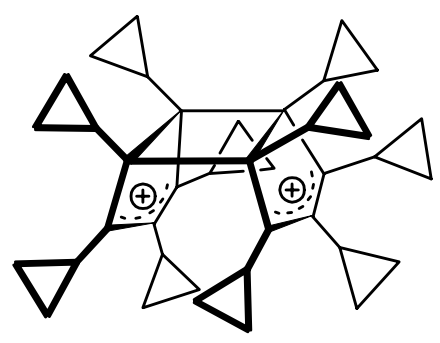

syn-73

$C_{2 \mathrm{v}}$

Schema 43. Hypothetische [2+2]-Cycloaddition des antiaromatischen Kations 12 zum Decacyclopropyl-substituierten tricyclischen Bisallyl-Kation 73.

Ein solches Dikation 73 würde auch die aus den NMR-Experimenten abgeleiteten Symmetrie-Bedingungen erfüllen. anti-73 besitzt eine Spiegelebene, die durch C-4 und C-9 verläuft, und zusätzlich eine zweizählige Drehachse senkrecht dazu. syn-73 weist zwei Spiegelebenen auf, die den Vierring je vertikal halbieren. Eine zweizählige Drehachse verläuft parallel zu den Spiegelebenen durch die Mitte des Vierrings. Also würde man für beide dieser dikationischen Spezies jeweils drei quartäre ${ }^{13} \mathrm{C}$-Signale erwarten. Auch deren Intensitätsverhältnis von 2:1:2 würde mit den Beobachtungen übereinstimmen. Lägen diese Dikationen in einem Gleichgewicht nebeneinander vor, so könnte man das bei der Temperaturvariation entdeckte dynamische Phänomen (siehe Seite 64) mit dem Auftreten eben dieser beiden Isomeren erklären. Dabei läge das thermodynamisch wohl weniger stabile syn-Dikation 73 in geringeren Mengen (kleineres Signal) als das anti-Dikation 73 vor (größeres Signal). Für deren gegenseitige Umwandlung sind unterschiedliche Reaktionswege 
denkbar. Erstens könnte eine Cycloreversion unter Bindungsbruch zwischen C-1,2 und C-6,7 zu zwei Cyclopentadienyl-Kationen 12 führen, die nach entsprechender Drehung und erneuter [2+2]-Cycloaddition jeweils das andere isomere Dikation $\mathbf{7 3}$ hervorbringen könnte (Schema 43).

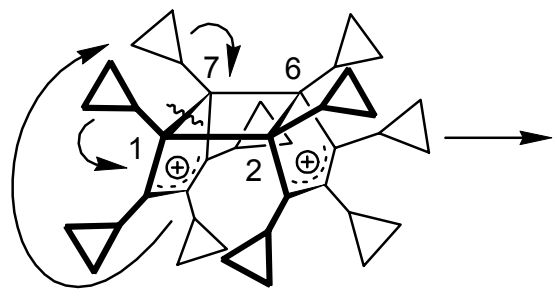

syn-73

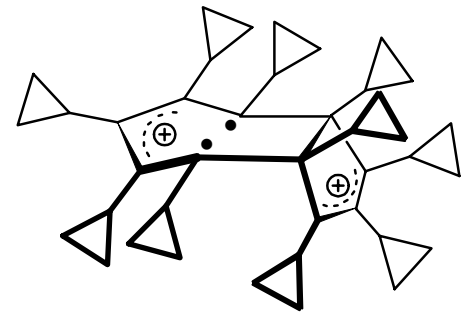

$\Delta \mathrm{E}=0.8 \mathrm{kcal} / \mathrm{mol}$

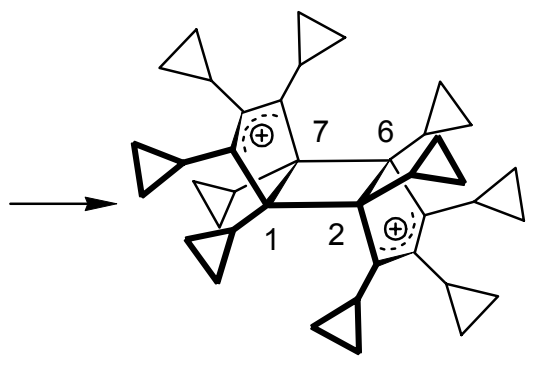

anti-73

Schema 44. Hypothetische Inversion des Bisallyl-Kations 73 ohne intermediäres Auftreten des Pentacyclopropylcyclopentadienyl-Kations $\mathbf{1 2}$ und die dazugehörige berechnete Inversionsenergie.

Zweitens könnte eine Isomerisierung durch eine konrotatorische Drehung entlang der Bindungen zwischen C-1,2 und C-6,7 erfolgen, wobei die beiden Cyclopropyl-Substituenten jeweils außen eine halbkreisförmige Bewegung durchführen und die Cyclopentenyl-Einheit sich im gleichen Maße gegenläufig dazu bewegen würde (Schema 44). Simultan dazu müsste allerdings die Bindung zwischen $\mathrm{C}-1$ und $\mathrm{C}-7$ (oder C-2 und C-6) homolytisch brechen. Die berechnete Energiedifferenz für die Umwandlung des syn-Isomers zum anti-Isomer von $\Delta G_{\mathrm{R}}=0.8 \mathrm{kcal} / \mathrm{mol}$ lässt diese Inversion zumindest unter thermodynamischen Gesichtspunkten möglich erscheinen. syn-73 und anti-73 sind demnach erstaunlicherweise nahezu isoenergetisch, obwohl im syn-Dikation $\mathbf{7 3}$ doch eine erheblich stärkere sterische Abstoßung zu erwarten wäre. Dazu käme eventuell noch eine abstoßende elektrostatische Wechselwirkung zwischen den beiden positiven Ladungen der Allyl-Kation-Systeme.

Unterstützt wird diese Hypothese durch Arbeiten von OLAH et al. Anfang der 80er Jahre. Sie berichteten über die Umlagerung des 3,10-Dimethyltricyclo[5.2.1.0 $\left.0^{2,6}\right]$ deca- 
4,8-dien-3,10-diyldikations $\mathbf{7 5}$ in das 3,10-Dimethyltricyclo[5.3.0.0 $0^{2,6}$ deca-4,8-dien3,10-diyldikation 76 .<smiles>CC1(O)C=CC2CC1C1C=CC2C1(C)O</smiles>

74

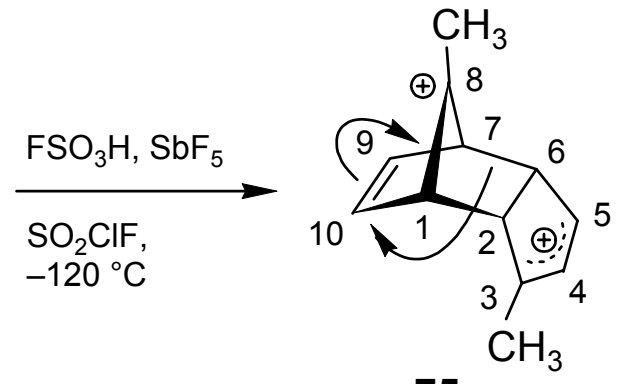

75
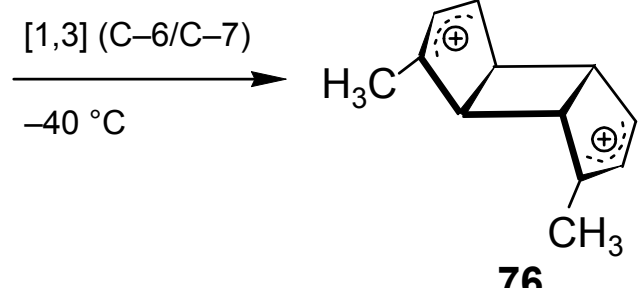

Schema 45. Von OLAH et al. untersuchte Umlagerung des Homoallyl-allyl-Dikations 75 zum Bisallyl-Dikation 76 bei $-40{ }^{\circ} \mathrm{C}$ in $\mathrm{SO}_{2} \mathrm{ClF}$.

Von diesen Überlegungen ausgehend, lassen sich unterschiedlich günstige Reaktionspfade zur Bildung von Cycloaddidtionsprodukten aus dem Pentacyclopropylcyclopentadienyl-Kation formulieren. So wäre es im Verlauf der Kationenpräparation auch möglich, dass ein Pentacyclopropylcyclopentadienyl-Kation zunächst mit einem noch nicht ionisierten Vorläufermolekül des Chlorids 36 in einer der DIELS-ALDER-Reaktion ähnlichen [4+2]-Cycloaddition unter Bildung der Monokationen 77a und 77b reagiert. Dabei wäre die Bildung des Allyl-Kations 77b sicherlich thermodynamisch günstiger, da hierbei das Pentacyclopropylcyclopentadienyl-Kation als elektronenarmes Dienophil und das Chlorid als elektronenreicheres Dien fungieren würde. Theoretische Rechnungen für die relativen freien Reaktionsenthalpien bestätigen diese Vermutung: $\Delta G_{\mathrm{R}}(\mathbf{7 7 a})=30.2 \mathrm{kcal} / \mathrm{mol}<\Delta G_{\mathrm{R}}(\mathbf{7 7} \mathbf{b})=$ $45.7 \mathrm{kcal} / \mathrm{mol}$. Beide Monokationen können dann durch weitere LEWIS-Säure-vermittelte Chloridabstraktion mit einer Wärmetönung von $\Delta G_{\mathrm{R}}(\mathbf{7 7 a})=30.7 \mathrm{kcal} / \mathrm{mol} \mathrm{bzw.} \Delta G_{\mathrm{R}}(\mathbf{7 7 a})=$ $15.7 \mathrm{kcal} / \mathrm{mol} \mathrm{zu}$ dem zu OLAH analogen Norbornenyl-allyl-Dikation 78 weiterreagieren. Letzteres könnte sich ebenso wie OLAHs Dikation durch eine 1,2-sigmatrope Umlagerung zum Bisallyldikation 73 umlagern - diese Umlagerung müsste mit $10.8 \mathrm{kcal} / \mathrm{mol}$ exotherm ablaufen. 
Diesen Werten stehen die berechneten relativen freien Reaktionsenthalpien für die direkte [4+2]- bzw. [2+2]-Cycloaddition zweier Pentacyclopropylcyclopentadienyl-Kationen gegenüber, wobei die direkte Dimerisierung zum Bisallyldikation 73 mit $\Delta G_{\mathrm{R}}=50.1 \mathrm{kcal} / \mathrm{mol}$ etwas günstiger wäre als die Reaktion zum Norbornenyl-allyl-Dikation $78\left(\Delta G_{\mathrm{R}}=\right.$ $60.9 \mathrm{kcal} / \mathrm{mol})$.

Das Vorliegen eines hinreichend stabilen endo-konfigurierten Norbornenyl-allylDikations 78 (kinetisches Diels-Alder-Addukt) ist an dieser Stelle wohl auszuschliessen, da dieses sechs unterschiedliche quartäre ${ }^{13} \mathrm{C}$-Signale im NMR-Spektrum in einem Verhältnis von $2: 2: 1: 2: 2: 1$ hervorrufen müsste, die nicht beobachtet wurden. Ein solches Norbornenylallyl-Dikation besäße auch eine niedrigere als aus den aufgezeichneten NMR-Spektren ableitbare Symmetrie - es hätte nur eine Spiegelebene durch C-10 und C-4. Die etwaige Betrachtung eines thermodynamisch begünstigten exo-Norbornenyl-allyl-Dikations erübrigt sich damit.

Betrachtet man zusammenfassend die errechneten Energiebeträge für die möglichen [2+2]- bzw. [4+2]-Cycloadditionen, so fällt auf, dass diese durchweg stark positiv ausfallen, die Reaktionen also deutlich endotherm verlaufen müssten. Diese Energiebeträge wären zu groß als dass sie in dem experimentellen Temperaturbereich von -140 bis $-110^{\circ} \mathrm{C}$ aufgebracht werden könnten, d. h. die diskutierten Reaktionen könnten würden nicht ablaufen. Aus den berechneten Energiebeträgen lässt sich aber auch noch ableiten, dass ein Cyclopentadienyl-Kation 12 offensichtlich energieärmer wäre als ein Bisallyl-Dikation wie 73. Dies ist vom chemischen Standpunkt aus betrachtet nicht unmittelbar einsichtig, da ein Cyclopentadienyl-Kation als antiaromatische Spezies grundsätzlich ein instabiles und damit hochenergetisches Molekül sein sollte. Dagegen ist ein Allyl-Kation wie beispielsweise das ebenfalls untersuchte Cyclopentenyl-Kation 60 (siehe Kapitel 4.4) sehr gut stabilisiert. Eine eventuelle elektrostatische Wechselwirkung innerhalb der Dikationen kann sicherlich nicht zu einer Endothermie beitragen, da die positiven Ladungen erstens delokalisiert wären und dazu noch weit voneinander entfernt. Außerdem müsste bei der formulierten Reaktion zwischen 
einem Cyclopentadienyl-Kation 12 und einem Chlorid 36 hin zum Monokation 77a oder 77b, bei der die Anzahl an positiven Ladungen im Molekül konstant bleibt, eine negative Reaktionsenergie beobachtet werden, was aber nicht der Fall ist. Beide Reaktionen sind ebenfalls deutlich endotherm. Zu erwarten wäre aber jeweils eine exotherme Reaktion, da ein antiaromatisches Kation in eine gut stabilisierte Spezies transformiert wird.

In der Reihe der denkbaren Cycloadditionen muss noch die Möglichkeit einer formalen [4+4]-Cycloaddition in Betracht gezogen werden, die $\mathrm{zu}$ einem bishomoaromatischen Dikation des Typs 79 führen würde. Auch dessen Bildung aus 78 durch eine 1,2-sigmatrope Umlagerung wäre möglich. Dieses könnte wiederrum als syn- oder anti-Isomer vorliegen. Für diese ebenfalls hochsymmetrischen Dikationen syn-79 $\left(C_{2 \mathrm{~h}}\right)$ und anti-79 $\left(C_{2 \mathrm{v}}\right)$ würde das Auftreten von jeweils drei quartären ${ }^{13} \mathrm{C}$-NMR-Signalen sprechen, deren integrales Verhältnis aber 1:2:2 wäre. Auch ein dynamisches Verhalten wäre zu erwarten, wenn man bedenkt, dass die Cyclopropylgruppen an den Brücken (an C-9,10) jeweils bisektierte Konformationen einnehmen können und so auf die schon weiter oben beschriebene Weise Rotamere bilden könnten. Allerdings spricht gegen das Vorliegen solcher Kationen, dass die chemische Verschiebung der Brückenkohlenstoffatome von Norbornenyl- oder Norbornadienyl-Kationen typischerweise bei 70 bis 80 ppm erwartet werden - beobachtet wurden dagegen ${ }^{13} \mathrm{C}$ Verschiebungen von 222 ppm. ${ }^{[94]}$

Unter den bisher diskutierten möglichen Dimerisierungsprodukten erfüllt das BisallylDikation 73 die theoretisch erwarteten physikalischen Eigenschaften am besten. Daher wurden die ${ }^{13} \mathrm{C}-\mathrm{NMR}-V$ erschiebungen dieser Spezies berechnet. Dabei stellte sich heraus, dass die chemischen Verschiebungen in der Tat besser übereinstimmen. Die noch bestehenden Abweichungen von 25-30 ppm können auf Elektronenkorrelation, die in der theoretischen Rechnung eventuell nicht genügend berücksichtigt werden. Diese NMRVerschiebungen unterstützen demnach das Vorliegen des Tricyclo[5.3.0.0 $0^{2,6}$ deca-4,8-dien3,10-diyldikations 73. 


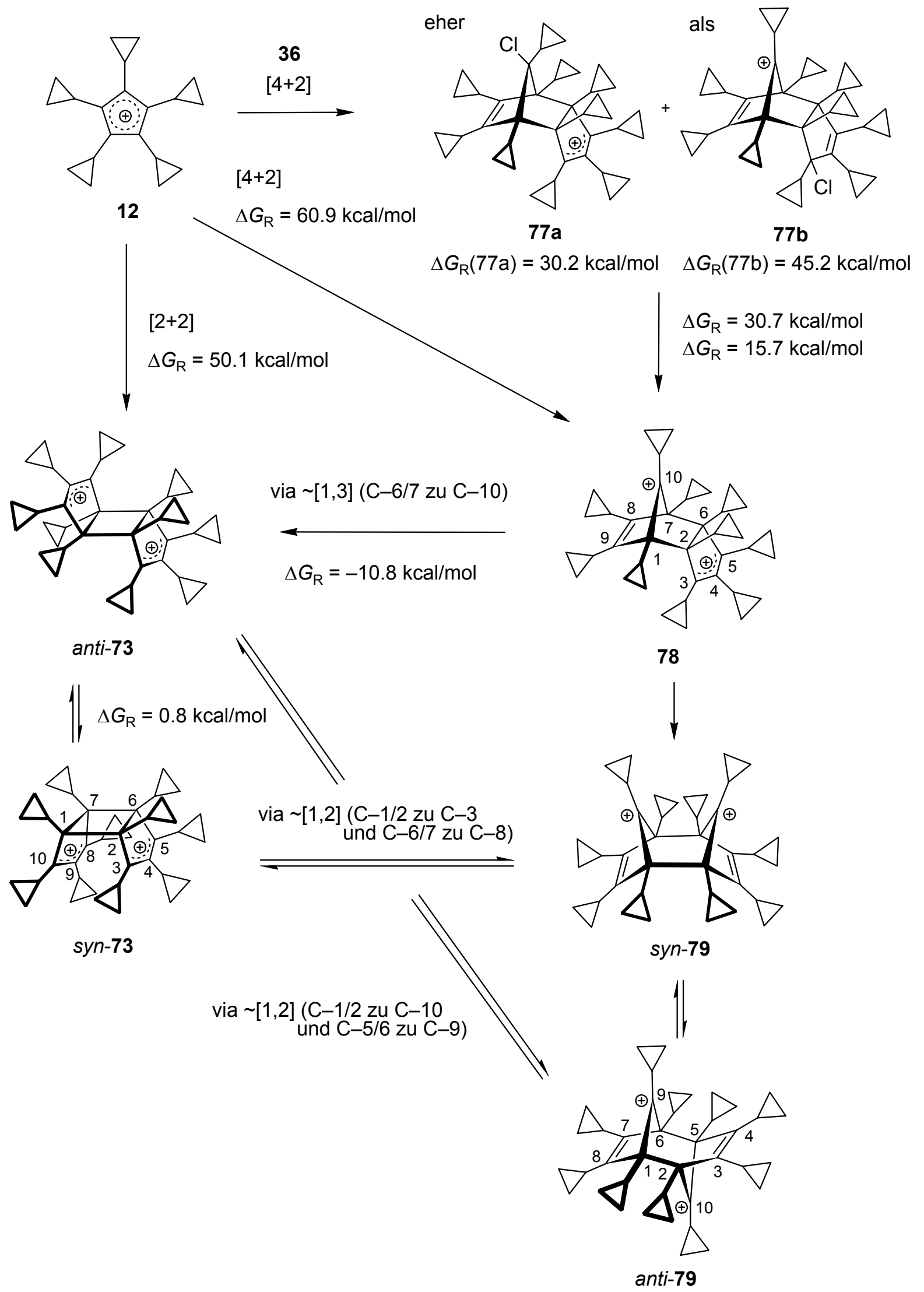

Schema 46. Mögliche Reaktionskanäle des Pentacyclopropylcyclopentadienyl-Kations 12. 
Tabelle 4. Vergleich der experimentellen und berechneten ${ }^{13} \mathrm{C}-\mathrm{NMR}-$ Verschiebungen des kationischen Bisallyl-Systems von anti-73 (auf B3LYP/6-311G**-Niveau).

\begin{tabular}{cccccc}
\hline & $\mathrm{C}-1$ & $\mathrm{C}-2$ & $\mathrm{C}-3$ & $\mathrm{C}-4$ & $\mathrm{C}-5$ \\
\hline $\begin{array}{c}\text { experimentell } \\
\text { berechnet für } \\
\text { anti-73 }\end{array}$ & $222.6,(221.9)$ & $(149.2), 148.3$ & $222.6,(221.9)$ & $83.2,(82.3)$ & $83.2,(82.3)$ \\
\hline
\end{tabular}

Es bliebe noch zu prüfen wie das Vorliegen eines durch Cycloadditionen gebildeten Dikations mit dem beim Quenchen der untersuchten NMR-Probe identifizierten Abfangprodukt 65 des monomeren Cyclopentadienyl-Kations 12 in Einklang gebracht werden kann (vgl. S. 52). Ein entsprechender Bismethylether $\mathbf{8 0}$ der aus dem Abfang der Dikationen mit Methanol erhalten würde, konnte nicht detektiert werden.

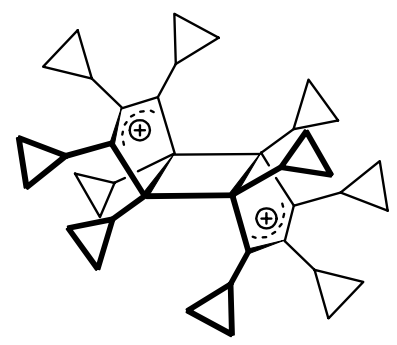

anti-73
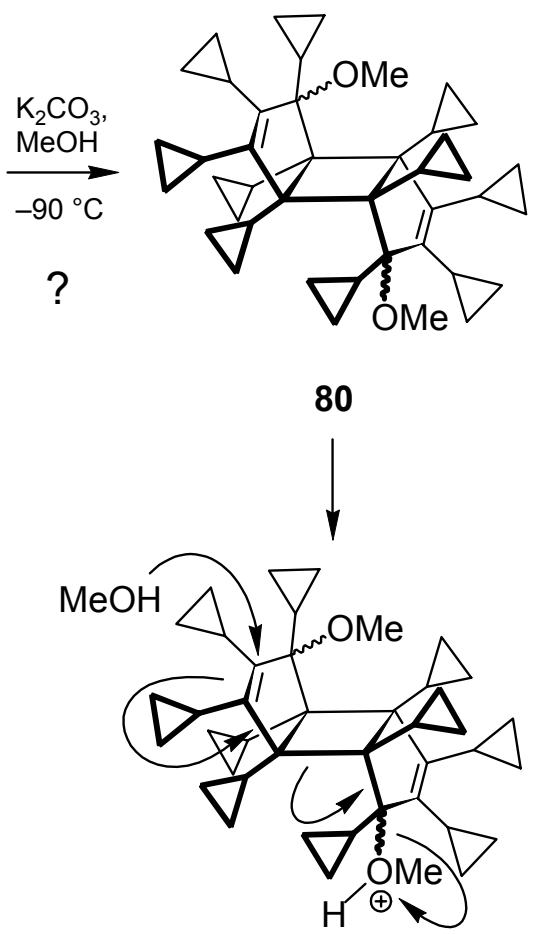

80

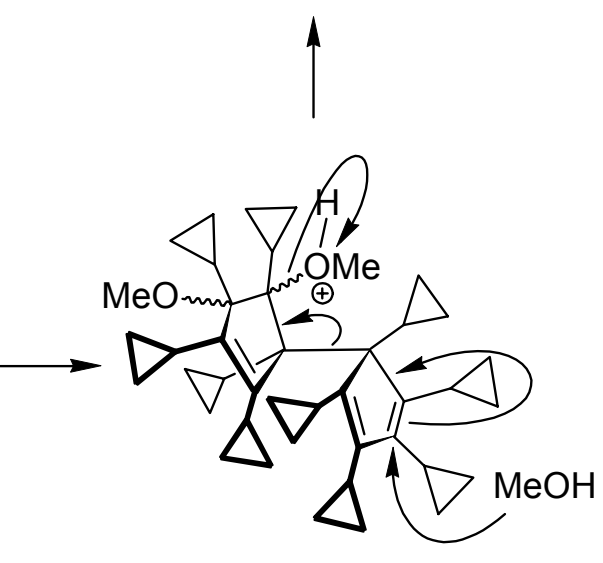

Schema 47. Hypothetischer Mechanismus zur Bildung des monomeren Abfangproduktes 65. 
Denkbar wäre in Umkehrung der Dikationen-Bildung eine unter Energiezufuhr (Abfang bei $-90{ }^{\circ} \mathrm{C}$ ) zum Pentacyclopropylcyclopentadienyl-Kation 12 zurückführende Cycloreversion und nachfolgende Reaktion mit Methanol (Schema 47). Die oben diskutierte starke Endothermie der Dikationen-Bildung würde umgekehrt eine Rückreaktion begünstigen. Vielleicht wäre aber doch der zunächst intermediär gebildete Bismethylether $\mathbf{8 0}$ für einen Nucleophil-assistierten Zerfall empfänglich. Bei dem Abfangexperiment wird, wie oben geschildert, zunächst auch eine große Menge Säure freigesetzt, die den Bismethylether 80 aktivieren könnte. Ein zweimaliger nucleophiler Angriff von Methanol könnte dann zwei Moleküle Methoxypentacyclopropylcyclopentadien 65 freisetzen. Andererseits haben OLAH et al. hätten bei dem von ihnen untersuchten System ein solches Phänomen nicht beobachten können. Allerdings wurde ein vergleichbarer Fall von Brown et al. berichtet, und er stellt einen Schlüsselschritt in ihrer Synthese von Cyclopentenolonen dar. Das strukturell ähnliche Cyclobutadicyclopentendion $\mathbf{8 1}$ erhielten sie durch eine doppelte vinyloge CLAISENKondensation von Methylhexenoat mit Dimethyloxalat und Kaliummethylat. Dieses konnten sie in Gegewart von Dimethylmalonat und einer Base in das Hydroxycyclopentenon 82 überführen. Sie vermuteten dabei einen nucleophilen Angriff des Malonatenolations und eine Folge von ClAISEN- und Retro-CLAISEN-Teilschritten (Schema 48). 


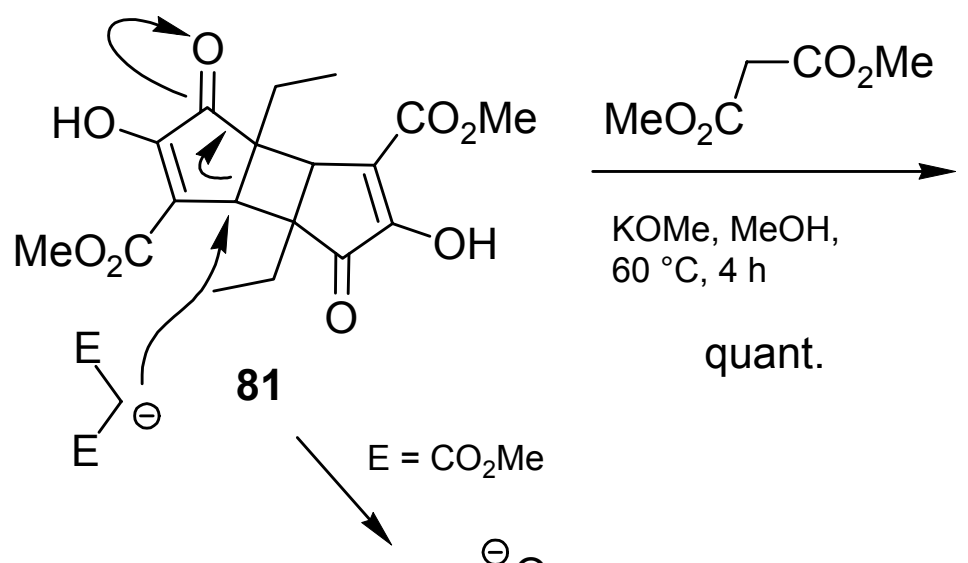

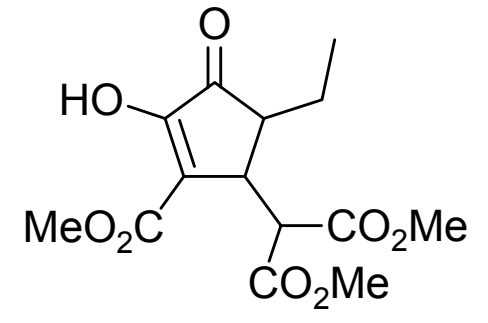

82

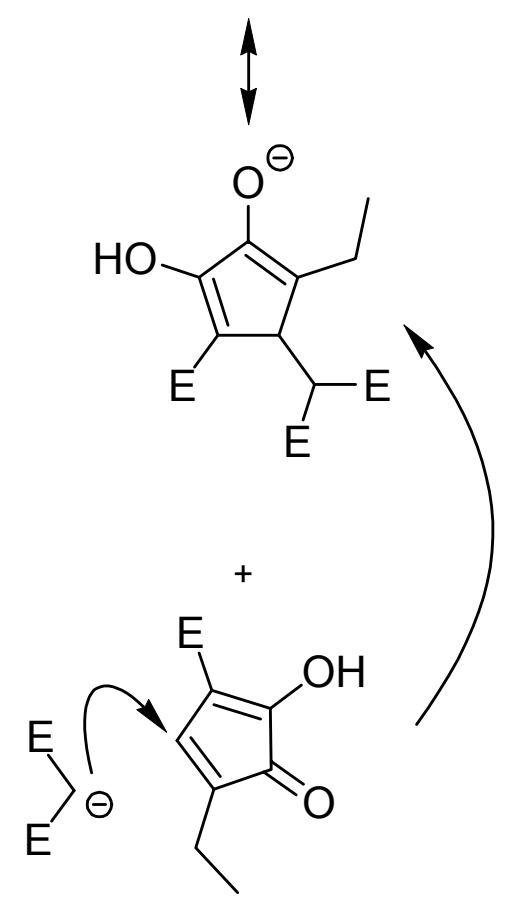

Schema 48. Bildung von monomerem Cyclopentenon 82 aus dem Cyclobutadicyclopentendion 81 .

Zusammenfassend lässt sich hier feststellen, dass die erhaltenen experimentellen Ergebnisse - nämlich Solvolyseversuche, NMR-spektroskopische Untersuchungen einer unter stable cation conditions hergestellten Kationenprobe und Abfangexperimente - einen klaren Rahmen für deren Interpretation vorgeben. In diesen fügt sich das Tricyclo[5.3.0.0 $\left.0^{2,6}\right]$ deca4,8-dien-3,10-diyldikation 73 nach der vorangegangenen Diskussion bisher am besten. Das angestrebte Pentacyclopropylcyclopentadienyl-Kation 12 kann demnach als reaktives Intermediat formuliert werden, das zunächst aus dem Chlorid $\mathbf{3 6}$ erzeugt und nachfolgend zum Bisallyl-Dikation $\mathbf{7 3}$ dimerisieren würde. Beim Abfangen von $\mathbf{7 3}$ könnte aus diesem der monomere Methylether $\mathbf{6 5}$ wieder freigesetzt werden. Mit den auf die beschriebene Art und 
Weise durchgeführten Experimenten und den bisher zur Verfügung stehenden theoretischen

Methoden lässt sich das Pentacyclopropylcyclopentadienyl-Kation 12 demnach nicht zweifelsfrei als hinreichend stabilisiertes antiaromatisches Carbokation erzeugen und charakterisieren/verstehen. Unter diesen Versuchsbedingungen besitzt 12 offensichtlich noch eine inherente Reaktivität. Sehr wohl wurden eindeutige Hinweise erbracht, die dessen Existenz als Zwischenstufe belegen. Diese Beobachtungen bedeuten aber nicht zwingenderweise, dass das Pentacyclopropylcyclopentadienyl-Kation 12 nicht auf anderem Wege erzeugt werden könnte.

\section{Tetra- und Pentacyclopropylcyclopentadien 14 und 15 als Bausteine für planar- chirale Heteroferrocen-Liganden}

Die Feinabstimmung von Liganden ist unzweifelhaft eines der wichtigsten Instrumente, um die Eigenschaften von Metallkomplexen $\mathrm{zu}$ beeinflussen und beispielsweise die katalytische Aktivität und die Stereoselektivität in Metall-katalysierten Reaktionen zu erhöhen. In der asymmetrischen Katalyse transferieren Liganden ihre chirale Information während der Reaktion nicht nur durch sterische Faktoren. Sie können durch die Koordination von unterschiedlichen Donoratomen am Metallzentrum im gleichen Maße auch elektronische Asymmetrie erzeugen. Die wichtigsten und meist eingesetzten heterozähnigen Liganden sind solche, die Stickstoff oder Phosphor als Donoratome tragen. Die $\pi$-Akzeptorstärke eines Phosphoratoms kann Metallzentren mit niedriger Oxidationsstufe stabilisieren, während die $\sigma$-Donorfähigkeit des Stickstoffs ein Metallzentrum eher zu oxidativen Additionsreaktionen befähigt. Diese Kombination kann dazu beitragen, intermediäre Oxidationsstufen und Geometrien innerhalb eines Katalysezyklus zu stabilisieren. Solche elektronischen Asymmetrien können auch zur Optimierung von Liganden für bestimmte Reaktionen durch gezielte Beeinflussung der Natur der Donoratome genutzt werden. So führt die direkte Bindung eines Phosphoratoms an ein benachbartes elektronegativeres Atom - wie Sauerstoff 
- zu einer Verringerung seiner $\sigma$-Elektronendonorfähigkeit und einer gleichzeitigen Steigerung seiner $\pi$-Akzeptoreigenschaft. Auf der anderen Seite kann die Anwesenheit einer Imino- statt einer Aminogruppe das Stickstoffatom mit einer größeren $\sigma$-Elektronendonorstärke ausstatten. In der Summe resultiert so eine größere Ungleichheit zwischen den Donoratomen. ${ }^{[38]}$

\subsection{Versuche zur Darstellung eines neuartigen N,P-Heteroferrocen-Liganden (16)}

Erst kürzlich stellten FU et al. eine neue Art von Ligand zur katalytischen (asymmetrischen) Acylierung von sekundären Alkoholen mit Diketenen bzw. von Alkoholen mit Ketenen vor, die auf Azaferrocenen basieren. ${ }^{[95]}$ Sie konnten erstmalig auch Indol als $\eta^{5}$-Ligand in einem Ferrocenkomplex wie 85a einsetzen (Abbildung 22). ${ }^{[96]}$

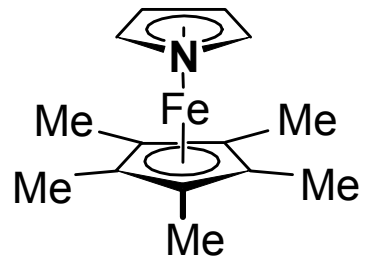

83

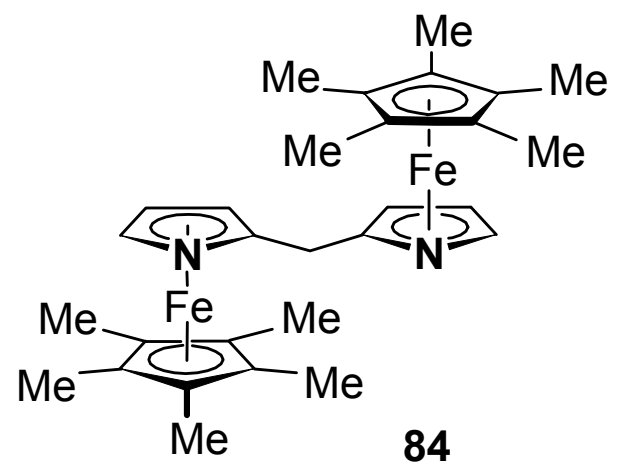

84

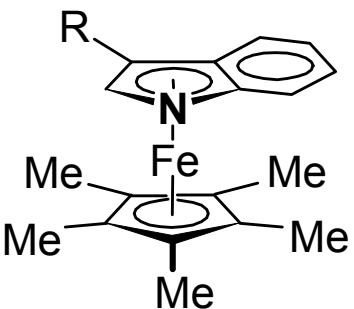

$$
\mathrm{R}=\mathrm{H}, \mathbf{8 5 a}
$$$$
\mathrm{R}=\mathrm{NMe}_{2}, \mathbf{8 5 b}
$$

Abbildung 22. Einige planar-chirale (außer 83) Azaferrocen-Liganden 84, 85 nach FU.

Mit dieser neuen Katalysator-Generation als Vorbild wäre es möglich, einen weiteren neuartigen Liganden auf Basis eines Indolylferrocens $\mathrm{zu}$ synthetisieren, nämlich den zweizähnigen, planar-chiralen N,P-Azaferrocenkomplex 16. Die Cyclopropyl-substituierten Cyclopentadiene 14 oder 15 und das Indol 87 könnten dabei als Bausteine für ein solches Ferrocen 16 dienen (Schema 49). Heteroferrocen-Liganden unterscheiden sich signifikant von anderen häufig angewandten Liganden, die ausschließlich tertiäre Heteroatome (Stickstoff 
oder Phophor) tragen. Ungleich einem $\mathrm{sp}^{3}$-hybridisierten Stickstoffatom eines typischen tertiären Amins, besäße das $\mathrm{sp}^{2}$-hybridisierte N-Atom eines Azaferrocens eine gesteigerte $\sigma$-Elektronendonorfähigkeit, die einem daran koordinierten Metall dazu verhelfen würde, z. B. eine oxidative Addition einfacher einzugehen. Außerdem würde ein solches Stickstoffatom zusätzlich stereogene Information tragen.

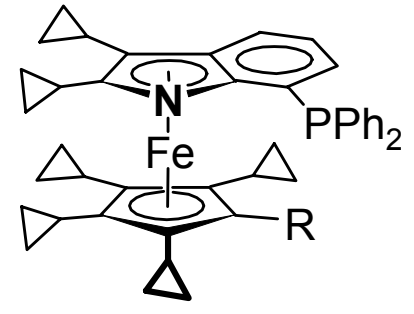

$$
\begin{gathered}
R=H, 16 \mathbf{a} \\
R=c \operatorname{Pr}, 16 \mathbf{b}
\end{gathered}
$$

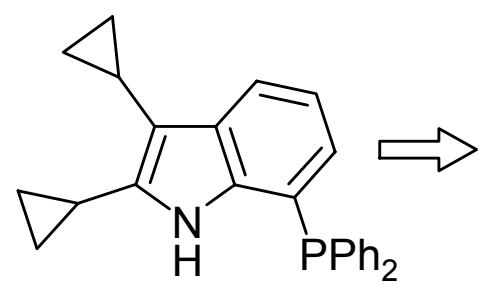

87<smiles>[X]c1ccccc1[N+](=O)[O-]</smiles>

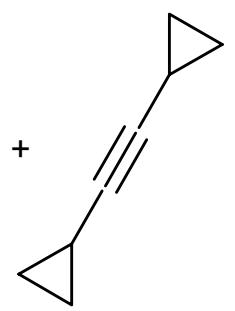

13

Schema 49. Retrosynthese des zweizähnigen, planar-chiralen Indolyl-Liganden 16.

Das Cyclopropyl-substituierte Indol-Grundgerüst konnte in einem einstufigen Prozess aus Dicyclopropylethin (13) und $o$-Brom- bzw. $o$-Iodnitrobenzol $(\mathbf{8 6 a}, \mathbf{b})$ durch eine BARTOLIReaktion zugänglich gemacht werden. ${ }^{[97]}$ Dazu wurde das Acetylen 13 durch eine Hydromagnesierung nach SATO in das Dicyclopropylethenylmagnesiumbromid 46 transformiert und dieses bei -40 bis $-35{ }^{\circ} \mathrm{C}$ mit $o$-Bromnitrobenzol (86a) oder $o$-Iodnitrobenzol (86b) in THF zur Reaktion gebracht. Nach wässriger Aufarbeitung der jeweiligen Reaktionsgemische und säulenchromatographischer Reinigung der Rohprodukte an Kieselgel konnten die 7-Halogenindole 88a und 88b in einer Ausbeute von 38 bzw. 33\% erhalten werden. 


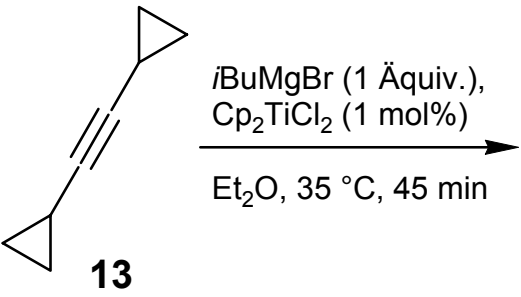

13

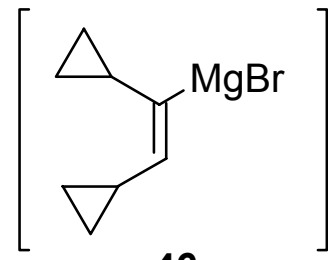

46<smiles>[X]c1ccccc1[N+](=O)[O-]</smiles>

86a $(X=B r)$

86b $(X=I)$

THF, $4 \mathrm{~h}$

-40 bis $-35^{\circ} \mathrm{C}$

$\mathrm{X}=\mathrm{Br}, 38 \%$ 88a

$X=I, 33 \% \mathbf{8 8 b}$

Schema 50. Synthese der 7-Halogenindole 88a und 88b durch BARTOLI-Reaktionen.

Beide Indole wurden nachfolgend in Toluol bei $120{ }^{\circ} \mathrm{C}$ Palladium-katalysiert mit Diphenylphosphan gekuppelt und ergaben so das Phosphanylindol 87 in einer Ausbeute von $52 \%$ im Falle des Bromindols 88a als Kupplungspartner und in 62\% Ausbeute bei Einsatz des Iodindols $\mathbf{8 8 b}$. $^{[98]}$
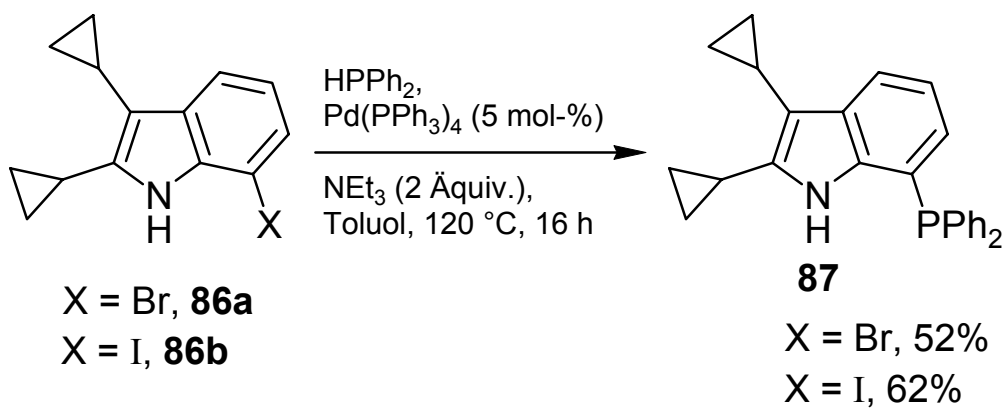

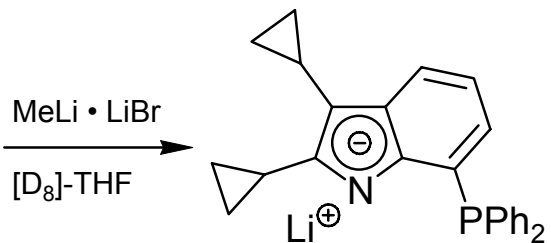

$87 a$

Schema 51. Palladium-katalysierte Kupplung von Diphenylphosphan und 86 zum N,PLiganden 87 und dessen Deprotonierung zum Indolylanion 87a.

Durch Deprotonierung des Phosphanylindols 87 mittels Methyllithium-LithiumbromidKomplex in Deuterotetrahydrofuran bei $0{ }^{\circ} \mathrm{C}$ gelang es, das Indolylanion 87a quantitativ zu bilden und NMR-spektroskopisch $\left({ }^{1} \mathrm{H},{ }^{13} \mathrm{C}\right.$ und $\left.{ }^{31} \mathrm{P}\right) \mathrm{zu}$ charakterisieren. Nachdem die Synthese des Indolferrocens 85a reproduziert werden konnte, wurde versucht, auch aus den eigens synthetisierten Bausteinen 87 und 14 oder 15 einen entsprechenden Komplex aufzubauen. Zahlreiche Ansätze dieses so erzeugten Indolylanions 87a jeweils mit den Anionen der Oligocyclopropylcyclopentadiene 14a oder 15a und Eisen(II)chlorid oder Eisen(II)acetylacetonat in THF analog dem Protokol von FU umzusetzen, führten nicht zur 
Isolierung des gewünschten Komplexes 16. Stattdessen konnten die Edukte teilweise zurückgewonnen werden. Grund dafür könnte sein, dass das zweizähnige Indolylanion 87 selber nicht als $\eta^{5}-\pi$-Ligand fungiert, sondern das Eisenatom in ungewünschter Weise als $\eta^{2}-\sigma$-Ligand komplexiert. Dies könnte dadurch verhindert werden, dass die Valenzen vor der Reaktion mit der Eisen(II)quelle durch Silberionen abgesättigt werden. Zu diesem Zweck wird häufig Silbercyanid eingesetzt. ${ }^{[99]}$ Andererseits wäre es möglich, zunächst die Halogenindole 88a,b zur Komplexbildung heranzuziehen und nachfolgend die Diphenylphosphyl-Gruppe einzuführen.

4.2. Versuche zur Darstellung eines gemischten Tetra- oder Pentayclopropyl-substituierten Ferrocens (89)

Über die Darstellung eines gemischten Ferrocens vom Typ 89 wäre es möglich, durch Metallierung des unsubstituierten Cyclopentadienylrings eine weitere Funktionalisierung, wie z. B. Aminierung oder Phosphorylierung, zu erreichen. ${ }^{[100]}$ Da solche Komplexe meist aus dem jeweiligen Cyclopentadienid und einer Eisen(II)-Quelle hergestellt werden, tritt grundsätzlich das Problem einer statistischen Verteilung der Produkte von 1:2:1 auf, nämlich der Bildung je eines Teiles des decasubstituierten und des unsubstituierten Ferrocens, sowie zweier Teile des gewünschten gemischten Komplexes. Dieses statistische Verhältnis verändert sich nur dann, wenn die eingesetzten Cyclopentadienide unterschiedliche Reaktivitäten aufweisen.
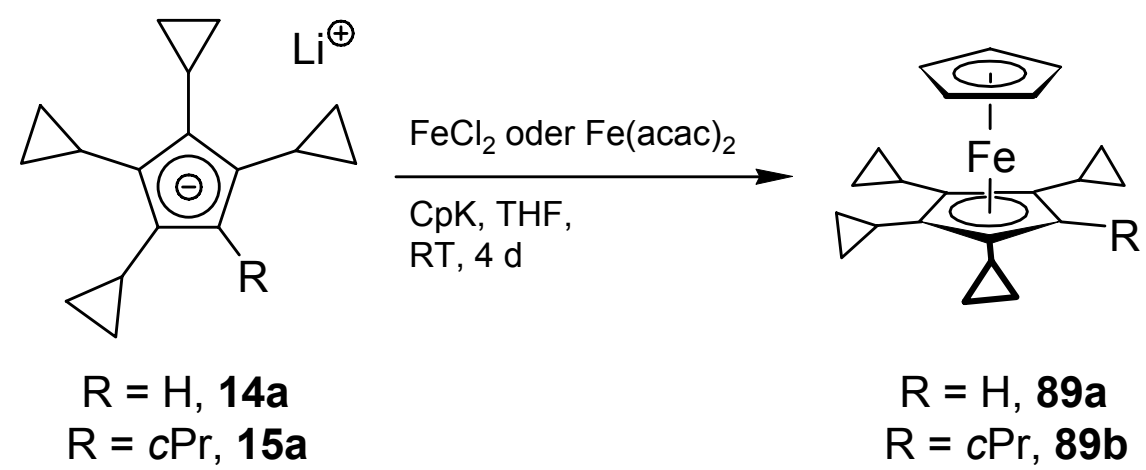

Schema 52. Versuch der Darstellung eines gemischten Ferrocens 89a oder 89b. 
Nach viertägigem Rühren einer Suspension aus dem Tetracyclopropyl-substituierten Cyclopentadienid 14a mit Cyclopentadienylkalium und Eisen(II)chlorid oder Eisen(II)acetylacetonat in THF bei Raumtemperatur konnte nur bei Verwendung von Eisen(II)chlorid ein Rohprodukt erhalten werden, dessen Massenspektrum auf den Komplex 89a eindeutig hinwies. ${ }^{[101]}$ Allerdings konnten NMR-spektroskopisch noch deutliche Spuren von nicht umgesetztem, unsubstituierten Cyclopentadien identifiziert werden. Mit Eisen(II)acetylacetonat konnte kein Komplex 89a detektiert werden, sondern nur normales Ferrocen. Zusätzlich wurde im Rohprodukt massenspektrometrisch ein Dimer des Tetracyclopropylcyclopentadiens 14 identifiziert, was darauf hinweist, dass das Reduktionspotential der eingesetzten Eisen(II)spezies dem Oxidationspotential des Tetracycloproyplcyclopentadienids 14a nahe kommt und so eine radikalische Dimersierung von 14 bewirken kann. Ähnliche Resultate wurden beim Einsatz von Pentacyclopropylcyclopentadienyllithium 15a erhalten. Mit Eisen(II)chlorid wurde hauptsächlich normales Ferrocen isoliert und kein Komplex 89b, was auf die geringere Reaktivität von 15a aufgrund seines höheren sterischen Anspruchs verglichen mit 14a hinweist. Bei Einsatz von Eisen(II)acetylacetonat konnte ganz analog das Dimerisierungsprodukt von 15 nachgewiesen werden, isoliert wurde ebenfalls nur normales Ferrocen. ${ }^{[102]}$

\subsection{Versuche zur Darstellung von Eisentricarbonyl-Cyclopentadien-Komplexen}

$\eta^{4}$-Eisenkomplexe von 1,3-Dienen repräsentieren einen besonders stabilen Bindungsmodus und sind auf einfache Art direkt durch Ligandenaustausch an Eisencarbonylen durch Erhitzen, Bestrahlung mit Licht oder Ultraschall darstellbarbar. Die einfachste Vorstufe ist Eisenpentacarbonyl, aber auch die reaktiveren Quellen wie Dieisennonacarbonyl oder Trieisendodecacarbonyl werden verwendet. Das stark polarisierbare $\pi$-System von Trieisencarbonyldien-Komplexen wie 90 ermöglicht sowohl einerseits die Reaktion mit Elektrophilen in Form einer elektrophilen Substitution in analoger Weise zu einer „klassischen“ elektrophilen aromatischen Substitutionen über eine 
$\eta^{3}$-allylkationische Zwischenstufe 91 als auch eine Addition von Nucleophilen über ein $\eta^{3}$-allylanionisches oder ein $\eta^{3}$-homoallylanionisches Intermediat 93 bzw. 94 (Schema 53). ${ }^{[103]}$

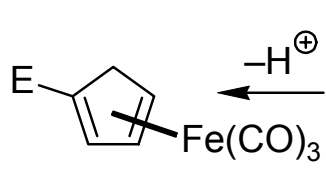

92<smiles>CC(C)C1C2C=CC(C2)C1C(=O)O</smiles>

91

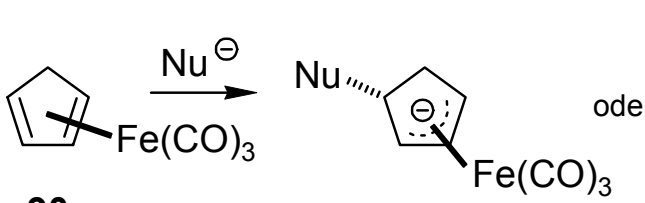

93

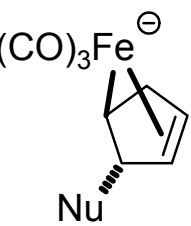

94

Schema 53. Elektrophile und nucleophile Kupplungen mit $\eta^{4}-1,3$-DieneisentricarbonylKomplexen.

Durch Rühren mit Dieisennonacarbonyl in Toluol bei $110{ }^{\circ} \mathrm{C}$ wurde versucht, sowohl Tetra- 14 als auch Pentacyclopropylcyclopentadien 15 zur Reaktion zu bringen. Erfolgreich war dies nur im Falle von 14. Dabei konnte der entsprechende Tetracyclopropylcyclopentadienyleisentricarbonyl-Komplex 95 in einer Ausbeute von 45\% in Form eines orangefarbenen Öls isoliert werden. Im Gegensatz dazu wurde beim Einsatz von 15 nur polymeres Material erhalten.

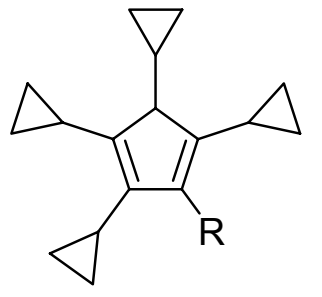

$\mathrm{R}=\mathrm{H}, 14$

$\mathrm{R}=c \operatorname{Pr}, 15$

$$
15
$$
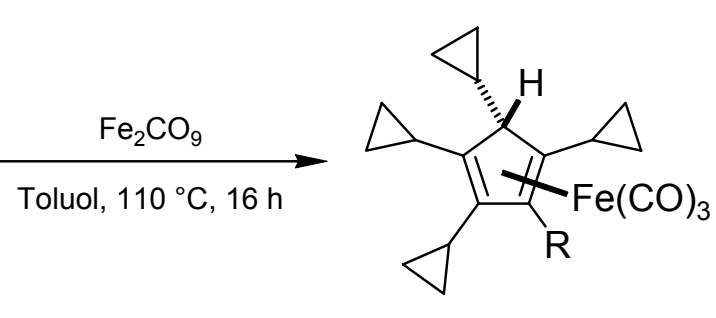

$\mathrm{R}=\mathrm{H}, 95 \mathrm{a}, 45 \%$

$\mathrm{R}=c \mathrm{Pr}, \mathbf{9 5 b}, 0 \%$
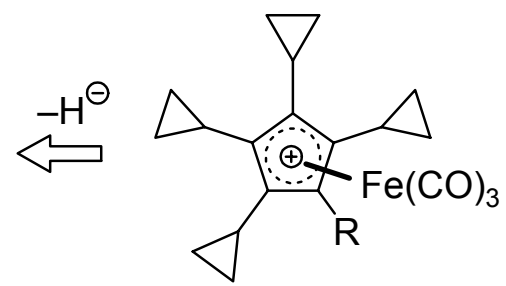

$\mathrm{R}=\mathrm{H}, \mathbf{9 6} \mathbf{a}$
$\mathrm{R}=c \operatorname{Pr}, \mathbf{9 6 b}$

Schema 54. Synthese des Tetracyclopropylcyclopentadienyleisentricarbonyl-Komplexes 95a

- Zugang zu einem Eisentricarbonyl-komplexierten Cyclopentadienyl-Kation 96?

Carbonyleisen-Cyclopentadienyl-Komplexe lassen sich bekanntermaßen durch Hydridabstraktion mittels Tritylsalzen auch in kationische Cyclopentadienyl-Eisen-Komplexe überführen. ${ }^{[104]}$ Ausgehend vom Komplex 95a könnte eventuell ein Eisentricarbonyl- 
komplexiertes Cyclopentadienyl-Kationen 96a erhalten werden. Bei einem solchen Cyclopentadienyl-Kation wäre aber die positive Ladung sicherlich nicht überwiegend im Ring, sondern vor allem auf das Eisenatom delokalisiert. Dies könnte zur Oxidation des Eisenatoms führen, wobei ein Tricarbonyleisen-Halbsandwich-Komplex mit einem Eisen(II)zentrum und einem Cyclopentadienylanion-Liganden entstünde. Entsprechende Reaktionen von Elektrophilen und Nucleophilen mit 96a wären sicherlich lohnend. Auch der Pentacyclopropyl-substituierte Komplex 95b könnte vielleicht besser durch Lichteinwirkung als durch Erhitzen zugänglich gemacht werden.

\section{Oligocyclopropylierte Diene als Substrate in DIELS-ALDER-Reaktionen}

Die Chemie von (substituierten) Cyclopentadienen weist viele Facetten auf. So sind Cyclopentadiene und seine Derivate nicht nur die am häufigsten verwendeten Liganden für Metallkomplexe. ${ }^{[63]}$ Als cyclische Diene fungieren sie sehr gut auch als Substrat in Cycloadditionen. ${ }^{[105]}$ DIELS-ALDER-Reaktionen mit Cyclopentadienen als Dienkomponente sind wohl bekannt und können wertvolle Schlüsselrollen in der Synthese von biologisch relevanten Molekülen einnehmen. ${ }^{[106]}$ Insofern wäre es lohnend, die Reaktivität der neuen Cyclopropyl-substituierten Cyclopentadiene 14 und 15 gegenüber Dienophilen auszuloten. Durch solche DIELS-ALDER-Reaktionen wären neuartige oligocyclopropylierte Bicyclo[2.2.1]hepten-Grundgerüste auf einfache Weise in einem Schritt zugänglich.

Auch mehrfach Cyclopropyl-substituierte Cyclohexen-Derivate können durch pericyclische Reaktionen aufgebaut werden, wenn man Dienophile mit 1,2,3,4-Tetracyclopropylbuta-1,3-dien (21) umsetzt. Dieses ist mit einer sehr guten Ausbeute in einem einstufigen Prozess aus Dicyclopropylethin (13) in Anlehnung an Protokolle von SATO et al. darstellbar. ${ }^{[107]} \mathrm{Zu}$ einer Lösung von Titantetraisopropylat und dem Alkin 13 in Diethylether wurde bei $-78^{\circ} \mathrm{C}$ eine etherische Lösung von Isopropylmagnesiumbromid getropft, die 
Mischung eine Stunde bei -50 bis $-40{ }^{\circ} \mathrm{C}$ gerührt und nachfolgend bei $-78{ }^{\circ} \mathrm{C}$ tropfenweise mit Methanol versetzt. So konnte das gewünschte 1,2,4,5-Tetracyclopropylbutadien (21) als diastereoselektiv gebildetes (E,E)-Isomer in 91\% Ausbeute isoliert werden (Schema 55).

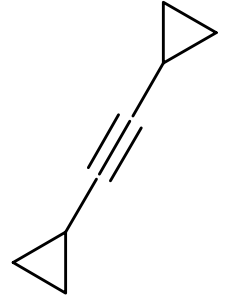

13
1) $\mathrm{Ti}(\mathrm{OiPr})_{4}(0.5$ Äquiv.) iPrMgBr (1.1 Äquiv.) $\mathrm{Et}_{2} \mathrm{O},-45^{\circ} \mathrm{C}, 1 \mathrm{~h}$

2) $\mathrm{MeOH} /$ Pentan $-78{ }^{\circ} \mathrm{C} \rightarrow \mathrm{RT}, 1 \mathrm{~h}$

$91 \%$

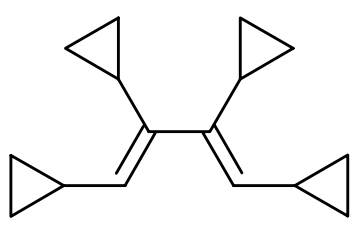

21

Schema 55. Einstufige Synthese von $(E, E)$-1,2,3,4-Tetracyclopropylbuta-1,3-dien (21) durch Titan-vermittelte Dimerisierung von Dicyclopropylethin (13).

5.1. DIELS-ALDER-Reaktionen mit Dimethylacetylendicarboxylat (97) und N-Phenylmaleimid (98)

Für die Reaktion der Diene Tetra- 14 und Pentacyclopropylcyclopentadien 15 sowie Tetracyclopropylbutadien 21 mit Dimethylacetylendicarboxylat 97 oder $N$-Phenylmaleimid 98 wurde eine Mischung von 14, 15 oder 21 in Toluol mit dem jeweiligen Dienophil bei Temperaturen zwischen 100 und $130^{\circ} \mathrm{C}$ für 16 bis 22 Stunden umgesetzt, und dabei die DIELS-ALDER-Produkte in Ausbeuten von 55 bis 77\% isoliert (Tabelle 5, Schema 56, 57 und $58)$. 
Tabelle 5. DIELS-ALDER-Reaktionen von Tetra- 14und Pentacyclopropylcyclopentadien 15 sowie 1,2,3,4-Tetracyclopropylbutadien (21) mit Dimethylacetylendicarboxylat (97) und $N$-Phenylmaleimid (98) in Toluol.

\begin{tabular}{ccccccc}
\hline Eintrag & Dien & Dienophil & Temp. $\left[{ }^{\circ} \mathrm{C}\right]$ & Zeit $[\mathrm{h}]$ & Produkt $^{[\mathrm{a}]}$ & Ausbeute (\%) \\
\hline 1 & $\mathbf{1 4}$ & $\mathbf{9 7}$ & 100 & 16 & $\mathbf{9 9}(1: 1)$ & 77 \\
2 & $\mathbf{9 8}$ & 100 & 16 & $\mathbf{1 0 0}(3.5: 1)$ & 56 \\
\hline 3 & $\mathbf{1 5}$ & $\mathbf{9 7}$ & 110 & 20 & $\mathbf{1 0 1}(1: 1)$ & 55 \\
4 & $\mathbf{9 8}$ & 110 & 20 & $\mathbf{1 0 2}$ & 62 \\
\hline 5 & $\mathbf{2 1}$ & $\mathbf{9 7}$ & 130 & 22 & $\mathbf{1 0 3}$ & 70 \\
\hline
\end{tabular}

${ }^{\text {[a] } V e r h a ̈ l t n i s ~ d e r ~ D i a s t e r e o m e r e n ~ i n ~ K l a m m e r n . ~}$

Orientierende Vorversuche im NMR-Röhrchen zeigten, dass die Reaktionen des Pentacyclopropylcyclopentadiens $\mathbf{1 5}$ eine etwas höhere Temperatur $\left(110^{\circ} \mathrm{C}\right.$, Einträge $\left.3-4\right)$ erfordern als die des Tetracyclopropylcyclopentadiens $14\left(100^{\circ} \mathrm{C}\right.$, Einträge 1-2). Für 1,2,3,4-Tetracyclopropylbutadien (21) musste die Reaktionstemperatur sogar auf $130{ }^{\circ} \mathrm{C}$ (Einträge 5-6) erhöht werden, um einen zügigen Umsatz zu erreichen. Dies passt zu der nicht cyclischen Struktur dieses Diens, obwohl dessen Vorzugskonformation wegen der Cyclopropylsubstituenten in 2,3-Position sicherlich nicht s-trans sein dürfte. 


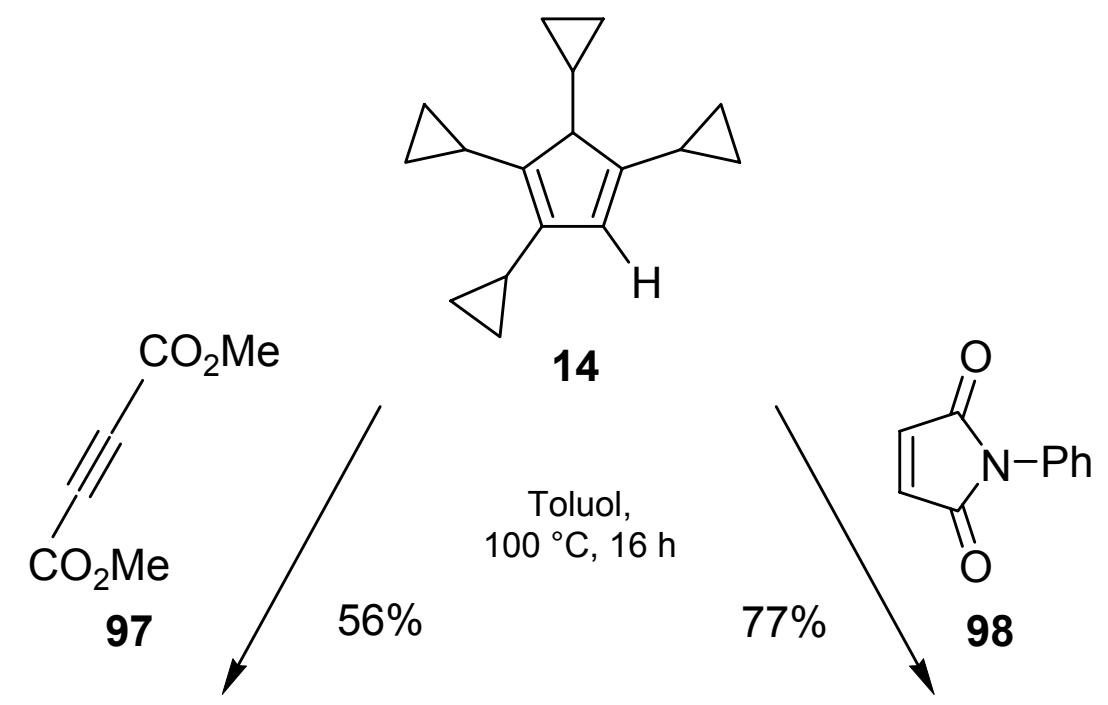

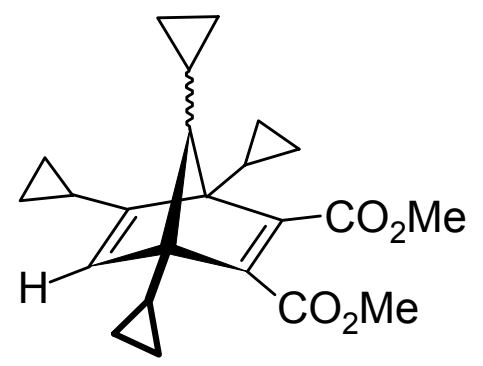

$99(1: 1)$

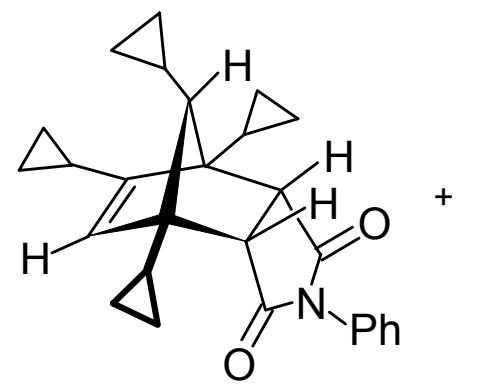

syn-100

\section{5:1}

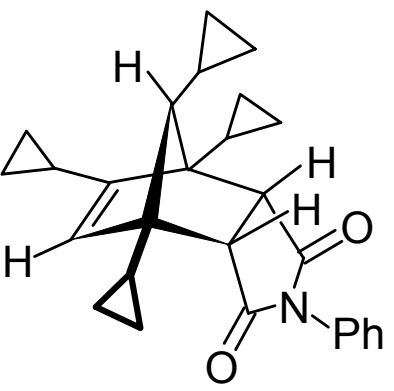

anti-100

Schema 56. DIELS-ALDER-Produkte 99 und 100 der Reaktionen zwischen Tetracyclopropylcyclopentadien 14 mit Dimethylacetylendicaboxylat 97 und $N$-Phenylmaleimid 98.

Bei der Reaktion des Tetracyclopropylcyclopentadiens 14 mit Dimethylacetylendicarboxylat 97 wurde das Norbornen 99 mit 77\% Ausbeute als 1:1 Gemisch zweier Diastereomere erhalten, die sich in der Konfiguration des Brückenkohlenstoffatoms unterscheiden (Schema 56, Tabelle 5, Eintrag 1). Das offenbar etwas reaktionsträgere $N$-Phenylmaleimid 98 lieferte anti- und syn-100 dagegen als Diastereomerengemisch in einem Verhältnis von 3.5:1 mit 56\% Ausbeute (Schema 56, Tabelle 5, Eintrag 2). Die bevorzugte Bildung des anti-Diastereomers anti-100a ist sicherlich auch auf den größeren sterischen Anspruch von 98 im Vergleich zu 97 zurückzuführen, der sich im Übergangszustand der Reaktion auswirkt. Nach Umkristallisieren aus Heptan/Dichlormethan konnte die Konstitution des Tetracyclopropylnorbornen-Derivats $\mathbf{1 0 0}$ auch durch eine Röntgen-Strukturanalyse bestätigt werden. Dabei liegen beide Diastereomere in einer Elementarzelle nebeneinander vor (Abbildung 23). 


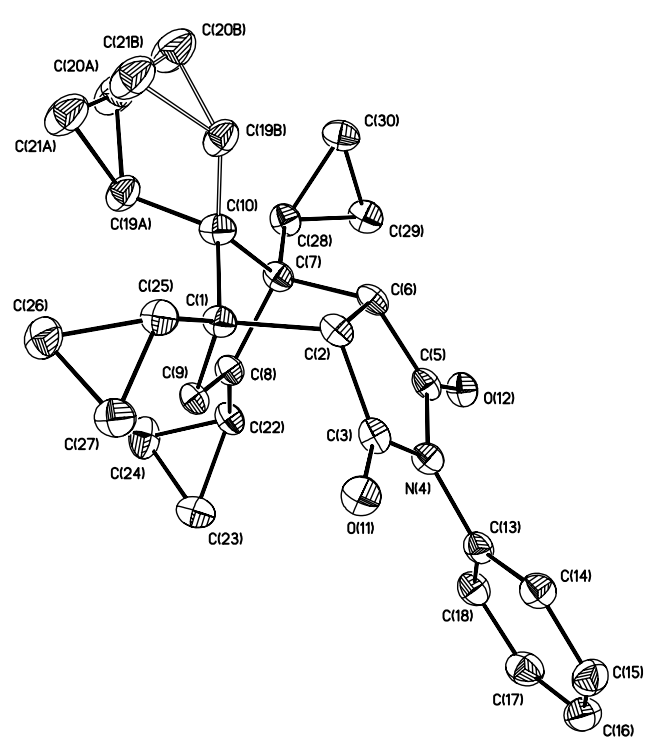

Abbildung 23. Struktur von 100 im Kristall (Schwingungselipsoide mit 30\% Aufenthaltswahrscheinlichkeit).

Das DIELS-ALDER-Produkt 101 aus 15 und Dimethylacetylendicarboxylat wurde ebenfalls als 1:1 Gemisch des syn- und anti-Diastereomers in 55\% Ausbeute erhalten (Schema 57, Tabelle 5, Eintrag 3). Das Norbornenderivat anti-102 aus dem Pentacyclopropylcyclopentadien (15) und $N$-Phenylmaleimid entstand dagegen als ein einziges Diastereomer in 62\% Ausbeute (Schema 57, Tabelle 5, Eintrag 4). In diesem Falle bewirken offenbar sowohl die sterischen Effekte des Pentacyclopropylcyclopentadiens als auch die des $N$-Phenylmaleimids eine selektive Bildung von 102. 


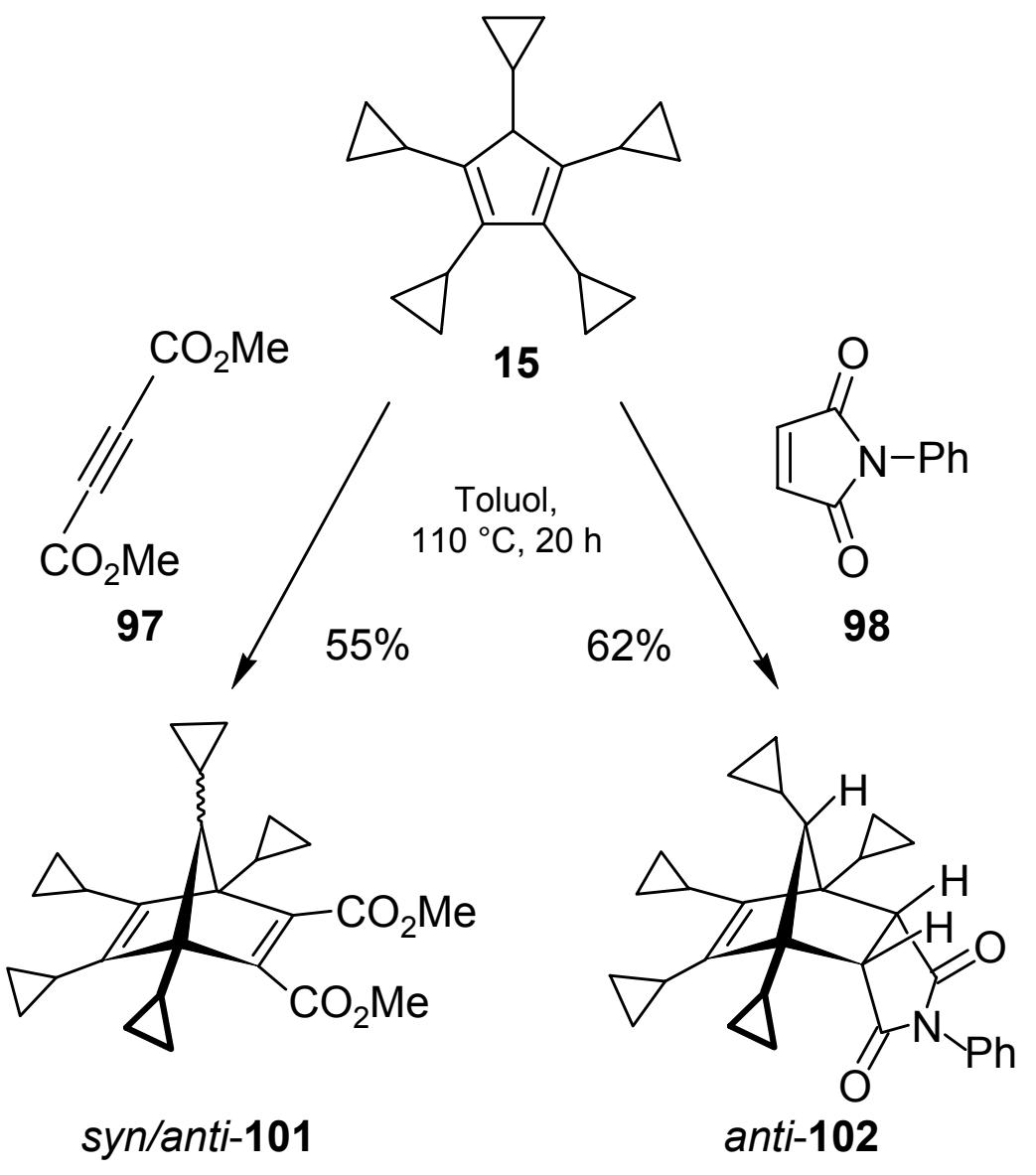

Schema 57. DIELS-ALDER-Produkte 101 und 102 der Reaktionen von Pentacyclopropylcyclopentadien (15) mit Dimethylacetylendicaboxylat (97) und $N$-Phenylmaleimid (98).

Ausgehend von 1,2,3,4-Tetracyclopropylbutadien (21) wurden das Cyclohexadiendicarboxylat 103 und das Cyclohexenderivat 104 in jeweils 70\% Ausbeute als einzelne Diastereomere erhalten (Schema 58, Tabelle 5, Einträge 5-6). Durch Umkristallisieren von 102 und 104 jeweils aus einem Gemisch von Heptan und Dichlormethan konnten jeweils geeignete Kristalle gewonnen werden, deren RöntgenStrukturanalysen die Konfigurationen bestätigten (Abbildung 24). Dabei wies 102 eine Elementarzelle mit zwei gleichen Diastereomeren auf, wovon eines vier stark fehlgeordnete Cyclopropylgruppen aufwies. 


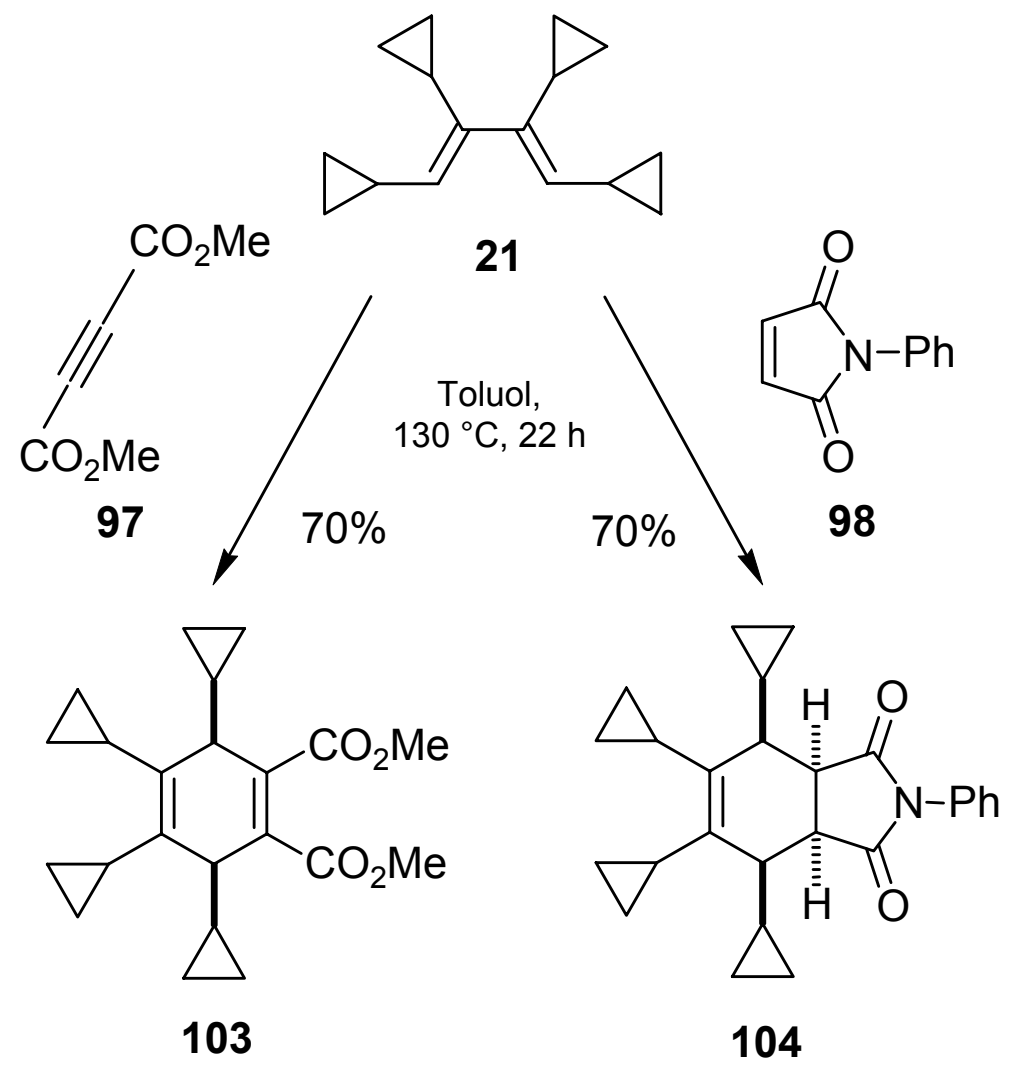

Schema 58. DIELS-ALDER-Produkte 103 und 104 der Reaktionen von 1,2,3,4-Tetracyclopropylbutadien (21) mit Dimethylacetylendicaboxylat (97) und $N$-Phenylmaleimid (98).
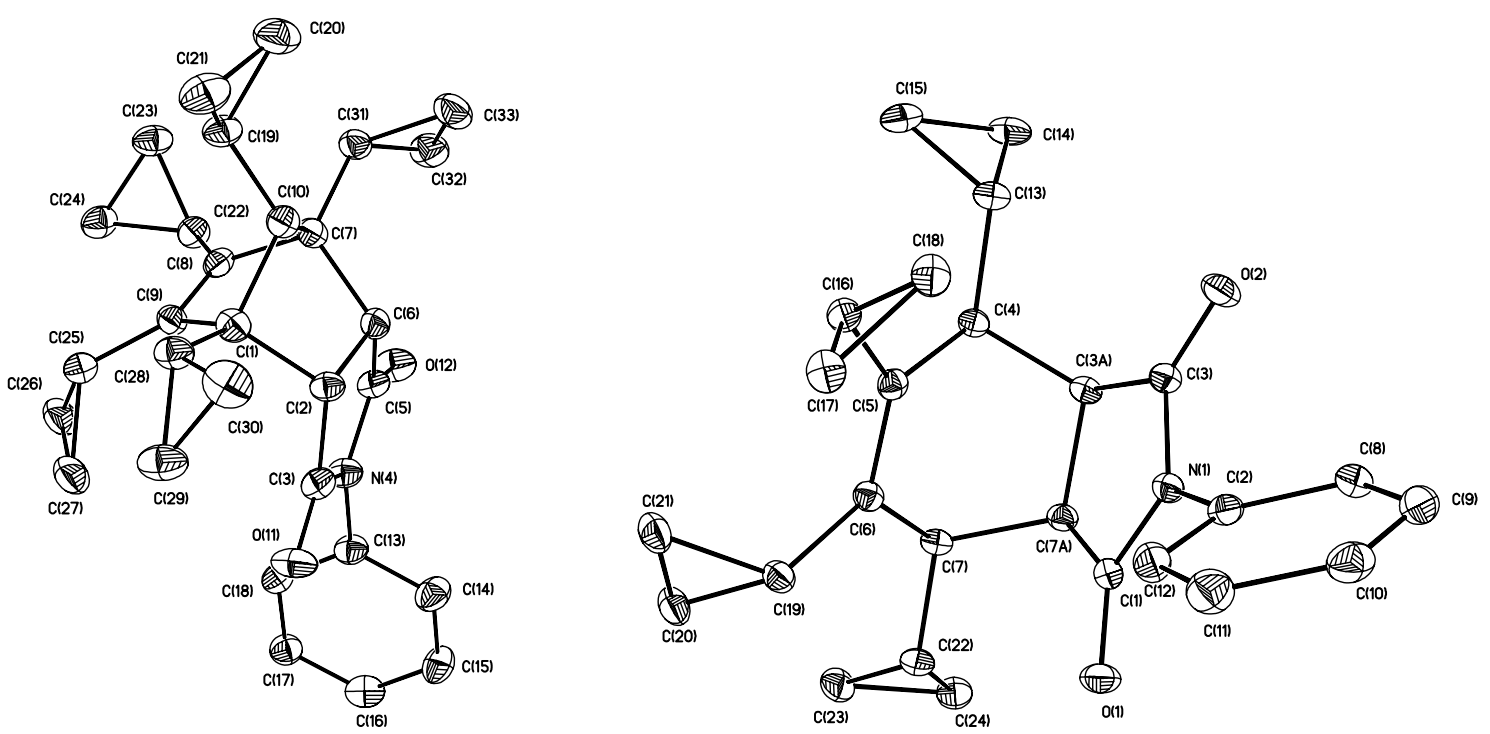

Abbildung 24. Strukturen von 102 (links) und 104 (rechts) im Kristall (Schwingungselipsoide mit 30\% Aufenthaltswahrscheinlichkeit). 


\subsection{Versuche zum Aufbau eines Heptacyclopropylnorbornadienyl-Gerüsts}

Mit diesen bisherigen Ergebnissen im Hinterkopf ist die Synthese eines Heptacyclopropylnorbornadienyl-Gerüsts denkbar, das als Vorstufe für ein stabilisiertes Heptacyclopropylnorbornadienyl-Kation 108 dienen könnte (Schema 59).

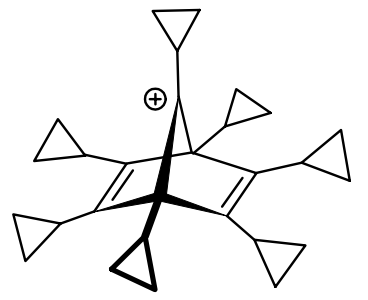

108

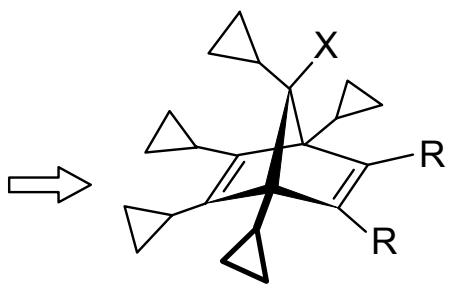

$$
101\left(\mathrm{R}=\mathrm{CO}_{2} \mathrm{Me}, \mathrm{X}=\mathrm{H}\right)
$$

101a $\left(R=\mathrm{CO}_{2} \mathrm{Me}, \mathrm{X}=\mathrm{Cl}\right)$

$105(\mathrm{R}=\mathrm{CHO}, \mathrm{X}=\mathrm{H})$

E

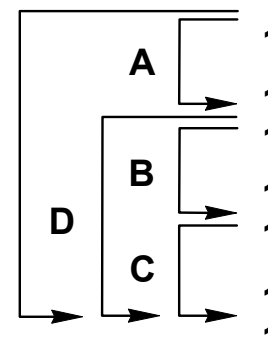

105a $(\mathrm{R}=\mathrm{CHO}, \mathrm{X}=\mathrm{Cl})$

$106(\mathrm{R}=$ Vinyl, $X=\mathrm{H})$

106a ( $R=$ Vinyl, $X=C l)$

$107(\mathrm{R}=c \mathrm{Pr}, \mathrm{X}=\mathrm{H})$

107a $(\mathrm{R}=c \operatorname{Pr}, \mathrm{X}=\mathrm{Cl})$

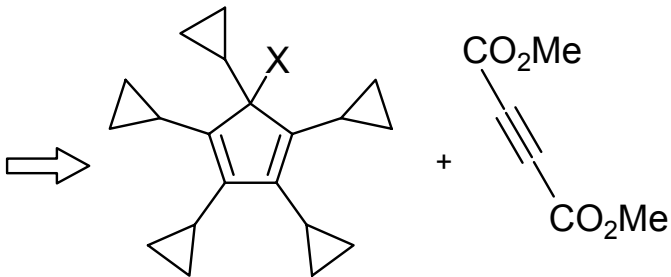

$15(X=H)$

$36(X=C l)$
97

Schema 59. Pentacyclopropylcyclopentadiene $\mathbf{1 5}$ oder $\mathbf{3 6}$ als Bausteine für ein Heptacyclopropylnorbornadienyl-Kation 108.

Dazu könnte man vom Norbornadienderivat 101 ausgehen und müsste die Estergruppierungen in Cyclopropylreste transformieren. Dies könnte durch eine KULINKOVICReaktion zum Bis(cyclopropylalkohol) und nachfolgende Defunktionalisierung der Hydroxygruppen bewerkstelligt werden (Schema 59, Weg E). ${ }^{[108]}$ Alternativ könnten die Esterfunktionen jeweils zum Aldehyd reduziert werden (Schema 59, Weg A), den man durch eine WitTiG-Reaktion in je eine Vinylgruppe umwandeln (Schema 59, Weg B) und diese dann nach SimmONS-SMith cyclopropanieren könnte (Schema 59, Weg C). Wenn man für die DIELS-ALDER-Reaktion von Pentacyclopropylcyclopentadien (15) ein Cyclopropylpropiolat 109 oder Cyclopropylpropional 110 als Dienophil einsetzte, ließe sich schon in diesem Schritt ein weiterer Cyclopropylrest einführen. 


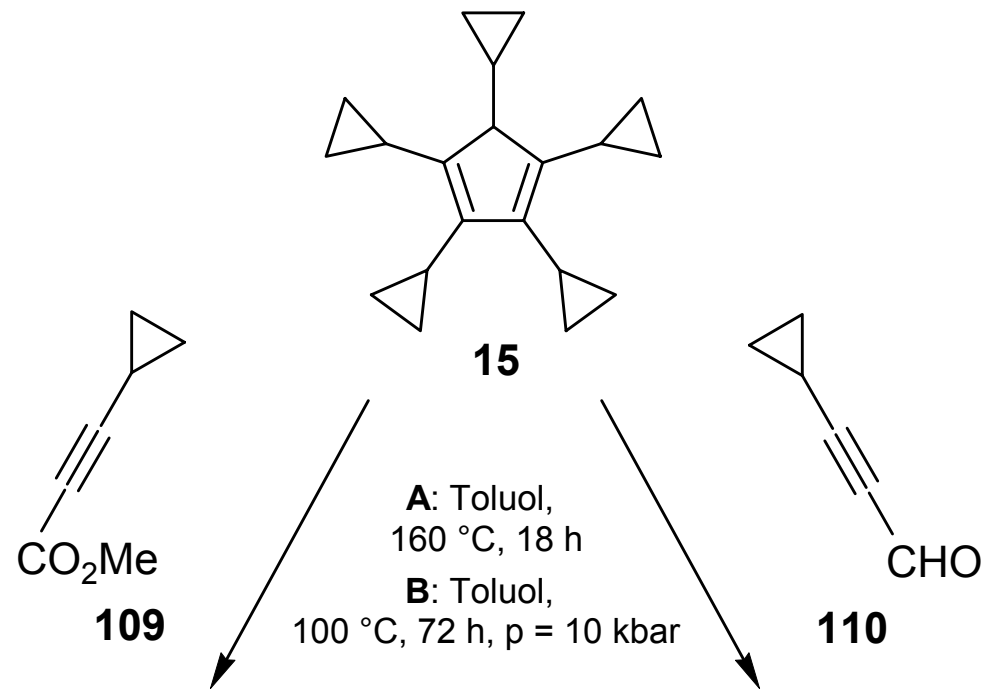

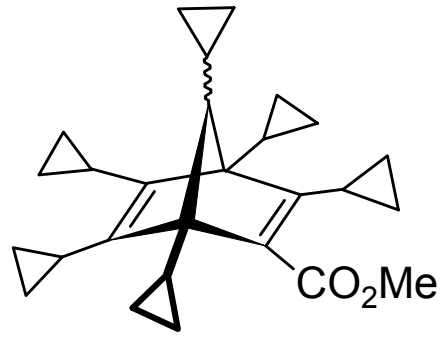

111

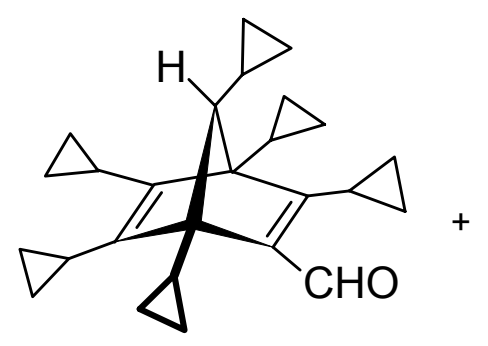

syn-112

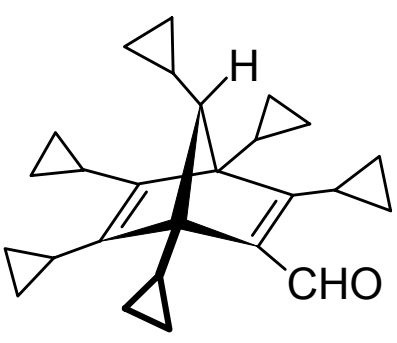

anti-112
A: $9 \%, 1: 2$
B: $89 \%, 2.5: 1$

$64 \%, 1: 2$

$86 \%, 2.5: 1$

Schema 60. DIELS-ALDER-Produkte der Reaktionen von Pentacyclopropylcyclopentadien 15 mit Cyclopropylpropiolsäuremethylester (109) und Cyclopropylpropinal (110).

Tatsächlich konnten bei den Reaktionen von 15 mit Cyclopropylpropiolsäuremethylester (109) bzw. Cyclopropylpropinal (110) in Toluol bei normalem Druck und einer Temperatur von $160{ }^{\circ} \mathrm{C}$ nach 18 Stunden die entsprechenden Norbornadiene 111 und 112 mit Ausbeuten von 9 und 64\% erhalten werden. Der Norbornadienylcarbaldehyd 112 wurde dabei als 1:2 Gemisch der beiden Diastereomeren syn-112a und anti-112b, mit unterschiedlicher Konfiguration am Brückenkohlenstoffatom erhalten (Schema 60, Tabelle 6, Einträge 1-2). 
Tabelle 6. DIELS-ALDER-Reaktion von Pentacyclopropylcyclopentadien 15 mit Cyclopropylpropiolsäuremethylester (109) und Cyclopropylpropinal (110) in Toluol.

\begin{tabular}{cccccc}
\hline Eintrag & Dienophil & Druck & Temp. $\left[{ }^{\circ} \mathrm{C}\right]$ & Zeit $[\mathrm{h}]$ & Ausbeute $(\%)^{[\mathrm{a}]}$ \\
\hline 1 & $\mathbf{1 0 9}$ & norm. Druck & 160 & 18 & $\mathbf{1 1 1}(1: 1), 9$ \\
2 & $\mathbf{1 1 0}$ & norm. Druck & 160 & 18 & $\mathbf{1 1 2 a}, \mathbf{1 1 2 b}(1: 2), 64$ \\
3 & $\mathbf{1 0 9}$ & $10 \mathrm{kbar}$ & 100 & 72 & $\mathbf{1 1 1}(1: 1), 89$ \\
4 & $\mathbf{1 1 0}$ & $10 \mathrm{kbar}$ & 100 & 72 & $\mathbf{1 1 2 a}, \mathbf{1 1 2 b}(2.5: 1), 86$ \\
\hline
\end{tabular}

${ }^{[\mathrm{a}]}$ Verhältnis der Diastereomeren in Klammern.

Bei einem Druck von $10 \mathrm{kbar}$ konnte die Ausbeute an den Norbornadienylcarbonsäureesters 111 auf $89 \%$ und die an dem Aldehyd 112 auf 86\% gesteigert werden (Schema 60, Tabelle 6, Einträge 3-4). Dabei kehrte sich das Diastereomerenverhältnis für syn-112 und anti-112 auf 2.5:1 um. Druckabhängigkeiten von DIELS-ALDER-Reaktionen sind intensiv studiert worden. Die beobachteten Beschleunigungen resultieren aus den negativen Aktivierungsvolumen solcher Reaktionen. Bei hohem Druck kommt es deshalb auch zu einer Bevorzugung des jeweils „kompakteren“ Übergangszustandskomplexes, wodurch ein Diastereomer schneller gebildet werden kann. ${ }^{[109]}$

Zur Erzeugung eines Norbornadienyl-Kations 108 würde eine entsprechend geeignete Abgangsgruppe in Position C-7 benötigt. Erste Versuche, das Wasserstoffatom an C-7 ausgehend vom Norbornadienylestern 101 durch Hydridabstraktion mittels der potenten Tritylsalze Trityltetrakis(pentafluorphenyl)borat oder Trityltetrakis[ $m$-di(trifluormethyl)phenyl]borat waren nicht erfolgreich. Wollte man ein Halogenatom in der Position einführen, so böte sich die Verwendung des Chlorpentacyclopropylcyclopentadiens 36 als Dien in der DIELS-ALDER-Reaktion an (Schema 59). Dies scheiterte aber an der erheblichen Temperaturempfindlichkeit des Chlorids. Dagegen konnte jedoch das weniger empfindliche 
Methoxypentacyclopropylcyclopentadien 65 mit Dimethylacetylendicarboxylat 97 in Toluol bei $160{ }^{\circ} \mathrm{C}$ zur Reaktion gebracht werden.

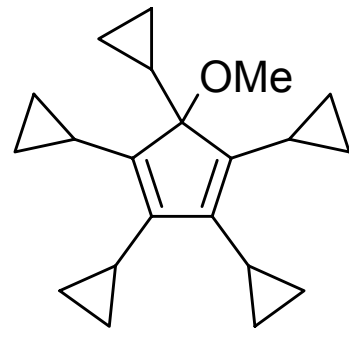

65

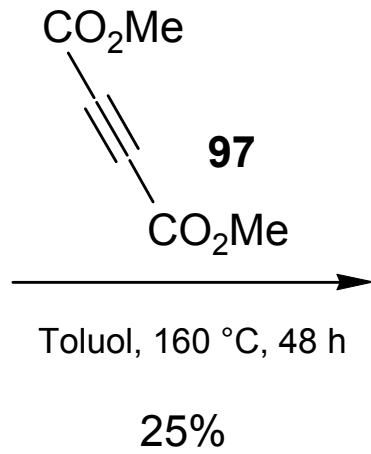

$25 \%$

Schema 61. DIELS-ALDER-Reaktion von Methoxypentacyclopropylcyclopentadien 65 mit Dimethylacetylendicaboxylat (97).

Das entsprechende Methoxynorbornadien 113 konnte daraus als 1:1 Gemisch zweier Diastereomere in 25\% Ausbeute isoliert werden. Diese Verbindung mit einer Methoxygruppe als potentielle Abgangsgruppe wäre aber eine eher untypische Kationvorstufe. Um das Heptacyclopropylnorbornadienyl-Kation $\mathbf{1 0 8}$ trotzdem zugänglich zu machen, stehen noch Versuche aus, die C-7 Position von 111 oder 112 mittels starker, nicht-nucleophiler Basen zu deprotonieren und durch Halogenierungsmittel wie $N$-Chlorsuccinimid zu funktionalisieren.

\subsection{Versuche zum Aufbau eines oligocyclopropylierten Tropylium-Grundgerüsts}

KATO et al. berichteten über eine Synthese von Troponen ausgehend von 7,7-Dihalogen-3,4-epoxybicyclo[4.1.0]heptanen durch Behandlung mit Trifluoressigsäure. ${ }^{[110]}$ Sie konnten beispielsweise nach Erhitzen des Epoxids 114 mit wässriger Trifluoressigsäure in Toluol bei $100{ }^{\circ} \mathrm{C}$ nach vier Stunden das Tropon 116 isolieren. Die intermediäre Tropyliumion-Zwischenstufe $\mathbf{1 1 5}$ konnten sie bei Raumtemperatur ebenfalls NMR-spektroskopisch charakterisieren (Schema 62). 
<smiles>CC(C)[C@]12CC3C(Cl)(Cl)[C@@]3(C)C[C@H]1O2</smiles>

114<smiles>CC(C)[C@@H]1C[C@H](O)[C@H](O)CC1(C)C</smiles><smiles>CO[13CH]=[18O]</smiles>

TFA/ $\mathrm{H}_{2} \mathrm{O}$ (1:5), Toluol $100{ }^{\circ} \mathrm{C}, 4 \mathrm{~h}$

$73 \%$<smiles>Cc1ccc(C(C)C)ccc1=O</smiles>

116<smiles>CC1=CC=C(C(C)C)C=CC1(O)Cl</smiles>

$\mathrm{H}_{2} \mathrm{O} \uparrow$<smiles>CC(C)C1=CC2C(Cl)(Cl)[C@@]2(C)C=C1</smiles><smiles>CC1=CC=C(C(C)C)C=CC1(Cl)Cl</smiles>

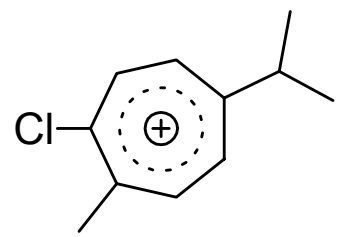

115

Schema 62. Saure Hydrolyse des Epoxids 114 zum Tropon 116 über das intermediäre Tropyliumion 115 nach KATO. ${ }^{[110]}$

Die Synthese eines entsprechenden Cyclopropyl-substituierten Tropons bzw. eines oligocyclopropylierten Tropyliumions erschien in analoger Weise möglich. In diesem Fall wäre auch ein Vergleich mit den schon von KOMATSU et al. hergestellten Cyclopropylsubstituierten Tropyliumionenen 117-122 möglich. Sie verglichen die $\mathrm{p} K_{\mathrm{R}}{ }^{+}$-Werte einer Reihe von Cyclopropyl-substituierten Tropyliumionen miteinander und fanden einen erheblichen Anstieg dieses Wertes mit zunehmender Zahl von Cyclopropylsubstituenten bis hin $\mathrm{zu}$ einem $\mathrm{p} K_{\mathrm{R}}{ }^{+}=+9.10$ für das Tetracyclopropyl-substituierte Tropyliumion, der den bisher höchsten bekannten Wert für ein Carbokation repräsentiert (Abbildung 25). ${ }^{[111]}$ Obwohl dieser Stabilisierungseffekt offenbar nicht linear mit der Zahl der Cyclopropylgruppen ansteigt, wäre es interessant, die Effekte von weiteren Cyclopropylgruppen auf die Stabilität des Tropyliumions zu untersuchen. 


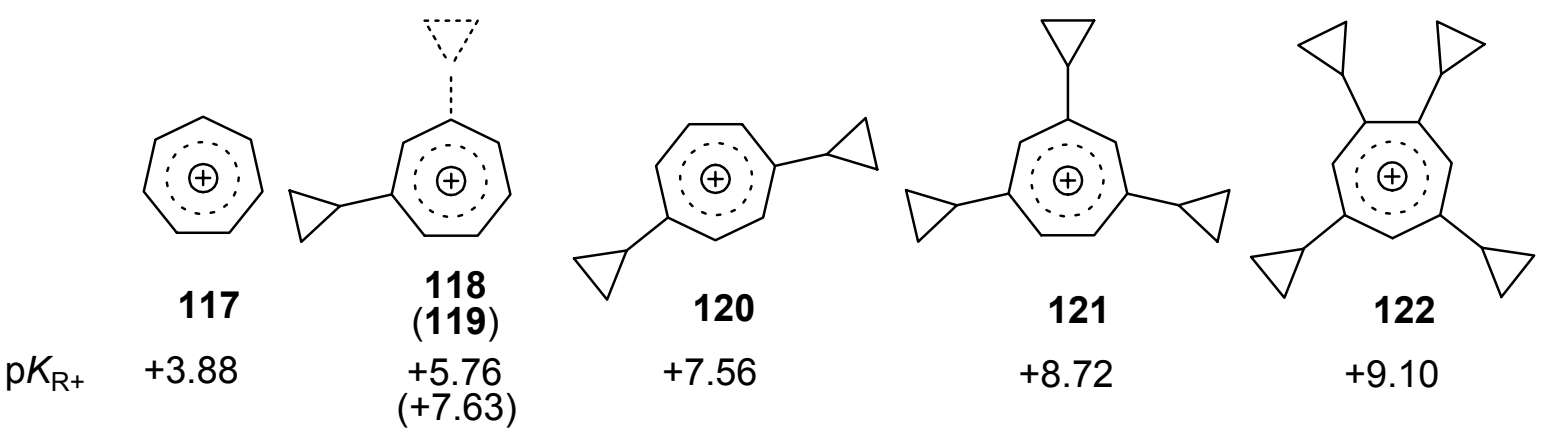

Abbildung 25. Ausgewählte $\mathrm{p} K_{\mathrm{R}}{ }^{+}$-Werte für einige Cyclopropyl-substituierte Tropyliumionen nach KomATsU. ${ }^{[11]}$

Die Reaktion von 1,2,3,4-Tetracyclopropylbutadien (21) mit Tetrachlorcyclopropen (123) in Toluol bei $160^{\circ} \mathrm{C}$ für 16 Stunden ergab in $51 \%$ Ausbeute das Bicycloheptenderivat 124, das mit $m$-Chlorperoxybenzoesäure in Dichlormethan epoxidiert werden konnte. Nach 90 min konnte das Epoxid 125 mit einer Ausbeute von 91\% isoliert werden (Schema 63).<smiles>C(=C(C=C(C1CC1)C1CC1)C1CC1)C1CC1</smiles>

21

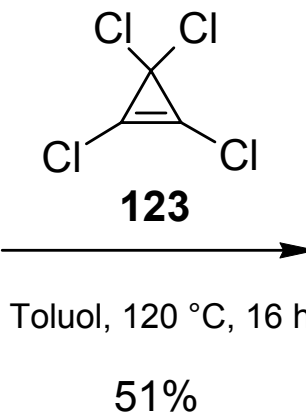

$51 \%$

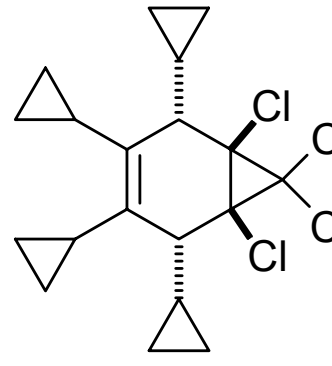

124<smiles>ClC1(C2CC2)C(C2CC2)C2(C3CC3)OC2(C2CC2)C(Cl)(Cl)C1(Cl)Cl</smiles>

125

Schema 63. Synthese des 8-Oxatricyclo[5.1.0 $\left.0^{1,7} \cdot 0^{3,5}\right]$ octans 125 durch Epoxidierung des DIELS-ALDER-Adduktes aus 1,2,3,4-Tetracyclopropylbutadien (21) und Tetrachlorcyclopropen (123).

Wurde nun das Epoxid 125 unter den von KATO angegebenen Bedingungen mit Trifluoressigsäure in Toluol erhitzt, so konnte nach wässriger Aufarbeitung nur polymeres Material erhalten werden. Auch separate Versuche, das intermediäre Tropyliumion im NMRRöhrchen direkt zu erzeugen und NMR-spektroskopisch $\mathrm{zu}$ charakterisieren, waren nicht erfolgreich (Schema 64). 


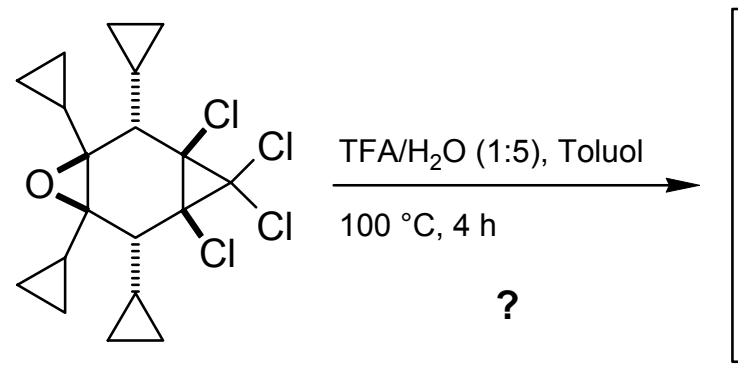

125

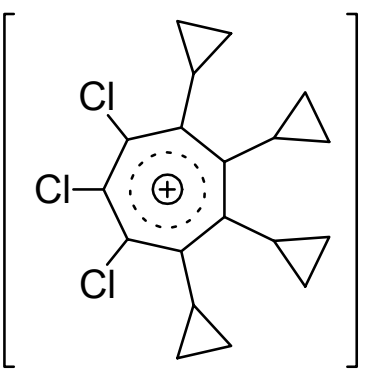

126<smiles>O=C1C(Cl)=C(C2CC2)C(C2CC2)=C(C2CC2)C1C1CC1</smiles>

127

Schema 64. Versuch der sauren Hydrolyse des Epoxids 125 zum Tropon 127 über das intermediäre Tropyliumion 126.

TAKEUCHI et al. berichteten über einen weiteren interessanten Zugang zu Cycloheptatrienen über eine Sequenz aus DIELS-ALDER-, Retro-DIELS-ALDER-Reaktion und einer sich anschließenden in situ Divinylcyclopropan-Umlagerung. Dazu verwendeten sie eine Reihe Methyl-substituierter Thiophen-1,1-dioxide wie 128, ließen diese mit Methylsubstituierten Cyclopropenen des Typs 129 bei Raumtemperatur in Dichlormethan reagieren und erhielten die entsprechenden Oligomethyltropylidene wie beispielsweise $\mathbf{1 3 0}$ mit Ausbeuten von durchschnittlich 60\% (Schema 65). ${ }^{[112]}$

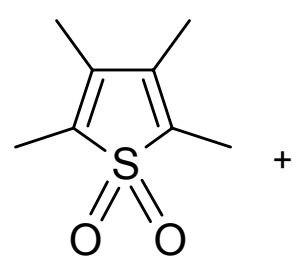

128

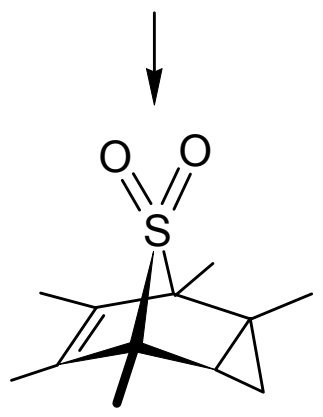

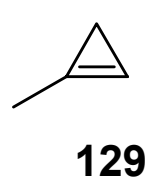

129

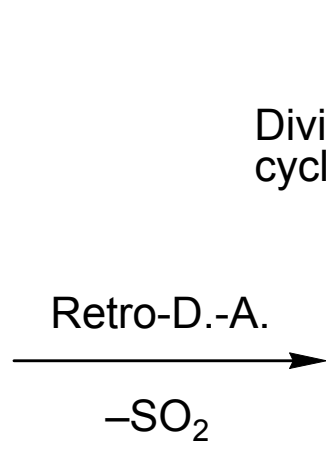

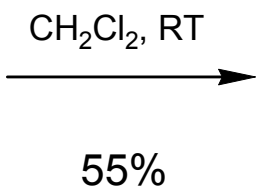

Divinyl-
cyclopropan- Umlag.<smiles>CC1=CCC(C)=C(C)C(C)=C1C</smiles>

130<smiles>CC1=C(C)C2CC2(C)C(C)=C1C</smiles>

Schema 65. Darstellung von Pentamethylcycloheptatrien 130 nach TAKEUCHI. 
Alle drei Schritte laufen dabei schon bei Raumtemperatur ab. Bei der DIELS-ALDER-Reaktion und der Divinylcyclopropan-Umlagerung ist dies sicher auf den Abbau der Ringspannung im Cyclopropen- bzw. Cyclopropanring zurückzuführen. Die Triebkraft der Cycloreversion liegt wohl im irreversiblen Ausstoß von Schwefeldioxid. ${ }^{[13]}$

Ein entsprechendes Tetracyclopropylthiophenoxid 132 konnte in Variation eines Protokolls von TILLEY et al. ${ }^{[114]}$ durch Einleiten von gasförmigem Schwefeldioxid zu einer Lösung aus in situ - durch Zugabe einer Lösung von $n$-Butyllithium in Hexan zu einer Mischung des Acetylens 13 und Biscyclopentadienylzirkondichlorid in THF - erzeugtem Tetracyclopropyl-substituiertem Zirkonacyclopentadien gewonnen werden. Das Sulfoxid 131 wurde als Rohprodukt ohne weitere Reinigung mit $m$-Chlorperoxybenzoesäure in Dichlormethan weiter oxidiert, wobei das Tetracyclopropylthiophendioxid 132 mit einer Ausbeute von 51\% über beide Stufen isoliert werden konnte (Schema 66).

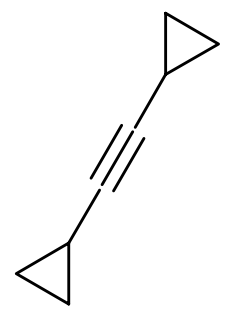

13
1) $\mathrm{Cp}_{2} \mathrm{ZrCl}_{2}$ (0.5 Äquiv.), $n$ BuLi (1.0 Äquiv.), $\mathrm{THF},-78^{\circ} \mathrm{C} \rightarrow \mathrm{RT}, 1 \mathrm{~h}$

2) $\mathrm{SO}_{2(\mathrm{~g})}, 30 \mathrm{~min}$

$m \mathrm{CPBA}$ (3 Äquiv.), $\mathrm{CH}_{2} \mathrm{Cl}_{2}$

RT, 12 h

$51 \%$

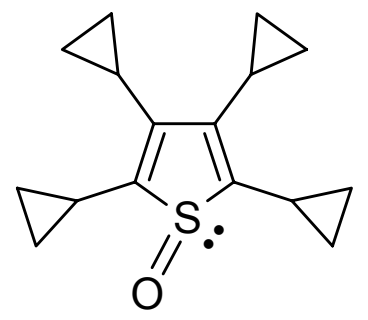

131

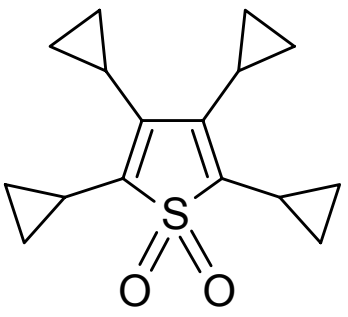

132

Schema 66. Darstellung von 2,3,4,5-Tetracyclopropylthiophen-1,1-dioxid (132) in Anlehnung an TILLEY.

Dieses Thiophendioxid 132 wurde anschließend zusammen mit Tetrachlorcyclopropen 123 bzw. Cyclopropylcyclopropen 133 in Chloroform bei Raumtemperatur gerührt, wobei 
allerdings noch keine Reaktion eintrat. Aber auch nach stufenweisem Erhitzen der jeweiligen Reaktionslösungen bis auf $65^{\circ} \mathrm{C}$ konnte kein Umsatz festgestellt werden. In beiden Fällen konnte die Bildung keines der gewünschten Cycloheptatriene 134 oder 135 detektiert werden. Stattdessen wurden zu 76\% das Thiophendioxid 132 zurückgewonnen.

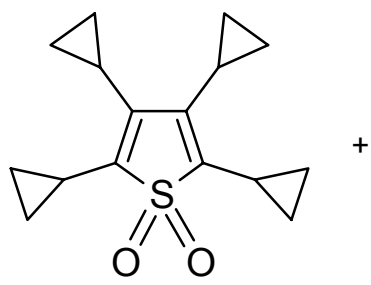

132

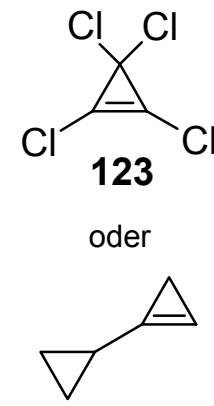

133

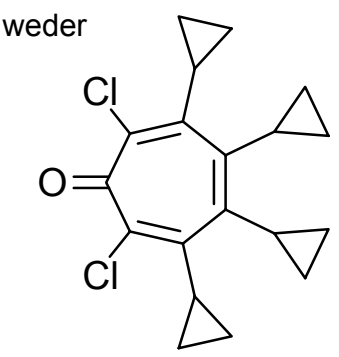

134

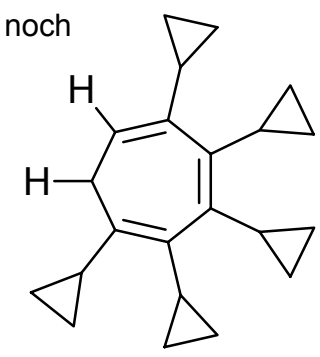

135

Schema 67. Versuch der Darstellung der Cycloheptatriene 134 und 135 durch eine Folge von DIELS-ALDER-Reaktion, Cycloreversion und Divinylcyclopropan-Umlagerung.

\section{Neue percyclopropylierte, fünfgliedrige Heterocyclen}

Etwa die Hälfte aller bekannten organischen Verbindungen besitzen Strukturen mit mindestens einer heterocyclischen Komponente. Die am häufigsten - neben Kohlenstoff und Wasserstoff - darin vorkommenden Elemente sind Stickstoff, Sauerstoff, und Schwefel. Heterocyclische Verbindungen haben eine weite Verbreitung in der Natur, wenn man beispielsweise an das Chlorophyll, das Hämin (beides Derivate des Porphyrins) oder an Nucleosidbasen und Vitamine denkt. Sie eröffnen auch zahlreiche Anwendungsbereiche in Form von Arzneimitteln, Pflanzenschutzstoffe, Farbstoffe oder Pigmente. ${ }^{[15]}$

Eine Reihe von klassischen Synthesen für Heterocyclen stehen zur Verfügung, für Pyrrole beispielsweise die KNORR-Synthese, die ausgehend von 1,3-Dicarbonylverbindungen wie 136 durch Nitrosierung und in situ Reduktion zur 2-Amino-1,3-dicarbonylverbindung 
137und dessen Kondensation mit einem zweiten Äquivalent 136 Pyrrole wie 138 liefert (Schema 68). ${ }^{[116]}$

1) $\mathrm{NaNO}_{2}, \mathrm{HOAc}$

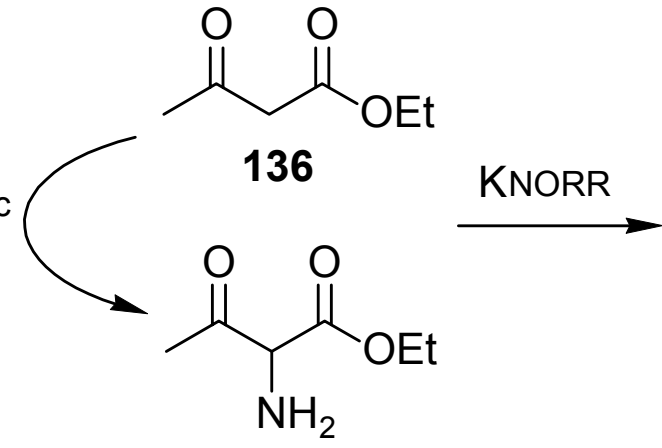

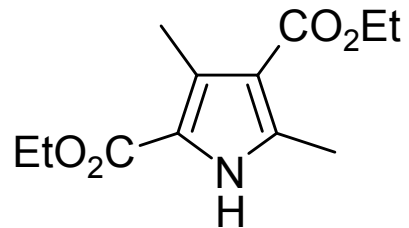

138

137

Schema 68. Beispiel einer KNORR-Synthese für Pyrrole aus 1,3-Dicarbonylverbindungen. ${ }^{[117]}$

Auch die PAAL-KNORR-Synthese bietet einen Zugang zu Pyrrolen des Typs 140. Dabei werden 1,4-Dicarbonylverbindungen wie z. B. 139 mit Ammoniak, primären Aminen, Hydroxylaminen oder Hydrazinen als Stickstoffquelle umgesetzt (Schema 69). Diese Reaktion ist - sofern die 1,4-Dicarbonylverbindung leicht zugänglich ist - so einfach durchzuführen, dass man sie zum Schutz von primären Aminen verwendet. Aus den aus Acetylaceton und Alkylaminen gebildeten 2,5-Dimethylpyrrolen lässt sich nämlich das primäre Amin durch Reaktion mit Hydroxylaminhydrochlorid wieder freisetzen. ${ }^{[18]}$

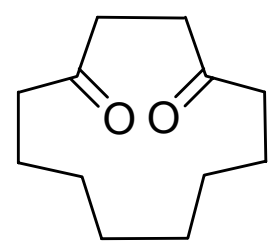

139

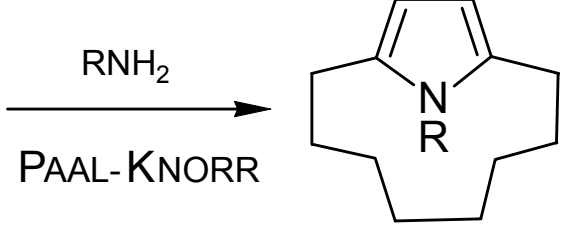

140

Schema 69. Beispiel einer PAAL-KNORR-Synthese für Pyrrole aus einer 1,4-Dicarbonylverbindung. ${ }^{[119]}$

Behandelt man eine 1,4-Dicarbonylverbindung mit einem sauren Katalysator wie Schwefelsäure, Phosphorpentoxid, Zinkchlorid oder einem sauren Ionenaustauscher, so erhält 
man das entsprechend substituierte Furan. ${ }^{[120]}$ Bei Behandlung mit Phosphorpentasulfid oder dem LAWESSON-Reagenz entstehen aus 1,4-Dicarbonylverbindungen die entsprechenden Thiophene. ${ }^{[121]}$

Unter der HANTZSCH-Pyrrol-Synthese versteht man die Reaktion eines $\alpha$-Halogenketons des Typs 141 mit einem $\beta$-Ketoester wie 142 und Ammoniak oder einem primären Amin beispielsweise zu 143. Allerdings können das $\alpha$-Halogenketon und der $\beta$-Ketoester schon alleine unter Bildung eines Furans wie beispielsweise 144 reagieren, was eine störende Nebenreaktion darstellen kann. Diese Umsetzung in Abwesenheit eines Amins beschreibt die FEIST-BENARY-Synthese und führt zu Furanen (Schema 70). ${ }^{[122]}$

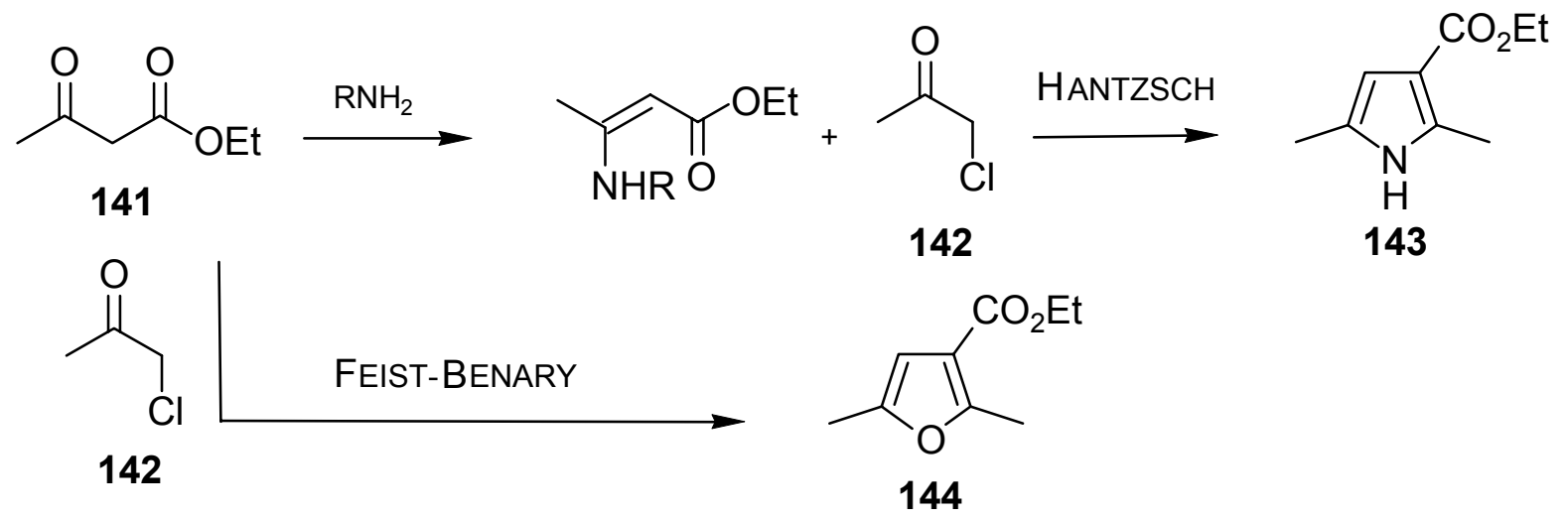

Schema 70. Beispiel einer HANTZSCH-Synthese für ein Pyrrol 144 aus einem $\alpha$-Halogenketon 141 und einem $\beta$-Ketoester 142 und die FEIST-BENARY-Reaktion als Konkurrenzreaktion zur Bildung von Furanen wie 143.

Weitere klassische Zugänge zu Thiophenen wie 147 sind die HinsBERG- und die GEWALD-Synthese. ${ }^{[123]}$ Bei ersterer werden Bis(alkyloxycarbonylmethyl)thioether wie 145 mit 1,2-Diketonen in basischem Medium zur Reaktion gebracht. Das primäre Reaktionsprodukt 146 kann nachfolgend sukzessive decarboxyliert werden. Bei letzterer werden $\alpha$-Mercaptocarbonylverbindungen des Typs 148 mit Malonitrilen umgesetzt, wobei 2-Aminothiophene wie 149 erhalten werden können (Schema 71). Alternativ kann auch eine einfache Carbonylverbindung in Gegenwart von elementarem Schwefel mit einem Nitril 
cyclisiert werden. Der Ringschluss zum Thiophen ist grundsätzlich reversibel und einige 2-Aminothiophene können durch Einwirkung von Basen wieder gespalten werden. ${ }^{[124]}$

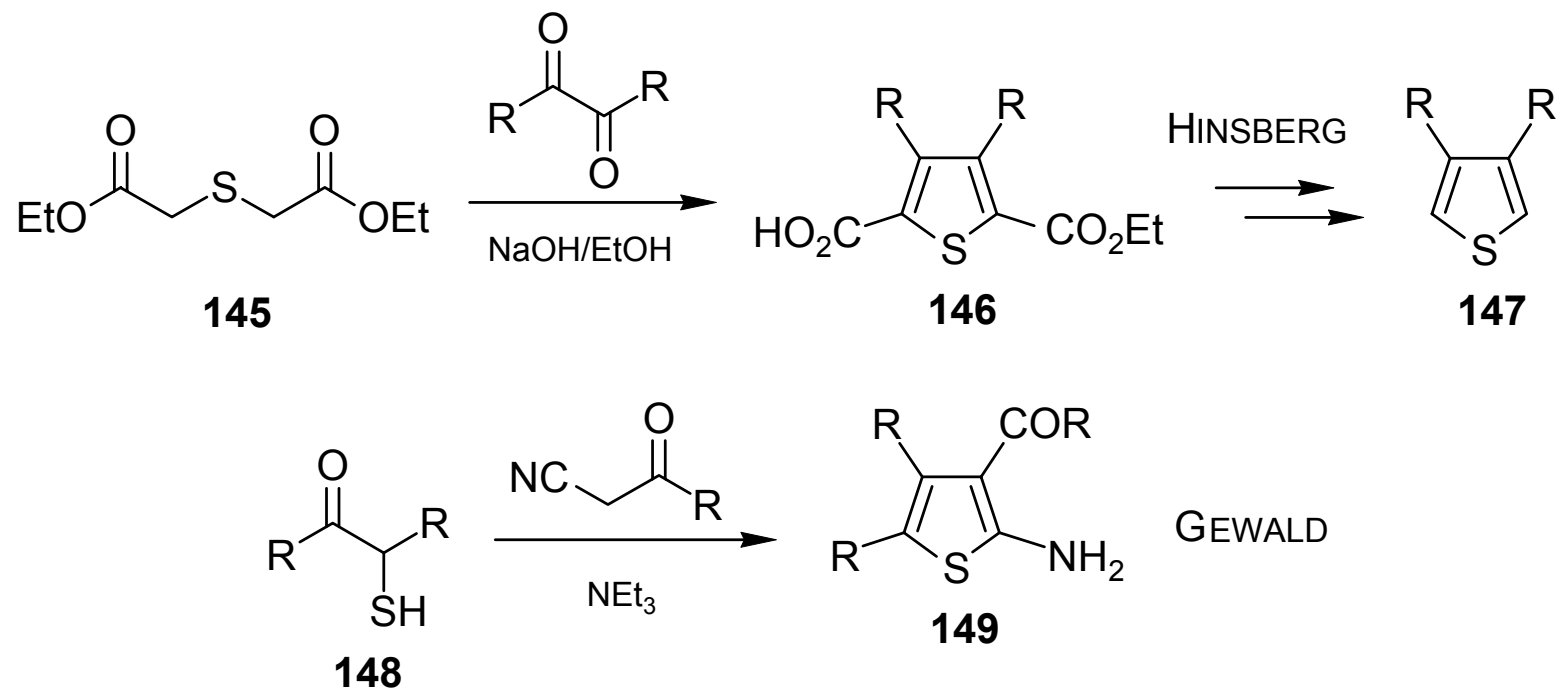

Schema 71. Beispiel einer HINSBERG- und einer GEWALD-Synthese für Thiophene.

\subsection{Cyclopropylierte fünfgliedrige Heterocyclen mit einem Heteroatom}

Dicyclopropylethin (13) zeigt seine Einsetzbarkeit als vielseitiger Baustein nicht nur dadurch, dass mit seiner Hilfe die eingangs vorgestellten neuen Cyclopropyl-substituierten Cyclopentadien-Liganden 14 und 15 sehr leicht zugänglich sind. Es sollte auch als Vorstufe für eine Reihe von Cyclopropyl-substituierten heterocyclischen Fünfringverbindungen dienen können.

Unter den oben vorgestellten passierten klassischen Fünfring-Heterocyclen-Synthesen erschien die PAAL-KNORR-Synthese ausgehend von einer 1,4-Dicarbonylverbindung wie 151 am flexibelsten im Hinblick auf die mögliche Produktpalette von Cyclopropyl-substituierten Fünfring-Heterocyclen, die durch den Einsatz von unterschiedlichen Aminen, sauren Katalysatoren oder Schwefel-Reagenzien erhalten werden könnten (Schema 72). 


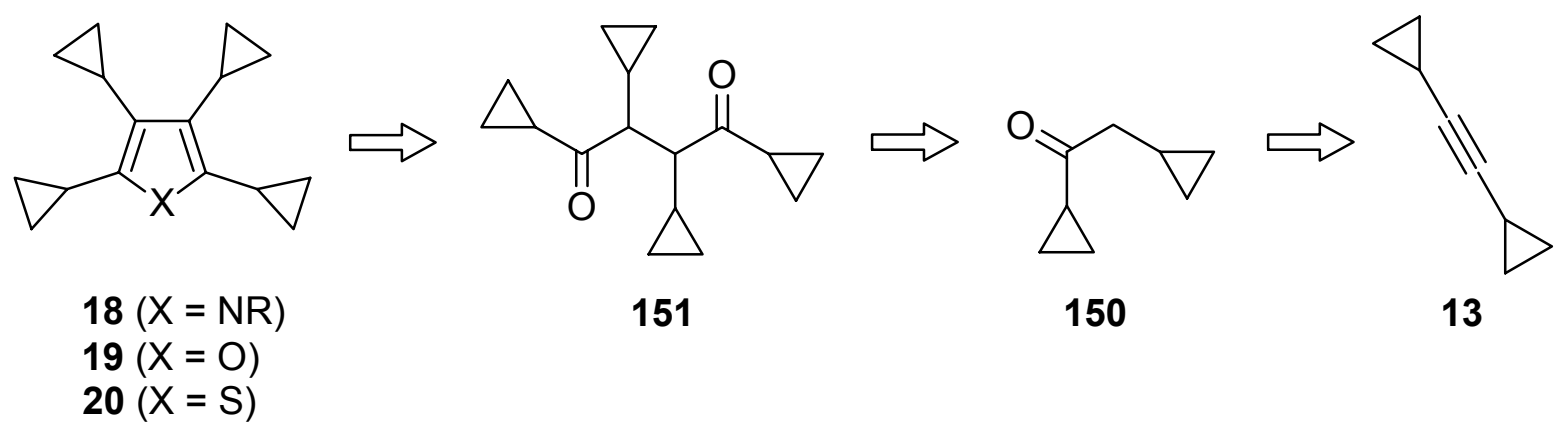

Schema 72. Retrosynthese des Tetracyclopropyl-substituierten 1,4-Diketons 151 als Substrat für eine PAAL-KNORR-Synthese von Cyclopropyl-substituierten Fünfring-Heterocyclen 18-20.

Um dies zu realisieren, wurde analog zu einem Protokoll von ELSNER und HERBERTz das Dicyclopropylethin (13) zunächst einer Hydratisierung unterzogen, indem man es mit katalytischen Mengen Quecksilber(II)oxid und konzentrierter Schwefelsäure in wässrigem Aceton bei $100{ }^{\circ} \mathrm{C}$ für 16 Stunden intensiv rührte. ${ }^{[125]}$ Nach wässriger Aufarbeitung des Reaktionsgemisches und Filtrieren des Rohproduktes über Kieselgel konnte 1,2-Dicyclopropylethanon (150) in einer Ausbeute von 96\% gewonnen werden.

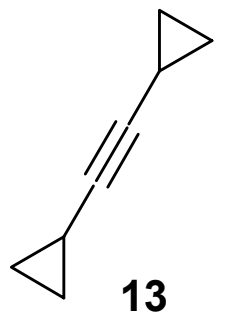

13

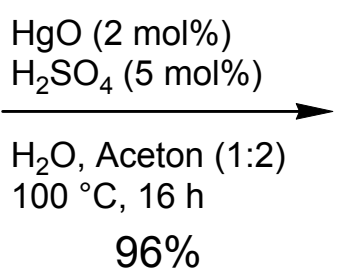

$96 \%$

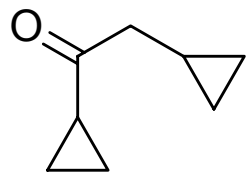

150

Schema 73. Darstellung von Dicyclopropylethanon 150 durch Quecksilber-katalysierte Hydratisierung von Dicyclopropylethin (13).

Für die nachfolgende Dimerisierung zum 1,4-Diketon wurde 151 zunächst mit Blei(IV)dioxid umgesetzt, da dies als eine gängige Methode für die Herstellung von 3,4-Dimethylhexan-2,5-dion aus Butanon bekannt ist. ${ }^{[126]}$ Nach mehrtägigem Rühren bei $100{ }^{\circ} \mathrm{C}$ konnte aber dünnschichtchromatographisch keinerlei Umsatz festgestellt werden. Daher wurde versucht, die $\alpha$-Methylenposition von $150 \mathrm{zu}$ chlorieren und durch eine 
nachfolgende Substitution des Halogens mit einem Äquivalent eines Enolats von 150 das Diketon 151 zugänglich zu machen. Die Chlorierung des Dicyclopropylethanons (150) mit $N$-Chlorsuccinimid in Gegenwart von katalytischen Mengen konz. Salzsäure in Tetrachlorkohlenstoff war jedoch nicht erfolgreich.

Analog zu dem Einelektronen-Oxidations-Prozess eines $\alpha$-Methylenketons mittels Blei(IV)dioxid ist auch eine oxidative Dimerisierung des Enolats eines solchen Ketons möglich. Dies bewerkstelligten KOBAYASHI et al. durch Verwendung von Kupfer(II)triflat. ${ }^{[127]}$
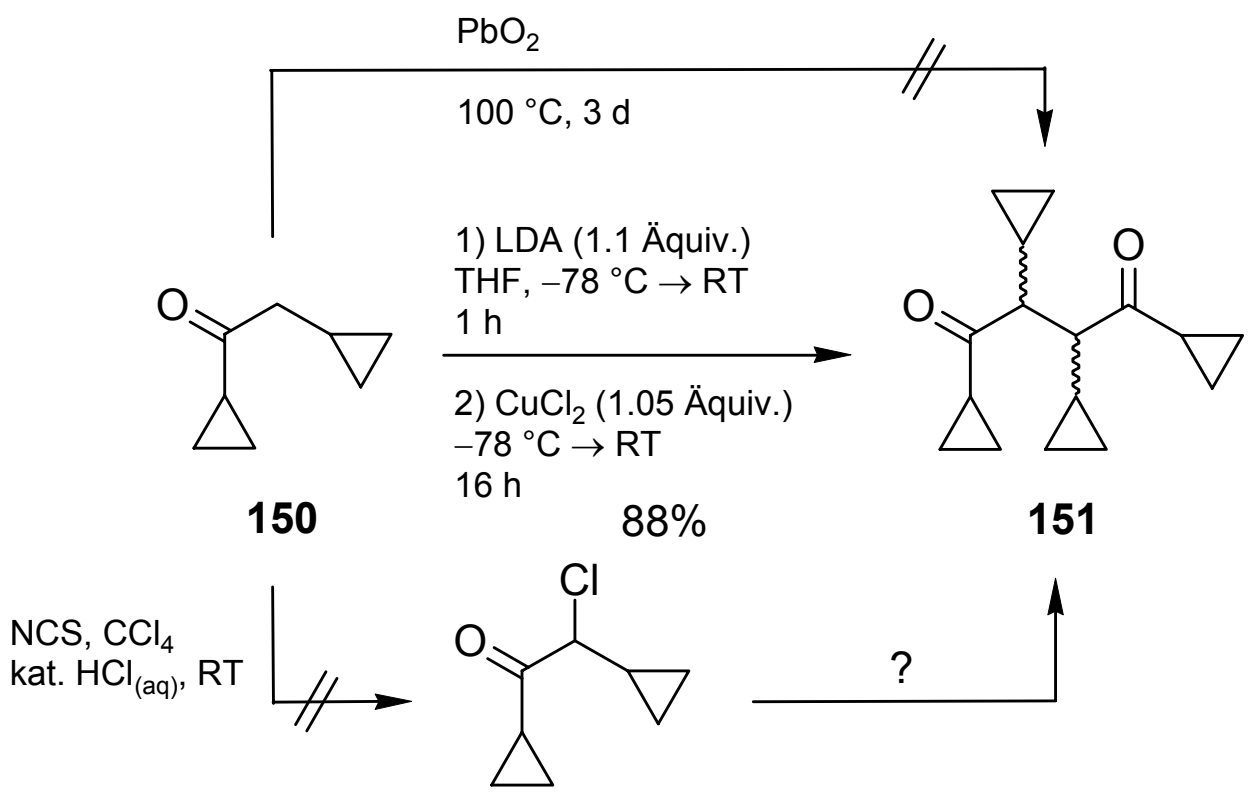

Schema 74. Darstellung von Tetracyclopropylbutandion 151 durch oxidative Dimerisierung des Enolats von Dicyclopropylethanon $\mathbf{1 5 0 .}$

Daran anknüpfend wurde Dicyclopropylethanon (150) mit Lithiumdiisopropylamid (LDA) in THF bei $-78^{\circ} \mathrm{C}$ innerhalb von einer Stunde deprotoniert. Zu der erhaltenen Lösung des Enolats wurde in Variation des Protokolls von KOBAYASHI bei dieser Temperatur ein Äquivalent wasserfreies Kupfer(II)chlorid hinzugefügt und über Nacht gerührt. Das gewünschte 1,2,3,4-Tetracyclopropylbutan-1,4-dion (151) wurde als Gemisch von zwei Diastereomeren im Verhältnis 1:1 in einer Ausbeute von 88\% isoliert (Schema 74). 
In Anlehnung an Protokolle von KUBÁČEK und GRAHAM für die Darstellung von Pyrrolen durch die PAAL-KNORR-Synthese wurde 151 mit einem Überschuss an Benzylamin bzw. Cyclopropylamin ohne weiteren Zusatz von Lösungsmittel mit wenigen Tropfen konz. Salzsäure bei $65{ }^{\circ} \mathrm{C}$ für 24 Stunden gerührt. ${ }^{[128]}$ 1-Benzyl-2,3,4,5-tetracyclopropyl-1H-pyrrol (18a) wurde dabei in 37 und wurde 1,2,3,4,5-Pentacyclopropyl-1H-pyrrol (18b) in 48\% Ausbeute gewonnen (Schema 75).

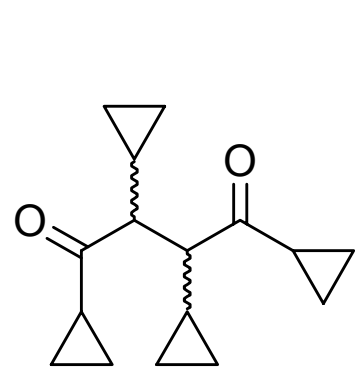

151

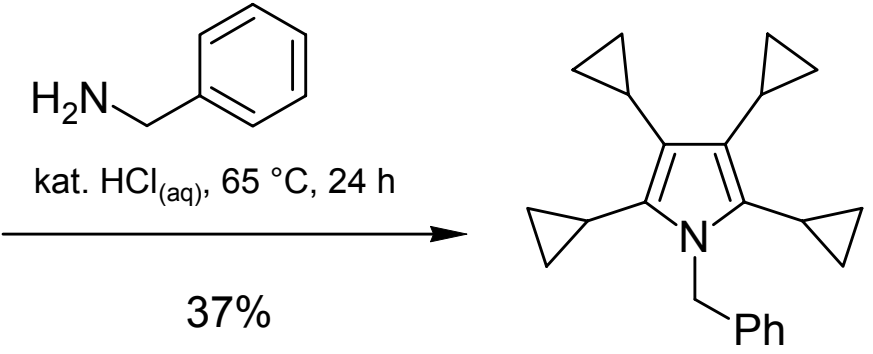

$18 a$

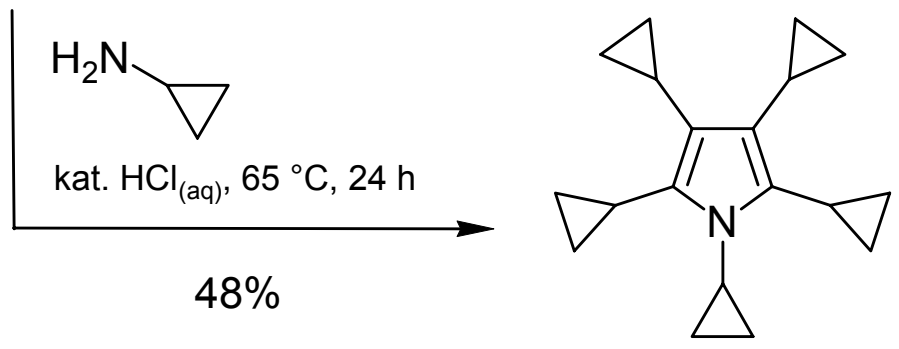

$18 b$

Schema 75. Darstellung von 1-Benzyl-2,3,4,5-tetracyclopropyl-1H-pyrrol (18a) und 1,2,3,4,5-Pentacyclopropyl-1H-pyrrol (18b) durch PAAL-KNORR-Synthesen.

Indem man das Diketon 151 mit Ammoniumacetat in einer Mischung aus Ethanol und Chloroform bei $65^{\circ} \mathrm{C}$ für 24 Stunden rührte, konnte 2,3,4,5-Tetracyclopropyl-1H-pyrrol (18c) in $43 \%$ Ausbeute erhalten werden (Schema 76). 


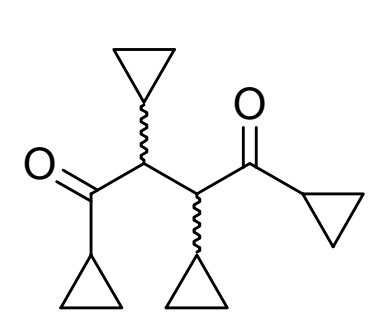

151
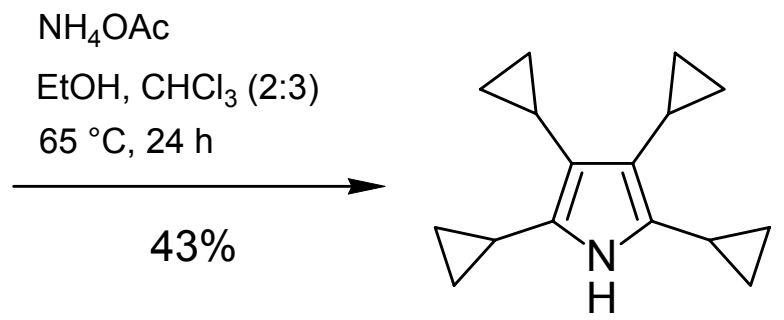

$18 \mathrm{c}$

Schema 76. Darstellung von 2,3,4,5-Tetracyclopropyl-1H-pyrrol (18c) durch eine PAALKNORR-Synthese. ${ }^{[129]}$

Pyrrol - wie auch Furan und Thiophen - ist ein sogenannter ElektronenüberschussHeteroaromat, da es sechs $\pi$-Elektronen auf nur fünf $\mathrm{sp}^{2}$-Zentren verteilt beherbergt. Es erfährt dadurch eine erhebliche Resonanzstabilisierung und geht sehr leicht elektrophile Substitutionsreaktionen ein. Pyrrol ist eine schwache Base von etwa der gleichen Stärke wie einfache Alkohole. Sein $\mathrm{p} K_{\mathrm{S}}$-Wert in wässriger Lösung beträgt 17.5 und kann durch Substituenten mit -M-Effekt in den 2- und/oder 5-Positionen deutlich erniedrigt werden. In jüngerer Zeit wird verstärkt versucht, Pyrrolanionen als cyclopentadienyl-analoge Liganden für Metallkomplexe zu verwenden. Solche Heterometallocene haben durch die senkrecht zum aromatischen $\pi$-System stehenden freien Elektronenpaare ihrerseits wieder interessante Koordinationsmöglichkeiten. ${ }^{[130]}$ Daher wurde versucht, auch das Tetracyclopropylpyrrol 18c zu deprotonieren. Dazu wurde es bei $0{ }^{\circ} \mathrm{C}$ mit Methyllithium-Lithiumbromid-Komplex in $\left[\mathrm{D}_{8}\right]$-Tetrahydrofuran behandelt, wobei sich die Lösung leuchtend gelb färbte (Schema 77).

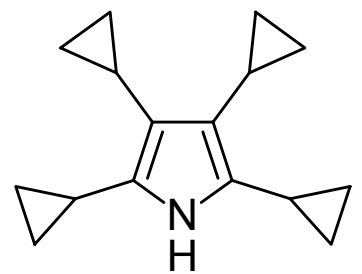

$18 c$

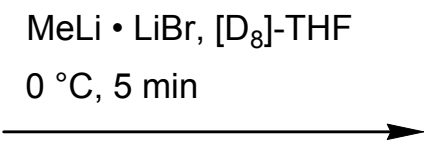

quant.

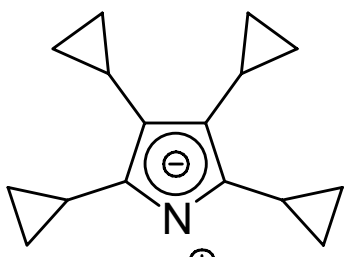

$\mathrm{Li}^{\oplus}$

18d

Schema 77. Deprotonierung von 2,3,4,5-Tetracyclopropyl-1H-pyrrol (18c) durch Methyllithium-Lithiumbromid-Komplex in $\left[\mathrm{D}_{8}\right]$-THF. 
Die so erhaltene Reaktionslösung wurde unmittelbar in ein NMR-Röhrchen transferiert und bei Raumtemperatur NMR-spektrokopisch untersucht. Die aufgezeichneten ${ }^{1} \mathrm{H}$ - und ${ }^{13} \mathrm{C}-\mathrm{NMR}-$ Spektren zeigten einen quantitativen Umsatz des Pyrrols 18c zum Tetracyclopropylpyrrolanion 18d an. Unter Ausschluss von Sauerstoff und Luftfeutigkeit weist die Lösung des Pyrrolanions 18d auch nach mehreren Wochen noch die gleichen charakteristischen NMR-Signale auf, was für die Stabilität von 18d spricht.

Die Darstellung des entsprechenden Cyclopropyl-substiuierten Furans gelang durch Rühren einer Mischung des 1,2,3,4-Tetracyclopropylbutan-1,4-dions (151) in Toluol mit einer katalytischen Menge konz. Schwefelsäure bei $65^{\circ} \mathrm{C}$. Das gewünschte 2,3,4,5-Tetracyclopropylfuran 19 wurde in 36\% Ausbeute erhalten (in Anlehnung an ${ }^{[131]}$; Schema 78).

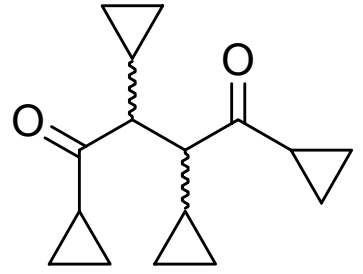

151 kat. $\mathrm{H}_{2} \mathrm{SO}_{4}$

Toluol, $65^{\circ} \mathrm{C}, 3 \mathrm{~d}$
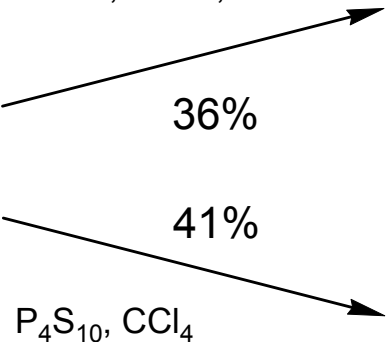

$70{ }^{\circ} \mathrm{C}, 3 \mathrm{~d}$

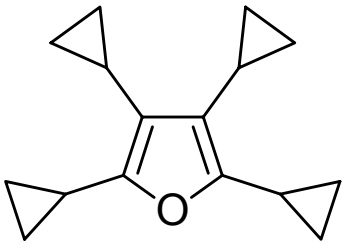

19

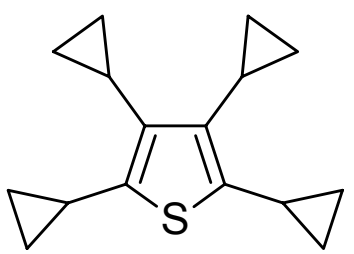

20

Schema 78. Darstellung von 2,3,4,5-Tetracyclopropylfuran (19) und 2,3,4,5-Tetracyclopropylthiophen (20) durch PAAL-KNORR-Synthesen.

Die Herstellung des Cyclopropyl-substituierten Thiophens 20 wurde durch dreitägiges Rühren einer Mischung von 1,2,3,4-Tetracyclopropylbutan-1,4-dion (151) und Phosphorpentasulfid in Tetrachlormethan bei $70^{\circ} \mathrm{C}$ bewerkstelligt, wobei das gewünschte 2,3,4,5-Tetracyclopropylthiophen $\mathbf{2 0}$ in einer Ausbeute von 41\% als farbloses Öl isoliert werden konnte (Schema 78). ${ }^{[132]}$ 


\subsection{Cyclopropylierte fünfgliedrige Heterocyclen mit zwei Heteroatomen}

Zur Synthese von fünfgliedrigen Heterocyclen mit zwei vicinalen Heteroatomen können Cyclisierungs- und Cycloadditionsreaktionen herangezogen werden. So ist es möglich, durch Reaktion von Alkinonen oder 1,3-Dicarbonylverbindungen wie 152 oder 154 mit Hydrazin oder Hydroxylamin bis zu dreifach substituierte Pyrazole bzw. Isoxazole des Typs $\mathbf{1 5 3}$ oder 155 herzustellen (Schema 79). ${ }^{[133,134]}$<smiles>[R]C#CC([R])=O</smiles>

152<smiles>[R]C(C(C)=O)C(C)=O</smiles>

154<smiles>[R]c1cc([R])[nH]n1</smiles>

$\mathrm{H}$

153<smiles>[R]c1c(C)noc1C</smiles>

155

Schema 79. Möglichkeiten zur Darstellung von Pyrazolen ${ }^{[133]}$ und Isoxazolen ${ }^{[134]}$.

Zur Darstellung eines Cyclopropyl-substituierten Pyrazols 158 bzw. Isoxazols 160 erschien eine Synthese ausgehend von einem Tricyclopropyl-substituierten 1,3-Diketon 157 praktikabel, zumal dieses aus dem schon vorhandenen Baustein Dicyclopropylethanon 150 zugänglich sein sollte.

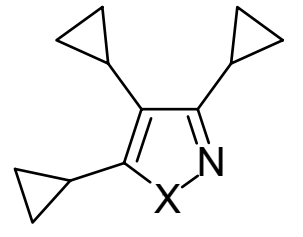

$158(\mathrm{X}=\mathrm{NH})$

$160(X=O)$

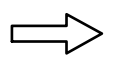<smiles>O=C(C1CC1)C(C1CC1)C1CC1</smiles>

157
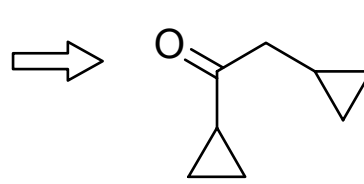

150

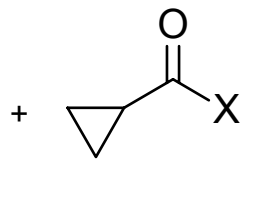

$71\left(\mathrm{X}=\mathrm{CO}_{2} \mathrm{Me}\right)$

$156(\mathrm{X}=\mathrm{Cl})$

Schema 80. Retrosynthese des 1,3-Diketons 157 als Cyclisierungssubstrat für Cyclopropylsubstituierte Fünfring-Heterocyclen mit zwei vicinalen Heteroatomen. 
Brachte man das Enolat von 150 - aus 150 und LDA bei $-78^{\circ} \mathrm{C}$ hergestellt - analog zu einem Protokoll von TIETZE und EICHER zur Darstellung von 1,2,3-Triphenylpropan1,3-dion ${ }^{[135]}$ bei $0{ }^{\circ} \mathrm{C}$ mit Cyclopropancarbonsäuremethylester in THF zur Reaktion, so konnte auch nach Auftauen der Reaktionsmischung und mehrstündigem Erhitzen unter Rückfluss kein Umsatz verzeichnet werden. Erst die Reaktion des aktiveren Cyclopropancarbonsäurechlorids $^{[136]}$ mit dem Enolat von 150 in THF brachte den gewünschten Erfolg. Nach Zugabe des Säurechlorids bei $-78{ }^{\circ} \mathrm{C}$ wurde die Mischung unter Rühren aufgetaut, so dass nach Aufarbeitung das gewünschte Diketon 157 mit einer Ausbeute von 32\% gewonnen wurde (Schema 81).

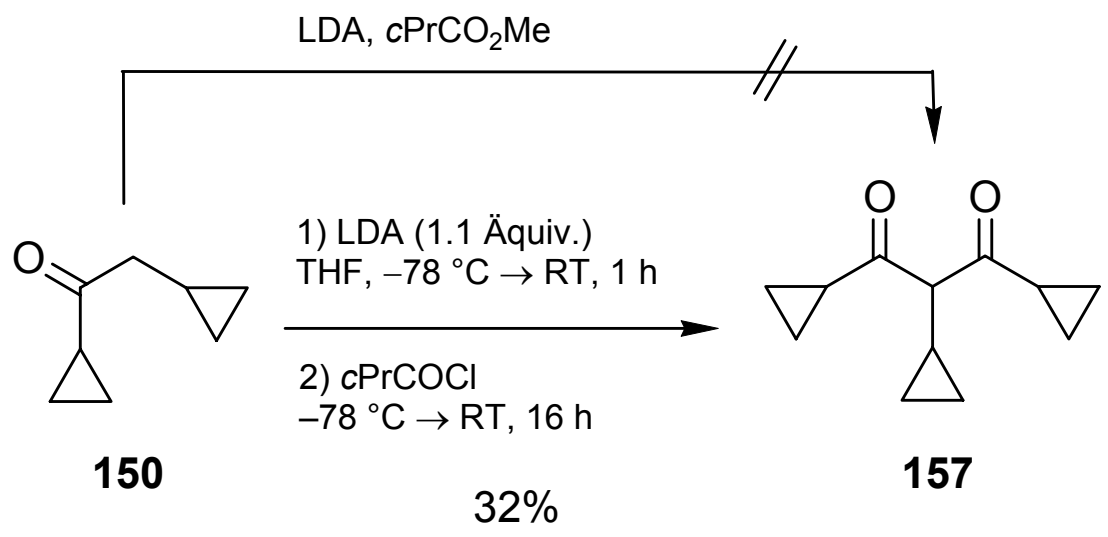

Schema 81. Darstellung von Tricyclopropylpropandion 157 aus Dicyclopropylethanon 150.

Nachfolgend konnte das Pyrazol 158 durch Reaktion von 157 mit einem Überschuss an Hydrazinhydrat gewonnen werden. Nach dreistündigem Erhitzen in Ethanol unter Rückfluss und anschließendem Rühren der abgekühlten Reaktionmischung bei Raumtemperatur für zwölf Stunden wurde das gewünschte 3,4,5-Tricyclopropyl-1H-pyrazol (158) in 89\% Ausbeute erhalten (in Anlehnung an ${ }^{[137]}$; Schema 82). 


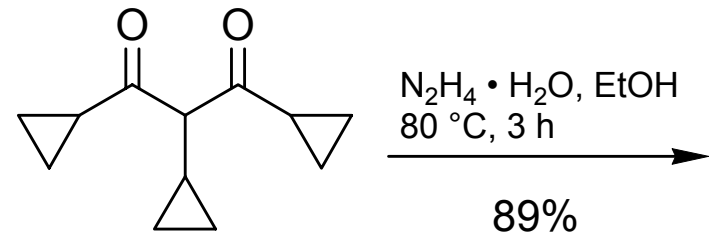

157

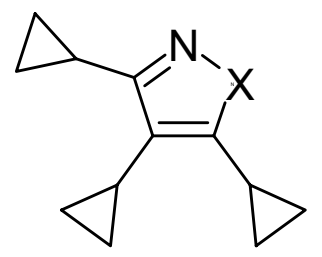

$\mathrm{X}=\mathrm{NH}, 158$

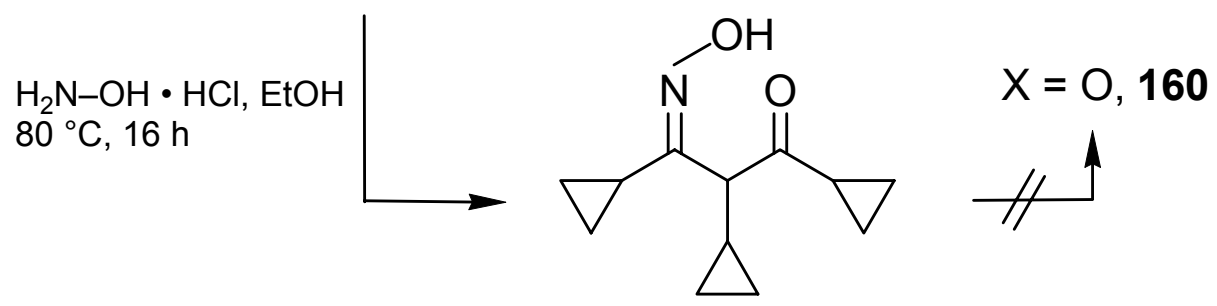

159

Schema 82. Darstellung des Tricyclopropylpyrazols 158 aus 157 und Hydrazin und versuchte Synthese des Tricyclopropylisoxazols $\mathbf{1 6 0}$.

In Analogie zur Darstellung des Cyclopropyl-substituierten Pyrazols 158 wurde versucht, das Isoxazol $\mathbf{1 6 0}$ durch Erhitzen von 157 mit einem Überschuss an Hydroxylaminhydrochlorid zu gewinnen. Trotz mehrerer Versuche konnte im Rohprodukt massenspektrometrisch $\left(\mathrm{M}_{\text {Isoxazol }}+18\right)$ und NMR-spektroskopisch (Hydroxylgruppe) nur das Oxim 159 als einfaches Kondensationsprodukt identifiziert werden.

Die Anwesenheit zusätzlicher Stickstoffatome im Ringsystem bewirkt, dass Diazole im Gegensatz zu Pyrrolen weniger „, $\pi$-elektronenreich“ sind als Benzol. Andererseits haben diese einen negativen induktiven Effekt und können so zur Stabilisierung von negativ geladenen Zwischenstufen beitragen. Man beobachtet bei Diazolen beispielsweise häufig eine nucleophile Substitution nach dem Additions-Eliminierungs-Mechanismus und eine Deprotonierung von Methylsubstituenten. 


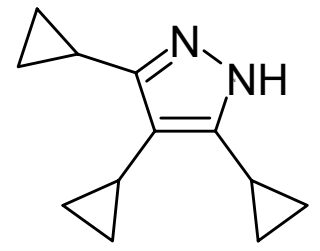

158

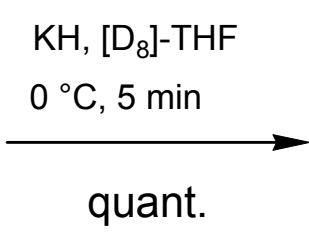

quant.

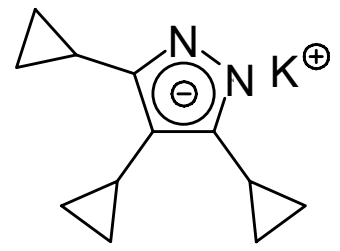

$158 \mathrm{a}$

Schema 83. Deprotonierung von 3,4,5-Tricyclopropyl-1H-pyrazol (158) mittels Kaliumhydrid in $\left[\mathrm{D}_{8}\right]$-THF.

Verglichen mit Pyrrolen sind Diazole aufgrund des zusätzlichen Stickstoffatoms auch stärkere Säuren, da die korrespondierenden Diazolanionen weit besser stabilisiert werden. Analog zum Pyrrol 18d wurde 3,4,5-Tricyclopropyl-1 $H$-pyrazol (154) daher ebenfalls deprotoniert und das korrespondierende Anion 158a NMR-spektroskopisch charakterisiert. Die Reaktion von 158 mit Kaliumhydrid in $\left[\mathrm{D}_{8}\right]$-THF bei $0{ }^{\circ} \mathrm{C}$ verlief quantitativ.

\section{Neuartige Cyclopropyl-substituierte cyclische und acyclische Oligoene}

7.1. Synthese des Octacyclopropyltricyclo[4.2.0.0.5 $0^{2,5}$ octadiens (165) als Vorstufe für Octacyclopropylcuban (169)

TAKAHASHI et al. berichteten kürzlich über eine neue Methode zur in situ Präparation von Cyclobutadienen durch intramolekulare Kupplung von 1-Zirkona-4-halogenbuta1,3-dienen in Gegenwart von Kupfer(I)chlorid. ${ }^{[138]}$ In analoger Weise konnte Dicyclopropylethin (13) mit Biscyclopentadienylzirkoniumdichlorid mittels $n$-Butyllithium in THF in das Zirkonacyclopentadien 161 transformiert werden. Dieses konnte in situ mit elementarem Iod zum intermediären 1-Zirkona-4-iodbuta-1,3-dien 162 bei $-78^{\circ} \mathrm{C}$ halogeniert und nachfolgend mit Kupfer(I)chlorid bei Raumtemperatur zur entsprechenden Kupferspezies 163 transmetalliert werden. 


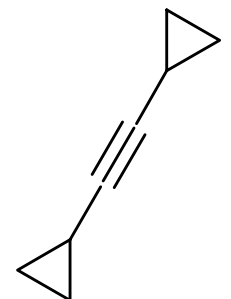

13

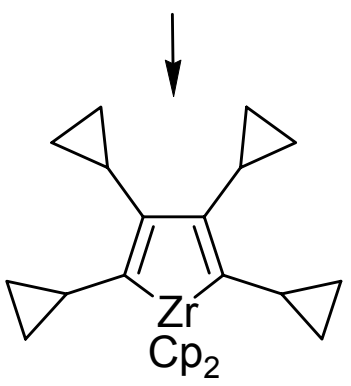

161

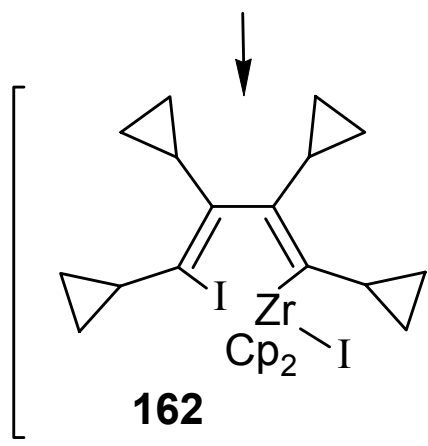

1) $\mathrm{Cp}_{2} \mathrm{ZrCl}_{2}$ (0.5 Äquiv.), $n$-BuLi (1.0 Äquiv.), $\mathrm{THF},-78^{\circ} \mathrm{C} \rightarrow \mathrm{RT}, 1 \mathrm{~h}$

2) $\mathrm{I}_{2}$ (1.0 Äquiv.), $-78^{\circ} \mathrm{C} \rightarrow \mathrm{RT}, 1 \mathrm{~h}$

3) $\mathrm{CuCl}$ (1.0 Äquiv.), $\mathrm{RT}, 1 \mathrm{~h}$

$38 \%$

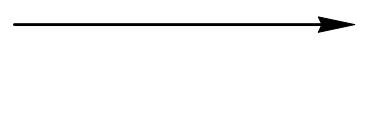

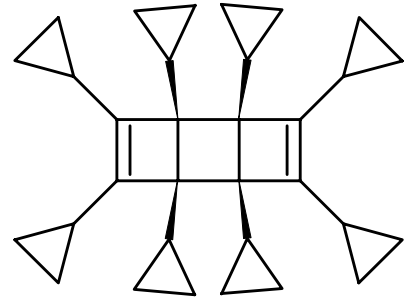

165
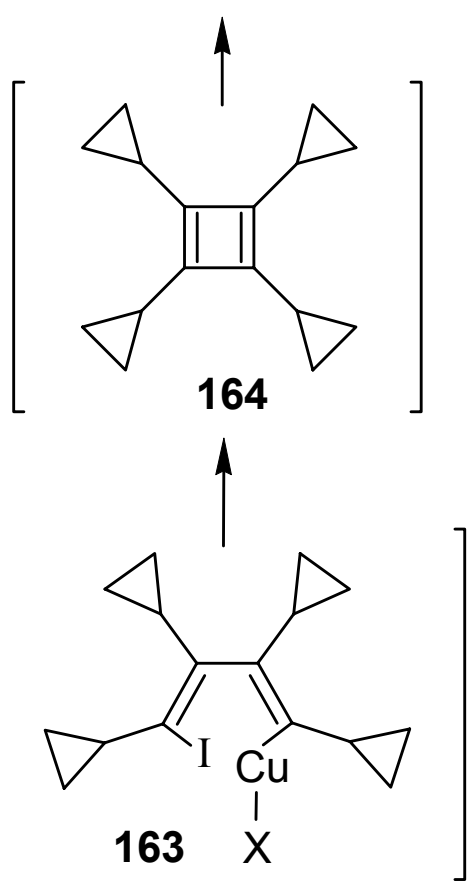

Schema 84. Darstellung des Tricyclooctadiens 165 durch intramolekulare Kupplung von in situ erzeugtem 1-Zirkona-4-iodbuta-1,3-dien 161 bei Raumtemperatur.

Diese 1-Kupfer-4-iodbuta-1,3-dien-Spezies 163 kuppelt nachfolgend intramolekular unter Freisetzung des Tetracyclopropylcyclobutadiens 164, das nachfolgend über [2+2]-Cycloaddition selektiv zum syn-Tricyclooctadien 165 dimerisiert, das in einer Ausbeute von $38 \%$ isoliert werden konnte.

Dieses Tricyclooctadien ist ein Isomer des entsprechendem Pentacyclo$\left[4 \cdot 2 \cdot 0 \cdot 0^{2,5} \cdot 0^{3,8} \cdot 0^{4,7}\right]$ octans - oder auch Cubans. Die Stammverbindung konnte erstmals von EATON und COLE in einer achtstufigen Synthese ausgehend von dem DiELs-ALDER-Addukt des 2-Bromcyclopentadienons synthetisiert werden. ${ }^{[139]}$ Auch ein syn-Tricyclooctadien kann eine Ausgangsverbindung für ein Cuban sein, in das es durch Bestrahlung mit Licht überführt 
werden könnte. Genau dies gelang GLEITER et al., indem sie den Tricyclus 166a in Pentan als Lösungsmittel mit einer Quecksilberdampf-Hochdrucklampe bestrahlten und 167a in Spuren nachweisen konnten. Auch das vierfach Propano-verbrückte Cuban 168 erhielten sie so in immerhin 7\% Ausbeute. In gleicher Weise konnten MILLER et al. das Octa(trifluormethyl)cuban 167b in einer Ausbeute von bemerkenswerten 20\% herstellen. ${ }^{[140]}$
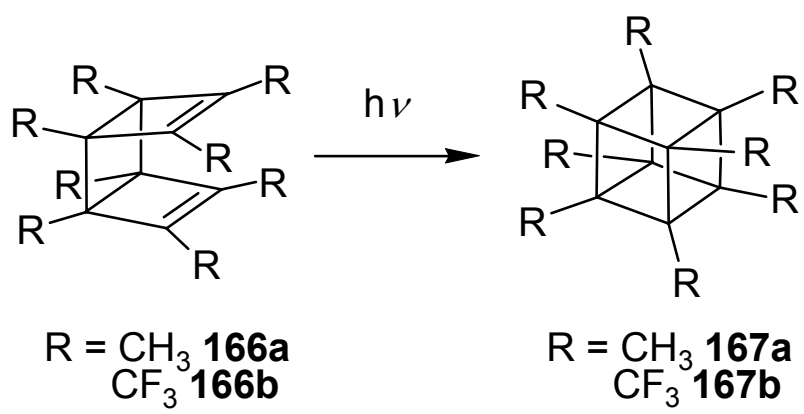

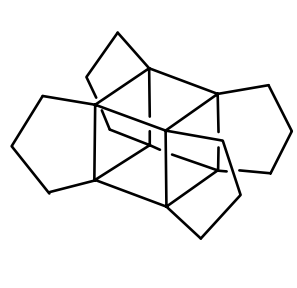

168

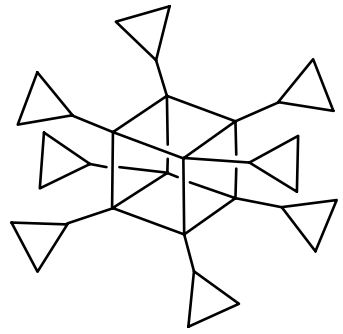

169

Schema 85. Mögliche Darstellung der Cubane 167, 168 und 169 durch Photolyse von synTricyclooctadienen.

Normalerweise cyclisiert ein Tricyclooctadien unter photolytischen Bedingungen nicht einfach so. Drei Gründe sprechen gegen eine solche Umlagerung. Erstens besteht ein erheblicher Unterschied in den Spannungsenergien zwischen Edukt und Produkt, die dessen Bildung benachteiligt. Cuban besitzt eine sehr hohe Spannungsenergie von $166 \mathrm{kcal} / \mathrm{mol}$; pro C-C-Bindung macht dies $14 \mathrm{kcal} / \mathrm{mol}$ aus (zum Vergleich mit dem Cyclopropanring: $9.4 \mathrm{kcal} / \mathrm{mol}$ ). Zweitens ist der Abstand der beiden Doppelbindungen mit $3.05 \AA$ für das unsubstituierte syn-Tricyclooctadien recht groß. Drittens macht eine ungünstige Energieabfolge der Molekülorbitale durch eine dominante „through-bond“-Wechselwirkung zwischen den Doppelbindungen die Reaktion zu einer potentiell Symmetrie-verbotenen Transformation. Die entscheidende Rolle, die der Spannungsenergiezunahme bei der Reaktion zukommt, zeigt sich am Besipiel des vierfach Propano-verbrückten Cubans 168. In dem entsprechenden Vorläufer konnte durch eine Einkristall-Röntgenstrukturanalyse der Abstand zwischen den beiden Doppelbindungen zu $2.65 \AA$ A bestimmt werden. Die kurzen Alkylbrücken bringen die Doppelbindungen hier nicht nur in engeren Kontakt, sondern sie reduzieren auch 
die Spannungsenergie-Differenz zwischen Edukt und Produkt zum Vorteil des Cubans 168. Es ist durchaus denkbar, dass auch im Octacyclopropyltricyclooctadien 169 die CyclopropylSubstituenten die Doppelbindungen näher zusammen zwingen. Entsprechende Umsetzungen des Tricyclus 165 unter photolytischen Bedingungen würden demnach lohnend erscheinen.

\subsection{Versuch der Darstellung von 1,2,3,4,5,6,7,8-Octacyclopropylcyclooctatetraen (176)}

Durch eine kleine Variation der Reaktionsbedingungen konnten TAKAHASHI et al. ebenfalls ausgehend von in situ erzeugtem Zirkonacyclopentadienen wie $\mathbf{1 7 1}$ in einer EintopfReaktion Cyclooctatetraene wie 175 herstellen. ${ }^{[141]}$

Dabei wurde 171 durch doppelte Transmetallierung mit Kupfer(I)chlorid in die Biskupferspezies 172 transformiert und diese nachfolgend mittels $N$-Bromsuccinimid monobromiert. Bei niedrigen Temperaturen war die 1-Kupfer-4-brombuta-1,3-dien-Spezies 173 aufgrund sterischer Hinderung nicht fähig, intramolekular zu reagieren, was zu dem oben beschriebenen Tricyclooctadien geführt hätte. Stattdessen reagiert es intermolekular mit einem weiteren Molekül 173 zu einer 1-Kupfer-8-bromocta-1,3,5,7-tetraen-Spezies 174, die wiederrum durch intramolekulare Kupplung das Cyclooctatetraen 175 freisetzt. 


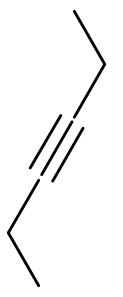

170<smiles>[R6][Z7]1(C)c(CC)c(CC)c(CC)c1CC</smiles>

171<smiles>[X]C([Y])=C(CC)C([Y])=C(CC)C(CC)=C(CC)CC</smiles>

1) $\mathrm{Cp}_{2} \mathrm{ZrCl}_{2}$ (0.5 Äquiv.), n-BuLi (1.0 Äquiv.), THF, $-78^{\circ} \mathrm{C} \rightarrow \mathrm{RT}, 1 \mathrm{~h}$

2) $\mathrm{CuCl}$ (1.0 Äquiv.), $\mathrm{RT}, 1 \mathrm{~h}$

3) NBS (0.5 Äquiv.), $-78^{\circ} \mathrm{C}, 1 \mathrm{~h}$

\section{$52 \%$}<smiles>[Y]C(Cl)=C(CC)C(CC)=C(CC)C(CC)=C(CC)C(CC)=C(Cl)Br</smiles>

\section{4}<smiles>[Y]C(Cl)=C(CC)C(CC)=C(Br)CC</smiles>

Schema 86. Darstellung des Cyclooctatetraens 175 durch intermolekulare Kupplung von in situ erzeugtem 1-Zirkona-4-brombuta-1,3-dien $\mathbf{1 7 0}$ bei niedrigen Temperaturen.

Dicyclopropylethin (13) wurde in analoger Weise umgesetzt. In der Tat konnte im Rohprodukt das entsprechende Octacyclopropylcyclooctatetraen 176 massenspektrometrisch nachgewiesen werden. Allerdings war es noch mit einer erheblichen Menge Tricyclooctadien 165 verunreinigt, das säulenchromatographisch nicht abgetrennt werden konnte. Durch eine nachträgliche Silberionen-katalysierte Isomerisierung des Tricyclooctadiens 165 könnte das Cyclooctatetraen 176 vielleicht in höherer Reinheit gewonnen werden. ${ }^{[142]}$ 


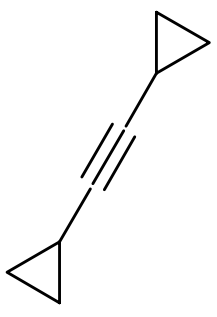

13
1) $\mathrm{Cp}_{2} \mathrm{ZrCl}_{2}$ (0.5 Äquiv.), $n$-BuLi (1.0 Äquiv.), THF, $-78^{\circ} \mathrm{C} \rightarrow \mathrm{RT}, 1 \mathrm{~h}$

2) $\mathrm{CuCl}$ (1.0 Äquiv.), $\mathrm{RT}, 1 \mathrm{~h}$

3) NBS (0.5 Äquiv.), $-78^{\circ} \mathrm{C}, 1 \mathrm{~h}$

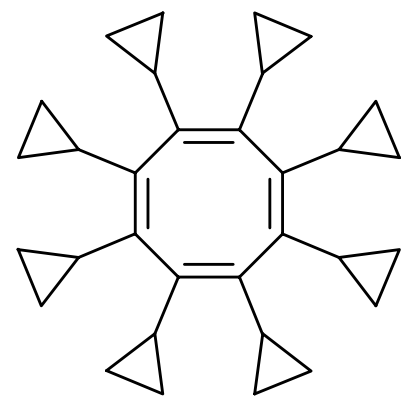

176

Schema 87. Versuch der Darstellung des Octacyclopropylcyclooctatetraens 176 analog einer Vorschrift von TAKAHASHI.

7.3. Cyclopropyl-substituierte 1,4-Dihalogenbutadiene als Kupplungskomponenten für oligocyclopropylierte acyclische Triene und Tetraene

Die schon zur Herstellung des 1,2,3,4-Tetracyclopropylbutadiens 21 angewandte Methode von SATO et al. konnte dahingehend modifiziert werden werden, dass auch Cyclopropyl-substituierte 1,4-Dihalogenbutadiene zugänglich wurden. Ein entsprechendes 1,2,3,4-Tetracyclopropyl-1,4-diiodbuta-1,3-dien (179) wurde in Anlehnung an Protokolle von SATO et al. in guter Ausbeute in einem einstufigen Prozess aus Dicyclopropylethin (13) dargestellt werden. ${ }^{[70]} \mathrm{Zu}$ einer Mischung aus Titantetraisopropylat und Dicyclopropylethin (13) in Diethylether wurde bei $-78^{\circ} \mathrm{C}$ eine etherische Lösung von Isopropylmagnesiumbromid getropft, eine Stunde bei -50 bis $-40{ }^{\circ} \mathrm{C}$ gerührt und nachfolgend bei $-78^{\circ} \mathrm{C}$ mit elementarem Iod versetzt. Nach wässriger Aufarbeitung des Reaktionsgemisches und säulenchromatographischer Reinigung des Rohproduktes an Kieselgel konnte das gewünschte 1,2,3,4-Tetracyclopropyldiiodbutadien 179 als diastereoselektiv gebildetes $(Z, Z)$-Isomer in $77 \%$ Ausbeute isoliert werden. Bemerkenswerterweise konnte auch das terminale Alkin 13 in dieser Reaktion unter den gleichen Bedingungen eingesetzt werden. Man erhielt dabei immerhin 28\% des 1,4-Dicyclopropyl-1,4-iodbuta-1,3-diens (178). Dabei muss sich intermediär ein 1,4-disubstituiertes Titanacyclopentadien regioselektiv gebildet haben, aus dem durch Halogenierung dann 178 freigesetzt wurde. 


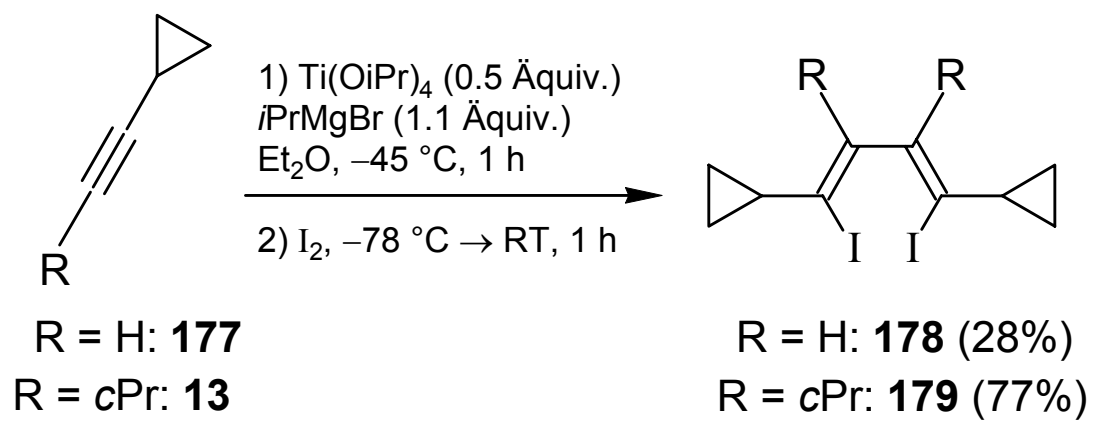

Schema 88. Einstufige Synthese von (Z,Z)-1,4-Diiodbuta-1,3-dienen 178 und 179 durch Titan-vermittelte Dimerisierung der Cyclopropylacetylene 177 und 13.

Diese Diiodbutadiene 178 und 179 könnten aufgrund ihrer regioselektiven Bildung als ideale Vorstufen für längerkettige acyclische Oligoene wie $(E, Z, Z, E)-23$ dienen, ${ }^{[143]}$ die durch eine sterische Abstoßung der Cyclopropylgruppen untereinander die Oligoene in eine helicale Konformation dirigieren könnten.

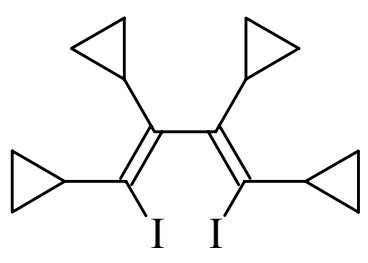

179

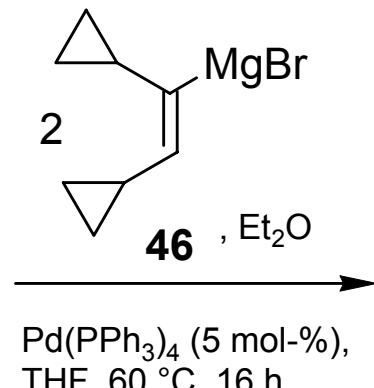

$\mathrm{THF}, 60^{\circ} \mathrm{C}, 16 \mathrm{~h}$

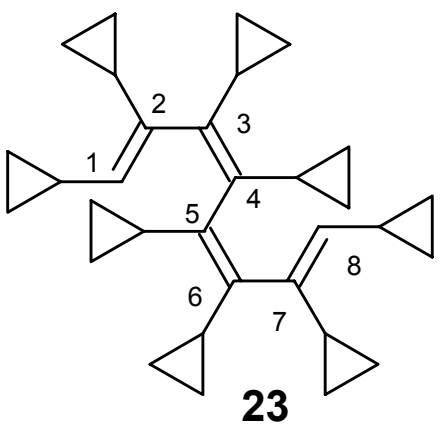

23

Schema 89. Versuchte Darstellung des 1,2,3,4,5,6,7,8-Cyclopropylocta-1,3,5,7-tetraens 23 durch Palladium-katalysierte Kreuzkupplung des Diiodbutadiens $\mathbf{1 7 9}$ mit dem 1,2-Dicyclopropylethenylmagnesiumbromid (46).

Daher wurde das Diiodbutadien 179 mit dem 1,2-Dicyclopropylethenylmagnesiumbromid (46) und einer katalytischen Menge Tetrakis(triphenylphosphan)palladium(0) in THF bei $60{ }^{\circ} \mathrm{C}$ zur Reaktion gebracht. Nach wässriger Aufarbeitung des Reaktionsgemisches und säulenchromatographischer Reinigung des Rohproduktes an Kieselgel konnte das Octacyclopropyloctatetraen 23 massenspektrometrisch eindeutig 
nachgewiesen werden; es war aber noch mit bisher nicht identifizierten Nebenprodukten verunreinigt. $\mathrm{Ob}$ dieses acyclische Octatetraen nun tatsächlich bevorzugt, eine helicale Konformation mit gleichsinnigen gauche-Anordnungen bezüglich der Bindungen $\mathrm{C}^{2}-\mathrm{C}^{3}$, $C^{4}-C^{5}$ und $C^{6}-C^{7}$ annehmen würde, bliebe durch eine Röntgen-Strukturanalyse zu prüfen. Für die Züchtung geeigneter Kristalle müsste $\mathbf{2 3}$ zuvor aber von den Verunreinigungen befreit werden.

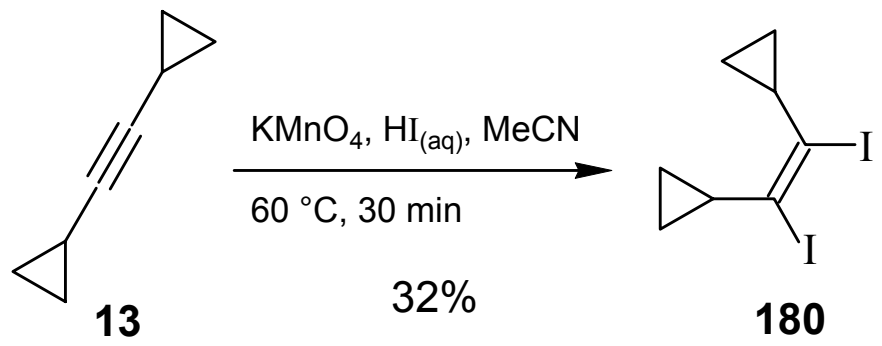

Schema 90. Darstellung des 1,2-Dicyclopropyl-1,2-diiodethens (180) durch Iodierung von Dicyclopropylethin (13).

Um auch die Möglichkeit der Synthese des Hexacyclopropylhexatriens zu untersuchen, wurde das (Z)-1,2-Dicyclopropyl-1,2-diiodethen (180) aus Dicyclopropylethin (13) durch Erhitzen mit Kaliumpermanganat und wässriger Iodwasserstoffsäure in Acetonitril analog einer Vorschrift von LIU dargestellt. ${ }^{[144]}$ Es konnte in einer Ausbeute von $32 \%$ als lichtempfindliches Öl erhalten werden. Dieses Diiodethen 180 sollte als Kupplungskomponente unter den oben beschriebenen Bedingungen ebenfalls Palladium-katalysiert mit der GRIGNARD-Verbindung 46 zur Reaktion gebracht werden. Nach wässriger Aufarbeitung des Reaktionsgemisches und säulenchromatographischer Reinigung des Rohproduktes an Kieselgel konnte aber kein $(E, Z, E)$-Hexatrien des Typs 22 isoliert werden. Stattdessen erhielt man eine große Menge Dicyclopropylethen, das aus der Hydrolyse der Vinyl-GRIGNARDVerbindung 46 hervorgeangen sein musste, und auch Dicycloproyplethin (13). Dieses entstand wahrscheinlich durch eine $\beta$-Halogeneliminierung aus der bei der oxidativen Addition des Palladiumkatalysators an $\mathbf{1 8 0}$ gebildeten 1-Palladium-2-iodethen-Spezies. 
Solche Nebenreaktionen sind bei Verwendung von 1,2-Dihalogenverbindungen als Kupplungskomponente in Übergangsmetall-katalysierten Reaktionen wohl bekannt. ${ }^{[145]}$

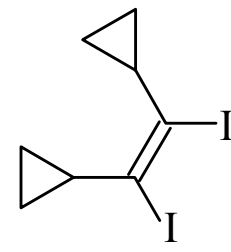

180

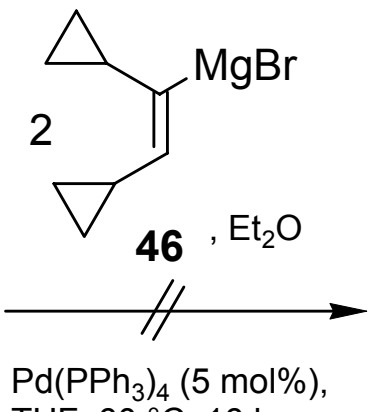

THF, $60^{\circ} \mathrm{C}, 16 \mathrm{~h}$

Schema 91. Versuchte Darstellung des 1,2,3,4,5,6-Hexacyclopropylhexatriens 22 durch Palladium-katalysierte Kreuzkupplung des Diiodethens $\mathbf{1 8 0}$ mit der Vinyl-GRIGNARDVerbindung 46.

8. Versuche zur Darstellung des 6,6-Dicyclopropyl-substituiertem Fulven 24 und die präparativ interessante Bildung von Allylidencyclobutenen

Fulvene bestehen generell aus einem ungeradzahligem Ring $(n=1,2,3 \ldots, \mathbf{1 8 1})$, in dem alle Kohlenstoffatome eine $\mathrm{sp}^{2}$-Hybridisierung aufweisen, und einer exocyclischen Doppelbindung. Pentafulvene $(n=2, \mathbf{1 8 2})$ sind isomer $\mathrm{zu}$ Benzolderivaten, besitzen aber Eigenschaften, die zwischen denen von aromatischen und olefinischen Systemen stehen. ${ }^{[146]}$ Sie weisen mit ihrem kreuzkonjugierten Triensystem interessante chemische und physikalische Charakteristika auf. So sorgte beispielsweise das große Dipolmoment von 6,6-disubstituierten Pentafulvenen für ein großes Interesse an deren quantenmechanischen Berechnungen. ${ }^{[147]}$ Die Natur der Bindung in Fulvenen kann qualitativ durch zwei mesomere Grenzstrukturen, eine kovalente und eine dipolare Struktur, verdeutlicht werden. Eine Abschätzung der Gewichtung beider Grenzstrukturen erlaubt nach OLAH ein Vergleich der chemischen Verschiebungen der C-Atome 1 und 6. Dabei weist eine große Differenz der 
Signale auf eine stärkere Gewichtung der dipolaren Grenzstruktur hin und entsprechend umgekehrt. $^{[48,148]}$

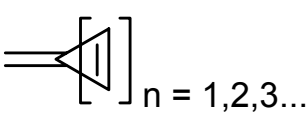

181

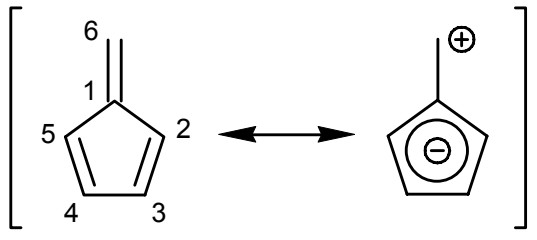

182

Schema 92. Generelle Konstitution von Fulvenen 181 und mesomere Grenzformeln von Pentafulven 182.

Das UV-Spektrum des unsubstituierten Pentafulvens weist im Bereich von 400 bis 200 nm zwei Hauptbanden auf, die auf $\pi \rightarrow \pi^{*}$-Übergänge zurückzuführen sind. ${ }^{[149]}$ Eine Verschiebung der Banden zu längeren Wellenlängen hin (bathochromer Effekt) zeigt ein elektronenreicheres Fulven an. Genau dies beobachtet man beim 6,6-Ethano-verbrückten, 2,3,4,5-Tetracyclopropylfulven $\mathbf{3 5}^{[36 \mathrm{a}]}$

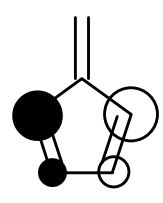

HOMO

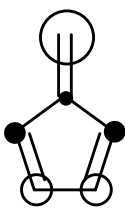

LUMO

Abbildung 26. Qualitative Verdeutlichung der AO-Koeffizienten des HOMO und des LUMO von unsubstituiertem Pentafulven 182.

Anhand der AO-Koeffizienten des HOMO und des LUMO von Fulvenen lässt sich deren beobachtete Reaktivität treffend erläutern. So wechselwirken Nucleophile mit dem LUMO und sollten selektiv an C-6 angreifen, da es die größte positive Ladungsdichte aufweist. Elektrophile sollten wegen ihrer Wechselwirkung mit dem HOMO jedoch selektiv in 2- oder 5-Position angreifen, da dort an denen die größte negative Ladungsdichte lokalisiert 
ist. Tatsächlich findet die Addition von Nucleophilen an Pentafulvene selektiv in Position 6 statt und liefert Cyclopentadiene wie 185. ${ }^{[36 a]}$ Dagegen addieren Triafulvene Nucleophile an Position 2 oder 3 und führten zu exo-Methylencyclopropanen wie 184. ${ }^{[150]}$
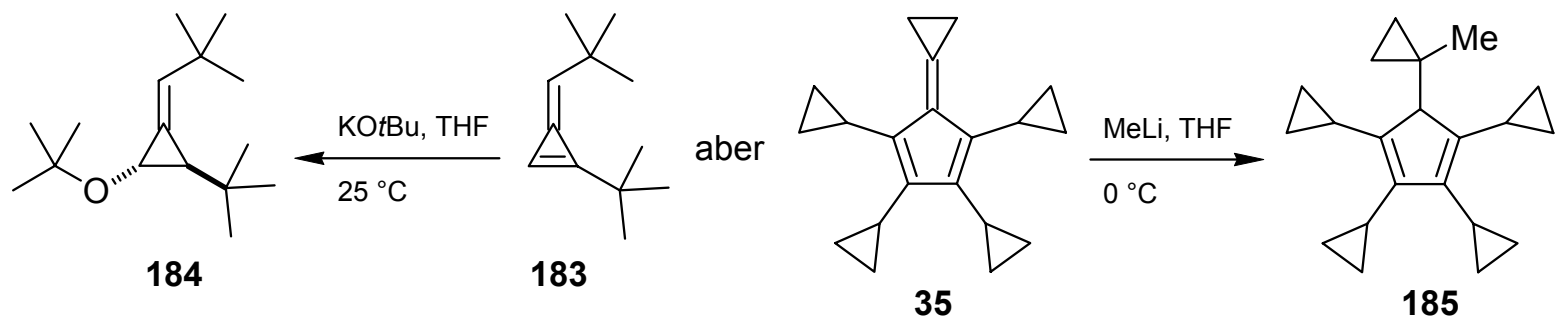

Schema 93. Regioselektive nucleophile Angriffe am Triafulven 183 und am Pentafulven 35.

Zur Synthese von Pentafulvenen geht man im Allgemeinen von den entsprechenden Cyclopentadienen aus und kondensiert sie mit Carbonylverbindungen. ${ }^{[151]}$ EMME konnte zwar ausgehend vom Tetracyclopropylcyclopentadienylanion 14a durch siebentägiges Rühren mit Paraformaldehyd das Tetracyclopropylfulven 186 in 22 bis 27\% Ausbeute erhalten, der Zugang zu 6,6-disubstituierten Fulvenen durch Reaktion mit Aceton oder Cyclopentanon nach LITTLE gelang auf diese Weise jedoch nicht. ${ }^{[37,151 b]}$ Die lange Reaktionszeit für $\mathbf{1 8 6}$ in Verbindung mit der moderaten Ausbeute der Reaktion weist deutlich auf die sterische Überfrachtung des Tetracyclopropylcyclopentadienylanions 14a hin. Dieser erklärt auch, warum die nächst höheren Homologen des Formaldehyds mit 14a keine Reaktion mehr eingehen. 


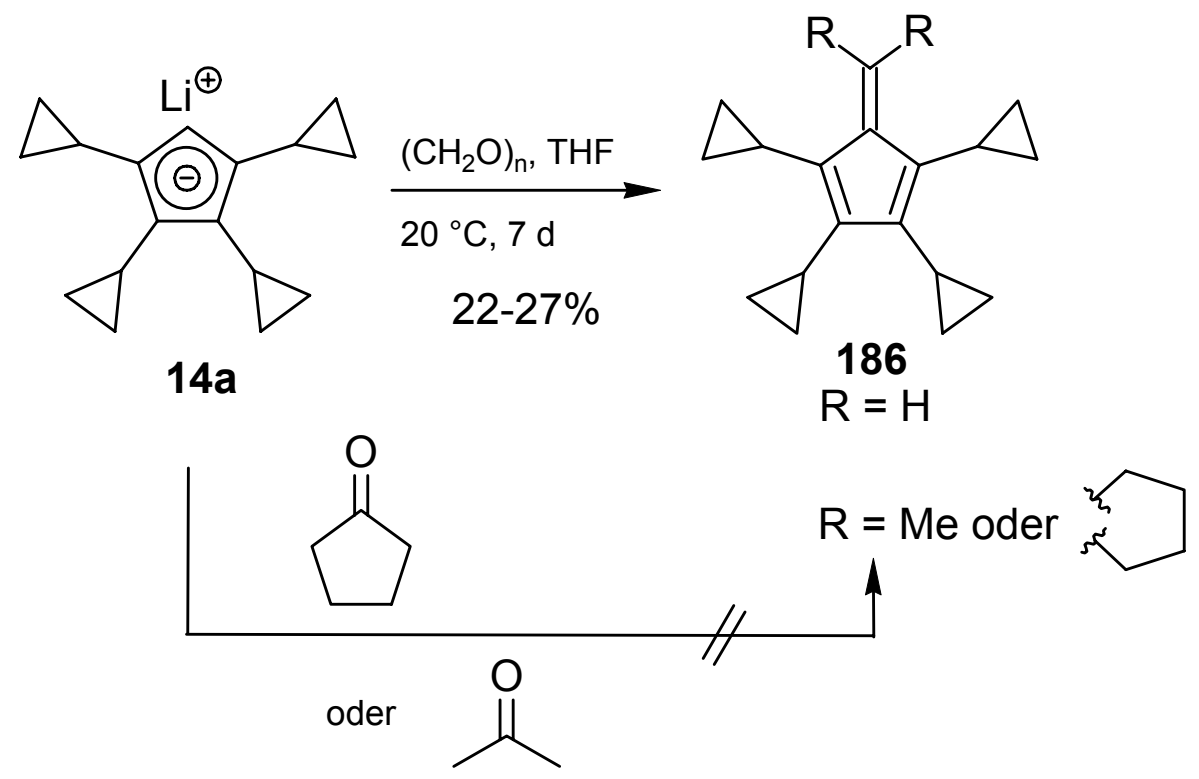

Schema 94. Synthese von 2,3,4,5-Tetracyclopropylfulven 186 und versuchte Darstellung von 6,6-Dimethyl-substituiertem und 6,6-Butano-verbrückten 2,3,4,5-Tetracyclopropylfulvenen.

Im Hinblick auf die gelungenen Synthesen der Tetracyclopropylfulvene $\mathbf{1 8 6}$ und $\mathbf{3 5}$ wäre eine Synthese des entsprechenden 6,6-Dicyclopropyl-substituierten Fulvens 24 von Interesse. Dieses könnte auch als Vorstufe für sein korrespondierendes Fulvenyl-Kation 187 dienen, wie sie aus 186 und 35 schon erzeugt wurden, und würden diese Reihe von FulvenylKationen abrunden. ${ }^{[152]}$

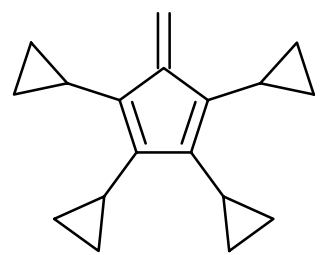

186

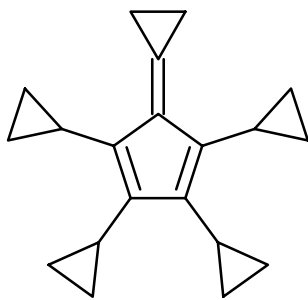

35

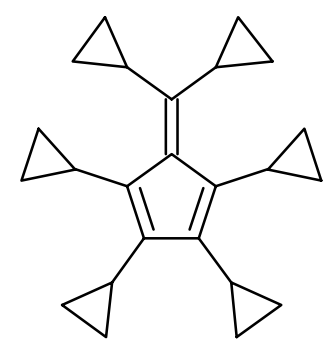

24

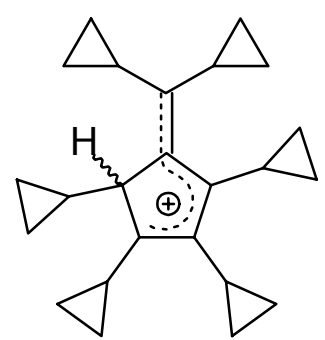

187

Schema 95. 2,3,4,5-Tetracyclopropylfulvene mit unterschiedlichem Substitutionsmuster an Position C-6.

Hexacyclopropylfulven 24 wäre nach den obigen Beobachtungen aller Wahrscheinlichkeit nach nun nicht durch eine simple Kondensation von 14a mit Dicyclopropylketon 
zugänglich. TOLSTIKOV et al. konnten aber durch einfaches Erhitzen von Cyclopropylethin (177) mit einer katalytischen Menge Palladium(II)acetat in THF das 2,4,6-Tricyclopropylfulven 188 in sehr guter Ausbeute darstellen. ${ }^{[153]}$

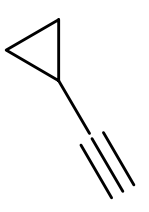

177
$\mathrm{Pd}(\mathrm{OAc})_{2}(5 \mathrm{~mol} \%)$

THF, $1 \mathrm{~h}, 80^{\circ} \mathrm{C}$

$100 \%$

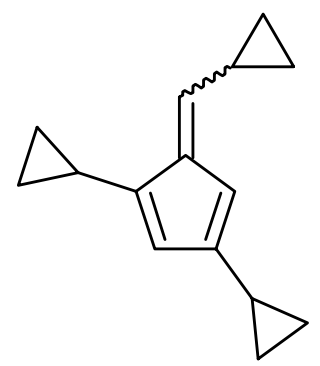

188

Schema 96. Darstellung des Tricyclopropylfulvens 188 nach TOLSTIKOV.

Solche sukzessiven Carbopalladierungen von zwei Molekülen eines Acetylens mit einem Alkenylpalladiumhalogenid sind zur Herstellung von Fulvenen in moderaten bis guten Ausbeuten gut geeignet. ${ }^{[154]}$ So konnten NEGISHI et al. aus 1-Brom-1-hexen (189) und 190 in 40\% Ausbeute das Cyclopentan-annelierte Fulven 191 darstellen und HECK et al. gelang es, aus 3-Hexin (170) und cis-1-Brom-2-ethoxyethen (192) analog das Tetraethylfulven 193 in einer Ausbeute von $26 \%$ herzustellen. ${ }^{[155,154 \mathrm{c}]}$<smiles>C#CCC(CC#C)(COCC)C(=O)OCC</smiles>

189<smiles>[CH2+]CO/C=C\Br</smiles>

192<smiles>CCCC/C=C/Br</smiles>

190

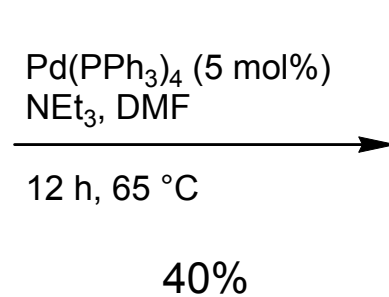

$40 \%$

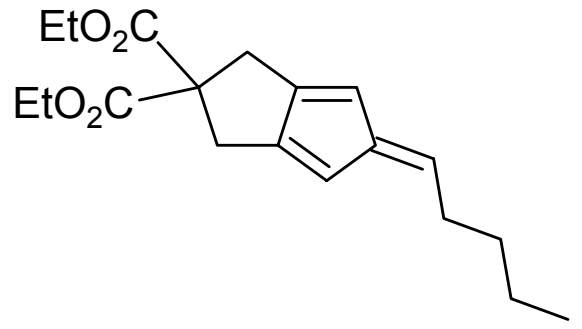

191

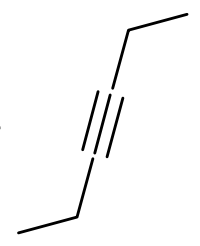

170
$\mathrm{Pd}(\mathrm{OAc})_{2}(5 \mathrm{~mol} \%)$ $\mathrm{P}(\mathrm{OTOl})_{3}, \mathrm{NEt}_{3}$, DMF

$29 \mathrm{~h}, 100^{\circ} \mathrm{C}$

$26 \%$

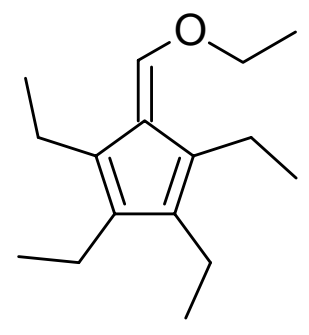

193

Schema 97. Bildung der Fulvene 191 und 193 durch sukzesssive Kupplung der Alkenylbromide 190 und 192 an die Acetylene 189 und 170. 
Die darauf aufbauende retrosynthetische Überlegung war demnach, das Hexacyclopropylfulven 24 aus einem Molekül 2,2-Dicyclopropylvinylhalogenid und zwei Molekülen Dicyclopropylethin (13) zusammen zu fügen (Schema 98).

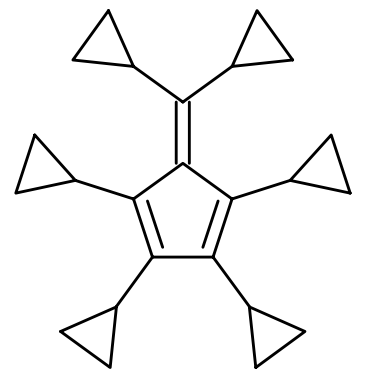

24

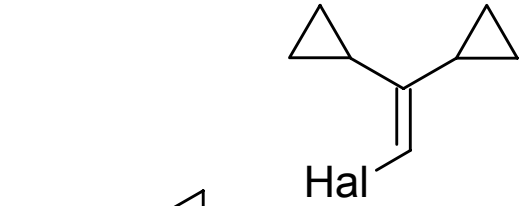

194

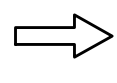

13

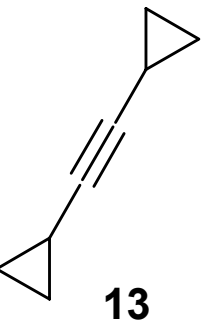

Schema 98. Retrosynthetische Zerlegung des Hexacyclopropylfulvens 24 in Dicyclopropylethin (13) und ein Vinylhalogenid 194.

Allerdings müssen bei dieser Strategie zwei Dinge beachtet werden: zum einen würde sich die Palladiumspezies nach der syn-Insertion in die ursprüngliche vinylische Doppelbindung zu 195 in einer „doppelten“ Cyclopropylmethyl-Stellung befinden. Von einer solchen Konstellationen ausgehend wäre eine Palladium-vermittelte CyclopropylmethylHomoallyl-Umlagerung ${ }^{[74]}$ mit einer nicht gewünschten Dreiringöffnung zu 196 typischerweise $\mathrm{zu}$ erwarten. Diese stünde mit der für die Fulvenbildung nötigen $\beta$-Hydrideliminierung in Konkurrenz. Von dieser Eventualität abgesehen könnte eine solche ringöffnende Umlagerung auch schon nach jeder syn-Insertion der Palladiumspezies an Dicyclopropylethin zu 197 geschehen, was natürlich ebenfalls nicht zum gewünschten Fulven führen würde. Allerdings ist eine solche Umlagerung bisher noch nicht beobachtet worden, wenn der Palladiumrest an einem $\mathrm{sp}^{2}$-hybridisierten Zentrum steht. In diesem Fall müsste ein Allen 198 als Intermediat entstehen, dessen Bildung jedoch aufgrund einer Energiebarriere von ca. $22 \mathrm{kcal} / \mathrm{mol}$ recht unwahrscheinlich wäre. 


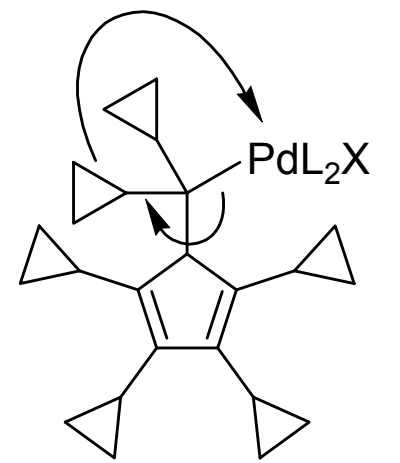

195

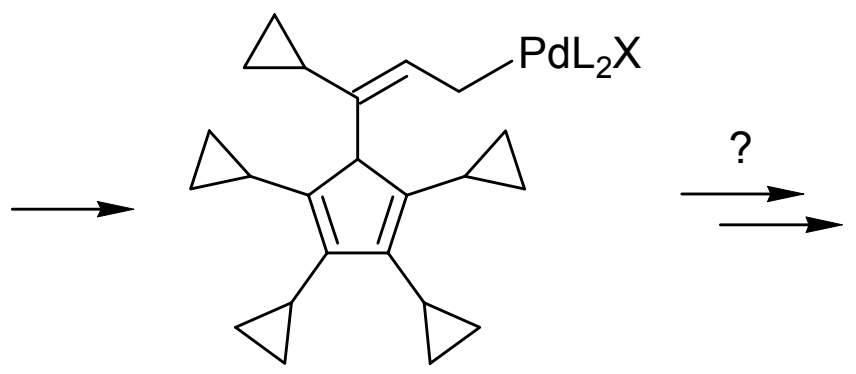

196

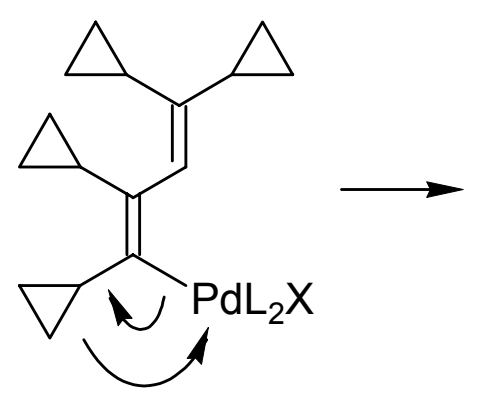

197

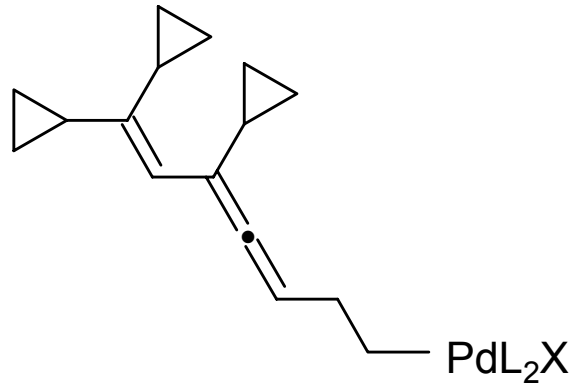

198

Schema 99. Mögliche Palladium-vermittelte Cyclopropylmethyl-Homoallyl-Umlagerungen der Intermediate 196 und 198.

Da die sukzessive Carbopalladierung von zwei Molekülen Dicyclopropylethin (13) bisher nicht untersucht war, wurde die Reaktion zunächst unter „klassischen“ HECKBedingungen mit 1-Brom-2,2-diphenylethen (199) als Kupplungpartner getestet. Diese Modellreaktion schloss so auch die Gefahr einer Öffnung der Cyclopropanringe in 6-Position aus. Bei $80^{\circ} \mathrm{C}$ wurde eine Mischung aus dem Vinylbromid 199 und zwei Äquivalenten Dicyclopropylethin zusammen mit Palladium(II)acetat als Katalysator, Triphenylphosphan und Triethylamin in Acetonitril für 24 Stunden gerührt. Nach wässriger Aufarbeitung des Reaktionsgemisches und säulenchromatographischer Reinigung des Rohproduktes an Kieselgel konnte das Fulven 206 allerdings nicht detektiert werden. Stattdessen wurde ein farbloses Öl isoliert, dessen Massenspektrum auf ein Molekül hinwies, das aus je einem Äquivalent 13 und 199 entstanden sein musste. In der Tat konnte auch ein Teil des eingesetzten Dicyclopropylethins zurückgewonnen werden. Das neue Produkt konnte anhand seiner NMR-Korrelationsspektren identifiziert werden - erstaunlicherweise handelte es sich 
um das Allylidencyclobuten 200, das mit einer Ausbeute 71\% entstanden war. Das gleiche Produkt wurde in 59\% Ausbeute isoliert werden, wenn statt Triethylamin Kaliumcarbonat als Base verwendet wurde.

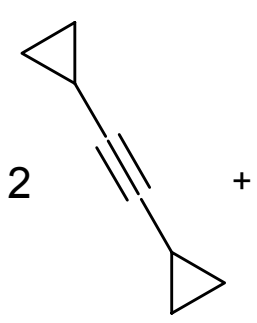

13

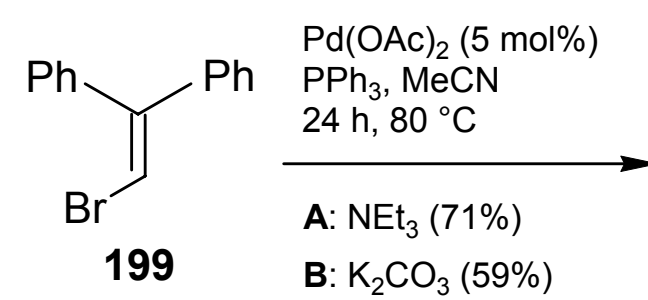

B: $\mathrm{K}_{2} \mathrm{CO}_{3}(59 \%)$

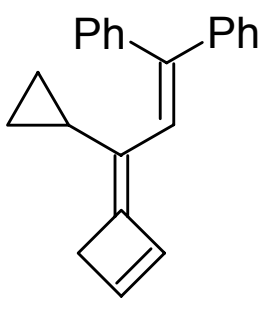

200

Schema 100. Überraschende Isolierung des Allylidencyclobutens 200 bei der HECK-Reaktion von Diphenylbromethen mit Dicyclopropylethin.

Die überraschende Bildung des Methylencyclobutens 200 ist insofern sehr bemerkenswert, da es doch offensichtlich am plausibelsten aus dem anfangs für unwahrscheinlich erklärten, zu 197 analogen Intermediat 201 entstanden sein muss. Offenbar ist die Energiebarriere zur Bildung einer zu 198 analogen Allenspezies 203 nicht so groß, als dass sie unter den gegebenen Bedingungen nicht überwunden werden konnte. Das Allylidencyclobutan 200 könnte durch eine 4-exo-dig Cyclisierung aus einem zu 204 analogen Intermediat 202 und anschließender $\beta$-Hydrideliminierung hervorgegangen sein. Dem gegenüber steht allerdings, dass 4-exo-dig Cyclisierungen nach BALDWIN weniger bevorzugt auftreten. Außerdem befände sich die Palladiumspezies nach erfolgter syn-Insertion an das Allen wieder in einer zur Umlagerung (zu 205) günstigen Position zu einem Cyclopropanring, die aber nicht beobachtet wurde. 


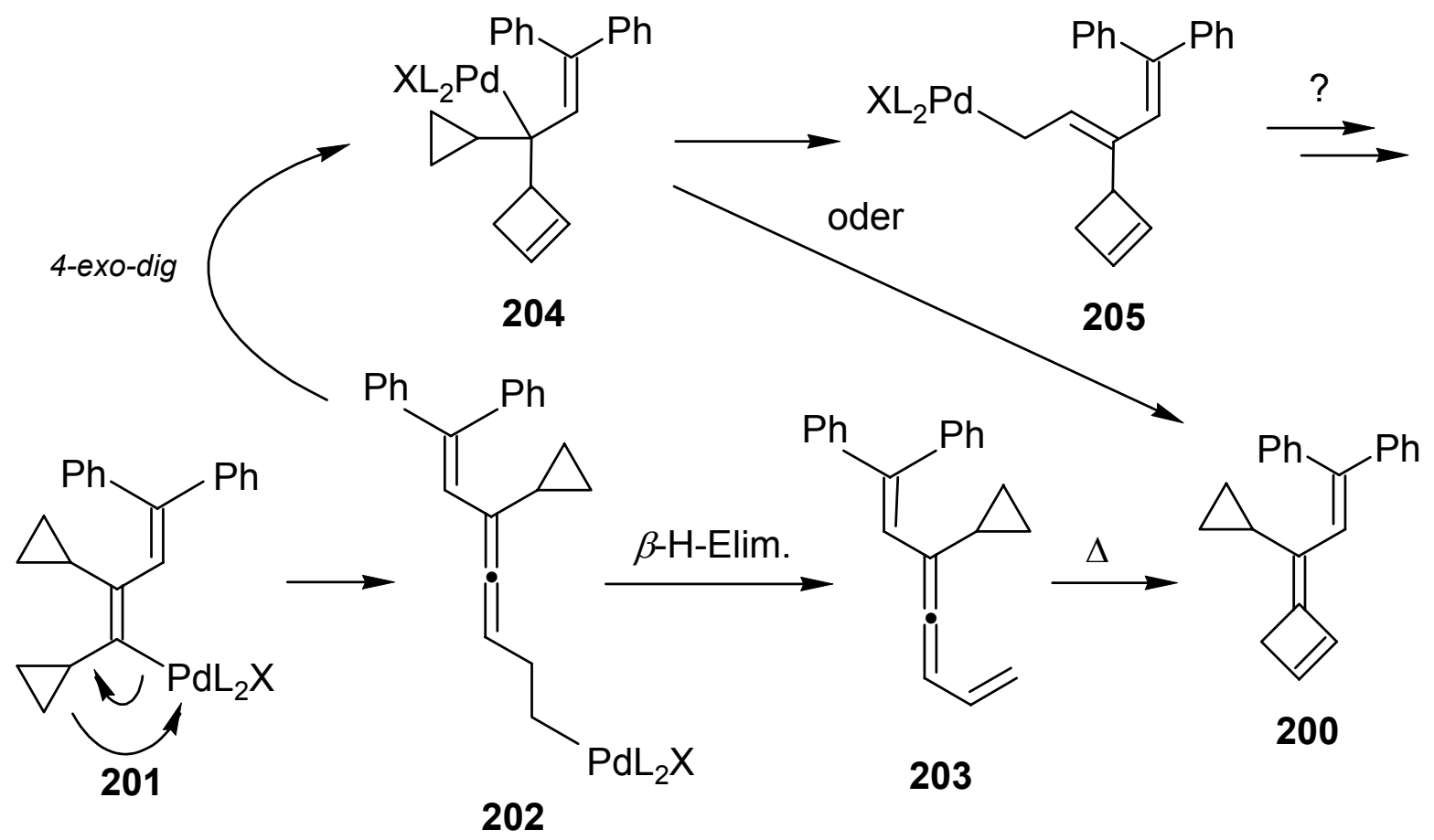

Schema 101. Möglicher Bildungsmechanismus für das Allylidencyclobutan 200.

Sehr wahrscheinlich ist dagegen ein Mechanismus, bei dem nach der ersten Carbopalladierung eine Cyclopropylmethyl-Homoallyl-Umlagerung stattfindet und eine Dehydropalladierung das Vinylallen 203 freisetzt, das im Anschluss daran thermisch zum detektierten Allylidencyclobutan $\mathbf{2 0 0}$ cyclisiert. Untersuchungen zur thermischen Elektroyclisierung von Vinylallenen durch DE LERA, ITO und PASTO et al. untermauern einen solchen Mechanismus. ${ }^{[156]}$

Um diese Reaktionsweise während der HECK-Reaktion eventuell zu verhindern, wurde als Base Silbercarbonat verwendet. Letzteres ist bekannt Doppelbindungs-Isomerisierungen bei Kreuzkupplungen zu unterdrücken - außerdem wird durch den Abfang von Halogenidionen mittels Silberionen eine positiv geladene Palladiumspezies gebildet, wodurch der Verlauf der Reaktion eher elektronisch kontrolliert abläuft. ${ }^{[156 c, 157]}$ Das dann elektrophilere Palladiumintermediat 201 könnte rascher mit einem weiteren Molekül Dicyclopropylethin reagieren, anstatt sich zu 202 umzulagern. In der Tat konnte so unter sonst unveränderten Bedingungen das erwartete Fulven 207 in geringer Ausbeute (8\%)isoliert werden. Dagegen lieferte die Reaktion des Vinylbromids 206 unter analogen Bedingungen statt des 
gewünschten Hexacyclopropylfulvens 24 nur große Mengen polymeren Materials und unumgesetztes Dicyclopropylethin, das Fulven $\mathbf{2 4}$ konnte nicht detektiert werden.

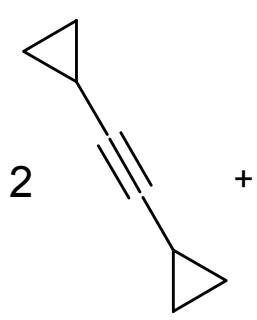

13

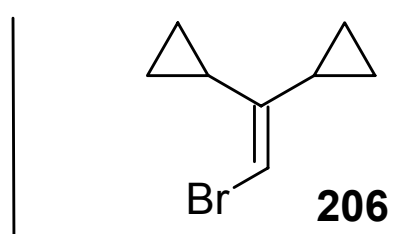

$\mathrm{Pd}(\mathrm{OAc})_{2}(5 \mathrm{~mol} \%)$

$\mathrm{PPh}_{3}, \mathrm{Ag}_{2} \mathrm{CO}_{3}, \mathrm{MeCN}$

$24 \mathrm{~h}, 80^{\circ} \mathrm{C}$

$8 \%$

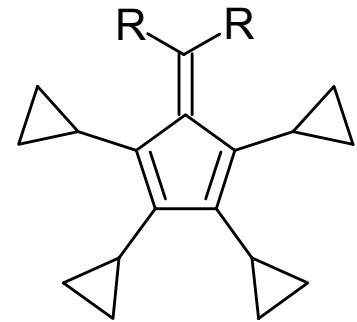

$\mathrm{R}=\mathrm{Ph}, 207$

Schema 102. Bildung des Fulvens 207 nach Wechsel der Base zu Silbercarbonat.

Um die Regioselektivität der Reaktion bei Verwendung von Alkyl-substituierten Cyclopropylacetylenen auszuloten, wurde 1-Cyclopropyl-1-propin (177) mit Triethylamin als Base eingesetzt. Nach wässriger Aufarbeitung des Reaktionsgemisches und säulenchromatographischer Reinigung des Rohprodukts an Kieselgel konnten 209 und 210 in gleichen Anteilen (jeweils 6\% Ausbeute) isoliert werden. Die Größe des Methylsubstituenten wirkt sich offensichtlich noch nicht auf eine bevorzugte Richtung der syn-Insertion der Vinylpalladiumspezies an das Acetylen 208 aus. Da eine Ethylgruppe dem sterischen Anspruch einer Cyclopropylgruppe eher gleicht, kann hier ein regioselektierender sterischer Effekt frühestens ab dem Verzweigungsgrad einer Isopropylgruppe erwartet werden. ${ }^{\text {[64a] }}$ 


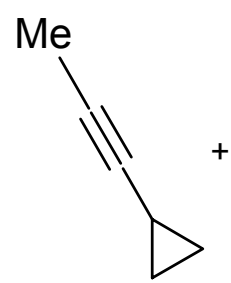

208<smiles>BrC=C(c1ccccc1)c1ccccc1</smiles>

199

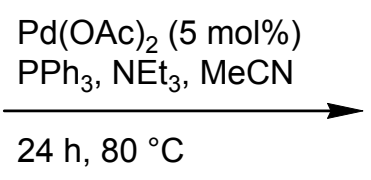

h, $80^{\circ} \mathrm{C}$

\section{8}
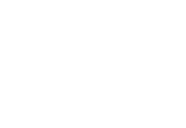

Schema 103. Untersuchung zur Regioselektivität der HECK-Reaktion von 208 und 199.

Die Bildung von 209 kann erneut durch eine thermische Elektrocyclisierung eines zu 203 analogen Vinylallens erklärt werden. Interessant ist aber die Entstehung des 2,5-Dicyclopropyl-substituierten Regioisomers 210. Nachdem die zweite syn-Insertion der zu 201 homologen Butadienylpalladiumspezies erfolgte, muss der nachfolgende Schritt zum 5-exo-trig Ringschluss offenbar schneller erfolgen als eine mögliche Umlagerung unter Öffnung des Cyclopropanringes.

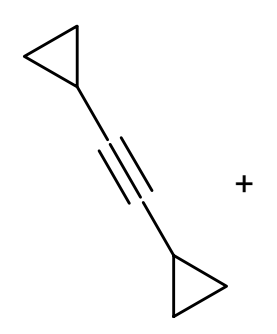

13

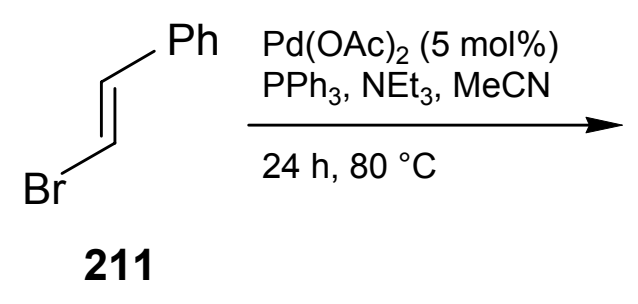

211

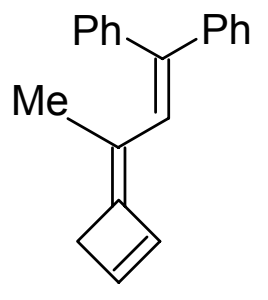

$6 \%$<smiles>CC1=C(C2CC2)C(=C(c2ccccc2)c2ccccc2)C(C2CC2)=C1C</smiles>

$6 \%$ 
substituierten Diinen oder von 1-Cyclopropyl-1-halogenethylenen, so ließe sich ein weit gefächertes Gebiet strukturell interessanter Oligoene erschließen. Auch der Einbau von Heteroatomen wäre bei Verwendung von Halogeniminen als Kupplungskomponente denkbar. 


\section{Experimenteller Teil}

\section{Allgemeines}

Schmelzpunkte: Schmelzpunktapparatur nach Dr. Trottoli der Fa. Büchi; die gemessenen Schmelzpunkte sind unkorrigiert). - IR-Spektroskopie: Bruker IFS 66 (FT-IR). - ${ }^{1} \mathrm{H}-\mathrm{NMR}-$ Spektroskopie: Mercury 200 (200 MHz), Bruker AM 250 (250 MHz), Bruker AMX 300 (300 MHz), Varian Unity 300 (300 MHz), Varian Inova 600 (600 MHz); Referenzen: $\delta=0.00$ ppm für Tetramethylsilan, 7.15 für [ $\left.\mathrm{D}_{5}\right]$-Benzol, 7.26 für Chloroform, 3.56 für $\left[\mathrm{D}_{8}\right]-$ Tetrahydrofuran. Charakterisierung der Signalaufspaltung: br $=$ verbreitertes Signal, $\mathrm{s}=$ Singulett, $\mathrm{d}=$ Dublett, $\mathrm{t}=$ Triplett, $\mathrm{q}=$ Quartett, quint $=$ Quintett, sept $=$ Septett, $\mathrm{m}=$ Multiplett, $\mathrm{m}_{\mathrm{c}}=$ zentriertes Multiplett, $\mathrm{dd}=$ doppeltes Dublett, $\mathrm{dt}=$ Dublett eines Tripletts, $\mathrm{ddd}=$ doppeltes Dublett eines Dubletts, $\mathrm{dq}=$ Dublett eines Quartetts, $\mathrm{tt}=$ Triplett eines Tripletts, $\mathrm{ddt}=$ doppeltes $\quad$ Dublett eines Tripletts, $\mathrm{AB}=$ Signalaufspaltung entspricht einem ABSpektrum. Spektren wurden nach erster Ordnung ausgewertet; die entsprechenden Kopplungskonstanten sind als Betrag in $\mathrm{Hz}$ angegeben. Abkürzungen für Zuordnung der Signale: $c \mathrm{Pr}-\mathrm{H}=$ cyclopropylisch, $\mathrm{Ar}-\mathrm{H}=$ aromatisch. Bei nicht eindeutiger Zuordnung wurden jeweils alle möglichen Wasserstoffatome angegeben. $-{ }^{13} \mathrm{C}-\mathrm{NMR}-$ Spektroskopie: Mercury 200 (50.3 MHz), Bruker AM 250 (62.9 MHz), Bruker AMX 300 (75.4 MHz), Varian Unity 300 (75.4 MHz), Varian Inova 600 (150 MHz); Referenzen: $\delta=77.0$ ppm für Deuterochloroform, 128.0 für [ $\left.\mathrm{D}_{6}\right]$-Benzol, 65.3 für $\left[\mathrm{D}_{8}\right]$-Tetrahydrofuran. Die Multiplizität der Signale wurde durch die DEPT-Aufnahmetechnik $($ DEPT $=$ distortionless enhancement by polarisation transfer) oder durch die APT-Aufnahmetechnik (APT = attached proton test) bestimmt und ist wie folgt angegeben; DEPT: $+=$ primäre oder tertiäre (positives DEPTSignal), $\quad-=$ sekundäre (negatives $\quad$ DEPT-Signal), $\mathrm{C}_{\text {quart }}=$ quartäre C-Atome (DEPTSignalintensität Null); APT: $+=$ primäre oder tertiäre (positives APT-Signal), $-=$ sekundäre oder quartäre (negatives APT-Signal) C-Atome. Abkürzungen und Zuordnung der Signale: $c$ Pr-C $=$ cyclopropylisch, Ar- $\mathrm{C}=$ aromatisch. Bei nicht eindeutiger Zuordnung wurden jeweils alle möglichen Kohlenstoffatome mit „,“ gekennzeichnet. $-{ }^{31}$ P-NMR-Spektroskopie: Varian 
500 (500 MHz); Referenzen: $\delta=0.00 \mathrm{ppm}$ für Phosphorsäure (85\%ig, extern). - MS: Finnigan MAT 95. - HRMS (hochauflösende MS): Finnigan MAT 95; korrekte HRMS = vorgewählter Ionen-Peak stimmt bei R >> 10000 mit einer Fehlerbreite von \pm 2 ppm mit der exakten Masse überein. - UV/Vis-Absorptionsspektroskopie: Zweistrahlspektrometer Varian Cary 219 (Geräteeinstellung und Spektrenauswertung durch HP 9845 B) und Spektrophotometer Perkin-Elmer Lambda 2. - Säulenchromatographie (SC): Chromatographische Trennungen erfolgten an Merck Kieselgel 60 (0.063-0.200 mm, 70-230 mesh) als Normal- bzw. an Macherey-Nagel Kieselgel 60 (0.040-0.063 mm, 46-70 mesh) als FlashChromatographie nach STILL. ${ }^{[158]}$ Die Säulendimensionen werden als "Säulendurchmesser $\times$ Höhe der Säule" angegeben. Alle Laufmittel wurden destilliert verwendet. - Dünnschichtchromatographie (DC): Macherey-Nagel Fertigfolien: Alugram Sil G/UV ${ }_{254}$; Merck DC-Fertigfolien: Kieselgel $60 \mathrm{~F}_{254}$ auf Aluminiumfolie. Detektion unter UV-Licht bei 254 nm, Entwicklung mit Molybdatophosphorsäure-Tauchreagenz (10proz. Lsg. in Ethanol). - Elementaranalysen: Mikroanalytisches Laboratorium des Instituts für Organische und Biomolekulare Chemie der Universität Göttingen. - Einkristall-Röntgen-Strukturanalysen wurden am Institut für Anorganische Chemie der Universität Göttingen durchgeführt. Die Daten wurden auf einem Stoe-Siemens-AED bzw. Stoe-Siemens-Huber-IPDS IIVierkreisdiffraktometer mit einem Siemens-CCD-Flächendetektor gesammelt. Die Intensitäten wurden mit $f$ - und $w$-Scans aufgenommen. Die Integration der Daten wurde mit dem Programm X-AREA ausgeführt. Die Strukturen wurden mit direkten Methoden gelöst $\left(\right.$ SHELXS-90/97) ${ }^{[159]}$ und nach dem Verfahren der kleinsten Fehlerquadratsummen gegen $\mathrm{F}^{2}$ verfeinert. Alle Nicht-Wasserstoffatome konnten anisotrop verfeinert werden. Alle Wasserstoffatome wurden geometrisch ideal positioniert und in die Verfeinerung einbezogen. Alle Fehlordnungen konnten mit Hilfe von Abstands- und ADP-Restraints aufgelöst und anisotrop verfeinert werden. - Wasserfreie Lösungsmittel wurden nach üblichen Laboratoriumsmethoden gewonnen. ${ }^{[160]}$ Alle Umsetzungen mit Übergangsmetallkomplexen und Organometallverbindungen wurden unter Sauerstoff- und Feuchtigkeitsausschluss durchgeführt. - Alle im Hauptteil beschriebenen und nicht im Experimentellen Teil aufgelisteten Verbindungen sind einschließlich ihrer Synthese und spektralen Daten in der 
Literatur beschrieben. Nicht gesondert aufgeführte Chemikalien sind kommerziell erhältlich und wurden ohne weitere Reinigung eingesetzt.

\section{Darstellung der Verbindungen}

\subsection{Synthese und Reinigung der verwendeten Reagenzien}

Sulfurylchloridfluorid $\left(\mathrm{SO}_{2} \mathrm{ClF}\right):^{[161]}$ Unter einer Schutzgasatmosphäre wurden $154 \mathrm{~g}$ wasserfreies Natriumfluorid $(3.67 \mathrm{~mol})$ in einem Kolben mit Tropftrichter und Destillationsbrücke in $250 \mathrm{ml}$ frisch destilliertem Acetonitril suspendiert und intensiv gerührt. Der Vorlagekolben wurde auf $-70{ }^{\circ} \mathrm{C}$ abgekühlt und über den Tropftrichter wurden dann $60 \mathrm{ml}$ Sulfurylchlorid $(0.74 \mathrm{~mol})$ innerhalb von einer Stunde zugetropft, wobei sich das Reaktionsgemisch schwach erwärmte. Nach beendetem Zutropfen wurde die Reaktionsmischung zunächst auf etwa $40-50{ }^{\circ} \mathrm{C}$ erwärmt bis ein hellgelber Vorlauf im Vorlagekolben kondensierte. Sobald $\mathrm{SO}_{2} \mathrm{ClF}$ als farblose Flüssigkeit kondensierte, wurde der Vorlagekolben gewechselt und die Temperatur langsam auf 60 bis $70^{\circ} \mathrm{C}$ erhöht, so dass die Kondensationsgeschwindigkeit des $\mathrm{SO}_{2} \mathrm{ClF}$ bis zum Ende der Reaktion konstant blieb. Die Badtemperatur sollte dabei $70{ }^{\circ} \mathrm{C}$ nicht überschreiten, um die Zersetzung von $\mathrm{SO}_{2} \mathrm{Cl}_{2}$ in $\mathrm{SO}_{2}$ und $\mathrm{Cl}_{2}$ gering zu halten. Das rohe $\mathrm{SO}_{2} \mathrm{ClF}$ wurde im Hochvakuum sorgfältig entgast und ein Vorlauf von etwa 5-6 $\mathrm{ml}$ abkondensiert. Die restliche Lösung wurde wiederholt auf $\mathrm{SbF}_{5}$ kondensiert $\left(0.5 \mathrm{ml} \mathrm{SbF}\right.$ pro $\left.25 \mathrm{ml} \mathrm{SO} \mathrm{SlF}_{2}\right)$, bis kein - aus $\mathrm{SbF}_{5} \cdot \mathrm{SO}_{2}$ bestehender - weißer Niederschlag mehr ausfiel und die Lösung klar und farblos blieb. Anschließend wurde das $\mathrm{SO}_{2} \mathrm{ClF}$ auf wasserfreies Kaliumfluorid kondensiert und über Nacht gerührt, um Spuren von $\mathrm{HF}$ zu entfernen. Zur Lagerung wurde das so gewonnene $\mathrm{SO}_{2} \mathrm{ClF}$ in ein Vorratsgefäß mit Teflonhahn umkondensiert. Vor der Verwendung zur Darstellung von Carbokationen wurde das $\mathrm{SO}_{2} \mathrm{ClF}$ in ein kalibriertes $10 \mathrm{ml}$-Gefäß mit Teflonhahn umkondensiert und am Hochvakuum nochmals sorgfältig entgast. Reines $\mathrm{SO}_{2} \mathrm{ClF}$ ist eine klare, farblose Flüssigkeit von unangenehm stechendem Geruch. Sdp. $7.1^{\circ} \mathrm{C}$, Schmp. $-124^{\circ} \mathrm{C} .{ }^{[6 \mathrm{~d}]}$ 
Sulfurylfluorid $\left(\mathrm{SO}_{2} \mathrm{~F}_{2}\right)$ : Handelsübliches Sulfurylfluorid („Vikane“von Dow Chemicals bzw. von der Kalichemie) wurde direkt aus der Gasbombe in ein auf $-120^{\circ} \mathrm{C}$ gekühltes kalibriertes Spitzbodengefäß kondensiert und im Hochvakuum bis zur Vakuumkonstanz entgast. Reines $\mathrm{SO}_{2} \mathrm{~F}_{2}$ ist bei etwa $-100{ }^{\circ} \mathrm{C}$ eine klare, farblose, leichtbewegliche Flüssigkeit und bei Raumtemperatur ein farbloses, stechend riechendes Gas. Sdp. $-55.4{ }^{\circ} \mathrm{C}, \mathrm{Schmp} .-136{ }^{\circ} \mathrm{C} .{ }^{[6 \mathrm{~d}]}$

Antimonpentafluorid $\left(\mathrm{SbF}_{5}\right)$ : Handelsübliches $\mathrm{SbF}_{5} \quad$ (ACROS, ALDRICH oder FLUOROCHEM) wurde in einer trockenen Apparatur, bestehend aus einem $100 \mathrm{~mL}-$ Einhalskolben mit NS 14.5, einem nicht an die Wasserleitung angeschlossenem Rückflußkühler und einem, mit Blaugel gefüllten, Trockenrohr ( $\left.\mathrm{kein} \mathrm{CaCl}_{2}\right)$ mit Gasableitung in den Abzug, ohne zu rühren 5-6h zum Sieden erhitzt, bis eine zähflüssige Konsistenz erreicht wurde. Das Erhitzen geschah im Luftbad eines Heizpilzes, wobei der $\mathrm{SbF}_{5}-\mathrm{Kolben}$ gerade nicht auf dem Boden des Heizpilzes aufsitzen sollte. Anschließend wurde über eine Destillationsbrücke mit senkrechtem Ablaufrohr, Trockenrohr und ohne Wasserkühlung in einen Vorlagekolben mit zusätzlichem Teflonhahn destilliert. Das $\mathrm{SbF}_{5}$ sollte farblos und von honigartiger Konsistenz in den Kolben abfließen. Alle Schliffe der Apparaturen wurden mit Teflonmanschetten abgedichtet. Das destillierte $\mathrm{SbF}_{5}$ wurde im Hochvakuum sorgfältig entgast, zweimal umkondensiert und in einem Vorratsgefäß mit Teflonhahn aufbewahrt. Vor der Verwendung zur Darstellung der Carbokationen wurde $\mathrm{SbF}_{5}$ in ein kalibriertes 10 ml-Gefäß mit Teflonhahn kondensiert und im Hochvakuum nochmals sorgfältig entgast. $\mathrm{SbF}_{5}$ ist eine klare, hochviskose Flüssigkeit von extrem hoher Reaktivität. Sdp. $142.7^{\circ} \mathrm{C}$, Schmp. $7^{\circ} \mathrm{C} .{ }^{[6 \mathrm{c}]}$

\subsection{Carbokationen-Präparation und Tieftemperatur-NMR-Messungen}

Allgemeine Arbeitsvorschrift zur Präparation von Carbokationen durch das HochvakuumMatrix-Cokondensationsverfahren (AAV 1): Der Glasreaktor (Abbildung 26) wurde über 
Nacht am Ölpumpenvakuum (ca. $10^{-2}$ mbar) evakuiert. In einem kalibrierten Glasgefäß mit Teflonhahn wurden $\mathrm{SO}_{2} \mathrm{ClF}$ und $\mathrm{SO}_{2} \mathrm{~F}_{2}$ in einem kalibrierten Spitzbodengefäß am Vakuumrechen bis zur Vakuumkonstanz entgast. Die Kationenvorstufe (ca. 50-150 mg) wurde in wenig Dichlormethan gelöst und mittels einer Kanüle durch den geöffneten Hahn 2 im Argongegenstrom auf dem Boden des Reaktors vorgelegt. Das Lösungsmittel wurde anschließend im Vakuum entfernt. An Hahn 3 wurde das kalibrierte Gefäß (mit Teflonhahn) mit $\mathrm{SbF}_{5}$ angebracht. Die Apparatur, alle Lösungsmittel und die Vorstufe wurden dann an der Quecksilber-Diffusionspumpe entgast, bis ein konstantes Vakuum von mindestens $10^{-5}$ mbar erreicht war. Danach wurde $1 \mathrm{ml} \mathrm{SO} \mathrm{SlF}_{2} \mathrm{ClF}$ auf den mit flüssigem Stickstoff gekühlten Boden kondensiert und Hahn 1 geschlossen. Die Kationenvorstufe wurde dann bei Raumtemperatur durch Schwenken im $\mathrm{SO}_{2} \mathrm{ClF}$ gelöst und anschließend wieder tiefgefroren. Der obere, siphonartig gebogene Teil des Glasrohrs, an den das 10mm-NMR-Röhrchen angeschmolzen war, wurde mit Verbandmull umwickelt. Der Boden der Apparatur wurde wieder in flüssigem Stickstoff gekühlt und nacheinander wurden und $0.7 \mathrm{ml} \mathrm{SO} \mathrm{SO}_{2}$ und $1 \mathrm{ml} \mathrm{SO} \mathrm{SO}_{2} \mathrm{ClF}$ auf den Reaktorboden kondensiert. In der Ummantelung der rechten Düse des Reaktors wurde Wasser durch elektrisch geheizte Drähte auf etwa $40^{\circ} \mathrm{C}$ erwärmt, wodurch ein Auskondensieren von $\mathrm{SbF}_{5}$ in der Düse verhindert wurde. Anschließend wurden auf die eingefrorene Kationen-

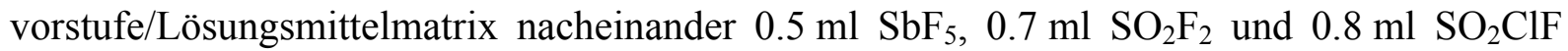
aufkondensiert. Das Wasser wurde aus den Ummantelungen abgesaugt und die Ummantelungen zur Entfernung von Wasserresten mit Methanol gespült. Das Gefäß für $\mathrm{SbF}_{5}$ wurde nach Schließen der Hähne 1 bis 3 vom Reaktor entfernt, der Reaktor vom Vakuumrechen abgenommen und in ein auf $-140^{\circ} \mathrm{C}$ gekühltes Kältebad getaucht. Zur späteren Kühlung des angeschmolzenen NMR-Röhrchens wurde ein Dewargefäß mit derselben Kältemischung bereitgestellt. 


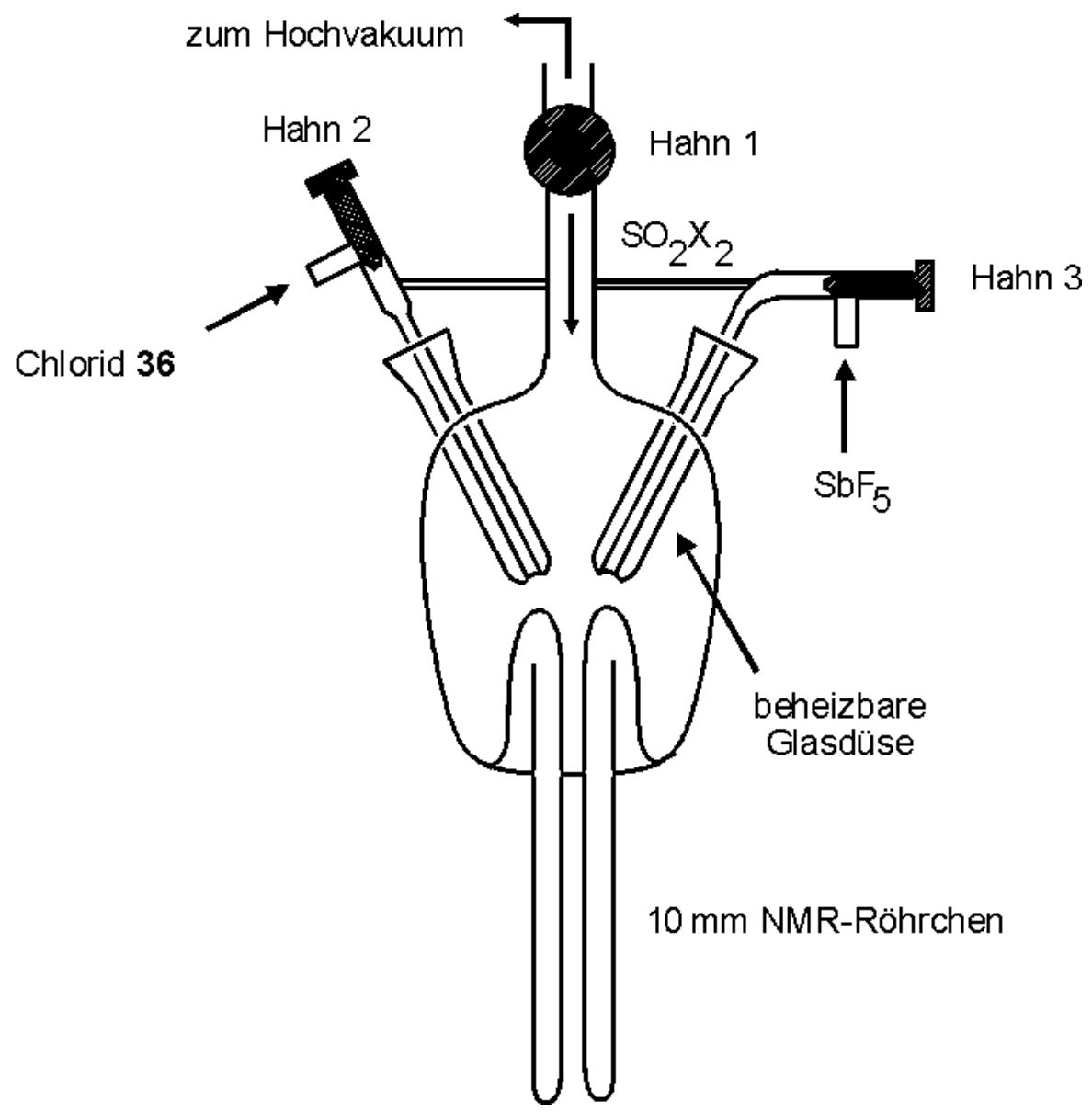

Abbildung 26. Apparat zur Darstellung von Carbokationen unter ,stable cation conditions“.

Der Reaktorinhalt wurde durch gelegentliches vorsichtiges Umschwenken des Reaktors aufgetaut. Als sich die Reaktionslösung verflüssigte, wurde das angeschmolzene NMRRöhrchen in die vorbereitete Kältemischung eingetaucht und der Verbandmull mit zähflüssiger Kältemischung bestrichen. Die Reaktionslösung wurde dann rasch aus dem Reaktor in das NMR-Röhrchen umgegossen. Ein 10 mm-NMR-Röhrchen sollte, entsprechend einer Füllmenge von ca. $4 \mathrm{ml}$, etwa 4.5 bis $5 \mathrm{~cm}$ hoch gefüllt sein. Dies entspricht bei einer Vorstufenmenge von etwa $0.5 \mathrm{mmol}$ einer etwa 0.17 molaren Carbokationenlösung. Röhrchen und Reaktor wurden sofort wieder gekühlt und der Verbandmull noch einige Zeit mit Kältemischung bestrichen, damit die Lösung im gebogenen Teil des Reaktors und im oberen Teil des NMR-Röhrchens abfließen konnte, ohne sich zu zersetzen. Der Verbandmull wurde entfernt, der Reaktor wieder an den Vakuumrechen angeschlossen und NMR-Röhrchen und Reaktorboden wurden umgehend durch vorbereitete Dewar-Gefäße mit flüssigem Stickstoff 
gekühlt. Anschließend wurde der Reaktor vollständig evakuiert, das eingefrorene NMRRöhrchen im Hochvakuum abgeschmolzen und bis zur NMR-Messung in flüssigem Stickstoff aufbewahrt.

\section{Tieftemperatur-NMR-Spektroskopie}

Die NMR-Spektren der Carbokationenlösungen wurden mit einem Bruker DRX 400 Spektrometer $\left({ }^{1} \mathrm{H}: 400.13 \mathrm{MHz},{ }^{13} \mathrm{C}: 100.63 \mathrm{MHz}\right.$, mit XWIN-NMR Suite 3.1) aufgezeichnet. Die Tieftemperatur- ${ }^{1} \mathrm{H}$ - und $-{ }^{13} \mathrm{C}-\mathrm{NMR}-$ Spektren wurden mit Hilfe eines $10 \mathrm{~mm}$ DualProbenkopfes mit ${ }^{1}$ H-Entkoppler-, ${ }^{13} \mathrm{C}$-Messspule und ${ }^{19}$ F-Lock-Kanal durchgeführt. Der Probenkopf wurde zur Messung der Carbokationenlösungen manuell auf die jeweilige Probe abgestimmt und geshimmt. Die Pulswinkel un die Transmitterleistung der verwendeten Hochleistungs- bzw. Entkopplerpulse wurden mit den in der Literatur bekannten Standardmethoden bestimmt. ${ }^{[162]}$ Die Temperatur der Carbokationenlösung im NMR-Gerät wurde über die Temperaturabhängigkeit der ${ }^{13} \mathrm{C}$-Verschiebung der Kohlenstoffatome C-3 und C-4 einer Kapillarprobe von reinem 2-Chlorbutan in $\mathrm{SO}_{2} \mathrm{ClF}$ bestimmt, das als Locksubstanz diente. Mit Hilfe der empirischen Funktion $\mathrm{T}=9296.16309-975.56567 \Delta \delta+24.90276 \Delta \delta^{2}$ kann die Temperatur auf $\pm 1.3{ }^{\circ} \mathrm{C}$ genau ermittelt werden. ${ }^{[163]}$ In die Formel geht die Verschiebungsdifferenz $\Delta \delta$ der C-Atome 3 und 4 ein. Während der Tieftemperatur-NMRMessungen wurde das Spektrometer mit kaltem Stickstoffgas gespült. Die Abkühlung erfolgte in Schritten von $20 \mathrm{~K}$, wonach jeweils die Abstimmung des Probenkopfes, die Homogenität des Magnetfeldes und die tatsächliche Temperatur im Probenraum überprüft wurden.

\subsection{Darstellung der Verbindungen aus Teil B Kapitel 3}

Allgemeine Arbeitsvorschrift zur Gewinnung von ${ }^{13} \mathrm{CO}_{2}$ aus ${ }^{13} \mathrm{C}$-Bariumcarbonat bzw. unmarkiertem $\mathrm{CO}_{2}$ aus Calciumcarbonat (AAV 2): In einem $100 \mathrm{ml}$ Schlenkkolben wurde die 
entsprechende Stoffmenge an Carbonat eingewogen, der Kolben mit einem Septum verschlossen und der Kolben evakuiert. Unter Eiskühlung wurden mittels einer Spritze $50 \mathrm{ml}$ konz. Schwefelsäure (Überschuss) durch das Septum langsam auf das Carbonat getropft. Das lebhaft entstehende $\mathrm{CO}_{2}$ wurde dann unter Kühlung mit flsg. $\mathrm{N}_{2}$ in den entsprechenden Reaktionskolben sublimiert.

Allgemeine Arbeitsvorschrift zur Gewinnung einer etherischen Lösung von Diazomethan aus $N$-Methyl-N-nitrosoharnstoff (AAV 3): Zu einer intensiv gerührten Suspension aus 40\%iger wässriger Kaliumhydroxid-Lösung (ca. $25 \mathrm{ml}$ pro $5 \mathrm{~g} N$-Methyl- $N$-nitrosoharnstoff) und Diethylether (für eine ca. $1 \mathrm{M}$ etherische Diazomethan-Lösung) in einem $100 \mathrm{ml}$ Erlenmeyerkolben wurde unter Eisbadkühlung portionsweise die entsprechende Menge an $\mathrm{N}$ Methyl- $N$-nitrosoharnstoff (für eine ca. $1 \mathrm{M}$ etherische Diazomethan-Lösung) so zugegeben, dass die Temp. $5{ }^{\circ} \mathrm{C}$ nicht überstieg und nachfolgend wurde noch 15 min gerührt. Die etherische Phase (Diazomethanlösung) wurde anschließend weitestgehend dekantiert, die restliche Lösung abpipettiert und über festen Kaliumhydroxidplätzchen für 30 min getrocknet (im Kühlschrank, Flasche ohne Schliff mit Korkstopfen).

$\left[1{ }^{1}-{ }^{13} C\right]$-Cyclopropancarbonsäure: In einem $100 \mathrm{ml}$ Schlenkkolben wurde auf eine tiefgefrorene Lösung (flsg. $\mathrm{N}_{2}$ ) von Cyclopropylmagnesiumbromid in $\nabla^{13} \mathrm{CO}_{2} \mathrm{H}$ Tetrahydrofuran - gewonnen durch tropfenweise Zugabe von $4.03 \mathrm{~g}$ Bromcyclopropan $(33.3 \mathrm{mmol}) \quad \mathrm{zu}$ einer Suspension von $1.62 \mathrm{~g}$ Magnesiumspänen $(66.6 \mathrm{mmol})$ in $40 \mathrm{ml}$ trockenem THF und nachfolgendes 30minütiges Rühren bei Raumtemp. - $30.3 \mathrm{mmol}{ }^{13} \mathrm{C}$-markiertes Kohlenstoffdioxidgas (aus $6.00 \mathrm{~g}{ }^{13} \mathrm{C}$ $\mathrm{BaCO}_{3}$ nach AAV 2) kondensiert. Nachdem die Mischung auf $-78{ }^{\circ} \mathrm{C}$ erwärmt wurde, entfernte man das Aceton/Trockeneis-Bad und ließ die Reaktionslösung $12 \mathrm{~h}$ rühren, wobei diese auftaute. Um einen etwaigen Druckausgleich zu gewähren, war der Reaktionsraum immer mit einem an der Apparatur befestigten Ballon in Kontakt. Nach Zugabe von $20 \mathrm{ml} 3 \mathrm{~N}$ Salzsäurelösung und $20 \mathrm{ml}$ gesättigte $\mathrm{NaCl}$-Lösung wurde die abgetrennte wässrige Phase mit 
$4 \times 20 \mathrm{ml}$ Diethylether extrahiert, die vereinigten organischen Phasen über $\mathrm{MgSO}_{4}$ getrocknet und die Lösungsmittel bei normalem Druck abdestilliert. Dabei blieben $2.40 \mathrm{~g}$ einer gelblichen Flüssigkeit zurück, die überwiegend aus $\left[1{ }^{1}-{ }^{13} \mathrm{C}\right]$-markierter Cyclopropancarbonsäure bestand, jedoch noch THF und Essigsäure enthielt, aber ohne weitere Reinigung in der nächsten Reaktion eingesetzt wurden. - Charakteristische NMRspektroskopische Daten: ${ }^{13} \mathrm{C}-\mathrm{NMR}\left(62.9 \mathrm{MHz}, \mathrm{CDCl}_{3}, \mathrm{DEPT}\right): \delta=12.8\left(+, \mathrm{d},{ }^{1} J_{\mathrm{CC}}=71.9 \mathrm{~Hz}\right.$, C-1), 181.3 ( $\mathrm{C}_{\text {quart }}, \mathrm{C}-1$ ', Satteliten sichtbar).

[2,3- $\left.{ }^{13} C_{2}\right]$ - und $\left[1,2,3-{ }^{13} C_{3}\right]$-Propinsäure (69a) und (69b): Kommerziell erhältliches [1,2-

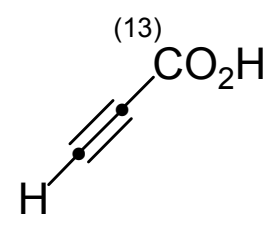

$\left.{ }^{13} \mathrm{C}\right]$-markiertes Acetylen (500 ml-Kolben mit Glassiegel als Sollbruchstelle, Eurisotop/Cambridge Isotopes Inc., ca. $21 \mathrm{mmol}$ ) wurde mit Hilfe von flsg. $\mathrm{N}_{2}$ auf den Boden eines $100 \mathrm{ml}$ Schlenkkolbens kondensiert und auf den Schlenkhahn wurde ein Ballon gesetzt, um einen etwaigen Druckausgleich zu gewährleisten. Unter Kühlung mittels flsg. $\mathrm{N}_{2}$ wurden $30 \mathrm{ml}$ trockenes Tetrahydrofuran langsam durch das Septum zugespritzt und nachfolgend $7.0 \mathrm{ml}$ einer Lösung von Methylmagnesiumchlorid in THF (3.15 M, Fluka, 22 mmol). Nachdem die gefrorene Mischung mit Hilfe eines Aceton/Trockeneis-Bades auf $-78^{\circ} \mathrm{C}$ erwärmt wurde, entfernte man dieses und ließ die Reaktionslösung $6 \mathrm{~h}$ rühren, wobei diese auftaute. Der Fortgang der Reaktion konnte anhand der Methanentwicklung im Druckausgleichsballon verfolgt werden. Die so hergestellte Lösung von Ethinylmagnesiumchlorid in THF wurde erneut mit flsg. $\mathrm{N}_{2}$ tiefgefroren und im Falle von 69a wurden 1.1 Äquiv. unmarkiertes $\mathrm{CO}_{2}$ (nach AAV 1 aus $2.3 \mathrm{~g} \mathrm{CaCO}_{3}, 23 \mathrm{mmol}$ ) auf die gefrorene Oberfläche sublimiert, für 69b wurden 1.05 Äquiv. ${ }^{13} \mathrm{C}$-markiertes $\mathrm{CO}_{2}$ - hergestellt nach AAV 2 aus $4.4 \mathrm{~g}{ }^{13} \mathrm{C}-\mathrm{BaCO}_{3}$, $22 \mathrm{mmol}$ - verwendet. Nachdem die gefrorene Mischung mit Hilfe eines Aceton/TrockeneisBades auf $-78^{\circ} \mathrm{C}$ erwärmt wurde, entfernte man dieses und ließ die Reaktionslösung $12 \mathrm{~h}$ rühren, wobei diese auftaute. Nach Zugabe von $20 \mathrm{ml} 3 \mathrm{~N}$ wässriger Salzsäurelösung und $20 \mathrm{ml}$ gesättigter NaCl-Lösung wurde die abgetrennte wässrige Phase mit $4 \times 20 \mathrm{ml}$ Diethylether extrahiert, die vereinigten organischen Phasen über $\mathrm{MgSO}_{4}$ getrocknet und die 
Lösungsmittel bei normalem Druck abdestilliert, wobei man $1.9 \mathrm{~g}$ bzw. $2.1 \mathrm{~g}$ der rohen (noch wenig THF und HOAc enthaltenden), aber ausreichend reinen $\left[2,3-{ }^{13} \mathrm{C}_{2}\right]$ - bzw. $\left[1,2,3-{ }^{13} \mathrm{C}_{3}\right]$ markierten Propinsäure als gelbe Flüssigkeit erhielt, die jeweils gleich weiter zur Hydrierung eingesetzt wurde.

[2,3- $\left.{ }^{13} C_{2}\right]$-Propinsäure (69a): charakteristische NMR-spektroskopische Daten: ${ }^{1} \mathrm{H}-\mathrm{NMR}$ $\left(250 \mathrm{MHz}, \mathrm{CDCl}_{3}\right): \delta=2.87\left(\mathrm{~d},{ }^{1} J_{\mathrm{CH}}=10.3 \mathrm{~Hz}, 1 \mathrm{H}, 3-\mathrm{H}\right),{ }^{13} \mathrm{C}-\mathrm{NMR}\left(62.9 \mathrm{MHz}, \mathrm{CDCl}_{3}\right): \delta=$ 74.9, 75.3 (s, 2 C, C-2,3). $-{ }^{13} \mathrm{C}-\mathrm{NMR}\left(62.9 \mathrm{MHz}, \mathrm{C}_{6} \mathrm{D}_{6}\right): \delta=75.9,75.2,75.1$ (d, $\left.2 \mathrm{C}, \mathrm{C}-2,3\right)$.

[1,2,3- $\left.{ }^{13} C_{3}\right]$-Propinsäure (69b): charakteristische NMR-spektroskopische und massenspektrometrische Daten: ${ }^{13} \mathrm{C}-\mathrm{NMR}\left(62.9 \mathrm{MHz}, \mathrm{CDCl}_{3}\right): \delta=74.2(\mathrm{~s}, \mathrm{C}-3), 75.3\left(\mathrm{~d},{ }^{1} J_{\mathrm{CC}}=24.1 \mathrm{~Hz}\right.$, C-2), $153.8\left(\mathrm{dd},{ }^{1} J_{\mathrm{CC}}=136.5 \mathrm{~Hz},{ }^{2} J_{\mathrm{CC}}=24.1 \mathrm{~Hz}, \mathrm{C}-1\right) .-\mathrm{MS} \quad(\mathrm{EI}, 70 \mathrm{eV}), \mathrm{m} / z \quad(\%)$ : 75/74/73/72/71 (3/5/69/64/58) $\left[\mathrm{M}^{+}\right]$.

[2,3- $\left.{ }^{13} C_{2}\right]$ - und $\left[1,2,3-{ }^{13} C_{3}\right]$-Acrylsäure (70a) und (70b): Eine Lösung der jeweiligen rohen ${ }^{(13)} \mathrm{CO}_{2} \mathrm{H} \quad$ zweifach bzw. dreifach ${ }^{13} \mathrm{C}$-markierten Propinsäure 69a bzw. 69b wurde in $5 \mathrm{ml}$ Methanol zusammen mit 5 mol-\% Lindlar-Katalysator (5\% Pd auf $\mathrm{BaSO}_{4}$, Aldrich) in einer Wasserstoffatmosphäre bei normalem Druck intensiv gerührt bis jeweils genau 1.00 Äquiv. $\mathrm{H}_{2}$ aufgenommen worden war. Der Fortgang der Hydrierung konnte durch ${ }^{13} \mathrm{C}$-NMR-spektroskopische Untersuchungen eines Aliquots der Reaktionsmischung verfolgt werden, hieraus wurde zusammen mit dem $\mathrm{H}_{2}$-Konsum auch die Ausbeute bestimmt. Danach wurden die jeweiligen Lösung über ein Schicht Celite $(1 \times 0.5 \mathrm{~cm}$, zuoberst $)$ und Kieselgel $(1 \times 0.5 \mathrm{~cm}$, zuunterst $)$ mit $\mathrm{Et}_{2} \mathrm{O}$ als Eluent filtriert und die Lösungsmittel bei normalem Druck entfernt. Man erhielt 992 mg (13.4 mmol, 64\% bezogen auf Acetylen) bzw. $1.09 \mathrm{~g}$ (14.6 mmol, 69\% bezogen auf Acetylen) der rohen (noch wenig $\mathrm{MeOH}$ enthaltenden), aber ausreichend reinen $\left[2,3-{ }^{13} \mathrm{C}_{2}\right]-$ bzw. $\left[1,2,3-{ }^{13} \mathrm{C}_{3}\right]$-markierten Acrylsäure als gelbliche Flüssigleiten, die jeweils gleich weiter zur Veresterung und Cyclopropanierung eingesetzt wurden. 
[2,3- $\left.{ }^{13} C_{2}\right]$-Acrylsäure (70a): charakteristische NMR-spektroskopische Daten: ${ }^{13} \mathrm{C}-\mathrm{NMR}$ $\left(62.9 \mathrm{MHz}, \mathrm{CDCl}_{3}\right): \delta=128.1\left(\mathrm{~d},{ }^{1} J_{\mathrm{CC}}=68.1 \mathrm{~Hz}, \mathrm{C}-2\right), 132.2\left(\mathrm{~d},{ }^{1} J_{\mathrm{CC}}=68.1 \mathrm{~Hz}, \mathrm{C}-3\right)$.

[1,2,3- $\left.{ }^{13} C_{3}\right]$-Acrylsäure (70b): charakteristische NMR-spektroskopische und massenspektrometrische Daten: ${ }^{13} \mathrm{C}-\mathrm{NMR} \quad\left(62.9 \mathrm{MHz}, \quad \mathrm{CDCl}_{3}\right): \delta=128.4 \quad\left(\mathrm{dd}, \quad{ }^{1} J_{\mathrm{CC}}=69.4 \mathrm{~Hz}\right.$, $\left.{ }^{1} J_{\mathrm{CC}}=69.0 \mathrm{~Hz}, \quad \mathrm{C}-2\right), \quad 131.1 \quad\left(\mathrm{dd}, \quad{ }^{1} J_{\mathrm{CC}}=69.0 \mathrm{~Hz}, \quad{ }^{2} J_{\mathrm{CC}}=1.4 \mathrm{~Hz}, \quad \mathrm{C}-3\right), \quad 168.1 \quad(\mathrm{dd}$, $\left.{ }^{1} J_{\mathrm{CC}}=69.4 \mathrm{~Hz},{ }^{2} J_{\mathrm{CC}}=1.4 \mathrm{~Hz}, \mathrm{C}-1\right) .-\mathrm{MS}(\mathrm{EI}, 70 \mathrm{eV}), m / z(\%): 78 / 77 / 76 / 75 / 74 / 73 / 72 / 71$ $(1 / 6 / 4 / 100 / 8 / 1 / 2 / 2)\left[\mathrm{M}^{+}\right]$.

$\left[1{ }^{,-13}\right.$ C]-Cyclopropancarbonsäuremethylester (71a): Zu einer intensiv gerührten Lösung von

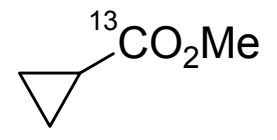
$2.40 \mathrm{~g}$ rohen $\left[1{ }^{\prime}-{ }^{13} \mathrm{C}\right]$-Cyclopropancarbonsäure in $30 \mathrm{ml}$ Diethylether wurde bei $0{ }^{\circ} \mathrm{C}$ solange eine etherische Diazomethan-Lösung (nach AAV 3 aus $3.75 \mathrm{~g} N$-Methyl- $N$-nitrosoharnstoff, $36.4 \mathrm{mmol}$ ) getropft bis die Gasentwicklung beendet war. Daraufhin wurden die Lösungsmittel bei normalem Druck abdestilliert und der Rückstand destillativ wobei man $1.38 \mathrm{~g}$ des $\left[1\right.$ ' $\left.{ }^{13} \mathrm{C}\right]$-markierten Cyclopropancarbonsäuremethylesters (71a) (13.6 mmol, 45\% bezogen auf ${ }^{13} \mathrm{CO}_{2}$ ) als farblose Flüssigkeit (Sdb. 123-126 $\left.{ }^{\circ} \mathrm{C}\right)$ erhielt. - Charakteristische NMR-spektroskopische und massenspektrometrische Daten: ${ }^{1} \mathrm{H}-\mathrm{NMR}\left(250 \mathrm{MHz}, \mathrm{CDCl}_{3}\right): \delta=3.67\left(\mathrm{~d},{ }^{3} J_{\mathrm{CH}}=3.9 \mathrm{~Hz}, 3 \mathrm{H}\right.$, OMe). $-{ }^{13} \mathrm{C}-\mathrm{NMR}\left(62.9 \mathrm{MHz}, \mathrm{CDCl}_{3}, \mathrm{DEPT}\right): \delta=12.6\left(+, \mathrm{d},{ }^{1} J_{\mathrm{CC}}=76.6 \mathrm{~Hz}, \mathrm{C}-1\right), 175.4$ (C quart $_{1}$ C-1'). - MS (EI, $\left.70 \mathrm{eV}\right), m / z(\%): 102 / 101 / 100 / 99(2 / 22 / 100 / 1)\left[\mathrm{M}^{+}\right]$.

[1,2- $\left.{ }^{13} C_{2}\right]$ - und $\left[1,1^{1}, 2-{ }^{13} C_{3}\right]$-Cyclopropancarbonsäuremethylester (71b) und (71c): Die rohen, zweifach bzw. dreifach ${ }^{13} \mathrm{C}$-markierten Acrylsäuren 70a und 70b $\nabla^{(13)} \mathrm{CO}_{2} \mathrm{Me}$ wurden jeweils in wasserfreiem Dichlormethan (1.0 Volumenteile) gelöst und bei $0{ }^{\circ} \mathrm{C}$ intensiv gerührt. Dazu wurde jeweils solange eine Lösung von Diazomethan (nach AAV 3, insgesamt 5.0 Äquiv.) in Diethylether (3 Volumenteile) getropft bis die Gasentwicklung nachließ und die Reaktionslösung leicht gelb gefärbt war. 
Dann gab man je 10 mol-\% Palladiumacetat hinzu und tropfte die restliche DiazomethanLösung bei $0{ }^{\circ} \mathrm{C}$ unter Rühren hinzu. Nach beendeter Zugabe wurde noch je $3 \mathrm{~h}$ bei dieser Temp. gerührt und die Reaktionsmischung durch eine dünne Schicht Celite $(1 \times 0.5 \mathrm{~cm}$, zuoberst $)$ und Kieselgel $(1 \times 0.5 \mathrm{~cm}$, zuunterst $)$ mit $\mathrm{Et}_{2} \mathrm{O}$ als Eluent filtriert, die Lösungsmittel bei normalem Druck abdestilliert und der Rückstand destillativ gereinigt. Man erhielt $1.25 \mathrm{~g}$ (12.2 mmol, 91\%) bzw. $1.40 \mathrm{~g}(13.6 \mathrm{mmol}, 93 \%)$ der $\left[1,2-{ }^{13} \mathrm{C}_{2}\right]-$ bzw. $\left[1,1^{\prime}, 2-{ }^{13} \mathrm{C}_{3}\right]-$ markierten Cyclopropancarbonsäuremethylester als farblose Flüssigkeiten (Sdb. $123-127^{\circ} \mathrm{C}$ ).

[1,2- $\left.{ }^{13} C_{2}\right]$-Cyclopropancarbonsäuremethylester (71b): charakteristische NMR-spektroskopische Daten: ${ }^{13} \mathrm{C}-\mathrm{NMR}\left(62.9 \mathrm{MHz}, \mathrm{CDCl}_{3}\right): \delta=8.3\left(\mathrm{~d},{ }^{1} J_{\mathrm{CC}}=10.6 \mathrm{~Hz}, \mathrm{C}-3\right), 12.6(\mathrm{~d}$, $\left.{ }^{1} J_{\mathrm{CC}}=10.6 \mathrm{~Hz}, \mathrm{C}-2\right)$.

[1,1',2- $\left.{ }^{13} C_{2}\right]$-Cyclopropancarbonsäuremethylester (71c): charakteristische NMR-spektroskopische und massenspektrometrische Daten: ${ }^{1} \mathrm{H}-\mathrm{NMR}\left(250 \mathrm{MHz}, \mathrm{CDCl}_{3}\right): \delta=3.66(\mathrm{~d}$, $\left.{ }^{3} J_{\mathrm{CH}}=3.9 \mathrm{~Hz}, 3 \mathrm{H}, \mathrm{OMe}\right) .-{ }^{13} \mathrm{C}-\mathrm{NMR}\left(62.9 \mathrm{MHz}, \mathrm{CDCl}_{3}\right): \delta=8.3\left(\mathrm{dd},{ }^{1} J_{\mathrm{CC}}=10.7 \mathrm{~Hz}\right.$, $\left.{ }^{2} J_{\mathrm{CC}}=1.3 \mathrm{~Hz}, \mathrm{C}-3\right), 12.7\left(\mathrm{~d},{ }^{1} J_{\mathrm{CC}}=76.8 \mathrm{~Hz},{ }^{1} J_{\mathrm{CC}}=10.7 \mathrm{~Hz}, \mathrm{C}-2\right), 175.4\left(\mathrm{dd},{ }^{1} J_{\mathrm{CC}}=76.8 \mathrm{~Hz}\right.$, $\left.{ }^{2} J_{\mathrm{CC}}=1.3 \mathrm{~Hz}, \mathrm{C}-1\right)$. - MS (EI, $\left.70 \mathrm{eV}\right), m / z$ (\%):104/103/102/101/100/99/98/97 (8/9/34/7/$15 / 8 / 13 / 4)\left[\mathrm{M}^{+}\right]$.

$\left[1-{ }^{13} C\right]-, \quad\left[1^{\prime}, 2^{\prime}-{ }^{13} C_{2}\right]-\quad$ und $\left[1,1,2{ }^{\prime}{ }_{-}^{13} C_{3}\right]-1,2,3,4,5$-Pentacyclopropylcyclopenta-1,3-dien
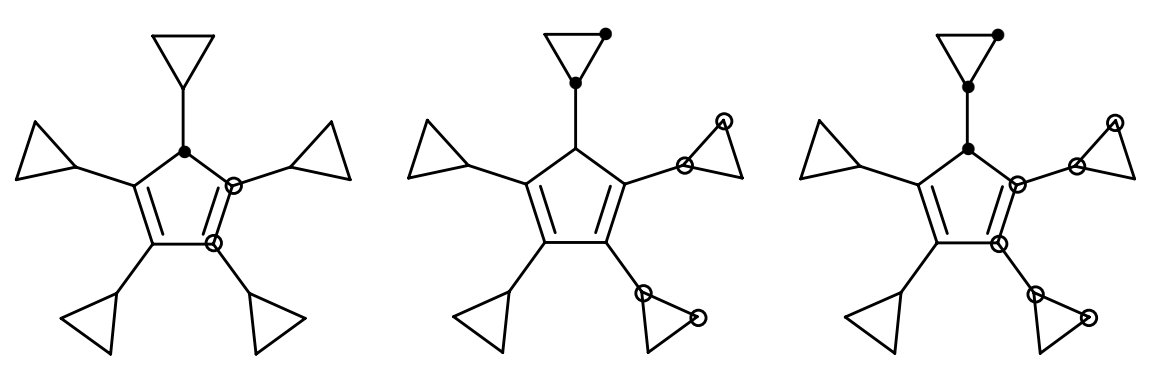

(15a), (15b) bzw.

(15c): Innerhalb von

$5 \mathrm{~h} \quad$ wurden $\quad 6.71 \mathrm{~g}$

Cer(III)chloridhepta-

hydrat $\quad(18.0 \mathrm{mmol})$

i. Vak. (0.1 mbar) durch Erhitzen auf $200{ }^{\circ} \mathrm{C}$ getrocknet. Anschließend ließ man unter Argon auf Raumtemperatur abkühlen, suspendierte das feinpulvrige $\mathrm{CeCl}_{3}$ in $300 \mathrm{ml}$ Tetrahydrofuran und rührte die weiße Suspension $1 \mathrm{~h}$ bei Raumtemperatur. In einem $100 \mathrm{ml}$ 
Zweihalskolben mit Rückflusskühler wurden $\mathrm{zu} 35.8 \mathrm{ml}$ einer $0.67 \mathrm{M}$ Lösung von Isobutylmagnesiumbromid in Diethylether $(24.0 \mathrm{mmol}) 2.68 \mathrm{~g}(25.2 \mathrm{mmol})$ Dicyclopropylethin (13) und $75 \mathrm{mg}$ Biscyclopentadienyltitanocendichlorid $(0.30 \mathrm{mmol})$ gegeben und die Mischung 30 min unter Rückfluss erhitzt. Die resultierende tiefgrüne Lösung tropfte man zu der Suspension von $\mathrm{CeCl}_{3}$ in THF und rührte die Mischung noch $1 \mathrm{~h}$. $\mathrm{Zu}$ dieser jeweils so hergestellten beigefarbenen Suspension wurden je $12.0 \mathrm{mmol}$ des entsprechenden Cyclopropylcarbonsäuremethylesters $(1.21 \mathrm{~g}, 1.23 \mathrm{~g}, 1.24 \mathrm{~g})$ gegeben, eine weitere Stunde gerührt und anschließend unter kräftigem Rühren mit $70 \mathrm{ml}$ einer Mischung von Wasser und Eisessig (6:1) versetzt und weitere $30 \mathrm{~min}$ gerührt. Die organische Phase wurde mit verdünnter wässriger $\mathrm{NaHCO}_{3}$-Lösung $(3 \times 700 \mathrm{ml})$ gewaschen und die vereinigten wässrigen Phasen mit Pentan $(3 \times 50 \mathrm{ml})$ extrahiert. Die vereinigten organischen Phasen wurden über $\mathrm{MgSO}_{4}$ getrocknet und das Lösungsmittel i. Vak. abdestilliert. Säulenchromatographie des Rohproduktes an $150 \mathrm{~g}$ Kieselgel (Säule $4 \times 30 \mathrm{~cm}$, Pentan) lieferte die Cyclopentadiene $\left(R_{\mathrm{f}}=0.45\right.$ in Hexan) als farblose Öle (15a: $2.22 \mathrm{~g}, 8.30 \mathrm{mmol}, 61 \%$; 15b: $1.74 \mathrm{~g}, 6.47 \mathrm{mmol}$, 53\%; 15c: $2.01 \mathrm{~g}, 7.48 \mathrm{mmol}, 55 \%)$.

$\left[1-{ }^{13} \mathrm{C}\right]-1,2,3,4,5-P e n t a c y c l o p r o p y l c y c l o p e n t a-1,3-d i e n \quad$ (15a): charakteristische NMRspektroskopische und massenspektrometrische Daten: ${ }^{13} \mathrm{C}-\mathrm{NMR}\left(62.9 \mathrm{MHz}, \mathrm{CDCl}_{3}, \mathrm{DEPT}\right)$ : $\delta=58.5(\mathrm{C}-5), 142.2,143.9(4 \mathrm{C}, \mathrm{C}-1,4, \mathrm{C}-2,3) .-\mathrm{MS}(\mathrm{EI}, 70 \mathrm{eV}), \mathrm{m} / z(\%): 271 / 270 / 269 /-$ 268/267/266 (1/2/16/100/4/1) $\left[\mathrm{M}^{+}\right]$.

[1',2 $\left.{ }^{\prime}{ }^{13} C_{2}\right]-1,2,3,4,5-P e n t a c y c l o p r o p y l c y c l o p e n t a-1,3-$ dien (15b): charakteristische NMRspektroskopische und massenspektrometrische Daten: ${ }^{13} \mathrm{C}-\mathrm{NMR}\left(62.9 \mathrm{MHz}, \mathrm{CDCl}_{3}\right): \delta=4.1$ (d, 2 C, $c$ Pr-C), 6.1 (d, 2 C, $c$ Pr-C), 6.2 (d, 2 C, $c$ Pr-C), 6.5 (d, 2 C, $c$ Pr-C), 7.8 (d, 2 C, $c$ PrC), 8.8 (d, 2 C, $c$ Pr-C), 9.8 (d, 2 C, $c$ Pr-C), 12.5 (d, $c$ Pr-C), Kopplungskonst. für diese $c$ Pr-C: ${ }^{1} J_{\mathrm{CC}}=13.3,12.8,12.4$ Hz. - MS (EI, $\left.70 \mathrm{eV}\right), m / z(\%): 272 / 271 / 270 / 269 / 268 / 267$ (2/3/4/18/$100 / 4)\left[\mathrm{M}^{+}\right]$. 
$\left[1,1,2{ }^{\prime}-{ }^{13} C_{3}\right]-1,2,3,4,5$-Pentacyclopropylcyclopenta-1,3-dien (15c): charakteristische NMRspektroskopische und massenspektrometrische Daten: ${ }^{13} \mathrm{C}-\mathrm{NMR}\left(62.9 \mathrm{MHz}, \mathrm{CDCl}_{3}\right): \delta=4.1$ (dd, 2 C, $c$ Pr-C, n. b.), 6.1 (dd, 2 C, $c$ Pr-C), 6.2 (dd, 2 C, $c$ Pr-C), 6.5 (dd, 2 C, $c$ Pr-C), 7.8 (dd, $2 \mathrm{C}, \quad c \operatorname{Pr}-\mathrm{C}), 8.8$ (dd, $2 \mathrm{C}, c \operatorname{Pr}-\mathrm{C}), 9.8$ (dd, $2 \mathrm{C}, c \operatorname{Pr}-\mathrm{C}), 12.5$ (dd, $c \operatorname{Pr}-\mathrm{C}, \quad \mathrm{n} . \mathrm{b})$, Kopplungskonst. für diese $c$ Pr-C: ${ }^{1} J_{\mathrm{CC}}=13.3,12.8,12.4 \mathrm{~Hz}, 58.5\left(\mathrm{dd},{ }^{1} J_{\mathrm{CC}}=41.8 \mathrm{~Hz}\right.$, ${ }^{2} J_{\mathrm{CC}}$ n. b., C-5), 142.2, 143.9 (dd, $\left.{ }^{1} J_{\mathrm{CC}}=59.8,57.4 \mathrm{~Hz},{ }^{2} J_{\mathrm{CC}}=2.0,1.5 \mathrm{~Hz}, 4 \mathrm{C}, \mathrm{C}-1,4, \mathrm{C}-2,3\right)$. - MS (EI, $70 \mathrm{eV}), m / z(\%): 273 / 272 / 271 / 270 / 269 / 268 / 267(1 / 1 / 2 / 17 / 100 / 6 / 1)\left[\mathrm{M}^{+}\right]$.

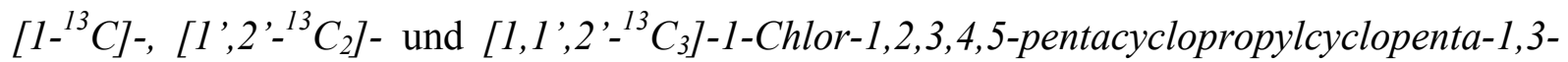
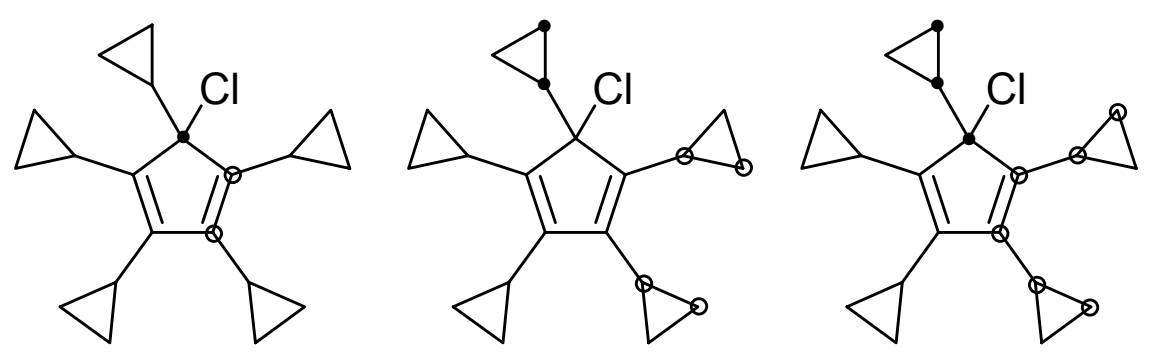
dien (36a), (36b) bzw. (36c): $\mathrm{Zu} \quad$ einer jeweiligen Lösung von $2.41 \mathrm{mmol}$ des ${ }^{13} \mathrm{C}$ markierten Pentacyclopropylcyclopentadiens in $30 \mathrm{ml}$ THF tropfte man bei $0{ }^{\circ} \mathrm{C}$ je $1.21 \mathrm{ml}$ einer $2.2 \mathrm{M}$ etherischen Methyllithiumlösung $(2.65 \mathrm{mmol})$ und rührte die Mischung $30 \mathrm{~min}$ bei Raumtemperatur. Diese jeweiligen Lösungen wurden erneut auf $0{ }^{\circ} \mathrm{C}$ abgekühlt, mit $644 \mathrm{mg}$ (4.82 mmol) $\mathrm{N}$-Chlorsuccinimid versetzt und $18 \mathrm{~h}$ bei Raumtemp. gerührt. Das Lösungsmittel wurde jeweils i. Vak. entfernt, der Rückstand in $30 \mathrm{ml}$ wasserfreiem Pentan aufgenommen und unter Feuchtigkeitsausschluss durch eine Glasfritte (P3) filtriert. Nach Entfernen des Lösungsmittels i. Vak. erhielt man die entsprechenden Chloride als feuchtigkeits- und luftempfindliche, orange Öle (36a: 2.38 g, 7.89 mmol, 95\%; 36b: 1.82 g, 6.02 mmol, 93\%; 36c: $2.14 \mathrm{~g}, 7.03 \mathrm{mmol}, 94 \%)$.

[1- ${ }^{13}$ C]-1-Chlor-1,2,3,4,5-pentacyclopropylcyclopenta-1,3-dien (36a): charakteristische NMR-spektroskopische und massenspektrometrische Daten: ${ }^{13} \mathrm{C}-\mathrm{NMR}\left(62.9 \mathrm{MHz}, \mathrm{C}_{6} \mathrm{D}_{6}\right): \delta=$ 81.8 (C-1), 141.6, 145.7 (4 C, C-2,5, C-3,4). - MS (EI, 70 eV), m/z (\%):306/305/304/303/-

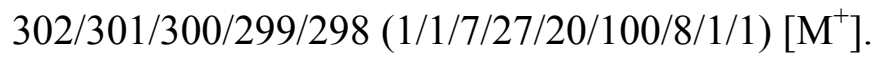


[1',2'-13 $\left.C_{2}\right]$-1-Chlor-1,2,3,4,5-pentacyclopropylcyclopenta-1,3-dien (36b): charakteristische NMR-spektroskopische und massenspektrometrische Daten: ${ }^{13} \mathrm{C}-\mathrm{NMR}\left(62.9 \mathrm{MHz}, \mathrm{C}_{6} \mathrm{D}_{6}\right): \delta=$ $6.1(\mathrm{~d}, 2 \mathrm{C}, c \operatorname{Pr}-\mathrm{C}), 6.9$ (d, 2 C, $c \operatorname{Pr}-\mathrm{C}), 7.2$ (d, 2 C, $c$ Pr-C), 7.5 (d, 2 C,$c \operatorname{Pr}-\mathrm{C}), 8.4$ (d, 2 C, $c \operatorname{Pr}-\mathrm{C}), 9.1$ (d, $2 \mathrm{C}, c \operatorname{Pr}-\mathrm{C}), 17.6(\mathrm{~d}, c \operatorname{Pr}-\mathrm{C})$, Kopplungskonst. für diese $c \operatorname{Pr}-\mathrm{C}:{ }^{1} J_{\mathrm{CC}}=14.3$,

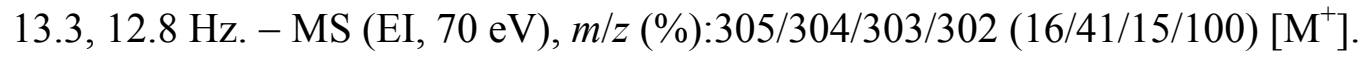

[1,1',2 $\left.{ }^{,}{ }^{13} C_{3}\right]$-1-Chlor-1,2,3,4,5-pentacyclopropylcyclopenta-1,3-dien (36c): charakteristische NMR-spektroskopische und massenspektrometrische Daten: ${ }^{13} \mathrm{C}-\mathrm{NMR}\left(62.9 \mathrm{MHz}, \mathrm{C}_{6} \mathrm{D}_{6}\right): \delta=$ 6.1 (dd, 2 C, $c$ Pr-C), 6.9 (dd, 2 C, $c$ Pr-C), 7.2 (dd, 2 C, $c$ Pr-C), 7.5 (dd, 2 C, $c$ Pr-C), 8.4 (dd, $2 \mathrm{C}, c \operatorname{Pr}-\mathrm{C}), 9.1(\mathrm{dd}, 2 \mathrm{C}, c \operatorname{Pr}-\mathrm{C}), 17.6(\mathrm{dd}, c \operatorname{Pr}-\mathrm{C})$, Kopplungskonst. für diese $c \operatorname{Pr}-\mathrm{C}$ : ${ }^{1} J_{\mathrm{CC}}=14.3,13.3,12.8 \mathrm{~Hz}, 81.8\left(\mathrm{dd},{ }^{1} J_{\mathrm{CC}}=45.5 \mathrm{~Hz},{ }^{2} J_{\mathrm{CC}}=1.8 \mathrm{~Hz}, \mathrm{C}-1\right), 141.6,145.7$ (dd, $\left.{ }^{1} J_{\mathrm{CC}}=63.0,57.6 \mathrm{~Hz},{ }^{2} J_{\mathrm{CC}}=2.0 \mathrm{~Hz}, 4 \mathrm{C}, \mathrm{C}-2,5, \mathrm{C}-3,4\right) .-\mathrm{MS}(\mathrm{EI}, 70 \mathrm{eV}), \mathrm{m} / z(\%): 309 / 308 /-$ 307/306/305/304/303/302 (3/2/1/6/34/18/100/14) $\left[\mathrm{M}^{+}\right]$.

\subsection{Darstellung der Verbindungen aus Teil B Kapitel 4}

7-Brom-2,3-dicyclopropyl-1H-indol (88a): Zu einer Mischung aus $4.00 \mathrm{~g}$ Dicyclopropylethin<smiles>Brc1cccc2c(C3CC3)c(C3CC3)[nH]c12</smiles>
(13) (37.7 mmol) und $50.0 \mathrm{ml}$ einer Lösung von Isobutylmagnesiumbromid in Diethylether $(0.75 \mathrm{M}, 37.5 \mathrm{mmol})$ wurden im Abstand von $20 \mathrm{~min}$ jeweils $100 \mathrm{mg}$ Biscyclopentadienyltitanocendichlorid (ges. $816 \mu \mathrm{mol}$ ) gegeben und die Reaktionslösung 45 min unter Rückfluss erhitzt (Beendigung der Gasentwicklung!). Die so hergestellte Lösung von 1,2-Dicyclopropylvinylmagnesiumbromid in Diethylether wurde danach zügig zu einer Lösung aus $2.54 \mathrm{~g}$ 1-Brom-2-nitrobenzol (86a) (12.5 mmol) in $100 \mathrm{ml}$ wasserfreiem Tetrahydrofuran bei -40 bis $-35^{\circ} \mathrm{C}$ gegeben. Nach $4 \mathrm{~h}$ Rühren bei dieser Temp. wurde auf $50 \mathrm{ml}$ gesättigte $\mathrm{NH}_{4} \mathrm{Cl}$-Löung gegeben, die Phasen getrennt und die wässrige Phase mit $3 \times 30 \mathrm{ml} \mathrm{Et}_{2} \mathrm{O}$ extrahiert. Die vereinigten organischen Extrakte wurden mit $30 \mathrm{ml}$ gesättigter $\mathrm{NaCl-Lösung} \mathrm{gewaschen,} \mathrm{über} \mathrm{MgSO}_{4}$ getrocknet und die Lösungsmittel i. Vak. entfernt. Der 
Rückstand wurde säulenchromatographisch an $90 \mathrm{~g}$ Kieselgel mit Pentan/Et ${ }_{2} \mathrm{O}=15: 1$ als Eluent $\left(R_{\mathrm{f}}=0.68\right)$ gereinigt (Säule $\left.3.5 \times 25 \mathrm{~cm}\right)$, wobei man $1.31 \mathrm{~g}$ des Produktes $(4.74 \mathrm{mmol}$, $38 \%$ ) als leicht gelbliches Öl erhielt. - IR (Film): $v=3452 \mathrm{~cm}^{-1}(\mathrm{~N}-\mathrm{H}), 3081(\mathrm{C}-\mathrm{H}), 3003$ (C-H), 1574, 1554, 1490, 1452, 1419, 1329, 1310, 1297, 1200, 1190, 1047, 1021, 973, 885, 818, $773(\mathrm{C}-\mathrm{H}), 735(\mathrm{C}-\mathrm{H}), 580,424 .-{ }^{1} \mathrm{H}-\mathrm{NMR}\left(250 \mathrm{MHz}, \mathrm{CDCl}_{3}\right): \delta=0.71-1.14(\mathrm{~m}, 8 \mathrm{H}$, $c$ Pr-H), $1.86\left(\mathrm{~m}_{\mathrm{c}}, 1 \mathrm{H}, c \operatorname{Pr}-\mathrm{H}\right), 2.21\left(\mathrm{~m}_{\mathrm{c}}, 1 \mathrm{H}, c \mathrm{Pr}-\mathrm{H}\right), 6.95\left(\mathrm{t},{ }^{3} \mathrm{~J}=7.9 \mathrm{~Hz}, 1 \mathrm{H}, 5-\mathrm{H}\right), 7.24 *$ $\left(\mathrm{dd},{ }^{3} J=7.9 \mathrm{~Hz},{ }^{4} J=1.5 \mathrm{~Hz}, 1 \mathrm{H} 4-\mathrm{H}\right), 7.56^{*}\left(\mathrm{dd},{ }^{3} J=7.9 \mathrm{~Hz},{ }^{4} J=1.5 \mathrm{~Hz}, 1 \mathrm{H}, 6-\mathrm{H}\right), 7.60$ $\left(\mathrm{s}_{\mathrm{br}}, 1 \mathrm{H}, \mathrm{NH}\right) .-{ }^{13} \mathrm{C}-\mathrm{NMR}\left(62.9 \mathrm{MHz}, \mathrm{CDCl}_{3}, \mathrm{DEPT}\right): \delta=5.2(-, 2 \mathrm{C}, c \operatorname{Pr}-\mathrm{C}), 5.6(+, c \operatorname{Pr}-\mathrm{C})$, 6.9 (-, 2 C,$c \operatorname{Pr}-\mathrm{C}), 7.8$ (+, $c$ Pr-C $), 103.9 *\left(\mathrm{C}_{\text {quart }}, \mathrm{C}-7\right), 115.1 *\left(\mathrm{C}_{\text {quart }}, \mathrm{C}-2\right), 117.7 * *(+, \mathrm{C}-4)$, $120.3 * *(+, \mathrm{C}-5), 123.2 * *(+, \mathrm{C}-6), 130.5 *\left(\mathrm{C}_{\text {quart }}, \mathrm{C}-3\right), 132.9 *\left(\mathrm{C}_{\text {quart }}, \mathrm{C}-3 \mathrm{a}\right), 138.4 *\left(\mathrm{C}_{\text {quart }}\right.$,

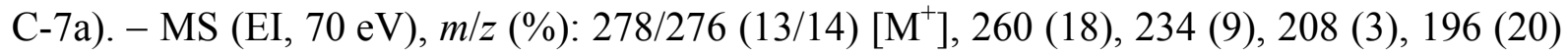
$\left[\mathrm{M}^{+}-\mathrm{Br}\right], 181(100), 167$ (91), $154(22), 139$ (8), 77 (9) $\left[\mathrm{C}_{6} \mathrm{H}_{5}^{+}\right], 63$ (5), 41 (6) $\left[\mathrm{C}_{3} \mathrm{H}_{5}^{+}\right] .-$ $\mathrm{C}_{14} \mathrm{H}_{14} \mathrm{BrN}$ (276.18). ber. C 60.89\%, H 5.11\%, N 5.07\%; gef. C 60.95\%, H 5.21\%, N 4.87\%.

7-Iod-2,3-dicyclopropyl-1H-indol (88b): Zu einer Mischung aus $4.00 \mathrm{~g}$ Dicyclopropylethin

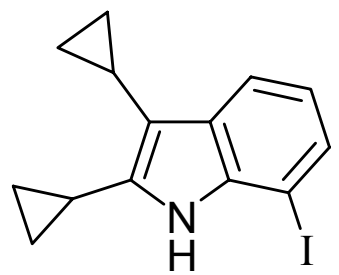
(13) (37.7 mmol) und $50.0 \mathrm{ml}$ einer Lösung von Isobutylmagnesiumbromid in Diethylether $(0.75 \mathrm{M}, 37.5 \mathrm{mmol})$ wurden im Abstand von 20 min jeweils $100 \mathrm{mg}$ Biscyclopentadienyltitanocendichlorid (ges. $816 \mu \mathrm{mol})$ gegeben und die Reaktionslösung 45 min unter Rückfluss erhitzt (Beendigung der Gasentwicklung!). Die so hergestellte Lösung von 1,2-Dicyclopropylvinylmagnesiumbromid in Diethylether wurde danach zügig zu einer Lösung aus $3.13 \mathrm{~g}$ 1-Iod-2-nitrobenzol (86b) $(12.5 \mathrm{mmol})$ in $100 \mathrm{ml}$ trockenem Tetrahydrofuran bei -40 bis $-35^{\circ} \mathrm{C}$ gegeben. Nach $4 \mathrm{~h}$ Rühren bei dieser Temp. wurde auf $50 \mathrm{ml}$ gesättigte $\mathrm{NH}_{4} \mathrm{Cl}$-Löung gegeben, die Phasen getrennt und die wässrige Phase mit $3 \times 30 \mathrm{ml} \mathrm{Et}_{2} \mathrm{O}$ extrahiert. Die vereinigten organischen Extrakte wurden mit $30 \mathrm{ml}$ gesättigter $\mathrm{NaCl}$-Lösung gewaschen, über $\mathrm{MgSO}_{4}$ getrocknet und die Lösungsmittel i. Vak. entfernt. Der Rückstand wurde säulenchromatographisch an $90 \mathrm{~g}$ Kieselgel mit Pentan/Et $2 \mathrm{O}=20: 1$ als Eluent $\left(R_{\mathrm{f}}=0.61\right)$ gereinigt (Säule $3.5 \times 25 \mathrm{~cm}$ ), wobei man $1.35 \mathrm{~g}$ des Produktes $(4.18 \mathrm{mmol}, 33 \%$ ) als leicht gelbliches 
Öl erhielt. - IR (Film): v = $3269 \mathrm{~cm}^{-1}(\mathrm{~N}-\mathrm{H}), 3081(\mathrm{C}-\mathrm{H}), 3006(\mathrm{C}-\mathrm{H}), 1667,1572,1488$, 1449, 1415, 1378, 1328, 1240, 1195, 1033, 997, 958, 882, 819, 773, 737, 664. - ${ }^{1} \mathrm{H}-\mathrm{NMR}$ $\left(250 \mathrm{MHz}, \mathrm{CDCl}_{3}\right): \delta=0.70-1.12(\mathrm{~m}, 8 \mathrm{H}, c \operatorname{Pr}-\mathrm{H}), 1.85\left(\mathrm{~m}_{\mathrm{c}}, 1 \mathrm{H}, c \operatorname{Pr}-\mathrm{H}\right), 2.20\left(\mathrm{~m}_{\mathrm{c}}, 1 \mathrm{H}\right.$, $c \operatorname{Pr}-\mathrm{H}), 6.84\left(\mathrm{t},{ }^{3} J=7.7 \mathrm{~Hz}, 1 \mathrm{H}, 5-\mathrm{H}\right), 7.44^{*}\left(\mathrm{~d},{ }^{3} J=7.7 \mathrm{~Hz}, 1 \mathrm{H}, 4-\mathrm{H}\right), 7.49$ (sbr, $\left.1 \mathrm{H}, \mathrm{NH}\right)$, $7.59 *\left(\mathrm{~d},{ }^{3} J=7.7 \mathrm{~Hz}, 1 \mathrm{H}, 6-\mathrm{H}\right) .-{ }^{13} \mathrm{C}-\mathrm{NMR}\left(62.9 \mathrm{MHz}, \mathrm{CDCl}_{3}, \mathrm{DEPT}\right): \delta=5.2(-, 2 \mathrm{C}$, $c \operatorname{Pr}-\mathrm{C}), 5.8(+, c \operatorname{Pr}-\mathrm{C}), 6.9(-, 2 \mathrm{C}, c \operatorname{Pr}-\mathrm{C}), 7.8(+, c \operatorname{Pr}-\mathrm{C}), 76.0^{*}\left(\mathrm{C}_{\text {quart }}, \mathrm{C}-7\right), 115.4^{*}\left(\mathrm{C}_{\text {quart }}\right.$, C-2), 118.6** (+, C-4), 120.9** (+, C-5), 129.4** (+, C-6), 136.0* (C ${ }^{*}$ quart, C-3a), 138.1* (C $\left.\mathrm{C}_{\text {quart }}, 2 \mathrm{C}, \mathrm{C}-3, \mathrm{C}-7 \mathrm{a}\right) .-\mathrm{MS}(\mathrm{EI}, 70 \mathrm{eV}), \mathrm{m} / \mathrm{z}(\%): 323(100)\left[\mathrm{M}^{+}\right], 308(24)\left[\mathrm{M}^{+}-\mathrm{CH}_{3}\right], 294$ (14) $\left[\mathrm{M}^{+}-\mathrm{C}_{2} \mathrm{H}_{5}\right], 282(8)\left[\mathrm{M}^{+}-\mathrm{C}_{3} \mathrm{H}_{5}\right], 196(22)\left[\mathrm{M}^{+}-\mathrm{I}\right], 181$ (92), 167 (80), 154 (16), 139 (8), $127(6)\left[\mathrm{I}^{+}\right] 115\left(39,83\right.$ (7), 51 (2), 41 (1) $\left[\mathrm{C}_{3} \mathrm{H}_{5}^{+}\right] .-\mathrm{C}_{14} \mathrm{H}_{14} \mathrm{IN}(323.15)$.

2,3-Dicyclopropyl-7-diphenylphosphanyl-1H-indol (87): Variante A: In einem dickwandigen

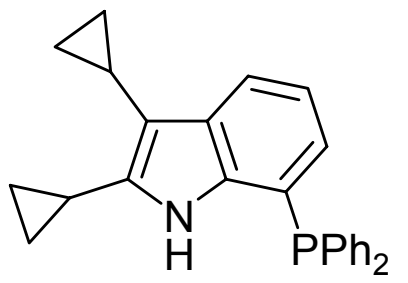
Pyrex-Schraubglasgefäß wurden nacheinander 116 mg Tetrakis(triphenylphosphan)palladium $(100 \mu \mathrm{mol}), 410 \mathrm{mg}$ Diphenylphosphan (2.20 mmol), $551 \mathrm{mg}$ 7-Brom-2,3-dicyclopropyl-1Hindol (88a) $(2.00 \mathrm{mmol}), 6 \mathrm{ml}$ trockenes Toluol und $408 \mathrm{mg}$ Triethylamin $(4.00 \mathrm{mmol})$ zusammengegeben und $5 \mathrm{~min}$ ein Argonstrom durch diese Mischung geleitet. Danach wurde das Reaktionsgefäß fest verschlossen und $16 \mathrm{~h}$ bei $120{ }^{\circ} \mathrm{C}$ gerührt. Nach Abkühlen gab man auf $30 \mathrm{ml} \mathrm{NaCl-Lösung} \mathrm{und} \mathrm{extrahierte} \mathrm{mit} 3 \times 20 \mathrm{ml} \mathrm{Et}_{2} \mathrm{O}$. Die vereinigten organischen Lösungen wurden über $\mathrm{MgSO}_{4}$ getrocknet und die Lösungsmittel i. Vak. entfernt. Der Rückstand wurde säulenchromatographisch an $40 \mathrm{~g}$ Kieselgel mit Pentan $/ \mathrm{Et}_{2} \mathrm{O}=15: 1$ als Eluent $\left(R_{\mathrm{f}}=0.64\right)$ gereinigt (Säule $\left.2.5 \times 18 \mathrm{~cm}\right)$, wobei man $397 \mathrm{mg}$ des Produktes $(1.04 \mathrm{mmol}, 52 \%)$ als farbloses, zähes Öl erhielt. - Variante B: In einem dickwandigen Pyrex-Schraubglasgefäß wurden nacheinander $98 \mathrm{mg}$ Tetrakis(triphenylphosphan)palladium (84.8 $\mu \mathrm{mol}), 348 \mathrm{mg}$ Diphenylphosphan (1.87 mmol), $550 \mathrm{mg}$ 7-Iod-2,3dicyclopropyl-1H-indol (88b) $(1.78 \mathrm{mmol}), 10 \mathrm{ml}$ trockenes Toluol und $363 \mathrm{mg}$ Triethylamin (3.56 mmol) zusammengegeben und $5 \mathrm{~min}$ ein Argonstrom durch diese Mischung geleitet. Danach wurde das Reaktionsgefäß fest verschlossen und $16 \mathrm{~h}$ bei $120^{\circ} \mathrm{C}$ gerührt. Nach 
Abkühlen gab man auf $30 \mathrm{ml} \mathrm{NaCl-Lösung} \mathrm{und} \mathrm{extrahierte} \mathrm{mit} 3 \times 20 \mathrm{ml} \mathrm{Et}_{2} \mathrm{O}$. Die vereinigten organischen Lösungen wurden über $\mathrm{MgSO}_{4}$ getrocknet und die Lösungsmittel i. Vak. entfernt. Der Rückstand wurde säulenchromatographisch an $45 \mathrm{~g}$ Kieselgel mit Pentan $/ \mathrm{Et}_{2} \mathrm{O}=15: 1$ als Eluent $\left(R_{\mathrm{f}}=0.64\right)$ gereinigt (Säule $2.5 \times 19 \mathrm{~cm}$ ), wobei man $420 \mathrm{mg}$ des Produktes (1.10 mmol, 62\%) als farbloses, zähes Öl erhielt. - IR (Film): $v=3443 \mathrm{~cm}^{-1}$ (N-H), 3069 (C-H), 3052 (C-H), $3001(\mathrm{C}-\mathrm{H})$, 2235, 2081, 1774, 1604, 1480, 1464, 1451, 1433 (P-Ph), 1408, 1328, 1285, 1157, 1096, 1044, 1026, 899, 784 (C-H), 744 (C-H), 696, 596, 502, 482. $-{ }^{1} \mathrm{H}-\mathrm{NMR}\left(250 \mathrm{MHz},\left[\mathrm{D}_{8}\right]-\mathrm{THF}\right): \delta=0.63-0.76(\mathrm{~m}, 4 \mathrm{H}, c \mathrm{Pr}-\mathrm{H}), 0.81-0.94$ $(\mathrm{m}, 4 \mathrm{H}, c \operatorname{Pr}-\mathrm{H}), 1.83\left(\mathrm{~m}_{\mathrm{c}}, 1 \mathrm{H}, c \operatorname{Pr}-\mathrm{H}\right), 2.12\left(\mathrm{~m}_{\mathrm{c}}, 1 \mathrm{H}, c \operatorname{Pr}-\mathrm{H}\right), 6.76\left(\mathrm{dt},{ }^{3} J=7.3 \mathrm{~Hz},{ }^{4} J=\right.$ $0.9 \mathrm{~Hz}, 1 \mathrm{H}, 4-\mathrm{H}), 6.93\left(\mathrm{t},{ }^{3} J=7.4 \mathrm{~Hz}, 1 \mathrm{H}, 5 \mathrm{H}\right), 7.23-7.36(\mathrm{~m}, 10 \mathrm{H}, \mathrm{Ar}-\mathrm{H}), 7.60\left(\mathrm{~d},{ }^{3} J=\right.$ $7.9 \mathrm{~Hz}, 1 \mathrm{H}, 6-\mathrm{H}), 8.78$ (s $\mathrm{br}, 1 \mathrm{H}, \mathrm{NH}) .-{ }^{13} \mathrm{C}-\mathrm{NMR}\left(62.9 \mathrm{MHz},\left[\mathrm{D}_{8}\right]-\mathrm{THF}, \mathrm{DEPT}\right): \delta=6.1(-$, $2 \mathrm{C}, c \operatorname{Pr}-\mathrm{C}), 6.7$ (+, $c \operatorname{Pr}-\mathrm{C}), 7.5$ (-, $2 \mathrm{C}, c \operatorname{Pr}-\mathrm{C}), 8.9$ (+, $c \operatorname{Pr}-\mathrm{C}), 114.3\left(\mathrm{C}_{\text {quart }}, \mathrm{C}-7 \mathrm{a}\right), 117.5$ $\left(\mathrm{C}_{\text {quart }}, \mathrm{d},{ }^{1} J_{\mathrm{CP}}=12 \mathrm{~Hz}, \mathrm{C}-7\right), 120.4\left(+, \mathrm{d},{ }^{2} J_{\mathrm{CP}}=25 \mathrm{~Hz}, 2 \mathrm{C}, \mathrm{C}_{\text {ortho }}\right), 127.9\left(+, \mathrm{d},{ }^{3} J_{\mathrm{CP}}=10 \mathrm{~Hz}\right.$, C-5), $129.5\left(+, 7\right.$ C $\left., \mathrm{C}_{\text {para }}, \mathrm{C}_{\text {meta }}, \mathrm{C}-4\right), 130.5\left(\mathrm{C}_{\text {quart }}, \mathrm{d},{ }^{3} J_{\mathrm{CP}}=4 \mathrm{~Hz}, \mathrm{C}-3 \mathrm{a}\right), 134.5\left(+, \mathrm{d},{ }^{2} J_{\mathrm{CP}}=\right.$ $\left.20 \mathrm{~Hz}, 2 \mathrm{C}, \mathrm{C}_{\text {ortho }}\right), 134.7\left(+, \mathrm{d},{ }^{2} J_{\mathrm{CP}}=20 \mathrm{~Hz}, \mathrm{C}-6\right), 138.5\left(\mathrm{C}_{\text {quart }}, \mathrm{d},{ }^{1} J_{\mathrm{CP}}=11 \mathrm{~Hz}, 2 \mathrm{C}, \mathrm{C}_{\mathrm{ipso}}\right)$, 139.3 ( $\left.\mathrm{C}_{\text {quart }}, \mathrm{C}-2\right), 139.5$ (C $\left.\mathrm{C}_{\text {quart }}, \mathrm{C}-3\right) .-{ }^{31} \mathrm{P}-\mathrm{NMR}\left(202.5 \mathrm{MHz},\left[\mathrm{D}_{8}\right]-\mathrm{THF}\right): \delta=-14.9 \mathrm{ppm} .-$ MS (EI, $70 \mathrm{eV}), m / z(\%): 381(18)\left[\mathrm{M}^{+}\right], 262$ (100), 196 (9) $\left[\mathrm{M}^{+}-\mathrm{C}_{12} \mathrm{H}_{10} \mathrm{P}\right], 183$ (58), 152 (7), 115 (2), 108 (20), 91 (5), 74 (18), 59 (22), 41 (10) $\left[\mathrm{C}_{3} \mathrm{H}_{5}^{+}\right] .-\mathrm{C}_{26} \mathrm{H}_{24} \mathrm{NP}$ (381.46): 381.1646 (korrekte HRMS).

2,3-Dicyclopropyl-7-diphenylphosphanyl-1H-indolyllithium (87a): In einem Schlenk-

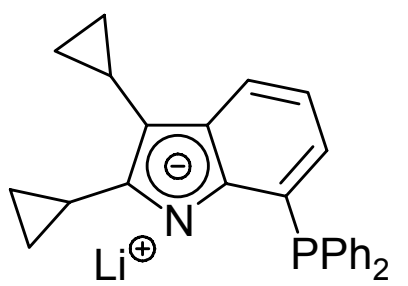
Spitzkolben wurden $0.12 \mathrm{ml}$ einer Lösung von MethyllithiumLithiumbromid-Komplex in Diethylether $(2.20 \mathrm{M}, 264 \mu \mathrm{mol})$ vorgelgt. Nach weitestgehendem Entfernen des Lösungsmittel durch Überleiten von Argon wurde restliches Lösungsmittel i. Vak. entfernt, der Kolben mit Argon belüftet und $0.20 \mathrm{ml}\left[\mathrm{D}_{8}\right]$-Tetrahydrofuran hinzugegeben. $\mathrm{Zu}$ dieser Lösung von $\mathrm{MeLi} \cdot \mathrm{LiBr}$ in $\left[\mathrm{D}_{8}\right]-\mathrm{THF}$ wurden unter Eiskühlung $98 \mathrm{mg} \quad$ 2,3-Dicyclopropyl-7-diphenylphosphanyl-1H-indol $\quad(\mathbf{8 7}) \quad(256 \mu \mathrm{mol}) \quad$ in $\quad 0.30 \mathrm{ml}$ 
$\left[D_{8}\right]$-THF gelöst tropfenweise zugegeben, wobei sich die Lösung intensiv gelb färbte. Die so gewonnene Lösung des Lithiumindolids 87a wurde anschließend bei Raumtemp. NMRspektroskopisch untersucht. $-{ }^{1} \mathrm{H}-\mathrm{NMR}\left(250 \mathrm{MHz},\left[\mathrm{D}_{8}\right]-\mathrm{THF}\right): \delta=0.59-0.84(\mathrm{~m}, 8 \mathrm{H}$, $c \operatorname{Pr}-\mathrm{H}), 1.93\left(\mathrm{~m}_{\mathrm{c}}, 1 \mathrm{H}, c \operatorname{Pr}-\mathrm{H}\right), 2.30\left(\mathrm{~m}_{\mathrm{c}}, 1 \mathrm{H}, c \operatorname{Pr}-\mathrm{H}\right), 6.45^{*}\left(\mathrm{dt},{ }^{3} J=7.2 \mathrm{~Hz},{ }^{4} J=1.0 \mathrm{~Hz}, 1 \mathrm{H}\right.$, $5-\mathrm{H}), 6.59^{*}\left(\mathrm{t},{ }^{3} J=7.3 \mathrm{~Hz}, 1 \mathrm{H}, \mathrm{Ar}-\mathrm{H}\right), 7.16-7.42 *(\mathrm{~m}, 10 \mathrm{H}, \mathrm{Ar}-\mathrm{H}, 6-\mathrm{H}), 7.41^{*}\left(\mathrm{~d},{ }^{3} J=\right.$ 7.6 Hz, $1 \mathrm{H}, 4-\mathrm{H}) .-{ }^{13} \mathrm{C}-\mathrm{NMR}\left(62.9 \mathrm{MHz},\left[\mathrm{D}_{8}\right]-\mathrm{THF}, \mathrm{DEPT}\right): \delta=6.2(-, 2 \mathrm{C}, c \operatorname{Pr}-\mathrm{C}), 7.4(-$, $2 \mathrm{C}, c \operatorname{Pr}-\mathrm{C}), 7.9(+, c \operatorname{Pr}-\mathrm{C}), 12.2(+, c \operatorname{Pr}-\mathrm{C}), 110.5,\left(\mathrm{C}_{\text {quart }}, \mathrm{d},{ }^{3} \mathrm{~J}=3 \mathrm{~Hz}, \mathrm{C}-3 \mathrm{a}\right), 115.2,\left(\mathrm{C}_{\text {quart }}, \mathrm{d}\right.$, $\left.{ }^{2} J=7 \mathrm{~Hz}, \mathrm{C}-7 \mathrm{a}\right), 115.4(+, \mathrm{C}-4), 118.3(+, \mathrm{C}-5), 122.0(+, \mathrm{C}-6), 128.9,129.0\left(+, 4 \mathrm{C}, \mathrm{C}_{\text {para }}\right.$, $\left.\mathrm{C}_{\text {meta }}\right), 129.4,129.5,129.7$ (+, $\left.2 \mathrm{C}, \mathrm{C}_{\text {meta }}\right), 132.8,\left(\mathrm{C}_{\text {quart }}, \mathrm{d},{ }^{1} J=7 \mathrm{~Hz}, \mathrm{C}-7\right), 134.2,\left(+, \mathrm{d},{ }^{2} J=\right.$ $\left.16 \mathrm{~Hz}, 2 \mathrm{C}, \mathrm{C}_{\text {ortho }}\right), 134.6,\left(+, \mathrm{d},{ }^{2} J=20 \mathrm{~Hz}, 2 \mathrm{C}, \mathrm{C}_{\text {ortho }}\right), 138.6,\left(\mathrm{C}_{\text {quart }}, \mathrm{d},{ }^{1} J=12 \mathrm{~Hz}, \mathrm{C}_{\text {ipso }}\right)$, $139.2\left(\mathrm{C}_{\text {quart }}, \mathrm{C}-2\right), 151.3\left(\mathrm{C}_{\text {quart }}, \mathrm{C}-3\right), 152.4\left(\mathrm{C}_{\text {quart }}, \mathrm{d},{ }^{1} J=26 \mathrm{~Hz}, \mathrm{C}_{\text {ipso }}\right) .-{ }^{31} \mathrm{P}-\mathrm{NMR}$ (202.5 MHz, [D $\left.\mathrm{D}_{8}\right]$-THF): $\delta=-18.3$ ppm. $-\mathrm{C}_{26} \mathrm{H}_{23} \mathrm{NPLi}(386.46)$

$\eta^{4}$-1,2,4,5-Tetracyclopropylcyclopenta-1,3-dieneisen(0)tricarbonyl $\quad(\mathbf{9 5 a}): \quad$ Unter einer

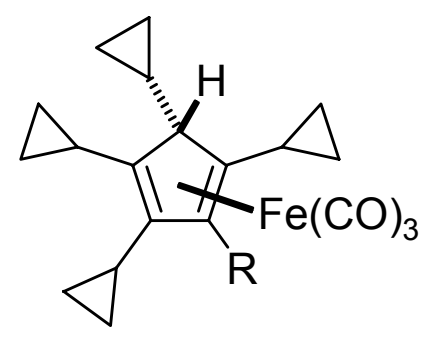

Schutzgasatmosphäre wurde eine Mischung aus $300 \mathrm{mg}$ 1,2,4,5Tetracyclopropylcyclopenta-1,3-dien (14) (1.30 mmol) und $546 \mathrm{mg}$ Dieisennonacarbonyl $(1.50 \mathrm{mmol})$ in $6 \mathrm{ml}$ wasserfreiem Toluol bei $110^{\circ} \mathrm{C}$ für $16 \mathrm{~h}$ gerührt. Anschließend wurde die abgekühlte Reaktionslösung i. Vak. weitestgehend eingeengt. Der Rückstand wurde säulenchromatographisch an $35 \mathrm{~g}$ Kieselgel mit Pentan als Eluent $\left(R_{\mathrm{f}}=0.59\right)$ gereinigt (Säule $\left.2.5 \times 12 \mathrm{~cm}\right)$, wobei man $212 \mathrm{mg}$ des Produktes $(0.59 \mathrm{mmol}$, 45\%) als orangefarbenes Öl erhielt, das bei tieferen Temp. zu einem amorphen Feststoff erstarrte. - IR (Film): $v=3083 \mathrm{~cm}^{-1}(\mathrm{C}-\mathrm{H}), 3006(\mathrm{C}-\mathrm{H}), 2076(\mathrm{C}=\mathrm{O}), 1949(\mathrm{C}=\mathrm{O}), 1702$, 1429, 1022, 888, 819, 621, 608, 565. - ${ }^{1} \mathrm{H}-\mathrm{NMR}\left(600 \mathrm{MHz}, \mathrm{CDCl}_{3}\right): \delta=-0.09\left(\mathrm{~m}_{\mathrm{c}}, 1 \mathrm{H}\right.$, $c$ Pr-H), $0.12\left(\mathrm{~m}_{\mathrm{c}}, 2 \mathrm{H}, c \operatorname{Pr}-\mathrm{H}\right), 0.27\left(\mathrm{~m}_{\mathrm{c}}, 1 \mathrm{H}, c \operatorname{Pr}-\mathrm{H}\right), 0.30-0.43$ (m, $\left.3 \mathrm{H}, c \operatorname{Pr}-\mathrm{H}\right), 0.51-0.64$ (m, $3 \mathrm{H}, c$ Pr-H), 0.64-0.88 (m, $3 \mathrm{H}, c \operatorname{Pr}-\mathrm{H}), 0.95\left(\mathrm{~m}_{\mathrm{c}}, 1 \mathrm{H}, c \operatorname{Pr}-\mathrm{H}\right), 1.05\left(\mathrm{~m}_{\mathrm{c}}, 1 \mathrm{H}, c \operatorname{Pr}-\mathrm{H}\right)$, $1.14\left(\mathrm{~m}_{\mathrm{c}}, 1 \mathrm{H}, c \operatorname{Pr}-\mathrm{H}\right), 1.29\left(\mathrm{~m}_{\mathrm{c}}, 1 \mathrm{H}, c \operatorname{Pr}-\mathrm{H}\right), 1.48\left(\mathrm{~m}_{\mathrm{c}}, 1 \mathrm{H}, c \operatorname{Pr}-\mathrm{H}\right), 1.79\left(\mathrm{~m}_{\mathrm{c}}, 1 \mathrm{H}, c \operatorname{Pr}-\mathrm{H}\right)$, $1.91\left(\mathrm{~d},{ }^{3} \mathrm{~J}=8.6 \mathrm{~Hz}, 1 \mathrm{H}, 5-\mathrm{H}\right), 1.98\left(\mathrm{~m}_{\mathrm{c}}, 1 \mathrm{H}, c \mathrm{Pr}-\mathrm{H}\right), 4.68(\mathrm{~s}, 1 \mathrm{H}, 3-\mathrm{H}) .-{ }^{13} \mathrm{C}-\mathrm{NMR}$ 
(62.9 MHz, $\left.\mathrm{CDCl}_{3}, \mathrm{APT}\right): \delta=3.7(-, c \operatorname{Pr}-\mathrm{C}), 5.2(-, c \operatorname{Pr}-\mathrm{C}), 5.7$ (-, $c$ Pr-C), 7.3 (-, $c$ Pr-C), 7.8 (-, cPr-C), 8.2 (-, cPr-C), 9.1 (+, $c$ Pr-C), 10.6 (-, $c$ Pr-C), 10.7 (+, $c$ Pr-C) $, 12.5,(+, c \operatorname{Pr}-\mathrm{C})$ 13.6 (-, cPr-C), 20.2 (+, cPr-C), 69.3 (+, C-5), 75.5 (+,C-3), 110.1* (-, C-1), 138.8* (-,C-4),

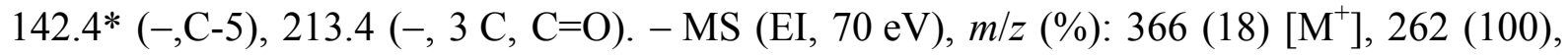
196 (9) $\left[\mathrm{M}^{+}-\mathrm{C}_{12} \mathrm{H}_{10}\right], 183$ (58), 152 (7), 115 (2), 108 (20), 91 (5), 74 (18), 59 (22), 41 (10) $\left[\mathrm{C}_{3} \mathrm{H}_{5}^{+}\right] .-\mathrm{C}_{20} \mathrm{H}_{22} \mathrm{O}_{3} \mathrm{Fe}$ (366.14): 366.0918 (korrekte HRMS).

\subsection{Darstellung der Verbindungen aus Teil B Kapitel 5}

Allgemeine Arbeitsvorschrift zur Umsetzung der Cyclopentadiene 14 und 15 sowie des Butadiens 21 mit Dienophilen unter normalem Druck (AAV 4): In einem dickwandigen PyrexSchraubglasgefäß mit Magnetrührstab wurden nacheinander die angegebenen Mengen des Cyclopentadiens bzw. Butadiens und des Dienophils eingewogen und mit dem entsprechenden Volumen Toluol versetzt. Durch fünfminütiges Durchleiten von Argon wurde diese Mischung von Sauerstoff befreit, das Gefäß fest verschlossen und bei der angegeben Temp. für die entsprechende Zeit gerührt. Anschließend wurde das Lösungsmittel von der noch warmen Reaktionsmischung i. Vak. entfernt und der Rückstand säulenchromatographisch an Kieselgel mit einem Gemisch aus Pentan/Et $2 \mathrm{O}$ gereinigt.

Allgemeine Arbeitsvorschrift zur Umsetzung der Cyclopentadiene 14 und 15 sowie des Butadiens 21 mit Dienophilen unter hohem Druck (AAV 5): In einem einseitig zugeschmolzenen Teflonschrumpfschlauch wurden nacheinander die angegebenen Mengen des Cyclopentadiens bzw. Butadiens und des Dienophils eingewogen und mit dem entsprechenden Volumen an Lösungsmittel versetzt. Zur Unterdrückung möglicher Radikalbildung wurde eine Spur Hydrochinon hinzugefügt und der Teflonschrumpfschlauch vollständig zugeschmolzen. Anschließend wurde diese Reaktionsmischung bei der angegeben Temp. für die entsprechende Zeit in einer beheizbaren Hochdruckpresse bei einem Druck von 
10 kbar umgesetzt. Nach Entfernen des Lösungsmittels i. Vak. wurde der Rückstand säulenchromatographisch an Kieselgel mit einem Gemisch aus Pentan/Et ${ }_{2} \mathrm{O}$ gereinigt.

(E,E)-1,2,3,4-Tetracyclopropylbuta-1,3-dien (21): Zu einer Lösung von $1 \mathrm{~g}$ Dicyclopropyl-

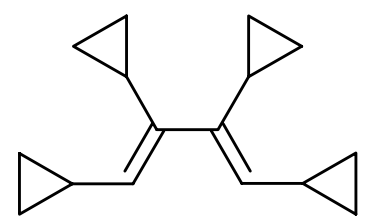
ethin (13) (9.42 mmol) und $2.67 \mathrm{~g}$ Titantetraisopropylat $(9.42 \mathrm{mmol})$ in $70 \mathrm{ml}$ wasserfreiem Diethylether wurden bei $-78^{\circ} \mathrm{C} 14.7 \mathrm{ml}$ einer Lösung von Isopropylmagnesiumbromid in $\mathrm{Et}_{2} \mathrm{O}(1.41 \mathrm{M}$, $20.7 \mathrm{mmol}$ ) tropfenweise hinzugefügt, wobei sich die Reaktionsmischung goldgelb färbte. Nach 1.5 stündigem Rühren bei -50 bis $-40{ }^{\circ} \mathrm{C}$ wurde die nun schwarze Reaktionslösung auf $-78{ }^{\circ} \mathrm{C}$ abgekühlt und eine Mischung von $5 \mathrm{ml}$ Methanol und $7 \mathrm{ml} \mathrm{Et}_{2} \mathrm{O}$ hinzugetropft. Anschließend entfernte man das Kältebad und ließ die Reaktionslösung auftauen. Es wurde auf $50 \mathrm{ml} 3 \mathrm{M}$ wässrige Salzsäurelösung gegeben, die Phasen getrennt und die wässrige Phase mit $3 \times 15 \mathrm{ml} \mathrm{Et}_{2} \mathrm{O}$ extrahiert. Die vereinigten organischen Lösungen wurden mit $30 \mathrm{ml}$ gesättigter $\mathrm{NaCl}$-Lösung gewaschen, über $\mathrm{MgSO}_{4}$ getrocknet und die Lösungsmittel i. Vak. entfernt. Der Rückstand wurde säulenchromatographisch an $35 \mathrm{~g}$ Kieselgel mit Pentan als Eluent $\left(R_{\mathrm{f}}=0.73\right)$ gereinigt (Säule $3 \times 35 \mathrm{~cm}$ ), wobei man $906 \mathrm{mg}$ des Produktes (4.23 mmol, 90\%) als farbloses Ö1 erhielt, das bei niedrigerer Temp. zu einem amorphen Feststoff erstarrte. - IR (Film): $v=3080 \mathrm{~cm}^{-1}$ (C-H), 3003 (C-H), 2868 (C-H), 1457, 1428, 1387, 1292, 1174, 1097, 1044, 1018, 978, 965, $945,891,809,736,668,600,572,518 .-{ }^{1} \mathrm{H}-\mathrm{NMR}\left(250 \mathrm{MHz}, \mathrm{CDCl}_{3}\right): \delta=0.22-0.50(\mathrm{~m}$, $8 \mathrm{H}, c \operatorname{Pr}-\mathrm{H}), 0.57-0.87$ (m, $8 \mathrm{H}, c \mathrm{Pr}-\mathrm{H}), 1.51\left(\mathrm{~m}_{\mathrm{c}}, 2 \mathrm{H}, c \mathrm{Pr}-\mathrm{H}\right), 1.77\left(\mathrm{~m}_{\mathrm{c}}, 2 \mathrm{H}, c \mathrm{Pr}-\mathrm{H}\right), 4.75$ (d, $\left.{ }^{3} J=9.5 \mathrm{~Hz}, 2 \mathrm{H}, 1-\mathrm{H}, 4-\mathrm{H}\right) .-{ }^{13} \mathrm{C}-\mathrm{NMR}\left(62.9 \mathrm{MHz}, \mathrm{CDCl}_{3}, \mathrm{DEPT}\right): \delta=6.1(-, 4 \mathrm{C}, c \operatorname{Pr}-\mathrm{C})$, $7.2(-, 4 \mathrm{C}, c \mathrm{Pr}-\mathrm{C}), 10.3(+, 2 \mathrm{C}, c \mathrm{Pr}-\mathrm{C}), 11.0(+, 2 \mathrm{C}, c \mathrm{Pr}-\mathrm{C}), 133.1(+, 2 \mathrm{C}, \mathrm{C}-1,4), 139.2$ $\left(\mathrm{C}_{\text {quart }}, 2 \mathrm{C}, \mathrm{C}-2,3\right) .-\mathrm{MS}(\mathrm{EI}, 70 \mathrm{eV}), \mathrm{m} / z(\%): 214(28)\left[\mathrm{M}^{+}\right], 199(4)\left[\mathrm{M}^{+}-\mathrm{CH}_{3}\right], 185$ (18), $173(22)\left[\mathrm{M}^{+}-\mathrm{C}_{3} \mathrm{H}_{5}\right], 157$ (44), 143 (52), 129 (72), 117 (56), 105 (45), 91 (100), 79 (58), 67 (30), 55 (24), 41 (29) $\left[\mathrm{C}_{3} \mathrm{H}_{5}{ }^{+}\right] .-\mathrm{C}_{16} \mathrm{H}_{22}$ (214.35): ber. C 89.65\%, H 10.35\%; gef. C 89.68\%, H $10.25 \%$. 


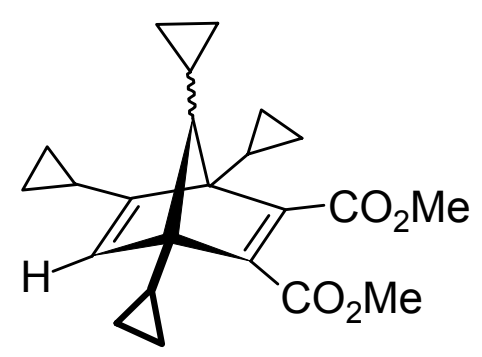

Nach AAV 4 wurden 1.00 g 1,2,4,5-Tetracyclopropylcyclopenta-1,3-dien (14) (4.42 mmol) mit $628 \mathrm{mg}$ Acetylendicarbonsäuredimethylester (97) (4.42 mmol) in $8 \mathrm{ml}$ Toluol für $2 \mathrm{~d}$ bei $90{ }^{\circ} \mathrm{C}$ gerührt. Säulenchromatographische Reinigung des Rohproduktes an $120 \mathrm{~g}$ Kieselgel (Säule $3.5 \times 30 \mathrm{~cm}$ Pentan $\left./ \mathrm{Et}_{2} \mathrm{O}=5: 1, R_{\mathrm{f}}=0.21\right)$ lieferte $1.30 \mathrm{~g}$ des Produkts $(3.40 \mathrm{mmol}, 77 \%)$ als $1: 1 \mathrm{Gemisch}$ zweier Diastereomere in Form eines farblosen, amorphen Feststoffs vom Schmp. $79^{\circ} \mathrm{C}$. - IR (Film): $v=3439 \mathrm{~cm}^{-1}, 3079(\mathrm{C}-\mathrm{H}), 3005(\mathrm{C}-\mathrm{H}), 2950(\mathrm{C}-\mathrm{H}), 2907(\mathrm{C}-\mathrm{H}), 2841(\mathrm{C}-\mathrm{H})$, $1726(\mathrm{C}=\mathrm{O}), 1721(\mathrm{C}=\mathrm{O}), 1636,1612,1458,1433,1395,1302,1243,1212,1193,1118$, 1051, 1020, 969, 891, 859, 829, 779, 733, 713, 648. - ${ }^{1} \mathrm{H}-\mathrm{NMR}\left(200 \mathrm{MHz}, \mathrm{CDCl}_{3}\right): \delta=$ -0.08-0.12 (m, 3 H, cPr-H), 0.19-0.78 (m, $28 \mathrm{H}, c \operatorname{Pr}-\mathrm{H}), 0.79-0.98$ (m, $4 \mathrm{H}, c \operatorname{Pr}-\mathrm{H}), 1.37$ $\left(\mathrm{m}_{\mathrm{c}}, 1 \mathrm{H}, c \operatorname{Pr}-\mathrm{H}\right), 1.43^{*}\left(\mathrm{~d},{ }^{3} J=9.2 \mathrm{~Hz}, 1 \mathrm{H}, 7-\mathrm{H}\right), 1.45-1.66(\mathrm{~m}, 4 \mathrm{H}, c \operatorname{Pr}-\mathrm{H}), 1.70^{*}(\mathrm{~d}$, $\left.{ }^{3} J=9.2 \mathrm{~Hz}, 1 \mathrm{H}, 7-\mathrm{H}\right), 3.67,3.68,3.69,3.74$ (s, $\left.12 \mathrm{H}, \mathrm{OMe}\right), 5.42,5.59$ (s, $\left.2 \mathrm{H}, 6-\mathrm{H}\right) .-$ ${ }^{13} \mathrm{C}-\mathrm{NMR}\left(62.9 \mathrm{MHz}, \mathrm{CDCl}_{3}, \mathrm{DEPT}\right): \delta=2.1$ (-, $\left.2 \mathrm{C}, c \mathrm{Pr}-\mathrm{C}\right), 2.3(-, 2 \mathrm{C}, c \mathrm{Pr}-\mathrm{C}), 2.4(-, 2 \mathrm{C}$, $c$ Pr-C), 2.5 (-, 2 C, $c$ Pr-C), 2.8 (-, 2 C , $c$ Pr-C), 3.8 (-, 2 C, $c$ Pr-C), 4.1 (-, 2 C, $c$ Pr-C), 4.4 (-, $2 \mathrm{C}, c \operatorname{Pr}-\mathrm{C}), 5.0$ (-, $2 \mathrm{C}, c \operatorname{Pr}-\mathrm{C}), 5.4$ (-, $2 \mathrm{C}, c \operatorname{Pr}-\mathrm{C}), 5.9$ (-, $2 \mathrm{C}, c \operatorname{Pr}-\mathrm{C}), 6.0$ (-, $2 \mathrm{C}, c \operatorname{Pr}-\mathrm{C})$, 6.3 (-, 2 C,$c \operatorname{Pr}-\mathrm{C}), 6.5$ (-, 2 C,$c \operatorname{Pr}-\mathrm{C}), 6.6$ (-, 2 C, $c$ Pr-C), 8.5 (+, 2 C, cPr-C), 8.9 (+, 2 C, $c$ Pr-C), $9.0(+, 2 \mathrm{C}, c \operatorname{Pr}-\mathrm{C}), 9.4$ (+, $2 \mathrm{C}, c \operatorname{Pr}-\mathrm{C}), 9.5$ (+, $2 \mathrm{C}, c \operatorname{Pr}-\mathrm{C}), 9.7$ (+, $2 \mathrm{C}, c \operatorname{Pr}-\mathrm{C}), 10.1$ (+, $2 \mathrm{C}, c \operatorname{Pr}-\mathrm{C}), 10.2(+, 2 \mathrm{C}, c \operatorname{Pr}-\mathrm{C}), 51.6(+, 2 \mathrm{C}, c \operatorname{Pr}-\mathrm{C}), 51.7$ (+, $2 \mathrm{C}, c \operatorname{Pr}-\mathrm{C}), 67.0,67.1$,

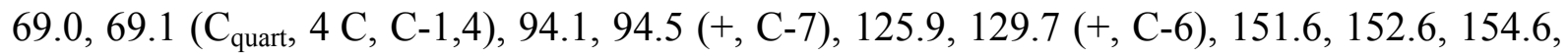
155.7, 160.6, 164.7, 165.9, 166.2, 166.3, $166.42\left(\mathrm{C}_{\text {quart }}, 10 \mathrm{C}, \mathrm{C}-2,3,5, \mathrm{C}=\mathrm{O}\right) .-\mathrm{MS}(\mathrm{EI}$, $70 \mathrm{eV}), m / z(\%): 368$ (2) $\left[\mathrm{M}^{+}\right], 327$ (18), 309 (100) $\left[\mathrm{M}^{+}-\mathrm{C}_{2} \mathrm{H}_{3} \mathrm{O}_{2}\right], 295$ (8), 249 (6), 235 (5), 207 (5), 179 (8), 165 (10), 153 (5), 115 (4), 91 (3), 59 (3) $\left[\mathrm{C}_{2} \mathrm{H}_{3} \mathrm{O}_{2}^{+}\right], \quad 41$ (4) $\left[\mathrm{C}_{3} \mathrm{H}_{5}{ }^{+}\right] .-\mathrm{C}_{23} \mathrm{H}_{28} \mathrm{O}_{4}$ (382.49): ber. C 74.97\%, H 7.66\%; gef. C 74.92\%, H 7.40\%. 
endo-1,7,8,10-Tetracyclopropyl-4-phenyl-4-aza-tricyclo[5.2.1.0 $\left.0^{2,6}\right]$ dec-8-en-3,5-dion

(100):

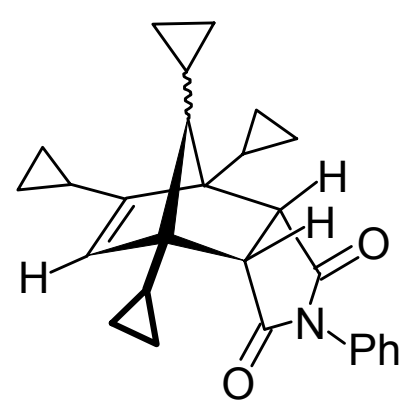

Nach AAV 4 wurden 400 mg 1,2,4,5-Tetracyclopropylcyclopenta1,3-dien (14) (1.77 mmol) mit $306 \mathrm{mg} \mathrm{N}$-Phenylmaleimid (98) $(1.77 \mathrm{mmol})$ in $6 \mathrm{ml}$ Toluol für $16 \mathrm{~h}$ bei $120^{\circ} \mathrm{C}$ gerührt. Säulenchromatographische Reinigung des Rohprodukts an $40 \mathrm{~g}$ Kieselgel (Säule $3.5 \times 10 \mathrm{~cm}, \operatorname{Pentan} / \mathrm{Et}_{2} \mathrm{O}=15: 1, R_{\mathrm{f}}=0.06$ ) lieferte $398 \mathrm{mg}$ des Produkts $(996 \mu \mathrm{mol}, 56 \%)$ als 3.5:1 Gemisch zweier Diastereomere in Form eines farblosen, amorphen Feststoffs. - IR (Film): $v=$ $3079 \mathrm{~cm}^{-1}(\mathrm{C}-\mathrm{H}), 3004(\mathrm{C}-\mathrm{H}), 1770(\mathrm{C}=\mathrm{O}), 1708(\mathrm{C}=\mathrm{O}), 1599,1500,1456,1378,1292$, 1184, 1019, 961, 877, 825, 758, 733, 714, 697, 619, 503. - ${ }^{1} \mathrm{H}-\mathrm{NMR}\left(250 \mathrm{MHz}, \mathrm{CDCl}_{3}\right): \delta=$ 0.06-1.06 (m, $15 \mathrm{H}, c \operatorname{Pr}-\mathrm{H}), 1.18\left(\mathrm{~m}_{\mathrm{c}}, 1 \mathrm{H}, c \operatorname{Pr}-\mathrm{H}\right), 1.31$ (d, $\left.{ }^{3} J=5.5 \mathrm{~Hz}, 1 \mathrm{H}, 10-\mathrm{H}:\right), 1.41$ $\left(\mathrm{m}_{\mathrm{c}}, 3 \mathrm{H}, c \operatorname{Pr}-\mathrm{H}\right), 1.52\left(\mathrm{~m}_{\mathrm{c}}, 2 \mathrm{H}, c \operatorname{Pr}-\mathrm{H}\right), 2.84,3.06\left(\mathrm{~d},{ }^{3} \mathrm{~J}=7.6 \mathrm{~Hz}, 2 \mathrm{H}, 2,6-\mathrm{H}\right), 3.23,3.40(\mathrm{~d}$, $\left.{ }^{3} J=7.6 \mathrm{~Hz}, 2 \mathrm{H}, 2,6-\mathrm{H}\right), 5.03,5.12(\mathrm{~s}, 2 \mathrm{H}, 8-\mathrm{H}), 7.14$ (d, ${ }^{3} J=7.5 \mathrm{~Hz}, 2 \mathrm{H}$, Ar- $\mathrm{H}_{\text {ortho}}$ ), 7.28-7.49 (m, $3 \mathrm{H}, \mathrm{Ar}-\mathrm{H}) .-{ }^{13} \mathrm{C}-\mathrm{NMR}\left(62.9 \mathrm{MHz}, \mathrm{CDCl}_{3}, \mathrm{DEPT}\right): \delta=-0.6,1.4,2.7,3.3$, 3.4, 3.5, 5.7, (-, 7 C, $c$ Pr-C), 6.3, 9.1, (+, 2 C, cPr-C), 9.8 (-, cPr-C), 10.1, 11.0, 11.7 (+, 3 C, $c \operatorname{Pr}-\mathrm{C}), 46.3^{*}, 46.7^{*}(+, 2 \mathrm{C}, \mathrm{C}-2,6), 51.0^{*}, 51.4^{*}(+, 2 \mathrm{C}, \mathrm{C}-2,6), 59.7^{*}, 60.2^{*}\left(\mathrm{C}_{\text {quart }}, 2 \mathrm{C}\right.$, C-1,7), 62.4*, 64.1* (C quart $2 \mathrm{C}, \mathrm{C}-1,7), 77.6,78.3$ (+, 2 C, C-10), 118.6, 125.0, 125.8, 126.3, 128.6, 128.7, 128.8, 127.6, 127.9, 128.0, 132.0, 133.9 (+, 12 C, Ar-C, C-8), 152.4, 169.3 $\left(\mathrm{C}_{\text {quart }}, 2 \mathrm{C}, \mathrm{Ar-} \mathrm{C}_{\mathrm{ipso}}, \mathrm{C}-8, \mathrm{C}-9\right), 175.7,175.8,176.3,176.5\left(\mathrm{C}_{\text {quart }}, 4 \mathrm{C}, \mathrm{C}=\mathrm{O}\right) .-\mathrm{MS}(\mathrm{EI}$, $70 \mathrm{eV}), m / z(\%): 399(3)\left[\mathrm{M}^{+}\right], 358$ (1), 293 (1), 255 (1), 226 (100) $\left[\mathrm{C}_{17} \mathrm{H}_{22}{ }^{+}\right], 197$ (36), 173

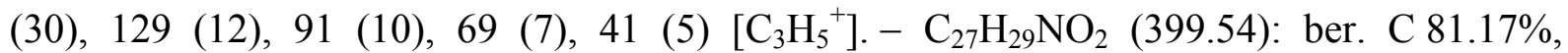
H 7.32\%, N 3.51\%; gef. C 81.19\%, H 7.16\%, N 3.53\%.

\section{1,4,5,6,7-Pentacyclopropylbicyclo[2.2.1] hepta-2,5-dien-2,3-dicarbonsaüredimethylester}

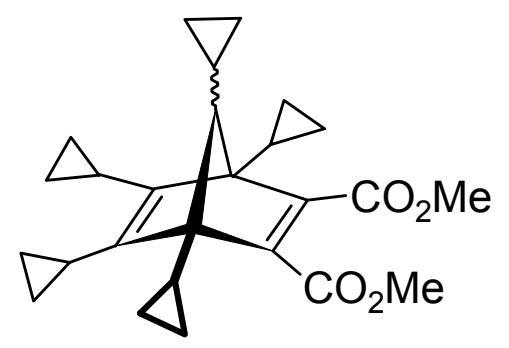

(101): Nach AAV 4 wurden $262 \mathrm{mg} \quad 1,2,3,4,5-$ Pentacyclopropylcyclopentadien (15) $\quad(983 \mu \mathrm{mol}) \quad$ mit $140 \mathrm{mg} \quad$ Acetylendicarbonsäuredimethylester

$(985 \mu \mathrm{mol})$ in $5 \mathrm{ml}$ Toluol bei $100^{\circ} \mathrm{C}$ für $20 \mathrm{~h}$ gerührt. 
Säulenchromatographische Reinigung des Rohproduktes an $20 \mathrm{~g}$ Kieselgel (Säule $3.5 \times 10 \mathrm{~cm}$, Pentan $\left./ \mathrm{Et}_{2} \mathrm{O}=10: 1, R_{\mathrm{f}}=0.16\right)$ lieferte $220 \mathrm{mg}$ des Produktes $(539 \mu \mathrm{mol}, 55 \%)$ als 1:1 Gemisches zweier Diastereomere in Form eines farblosen Öls. - IR (Film): v= $3433 \mathrm{~cm}^{-1}, 3081(\mathrm{C}-\mathrm{H}), 3007(\mathrm{C}-\mathrm{H}), 2950(\mathrm{C}-\mathrm{H}), 2901(\mathrm{C}-\mathrm{H}), 1718(\mathrm{C}=\mathrm{O}), 1602,1457$, 1434, 1393, 1294, 1245, 1193, 1119, 1023, 965, 882, 828, 780, 736. - ${ }^{1}$ H-NMR (250 MHz, $\left.\mathrm{CDCl}_{3}\right): \delta=0.17-0.21(\mathrm{~m}, 2 \mathrm{H}, c \operatorname{Pr}-\mathrm{H}), 0.30-0.88(\mathrm{~m}, 19 \mathrm{H}, c \operatorname{Pr}-\mathrm{H}, 7-\mathrm{H}), 1.15-1.25(\mathrm{~m}, 1 \mathrm{H}$, $c$ Pr-H), 1.25-1.40 (m, 2 H, cPr-H), 1.40-1.60 (m, 2 H, cPr-H), 3.68 (s, 3 H, OMe), 3.72 (s, $3 \mathrm{H}, \mathrm{OMe}) .-{ }^{13} \mathrm{C}-\mathrm{NMR}\left(62.9 \mathrm{MHz}, \mathrm{CDCl}_{3}, \mathrm{DEPT}\right): \delta=2.9,3.0(-, 2 \mathrm{C}, c \operatorname{Pr}-\mathrm{C}), 3.4(-$, cPr-C), 3.9 (-, cPr-C), 5.7 (-, 2 C, $c$ Pr-C), 6.0, 6.1 (-, 2 C, $c$ Pr-C), 7.4 (-, cPr-C), 7.9 (-, cPr-C), 8.7, 8.9 (+, 2 C, $c$ Pr-C), 9.5 (+, $c$ Pr-C), 10.9 (+, cPr-C), $11.4(+, c \operatorname{Pr}-\mathrm{C}), 51.6(+, 2$ C, OMe), 68.6, 68.8 ( $\left.\mathrm{C}_{\text {quart }}, 2 \mathrm{C}, \mathrm{C}-1,4\right), 89.9,92.4$ (+, 2 C, C-7), 148.7, 151.3, 153.2, 154.0 $\left(\mathrm{C}_{\text {quart }}, 4 \mathrm{C}, \mathrm{C}-2,3, \mathrm{C}-5,6\right), 166.8\left(\mathrm{C}_{\text {quart }}, 2 \mathrm{C}, \mathrm{C}=\mathrm{O}\right) .-\mathrm{MS}(\mathrm{EI}, 70 \mathrm{eV}), \mathrm{m} / \mathrm{z}(\%): 408(16)\left[\mathrm{M}^{+}\right]$, 367 (7), 349 (30) $\left[\mathrm{M}^{+}-\mathrm{C}_{2} \mathrm{H}_{3} \mathrm{O}_{2}\right], 302$ (6), 289 (8) $\left[\mathrm{M}^{+}-2 \mathrm{C}_{2} \mathrm{H}_{3} \mathrm{O}_{2}\right], 229$ (15), 216 (11), 175 (8), 159 (7), 129 (9), 91 (14), 69 (100), 45 (74), 41 (61) [ $\left.\mathrm{C}_{3} \mathrm{H}_{5}^{+}\right] .-\mathrm{C}_{26} \mathrm{H}_{33} \mathrm{O}_{4}$ (408.54).

endo-1,7,8,9,10-Pentacyclopropyl-4-phenyl-4-azatricyclo[5.2.1.0 $\left.{ }^{2,6}\right]$ dec-8-en-3,5-dion (102):

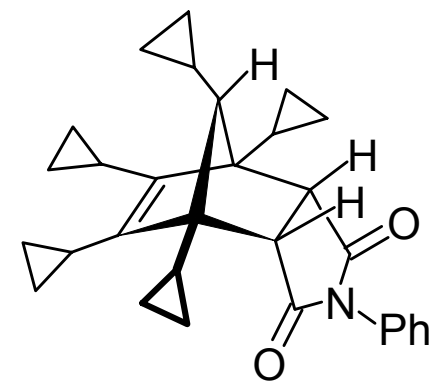

Nach AAV 4 wurden $522 \mathrm{mg}$ 1,2,3,4,5-Pentacyclopropylcyclopentadien (15) (1.96 mmol) mit $339 \mathrm{mg} N$-Phenylmaleimid (98) $(1.96 \mathrm{mmol})$ in $6 \mathrm{ml}$ Toluol für $20 \mathrm{~h}$ bei $100^{\circ} \mathrm{C}$ gerührt. Säulenchromatographische Reinigung des Rohprodukts an $25 \mathrm{~g}$ Kieselgel (Säule $2.5 \times 11 \mathrm{~cm}, \operatorname{Pentan} \mathrm{Et}_{2} \mathrm{O}=10: 1, R_{\mathrm{f}}=0.08$ ) lieferte $533 \mathrm{mg}$ des Produkts $(1.21 \mathrm{mmol}, 62 \%)$ als farblosen, amorphen Feststoff vom Schmp. $139{ }^{\circ}$ C. - IR (Film): v = $3452 \mathrm{~cm}^{-1}, 3074(\mathrm{C}-\mathrm{H}), 3003$ (C-H), 2901 (C-H), 1765 $(\mathrm{C}=\mathrm{O}), 1705(\mathrm{C}=\mathrm{O}), 1597,1501,1456,1430,1382,1294,1275,1194,1108,1096,1073$, $1048,1020,968,950,933,910,892,877,862,824,788,772,738,722,708,693,654,633$, 622, 596, 567, 507, 493, 469. - ${ }^{1} \mathrm{H}-\mathrm{NMR}\left(600 \mathrm{MHz}, \mathrm{CDCl}_{3}\right): \delta=0.19\left(\mathrm{~m}_{\mathrm{c}}, 2 \mathrm{H}, c \mathrm{Pr}-\mathrm{H}\right), 0.35$ $\left(\mathrm{m}_{\mathrm{c}}, 2 \mathrm{H}, c \operatorname{Pr}-\mathrm{H}\right), 0.37-0.43(\mathrm{~m}, 4 \mathrm{H}, c \mathrm{Pr}-\mathrm{H}), 0.52\left(\mathrm{~m}_{\mathrm{c}}, 1 \mathrm{H}, c \mathrm{Pr}-\mathrm{H}\right), 0.66\left(\mathrm{~d},{ }^{3} J=9.8 \mathrm{~Hz}, 1 \mathrm{H}\right.$, 10-H), 0.66-0.77 (m, 8 H, $c$ Pr-H), $0.79\left(\mathrm{~m}_{\mathrm{c}}, 2 \mathrm{H}, c \operatorname{Pr}-\mathrm{H}\right), 1.31,1.37,1.48\left(\mathrm{~m}_{\mathrm{c}}, 6 \mathrm{H}, c \operatorname{Pr}-\mathrm{H}\right)$, 
2.75 (s, 2 H, 2-H, 6-H), $7.13\left(\mathrm{~d},{ }^{3} J=8.0 \mathrm{~Hz}, 2 \mathrm{H}, \mathrm{H}_{\mathrm{ortho}}\right), 7.31$ (t, $\left.{ }^{3} J=8.0 \mathrm{~Hz}, 1 \mathrm{H}, \mathrm{H}_{\text {para }}\right), 7.40$ $\left(\mathrm{t},{ }^{3} J=8.0 \mathrm{~Hz}, 2 \mathrm{H}, \mathrm{H}_{\text {meta }}\right) .-{ }^{13} \mathrm{C}-\mathrm{NMR}\left(150 \mathrm{MHz}, \mathrm{CDCl}_{3}, \mathrm{DEPT}\right): \delta=0.6,3.1(-, 4 \mathrm{C}$, cPr-C), 4.9 (-, 2 C , cPr-C), 5.7 (-, 2 C,$c$ Pr-C), 6.3 (+, cPr-C), 6.5 (-, 2 C, $c$ Pr-C), 9.3 (+, 2 C, cPr-C), 11.1 (+, 2 C, cPr-C), 46.9 (+, 2 C, C-2, C-6), 63.4 (C quart, 2 C, C-1, C-7), $75.9(+$, C-10), 126.5 (+, 2 C, Ar- $\left.\mathrm{C}_{\text {meta }}\right), 128.3$ (+, Ar-C $\left.\mathrm{C}_{\text {para }}\right), 129.0$ (+, $\left.2 \mathrm{C}, \mathrm{Ar}-\mathrm{C}_{\text {ortho}}\right), 132.1$ ( $\mathrm{C}_{\text {quart }}$, Ar- $\left.\mathrm{C}_{\mathrm{ipso}}\right), 141.5$ ( $\left.\mathrm{C}_{\text {quart }}, 2 \mathrm{C}, \mathrm{C}-8, \mathrm{C}-9\right), 176.1$ (C $\left.\mathrm{C}_{\text {quart }}, 2 \mathrm{C}, \mathrm{C}-3, \mathrm{C}-5\right) .-\mathrm{MS}(\mathrm{EI}, 70 \mathrm{eV}), \mathrm{m} / \mathrm{z}$ (\%): 439 (60) $\left[\mathrm{M}^{+}\right], 265$ (100) $\left[\mathrm{C}_{20} \mathrm{H}_{25}{ }^{+}\right], 237$ (72), 225 (52) $\left[\mathrm{C}_{20} \mathrm{H}_{25}{ }^{+}-\mathrm{C}_{3} \mathrm{H}_{5}^{+}\right], 181$ (37), 155 (22), 129 (22), 115 (12), 91 (21), 69 (56), 41 (30) $\left[\mathrm{C}_{3} \mathrm{H}_{5}^{+}\right] .-\mathrm{C}_{30} \mathrm{H}_{33} \mathrm{NO}_{2}$ (439.60): ber. C $81.97 \%$, H 7.57\%, N 3.19\%; gef. C 81.78\%, H 7.34\%, N 3.04\%.

cis-3,4,5,6-Tetracyclopropylcyclohexa-1,4-dien-1,2-dicarbonsaüredimethylester (103): Nach

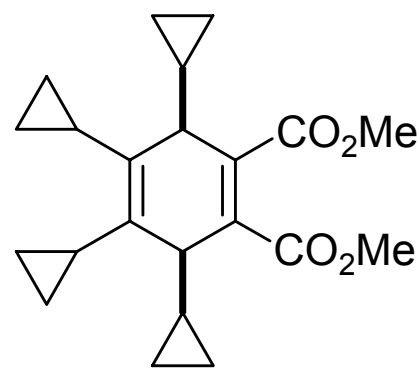
AAV 4 wurden $1.00 \mathrm{~g}$ 1,2,3,4-Tetracyclopropylbuta-1,3-dien (21) (4.67 mmol) mit $663 \mathrm{mg}$ Acetylendicarbonsäuredimethylester (97) $(4.67 \mathrm{mmol})$ in $8 \mathrm{ml}$ Toluol für $22 \mathrm{~h}$ bei $130{ }^{\circ} \mathrm{C}$ gerührt. Säulenchromatographische Reinigung des Rohproduktes an $110 \mathrm{~g}$ Kieselgel (Säule $3.5 \times 35 \mathrm{~cm}$, Pentan $\left./ \mathrm{Et}_{2} \mathrm{O}=10: 1, R_{\mathrm{f}}=0.30\right)$ lieferte $1.16 \mathrm{~g}$ des Produkts $(3.25 \mathrm{mmol}, 70 \%)$ in Form eines farblosen, amorphen Feststoffs vom Schmp. $65^{\circ} \mathrm{C}$. - IR (Film): $v=3433 \mathrm{~cm}^{-1}, 3082(\mathrm{C}-\mathrm{H})$, $3005(\mathrm{C}-\mathrm{H}), 2949(\mathrm{C}-\mathrm{H}), 2903(\mathrm{C}-\mathrm{H}), 2841(\mathrm{C}-\mathrm{H}), 1722(\mathrm{C}=\mathrm{O})$, 1663, 1636, 1457, 1387, $1348,1264,1196,1153,1116,1061,1022,972,947,916,888,862,831,795,761,747,667$, 561. - ${ }^{1} \mathrm{H}-\mathrm{NMR}\left(250 \mathrm{MHz}, \mathrm{C}_{6} \mathrm{D}_{6}\right): \delta=0.21\left(\mathrm{~m}_{\mathrm{c}}, 1 \mathrm{H}, c \mathrm{Pr}-\mathrm{H}\right), 0.31-0.71(\mathrm{~m}, 15 \mathrm{H}, c \mathrm{Pr}-\mathrm{H})$, $0.89\left(\mathrm{~m}_{\mathrm{c}}, 2 \mathrm{H}, c \mathrm{Pr}-\mathrm{H}\right), 1.60\left(\mathrm{~m}_{\mathrm{c}}, 2 \mathrm{H}, c \operatorname{Pr}-\mathrm{H}\right), 2.65\left(\mathrm{~d},{ }^{3} J_{\mathrm{H}}=8.1 \mathrm{~Hz}, 2 \mathrm{H}, 3,6-\mathrm{H}\right), 3.42(\mathrm{~s}, 6 \mathrm{H}$, OMe). $-{ }^{13} \mathrm{C}-\mathrm{NMR}\left(62.9 \mathrm{MHz}, \mathrm{C}_{6} \mathrm{D}_{6}, \mathrm{DEPT}\right): \delta=4.0,4.6,6.1,7.2,8.1(-, 8 \mathrm{C}, c \mathrm{Pr}-\mathrm{C}), 10.3$, 13.7, 17.8 (+, 4 C , cPr-C), 43.2 (+, 2 C, C-3,6), 51.6, 52.7 (+, 2 C, OMe), 132.4, 135.2, 138.5, $152.0\left(\mathrm{C}_{\text {quart }}, 4 \mathrm{C}, \mathrm{C}-1,2, \mathrm{C}-3,4\right), 168.7\left(\mathrm{C}_{\text {quart }}, 2 \mathrm{C}, \mathrm{C}=\mathrm{O}\right)$. - MS (EI, $\left.70 \mathrm{eV}\right), \mathrm{m} / z(\%): 356(97)$ $\left[\mathrm{M}^{+}\right], 325$ (36), 297 (63) $\left[\mathrm{M}^{+}-\mathrm{C}_{2} \mathrm{H}_{3} \mathrm{O}_{2}\right], 283$ (100), 265 (72), 255 (42), 237 (84), 195 (39), 181 (37), 165 (44), 155 (39), 128 (32), 91, (22), 77 (11), 59 (24) $\left[\mathrm{C}_{2} \mathrm{H}_{3} \mathrm{O}_{2}{ }^{+}\right], 41$ (41) $\left[\mathrm{C}_{3} \mathrm{H}_{5}{ }^{+}\right] .-\mathrm{C}_{22} \mathrm{H}_{28} \mathrm{O}_{4}$ (356.47): ber. C 74.13\%, H 7.92\%; gef. C 74.48\%, H 7.70\%. 
syn-4,5,6,7-Tetracyclopropyl-2-phenyl-3a,4,7,7a-tetrahydroisoindol-1,3-dion (104): Nach

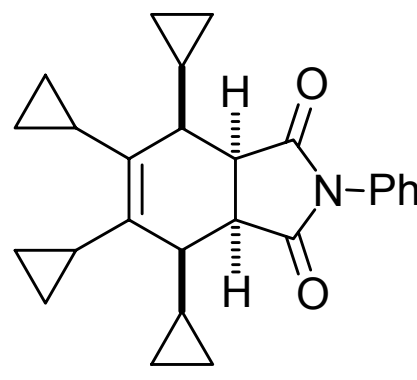

AAV 4 wurden $1.00 \mathrm{~g}$ 1,2,3,4-Tetracyclopropylbuta-1,3-dien

(4.67 mmol) mit $\quad 815 \mathrm{mg} \quad N$-Phenylmaleimid

(98)

(4.71 mmol) in $8 \mathrm{ml}$ Toluol für $22 \mathrm{~h}$ bei $130{ }^{\circ} \mathrm{C}$ gerührt. Säulenchromatographische Reinigung des Rohproduktes an $110 \mathrm{~g}$ Kieselgel (Säule $3.5 \times 35 \mathrm{~cm}$, Pentan $/ \mathrm{Et}_{2} \mathrm{O}=5: 1, \quad R_{\mathrm{f}}=0.31$ )

lieferte $1.26 \mathrm{~g}$ des Produkts $(3.26 \mathrm{mmol}, 70 \%)$ in Form eines farblosen, amorphen Feststoffs vom Schmp. $152{ }^{\circ} \mathrm{C} .-$ IR (Film): $v=3078 \mathrm{~cm}^{-1}(\mathrm{C}-\mathrm{H}), 3002(\mathrm{C}-\mathrm{H}), 2835(\mathrm{C}-\mathrm{H}), 1770$ $(\mathrm{C}=\mathrm{O}), 1707(\mathrm{C}=\mathrm{O}), 1598,1500,1456,1428,1379,1322,1243,1166,1105,1028,909,880$, 869, 821, 754, 733, 703, 690, 624, 615, 583, 463. - ${ }^{1} \mathrm{H}-\mathrm{NMR}\left(300 \mathrm{MHz}, \mathrm{CDCl}_{3}\right): \delta=0.13$ $\left(\mathrm{m}_{\mathrm{c}}, 4 \mathrm{H}, c \operatorname{Pr}-\mathrm{H}\right), 0.42\left(\mathrm{~m}_{\mathrm{c}}, 2 \mathrm{H}, c \operatorname{Pr}-\mathrm{H}\right), 0.53\left(\mathrm{~m}_{\mathrm{c}}, 2 \mathrm{H}, c \operatorname{Pr}-\mathrm{H}\right), 0.62-0.89(\mathrm{~m}, 8 \mathrm{H}, c \operatorname{Pr}-\mathrm{H})$, $1.23\left(\mathrm{~d},{ }^{3} J=10.6 \mathrm{~Hz},{ }^{3} J=3.7 \mathrm{~Hz}, 2 \mathrm{H}, 4,7-\mathrm{H}\right), 1.31-1.45(\mathrm{~m}, 2 \mathrm{H}, c \operatorname{Pr}-\mathrm{H}), 1.76\left(\mathrm{~m}_{\mathrm{c}}, 2 \mathrm{H}\right.$, $c$ Pr-H), $3.21\left(\mathrm{dd},{ }^{3} J=3.7 \mathrm{~Hz},{ }^{4} J=1.9 \mathrm{~Hz}, 2 \mathrm{H}, 3 \mathrm{a}, 7 \mathrm{a}-\mathrm{H}\right), 7.12-7.19$ (m, $2 \mathrm{H}$, Ar- $\mathrm{H}_{\text {ortho}}$ ), 7.29-7.37 (m, $\left.1 \mathrm{H}, \mathrm{Ar}-\mathrm{H}_{\text {para }}\right), 7.29-7.37$ (m, $2 \mathrm{H}$, Ar- $\left.\mathrm{H}_{\text {meta }}\right) .-{ }^{13} \mathrm{C}-\mathrm{NMR}\left(75 \mathrm{MHz}, \mathrm{CDCl}_{3}\right.$, DEPT): $\delta=4.4$ (-, 2 C, $c$ Pr-C), 8.1 (-, 2 C,$c \operatorname{Pr}-\mathrm{C}), 8.3$ (-, 2 C, $c$ Pr-C), 8.7 (-, 2 C, $c$ Pr-C), $10.4(+, 2 \mathrm{C}, c \operatorname{Pr}-\mathrm{C}), 12.5$ (+, 2 C,$c \operatorname{Pr}-\mathrm{C}), 45.5$ (+, 2 C, C-4,7), 48.2 (+, 2 C, C-3a,7a), 126.3 (+, 2 C, $\left.\mathrm{C}_{\text {ortho }}\right), 128.3\left(+, \mathrm{C}_{\text {para }}\right), 128.9\left(+, 2 \mathrm{C}, \mathrm{C}_{\text {meta }}\right), 131.9\left(\mathrm{C}_{\text {quart }}, \mathrm{C}_{\mathrm{ipso}}\right), 138.9\left(\mathrm{C}_{\text {quart }}, 2 \mathrm{C}\right.$, C-5,6), $177.3\left(\mathrm{C}_{\text {quart }}, 2 \mathrm{C}, \mathrm{C}=\mathrm{O}\right) .-\mathrm{MS}(\mathrm{EI}, 70 \mathrm{eV}), \mathrm{m} / z(\%): 387$ (50) $\left[\mathrm{M}^{+}\right], 358$ (60), 344 (21), 330 (10), 316 (9), 302 (4), 268 (3), 228 (18), 212 (28) [ $\left.\mathrm{C}_{16} \mathrm{H}_{20}{ }^{+}\right], 199$ (38), 169 (45), 159 (82), 145 (90), 131 (100), 117 (93), 91 (79), 77 (36) $\left[\mathrm{C}_{6} \mathrm{H}_{5}^{+}\right], 67$ (22), 41 (28) $\left[\mathrm{C}_{3} \mathrm{H}_{5}^{+}\right] .-$ $\mathrm{C}_{26} \mathrm{H}_{29} \mathrm{NO}_{2}$ (387.53): ber. C 80.59\%, H 7.54\%, N 3.61\%; gef. C 80.83\%, H 7.30\%, N 3.75\%.

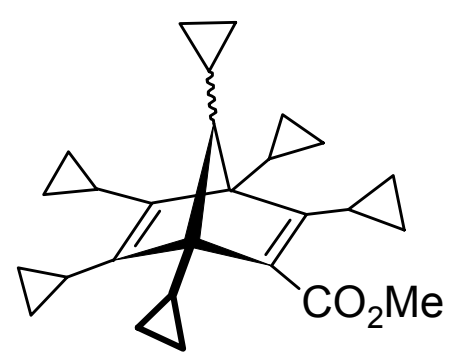

Variante A: Nach AAV 4 wurden $227 \mathrm{mg}$ 1,2,3,4,5Pentacyclopropylcyclopentadien (15) $(850 \mu \mathrm{mol})$ mit $151 \mathrm{mg}$ Cyclopropylacetylencarbonsäuremethylester (109) (1.22 mmol) in $5 \mathrm{ml}$ Toluol für $18 \mathrm{~h}$ bei $160^{\circ} \mathrm{C}$ gerührt. Säulenchromatographische Reinigung des Rohproduktes an $15 \mathrm{~g}$ 
Kieselgel (Säule $2.5 \times 6 \mathrm{~cm}$, Pentan/ $\mathrm{Et}_{2} \mathrm{O}=15: 1, R_{\mathrm{f}}=0.36$ ) lieferte $30 \mathrm{mg}$ des Produkts $(77 \mu \mathrm{mol}, 9 \%)$ als 1:1 Gemisch zweier Diastereomeren in Form eines farblosen Öls. Variante B: Nach AAV 5 wurden $150 \mathrm{mg}$ 1,2,3,4,5-Pentacyclopropylcyclopentadien (15) (563 $\mu \mathrm{mol})$ mit $180 \mathrm{mg}$ Cyclopropylacetylencarbonsäuremethylester (109) (1.45 mmol) in $2.5 \mathrm{ml}$ Toluol für $3 \mathrm{~d}$ bei $100^{\circ} \mathrm{C}$ und $10 \mathrm{kbar}$ umgesetzt. Säulenchromatographische Reinigung des Rohproduktes an $20 \mathrm{~g}$ Kieselgel (Säule $2.5 \times 10 \mathrm{~cm}$, Pentan/ $\mathrm{Et}_{2} \mathrm{O}=15: 1$, $\left.R_{\mathrm{f}}=0.36\right)$ lieferte $195 \mathrm{mg}$ des Produkts $(499 \mu \mathrm{mol}, 89 \%)$ als 1:1 Gemisch zweier Diastereomeren in Form eines farblosen Öls. - IR (Film): v=3081 cm ${ }^{-1}(\mathrm{C}-\mathrm{H}), 3006(\mathrm{C}-\mathrm{H})$, $2946(\mathrm{C}-\mathrm{H}), 2893(\mathrm{C}-\mathrm{H}), 1716(\mathrm{C}=\mathrm{O}), 1460,1431,1284,1258,1228,1200,1136,1096$, 1048, 1022, 880, 823. $-{ }^{1} \mathrm{H}-\mathrm{NMR}\left(250 \mathrm{MHz}, \mathrm{CDCl}_{3}\right): \delta=0.11\left(\mathrm{~m}_{\mathrm{c}}, 4 \mathrm{H}, c \operatorname{Pr}-\mathrm{H}\right), 0.29-1.46$ $(\mathrm{m}, 50 \mathrm{H}, 7-\mathrm{H}, c \operatorname{Pr}-\mathrm{H}), 1.61,1.92,2.21\left(\mathrm{~m}_{\mathrm{c}}, 8 \mathrm{H}, c \mathrm{Pr}-\mathrm{H}\right), 2.73,2.77$ (s, $\left.6 \mathrm{H}, \mathrm{OMe}\right)$. ${ }^{13} \mathrm{C}-\mathrm{NMR}\left(62.9 \mathrm{MHz}, \mathrm{CDCl}_{3}, \mathrm{DEPT}\right): \delta=2.9,3.2,3.3,3.4,3.5,3.7,4.3,5.5,5.8,5.9,6.0$, 6.1, 6.3, 6.7, 6.8, 7.1, 7.3, 7.6, 7.9, 8.1 (-, $24 \mathrm{C}, c \operatorname{Pr}-\mathrm{C}), 8.7,9.0,9.3,9.4,10.4,10.9,11.2$, 11.3, 11.6, 11.9 (+, $12 \mathrm{C}, c \mathrm{Pr}-\mathrm{C}), 50.9,51.2(+, 2 \mathrm{C}, \mathrm{OMe}), 66.9,67.5,68.7,69.0\left(\mathrm{C}_{\text {quart }}, 4 \mathrm{C}\right.$, C-1, C-4), 88.9, 89.9 (+, 2 C, C-7), 138.4, 142.3, 148.2, 148.6, 152.6, 153.5, 163.1, 163.9, 169.4, $170.1\left(\mathrm{C}_{\text {quart }}, 10 \mathrm{C}, \mathrm{C}-2,3,5,6, \mathrm{C}=\mathrm{O}\right) .-\mathrm{MS}(\mathrm{EI}, 70 \mathrm{eV}), \mathrm{m} / z(\%): 390(100)\left[\mathrm{M}^{+}\right], 359$ (6) $\left[\mathrm{M}^{+}-\mathrm{OCH}_{3}\right], 349$ (22) $\left[\mathrm{M}^{+}-\mathrm{C}_{3} \mathrm{H}_{5}\right], 331$ (20) $\left[\mathrm{M}^{+}-\mathrm{CO}_{2} \mathrm{CH}_{3}\right], 303$ (6), 284 (12), 255 (12), 219 (8), 179 (9), 167 (15), 155 (5), 91 (4), 84 (37), 73 (2), 41 (3) $\left[\mathrm{C}_{3} \mathrm{H}_{5}^{+}\right] .-\mathrm{C}_{27} \mathrm{H}_{34} \mathrm{O}_{2}$ (390.57).

1,3,4,5,6,7-Hexacyclopropylbicyclo[2.2.1]hepta-2,5-dien-2-carbaldehyd (112): Variante A:
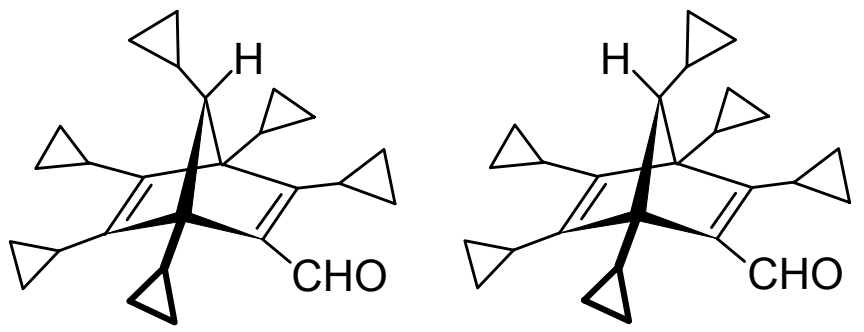

Nach AAV 4 wurden 225 mg 1,2,3,4,5Pentacyclopropylcyclopentadien (845 $\mu \mathrm{mol})$ mit $88 \mathrm{mg}$ Cyclopropylacetylencarbaldehyd $(935 \mu \mathrm{mol})$ in $5 \mathrm{ml}$ Toluol für $18 \mathrm{~h}$ bei $160^{\circ} \mathrm{C}$ gerührt. Säulenchromatographische Reinigung des Rohproduktes an 20 g Kieselgel (Säule $2.5 \times 12 \mathrm{~cm}, \quad$ Pentan $\left./ \mathrm{Et}_{2} \mathrm{O}=10: 1\right)$ lieferte $67 \mathrm{mg}$ des einen Diastereomers $\left(R_{\mathrm{f}}=0.39\right.$, 
$186 \mu \mathrm{mol}, 22 \%)$ und $128 \mathrm{mg}$ des anderen Diastereomers $\left(R_{\mathrm{f}}=0.32,355 \mu \mathrm{mol}, 42 \%\right)$ als farblose Öle. - Variante B: Nach AAV 5 wurden $150 \mathrm{mg}$ 1,2,3,4,5-Pentacyclopropylcyclopentadien $(563 \mu \mathrm{mol})$ mit $180 \mathrm{mg}$ Cyclopropylacetylencarbonsäuremethylester (1.45 mmol) in $2.5 \mathrm{ml}$ Toluol für $3 \mathrm{~d}$ bei $100^{\circ} \mathrm{C}$ und $10 \mathrm{kbar}$ umgesetzt. Säulenchromatographische Reinigung des Rohproduktes an $15 \mathrm{~g}$ Kieselgel (Säule $2.5 \times 10 \mathrm{~cm}$, Pentan $/ \mathrm{Et}_{2} \mathrm{O}=$ 15:1) lieferte $126 \mathrm{mg}$ des einen Diastereomers (DS 1) $\left(R_{\mathrm{f}}=0.22,349 \mu \mathrm{mol}, 62 \%\right)$ und $49 \mathrm{mg}$ des anderen Diastereomers (DS 2) $\left(R_{\mathrm{f}}=0.17,136 \mu \mathrm{mol}, 24 \%\right)$ als farblose Öle. - IR (Film): $v=3434,3081 \mathrm{~cm}^{-1}(\mathrm{C}-\mathrm{H}), 3005(\mathrm{C}-\mathrm{H}), 2896(\mathrm{C}-\mathrm{H}), 1764(\mathrm{C}=\mathrm{O}), 1742(\mathrm{C}=\mathrm{O}), 1661$, $1460,1427,1389,1306,1270,1229,1172,1128,1049,1023,946,885,825,736,564 .-$ DS 1: ${ }^{1} \mathrm{H}-\mathrm{NMR}\left(250 \mathrm{MHz}, \mathrm{CDCl}_{3}\right): \delta=0.05-0.24(\mathrm{~m}, 2 \mathrm{H}, c \operatorname{Pr}-\mathrm{H}), 0.31-1.11(\mathrm{~m}, 23 \mathrm{H}$, $c$ Pr-H), 1.11-1.41 (m, 4 H, $c$ Pr-H), 1.53, $1.74\left(\mathrm{~m}_{\mathrm{c}}, 2 \mathrm{H}, c \operatorname{Pr}-\mathrm{H}\right), 10.04$ (s, $\left.1 \mathrm{H}, \mathrm{CHO}\right) .-$ ${ }^{13} \mathrm{C}-\mathrm{NMR}\left(62.9 \mathrm{MHz}, \mathrm{CDCl}_{3}, \mathrm{DEPT}\right): \delta=3.6,3.7,3.8,4.3,5.6,6.4,6.7,7.4,7.8,7.9(-$, $11 \mathrm{C}, c \operatorname{Pr}-\mathrm{C}), 8.9$ (+, $c$ Pr-C), 9.0 (-, $c \operatorname{Pr}-\mathrm{C}), 9.4,9.9,10.8,11.7$ (+, $5 \mathrm{C}, c \operatorname{Pr}-\mathrm{C}), 66.4,70.3$ $\left(\mathrm{C}_{\text {quart }}, 2 \mathrm{C}, \mathrm{C}-1,4\right), 88.2$ (+, C-7), 147.4, 149.7, $150.8\left(\mathrm{C}_{\text {quart }}, 3 \mathrm{C}, \mathrm{C}-3,5,6\right), 185.1,187.6$ $\left(\mathrm{C}_{\text {quart }}, 2 \mathrm{C}, \mathrm{C}-2, \mathrm{C}=\mathrm{O}\right) .-\mathrm{DS} 2:{ }^{1} \mathrm{H}-\mathrm{NMR}\left(250 \mathrm{MHz}, \mathrm{CDCl}_{3}\right): \delta=0.04-0.26(\mathrm{~m}, 2 \mathrm{H}, c \mathrm{Pr}-\mathrm{H})$, 0.27-0.89 (m, $20 \mathrm{H}, c \mathrm{Pr}-\mathrm{H}), 0.90-1.20(\mathrm{~m}, 5 \mathrm{H}, c \mathrm{Pr}-\mathrm{H}), 1.24\left(\mathrm{~d},{ }^{3} J=9.2 \mathrm{~Hz}, 1 \mathrm{H}, 7-\mathrm{H}\right)$, 1.31-1.51 (m, $2 \mathrm{H}, c \operatorname{Pr}-\mathrm{H}), 2.02\left(\mathrm{~m}_{\mathrm{c}}, 1 \mathrm{H}, c \mathrm{Pr}-\mathrm{H}\right), 10.07$ (s, $\left.1 \mathrm{H}, \mathrm{CHO}\right) .-{ }^{13} \mathrm{C}-\mathrm{NMR}$ (62.9 MHz, $\left.\mathrm{CDCl}_{3}, \mathrm{DEPT}\right): \delta=3.6,3.7,4.4,4.7,5.8,6.0,6.1,6.9,8.4,8.5(-, 11 \mathrm{C}, c \operatorname{Pr}-\mathrm{C})$, 8.9 (+, $c$ Pr-C), 9.4 (-, cPr-C), 9.6, 9.8, 11.5, 11.8, 12.6 (+, $5 \mathrm{C}, c \operatorname{Pr}-\mathrm{C}), 65.5,70.4\left(\mathrm{C}_{\text {quart }}, 2 \mathrm{C}\right.$, $\mathrm{C}-1,4), 88.7$ (+, C-7), 146.5, 152.0, $154.6\left(\mathrm{C}_{\text {quart }}, 3 \mathrm{C}, \mathrm{C}-3,5,6\right), 182.7,187.8\left(\mathrm{C}_{\text {quart }}, 2 \mathrm{C}, \mathrm{C}-2\right.$, $\mathrm{C}=\mathrm{O}) .-\mathrm{MS}(\mathrm{EI}, 70 \mathrm{eV}), \mathrm{m} / z(\%): 360$ (100) $\left[\mathrm{M}^{+}\right], 331$ (18) $\left[\mathrm{M}^{+}-\mathrm{CHO}\right], 319$ (58) $\left[\mathrm{M}^{+}-\mathrm{C}_{3} \mathrm{H}_{5}\right], 291$ (12), 261 (9), 247 (11), 237 (19), 225 (17), 181 (20), 165 (33), 155 (20), 115 (18), 91 (24), 79 (19), 55 (14), 41 (26) $\left[\mathrm{C}_{3} \mathrm{H}_{5}{ }^{+}\right] .-\mathrm{C}_{26} \mathrm{H}_{32} \mathrm{O}$ (360.54). 


\section{1,4,5,6,7-Pentacyclopropyl-7-methoxybicyclo[2.2.1] hepta-2,5-dien-2,3-dicarbonsaüredi-}

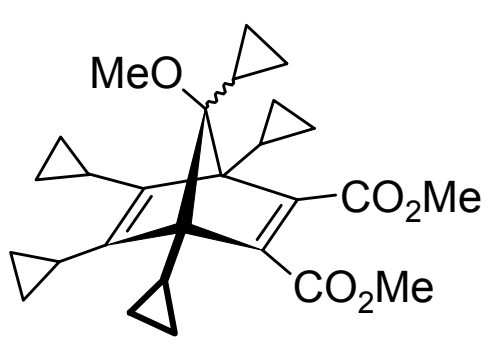

methylester (113): Nach AAV 4 wurden $94 \mathrm{mg}$ 1,2,3,4,5-Pentacyclopropyl-5-methoxycyclopenta-1,3-dien (65) $(318 \mu \mathrm{mol}) \quad$ mit $56 \mathrm{mg}$ Acetylendicarbonsäuredimethylester (97) $(398 \mu \mathrm{mol})$ in $3 \mathrm{ml}$ Toluol für $48 \mathrm{~h}$ bei $160{ }^{\circ} \mathrm{C}$ gerührt. Säulenchromatographische Reinigung des Rohproduktes an $10 \mathrm{~g}$ Kieselgel (Säule $2 \times 4 \mathrm{~cm}$, Pentan $/ \mathrm{Et}_{2} \mathrm{O}=10: 1, R_{\mathrm{f}}=0.20$ ) lieferte $35 \mathrm{mg}$ des Produkts $(79.8 \mu \mathrm{mol}, 25 \%)$ als 1:1 Gemisch zweier Diastereomeren in Form eines farblosen Öls. - IR (Film): v= $3087 \mathrm{~cm}^{-1}(\mathrm{C}-\mathrm{H}), 3007(\mathrm{C}-\mathrm{H}), 2949(\mathrm{C}-\mathrm{H}), 2836(\mathrm{C}-\mathrm{H})$, $1722(\mathrm{C}=\mathrm{O}), 1600,1433,1285,1244,1194,1108,1032,872,828,693 .-{ }^{1} \mathrm{H}-\mathrm{NMR}$ $\left(250 \mathrm{MHz}, \mathrm{CDCl}_{3}\right): \delta=0.17-0.35(\mathrm{~m}, 6 \mathrm{H}, c \operatorname{Pr}-\mathrm{H}), 0.39-0.56(\mathrm{~m}, 11 \mathrm{H}, c \operatorname{Pr}-\mathrm{H}), 0.56-0.88$ (m, $22 \mathrm{H}, c \mathrm{Pr}-\mathrm{H}), 1.22-1.45$ (m, $11 \mathrm{H}, c \mathrm{Pr}-\mathrm{H}), 3.25,3.35$ (s, $6 \mathrm{H}, 7-\mathrm{OMe}), 3.70,3.72$ (s, $12 \mathrm{H}$, $\left.\mathrm{CO}_{2} \mathrm{Me}\right) .-{ }^{13} \mathrm{C}-\mathrm{NMR}\left(62.9 \mathrm{MHz}, \mathrm{CDCl}_{3}, \mathrm{DEPT}\right): \delta=3.0,3.5,3.9,4.2,6.2,7.5(-, 20 \mathrm{C}$, $c$ Pr-C), 8.9, 9.2, 9.4, 9.6, 12.6, $12.9(+, 10 \mathrm{C}, c \mathrm{Pr}-\mathrm{C}), 51.8\left(+, 2 \mathrm{C}, 7-\mathrm{OCH}_{3}\right), 58.3(+, 4 \mathrm{C}$, $\left.\mathrm{CO}_{2} \mathrm{CH}_{3}\right), 70.1,70.3\left(\mathrm{C}_{\text {quart }}, 4 \mathrm{C}, \mathrm{C}-1,4\right), 113.4,113.5$ ( $\left.\mathrm{C}_{\text {quart }}, 2 \mathrm{C}, \mathrm{C}-7\right), 147.9^{*}, 148.7 *\left(\mathrm{C}_{\text {quart }}\right.$, $4 \mathrm{C}, \mathrm{C}-5,6), 150.7^{*}, 151.8^{*}\left(\mathrm{C}_{\text {quart }}, 4 \mathrm{C}, \mathrm{C}-2,3\right), 166.5,166.7\left(\mathrm{C}_{\text {quart }}, 4 \mathrm{C}, \mathrm{C}=\mathrm{O}\right) .-\mathrm{MS}(\mathrm{EI}$, $70 \mathrm{eV}), m / z(\%): 438$ (3) $\left[\mathrm{M}^{+}\right], 407$ (4) $\left[\mathrm{M}^{+}-\mathrm{CH}_{3} \mathrm{O}\right], 397$ (8) $\left[\mathrm{M}^{+}-\mathrm{C}_{3} \mathrm{H}_{5}\right], 379$ (100) $\left[\mathrm{M}^{+}-\mathrm{C}_{2} \mathrm{H}_{3} \mathrm{O}_{2}\right], 347$ (3), 315 (9), 291 (9), 247 (5), 205 (5), 178 (6), 165 (8), 152 (4), 141 (2), 115 (6), 85 (2), 69 (18), 41 (7) $\left[\mathrm{C}_{3} \mathrm{H}_{5}{ }^{+}\right] .-\mathrm{C}_{27} \mathrm{H}_{34} \mathrm{O}_{5}$ (438.55): ber. $\mathrm{C} 72.87 \%$, H 8.47\%; gef. C $74.13 \%, \mathrm{H} 7.57 \%$.

syn-1,6,7,7-Tetrachlor-2,3,4,5-tetracyclopropylbicyclo[4.1.0]hept-3-en (124): Nach AAV 4

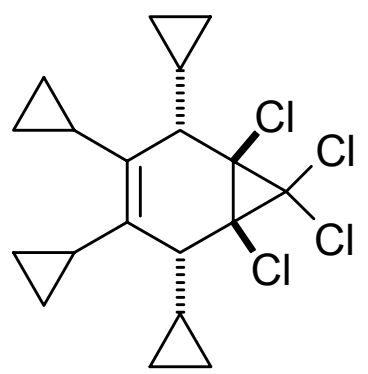
wurden $\quad 300 \mathrm{mg} \quad$ 1,2,3,4-Tetracyclopropylbuta-1,3-dien (1.33 mmol) mit $237 \mathrm{mg}$ Tetrachlorcyclopropen (123) (1.33 mmol) in $5 \mathrm{ml}$ Toluol für $16 \mathrm{~h}$ bei $120^{\circ} \mathrm{C}$ gerührt. Säulenchromatographische Reinigung des Rohproduktes an 25 g Kieselgel (Säule $1.5 \times 11 \mathrm{~cm}$, Pentan $/ \mathrm{Et}_{2} \mathrm{O}=50: 1, R_{\mathrm{f}}=0.75$ ) lieferte $264 \mathrm{mg}$ des Produkts $(673 \mu \mathrm{mol}, 51 \%)$ in Form eines farblosen Öls. - IR (Film): $v=3081 \mathrm{~cm}^{-1}$ 
(C-H), $3005(\mathrm{C}-\mathrm{H}), 2874(\mathrm{C}-\mathrm{H})$, 1697, 1460, 1430, 1378, 1309, 1243, 1170, 1098, 1050, 1027, 966, 938, 869, 825, 730, $656(\mathrm{C}-\mathrm{Cl}), 607 .-{ }^{1} \mathrm{H}-\mathrm{NMR}\left(250 \mathrm{MHz}, \mathrm{C}_{6} \mathrm{D}_{6}\right): \delta=0.18-0.76$ (m, $16 \mathrm{H}, c \operatorname{Pr}-\mathrm{H}), 1.11-1.34$ (m, $4 \mathrm{H}, c \operatorname{Pr}-\mathrm{H}), 1.11-1.34$ (m, $3 \mathrm{H}, c \operatorname{Pr}-\mathrm{H}), 1.61\left(\mathrm{~d},{ }^{3} J=9.5 \mathrm{~Hz}\right.$, $2 \mathrm{H}, 2,5-\mathrm{H}) .-{ }^{13} \mathrm{C}-\mathrm{NMR}\left(62.9 \mathrm{MHz}, \mathrm{C}_{6} \mathrm{D}_{6}, \mathrm{DEPT}\right): \delta=5.5,6.6,7.9,8.2(-, 8 \mathrm{C}, c \operatorname{Pr}-\mathrm{C}), 13.4$ (+, 4 C,$c \operatorname{Pr}-\mathrm{C}), 49.9$ (+, 2 C $, \mathrm{C}-2,5), 63.3$ (C $\left.\mathrm{C}_{\text {quart }}, 2 \mathrm{C}, \mathrm{C}-1,6\right), 73.1\left(\mathrm{C}_{\text {quart }}, \mathrm{C}-7\right), 137.5\left(\mathrm{C}_{\text {quart }}\right.$, 2 C, C-3,4). - MS (EI, $70 \mathrm{eV}), m / z(\%): 398 / 396 / 394 / 392 / 390(<1 /<1 / 1 / 2 / 2)\left[\mathrm{M}^{+}\right], 371(28)$, 363/361/359/357 (<1/6/16/18) [M+ - Cl], 320 (4), 279 (12), 258 (30), 229 (28), 215 (43), 189 (55), 161 (100), 131 (44), 119 (51), 105 (54), 91 (86), 69 (82), 41 (57) $\left[\mathrm{C}_{3} \mathrm{H}_{5}^{+}\right] .-\mathrm{C}_{19} \mathrm{H}_{22} \mathrm{Cl}_{4}$ (392.19).

1,7,8,8-Tetrachlor-2,3,5,6-tetracyclopropyl-4-oxatricyclo[5.1.0.0 $\left.0^{3,5}\right]$ octan (125): Zu einer

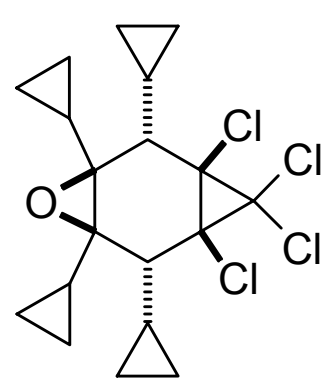
Lösung aus $258 \mathrm{mg}$ 1,6,7,7-Tetrachlor-2,3,4,5-tetracyclopropylbicyclo[4.1.0]hept-3-en (124) $(650 \mu \mathrm{mol})$ in $5 \mathrm{ml}$ Dichlormethan wurden bei Raumtemp. $450 \mathrm{mg} m$-Chlorperoxybenzoesäure (ca. 77\%ig, $1.95 \mathrm{mmol}$ ) gegeben und die Reaktionsmischung $1.5 \mathrm{~h}$ bei dieser Temp. gerührt. Danach wurde auf $5 \mathrm{ml}$ Wasser gegeben und die abgetrennte organische Phase mit je $5 \mathrm{ml}$ gesättigter $\mathrm{NaHCO}_{3^{-}}$, $\mathrm{Na}_{2} \mathrm{SO}_{3}$ - und $\mathrm{NaCl}-\mathrm{Lösung}$ gewaschen. Nach Trocknen der organischen Phase über $\mathrm{MgSO}_{4}$ und Entfernen des Lösungsmittels i. Vak. wurde der Rückstand säulenchromatographisch an $20 \mathrm{~g}$ Kieselgel (Säule $2.5 \times 12 \mathrm{~cm}$ ) gereinigt $\left(\right.$ Pentan $\left./ \mathrm{Et}_{2} \mathrm{O}=15: 1, R_{\mathrm{f}}=0.15\right)$. Man erhielt $242 \mathrm{mg}$ des Produktes (592 $\mu \mathrm{mol}, 91 \%)$ als farblosen, amorphen Feststoff. - IR (Film): $v=$ $3081 \mathrm{~cm}^{-1}$ (C-H), 3007 (C-H), 1464, 1431, 1287, 1219, 1141, 1114, 1092, 1024, 972, 869, 834, 709, $662(\mathrm{C}-\mathrm{Cl}), 567(\mathrm{C}-\mathrm{Cl}) .-{ }^{1} \mathrm{H}-\mathrm{NMR}\left(250 \mathrm{MHz}, \mathrm{CDCl}_{3}\right): \delta=0.30\left(\mathrm{~m}_{\mathrm{c}}, 4 \mathrm{H}, c \mathrm{Pr}-\mathrm{H}\right)$, 0.46-0.63 (m, 4 H, $c$ Pr-H), 0.63-0.85 (m, 8 H, $c$ Pr-H), 0.86 (d, ${ }^{3} J=9.8$ Hz, 2 H, 2,6-H), 1.29 $\left(\mathrm{m}_{\mathrm{c}}, 2 \mathrm{H}, c \mathrm{Pr}-\mathrm{H}\right), 2.52\left(\mathrm{~m}_{\mathrm{c}}, 2 \mathrm{H}, c \mathrm{Pr}-\mathrm{H}\right) .-{ }^{13} \mathrm{C}-\mathrm{NMR}\left(62.9 \mathrm{MHz}, \mathrm{CDCl}_{3}, \mathrm{DEPT}\right): \delta=4.3,4.4$, 5.2, 6.7 (-, $8 \mathrm{C}, c \operatorname{Pr}-\mathrm{C}), 10.6,11.0(+, 4 \mathrm{C}, c \mathrm{Pr}-\mathrm{C}), 54.6(+, 2 \mathrm{C}, \mathrm{C}-2,5), 62.2\left(\mathrm{C}_{\text {quart }}, 2 \mathrm{C}\right.$, C-1,7), 66.5 (C quart $\left._{1}, 2 \mathrm{C}, \mathrm{C}-3,4\right), 73.3$ (C $\left.\mathrm{C}_{\text {quart }}, \mathrm{C}-8\right) .-\mathrm{MS} \quad(\mathrm{EI}, 70 \mathrm{eV}), \mathrm{m} / \mathrm{z}$ (\%): 410/408/406/404/402 $(<1 /<1 / 4 / 12 / 25 / 12) \quad\left[\mathrm{M}^{+}\right], 377 / 375 / 373371 \quad(<1 / 9 / 24 / 26) \quad\left[\mathrm{M}^{+}-\mathrm{Cl}\right]$, 


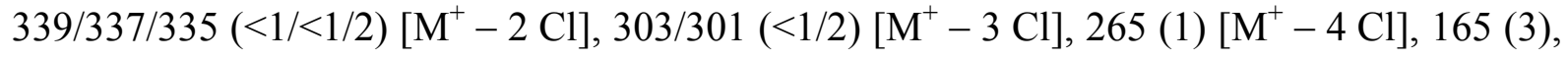
139 (3), 115 (3), 91 (15), 69 (100), 41 (34) $\left[\mathrm{C}_{3} \mathrm{H}_{5}{ }^{+}\right] .-\mathrm{C}_{19} \mathrm{H}_{22} \mathrm{OCl}_{4}$ (402.80): ber. C 55.91\%, H 5.43\%; gef. C $56.00 \%$, H 5.21\%.

2,3,4,5-Tetracyclopropylthiophen-1,1-dioxid (132): $\mathrm{Zu}$ einer Lösung von $1.38 \mathrm{~g}$

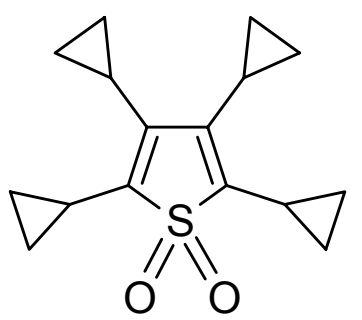
Biscyclopentadienylzirconiumdichlorid $(4.71 \mathrm{mmol})$ und $1.00 \mathrm{~g}$ Dicyclopropylethin $(9.42 \mathrm{mmol}) \quad$ in $60 \mathrm{ml}$ wasserfreiem Tetrahydrofuran wurden bei $-78^{\circ} \mathrm{C} 4.00 \mathrm{ml}$ einer Lösung von $n$ Butyllithium in Hexan (2.35 M, $9.40 \mathrm{mmol})$ getropft, das Kältebad entfernt und $1 \mathrm{~h}$ weiter gerührt, wobei die Lösung auftaute. Daraufhin wurde bei Raumtemp. 30 min lang trockenes Schwefeldioxidgas durch die Lösung geleitet und die Reaktionsmischung anschließend auf $50 \mathrm{ml}$ Wasser gegeben. Die abgetrennte wässrige Phase wurde mit $3 \times 15 \mathrm{ml}$ Dichlormethan extrahiert und die vereinigten organischen Phasen wurden mit $30 \mathrm{ml}$ gesättigter $\mathrm{NaHCO}_{3}$-Lösung gewaschen, über $\mathrm{MgSO}_{4}$ getrocknet und die Lösungsmittel i. Vak. entfernt. Man erhielt $2.50 \mathrm{~g}$ eines Rohproduktes (hauptsächlich das entsprechende Sulfoxid, $R_{\mathrm{f}}=0.92$ in $\mathrm{Et}_{2} \mathrm{O}$ und $R_{\mathrm{f}}=0.50$ in $\mathrm{CH}_{2} \mathrm{Cl}_{2}$ ), welches in $50 \mathrm{ml}$ Dichlormethan gelöst und mit $3.17 \mathrm{~g} \mathrm{~m}$-Chlorperbenzoesäure (ca. 77\%ig, $14.1 \mathrm{mmol}$ ) versetzt wurde. Nach 12stündigem Rühren bei Raumtemp. wurde diese Reaktionsmischung auf $50 \mathrm{ml}$ gesättigte $\mathrm{NaHCO}_{3}$-Lösung gegeben, die abgetrennte wässrige Phase mit $3 \times 15 \mathrm{ml}$ Dichlormethan extrahiert, die vereinigten organischen Phasen über $\mathrm{MgSO}_{4}$ getrocknet und die Lösungsmittel i. Vak. entfernt. Säulenchromatographische Reinigung des Rohproduktes an $80 \mathrm{~g}$ Kieselgel mit Dichlormethan als Eluent (Säule $\left.3 \times 25 \mathrm{~cm}, R_{\mathrm{f}}=0.61\right)$ ergab $673 \mathrm{mg}$ des Produktes $(2.44 \mathrm{mmol}, 51 \%)$ als farblosen amorphen Feststoff. - IR (Film): v = $3093 \mathrm{~cm}^{-1}(\mathrm{C}-\mathrm{H}), 3078(\mathrm{C}-\mathrm{H}), 3010(\mathrm{C}-\mathrm{H}), 1650,1601,1461$, $1427,1385,1342,1281,1253,1226,1206,1187,1175,1155,1114,1097,1065,1032,1024$, 1004, 965, 933, 891, 872, 824, 791, 777, 764, 738, 701, 621, 574, 521, 505, 431. - ${ }^{1} \mathrm{H}-\mathrm{NMR}$ $\left(250 \mathrm{MHz}, \mathrm{CDCl}_{3}\right): \delta=0.54-0.97(\mathrm{~m}, 16 \mathrm{H}, c \mathrm{Pr}-\mathrm{H}), 1.73,2.03\left(\mathrm{~m}_{\mathrm{c}}, 4 \mathrm{H}, c \operatorname{Pr}-\mathrm{H}\right) .-{ }^{13} \mathrm{C}-\mathrm{NMR}$ (62.9 MHz, $\left.\mathrm{CDCl}_{3}, \mathrm{DEPT}\right): \delta=6.1$ (-, $\left.2 \mathrm{C}, c \operatorname{Pr}-\mathrm{C}\right), 6.8$ (-, $\left.2 \mathrm{C}, c \mathrm{Pr}-\mathrm{C}\right), 7.7(-, 2 \mathrm{C}, c \operatorname{Pr}-\mathrm{C})$, 
$7.9(-, 2 \mathrm{C}, c \operatorname{Pr}-\mathrm{C}), 14.4(+, 2 \mathrm{C}, c \operatorname{Pr}-\mathrm{C}), 15.9$ (+, $2 \mathrm{C}, c \operatorname{Pr}-\mathrm{C}), 128.6\left(\mathrm{C}_{\text {quart }}, 2 \mathrm{C}, c \operatorname{Pr}-\mathrm{C}\right), 139.0$

$\left(\mathrm{C}_{\text {quart }}, 2 \mathrm{C}, c \mathrm{Pr}-\mathrm{C}\right) .-\mathrm{MS}(\mathrm{EI}, 70 \mathrm{eV}), m / z(\%): 276(100)\left[\mathrm{M}^{+}\right], 251(5)\left[\mathrm{M}^{+}-\mathrm{C}_{3} \mathrm{H}_{5}\right], 235$ (20), 199 (13), 169 (52), 155 (82), 141 (76), 128 (72), 115 (66), 91 (55), 77 (31), 69 (45), 55 (12), 41 (39) $\left[\mathrm{C}_{3} \mathrm{H}_{5}^{+}\right] .-\mathrm{C}_{16} \mathrm{H}_{20} \mathrm{O}_{2} \mathrm{~S}$ (276.40). ber. C 69.53\%, H 7.29\%; gef. C 69.60\%, H $7.05 \%$.

\subsection{Darstellung der Verbindungen aus Teil B Kapitel 6}

1,2-Dicyclopropylethanon (150): In ein dickwandigen Pyrex-Schraubgefäß wurden<smiles>O=C(CC1CC1)C1CC1</smiles>
nacheinander $204 \mathrm{mg}$ Quecksilber(II)oxid (2 mol-\% bez. auf das Alkin, $942 \mu \mathrm{mol}$ ), $5 \mathrm{ml}$ Wasser, $283 \mathrm{mg}$ konz. Schwefelsäure (98\%ig, 6 mol-\%, $2.83 \mathrm{mmol}), 5.00 \mathrm{~g}$ Dicyclopropylethin (13) (47.1 mmol) und $10 \mathrm{ml}$ Aceton gefüllt und das Gefäß fest verschlossen. Die Reaktionsmischung wurde $16 \mathrm{~h}$ bei $100{ }^{\circ} \mathrm{C}$ kräftig gerührt, auf Raumtemp. abgekühlt und auf $50 \mathrm{ml}$ Wasser gegeben. Nach erfolgter Phasentrennung wurde die wässrige Phase mit $3 \times 10 \mathrm{ml} \mathrm{Et}_{2} \mathrm{O}$ extrahiert. Die vereinigten organischen Phasen wurden anschließend mit $30 \mathrm{ml}$ gesättigter NaCl-Lösung gewaschen, über $\mathrm{MgSO}_{4}$ getrocknet und über $10 \mathrm{~g}$ Kieselgel filtriert. Nach Entfernen der Lösungsmittel i. Vak. erhielt man $5.63 \mathrm{~g}$ des Produktes 150 (45.3 mmol, 96\%) als leicht gelbliche Flüssigkeit von für weitere Umsetzungen hinreichender Reinheit, deren NMR-spektroskopische Daten mit den Literaturangaben überstimmte. ${ }^{[164]}-\mathrm{C}_{8} \mathrm{H}_{12} \mathrm{O}$ (124.18).

1,2,3,4-Tetracyclopropylbutan-1,4-dion (151): Unter einer Schutzgasatmosphäre wurden<smiles>O=C(C1CC1)C(C(=O)C1CC1)C1CC1</smiles>
$3.80 \mathrm{ml}$ einer Lösung von $n$-Butyllithium in Hexan $(2.35 \mathrm{M}$, $8.93 \mathrm{mmol}$ ) bei $0{ }^{\circ} \mathrm{C} \mathrm{zu}$ einer stark gerührten Lösung von $904 \mathrm{mg}$ Diisopropylamin $(9.38 \mathrm{mmol})$ in $40 \mathrm{ml}$ wasserfreiem THF getropft, das Eisbad entfernt und weitere 30 min gerührt, wobei die Reaktionsmischung auftaute. Diese Lösung wurde bei $-78{ }^{\circ} \mathrm{C}$ tropfenweise mit $1.06 \mathrm{~g}$ 1,2- 
Dicyclopropylethanon (150) (8.54 mmol) gelöst in $5 \mathrm{ml}$ THF versetzt. Nach einstündigem Rühren bei dieser Temp. wurden $1.16 \mathrm{~g}$ wasserfreies Kupfer(II)chlorid $(8.63 \mathrm{mmol})$ in einer Portion hinzugegeben und weitere $16 \mathrm{~h}$ gerührt, wobei die Reaktionsmischung auftaute. Es wurde auf $50 \mathrm{ml}$ Wasser gegeben, die Phasen getrennt und die wässrige Phase mit $3 \times 15 \mathrm{ml}$ $\mathrm{Et}_{2} \mathrm{O}$ extrahiert. Die vereinigten organischen Phasen wurden mit $30 \mathrm{ml}$ gesättigter $\mathrm{NaCl}$ Lösung gewaschen, über $\mathrm{MgSO}_{4}$ getrocknet und die Lösungsmittel unter vermindertem Druck abdestilliert. Der Rückstand wurde säulenchromatographisch an $105 \mathrm{~g}$ Kieselgel mit Pentan $/ \mathrm{Et}_{2} \mathrm{O}=15: 1$ als Laufmittel $\left(R_{\mathrm{f}}=0.14\right)$ gereinigt (Säule $\left.3 \times 35 \mathrm{~cm}\right)$, wobei man $922 \mathrm{mg}$ des Produktes (3.74 mmol, 88\%) als farblosen, amorphen Feststoff erhielt. - IR (Film): $v=$ $3355 \mathrm{~cm}^{-1}, 3080(\mathrm{C}-\mathrm{H}), 3005(\mathrm{C}-\mathrm{H}), 2915(\mathrm{C}-\mathrm{H}), 1688(\mathrm{C}=\mathrm{O}), 1443,1417,1383,1237$, 1195, 1168, 1076, 1020, 942, 897, 828, 645, 610, 553. $-{ }^{1} \mathrm{H}-\mathrm{NMR}\left(250 \mathrm{MHz}, \mathrm{CDCl}_{3}\right): \delta=$ 0.16-1.17 (m, 36 H, cPr-H), 1.93-2.15 (m, $4 \mathrm{H}, c \operatorname{Pr}-\mathrm{H}), 2.30,2.52\left(\mathrm{dd},{ }^{3} J=7.1,{ }^{4} J=2.5 \mathrm{~Hz}\right.$, $4 \mathrm{H}, 2,3-\mathrm{H}$, cis/trans). $-{ }^{13} \mathrm{C}-\mathrm{NMR}\left(62.9 \mathrm{MHz}, \mathrm{CDCl}_{3}, \mathrm{DEPT}\right): \delta=3.3(-, 2 \mathrm{C}, c \mathrm{Pr}-\mathrm{C}), 3.5(-$, $2 \mathrm{C}, c \operatorname{Pr}-\mathrm{C}), 5.9$ (-, $2 \mathrm{C}, c \operatorname{Pr}-\mathrm{C}), 6.1$ (-, $2 \mathrm{C}, c \operatorname{Pr}-\mathrm{C}), 11.0$ (-, $2 \mathrm{C}, c \operatorname{Pr}-\mathrm{C}), 11.1$ (-, $2 \mathrm{C}, c \operatorname{Pr}-\mathrm{C})$, 11.3 (-, 2 C, $c$ Pr-C), 11.5 (-, 2 C, $c \operatorname{Pr}-\mathrm{C}), 11.7$ (+, 2 C, $c \operatorname{Pr}-\mathrm{C}), 11.9$ (+, 2 C, $c$ Pr-C), 20.7 (+, $2 \mathrm{C}, c \operatorname{Pr}-\mathrm{C}), 21.0$ (+, $2 \mathrm{C}, c \mathrm{Pr}-\mathrm{C}), 58.9,59.9$ (+, 4 C, C-2,3, cis/trans), 212.1, 212.8 ( $\mathrm{C}_{\text {quart }}$, $4 \mathrm{C}, \mathrm{C}=\mathrm{O}$, cis/trans). - MS (EI, $70 \mathrm{eV}), m / z$ (\%): 246 (1) $\left[\mathrm{M}^{+}\right], 217$ (1), 177 (5) $\left[\mathrm{M}^{+}-\mathrm{C}_{4} \mathrm{H}_{5} \mathrm{O}\right], 149$ (1), 131 (1), 93 (4), 79 (3), 69 (100) $\left[\mathrm{C}_{3} \mathrm{H}_{5} \mathrm{CO}^{+}\right], 41$ (38) $\left[\mathrm{C}_{3} \mathrm{H}_{5}^{+}\right]$. $\mathrm{C}_{16} \mathrm{H}_{22} \mathrm{O}_{2}$ (246.34): ber. C 78.01\%, H 9.00\%; gef. C 78.29\%, H 8.76\%.

1-Benzyl-2,3,4,5-tetracyclopropyl-1H-pyrrol (18a): In einem Schraubdeckelgläschen mit

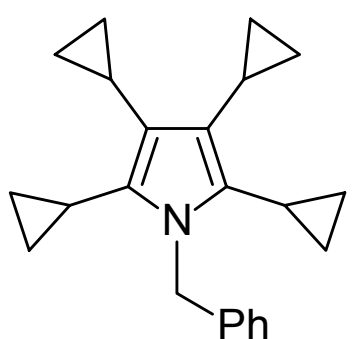
Magnetrührstäbchen wurden $350 \mathrm{mg}$ 1,2,3,4-Tetracyclopropylbutan1,4-dion (151) (1.42 mmol) in $1.35 \mathrm{~g}$ Benzylamin (9 Äquiv.) gelöst und mit 3 Trpf. konz. Salzsäure (37\%ig) versetzt. Diese Mischung wurde für 5 min mit Argon gespült, das Gläschen fest verschlossen und $3 \mathrm{~d}$ bei $65^{\circ} \mathrm{C}$ gerührt. Nach Abkühlen auf Raumtemp. wurde die Reaktionslösung durch ein Lage von $\mathrm{MgSO}_{4}(0.5 \times 0.5 \mathrm{~cm}$, zuoberst $)$ und Kieselgel $(0.5 \times 0.5 \mathrm{~cm}$, zuunterst $)$ filtriert mit $25 \mathrm{ml}$ Dichlormethan als Eluent und das Filtrat 
weitestgehend i. Vak. eingeengt. Der Rückstand wurde säulenchromatographisch an $20 \mathrm{~g}$ Kieselgel mit Pentan $/ \mathrm{Et}_{2} \mathrm{O}=15: 1$ als Laufmittel $\left(R_{\mathrm{f}}=0.82\right)$ gereinigt (Säule $\left.3 \times 10 \mathrm{~cm}\right)$, wobei $169 \mathrm{mg}$ des Produktes (532 $\mu \mathrm{mol}, 37 \%)$ als farbloser, amorpher Feststoff erhalten wurde. - IR (Film): $v=3082 \mathrm{~cm}^{-1}(\mathrm{C}-\mathrm{H}), 3004(\mathrm{C}-\mathrm{H}), 2976(\mathrm{C}-\mathrm{H}), 2929(\mathrm{C}-\mathrm{H}), 1692,1605,1536$, $1496,1453,1427,1388,1357,1346,1309,1249,1175,1098,1076,1042,1023,991,955$, 920, 891, 873, 820, 734, 570, 456. $-{ }^{1} \mathrm{H}-\mathrm{NMR}\left(250 \mathrm{MHz}, \mathrm{CDCl}_{3}\right): \delta=0.40-0.90(\mathrm{~m}, 16 \mathrm{H}$, $c \operatorname{Pr}-\mathrm{H}), 1.33,1.56\left(\mathrm{~m}_{\mathrm{c}}, 4 \mathrm{H}, c \mathrm{Pr}-\mathrm{H}\right), 5.38\left(\mathrm{~s}, 2 \mathrm{H}, \mathrm{PhCH}_{2}\right), 6.86\left(\mathrm{~d},{ }^{3} J=7.3 \mathrm{~Hz}, 2 \mathrm{H}, \mathrm{H}_{\text {ortho }}\right)$, 7.16-7.34 (m, $3 \mathrm{H}, \mathrm{Ar}-\mathrm{H}) .-{ }^{13} \mathrm{C}-\mathrm{NMR}\left(62.9 \mathrm{MHz}, \mathrm{CDCl}_{3}, \mathrm{DEPT}\right): \delta=5.4,6.1(-, 8 \mathrm{C}$,

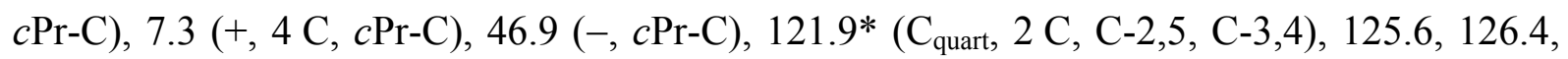
$128.3(+, 5 \mathrm{C}, \mathrm{Ar}-\mathrm{C}), 129.5 *\left(\mathrm{C}_{\text {quart }}, 2 \mathrm{C}, \mathrm{C}-2,5, \mathrm{C}-3,4\right), 140.4\left(\mathrm{C}_{\text {quart }}, \mathrm{C}_{\mathrm{ipso}}\right) .-\mathrm{MS}\left(\mathrm{DCI}, \mathrm{NH}_{3}\right)$, $m / z(\%): 318(15)\left[\mathrm{M}^{+}+\mathrm{H}\right] .-\mathrm{MS}(\mathrm{EI}, 70 \mathrm{eV}), m / z(\%): 317(66)\left[\mathrm{M}^{+}\right], 302$ (57), $288(38)$, 276 (8), 251 (8), 226 (18) $\left[\mathrm{M}^{+}-\mathrm{C}_{7} \mathrm{H}_{7}\right], 212$ (4) $\left[\mathrm{M}^{+}-\mathrm{C}_{7} \mathrm{H}_{7}-\mathrm{N}\right], 196$ (13), 182 (8), 170 (10), 167 (6), 143 (6), 128 (9), 115 (10), 91 (100) $\left[\mathrm{C}_{7} \mathrm{H}_{7}^{+}\right], 77$ (8), 69 (8), 65 (14), 51 (4), 41 (14) $\left[\mathrm{C}_{3} \mathrm{H}_{5}^{+}\right] .-\mathrm{C}_{23} \mathrm{H}_{27} \mathrm{~N}$ (317.48): ber. C 87.02\%, H 8.57\%, N 4.41\%; gef. C 86.90\%, H 8.41\%, $\mathrm{N} 4.35 \%$.

1,2,3,4,5-Pentacyclopropyl-1H-pyrrol (18b): In einem Schraubdeckelgläschen mit

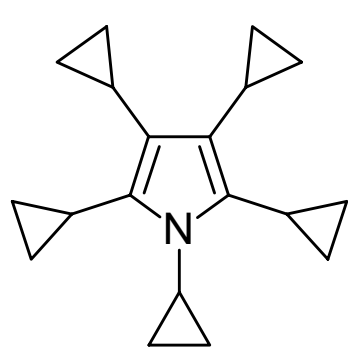
Magnetrührstäbchen wurden 300 mg 1,2,3,4-Tetracyclopropylbutan1,4-dion (151) (1.22 mmol) in $697 \mathrm{mg}$ Cyclopropylamin (8 Äquiv.) gelöst und mit 3 Trpf. konz. Salzsäure (37\%ig) versetzt. Diese Mischung wurde für 5 min mit Argon gespült, das Gläschen fest verschlossen und $1 \mathrm{~d}$ bei $65^{\circ} \mathrm{C}$ gerührt. Nach Abkühlen auf Raumtemp. wurde die Reaktionslösung durch ein Lage von $\mathrm{MgSO}_{4}(0.5 \times 0.5 \mathrm{~cm}$, zuoberst $)$ und Kieselgel $(0.5 \times 0.5 \mathrm{~cm}$, zuunterst $)$ mit $20 \mathrm{ml}$ Dichlormethan als Eluent filtriert und das Filtrat weitestgehend i. Vak. eingeengt. Der Rückstand wurde säulenchromatographisch an $20 \mathrm{~g}$ Kieselgel mit Pentan $/ \mathrm{Et}_{2} \mathrm{O}=15: 1$ als Eluent $\left(R_{\mathrm{f}}=0.87\right)$ gereinigt (Säule $3 \times 10 \mathrm{~cm}$ ), wobei $158 \mathrm{mg}$ des Produktes $(591 \mu \mathrm{mol}, 48 \%)$ als farbloser, amorpher Feststoff erhalten werden konnten. - IR (Film): v = $3085 \mathrm{~cm}^{-1}(\mathrm{C}-\mathrm{H}), 3006(\mathrm{C}-\mathrm{H}), 2792,1692,1649,1572$, 
1452, 1411, 1289, 1250, 1217, 1182, 1101, 1055, 1027, 993, 932, 891, 868, 820. - ${ }^{1}$ H-NMR $\left(250 \mathrm{MHz}, \mathrm{CDCl}_{3}\right): \delta=0.67-1.16(\mathrm{~m}, 19 \mathrm{H}, c \operatorname{Pr}-\mathrm{H}), 1.36\left(\mathrm{~m}_{\mathrm{c}}, 1 \mathrm{H}, c \operatorname{Pr}-\mathrm{H}\right), 1.51-1.76(\mathrm{~m}$, $4 \mathrm{H}, c \mathrm{Pr}-\mathrm{H}), 2.98\left(\mathrm{~m}_{\mathrm{c}}, 1 \mathrm{H}, c \mathrm{Pr}-\mathrm{H}\right) .-{ }^{13} \mathrm{C}-\mathrm{NMR}\left(62.9 \mathrm{MHz}, \mathrm{CDCl}_{3}, \mathrm{DEPT}\right): \delta=6.8(-, 4 \mathrm{C}$, $c$ Pr-C), $7.2(+, 2$ C,$c \operatorname{Pr}-\mathrm{C}), 7.3$ (-, 4 C,$c \operatorname{Pr}-\mathrm{C}), 7.8$ (+, 2 C, $c$ Pr-C), 8.7 (-, 2 C,$c \operatorname{Pr}-\mathrm{C}), 27.5$ (+, $c$ Pr-C), 121.1, $131.4\left(\mathrm{C}_{\text {quart }}, 4 \mathrm{C}, \mathrm{C}-2,5, \mathrm{C}-3,4\right) .-\mathrm{MS}(\mathrm{EI}, 70 \mathrm{eV}), \mathrm{m} / z(\%): 267$ (100) $\left[\mathrm{M}^{+}\right], 252$ (42), 238 (82), $226(31)\left[\mathrm{M}^{+}-\mathrm{C}_{3} \mathrm{H}_{5}\right], 210$ (28), 196 (25), 182 (19), 168 (16), 156 (7), 128 (5), 115 (6), 91 (4), 69 (100), 41 (10) $\left[\mathrm{C}_{3} \mathrm{H}_{5}^{+}\right] .-\mathrm{C}_{19} \mathrm{H}_{25} \mathrm{~N}$ (267.42): ber. $\mathrm{C} 85.43 \%$, H 9.42\%, N 5.24\%; gef. C 85.11\%, H 9.31\%, N 5.13\%.

2,3,4,5-Tetracyclopropyl-1H-pyrrol (18c): In einem Schraubdeckelgläschen mit

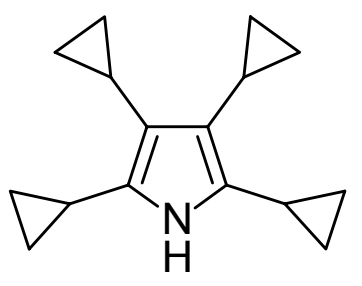

Magnetrührstäbchen wurden $300 \mathrm{mg}$ 1,2,3,4-Tetracyclopropylbutan1,4-dion (151) (1.22 mmol) und $939 \mathrm{mg}$ Ammoniumacetat (12.2 mmol) in $2 \mathrm{ml}$ Ethanol und $3 \mathrm{ml}$ Chloroform gelöst. Diese Mischung wurde $5 \mathrm{~min}$ mit Argon durchspült, das Gläschen fest verschlossen und $3 \mathrm{~d}$ bei $65^{\circ} \mathrm{C}$ gerührt. Nach Abkühlen auf Raumtemp. wurde die Reaktionslösung durch ein Lage von $\mathrm{MgSO}_{4}(0.5 \times 0.5 \mathrm{~cm}$, zuoberst $)$ und Kieselgel $(0.5 \times 0.5 \mathrm{~cm}$, zuunterst $)$ mit Dichlormethan als Eluent filtriert und das Filtrat weitestgehend i. Vak. eingeengt. Der Rückstand wurde säulenchromatographisch an $20 \mathrm{~g}$ Kieselgel mit Pentan $/ \mathrm{Et}_{2} \mathrm{O}=5: 1$ als Laufmittel $\left(R_{\mathrm{f}}=0.91, R_{\mathrm{f}}=0.59\right.$ in Pentan $\left./ \mathrm{Et}_{2} \mathrm{O}=15: 1\right)$ gereinigt (Säule $3 \times 10 \mathrm{~cm}$ ), wobei $119 \mathrm{mg}$ des Produktes $(525 \mu \mathrm{mol}, 43 \%)$ als farbloses Öl erhalten wurden, das bei niedriger Temp. zu einem amorphen Feststoff erstarrte. - IR (Film): $v=3366 \mathrm{~cm}^{-1}$ (N-H), $3088(\mathrm{C}-\mathrm{H}), 3008(\mathrm{C}-\mathrm{H})$, 1694, 1446, 1405, 1384, 1272, 1215, 1182, 1081, 1060, 1031, 976, 933, 900, 863, 816, 709, 627, 602, 483. $-{ }^{1} \mathrm{H}-\mathrm{NMR}\left(250 \mathrm{MHz}, \mathrm{CDCl}_{3}\right): \delta=$ 0.44-0.98 (m, $16 \mathrm{H}, c \operatorname{Pr}-\mathrm{H}), 1.65,1.89\left(\mathrm{~m}_{\mathrm{c}}, 4 \mathrm{H}, c \mathrm{Pr}-\mathrm{H}\right), 7.06(\mathrm{~s} b \mathrm{r}, 1 \mathrm{H}, \mathrm{NH}) .-{ }^{13} \mathrm{C}-\mathrm{NMR}$ (62.9 MHz, $\left.\mathrm{CDCl}_{3}, \mathrm{DEPT}\right): \delta=5.7,6.1$ (-, $\left.8 \mathrm{C}, c \operatorname{Pr}-\mathrm{C}\right), 6.5,7.3(+, 4 \mathrm{C}, c \operatorname{Pr}-\mathrm{C}), 122.1,126.7$ $\left(\mathrm{C}_{\text {quart }}, 4 \mathrm{C}, \mathrm{C}-2,5, \mathrm{C}-3,4\right) .-\mathrm{MS}(\mathrm{EI}, 70 \mathrm{eV}), \mathrm{m} / z(\%): 227(100)\left[\mathrm{M}^{+}\right], 212(44)\left[\mathrm{M}^{+}-\mathrm{NH}\right]$, 198 (65), 184 (21), 170 (25), 156 (17), 144 (8), 130 (8), 115 (4), 91 (2), 41 (3) [ $\left.\mathrm{C}_{3} \mathrm{H}_{5}^{+}\right]$. $\mathrm{C}_{16} \mathrm{H}_{21} \mathrm{~N}$ (227.35): 227.1674 (korrekte HRMS). 
2,3,4,5-Tetracyclopropyl-1H-pyrrolyllithium (18d): In einem Schlenk-Spitzkolben wurden

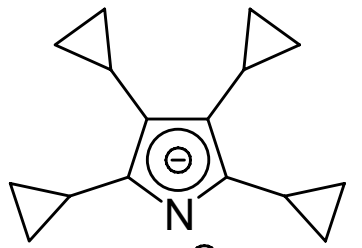

$\mathrm{Li}^{\oplus}$

$0.15 \mathrm{ml}$ einer Lösung von Methyllithium-Lithiumbromid-Komplex in

Diethylether $(2.20 \mathrm{M}, 330 \mu \mathrm{mol})$ vorgelgt. Nach weitestgehendem

Entfernen des Lösungsmittel durch Überleiten von Argon wurde restliches Lösungsmittel i. Vak. entfernt, der Kolben mit Argon belüftet und $0.30 \mathrm{ml}\left[\mathrm{D}_{8}\right]$-Tetrahydrofuran hinzugegeben. $\mathrm{Zu}$ dieser Lösung von $\mathrm{MeLi} \cdot \mathrm{LiBr}$ in $\left[\mathrm{D}_{8}\right]$-THF wurden unter Eiskühlung $50 \mathrm{mg}$ 2,3,4,5-Tetracyclopropyl-1H-pyrrol (18c) $(220 \mu \mathrm{mol})$ in $0.30 \mathrm{ml}\left[\mathrm{D}_{8}\right]$-THF gelöst tropfenweise zugegeben, wobei sich die Lösung leuchtend gelb färbte. Die so gewonnene Lösung des Lithiumpyrrolids $\mathbf{1 8 d}$ wurde sofort mit einer Spritze in ein NMR-Röhrchen transferiert und anschließend bei Raumtemp. NMRspektroskopisch untersucht. $-{ }^{1} \mathrm{H}-\mathrm{NMR}\left(250 \mathrm{MHz},\left[\mathrm{D}_{8}\right]-\mathrm{THF}\right): \delta=0.27-0.58(\mathrm{~m}, 16 \mathrm{H}$, $c$ Pr-H), 1.47, $1.72\left(\mathrm{~m}_{\mathrm{c}}, 4 \mathrm{H}, c \mathrm{Pr}-\mathrm{H}\right)$. Die Probe enthielt noch ca. 1 Äquiv. Diethylether und wenige nicht zugeordnete Signale. $-{ }^{13} \mathrm{C}-\mathrm{NMR}\left(62.9 \mathrm{MHz},\left[\mathrm{D}_{8}\right]\right.$-THF, DEPT): $\delta=6.6,7.1(-$, $8 \mathrm{C}, c \operatorname{Pr}-\mathrm{C}), 9.1,12.2(+, 4 \mathrm{C}, c \mathrm{Pr}-\mathrm{C}), 119.6,134.4\left(\mathrm{C}_{\text {quart }}, 4 \mathrm{C}, \mathrm{C}-2,5, \mathrm{C}-3,4\right) .-\mathrm{C}_{16} \mathrm{H}_{20} \mathrm{NLi}$ (231.35).

2,3,4,5-Tetracyclopropylfuran (19): In einem Schraubdeckelgläschen mit Magnetrührstäbchen

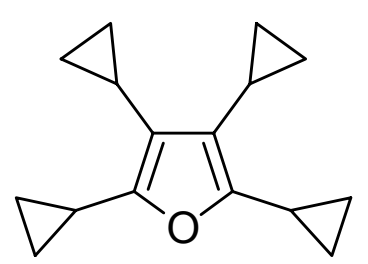

wurden $300 \mathrm{mg} \quad$ 1,2,3,4-Tetracyclopropylbutan-1,4-dion $(1.22 \mathrm{mmol})$ in $3 \mathrm{ml}$ Toluol gelöst und mit 3 Trpf. konz. Schwefelsäure (98\%ig) versetzt. Diese Mischung wurde 5 min mit Argon durchspült, das Gläschen fest verschlossen und $3 \mathrm{~d}$ bei $65^{\circ} \mathrm{C}$ gerührt. Nach Abkühlen auf Raumtemp. wurde die Reaktionslösung durch ein Lage von $\mathrm{MgSO}_{4}(0.5 \times 0.5 \mathrm{~cm}$, zuoberst $)$ und Kieselgel $(0.5 \times 0.5 \mathrm{~cm}$, zuunterst $)$ mit $15 \mathrm{ml}$ Dichlormethan als Eluent filtriert und das Filtrat weitestgehend i. Vak. eingeengt. Der Rückstand wurde säulenchromatographisch an $25 \mathrm{~g}$ Kieselgel mit Pentan/Et $\mathrm{Et}_{2} \mathrm{O}=15: 1$ als Laufmittel $\left(R_{\mathrm{f}}=0.97\right)$ gereinigt (Säule $\left.3 \times 12 \mathrm{~cm}\right)$, wobei $99 \mathrm{mg}$ des Produktes $(434 \mu \mathrm{mol}$, 36\%) als farbloses Öl erhalten wurden. - IR (Film): $v=3421 \mathrm{~cm}^{-1}, 3078(\mathrm{C}-\mathrm{H}), 3005(\mathrm{C}-\mathrm{H})$, $1751,1667,1583,1441,1418,1373,1274,1221,1194,1167,1081,1054,1036,1026,954$, 
932, 899, 883, 814, 774, 656. $-{ }^{1} \mathrm{H}-\mathrm{NMR}\left(250 \mathrm{MHz}, \mathrm{CDCl}_{3}\right): \delta=0.63-0.74(\mathrm{~m}, 12 \mathrm{H}, c \mathrm{Pr}-\mathrm{H})$,

0.74-0.89 (m, $4 \mathrm{H}, c \mathrm{Pr}-\mathrm{H}), 1.56,1.92\left(\mathrm{~m}_{\mathrm{c}}, 4 \mathrm{H}, c \mathrm{Pr}-\mathrm{H}\right) .-{ }^{13} \mathrm{C}-\mathrm{NMR}\left(62.9 \mathrm{MHz}, \mathrm{CDCl}_{3}\right.$, DEPT): $\delta=5.1(+, 2 \mathrm{C}, c \operatorname{Pr}-\mathrm{C}), 5.4,5.9(-, 8 \mathrm{C}, c \operatorname{Pr}-\mathrm{C}), 7.7(+, 2 \mathrm{C}, c \operatorname{Pr}-\mathrm{C}), 122.0,148.1$ $\left(\mathrm{C}_{\text {quart }}, 4 \mathrm{C}, \mathrm{C}-2,5, \mathrm{C}-3,4\right) .-\mathrm{MS}\left(\mathrm{DCI}, \mathrm{NH}_{3}\right), m / z(\%): 262(18)\left[\mathrm{M}^{+}+2 \mathrm{NH}_{3}\right], 245$ (100) $\left[\mathrm{M}^{+}+\mathrm{NH}_{3}\right], 229$ (44) $\left[\mathrm{M}^{+}+\mathrm{H}\right] .-\mathrm{C}_{16} \mathrm{H}_{20} \mathrm{O}$ (228.34). ber. $\mathrm{C} 84.16 \%, \mathrm{H} \mathrm{8.83 \%}$; gef. C $84.26 \%, \mathrm{H} 8.71 \%$.

2,3,4,5-Tetracyclopropylthiophen (20): In einem Schraubdeckelgläschen mit

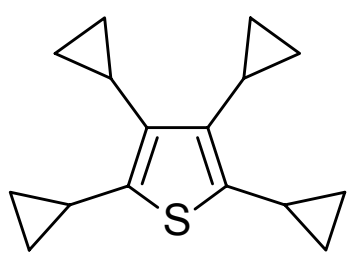
Magnetrührstäbchen wurden 156 mg 1,2,3,4-Tetracyclopropylbutan1,4-dion (151) $(633 \mu \mathrm{mol})$ und $563 \mathrm{mg}$ Phosphorpentasulfid (2.53 mmol) in $2 \mathrm{ml}$ Tetrachlormethan gelöst. Diese Mischung wurde 5 min mit Argon durchspült, das Gläschen fest verschlossen und die Mischung $3 \mathrm{~d}$ bei $70{ }^{\circ} \mathrm{C}$ gerührt. Nach Abkühlen auf Raumtemp. wurde die Reaktionslösung durch ein Lage von $\mathrm{MgSO}_{4}(0.5 \mathrm{~cm}$, zuoberst) und Kieselgel $(0.5 \mathrm{~cm}$, zuunterst) mit $20 \mathrm{ml}$ Dichlormethan als Eluent filtriert und das Filtrat weitestgehend i. Vak. eingeengt. Der Rückstand wurde säulenchromatographisch an $20 \mathrm{~g}$ Kieselgel mit Pentan/ $\mathrm{Et}_{2} \mathrm{O}=15: 1$ als Laufmittel $\left(R_{\mathrm{f}}=0.92, R_{\mathrm{f}}=0.76\right.$ in Pentan) gereinigt (Säule $3 \times 11 \mathrm{~cm}$ ), wobei man $64 \mathrm{mg}$ des Produktes $(262 \mu \mathrm{mol}, 41 \%)$ als farbloses Öl erhielt. - IR (Film): $v=3351 \mathrm{~cm}^{-1}, 3081(\mathrm{C}-\mathrm{H})$, 3004 (C-H), 1454, 1427, 1376, 1338, 1306, 1244, 1173, 1097, 1047, 1023, 973, 947, 894, 869, 821, 701, 543. - ${ }^{1} \mathrm{H}-\mathrm{NMR}\left(250 \mathrm{MHz}, \mathrm{CDCl}_{3}\right): \delta=0.61\left(\mathrm{~m}_{\mathrm{c}}, 4 \mathrm{H}, c \mathrm{Pr}-\mathrm{H}\right), 0.70\left(\mathrm{~m}_{\mathrm{c}}, 4 \mathrm{H}\right.$, $c \operatorname{Pr}-\mathrm{H}), 0.89\left(\mathrm{~m}_{\mathrm{c}}, 8 \mathrm{H}, c \operatorname{Pr}-\mathrm{H}\right), 1.66,2.17\left(\mathrm{~m}_{\mathrm{c}}, 4 \mathrm{H}, c \operatorname{Pr}-\mathrm{H}\right) .-{ }^{13} \mathrm{C}-\mathrm{NMR}\left(62.9 \mathrm{MHz}, \mathrm{CDCl}_{3}\right.$, DEPT): $\delta=6.2(-, 4 \mathrm{C}, c \operatorname{Pr}-\mathrm{C}), 9.5$ (-, $4 \mathrm{C}, c \operatorname{Pr}-\mathrm{C}), 9.7,10.1(+, 4 \mathrm{C}, c \operatorname{Pr}-\mathrm{C}), 138.5,140.3$

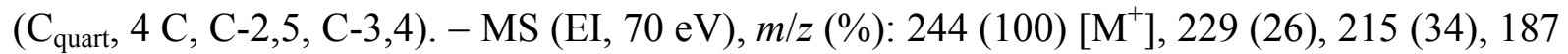
(24), 173 (19), 147 (12), 128 (9), 91 (3), 65 (2), 41 (3) $\left[\mathrm{C}_{3} \mathrm{H}_{5}{ }^{+}\right] .-\mathrm{C}_{16} \mathrm{H}_{20} \mathrm{~S}$ (244.40): 244.1286 (korrekte HRMS). 
1,2,3-Tricyclopropylpropan-1,3-dion (157): Unter einer Schutzgasatmosphäre wurden $3.80 \mathrm{ml}$<smiles>O=C(C1CC1)C(C(=O)C1CC1)C1CC1</smiles>
einer Lösung von $n$-Butyllithium in Hexan $(2.35 \mathrm{M}, 8.93 \mathrm{mmol})$ bei $0{ }^{\circ} \mathrm{C} \mathrm{zu}$ einer stark gerührten Lösung von $904 \mathrm{mg}$ Diisopropylamin (9.38 mmol) in $40 \mathrm{ml}$ wasserfreiem THF getropft, das Eisbad entfernt und weitere 30 min gerührt, wobei die Reaktionsmischung auftaute.

Diese Lösung wurde bei $-78{ }^{\circ} \mathrm{C}$ tropfenweise mit $1.06 \mathrm{~g}$ 1,2-Dicyclopropylethanon (150) (8.54 mmol) gelöst in $5 \mathrm{ml}$ THF versetzt. Nachdem einstündigem Rühren bei dieser Temp. wurden $934 \mathrm{mg}$ Cyclopropancarbonsäurechlorid $(8.93 \mathrm{mmol})$ tropfenweise hinzugegeben und weitere $16 \mathrm{~h}$ gerührt, wobei die Reaktionsmischung auftaute. Diese wurde auf $50 \mathrm{ml}$ gesättigte $\mathrm{NH}_{4} \mathrm{Cl}$-Lösung gegeben, die Phasen getrennt und die wässrige Phase mit $3 \times 15 \mathrm{ml} \mathrm{Et}_{2} \mathrm{O}$ extrahiert. Die vereinigten organischen Lösungen wurden mit $30 \mathrm{ml}$ gesättigter NaCl-Lösung gewaschen, über $\mathrm{MgSO}_{4}$ getrocknet und die Lösungsmittel i. Vak. entfernt. Der Rückstand wurde säulenchromatographisch an $65 \mathrm{~g}$ Kieselgel mit Pentan/ $\mathrm{Et}_{2} \mathrm{O}=15: 1$ als Eluent $\left(R_{\mathrm{f}}=0.11\right)$ gereinigt (Säule $\left.3 \times 25 \mathrm{~cm}\right)$, wobei man $523 \mathrm{mg}$ des Produktes $(2.72 \mathrm{mmol}, 32 \%)$ als farblose, schwer bewegliche Flüssigkeit erhielt. $-{ }^{1} \mathrm{H}-\mathrm{NMR}\left(250 \mathrm{MHz}, \mathrm{CDCl}_{3}\right): \delta=0.25$, 0.56, $0.67\left(\mathrm{~m}_{\mathrm{c}}, 5 \mathrm{H}, c \mathrm{Pr}-\mathrm{H}\right), 0.87-0.97$ (m, $\left.2 \mathrm{H}, c \operatorname{Pr}-\mathrm{H}\right), 1.05-1.14$ (m, $\left.2 \mathrm{H}, c \operatorname{Pr}-\mathrm{H}\right), 1.41-1.65$ (m, $2 \mathrm{H}, c \operatorname{Pr}-\mathrm{H}), 2.07,2.38\left(\mathrm{~m}_{\mathrm{c}}, 3 \mathrm{H}, c \mathrm{Pr}-\mathrm{H}\right), 2.99$ (d, $\left.{ }^{3} \mathrm{~J}=10.1 \mathrm{~Hz}, 1 \mathrm{H}, 2-\mathrm{H}\right) .-{ }^{13} \mathrm{C}-\mathrm{NMR}$ (62.9 MHz, $\left.\mathrm{CDCl}_{3}, \mathrm{DEPT}\right): \delta=4.2$ (-, $\left.2 \mathrm{C}, c \mathrm{Pr}-\mathrm{C}\right), 9.5$ (+, $\left.c \operatorname{Pr}-\mathrm{C}\right), 9.9$ (-, $\left.2 \mathrm{C}, c \operatorname{Pr}-\mathrm{C}\right), 11.5$, $11.9(-, 2 \mathrm{C}, c \operatorname{Pr}-\mathrm{C}) 14.9,19.7(+, 2 \mathrm{C}, c \operatorname{Pr}-\mathrm{C}) 73.6(+, c \operatorname{Pr}-\mathrm{C}), 206.6\left(\mathrm{C}_{\text {quart }}, 2 \mathrm{C}, \mathrm{C}=\mathrm{O}\right) .-\mathrm{MS}$ (EI, $70 \mathrm{eV}), m / z(\%): 192(2)\left[\mathrm{M}^{+}\right], 177$ (1), 164 (3), 151 (9) $\left[\mathrm{M}^{+}-\mathrm{C}_{3} \mathrm{H}_{5}\right], 123$ (11), $106(10)$, 91 (2), 79 (2), 69 (100) $\left[\mathrm{C}_{3} \mathrm{H}_{5} \mathrm{CO}^{+}\right], 53$ (4), 41 (54) $\left[\mathrm{C}_{3} \mathrm{H}_{5}^{+}\right] .-\mathrm{C}_{12} \mathrm{H}_{16} \mathrm{O}_{2}$ (192.26): 192.1150 (korrekte HRMS).

3,4,5-Tricyclopropyl-1H-pyrazol (158): Eine Mischung aus $523 \mathrm{mg}$ 1,2,3-Tricyclopropyl-<smiles>C1CC1c1n[nH]c(C2CC2)c1C1CC1</smilespropan-1,3-dion (157) (2.72 mmol), 2.72 g Hydrazinhydrat (20 Äquiv.) und $20 \mathrm{ml}$ Ethanol wurden $3 \mathrm{~h}$ unter Rückfluss erhitzt. Nach Entfernen des Heizbades wurde noch weitere $12 \mathrm{~h}$ gerührt, wobei die Reaktionsmischung abkühlte. Man gab diese auf $50 \mathrm{ml}$ Wasser, 
extrahierte die abgetrennte wässrige Phase mit $3 \times 10 \mathrm{ml}$ Dichlormethan, trocknete die vereinigten organischen Phasen über $\mathrm{MgSO} 4$ und entfernte die Lösungsmittel i. Vak. Säulenchromatographische Reinigung des Rückstandes an $30 \mathrm{~g}$ Kieselgel mit Pentan/Et $2 \mathrm{O}=$ 15:1 als Laufmittel (Säule $\left.3 \times 35 \mathrm{~cm}, R_{\mathrm{f}}=0.16\right)$ ergab $456 \mathrm{mg}$ des Produktes $(2.42 \mathrm{mmol}$, 89\%) als farblosen amorphen Feststoff. - IR (Film): $v=3168 \mathrm{~cm}^{-1}(\mathrm{C}-\mathrm{H}), 3125(\mathrm{C}-\mathrm{H}), 3002$ (C-H), 1585, 1519, 1489, 1460, 1426, 1382, 1322, 1285, 1208, 1154, 1087, 1049, 1020, 908, 891, 818, 739, 681, 605. - ${ }^{1} \mathrm{H}-\mathrm{NMR}\left(250 \mathrm{MHz}, \mathrm{CDCl}_{3}\right): \delta=0.58-0.90(\mathrm{~m}, 12 \mathrm{H}, c \mathrm{Pr}-\mathrm{H})$, 1.58, $1.88\left(\mathrm{~m}_{\mathrm{c}}, 4 \mathrm{H}, c \mathrm{Pr}-\mathrm{H}\right), 8.4\left(\mathrm{~s}_{\mathrm{br}}, 1 \mathrm{H}, \mathrm{NH}\right) .-{ }^{13} \mathrm{C}-\mathrm{NMR}\left(62.9 \mathrm{MHz}, \mathrm{CDCl}_{3}, \mathrm{DEPT}\right): \delta=$ $4.0(+, 2 \mathrm{C}, c \operatorname{Pr}-\mathrm{C}), 5.1,6.6,(-, 8 \mathrm{C}, c \operatorname{Pr}-\mathrm{C}), 6.7(+, 2 \mathrm{C}, c \mathrm{Pr}-\mathrm{C}), 117.2,148.3\left(\mathrm{C}_{\text {quart }}, 3 \mathrm{C}\right.$, C-3,4,5). - MS (EI, $70 \mathrm{eV}), m / z(\%): 188(75)\left[\mathrm{M}^{+}\right], 173$ (77) $\left[\mathrm{M}^{+}-\mathrm{NH}\right], 159$ (44) $\left[\mathrm{M}^{+}-\mathrm{NH}-\mathrm{N}\right], 145$ (24), 131 (32), 119 (30), 106 (30), 91 (24), 79 (25), 65 (30), 53 (23), 41 (199) $\left[\mathrm{C}_{3} \mathrm{H}_{5}^{+}\right] .-\mathrm{C}_{12} \mathrm{H}_{16} \mathrm{~N}_{2}$ (188.27). 188.1313 (korrekte HRMS).

3,4,5-Tricyclopropyl-1H-pyrazolylkalium (158a): In einer Schtuzgasatmosphäre wurden

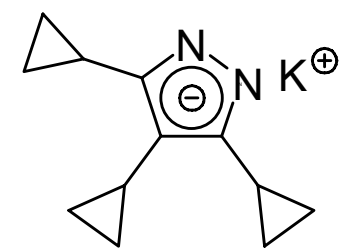
20 mg Kaliumhydrid (488 $\mu \mathrm{mol})$ in einem NMR-Röhrchen vorgelegt und $\quad 0.10 \mathrm{ml} \quad\left[\mathrm{D}_{8}\right]$-Tetrahydrofuran hinzugegeben. $\mathrm{Zu}$ dieser Suspension wurde unter Eiskühlung $50 \mathrm{mg}$ 3,4,5-Tetracyclopropyl$1 H$-pyrazol (158) $(266 \mu \mathrm{mol})$ in $0.40 \mathrm{ml}\left[\mathrm{D}_{8}\right]$-THF gelöst tropfenweise zugegeben, wobei sich die Lösung intensiv gelb färbte. Nach abgeschlossener Gasentwiklung wurde das NMR-Röhrchen verschlossen und die so gewonnene Lösung bei Raumtemp. NMR-spektroskopisch untersucht. - ${ }^{1} \mathrm{H}-\mathrm{NMR}\left(250 \mathrm{MHz},\left[\mathrm{D}_{8}\right]-\mathrm{THF}\right): \delta=0.37-0.81$ (m, $12 \mathrm{H}, c \operatorname{Pr}-\mathrm{H}), 1.57,1.84\left(\mathrm{~m}_{\mathrm{c}}, 3 \mathrm{H}, c \mathrm{Pr}-\mathrm{H}\right) .-{ }^{13} \mathrm{C}-\mathrm{NMR}\left(62.9 \mathrm{MHz},\left[\mathrm{D}_{8}\right]-\mathrm{THF}, \mathrm{DEPT}\right): \delta=6.4$, $7.3(-, 6 \mathrm{C}, c \operatorname{Pr}-\mathrm{C}), 6.6,9.8(+, 3 \mathrm{C}, c \operatorname{Pr}-\mathrm{C}), 114.9$ (C $\left.\mathrm{C}_{\text {quart }}, \mathrm{C}-4\right), 151.8\left(\mathrm{C}_{\text {quart }}, 2 \mathrm{C}, \mathrm{C}-3,5\right) .-$ $\mathrm{C}_{12} \mathrm{H}_{15} \mathrm{~N}_{2} \mathrm{~K}(226.27)$. 


\subsection{Darstellung der Verbindungen aus Teil B Kapitel 7}

syn-1,2,3,4,5,6,7,8-Octacyclopropyltricyclo[4.2.0.0 $\left.0^{2,5}\right]$ octa-3,7-dien (165): $\mathrm{Zu}$ einer Lösung

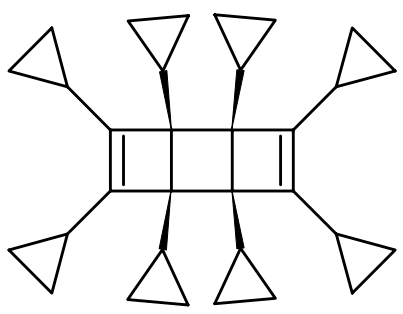
von $1.00 \mathrm{~g}$ Dicyclopropylethin (13) $(9.42 \mathrm{mmol})$ und $1.38 \mathrm{~g}$ Biscyclopentadienylzirconiumdichlorid $(4.72 \mathrm{mmol})$ in $60 \mathrm{ml}$ wasserfreiem Tetrahydrofuran wurden bei $-78^{\circ} \mathrm{C} 4.20 \mathrm{ml}$ einer Lösung von $n$-Butyllithium in $n$-Hexan $(2.35 \mathrm{M}, 9.89 \mathrm{mmol})$ tropfenweise hinzugefügt. Anschließend entfernte man das

Kältebad und ließ die Reaktionslösung noch $1 \mathrm{~h}$ rühren, wobei diese auftaute. Zu dieser roten Lösung wurden bei $-78^{\circ} \mathrm{C} 1.20 \mathrm{~g}$ Iod $(4.72 \mathrm{mmol})$ in einer Portion gegeben und die Reaktionslösung nach Entfernen des Kältebades noch 1 h gerührt, wobei diese auftaute. Dann gab man bei Raumtemp. 467 mg wasserfreies Kupfer(I)chlorid (4.72 mmol) in einer Portion hinzu und ließ noch $1 \mathrm{~h}$ bei dieser Temp. rühren. Man gab auf $50 \mathrm{ml}$ ges. $\mathrm{Na}_{2} \mathrm{~S}_{2} \mathrm{O}_{3}$-Lösung und extrahierte die abgetrennte wässrige Phase mit $3 \times 15 \mathrm{ml} \mathrm{Et}_{2} \mathrm{O}$. Die vereinigten organischen Lösungen wurden mit $30 \mathrm{ml}$ gesättigter $\mathrm{NaCl}$-Lösung gewaschen, über $\mathrm{MgSO}_{4}$ getrocknet und die Lösungsmittel i. Vak. entfernt. Der Rückstand wurde säulenchromatographisch an $65 \mathrm{~g}$ Kieselgel mit Pentan als Eluent $\left(R_{\mathrm{f}}=0.71\right)$ gereinigt (Säule $3 \times 21 \mathrm{~cm}$ ), wobei man $376 \mathrm{mg}$ des Produktes (max. $885 \mu$ mol, max. 38\%) als farblosen, amorphen Feststoff erhielt, der noch mit geringen Mengen an 1,2,3,4-Tetracyclopropylbuta1,3-dien (21) kontaminiert war. - IR (Film): $v=3083 \mathrm{~cm}^{-1}(\mathrm{C}-\mathrm{H}), 3004(\mathrm{C}-\mathrm{H}), 1657,1456$, $1425,1387,1266,1248,1174,1055,1016,986,904,893,827,738,705,671,529 .-$ UV (0.168 mg/10 ml Pentan): $\lambda=$ ca. $200 \mathrm{~nm}$ (breite Schulter), ca. 240.0 (breite Schulter); UV (0.154 mg/10 ml MeCN): $\lambda=$ ca. $200 \mathrm{~nm}$ (breite Schulter), ca. 240 (breite Schulter).${ }^{1} \mathrm{H}-\mathrm{NMR}\left(250 \mathrm{MHz}, \mathrm{CDCl}_{3}\right): \delta=0.31-0.44(\mathrm{~m}, 5 \mathrm{H}, c \mathrm{Pr}-\mathrm{H}), 0.44-0.67(\mathrm{~m}, 20 \mathrm{H}, c \mathrm{Pr}-\mathrm{H})$, 0.72-0.96 (m, 5 H, $c$ Pr-H), 1.01-1.29 (m, $10 \mathrm{H}, c \mathrm{Pr}-\mathrm{H}) .-{ }^{13} \mathrm{C}-\mathrm{NMR}\left(62.9 \mathrm{MHz}, \mathrm{CDCl}_{3}\right.$, DEPT): $\delta=3.1(-, 4 \mathrm{C}, c \operatorname{Pr}-\mathrm{C}), 4.5$ (-, $4 \mathrm{C}, c \operatorname{Pr}-\mathrm{C}), 6.6$ (-, $4 \mathrm{C}, c \operatorname{Pr}-\mathrm{C}), 7.0(-, 4 \mathrm{C}, c \operatorname{Pr}-\mathrm{C})$, $10.1(+, 4 \mathrm{C}, c \operatorname{Pr}-\mathrm{C}), 11.0(+, 4 \mathrm{C}, c \operatorname{Pr}-\mathrm{C}), 55.7$ ( $\left.\mathrm{C}_{\text {quart }}, 4 \mathrm{C}, \mathrm{C}-1,6, \mathrm{C}-2,5\right), 142.6\left(\mathrm{C}_{\text {quart }}, 4 \mathrm{C}\right.$, C-3,4, C-7,8). - MS (EI, $70 \mathrm{eV}), m / z(\%): 424$ (17) $\left[\mathrm{M}^{+}\right], 396(2)\left[\mathrm{M}^{+}-\mathrm{C}_{3} \mathrm{H}_{5}\right], 383$ (32), 355 (34), 344 (8), 325 (17), 313 (23), 297 (27), 257 (35), 243 (40), 229 (41), 179 (40), 165 (52), 
155 (57), 128 (66), 91 (95), 79 (54), 55 (44), 41 (100) $\left[\mathrm{C}_{3} \mathrm{H}_{5}^{+}\right] \cdot-\mathrm{C}_{32} \mathrm{H}_{40}$ (424.68): 424.3130 (korrekte HRMS).

(Z,Z)-1,4-Dicyclopropyl-1,4-diiodbuta-1,3-dien (178): Zu einer Mischung von $3.16 \mathrm{~g}$ einer<smiles>IC(=C/C(I)=C\C1CC1)C1CC1</smiles>
Cyclopropylethinlösung (177) (50\%ig in Toluol, $20.0 \mathrm{mmol}$ ) und $3.15 \mathrm{~g}$ Titantetraisopropylat $(11.0 \mathrm{mmol})$ in $70 \mathrm{ml}$ wasserfreiem Diethylether wurden bei $-78^{\circ} \mathrm{C} \quad 14.9 \mathrm{ml}$ einer Lösung von Isopropylmagnesiumbromid in $\mathrm{Et}_{2} \mathrm{O}(1.41 \mathrm{M}, 21.0 \mathrm{mmol})$ tropfenweise hinzugefügt, wobei sich die Reaktionsmischung goldgelb färbte. Nach 1.5 stündigem Rühren bei -50 bis $-40{ }^{\circ} \mathrm{C}$ wurde die nun schwarze Reaktionslösung auf $-78^{\circ} \mathrm{C}$ abgekühlt und $7.61 \mathrm{~g}$ Iod (30.0 mmol) in einer Portion hinzugefügt. Anschließend entfernte man das Kältebad und ließ die Reaktionslösung auftauen. Es wurde auf $50 \mathrm{ml}$ gesättigte $\mathrm{Na}_{2} \mathrm{~S}_{2} \mathrm{O}_{3}$-Löung gegeben, die Phasen getrennt und die wässrige Phase mit $3 \times 20 \mathrm{ml} \mathrm{Et}_{2} \mathrm{O}$ extrahiert. Die vereinigten organischen Lösungen wurden mit $30 \mathrm{ml}$ gesättigter $\mathrm{NaCl}$-Lösung gewaschen, über $\mathrm{MgSO}_{4}$ getrocknet und die Lösungsmittel i. Vak. entfernt. Der Rückstand wurde säulenchromatographisch an $55 \mathrm{~g}$ Kieselgel mit Pentan als Eluent $\left(R_{\mathrm{f}}=0.67\right)$ gereinigt (Säule $3 \times 15 \mathrm{~cm}$ ), wobei man $1.08 \mathrm{~g}$ des Produktes $(2.80 \mathrm{mmol}, 28 \%)$ als farbloses Öl erhielt. - IR (Film): $v=3080 \mathrm{~cm}^{-1}(\mathrm{C}-\mathrm{H})$, $3004(\mathrm{C}-\mathrm{H}), 2852(\mathrm{C}-\mathrm{H}), 1829,1736,1631,1447,1426,1265,1184,1100,1044,989$ $(\mathrm{C}-\mathrm{H}), 946(\mathrm{C}-\mathrm{H}), 856,810,739 .{ }^{1} \mathrm{H}-\mathrm{NMR}\left(250 \mathrm{MHz}, \mathrm{CDCl}_{3}\right): \delta=0.67-0.91(\mathrm{~m}, 8 \mathrm{H}$, $c$ Pr-H), $1.68\left(\mathrm{~m}_{\mathrm{c}}, 2 \mathrm{H}, c \mathrm{Pr}-\mathrm{H}\right), 6.49$ (s, $\left.2 \mathrm{H}, 2,3-\mathrm{H}\right) .-{ }^{13} \mathrm{C}-\mathrm{NMR}\left(62.9 \mathrm{MHz}, \mathrm{CDCl}_{3}, \mathrm{DEPT}\right)$ : $\delta=9.3(-, 4 \mathrm{C}, c \operatorname{Pr}-\mathrm{C}), 24.7(+, 2 \mathrm{C}, c \mathrm{Pr}-\mathrm{C}), 116.4\left(\mathrm{C}_{\text {quart }}, 2 \mathrm{C}, \mathrm{C}-1,4\right), 134.5(+, 2 \mathrm{C}$, C-2,3). - MS (EI, $70 \mathrm{eV}), m / z(\%): 386$ (80) $\left[\mathrm{M}^{+}\right], 358$ (3), 259 (5) $\left[\mathrm{M}^{+}\right.$- I], 231 (9), 217 (4), 193 (18), 155 (6), 132 (69) [M+ - 2 I], 117 (100), 104 (57), 91 (53), 66 (28), 51 (18), 41 (7) $\left[\mathrm{C}_{3} \mathrm{H}_{5}^{+}\right] .-\mathrm{C}_{10} \mathrm{H}_{12} \mathrm{I}_{2}$ (386.02): 385.9028 (korrekte HRMS). 
1,2,3,4-Tetracyclopropyl-1,4-diiodbuta-1,3-dien (179): Zu einer Lösung von 1.00 g Dicyclo-

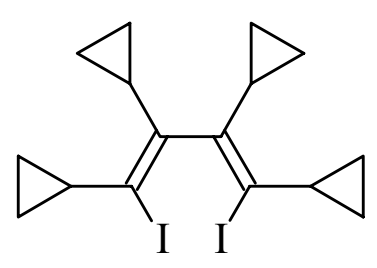

propylethin (13) $(9.42 \mathrm{mmol})$ und $2.67 \mathrm{~g}$ Titantetraisopropylat

$(9.42 \mathrm{mmol})$ in $70 \mathrm{ml}$ trockenem Diethylether wurden bei $-78{ }^{\circ} \mathrm{C}$ $14.7 \mathrm{ml}$ einer Lösung von Isopropylmagnesiumbromid in $\mathrm{Et}_{2} \mathrm{O}$ $(1.41 \mathrm{M}, 20.7 \mathrm{mmol})$ tropfenweise hinzugefügt, wobei sich die Reaktionsmischung goldgelb färbte. Nach 1.5 stündigem Rühren bei -50 bis $-40{ }^{\circ} \mathrm{C}$ wurde die nun schwarze Reaktionslösung auf $-78{ }^{\circ} \mathrm{C}$ abgekühlt und $7.19 \mathrm{~g}$ Iod $(28.3 \mathrm{mmol})$ in einer Portion hinzugefügt. Anschließend entfernte man das Kältebad und ließ die Reaktionslösung auftauen. Es wurde auf $50 \mathrm{ml}$ gesättigte $\mathrm{Na}_{2} \mathrm{~S}_{2} \mathrm{O}_{3}$-Löung gegeben, die Phasen getrennt und die wässrige Phase mit $3 \times 20 \mathrm{ml} \mathrm{Et}_{2} \mathrm{O}$ extrahiert. Die vereinigten organischen Lösungen wurden mit $30 \mathrm{ml}$ gesättigter NaCl-Lösung gewaschen, über $\mathrm{MgSO}_{4}$ getrocknet und die Lösungsmittel i. Vak. entfernt. Der Rückstand wurde säulenchromatographisch an $55 \mathrm{~g}$ Kieselgel mit Pentan als Eluent $\left(R_{\mathrm{f}}=0.45\right)$ gereinigt (Säule $3 \times 15 \mathrm{~cm}$ ), wobei man $1.68 \mathrm{~g}$ des Produktes $(3.60 \mathrm{mmol}, 77 \%)$ als farbloses Öl erhielt. $-{ }^{1} \mathrm{H}-\mathrm{NMR}\left(250 \mathrm{MHz}, \mathrm{CDCl}_{3}\right): \delta=$ 0.58-1.08 (m, $16 \mathrm{H}, c \mathrm{Pr}-\mathrm{H}), 1.68-1.95(\mathrm{~m}, 4 \mathrm{H}, c \mathrm{Pr}-\mathrm{H}) .-{ }^{13} \mathrm{C}-\mathrm{NMR}\left(62.9 \mathrm{MHz}, \mathrm{CDCl}_{3}\right.$, DEPT): $\delta=6.7$ (-, 2 C , cPr-C), 7.5 (-, 2 C, $c$ Pr-C), 10.7 (-, 2 C, $c$ Pr-C), 11.0 (-, 2 C, $c$ Pr-C), 15.1 (+, 2 C, $c$ Pr-C), 19.2 (+, 2 C, $c$ Pr-C), 112.6 (C quart $\left._{2} 2 \mathrm{C}, \mathrm{C}-1,4\right), 131.7$ ( $\left.\mathrm{C}_{\text {quart }}, 2 \mathrm{C}, \mathrm{C}-2,3\right)$. MS (EI, $70 \mathrm{eV}), m / z(\%): 466(36)\left[\mathrm{M}^{+}\right], 339$ (100) $\left[\mathrm{M}^{+}-\mathrm{I}\right], 283$ (6), 212 (11) [ $\left.\mathrm{M}^{+}-2 \mathrm{I}\right]$, 169 (28), 155 (42), 128 (40), 91 (34), 77 (25), 65 (12), 41 (16) $\left[\mathrm{C}_{3} \mathrm{H}_{5}{ }^{+}\right] .-\mathrm{C}_{16} \mathrm{H}_{20} \mathrm{I}_{2}$ (466.15): 465.9654 (korrekte HRMS).

(Z)-1,2-Dicyclopropyl-1,2-diiodethen (180): $\mathrm{Zu}$ einer Lösung aus $474 \mathrm{mg}$

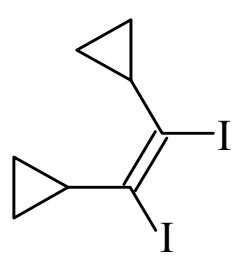
Kaliumpermanganat $(3.00 \mathrm{mmol})$ in $50 \mathrm{ml}$ Acetonitril wurden $3.30 \mathrm{ml}$ wässrige Iodwasserstoffsäure (57\%ig, $25 \mathrm{mmol})$ gegeben und $10 \mathrm{~min}$ bei Raumtemp. gerührt. Daraufhin gab man 1.06 g Dicyclopropylethin (13) (10 mmol) hinzu und rührte $30 \mathrm{~min}$ bei $60^{\circ} \mathrm{C}$. Die noch warme Reaktionslösung wurde weitestgehend i. Vak. eingeengt, mit gesättigter $\mathrm{K}_{2} \mathrm{CO}_{3}$-Lösung neutralisiert, mit $30 \mathrm{ml}$ gesättigter KI-Lösung versetzt und mit $3 \times 20 \mathrm{ml}$ Dichlormethan 
extrahiert. Die vereinigten organischen Lösungen wurden über $\mathrm{MgSO}_{4}$ getrocknet und die Lösungsmittel i. Vak. entfernt. Der Rückstand wurde säulenchromatographisch an $55 \mathrm{~g}$ Kieselgel mit Pentan als Eluent $\left(R_{\mathrm{f}}=0.67\right)$ gereinigt (Säule $3 \times 15 \mathrm{~cm}$ ), wobei man $1.15 \mathrm{~g}$ des Produktes $(3.20 \mathrm{mmol}, 32 \%)$ als farbloses, lichtempfindliches Öl erhielt, das bei tieferer Temp. zu einem amorphen Feststoff erstarrte. - IR (Film): $v=3084 \mathrm{~cm}^{-1}(\mathrm{C}-\mathrm{H}), 3045$ (C-H), 3006 (C-H), 1454, 1422, 1361, 1329, 1264, 1215, 1170, 1121, 1088, 1050, 1025, 989, 956, 894, 825, 768, 739, 705, 618. - ${ }^{1} \mathrm{H}-\mathrm{NMR}\left(250 \mathrm{MHz}, \mathrm{CDCl}_{3}\right): \delta=0.83-0.95\left(\mathrm{~m}_{\mathrm{c}}, 8 \mathrm{H}\right.$, $c$ Pr-H), $1.77\left(\mathrm{~m}_{\mathrm{c}}, 2 \mathrm{H}, c \mathrm{Pr}-\mathrm{H}\right) .-{ }^{13} \mathrm{C}-\mathrm{NMR}\left(62.9 \mathrm{MHz}, \mathrm{CDCl}_{3}, \mathrm{DEPT}\right): \delta=11.5(-, 4 \mathrm{C}$, $c$ Pr-C), 28.7 (+, 2 C, $c$ Pr-C), 107.1 (C quart $_{2} 2$ C, C-1,2). - MS (EI, 70 eV), m/z (\%): 360 (43) $\left[\mathrm{M}^{+}\right], 233(94)\left[\mathrm{M}^{+}-\mathrm{I}\right], 106(100)\left[\mathrm{M}^{+}-2 \mathrm{I}\right], 91$ (92), 78 (58), 65 (13), 51 (25), 41 (6) $\left[\mathrm{C}_{3} \mathrm{H}_{5}^{+}\right] .-\mathrm{C}_{8} \mathrm{H}_{10} \mathrm{I}_{2}$ (359.98): 359.8872 (korrekte HRMS).

\subsection{Darstellung der Verbindungen aus Teil B Kapitel 8}

Allgemeine Arbeitsvorschrift für Palladium-katalysierte Reaktionen von Halogenalkenen mit Cyclopropylacetylenen (AAV 6): In ein dickwandiges Pyrex-Schraubglasgefäß mit Magnetrührstab wurden nacheinander die angegebenen Mengen an Palladium(II)acetat, Triphenylphosphan, Halogenalken, Cyclopropylacetylen und Base gefüllt und mit dem entsprechenden Volumen an trockenem Acetonitril versetzt. Durch fünfminütiges Durchleiten von Argon wurde diese Mischung von Sauerstoff befreit, das Gefäß fest verschlossen und für die entsprechende Zeit bei der angegeben Temp. gerührt. Anschließend wurde die Reaktionsmischung durch ein $1 \mathrm{~cm}$ dicke Schicht von Celite filtriert, auf $30 \mathrm{ml}$ Wasser gegeben und mit $3 \times 20 \mathrm{ml} \mathrm{Et}{ }_{2} \mathrm{O}$ extrahiert. Die vereinigten organischen Phasen wurden über $\mathrm{MgSO}_{4}$ getrocknet und die Lösungsmittel i. Vak. entfernt. Der Rückstand wurde säulenchromatographisch an Kieselgel mit einem Gemisch aus Pentan/Et ${ }_{2} \mathrm{O}$ gereinigt. 
3-(1'-Cyclopropyl-3',3'-diphenylallyliden)-cyclobuten (200): Variante A: Nach AAV 6

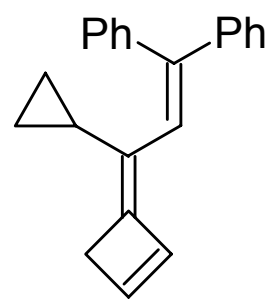
wurden $53 \mathrm{mg}$ Palladiumacetat $(236 \mu \mathrm{mol}), 186 \mathrm{mg}$ Triphenylphosphan (708 $\mu \mathrm{mol}), 1.02$ g 1-Brom-2,2-diphenylethen (199) (3.93 mmol), $500 \mathrm{mg}$ Dicyclopropylethin $(4.71 \mathrm{mmol}), 795 \mathrm{mg}$ Triethylamin $(7.86 \mathrm{mmol})$ in $8 \mathrm{ml}$ trockenem Acetonitril für $24 \mathrm{~h}$ bei $80^{\circ} \mathrm{C}$ gerührt. Säulenchromatographische Reinigung des Rohproduktes an $50 \mathrm{~g}$ Kieselgel (Säule $2.5 \times 20 \mathrm{~cm}$, Pentan, $R_{\mathrm{f}}=0.37$ ) lieferte $800 \mathrm{mg}$ des Produkts $(2.81 \mathrm{mmol}, 71 \%$ ) als farbloses Öl. - Variante B: Nach AAV 6 wurden 53 mg Palladiumacetat (236 $\mu \mathrm{mol}), 186 \mathrm{mg}$ Triphenylphosphan (708 $\mu \mathrm{mol}), 1.02 \mathrm{~g}$ 1-Brom-2,2-diphenylethen (3.93 mmol), $500 \mathrm{mg}$ Dicyclopropylethin $(4.71 \mathrm{mmol}), 1.10 \mathrm{~g}$ Kaliumcarbonat $(7.86 \mathrm{mmol})$ in $8 \mathrm{ml}$ trockenem Acetonitril für $24 \mathrm{~h}$ bei $80^{\circ} \mathrm{C}$ gerührt. Säulenchromatographische Reinigung des Rohproduktes an $50 \mathrm{~g}$ Kieselgel (Säule $2.5 \times 20 \mathrm{~cm}$, Pentan, $R_{\mathrm{f}}=0.37$ ) lieferte $655 \mathrm{mg}$ des Produkts (2.30 mmol, 59\%) als farbloses Öl. - IR (Film): v=3078 cm $\mathrm{cm}^{-1}(\mathrm{C}-\mathrm{H}), 3003(\mathrm{C}-\mathrm{H})$, $1637,1593,1511,1494,1442,1389,1330,1073,1049,1028,978,908,773$ (C-H), 751 $(\mathrm{C}-\mathrm{H}), 702,682,641,596 .-{ }^{1} \mathrm{H}-\mathrm{NMR}\left(250 \mathrm{MHz}, \mathrm{CDCl}_{3}\right): \delta=0.80\left(\mathrm{~m}_{\mathrm{c}}, 2 \mathrm{H}, c \mathrm{Pr}-\mathrm{H}\right), 1.03$ $\left(\mathrm{m}_{\mathrm{c}}, 2 \mathrm{H}, c \operatorname{Pr}-\mathrm{H}\right), 2.18\left(\mathrm{~m}_{\mathrm{c}}, 1 \mathrm{H}, c \operatorname{Pr}-\mathrm{H}\right), 4.10-4.17$ (m, $\left.2 \mathrm{H}, 4-\mathrm{H}\right), 4.89-5.14$ (m, $\left.1 \mathrm{H}, 2-\mathrm{H}\right)$, 6.06-6.25 (m, $1 \mathrm{H}, 1-\mathrm{H}), 7.14$ (s, $\left.1 \mathrm{H}, 2^{\prime}-\mathrm{H}\right), 7.31-7.55$ (m, $\left.6 \mathrm{H}, \mathrm{Ar}-\mathrm{H}\right), 7.85\left(\mathrm{~d},{ }^{3} J=8.2 \mathrm{~Hz}\right.$, $\left.2 \mathrm{H}, \mathrm{H}_{\text {ortho }}\right), 8.10\left(\mathrm{~d},{ }^{3} J=8.5 \mathrm{~Hz}, 2 \mathrm{H}, \mathrm{H}_{\text {ortho }}\right) .-{ }^{1} \mathrm{H}-\mathrm{NMR}\left(250 \mathrm{MHz}, \mathrm{C}_{6} \mathrm{D}_{6}\right): \delta=0.54-0.86(\mathrm{~m}$, $4 \mathrm{H}, c \operatorname{Pr}-\mathrm{H}), 2.04\left(\mathrm{~m}_{\mathrm{c}}, 1 \mathrm{H}, c \operatorname{Pr}-\mathrm{H}\right), 4.06-4.13(\mathrm{~m}, 2 \mathrm{H}, 4-\mathrm{H}), 4.96-5.18(\mathrm{~m}, 1 \mathrm{H}, 2-\mathrm{H})$, 6.02-6.23 (m, $1 \mathrm{H}, 1-\mathrm{H}), 7.18-7.38$ (m, $\left.6 \mathrm{H}, \mathrm{Ar}-\mathrm{H}, 2^{\prime}-\mathrm{H}\right), 7.42\left(\mathrm{t},{ }^{3} J=8.2 \mathrm{~Hz}, 1 \mathrm{H}, \mathrm{H}_{\text {para }}\right.$ ), 7.51 (d, $\left.{ }^{3} J=8.2 \mathrm{~Hz}, 2 \mathrm{H}, \mathrm{H}_{\text {ortho }}\right), 8.13$ (t, $\left.{ }^{3} J=8.5 \mathrm{~Hz}, 2 \mathrm{H}, \mathrm{H}_{\text {meta }}\right) .-{ }^{13} \mathrm{C}-\mathrm{NMR}(62.9 \mathrm{MHz}$, $\left.\mathrm{CDCl}_{3}, \mathrm{DEPT}\right): \delta=7.4(-, 2 \mathrm{C}, c \operatorname{Pr}-\mathrm{C}), 13.8(+, c \operatorname{Pr}-\mathrm{C}), 32.5(-, 4-\mathrm{C}), 124.3^{* *}(+, \mathrm{C}-1)$, $124.8^{* *}(+, \mathrm{C}-2), 125.8^{* *}\left(+, \mathrm{C}_{\mathrm{para}}\right), 126.0^{* *}\left(+, \mathrm{C}_{\mathrm{para}}\right), 126.5^{* *}\left(+, 2 \mathrm{C}, \mathrm{C}_{\mathrm{meta}}\right), 127.1^{* *}(+$, $\left.2 \mathrm{C}, \mathrm{C}_{\text {ortho }}\right), 128.2^{* *}\left(+, 2 \mathrm{C}, \mathrm{C}_{\text {ortho }}\right), 130.2^{* *}\left(+, 2 \mathrm{C}, \mathrm{C}_{\text {meta }}\right), 132.5^{*}$ ( $\left.\mathrm{C}_{\text {quart }}, 3-\mathrm{C}\right), 133.8^{*}$ $\left(\mathrm{C}_{\text {quart }}, 1^{\prime}-\mathrm{C}\right), 136.5^{* *}\left(+, 2^{\prime}-\mathrm{C}\right), 137.7^{*}\left(\mathrm{C}_{\text {quart }}, 3^{\prime}-\mathrm{C}\right), 138.8^{*}\left(\mathrm{C}_{\text {quart }}, \mathrm{C}_{\mathrm{ipso}}\right), 141.1^{*}\left(\mathrm{C}_{\text {quart }}\right.$, $\left.\mathrm{C}_{\mathrm{ipso}}\right) .-{ }^{13} \mathrm{C}-\mathrm{NMR}\left(62.9 \mathrm{MHz}, \mathrm{C}_{6} \mathrm{D}_{6}, \mathrm{DEPT}\right): \delta=7.5(-, 2 \mathrm{C}, c \mathrm{Pr}-\mathrm{C}), 14.3(+, c \operatorname{Pr}-\mathrm{C}), 32.7(-$, 4-C), 124.8** (+, C-1), 125.2** (+, C-2), 126.2** (+, $\left.\mathrm{C}_{\mathrm{para}}\right), 126.4^{* *}\left(+, \mathrm{C}_{\mathrm{para}}\right), 127.0^{* *}(+$, $\left.2 \mathrm{C}, \mathrm{C}_{\text {meta }}\right), 127.3^{* *}\left(+, 2 \mathrm{C}, \mathrm{C}_{\text {ortho }}\right), 128.5^{* *}\left(+, 2 \mathrm{C}, \mathrm{C}_{\text {ortho }}\right), 130.5^{* *}\left(+, 2 \mathrm{C}, \mathrm{C}_{\text {meta }}\right), 131.3^{*}$ $\left(\mathrm{C}_{\text {quart }}, \mathrm{C}-3\right), 133.3^{*}\left(\mathrm{C}_{\text {quart }}, \mathrm{C}-1^{\prime}\right), 134.2^{* *}\left(\mathrm{C}_{\text {quart }}, \mathrm{C}-3^{\prime}\right), 136.9^{*}\left(+, \mathrm{C}-2^{\prime}\right), 137.9^{*}\left(\mathrm{C}_{\text {quart }}\right.$, 
$\left.\mathrm{C}_{\mathrm{ipso}}\right), 141.1 *\left(\mathrm{C}_{\text {quart }}, \mathrm{C}_{\mathrm{ipso}}\right) .-\mathrm{MS}(\mathrm{EI}, 70 \mathrm{eV}), m / z(\%): 284(100)\left[\mathrm{M}^{+}\right], 269(16)\left[\mathrm{M}^{+}-\mathrm{CH}_{3}\right]$, $255(36)\left[\mathrm{M}^{+}-\mathrm{C}_{2} \mathrm{H}_{5}\right], 242$ (22) $\left[\mathrm{M}^{+}-\mathrm{C}_{3} \mathrm{H}_{5}\right], 228$ (14), 215 (9), 202 (6), 178 (8), 165 (12), 152 (2), 126 (8), $113(6), 91$ (10), 77 (3) $\left[\mathrm{C}_{6} \mathrm{H}_{5}^{+}\right], 51$ (1), 41 (1) $\left[\mathrm{C}_{3} \mathrm{H}_{5}^{+}\right] \cdot-\mathrm{C}_{22} \mathrm{H}_{20}(284.40)$ : ber. C $92.91 \%$, H 7.09\%; gef. C $92.62 \%$, H $6.91 \%$.

2,3,4,5-Tetracyclopropyl-6,6-diphenylpentafulven (207): Nach AAV 6 wurden 54 mg

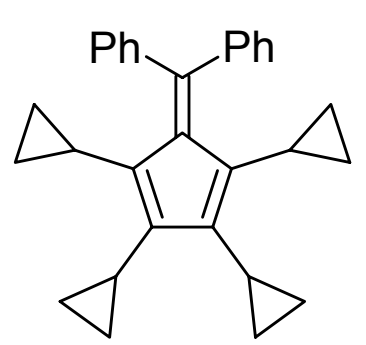
Palladiumacetat $(240 \mu \mathrm{mol}), 186 \mathrm{mg}$ Triphenylphosphan $(708 \mu \mathrm{mol})$, 1.22 g 1-Brom-2,2-diphenylethen (199) (4.71 mmol), 1.00 g Dicyclopropylethin (13) $(9.42 \mathrm{mmol}), 2.60 \mathrm{~g}$ Silbercarbonat $(9.45 \mathrm{mmol})$ in $8 \mathrm{ml}$ trockenem Acetonitril für $24 \mathrm{~h}$ bei $80^{\circ} \mathrm{C}$ gerührt. Säulenchromatographische Reinigung des Rohproduktes an 25 g Kieselgel (Säule $2.5 \times 10 \mathrm{~cm}$, Pentan, $\left.R_{\mathrm{f}}=0.23\right)$ lieferte $74 \mathrm{mg}$ des Produkts $(189 \mu \mathrm{mol}, 8 \%$ ) als farbloses Öl. $-{ }^{1} \mathrm{H}-\mathrm{NMR}\left(250 \mathrm{MHz}, \mathrm{CDCl}_{3}\right): \delta=0.01-0.26(\mathrm{~m}, 8 \mathrm{H}, c \mathrm{Pr}-\mathrm{H}), 0.56-1.01(\mathrm{~m}$, $10 \mathrm{H}, c \operatorname{Pr}-\mathrm{H}), 1.45-1.66$ (m, $2 \mathrm{H}, c \mathrm{Pr}-\mathrm{H}), 7.12-7.44$ (m, $10 \mathrm{H}, \mathrm{Ar}-\mathrm{H}) .-{ }^{13} \mathrm{C}-\mathrm{NMR}(62.9 \mathrm{MHz}$, $\left.\mathrm{CDCl}_{3}, \mathrm{DEPT}\right): \delta=6.8(-, 4 \mathrm{C}, c \mathrm{Pr}-\mathrm{C}), 9.1$ (-, $\left.4 \mathrm{C}, c \operatorname{Pr}-\mathrm{C}\right), 10.3$ (+, $\left.2 \mathrm{C}, c \operatorname{Pr}-\mathrm{C}\right), 11.4(+, 2 \mathrm{C}$, cPr-C), 127.1** (+, 4 C, $\left.\mathrm{C}_{\text {ortho }}\right), 127.8\left(+, 2 \mathrm{C}, \mathrm{C}_{\text {para }}\right), 132.4^{*}\left(\mathrm{C}_{\text {quart }}, 2 \mathrm{C}, \mathrm{C}_{\mathrm{ipso}}\right), 132.7^{* *}(+$, $\left.4 \mathrm{C}, \mathrm{C}_{\text {meta }}\right), 144.6^{*}\left(\mathrm{C}_{\text {quart }}, 2 \mathrm{C}, \mathrm{C}-2,5\right), 146.3^{* * *}\left(\mathrm{C}_{\text {quart }}, \mathrm{C}-1\right), 148.1^{*}\left(\mathrm{C}_{\text {quart }}, 2 \mathrm{C}, \mathrm{C}-3,4\right)$, 148.4*** (C quart, $\mathrm{C}-6) .-\mathrm{MS}(\mathrm{EI}, 70 \mathrm{eV}), m / z(\%): 390(2)\left[\mathrm{M}^{+}\right], 362$ (4), 334 (2), 313 (6), 284 (100) $\left[\mathrm{M}^{+}-\mathrm{C}_{8} \mathrm{H}_{10}\right], 255$ (38), 243 (27) $\left[\mathrm{M}^{+}-\mathrm{C}_{8} \mathrm{H}_{10}-\mathrm{C}_{3} \mathrm{H}_{5}\right], 228$ (17), 215 (13), 178 (7), 165 (12), 152 (2), 126 (12), 91 (10), 57 (6), 41 (4) $\left[\mathrm{C}_{3} \mathrm{H}_{5}^{+}\right] .-\mathrm{C}_{30} \mathrm{H}_{30}$ (390.57): 390.2348 (korrekte HRMS).

3-(1'-Methyl-3',3'-diphenylallyliden)-cyclobuten (209) und 2,4-Dimethyl-2,3-dicyclopropyl6,6-diphenylpentafulven (210): Nach AAV 6 wurden $51 \mathrm{mg}$ Palladiumacetat $(230 \mu \mathrm{mol})$, 179 mg Triphenylphosphan (680 $\mu \mathrm{mol}), 1.18 \mathrm{~g}$ 1-Brom-2,2-diphenylethen (199) (4.55 mmol), 467 mg 1-Cyclopropyl-1-propin (208) (4.71 mmol), $921 \mathrm{mg}$ Triethylamin $(9.10 \mathrm{mmol})$ in $6 \mathrm{ml}$ trockenem Acetonitril für $24 \mathrm{~h}$ bei $80^{\circ} \mathrm{C}$ gerührt. Säulenchromatographische Reinigung des Rohproduktes an $30 \mathrm{~g}$ Kieselgel (Säule $2.5 \times 12 \mathrm{~cm}$, Pentan) lieferte $69 \mathrm{mg} 209$ 
$\left(R_{\mathrm{f}}=0 . \mathrm{XX}, 270 \mu \mathrm{mol}, 6 \%\right)$ als farbloses Öl und $87 \mathrm{mg} 210\left(R_{\mathrm{f}}=0.76,260 \mu \mathrm{mol}, 6 \%\right)$ in Form eines orangegelben Öls.

209: ${ }^{1} \mathrm{H}-\mathrm{NMR}\left(250 \mathrm{MHz}, \mathrm{CDCl}_{3}\right): \delta=2.61$ (s, $\left.3 \mathrm{H}, \mathrm{Me}\right), 3.91-4.05(\mathrm{~m}, 2 \mathrm{H}, 4-\mathrm{H}), 5.01-5.23$<smiles>CC(C=C(c1ccccc1)c1ccccc1)=C1C=CC1</smiles>
(m, 1 H, 2-H), 6.07-6.28 (m, 1 H, 1-H), 7.36 (s, 1 H, 2'-H), 7.38-7.68 $(\mathrm{m}, 6 \mathrm{H}, \mathrm{Ar}-\mathrm{H}), 7.98\left(\mathrm{~d},{ }^{3} J=8.6 \mathrm{~Hz}, 2 \mathrm{H}, \mathrm{H}_{\mathrm{ortho}}\right), 8.15\left(\mathrm{~d},{ }^{3} \mathrm{~J}=8.6 \mathrm{~Hz}\right.$, $\left.2 \mathrm{H}, \mathrm{H}_{\text {ortho }}\right) \cdot-{ }^{13} \mathrm{C}-\mathrm{NMR}\left(62.9 \mathrm{MHz}, \mathrm{CDCl}_{3}, \mathrm{DEPT}\right): \delta=20.1(+, \mathrm{Me})$, 32.7 (-, C-4), $115.6(+, \mathrm{C}-2), 124.1\left(+, \mathrm{C}_{\text {ortho }}\right), 124.6\left(+, \mathrm{C}_{\mathrm{para}}\right), 125.8(+$, $\left.\mathrm{C}_{\text {meta }}\right), 126.6\left(+, \mathrm{C}_{\text {ortho }}\right), 127.0\left(+, \mathrm{C}_{\text {meta }}\right), 128.2\left(+, 2 \mathrm{C}, \mathrm{C}_{\text {ortho }}\right), 130.1(+$, $\left.3 \mathrm{C}, \mathrm{C}_{\text {meta }}, \mathrm{C}_{\text {para }}\right), 130.3$ (+, C-2'), 130.7 ( $\left.\mathrm{C}_{\text {quart }}, \mathrm{C}-3^{\prime}\right), 132.1\left(\mathrm{C}_{\text {quart }}, \mathrm{C}_{\mathrm{ipso}}\right), 132.6\left(\mathrm{C}_{\text {quart }}, \mathrm{C}_{\mathrm{ipso}}\right)$, 135.8 (+, C-1), $138.6\left(\mathrm{C}_{\text {quart }}, \mathrm{C}-3\right), 140.9$ (C $\mathrm{C}_{\text {quart }}, \mathrm{C}-1$ ') $)$ - MS (EI, $\left.70 \mathrm{eV}\right), \mathrm{m} / z(\%): 258$ (100) $\left[\mathrm{M}^{+}\right], 243$ (55) $\left[\mathrm{M}^{+}-\mathrm{CH}_{3}\right], 228$ (16), 215 (25) $\left[\mathrm{M}^{+}-\mathrm{C}_{3} \mathrm{H}_{7}\right], 202$ (9), 179 (8), 165 (18), 152 (3), 115 (8), 91 (15), 77 (3) $\left[\mathrm{C}_{6} \mathrm{H}_{5}^{+}\right] .-\mathrm{C}_{20} \mathrm{H}_{18}$ (258.36): 258.1409 (korrekte HRMS).

210: ${ }^{1} \mathrm{H}-\mathrm{NMR}\left(250 \mathrm{MHz}, \mathrm{CDCl}_{3}\right): \delta=-0.06-0.09(\mathrm{~m}, 4 \mathrm{H}, c \mathrm{Pr}-\mathrm{H}), 0.74-0.95(\mathrm{~m}, 1 \mathrm{H}$,

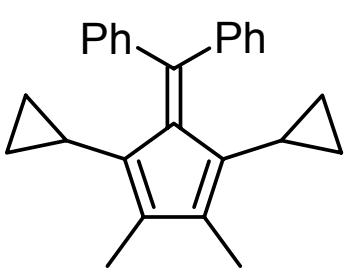
cPr-H), 1.95, 1.96 (s, $6 \mathrm{H}, \mathrm{Me}), 7.13-7.40$ (m, $10 \mathrm{H}, \mathrm{Ar}-\mathrm{H}) .-$ ${ }^{13} \mathrm{C}-\mathrm{NMR}\left(62.9 \mathrm{MHz}, \mathrm{CDCl}_{3}\right.$, DEPT): $\delta=8.2(-, 4 \mathrm{C}, c \operatorname{Pr}-\mathrm{C}), 12.7$ (+, 2 C, cPr-C), 13.7 (+, 2 C, Me), 127.1* (+, 4 C, $\left.\mathrm{C}_{\text {ortho}}\right), 127.9$ (+, $\left.2 \mathrm{C}, \mathrm{C}_{\text {para }}\right), 130.9\left(\mathrm{C}_{\text {quart }}, 2 \mathrm{C}, \mathrm{C}-2,3\right), 132.8^{*}\left(+, 4 \mathrm{C}, \mathrm{C}_{\text {meta }}\right), 142.5^{* *}$ ( $\left.\mathrm{C}_{\text {quart }}, 2 \mathrm{C}, \mathrm{C}-1,4\right), 144.6$ ( $\left.\mathrm{C}_{\text {quart }}, 2 \mathrm{C}, \mathrm{C}_{\mathrm{ipso}}\right), 146.0$ ( $\left.\mathrm{C}_{\text {quart }}, \mathrm{C}-1\right), 148.0$ ( $\left.\mathrm{C}_{\text {quart }}, \mathrm{C}-6\right) .-\mathrm{MS}$ (EI, $70 \mathrm{eV}), m / z(\%): 338(25)\left[\mathrm{M}^{+}\right], 323(35)\left[\mathrm{M}^{+}-\mathrm{CH}_{3}\right], 297(16)\left[\mathrm{M}^{+}-\mathrm{C}_{3} \mathrm{H}_{5}\right], 261$ (100) $\left[\mathrm{M}^{+}-\mathrm{C}_{6} \mathrm{H}_{5}\right], 252$ (13), 231 (16), 215 (22), 202 (16), 178 (9), 165 (15), 91 (17), 77 (6) $\left[\mathrm{C}_{6} \mathrm{H}_{5}^{+}\right], 41$ (7) $\left[\mathrm{C}_{3} \mathrm{H}_{5}^{+}\right] .-\mathrm{C}_{26} \mathrm{H}_{26}$ (338.49): 338.2035 (korrekte HRMS). 
3-(1'-Cyclopropyl-3'-phenylallyliden)-cyclobuten (212): Nach AAV 6 wurden 53 mg

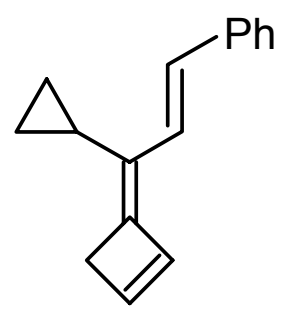

Palladiumacetat $(236 \mu \mathrm{mol}), 186 \mathrm{mg}$ Triphenylphosphan $(708 \mu \mathrm{mol})$, 822 mg E-1-Brom-2-phenylethen (211) (4.49 mmol), 500 mg Dicyclopropylethin (13) (4.71 mmol), $795 \mathrm{mg}$ Triethylamin $(7.86 \mathrm{mmol})$ in $8 \mathrm{ml}$ trockenem Acetonitril für $24 \mathrm{~h}$ bei $80^{\circ} \mathrm{C}$ gerührt. Säulenchromatographische Reinigung des Rohproduktes an $25 \mathrm{~g}$ Kieselgel (Säule $2.0 \times 11 \mathrm{~cm}$, Pentan, $\left.R_{\mathrm{f}}=0.49\right)$ lieferte $364 \mathrm{mg}$ des Produkts $(1.75 \mathrm{mmol}, 39 \%)$ als farbloses Öl. - ${ }^{1} \mathrm{H}-\mathrm{NMR}\left(250 \mathrm{MHz}, \mathrm{CDCl}_{3}\right): \delta=0.88\left(\mathrm{~m}_{\mathrm{c}}, 2 \mathrm{H}, c \mathrm{Pr}-\mathrm{H}\right), 1.12\left(\mathrm{~m}_{\mathrm{c}}, 2 \mathrm{H}, c \operatorname{Pr}-\mathrm{H}\right), 2.25\left(\mathrm{~m}_{\mathrm{c}}\right.$, $1 \mathrm{H}, c \mathrm{Pr}-\mathrm{H}), 4.19\left(\mathrm{~m}_{\mathrm{c}}, 2 \mathrm{H}, 4-\mathrm{H}\right), 4.99-5.19$ (m, $\left.1 \mathrm{H}, 2-\mathrm{H}\right), 6.21\left(\mathrm{~m}_{\mathrm{c}}, 1 \mathrm{H}, 1-\mathrm{H}\right), 7.29 *\left(\mathrm{~d},{ }^{3} J=\right.$ 8.6 Hz, $\left.1 \mathrm{H}, 3^{\prime}-\mathrm{H}\right), 7.42-7.63$ (m, $\left.3 \mathrm{H}, \mathrm{Ar}-\mathrm{H}\right), 7.76^{*}$ (d, ${ }^{3} J=8.3 \mathrm{~Hz}, 1 \mathrm{H}, \mathrm{H}_{\text {ortho }}$ ), 7.90* (d, $\left.{ }^{3} J=8.8 \mathrm{~Hz}, 1 \mathrm{H}, 2{ }^{\prime}-\mathrm{H}\right), 8.12^{*}\left(\mathrm{~d},{ }^{3} J=8.8 \mathrm{~Hz}, 1 \mathrm{H}, \mathrm{H}_{\text {ortho }}\right) .-{ }^{13} \mathrm{C}-\mathrm{NMR}\left(62.9 \mathrm{MHz}, \mathrm{CDCl}_{3}\right.$, DEPT): $\delta=7.3$ (-, 2 C, $c$ Pr-C), 13.8 (+, $c$ Pr-C), 32.4 (-, C-4), 115.4 (+, C-2), 124.0, 124.1, 124.6, 124.7, 125.9, 128.4 (+, 7 C, Ar-C, C-2', C-3'), 132.2* ( $\left.\mathrm{C}_{\text {quart }}, \mathrm{C}_{\mathrm{ipso}}\right), 134.2^{*}\left(\mathrm{C}_{\text {quart }}\right.$, C-1'), 136.5 (+, C-1), 138.2* (C quart, $\mathrm{C}-3) .-\mathrm{MS}(\mathrm{EI}, 70 \mathrm{eV}), \mathrm{m} / z(\%): 208(85)\left[\mathrm{M}^{+}\right], 193$ (60) $\left[\mathrm{M}^{+}-\mathrm{CH}_{3}\right], 178(100)\left[\mathrm{M}^{+}-\mathrm{C}_{2} \mathrm{H}_{6}\right], 167$ (24) $\left[\mathrm{M}^{+}-\mathrm{C}_{3} \mathrm{H}_{5}\right], 152$ (22), 139 (5), 115 (8), 89 (13), 77 (2) $\left[\mathrm{C}_{6} \mathrm{H}_{5}^{+}\right], 63$ (3), 51 (1). $-\mathrm{C}_{16} \mathrm{H}_{16}(208.30)$. 


\section{Zusammenfassung und Ausblick}

Ziel dieser Arbeit war zum einen die Erzeugung des antiaromatischen Pentacyclopropylcyclopentadienyl-Kations 12 unter „stable cation conditions“ sowie dessen Nachweis mittels Tieftemperatur-NMR-Spektroskopie. Weiterhin sollte ausgehend von Dicyclopropylethin (13) bzw. Tetra- 14 und Pentacyclopropylcyclopentadien 15 die mögliche Produktpalette an cyclopropylierten Oligoenen, Carbo- und auch Heterocyclen untersucht werden.

Aus dem seit kurzem zugänglichen Pentacyclopropylcyclopentadienylchlorid $\mathbf{3 6}$ konnte durch Hochvakuum-Matrix-Cokondensation mit Antimonpentafluorid und einem Gemisch aus Sulfurylfluorid und Sulfurylchloridfluorid eine Carbokationen-Lösung gewonnen werden, die in einem Temperaturbereich von -140 bis $-110{ }^{\circ} \mathrm{C}$ NMR-spektroskopisch untersucht wurde. Die aufgenommenen Spektren wiesen auf eine hoch symmetrische Konstitution des erzeugten Carbokations hin, das aus einem vollständig Cyclopropyl-substituierten, fünfgliedrigen Carbocyclus bestehen musste. Neben den $\mathrm{CH}$ - und $\mathrm{CH}_{2}$-Signalen für die Cyclopropanringe konnten drei, ins tiefere Feld verschobene, quartäre ${ }^{13} \mathrm{C}$-Signale detektiert werden, die jeweils als Paar zweier Signale unterschiedlicher Intensität auftraten, und deren Intensitäten sich in erster Näherung wie 2:1:2 verhielten. Bei stufenweiser Erwärmung der Kationenprobe auf $-110^{\circ} \mathrm{C}$ wurde ein dynamisches Phänomen beobachtet, das sowohl die Linienbreite der Signale als auch die Differenz der chemischen Verschiebungen zwischen den jeweiligen Haupt- und Nebensignalen verminderte. Der Abfangversuch einer repräsentativen NMR-Probe mit Methanol und Kaliumcarbonat bei $-90^{\circ} \mathrm{C}$ lieferte ein Rohprodukt, das vorwiegend das Methoxycyclopentadien $\mathbf{6 5}$ enthielt.

Diese experimentellen Daten wurden zunächst mit der Bildung des Pentacyclopropylcyclopentadienyl-Kations 12d interpretiert, das laut quantenchemischen Berechnungen in einem Singulett-Grundzustand vorliegen sollte. Eine bei tiefen Temperaturen sicherlich eingeschränkte Drehbarkeit der Cyclopropyl-Substituenten lieferte dazu eine konsistente 
Erklärung für das dynamische Auftreten von Rotationskonformeren, die bei der jeweiligen Temperatur in unterschiedlichen Verhältnissen vorlagen. Die für 12d berechneten ${ }^{13} \mathrm{C}-\mathrm{NMR}$ Verschiebungen wiesen, verglichen mit den experimentellen Werten, aber eine Diskrepanz von bis zu 70 ppm auf. Sie deuteten vielmehr auf das Vorliegen eines Bisallyl-Dikations 73 hin, das rein formal aus zwei Molekülen 12 im Sinne einer [2+2]-Cycloaddition entstanden sein konnte. Ein solches Dikation 73 wäre - genauso wie 12 - hoch symmetrisch und könnte ebenfalls konformationsisomere Spezies bilden. Allerdings wäre die Bildung des monomeren Abfangproduktes 65 aus 73 nur durch einen Reaktionsmodus zu erklären, der mit einer eventuell Nucleophil-vermittelten - Cycloreversion einhergeht.

Das Pentacyclopropylcyclopentadienyl-Kation 12 könnte demnach als hinreichend reaktives Intermediat formuliert werden, das zunächst aus dem Chlorid $\mathbf{3 6}$ erzeugt und nachfolgend zum Bisallyl-Dikation 73 dimerisieren würde. Die mit diesen experimentellen Methoden erzeugten Versuchsbedingungen und die zur Verfügung stehenden quantenchemischen Berechnungen erlauben die Charakterisierung des Pentacyclopropylcyclopentadienyl-Kations 12 als hinreichend stabilisiertes, antiaromatisches Carbokation nicht eindeutig. Trotzdem es offensichtlich eine bisher nicht vollständig erklärbare Reaktivität aufweist, wurden eindeutige Hinweise erbracht, die dessen Existenz als Zwischenstufe belegen. Diese Beobachtungen bedeuten aber nicht notwendigerweise, dass das Pentacyclopropylcyclopentadienyl-Kation 12 nicht auf anderem Wege generiert werden könnte. So könnten spektroskopische Untersuchungen an einer Festkörpermatrix zum gewünschten Erfolg führen.

Die BARTOLI-Reaktion des 1,2-Dicyclopropylethenylmagnesiumbromids (46) mit $o$-Brom- bzw o-Iodnitrobenzol lieferte mit Ausbeuten von bis zu 38\% die 7-Halogenindole 88a bzw. 88b. Beide konnten Palladium-katalysiert mit Diphenylphosphan gekuppelt werden, wobei man mit bis zu 62\% das Phosphanylindol 87 erhielt. Die Darstellung eines planarchiralen Heteroferrocens wie 16a durch Umsetzung der Anionen von 14 und 87 mit einer Eisen(II)-Quelle gelang bisher nicht. Dagegen konnte der Tricarbonyleisen-Komplex 95a 
durch Erhitzen von 14 mit Dieisennonacarbonyl in Toluol in 45\% Ausbeute gewonnen werden. Die Reaktionen der Cyclopentadiene 14, 15 und des 1,2,3,4-Tetracyclopropylbuta1,3-diens (21) mit Dimethylacetylendicarboxylat (97) oder $N$-Phenylmaleimid (98) lieferten die entsprechenden DiEls-ALDER-Addukte 99-104 mit Ausbeuten von 55-77\%. Die oligocyclopropylierten Bicyclo[2.2.1]heptene 99, 100 und 101 wurden dabei jeweils als Gemische zweier Diastereomere erhalten, die sich in der Konfiguration an C-7 unterschieden - 102 und die Tetracyclopropyl-substituierten Cyclohexene 103 und 104 wurden dagegen jeweils als einzelne Diastereomere isoliert werden. Die Konfigurationen von 100, 102 sowie von 104 konnten durch Röntgenstrukturanalysen bestätigt werden. Im Hinblick auf ein percyclopropyliertes Norbornadien-Gerüst wurde das Pentacyclopropylcyclopentadien $\mathbf{1 5}$ und sein Methoxy-Derivat 65 mit Cyclopropylpropinsäuremethylester (109) und Cyclopropylpropinal (110) bei $160{ }^{\circ} \mathrm{C}$ umgesetzt, wobei man 111, 112 und 113 in Ausbeuten von 9\%, 64\% bzw. 25\% erhielt. Unter hohem Druck konnte die Ausbeute von 112 nur geringfügig auf 86\%, die von 111 dagegen deutlich auf 89\% gesteigert werden. Um ein durch CyclopropylSubstituenten stabilisiertes Cycloheptatrienyl-Kation zugänglch zu machen, wurde Tetracyclopropylbutadien 21 mit Tetrachlorcyclopropen in 51\% zum Bicyclo[4.1.0]hepten 124 umgesetzt und nachfolgend mit m-Chlorperoxybenzoesäure in 91\% Ausbeute zum Epoxid 125 oxidiert. Letzteres konnte aber durch Reaktion mit Trifluoressigsäure nicht in ein Tropyliumion überführt werden.

Das durch nahezu quantitativ verlaufende Quecksilber-katalysierte Hydratisierung von Dicyclopropylethin (13) gewonnene Dicyclopropylethanon (150) konnte mit 88\% Ausbeute in das Tetracyclopropyl-substituierte 1,4-Diketon 151 überführt werden, das als Edukt für eine PAAL-KNORR-Synthesen von Fünfring-Heterocyclen mit einem Heteroatom diente. Die Pyrrole 18a-c wurden dabei in Ausbeuten von 37-48\% isoliert. Das Furan 19 und das Thiophen 20 erhielt man in Ausbeuten von 36\% bzw. 41\%. Durch Reaktion des Enolats von 150 mit Cyclopropancarbonsäurechlorid konnte das 1,3-Diketon 157 hergestellt und mit einer Ausbeute von 89\% in das Pyrazol 158 transformiert werden. Durch die intramolekulare Kupplung eines aus $\mathbf{1 3}$ in situ erzeugten Tetracyclopropyl-Zirkonacyclopentadiens lies sich 
das Tricyclo[4.2.0.0 $0^{2,5}$ ]octadien 165 in 38\% Ausbeute herstellen, das als Vorstufe für eine photochemische Erzeugung des Octacyclopropylcubans 169 dienen könnte. Durch Palladiumkatalysierte Kreuzkupplungen der aus Dicyclopropylethin (13) erhältlichen Diiodide 179 und 180 mit 1,2-Dicyclopropylethenylmagnesiumbromids (46) wurde versucht, das percyclopropylierte 1,3,5-Hexatrien 22 und 1,3,5,7-Octatetraen 23 zu erzeugen. Dabei konnte allerdings nur das Octatetraen 23, und das auch nur massenspektrometrisch, nachgewiesen werden. Bei der versuchten Kreuzkupplung von $\mathbf{1 8 0}$ konnte statt des Hextriens 22 nur Dicyclopropylethin (13) als Eliminierungsprodukt von 180 identifiziert werden. Beim Versuch, das Fulven 207 durch eine sukzessive, Palladium-katalysierte Kupplung des 2,2-Diphenyl-1-bromethens (199) an zwei Äquivalente Dicyclopropylethin (13) darzustellen, konnte ein bemerkenswerter Reaktionsmodus beobachtet werden. Als Produkt wurde nämlich das Allylidencyclobuten $\mathbf{2 0 0}$ in einer Ausbeute von bis zu 71\% erhalten, das nach der Carbometallierung eines Äquivalentes $\mathbf{1 3}$ über eine Cyclopropylmethyl-HomoallylUmlagerung mit anschließender Elektrocyclisierung eines Vinylallens gebildet worden sein musste. Dieser Reaktionsweg konnte durch Einsatz von Silbercarbonat unterdrückt werden, wobei man das Fulven 207 in einer Ausbeute von 8\% erhielt. Erste Versuche, den Einfluss der Größe des Alkylrestes auf die Regioselektivität der Carbometallierung zu untersuchen, lieferte mit dem Acetylen 208 und Triethylamin als Base ein Gemisch von je 6\% des Allylidencyclobutens 209 und des Fulvens 210. Der Einsatz des Bromstyrols 211 zusammen mit 13 lieferte - in jetzt erwarteter Weise - das Allylidencyclobuten 212 in 39\% Ausbeute. Die Einbeziehung eines $\mathrm{sp}^{2}$-Kohlenstoffzentrums in eine Palladium-vermittelte Cyclopropylmethyl-Homoallyl-Umlagerung stellt in dieser Art einen bisher noch nicht beobachteten Reaktionsweg dar. Weitergehende Untersuchungen unter Verwendung von diversen Cyclopropyl-substituierten Acetylenen oder Diinen könnten hier die Synthese einer Reihe von strukturell interessanten Oligoenen ermöglichen. 


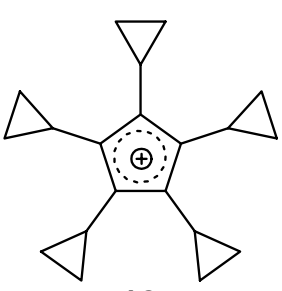

12

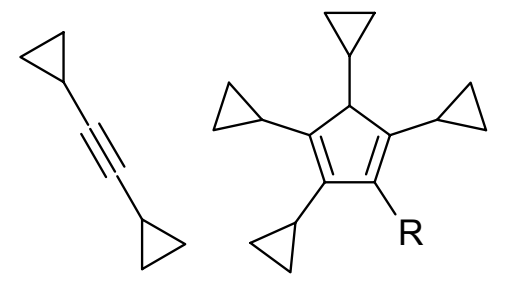

13
$14(\mathrm{R}=\mathrm{H})$

$15(\mathrm{R}=c \mathrm{Pr})$

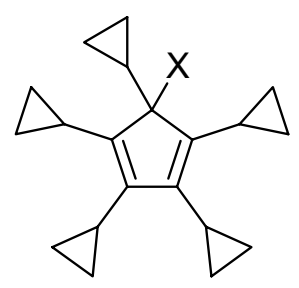

$36(\mathrm{X}=\mathrm{Cl})$

$65(\mathrm{X}=\mathrm{OMe})$

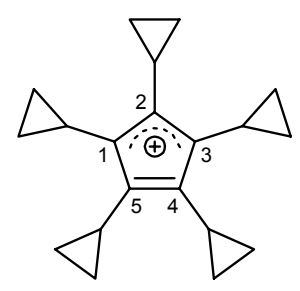

12d/S

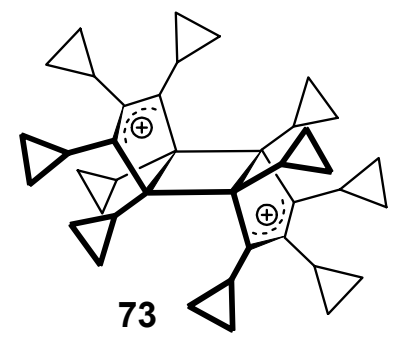

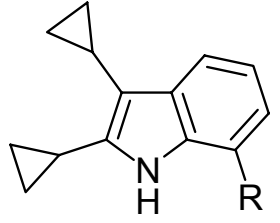

$87\left(R=P P h_{2}\right)$

88a $(\mathrm{R}=\mathrm{Br})$

88b $(R=I)$

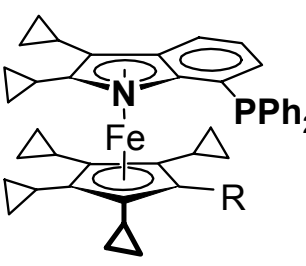

$16 a(R=H)$

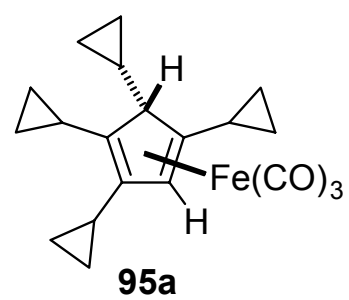<smiles>Br[Mg]C(=CC1CC1)C1CC1</smiles>

46

$97\left(\mathrm{R}=\mathrm{CO}_{2} \mathrm{Me}\right)$ $109(\mathrm{R}=c \mathrm{Pr})$<smiles>[R]C#CC(C)=O</smiles><smiles>O=C1C=CC(=O)N1c1ccccc1</smiles>

98

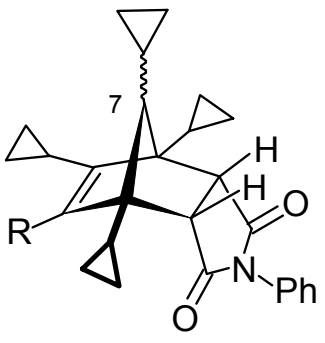

$$
100(\mathrm{R}=\mathrm{H})
$$

$102(\mathrm{R}=c \mathrm{Pr})$

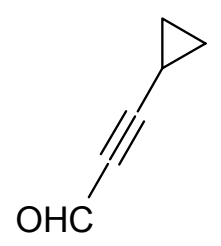

110

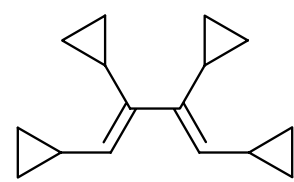

21

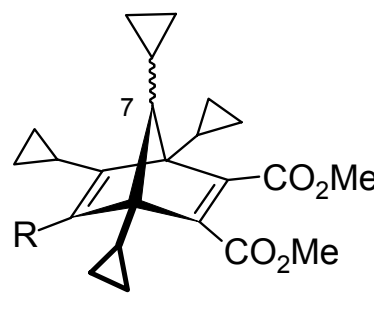

$99(\mathrm{R}=\mathrm{H})$

$101(\mathrm{R}=c \mathrm{Pr})$<smiles>COC(=O)C1=C(C(C)=O)C(C2CC2)C(C2CC2)=C(C2CC2)C1C1CC1</smiles>

103<smiles>C[C@@]12C(=O)N(c3ccccc3)C(=O)[C@H]1C(C1CC1)C(C1CC1)=C(C1CC1)C2C1CC1</smiles>

104
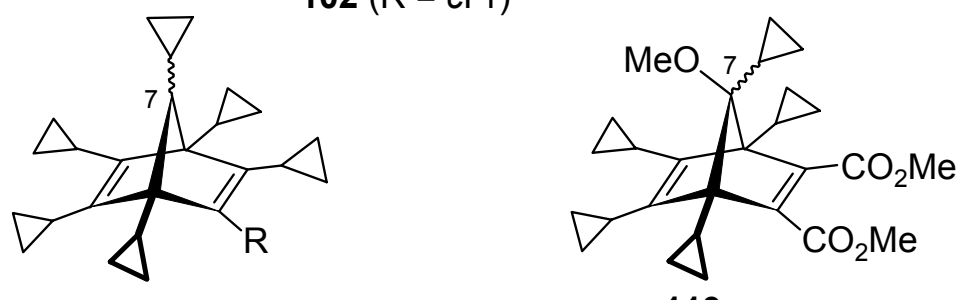

$$
\begin{aligned}
& 111\left(\mathrm{R}=\mathrm{CO}_{2} \mathrm{Me}\right) \\
& 112(\mathrm{R}=\mathrm{CHO})
\end{aligned}
$$<smiles>ClC1(Cl)C2(Cl)C(C3CC3)C(C3CC3)=C(C3CC3)[C@H](C3CC3)C12Cl</smiles>

124<smiles>ClC(Cl)(Cl)C1(Cl)[C@H](C2CC2)C2(C3CC3)OC2(C2CC2)[C@@H](C2CC2)C1(Cl)C1CC1</smiles>

125 
<smiles>O=C(CC1CC1)C1CC1</smiles>

150<smiles>O=C(C1CC1)C(C(=O)C1CC1)C1CC1</smiles>

151

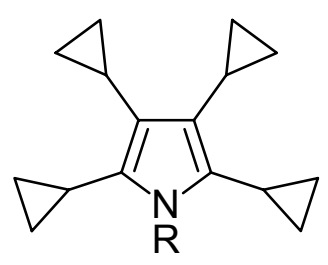

$18 \mathbf{a}(\mathrm{R}=\mathrm{Bn})$

18b $(\mathrm{R}=\mathrm{cPr})$

$18 c(R=H)$

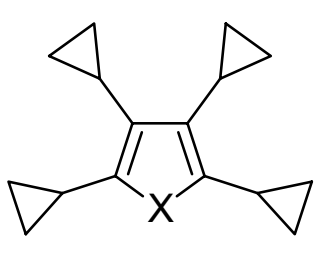

$19(X=0)$

$20(X=S)$<smiles>O=C(C1CC1)C(C(=O)C1CC1)C1CC1</smiles>

157<smiles>IC(=C1CC1)C(=C(I)C1CC1)C(C1CC1)C1CC1</smiles>

179

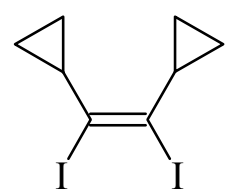

180

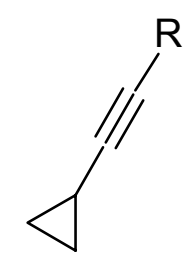

$13(\mathrm{R}=\mathrm{cPr})$ $208(R=M e)$

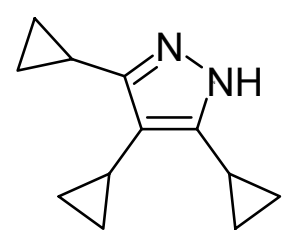

158

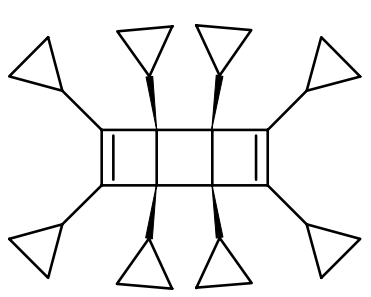

165

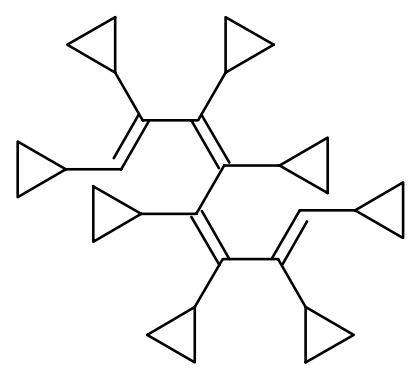

23

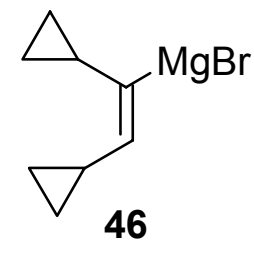

$\prod_{\mathrm{Br}^{\mathrm{R}}}^{\mathrm{R}}$

$199\left(\mathrm{R}=\mathrm{R}^{\prime}=\mathrm{Ph}\right)$

$(Z)-211\left(R=H, R^{\prime}=P h\right)$

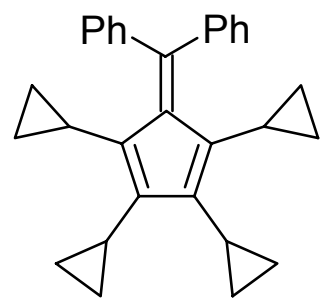

207

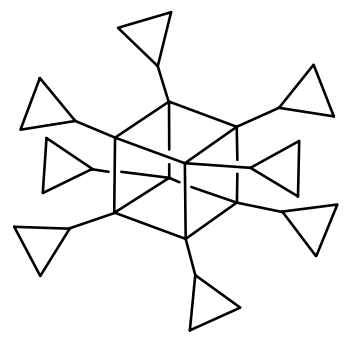

169

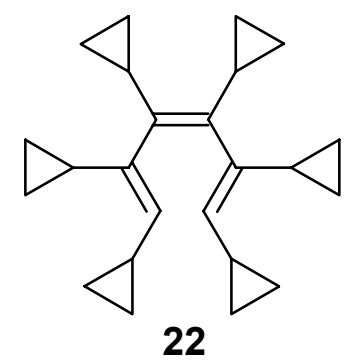

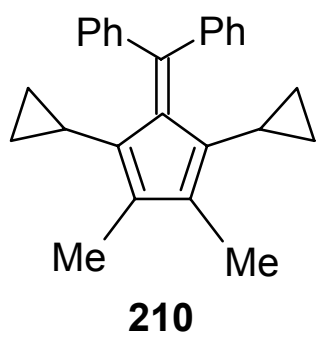

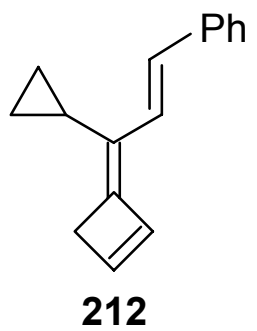




\section{E. Literatur und Anmerkungen}

[1] J. Stieglitz, J. Am. Chem. Soc. 1899, 21, 110.

${ }^{[2]}{ }^{[2 \mathrm{a}]}$ J. F. Norris, J. Am. Chem. Soc. 1901, 25, 117. - ${ }^{[2 \mathrm{~b}]}$ F. Kehrmann, F. Wentzel, Ber. Dtsch. Chem. Ges. 1901, 34, 3815. - ${ }^{[2 c]}$ A. von Baeyer, V. Villinger, Ber. Dtsch. Chem. Ges. 1901, 34, 1189-1208. - ${ }^{[2 \mathrm{~d}]}$ A. von Baeyer, V. Villinger, Ber. Dtsch. Chem. Ges. 1901, 34, 3013. - ${ }^{[2 \mathrm{e}]}$ A. von Baeyer, V. Villinger, Ber. Dtsch. Chem. Ges. 1902, 35, 2018. - ${ }^{[2 f]}$ A. von Baeyer, Ber. Dtsch. Chem. Ges. 1905, 38, 569. - ${ }^{[2 \mathrm{~g}]}$ M. Gomberg, L. H. Cone, Ber. Dtsch. Chem. Ges. 1902, 35, 2397. - ${ }^{[2 \mathrm{~h}]}$ M. Gomberg, L. H. Cone, Justus Liebigs Ann. Chem. 1909, 370, 142. - ${ }^{[2 \mathrm{i}]}$ M. Gomberg, L. H. Cone, Justus Liebigs Ann. Chem. 1910, 376, 183. - ${ }^{[2 \mathrm{j}]}$ P. Walden, Chem. Ber. 1902, 35, 2018.

${ }^{[3]}{ }^{[3 \mathrm{a}]}$ H. Meerwein, K. van Emster, Chem. Ber. 1922, 55, 2500-2528. - ${ }^{[3 \mathrm{~b}]}$ H. Meerwein, K. van Emster, Justus Liebigs Ann. Chem. 1927, 453, 16.

${ }^{[4]}{ }^{[4 a]}$ E. D. Hughes, C. K. Ingold, C. S. Patel, J. Chem. Soc. 1933, 526. - ${ }^{[4 b]}$ C. K. Ingold, Structure and Mechanism in Organic Chemistry, Cornell University Press, 1953, Ithaca, New York; $2^{\text {nd }}$ Ed., 1969. $-{ }^{[4 c]}$ F. C. Witmore, J. Am. Chem. Soc. 1932, 54, 3274-3278. - ${ }^{[4 \mathrm{~d}]}$ F. C. Witmore, Chem. Eng. News 1948, 26, 668.

${ }^{[5]}{ }^{[5 \mathrm{a}]}$ G. A. Olah, Angew. Chem. 1963, 75, 800.- ${ }^{[5 b]}$ G. A. Olah, Science 1970, 168, 1298. - ${ }^{[5 \mathrm{c}]}$ Nobel-Vorlesung: G. A. Olah, Angew. Chem. 1995, 107, 1519-1532; Angew. Chem. Int. Ed. Engl. 1995, 34, 1395-1408. - ${ }^{[5 \mathrm{~d}]}$ G. A. Olah, J. Org. Chem. 2001, 66, 5943-5957.

[6] Übersichten: ${ }^{[6 \mathrm{a}]}$ G. A. Olah, Topics Curr. Chem. 1979, 80, 19-88. - ${ }^{[6 \mathrm{~b}]}$ G. A. Olah, P. v. R. Schleyer, Carbonium Ions, Vol. I-V, Wiley Interscience, New York, 1968, 1970, 1972, 1973, 1976. $-{ }^{[6 c]}$ G. A. Olah, G. J. Sommer, G. K. S. Prakash, Superacids, John Wiley, New York, 1985. - ${ }^{[6 \mathrm{~d}]}$ D. Lenoir, H.-U. Siehl in Houben-Weyl, Methoden der Organischen Chemie, Hrsg.: M. Hanack, Thieme, Stuttgart, 1990, Vol. E19c. $-{ }^{[6 \mathrm{e}]}$ P. v. R. Schleyer, G. K. S. Prakash, Stable Carbocation Chemistry, John Wiley \& Sons, New York, 1997. - ${ }^{[6 f]}$ G. A. Olah, G. K. S. Prakash, Across Conventional Lines, Vol. 1-2, 
World Scientific Publishing, Singapore, 2003, Vol. 11 in the World Scientific Series in $20^{\text {th }}$ Century Chemistry.

[7] Zitat von G. A. Olah: "We feel that it is timely to recognize that as all carbocations are delocalized by varying degrees of $\pi$ - and $\sigma$-conjugation/hyperconjugation it is increasingly becoming unnecessary to label carbocations as classical or nonclassical. The terms classical (i.e. trivalent carbenium) and nonclassical (i.e. higher coordinate carbonium) ions define only the limits of a spectrum that all carbocations fall within." aus: G. A. Olah, N. J. Head, G. Rasul, G. K. S. Prakash, J. Am. Chem. Soc. 1995, 117, 875.

${ }^{[8]}{ }^{[8 \mathrm{a}]}$ M. Gomberg, Ber. Dtsch. Chem. Ges. 1900, 33, 3159. - ${ }^{[8 b]}$ M. Gomberg, Ber. Dtsch. Chem. Ges. 1901, 34, 2727. - ${ }^{[8 c]}$ M. Gomberg, Ber. Dtsch. Chem. Ges. 1902, 35, 1836. $-{ }^{[8 \mathrm{~d}]}$ M. Gomberg, Ber. Dtsch. Chem. Ges. 1902, 35, 3918. - ${ }^{[8 \mathrm{e}]}$ M. Gomberg, Ber. Dtsch. Chem. Ges. 1903, 36, 378.

${ }^{[9]}{ }^{[9 a]}$ N. C. Deno, H. G. Richey, J. S. Liu, D. N. Lincoln, J. O. Turner, J. Am. Chem. Soc. 1965, 87, 4533-4538. $-{ }^{[9 b]}$ C. U. Pittman, G. A. Olah, J. Am. Chem. Soc. 1965, 87, 5123-5132. $-{ }^{[9 c]}$ T. S. Sorensen, I. J. Miller, K. Ranganayakulu, Aust. J. Chem. 1973, 26, 311-317. - ${ }^{[9 \mathrm{~d}]}$ G. A. Olah, Angew. Chem. 1973, 85, 183-234.

[10] Eigenschaften: ${ }^{[10 \mathrm{a}]}$ A. de Meijere, Angew. Chem. 1979, 91, 867-884; Angew. Chem. Int. Ed. Engl. 1979, 18, 809-826. - ${ }^{[10 \mathrm{~b}]}$ K. B. Wiberg, Houben-Weyl, Methods of Organic Chemistry, Vol. E 17a (Hrsg.: A. de Meijere), Thieme, Stuttgart, 1997, S. 1-26. ${ }^{[10 \mathrm{c}]}$ T. T. Tidwell in The Chemistry of the Cyclopropyl Group (Hrsg.: Z. Rappoport), Wiley, Chichester, 1987, S. 565-632. $-{ }^{[10 \mathrm{~d}]}$ G. Boche, H. M. Wakbrosky, Cyclopropane derived reactive Intermediates, Eds.: S. Patai, Z. Rappoport, John Wiley \& Sons, New York, 1990. - Reaktivität: ${ }^{[10 e]}$ A. de Meijere, Synlett 1990, 20-32. ${ }^{[10 f]}$ A. de Meijere in Strain and in its Implications in Organic Chemistry, (Hrsg.: A. de Meijere, S. Blechert), Kluwer, Dordrecht, 1989. - ${ }^{[10 \mathrm{~g}]}$ Top. Curr. Chem., Small Ring Compounds in Organic Synthetis $I-I V$, Hrsg.: A. de Meijere, 1986, 1987, 1988, 1990, Vols. 133, 135, 144, 155. 
${ }^{[11]}{ }^{[11 \mathrm{a}]}$ A. D. Walsh, Trans. Faraday Soc. 1949, 45, 179. - ${ }^{[11 \mathrm{~b}]}$ A. D. Walsh, T. M. Sudgen, Nature 1947, 160, 367. - ${ }^{[11 b]}$ E. Honegger, E. Heilbronner, A. Schmelzer, und W. JianQi, Isr. J. Chem. 1982, 22, 3.

[12] E. Honneger, E. Heilbronner, A. Schmelzer, Nouv. J. Chim. 1982, 6, 519.

${ }^{[13]}{ }^{[13 a]}$ T. Förster, Z. Phys. Chem. 1939, 45, 179. $-{ }^{[13 b]}$ C. A. Coulson, W. E. Moffit, J. Chem. Phys. 1947, 15, 151. $-{ }^{[13 c]}$ C. A. Coulson, T. H. Goodwin, J. Chem. Soc. 1962, $2851-2854$.

[14] K. B. Wiberg, Acc. Chem. Res. 1996, 29, 229-234.

${ }^{[15]}{ }^{[15 \mathrm{a}]}$ A. de Meijere, W. Lüttke, Tetrahedron 1969, 25, 1531. $-{ }^{[15 b]}$ B. R. Ree, J. C. Martin, J. Am. Chem. Soc. 1970, 92, 1660.- ${ }^{[15 c]}$ A. de Meijere, O. Schallner, C. Weitemeyer, W. Spielmann, Chem. Ber. 1979, 112, 908.

[16] Rotationsbarriere z. B. für das 2-(Cyclopropyl)propyl-Kation: $13.7 \mathrm{kcal} / \mathrm{mol}$ bei -49 bis $-21^{\circ} \mathrm{C}$; für das 1-(Cyclopropyl)cyclohexenyl-Kation: $11.1 \mathrm{kcal} / \mathrm{mol}$ bei $-45^{\circ} \mathrm{C}$.

[17] G. A. Olah, R. J. Spear, J. Am. Chem. Soc. 1975, 97, 1539, und dort zit. Literatur.

[18] L. R. Schmitz und T. S. Sorensen, J. Am. Chem. Soc. 1982, 104, 2605.

[19] Für $\mathrm{Ph}_{3} \mathrm{C}_{3}^{+}$siehe: R. Breslow, H. Höver, H. W. Chang, J. Am. Chem. Soc. 1961, 83, 2375. - Für $\mathrm{Me}_{3} \mathrm{C}_{3}^{+}$siehe: J. Ciabattoni, E. C. Nathan III, Tetrahedron Lett. 1969, 4997. - Für $c \operatorname{Pr}_{3} \mathrm{C}_{3}^{+}$siehe: K. Komatsu, I. Tomioka, K. Okamoto, Tetrahedron Lett. 1980, 947; R. A. Moss, R. C. Munjal, Tetrahedron Lett. 1980, 1221.

[20] H. H. Freedman in Carbonium Ions, Vol. IV, (Ed.: G. A. Olah, P. von R. Schleyer), Wiley-Interscience, New York, 1973, S. 1501-1578.

[21] Für eine Übersicht zu Aspekten der Aromatizität siehe: ${ }^{[21 a]}$ V. I. Minkin, M. N. Glukhovtsev, B. Y. Simkin, Aromaticity and Antiaromaticity, Wiley, New York, 1994. - ${ }^{[21 b]}$ P. J. Garratt, Aromaticity, Wiley, New York, 1986. $-{ }^{[21 c]}$ Autoren in Chem. Rev. 2001, 101, und dort zit. Literatur.

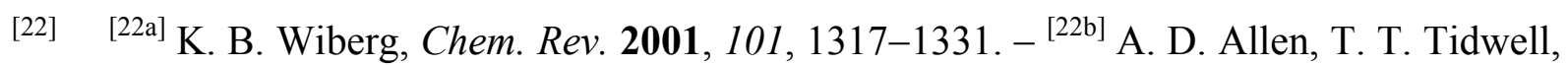
Chem. Rev. 2001, 101, 1333-1348. $-{ }^{[22 \mathrm{c}]}$ A. D. Allen, M. Sumonja, T. T. Tidwell, J. 
Am. Chem. Soc. 1997, 119, 2371-2375. - ${ }^{[22 \mathrm{~d}]}$ E. D. Bergmann, B. Pullmann, Aromaticity, Pseudoaromaticity and Antiaromaticity, Academic Press, New York, 1971.

[23] R. Breslow, J. M. Hoffman, J. Am. Chem. Soc. 1972, 94, 2110-2111.

[24] M. Saunders, R. Berger, A. Jaffe, J. M. McGridge, J. O’Neill, R. Breslow, J. M. Hoffman, C. Perchonok, E. Wasserman, R. S. Hutton, V. J. Kuck, J. Am. Chem. Soc. 1973, 95, 3017-3018.

${ }^{[25]}{ }^{[25 a]}$ M. J. S. Dewar, C. de Llano, J. Am. Chem. Soc. 1969, 91, 789. $-{ }^{[25 b]}$ M. J. S. Dewar, The Molecular Orbital Theory of Organic Chemistry, McGraw Hill, New York, 1969. - ${ }^{[25 c]}$ E. Hückel, Z. Physik 1931, 70, 204. - ${ }^{[25 \mathrm{~d}]}$ E. Hückel, Z. Physik 1932, 76, 628. - ${ }^{[25 e]}$ E. Hückel, Z. Physik 1933, 83, 632.

${ }^{[26]}{ }^{[26 a]}$ R. Breslow, Chem. Eng. News 1965, 90-99. $-{ }^{[26 b]}$ R. Breslow, Acc. Chem. Res. $1973,393-398$.

[27] T. M. Krygowski, M. K. Cyranski, Z. Czarnocki, G. Häfelinger, A. R. Katritzky, Tetrahedron 2000, 56, 1783-1796.

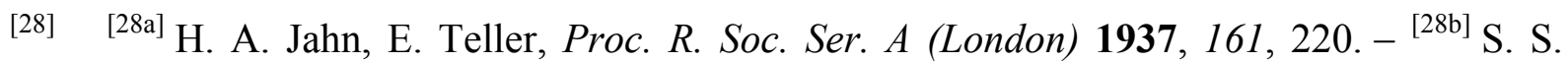
Shaik, P. C. Hiberty, J. Am. Chem Soc. 1985, 107, 3089-3095.

[29] M. N. Glukhovtsev, B. Reindl, P. v. R. Schleyer, Mendeleev Commun. 1993, 100-102.

[30] Rechnungen: ${ }^{[30 \mathrm{a}]}$ R. E. Williams, Inorg. Chem. 1971, 10, 210. - ${ }^{[30 \mathrm{~b}]}$ W. D. Stohrer, R. J. Hoffman, J. Am. Chem. Soc. 1972, 94, 1661. - Experimentelle Hinweise: ${ }^{[30 c]}$ S. Masamune, M. Sakai, H. Ona, J. Am. Chem. Soc. 1972, 94, 8955. - ${ }^{[30 \mathrm{~d}]}$ G. Maier, H. Rang, H. O. Kalinowsi, Angew. Chem. 1989, 101, 1293-1295.

[31] R. Breslow, S. Mazur, J. Am. Chem. Soc. 1973, 95, 584-585.

[32] Für $\mathrm{C}_{5} \mathrm{Cl}_{5}{ }^{+}$siehe: ${ }^{[32 \mathrm{a}]}$ R. Breslow, R. Hill, E. Wasserman, J. Am. Chem. Soc. 1964, 86, 5349-5350. - ${ }^{[32 b]}$ H. Vancik, I. Novak, D. Kidemet, J. Phys. Chem. A 1997, 101, 1523-1525. - Für $\mathrm{C}_{5} \mathrm{NR}_{4}{ }^{+}$siehe: ${ }^{[32 \mathrm{c}]}$ R. Gompper, H. Glöckner, Angew. Chem. 1984, 96, 48-79; Angew. Chem. Int. Ed. Engl. 1984, 9, 53-55. - ${ }^{[32 \mathrm{~d}]}$ R. Gompper, T. Geßner, Angew. Chem. 1985, 97, 996-998. - Für $\mathrm{C}_{5} \mathrm{Ar}_{5}{ }^{+}$siehe: ${ }^{[32 \mathrm{e}]} \mathrm{R}$. Breslow, H. W. Chang, $J$. Am. Chem. Soc. 1961, 83, 3727-3728. $-{ }^{[32 f]}$ R. Breslow, H. W. Chang, W. A. Yager, J. 
Am. Chem. Soc. 1963, 85, 2033-2034. $-{ }^{[32 \mathrm{~g}]}$ W. Broser, H. Kurreck, P. Siegle, Chem. Ber. 1967, 100, 788-794. $-{ }^{[32 \mathrm{~h}]}$ W. Broser, P. Siegle, H. Kurreck, Chem. Ber. 1968, $101,69-83$.

[33] H. Sitzmann, H. Bock, R. Boese, T. Dezember, Z. Havlas, W. Kaim, M. Moscherosch, L. Zanathy, J. Am. Chem. Soc. 1993, 115, 12003-12009.

${ }^{[34]}{ }^{[34 a]}$ J. B. Lambert, L. Lin, V. Rassolov, Angew. Chem. 2002, 114, 1487-1489; Angew. Chem. Int. Ed. 2002, 41, 1429-1431. - ${ }^{[34 b]}$ M. Otto, D. Scheschkewitz, T. Kato, M. M. Midland, J. B. Lambert, G. Bertrand, Angew. Chem. 2002, 114, 2379-2380; Angew. Chem. Int. Ed. 2002, 41, 2275-2276. - ${ }^{[34 c]}$ T. Müller, Angew. Chem. 2002, 114, 2380-2382; Angew. Chem. Int. Ed. 2002, 41, 2276-2278. - ${ }^{[34 d]}$ J. B. Lambert, Angew. Chem. 2002, 114, 2382; Angew. Chem. Int. Ed. 2002, 41, 2278.

[35] K. Komatsu, persönliche Mitteilung, 2004.

${ }^{[36]}{ }^{[36 a]}$ S. Redlich, Diplomarbeit, Universität Göttingen, 2001. - ${ }^{[36 b]}$ I. Emme, S. Redlich, T. Labahn, J. Magull, A. de Meijere, Angew. Chem. 2002, 114, 811-814; Angew. Chem. Int. Ed. 2002, 41,786-789.

[37] I. Emme, Dissertation, Universität Göttingen, 2000.

[38] Für eine Übersicht siehe: P. J. Guiry, C. P. Saunders, Adv. Synth. Catal. 2004, 346, $497-537$.

[39] Für 2,5-Dicyclopropylthiophen siehe: S. Kozhushkov, T. Haumann, R. Boese, B. Knieriem, S. Scheib, P. Bäuerle, A. de Meijere, Angew.Chem. 1995, 107, 859-862.

[40] Für einen Überblick zu diversen cyclopropylierten Derivate des Pentafulvens und Cyclopentadiens siehe: A. de Meijere, S. I. Kozhushkov, A. A. Fokin, I. Emme, S. Redlich, P. R. Schreiner, Pure Appl. Chem. 2003, 75, 549-562.

${ }^{[41] \quad[41 \mathrm{a}]}$ R. Breslow, J. M. Hoffmann, Jr., J. Am. Chem. Soc. 1972, 94, 2110-2111. - ${ }^{[41 b]}$ R. Breslow, Acc. Chem. Res. 1973, 6, 393-398. $-{ }^{[41 c]}$ R. Breslow, S. Mazur, J. Am. Chem. Soc. 1973, 95, 584-585. $-{ }^{[41 \mathrm{~d}]}$ M. Saunders, R. Berger, A. Jaffe, J. M. McBride, J. O’Neill, R. Breslow, J. M. Hoffman, C. Perchonock, E. Wasserman, R. S. Hutton, V. J. Kuck, J. Am. Chem. Soc. 1973, 95, 3017-3018. 


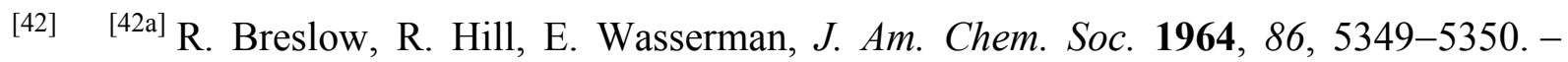
${ }^{[42 b]}$ H. Vancik, I. Novak, D. Kidemet, J. Phys. Chem. A 1997, 101, 1523-1525.

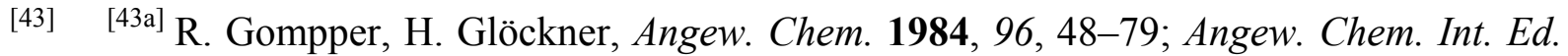
Engl. 1984, 9, 53-55. - ${ }^{[43 b]}$ R. Gompper, T. Geßner, Angew. Chem. 1985, 97, 996-998.

${ }^{[44]}{ }^{[44 a]}$ R. Breslow, H. W. Chang, J. Am. Chem. Soc. 1961, 83, 3727-3728. $-{ }^{[44 b]}$ R. Breslow, H. W. Chang, W. A. Yager, J. Am. Chem. Soc. 1963, 85, 2033-2034.${ }^{[44 \mathrm{c}]}$ W. Broser, H. Kurreck, P. Siegle, Chem. Ber. 1967, 100, 788-794. $-{ }^{[44 \mathrm{~d}]}$ W. Broser, P. Siegle, H. Kurreck, Chem. Ber. 1968, 101, 69-83.

[45] H. Sitzmann, H. Bock, R. Boese, T. Dezember, Z. Havlas, W. Kaim, M. Moscherosch, L. Zanathy, J. Am. Chem. Soc. 1993, 115, 12003-12009.

${ }^{[46]}{ }^{[46 a]}$ J. B. Lambert, L. Lin, V. Rassolov, Angew. Chem. 2002, 114, 1487-1489; Angew. Chem. Int. Ed. 2002, 41, 1429-1431. - ${ }^{[46 b]}$ M. Otto, D. Scheschkewitz, T. Kato, M. M. Midland, J. B. Lambert, G. Bertrand, Angew. Chem. 2002, 114, 2379-2380; Angew. Chem. Int. Ed. 2002, 41, 2275-2276. - ${ }^{[46 c]}$ T. Müller, Angew. Chem. 2002, 114, 2380-2382; Angew. Chem. Int. Ed. 2002, 41, 2276-2278. - ${ }^{[46 \mathrm{~d}]}$ J. B. Lambert, Angew. Chem. 2002, 114, 2382; Angew. Chem. Int. Ed. 2002, 41, 2278.

[47] B. Reindl, P. v. R. Schleyer, J. Comput. Chem. 1998, 19, 1402-1420.

[48] P. Jutzi, A. Mix, Chem. Ber. 1992, 125, 951-954.

[49] A. A. Frost, B. Musulin, J. Chem. Phys. 1953, 21, 572-573.

[50] $\mathrm{HOMO}=$ highest $o$ ccupied molecular orbital.

[51] $\mathrm{SOMO}=$ singly occupied molecular orbital.

[52] $\quad$ LUMO $=$ lowest $u$ noccupied molecular orbital.

[53] Die Rechnungen wurden von Prof. Dr. P. R. Schreiner (Justus-Liebig-Universität in Gießen) durchgeführt.

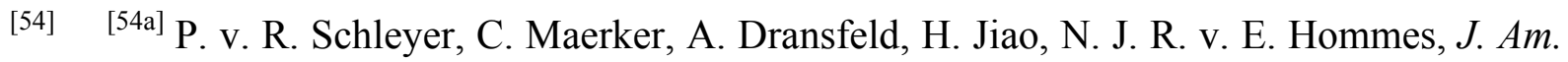
Chem. Soc. 1996, 118, 6317-6318. $-{ }^{[54 b]}$ M. Saunders, P. v. R. Schleyer, M. Bühl, W. Thiel, H. Jiao, J. Am. Chem. Soc. 1989, 111, 3007-3010. $-{ }^{[54 c]}$ P. v. R. Schleyer, P. Freeman, H. Jiao, B. Goldfuß, Angew. Chem. 1995, 107, 332-335; Angew. Chem. Int. 
Ed. Engl. 1995, 20, 991-994. - ${ }^{[54 d]}$ T. Heine, P. v. R. Schleyer, C. Lorminboeuf, G. Seifert, R. Reviakine, J. Weber, J. Phys. Chem. A 2003, 107, 6470-6475.

N. C. Baird, J. Am. Chem. Soc. 1972, 94, 4941-4948.

${ }^{[56]}{ }^{[56 a]}$ B. Reindl, P. von R. Schleyer, J. Comp. Chem. 1998, 19, 1402-1420. - ${ }^{[56 b]}$ Y. Mo, H. Jiao, Z. Lin, P. von R. Schleyer, Chem. Phys. Letters 1998, 383-390. $-{ }^{[56 c]}$ M. N. Glukhovtsev, B. Reindl, P. von R. Schleyer, Mendeleev Commun. 1993, 100-102.${ }^{[56 \mathrm{~d}]}$ E. P. F. Lee, T. G. Wright, Phys. Chem. Chem. Phys. 1999, 1, 219-225. - ${ }^{[56 \mathrm{e}]}$ V. Gogonea, P. v. R. Schleyer, P. R. Schreiner, Angew. Chem. 1998, 110, 2045-2049.

[57] G. A. Olah, Y. Halpern, J. Org. Chem. 1971, 36, 2354.

[58] G. A. Olah, Angew. Chem. 1973, 85, 183; Angew. Chem. Int. Ed. Engl. 1973, 12, 173.

[59] J. W. Larsen, P. A. Bouis, C. R. Watson, M. Pagni, J. Am. Chem. Soc. 1974, 96, 2284.

[60] E. M. Arnett, T. C. Hofelich, J. Am. Chem. Soc. 1983, 105, 2889.

${ }^{[61]}{ }^{[61 \mathrm{a}]}$ N. N. Lichtin in Carbonium Ions (G. A. Olah, P. von R. Schleyer), Bd. 1, Kap. 4, Wiley, New York, 1968. $-{ }^{[61 b]}$ R. J. Gillespie, E. A. Robinson in Carbonium Ions (G. A. Olah, P. von R. Schleyer), Bd. 1, Kap. 3, Wiley, New York, 1968.

[62] H. Vancik, D. E. Sunko, J. Am. Chem. Soc. 1989, 111, 3742.

[63] Übersichten zu substituierten Cyclopentadienylliganden und ihren Komplexen: ${ }^{[63 a]} \mathrm{J}$. Okuda, Top. Curr. Chem. 1992, 160, 97-145. $-{ }^{[63 b]}$ C. Janiak, H. Schumann, $A d v$. Organomet. Chem. 1991, 33, 291-393. - ${ }^{[63 c]}$ J. Okuda, T. Eberle in Metallocenes (Hrsg.: A. Togni, R.-L. Halterman), Wiley, New York, 1988, S. 415. - Potentielle Vorläufer für Di- und Tricyclopropylcyclopentadiene sind beschrieben worden: ${ }^{[63 \mathrm{~d}]} \mathrm{B}$. L. Flynn, A. de Meijere, J. Org. Chem. 1999, 64, 400-404. - ${ }^{[63 e]}$ H. Schirmer, F. J. Funke, S. Müller, M. Noltemeyer, B. L. Flynn, A. de Meijere, Eur. J. Org. Chem. 1999, 2025-2031.

${ }^{[64]}{ }^{[64 a]}$ Der sterische Effekt einer Cyclopropylgruppe wird durch einen Satz von Substituentenkonstanten $\mathscr{\mathscr { f }}_{\mathrm{f}}(\mathrm{R})$ sehr passend beschrieben [H. D. Beckhaus, Angew. Chem. 1978, 90, 633-635; Angew. Chem. Int. Ed. Engl. 1978, 17, 593-594: $\mathscr{O}_{\mathrm{f}}(\mathrm{R})=$ $0.00(\mathrm{Me}), 0.86(\mathrm{Et}), 1.33(c \mathrm{Pr}), 1.81(c \mathrm{Pent}), 2.29(i \mathrm{Pr}), 3.82(t \mathrm{Bu})]$. 


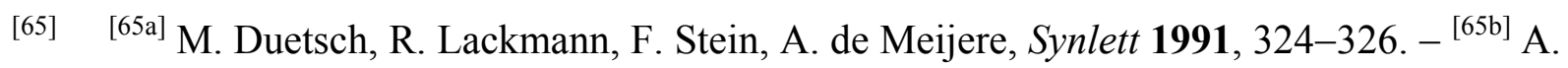
de Meijere, Pure Appl. Chem. 1996, 68, 61-72. $-{ }^{[65 c]}$ B. L. Flynn, F. J. Funke, C. C. Silveira, A. de Meijere, Synlett 1995, 1007-1010.

${ }^{[66]}{ }^{[66 a]}$ P. L. Pauson in Organometallics in Organic Synthesis, (Hrsg.: A. de Meijere, H. tom Dieck), Springer, Berlin, 1987, 233. $-{ }^{[66 b]}$ Neuere Entwicklungen: O. Geis, H. G. Schmalz, Angew. Chem. 1998, 110, 955-958; Angew. Chem. Int. Ed. 1998, 37, 911-914.

[67] Übersicht: C. Santelli-Rouvier, M. Santelli, Synthesis 1993, 429-442.

[68] V. Usieli, R. Victor, S. Sarel, Tetrahedron Lett. 1976, 31, 2705-2706.

[69] Mechanismus: R. B. Woodward, R. Hoffmann, Die Erhaltung der Orbitalsymmetrie, Verlag Chemie, Weinheim 1970, S. 45ff.

${ }^{[70]}{ }^{[70 a]}$ Y. Gao, F. Sato, J. Chem. Soc. Chem. Commun. 1995, 659-660. - ${ }^{[70 b]}$ Y. Gao, F. Sato, J. Chem. Soc. Chem. Commun. 1995, 1929. - ${ }^{[70 c]}$ F. Sato, H. Ishikawa, M. Sato, Tetrahedron Lett. 1981, 22, 85-88. - ${ }^{[70 d]}$ F. Sato, Y. Kobayashi, Org. Synth. 1990, 69, 106.

${ }^{[71]}{ }^{[71 a]}$ T. Imamoto, N. Takiyama, K. Nakamura, Tetrahedron Lett. 1985, 26, 4763-4766. ${ }^{[71 b]}$ T. Imamoto, T. Kusumoto, Y. Tawarayama, Y. Sugiura, T. Mita, Y. Hatanaka, M. Yokoyama, J. Org. Chem. 1984, 49, 3904-3912.

${ }^{[72]}{ }^{[72 a]}$ P. H. Campbell, N. W. K. Chiu, K. Deugau, I. J. Miller, T. S. Sorensen, J. Am. Chem. Soc. 1969, 91, 6404-6410. - ${ }^{[72 b]}$ T. S. Sorensen, I. J. Miller, C. M. Urness, Can. J. Chem. 1970, 48, 3374-3381.

[73] D. M. Brouwer, J. A. van Doorn, Recl. Trav. Chim. Pays-Bas 1970, 89, 333-352.

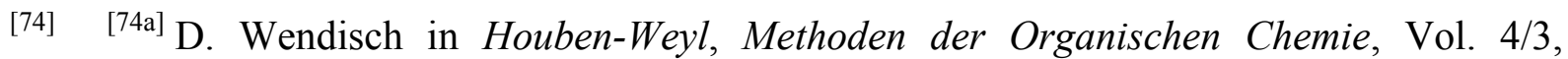
Thieme, Stuttgart 1971, S. 415-509. $-{ }^{[74 b]}$ M. Saunders, H. A. Jimenez-Vazquez, Chem. Rev. 1991, 91, 375.

${ }^{[75]}{ }^{[75 a]}$ siehe Lit.: ${ }^{[40]} .-{ }^{[75 b]}$ R. Haag, A. de Meijere in Modern Arene Chemistry, (Ed.: D. Astruc), Wiley-VCH, Weinheim, 2002, 32-52.

[76] G. A. Olah, Halonium Ions, Wiley, New York, 1975. 
${ }^{[77]}{ }^{[77 a]}$ E. Y.-X. Chen, T.-J. Marks, Chem. Rev. 2000, 100, 1391-1434. - ${ }^{[77 b]}$ C. A. Reed, Acc. Chem. Res. 1998, 31, 133-139. - ${ }^{[77 \mathrm{c}]}$ W. E. Piers, Chem. Eur. J. 1998, 4, 13-18. ${ }^{[77 d]}$ S. H. Strauss, Chem. Rev. 1993, 93, 927-942.

[78] M. R. Rosenthal, J. Chem. Educ. 1973, 50, 331.

${ }^{[79]}{ }^{[79 a]}$ P. A. Grieco, W. J. DuBay, L. J. Todd, Tetrahedron Lett. 1996, 37, 8707-8710. ${ }^{\left[{ }^{[7 b]}\right.}$ K. Fujiki, I. S. Kobayashi, A. Mori, A. Nagira, J. Nie, T. Sonoda, Y. Yagupolskii, Chem. Lett. 2000, 1, 62-63. $-{ }^{[79 c]}$ S. Moss, B. T. King, A. de Meijere, S. I. Kozhushkov, P. E. Eaton, J. Michl, Org. Lett. 2001, 3, 2375-2377.

[80] C. A. Reed, K.-C. Kim, R. D. Bolskar, L. Mueller, Science 2000, 289, 101-104.

[81] Für $\mathrm{C}_{60}{ }^{\bullet+}$ siehe: ${ }^{[80]}$. Für $\mathrm{Bu}_{3} \mathrm{Sn}^{+}$siehe: ${ }^{[81 \mathrm{a}]}$ I. Zharov, B. T. King, Z. Havlas, A. Pardi, J. Michl, J. Am. Chem. Soc. 2000, 122, 10253-10254. - Für Cu(CO) ${ }_{4}^{+}$siehe: ${ }^{[81 b]} \mathrm{S} . \mathrm{M}$. Ivanova, S. V. Ivanov, S. M. Miller, O. P. Anderson, K. A. Solntsev, S. H. Strauss, Inorg. Chem. 1999, 38, 3756.

[82] D. Stasko, C. A. Reed, J. Am. Chem. Soc. 2002, 124, 1148-1149.

[83] Herrn Prof. Siehl und Herrn Dr. Freudenberger danke ich sehr herzlich für die Forschungsaufenthalte in ihrem Arbeitskreis, die hilfreichen Diskussionen und Tipps und Tricks bei der Durchführung der experimentellen Arbeit.

[84] P. V. R. Schleyer, C. Maerker, P. Buzek, und S. Sieber, Accurate Carbocation Structures, in Stable Carbocation Chemistry, Eds.: G. K. S. Prakash und P. v. R. Schleyer, John Wiley \& Sons, New York, 1997, S. 19.

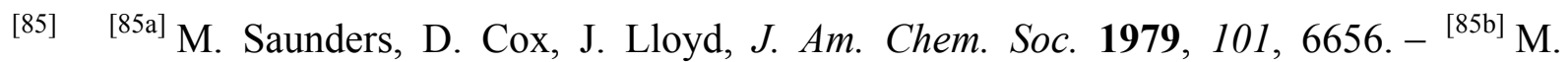
Saunders, D. Cox, W. Ohlmstead, J. Am. Chem. Soc. 1973, 95, 3018-3019. - ${ }^{[85 c]}$ J. C. Freudenberger, Dissertation, Universität Ulm, 2003.

[86] R. Tkachuk, C. C. Lee, Can. J. Chem. 1959, 37, 1644.

${ }^{[87]}{ }^{[87 a]}$ J. Buddrus, J. Lambert, Magn. Reson. Chem. 2002, 40, 3-23. - ${ }^{[87 b]}$ B. Reif, M. Köck, R. Kerssebaum, J. Schleucher, C. Griesinger, J. Magn. Res., Ser. B 1996, 112, $295-301$.

[88] N. Okazawa, T. S. Sorensen, Can. J. Chem. 1978, 56, 2355-2364. 
[89] M. Hesse, H. Meier, B. Zeeh, Spektroskopische Methoden in der Organischen Chemie, 5. Aufl., Georg Thieme Verlag, Stuttgart, 1995.

[90] R. E. Williams, Inorg. Chem. 1971, 10, 210.

${ }^{[91]}{ }^{[91 \mathrm{a}]}$ G. Maier, S. Pfriem, Angew.Chem. 1978, 79, 551-552. $-{ }^{[91 \mathrm{~b}]}$ G. Maier, F. Fleischer, H.-O. Kalinowski, Liebigs Ann. Org. Bioorg. Chem. 1995, 173-186.

[92] I. Emme, T. Labahn, A. de Meijere, J. Organomet. Chem. 2001, 624, 110-113.

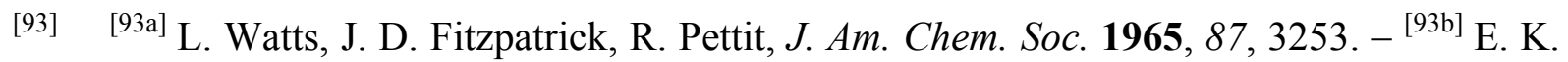
G. Schmidt, L. Brenner, R. Pettit, J. Am. Chem. Soc. 1970, 92, 3240.

[94] G. A. Olah, G. Liang, J. Am. Chem. Soc. 1975, 97, 6803-6806.

${ }^{[95]}{ }^{[95 a]}$ B. L. Hodrous, J. C. Ruble, G. C. Fu, J. Amer. Chem. Soc. 1999, 121, 2637-2638. ${ }^{\left[{ }^{[5 b}\right]}$ N. Kuhn, M. Schulten, E. Zauder, N. Augart, R. Boese, Chem. Ber. 1989, $1891-1896$.

[96] M. Suginome, G. C. Fu, Chirality 2000, 12, 318-324.

${ }^{[97]}{ }^{[97 a]}$ A. P. Dobbs, M. Voyle, N. Whittall, Synlett 1999, 10, 1594-1596. - ${ }^{[97 b]}$ A. P. Dobbs, J. Org. Chem. 2001, 66, 638-641.

${ }^{[98] \quad[98 \mathrm{a}]}$ O. Stelzer, M. Tepper, M. Hingst, A. Hebler, O. Herd, J. Organomet. Chem. 1996, 522, 69. - ${ }^{[98 b]}$ D. A. Allen, D. Venkataraman, J. Org. Chem. 2003, 68, 4590-4593.

[99] G. Salo, T. Guan, Organometallics 2003, 22, 5038-5049.

[100] N. Kataoka, Q. Shelby, J. P. Stambuli, J. F. Hartwig, J. Org. Chem. 2002, 67, $5553-5566$.

${ }^{[101] ~}{ }^{[101 a]}$ S.-G. Lee, H.-K. Lee, S. S. Lee, Y. K. Chung, Organometallics 1997, 16, 304-306. $-{ }^{[101 b]}$ J. Norinder, H. K. Cotton, J.-E. Bäckvall, J. Org. Chem. 2002, 67, 9096-9098. - ${ }^{[101 c]}$ J. Okuda, E. Herdtweck, Chem. Ber. 1988, 121, 1899-1905.

[102] Das Deca- bzw. Octacyclopropyldihydrofulvalen wurde schon früher durch oxidative Dimerisierung von Penta- bzw. Tetracyclopropylcyclopentadienyllithium mittels elementarem Iod dargestellt und charaktersiert; siehe Lit. ${ }^{[36 a]}$.

[103] M. F. Semmelhack in Organometallics in Synthesis, M. Schlosser (Ed.), $2^{\text {nd }}$ Ed., Wiley, Chichester, 2002, S. 1003-1122. 
[104] R. K. Kochhar, R. Pettit, J. Organomet. Chem. 1966, 6, 272.

${ }^{[105]}{ }^{[105 a]}$ I. Fleming, Grenzorbitale und Reaktionen organischer Verbindungen, Verlag Chemie, Weinheim, 1990. $-{ }^{[105 b]}$ F. Fringuelle, A. Taticchi, Dienes in the Diels-AlderReaction, John Wiley \& Sons, New York, 1990.

[106] K. C. Nicolaou, S. A. Snyder, T. Montagnon, G. E. Vassilikogiannakis, Angew. Chem. 2002, 114, 1742-1773; Angew. Chem. Int. Ed. 2002, 41, 1668-1698.

${ }^{[107]}{ }^{[107 a]}$ K. Sato, Y. Nishihara, S. Huo, Z. Xi, T. Takahashi, J. Organomet. Chem. 2001, 633, 18-26. - ${ }^{[107 b]}$ F. Sato, H. Urabe, S. Okamoto, Chem. Rev. 2000, 100, 2835-2886.

[108] O. G. Kulinkovich, A. de Meijere, Chem. Rev. 2000, 100, 2789-2834.

[109] L. F. Tietze, T. Hübsch, J. Oelze, C. Ott, W. Tost, G. Wörner, M. Buback, Chem. Ber. 1992, 125, 2249-2258.

[110] M. Kato, S. Yamamoto, S. Nomura, T. Miwa, Bull. Chem. Soc. Jpn. 1990, 63, 64-73.

[111] K. Komatsu, K. Takeuchi, M. Arima, Y. Waki, S. Shirai, K. Okamoto, Bull. Chem. Soc. Jpn. 1982, 55, 3257-3261.

[112] K. Takeuchi, Y. Yokomichi, T. Kurosaki, Y. Kimura, K. Okamoto, Tetrahedron 1979, $35,949-956$.

${ }^{[113]}{ }^{[113 a]}$ M. P. Schneider, A. Rau, J. Am. Chem. Soc. 1979, 101, 4426. - ${ }^{[113 b]}$ J. A. Berson, M. R. Willcott III, J. Amer. Chem. Soc. 1966, 88, 2494.

[114] B. Jiang, T. D. Tilley, J. Amer. Chem. Soc. 1999, 121, 9744-9745.

[115] A. R. Katritzky, C. W. Rees, E. S. V. Scriven (Eds.), Comprehensive Heterocyclic Chemistry II, Pergamon, Oxford, 1996.

[116] A. R. Katritzky, A. J. Boulton, Advances in Heterocyclic Chemistry, Academic Press, San Diego, 1963.

[117] H. Fischer, Org. Synth., Coll. Vol. 2, 1943, 202-204.

[118] S. P. Breukelmann, G. D. Meakins, M. D. Tirel, J. Chem Soc., Chem. Comm. 1982, $800-801$.

[119] H. Nozaki, T. Koyama, T. Mori, Tetrahedron 1969, 25, 5357-5364.

[120] L. T. Scott, J. O. Sharpless, Sythesis 1973, 209. 
[121] D. R. Shridhar, M. Jogibhukta, P. S. Rao, V. K. Handa, Synthesis 1982, 1061-1062.

[122] F. M. Dean, Adv. Heterocycl. Chem. 1982, 30, 167-238.

[123] Übersicht: ${ }^{[123 \mathrm{a}]}$ A. Weissberger, E. C. Taylor (Hrsg.), The Chemistry of Heterocyclic Compounds, Bd. 44, Wiley-Interscience, New York, 1985. - ${ }^{[123 b]}$ S. Gronowitz (Hrsg.), Thiophene and its Derivatives, Teil 1-3, Wiley-Interscience, New York, 1986. ${ }^{[123 c]}$ W.-D. Rudolf in Houben-Weyl, Methoden der Organischen Chemie, Bd. E6a, Hrsg. R. Kreher, G. Thieme Verlag, Stuttgart, 1994, S. 186-555.

[124] W. J. Middleton, V. A. Engelhardt, B. S. Fisher, J. Am. Chem. Soc. 1958, 80, $2822-2829$.

${ }^{[125]}{ }^{[125 \mathrm{a}]}$ P. Elsner, J. Chem. Soc. 1951, 893-895. - ${ }^{[125 b]}$ G. Herbertz, Chem. Ber. 1959, 92, $541-546$.

${ }^{[126]}{ }^{[126 a]}$ H. Wynberg, U. E. Wiersum, J. Chem. Soc., Chem. Commun. 1990, 6, 460-461. ${ }^{[126 b]}$ A. G. Davies, J. P. Goddard, E. Lusztyk, J. Lusztyk, J. Chem. Soc. Perkin Trans. 2 1982, $737-744$.

${ }^{[127]}{ }^{[127 a]}$ Y. Kobayashi, T. Taguchi, T. Morikawa, E. Tokuno, S. Sekiguchi, Chem. Pharm. Bull. 1980, 28, 262-267. - ${ }^{[127 b]}$ Y. Kobayashi, Tetrahedron Lett. 1977, 42, 3741-3742.

${ }^{[128]}{ }^{[128 a]}$ R. Klusák, P. Kubáček, Acta Chim. Scan. 1998, 52, 399-406. - ${ }^{[128 b]}$ G. SzakálQuin, D. G. Graham, J. Org. Chem. 1986, 51, 621-624.

[129] In Anlehnung an: V. Amarnath, D. C. Anthony, K. Amarnath, W. M. Valentine, L. A. Wetterau, D. G. Graham, J. Org. Chem. 1991, 56, 6924-6931.

${ }^{[130]}{ }^{[130 a]}$ N. Kuhn, K. Jendral, D. Bläser, R. Boese, Chem. Ber. 1991, 89-91. - ${ }^{[130 b]}$ X. Sava, L. Ricard, F. Mathey, P. Le Floch, Organometallics 2000, 19, 4899-4903. ${ }^{[130 c]}$ B. Deschamps, L. Ricard, F. Mathey, J. Organomet. Chem. 1997, 548, 17-22.

[131] H. Wynberg, U. E. Wiersum, J. Chem. Soc., Chem. Commun. 1990, 6, 460-461.

[132] Siehe dazu: S. N. Ivanov, B. V. Lichitskii, A. A. Dudinov, A. Y. Martykin, M. M. Krayushkin, Chem. Heterocycl. Compd. (Engl.Transl.) 2001, 37, 85-90; Khim. Geterotsikl. Soedin. 2001, 37, 89-94. 
${ }^{[133]}{ }^{[133 a]}$ A. N. Kost, I. I. Grandberg, Adv. Heterocycl. Chem. 1966, 6, 347-429. - ${ }^{[133 b]}$ W. Ried, M. Schön, Liebigs Ann. Chem. 1965, 689, 141-144.

[134] S. A. Lang, Y. I. Lin in Comprehensive Heterocyclic Chemistry, Hrsg. A. R. Katritzky, C. W. Rees, Bd. 6, Hrsg. K. T. Potts, Kap. 4.16, 1996, S. 1-130.

[135] L. F. Tietze, T. Eicher, Reaktionen und Synthesen im organisch-chemischen Praktikum und Forschungslaboratorium, 2. Aufl., Thieme, 1991, S. 194.

[136] In Variation des Protokolls von: T. C. Schwendemann, P. S. May, M. T. Merry, Y. Hou, C. Y. Meyers, J. Phys. Chem. A 1998, 102, 8690-8694.

[137] In Anlehnung an: C. Cativiela, J. L. Serrano, M. M. Zurbano, J. Org. Chem. 1995, 60, 3074-3083.

[138] H. Ubayama, W.-H. Sun, Z. Xi, T. Takahashi, Chem. Commun. 1998, 1931-1932.

[139] P. E. Eaton. T. W. Cole, J. Am. Chem. Soc. 1964, 86, 962-964; ibid. 3157-3158.

[140] Übersichten in: ${ }^{[140 \mathrm{a}]}$ R. Gleiter, S. Brand, Chem. Europ. J. 1998, 4, 2532-2538. ${ }^{[140 b]}$ G. W. Griffin, A. P. Marchand, Chem. Rev. 1989, 89, 997-1010. $-{ }^{[140 c]}$ P. E. Eaton, Angew. Chem. 1992, 104, 1447-1462.

[141] T. Takahashi, W.-H. Sun, K. Nakajima, Chem. Commun. 1999, 1595-1596.

[142] W. Merk, R. Pettit, J. Am. Chem. Soc. 1967, 89, 4788.

[143] U. Duerr, F. W. Heinemann, J. Organomet. Chem. 1997, 541, 307-320.

[144] J.-A. Chen, C.-S. Lin, L. K. Liu, J. Chin. Chem. Soc. (Taipei) 1996, 43, 95-100.

[145] Mit Pd siehe: S. Mataka, G.-B. Liu, M. Tashiro, Synthesis 1995, 2, 133-135. - Mit Ni siehe: C. Malanga, L. A. Aronica, L. Lardicci, Tetrahedron Lett. 1995, 9189-9192.

[146] K.-P. Zeller in Houben-Weyl, Methoden der Organischen Chemie, Vol. 5/2c, Thieme, Stuttgart 1985, S. 504-684.

${ }^{[147]}{ }^{[147 \mathrm{a}]}$ G. W. Wheland, D. E. Mann, J. Chem. Phys. 1949, 17, 264. - ${ }^{[147 b]}$ G. Berthier, J. Chem. Phys. 1953, 21, 953. $-{ }^{[147 \mathrm{c}]}$ M. A. Oglioruso, J. C. Schug, S. C. Kitching, Tetrahedron 1973, 29, 4065.

[148] G. A. Olah, G. K. S. Prakash, G. Liang, J. Org. Chem. 1977, 42, 661-666. 
[149] Das UV-Spektrum von Fulven in der Gasphase weist im Bereich von 450 bis $170 \mathrm{~nm}$ vier Hauptbanden auf: R. D. Brown, P. J. Domaille, J. E. Kent, Aust. J. Chem. 1970, 23, $1707-1720$.

[150] W. E. Billups, L.-J. Lin, Tetrahedron Lett. 1983, 24, 1683-1686.

${ }^{[151]}{ }^{[151 a]}$ J. H. Day, Chem. Rev. 1953, 53, 167. - ${ }^{[151 b]}$ K. J. Stone, R. D. Little, J. Org. Chem. 1984, 49, 1849-1853. - ${ }^{[151 \mathrm{c}]}$ P. Jutzi, T. Heidemann, B. Neumann, H. G. Stammler, Synthesis 1992, 1096-1098.

[152] Für das Fulvenyl-Kation aus 14 siehe Lit. ${ }^{[37]}$ und für das aus 15 siehe Lit. ${ }^{[36 a, 40]}$.

[153] U. M. Dzhemilev, R. I. Khusnutdinov, N. A. Shchadneva, O. M. Nefedov, G. A. Tolstikov, Bull. Acad. Sci. USSR Div. Chem. Sci. (Engl. Transl.) 1989, 38, 2171-2174; Izv. Akad. Nauk SSSR Ser. Khim. 1989, 10, 2360-2362.

${ }^{[154]}{ }^{[154 a]}$ M. Kotora, H. Matsumura, G. Gao, T. Takahashi, Org. Lett. 2001, 22, 3467-3470. $-{ }^{[154 b]}$ G. Dyker, P. Siemsen, S. Sostmann, A. Wiegand, I. Dix, P. G. Jones, Chem. Ber. 1997, 130, 261-266. - ${ }^{[154 c]}$ L. J. Silverberg, G. Wu, A. L. Rheingold, R. F. Heck, J. Organomet. Chem. 1991, 409, 411-420. - ${ }^{[154 \mathrm{~d}]}$ G. C. M. Lee, B. Tobias, J. M. Holmes, D. A. Harcourt, M. E. Garst, J. Amer. Chem. Soc. 1990, 112, 9330-9336.

[155] Ei-I. Negishi, L. S. Harring, Z. Owczarczyk, M. M. Mohamud, M. Ay, Tetrahedron Lett. 1992, 33, 3253-3256.

${ }^{[156] ~[156 a]}$ S. López, J. Rodríguez, J. G. Rey, A. R. de Lera, J. Amer. Chem. Soc. 1996, 118, 1881-1891. - ${ }^{[156 b]}$ M. Murakami, H. Amii, K. Itami, Y. Ito, Angew. Chem. 1995, 107, 1649-1650. - ${ }^{[156 c]}$ D. J. Pasto, W. Kong, J. Org. Chem. 1989, 54, 4028-4033.

${ }^{[157]}{ }^{[157 \mathrm{a}]}$ H. Bienayme, C. Yezeguelian, Tetrahedron 1994, 50, 3389-3396. $-{ }^{[157 b]}$ M. M. Abelmann, T. Oh, L. E. Overman, J. Org. Chem. 1987, 52, 4130-4133. - ${ }^{[157 c]} \mathrm{W}$. Cabri, I. Candiani, A. Bedeschi, S. Penco, R. Santi, J. Org. Chem. 1992, 57, $1481-1486$.

[158] W. C. Still, M. Kahn, A. Mitra, J. Org. Chem. 1978, 43, 2923-2925.

[159] G. M. Sheldrik, SHELXL-93/97, Programm für die Kristallstrukturverfeinerung, Universität Göttingen, 1997. 
[160] Autorenkollektiv, Organikum, 19. Aufl., VEB Dt. Verlag der Wissenschaften, Berlin, 1993.

[161] J. Schneider und W. Freitag, J. Am. Chem. Soc. 1976, 98, 978.

[162] Bruker, 150 Basic-NMR-Experiments, January 2000, Version 2.0.

[163] J. Schneider und W. Freitag, J. Am. Chem. Soc. 1976, 98, 478.

${ }^{[164]}{ }^{[164 a]}$ G. Koebrich, Chem. Ber. 1972, 105, 1683-1693. $-{ }^{[164 b]}$ A. V. Tarakanova, S. V. Baranova, T. I. Pekhk, O. B. Dogadin, N. S. Zefirov, J. Org. Chem. USSR (Engl. Transl.) 1987, 23, 464-470; Zh. Org. Khim. 1987, 23, 515-521. 


\section{F. Spektrenanhang}

\section{${ }^{1} \mathrm{H}$ - und ${ }^{13} \mathrm{C}-\mathrm{NMR}-$ Spektrum von}

1. 2,3-Dicyclopropyl-7-diphenylphosphanyl-1H-indol (87)

2. endo-1,7,8,9,10-Pentacyclopropyl-4-phenyl-4-azatricyclo[5.2.1.02,6]dec-8-en-3,5-dion (102)

3. syn-4,5,6,7-Tetracyclopropyl-2-phenyl-3a,4,7,7a-tetrahydroisoindol-1,3-dion (104)

4. 1,2,3,4,5-Pentacyclopropyl-1H-pyrrol (18b)

5. 2,3,4,5-Tetracyclopropylfuran (19)

6. 2,3,4,5-Tetracyclopropylthiophen (20)

7. 3-(1'-Cyclopropyl-3',3'-diphenylallyliden)-cyclobuten (200) 


\section{2,3-Dicyclopropyl-7-diphenylphosphanyl-1H-indol (87)}

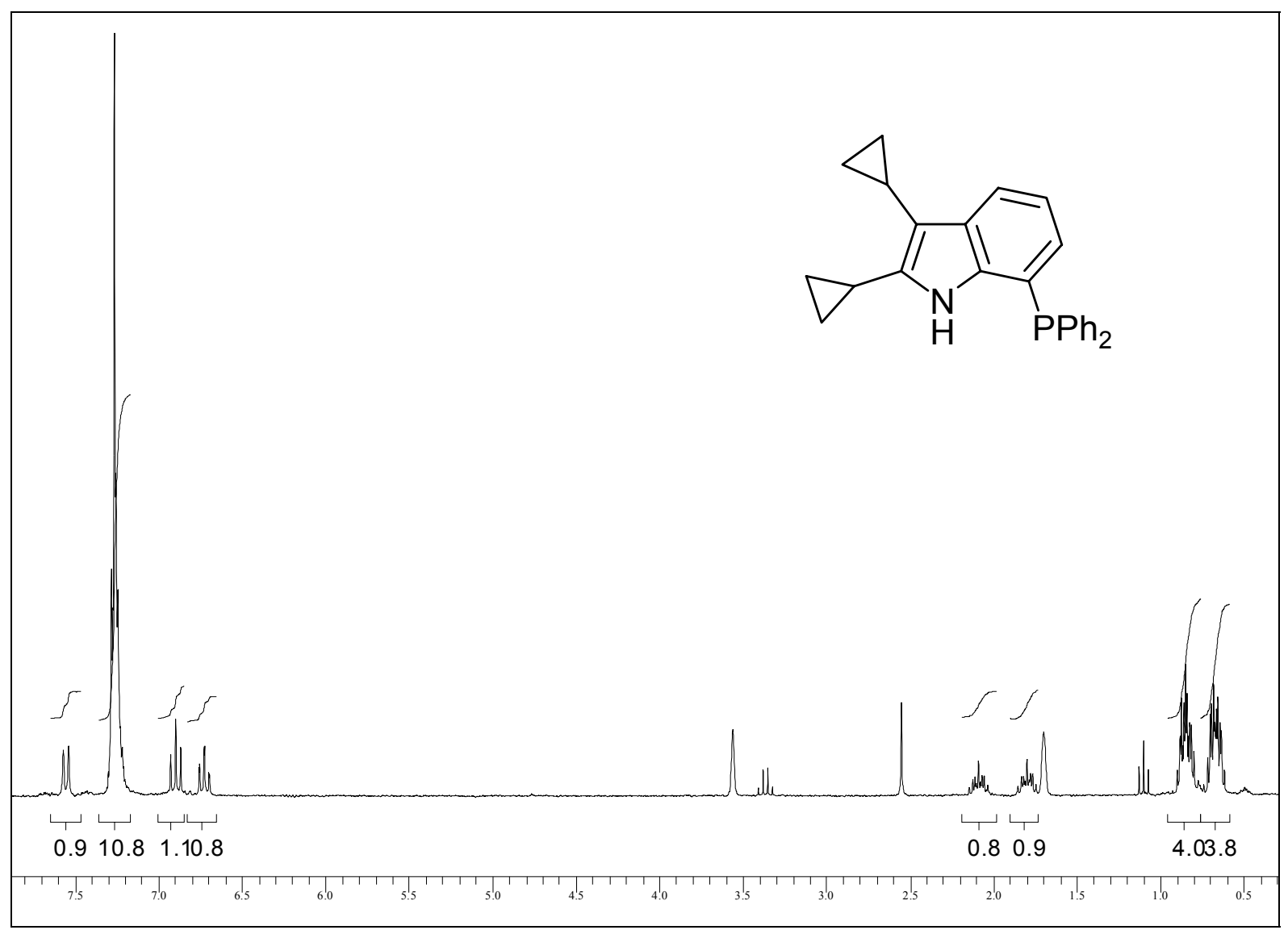

${ }^{1} \mathrm{H}-\mathrm{NMR}-$ Spektrum (250 MHz, [D 8 -THF).

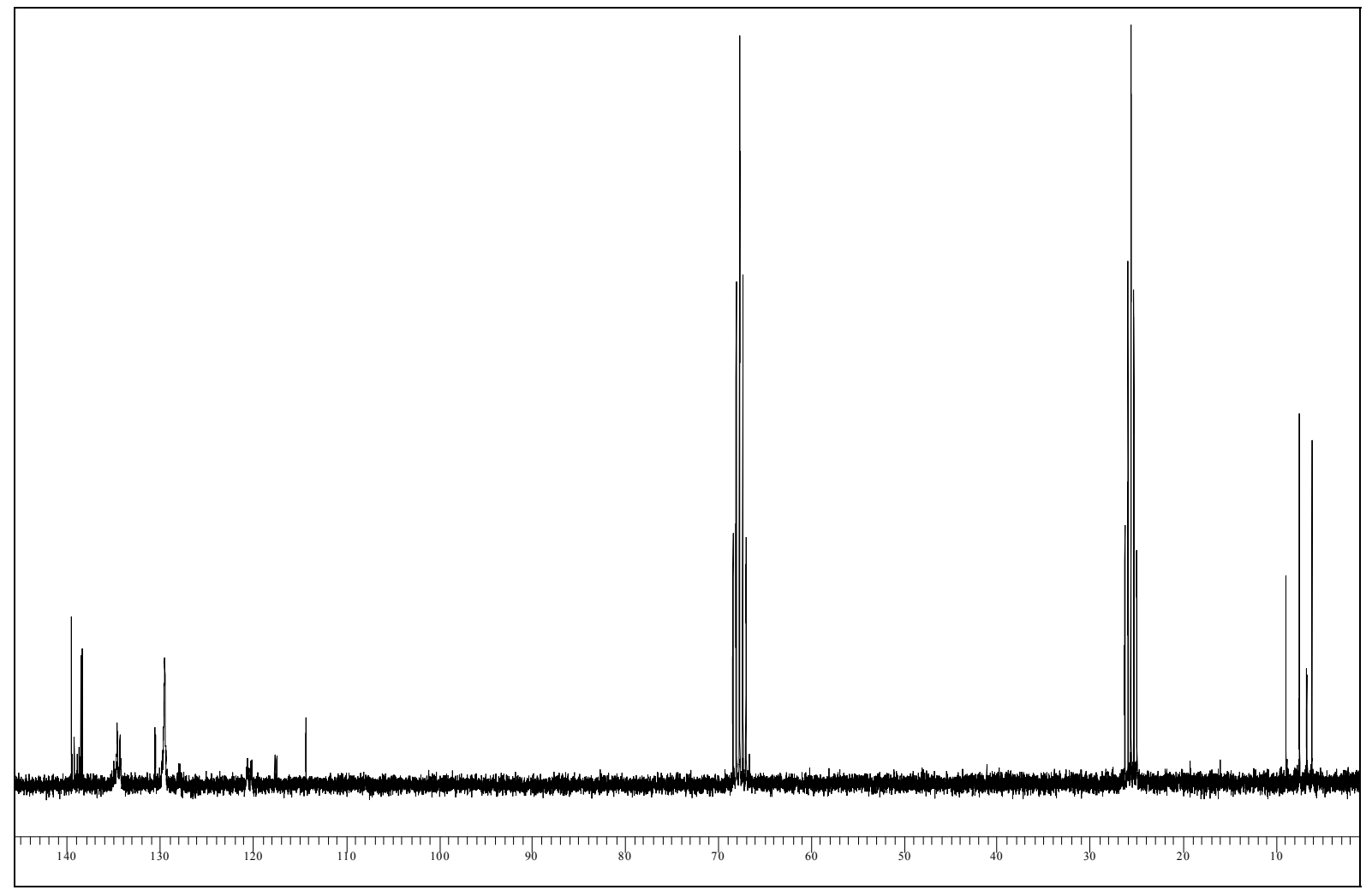

${ }^{13} \mathrm{C}-\mathrm{NMR}-$ Spektrum $\left(62.9 \mathrm{MHz},\left[\mathrm{D}_{8}\right]-\mathrm{THF}\right)$. 
2. endo-1,7,8,9,10-Pentacyclopropyl-4-phenyl-4-azatricyclo[5.2.1.0 $\left.{ }^{2,6}\right]$ dec-8-en-3,5-dion (102)

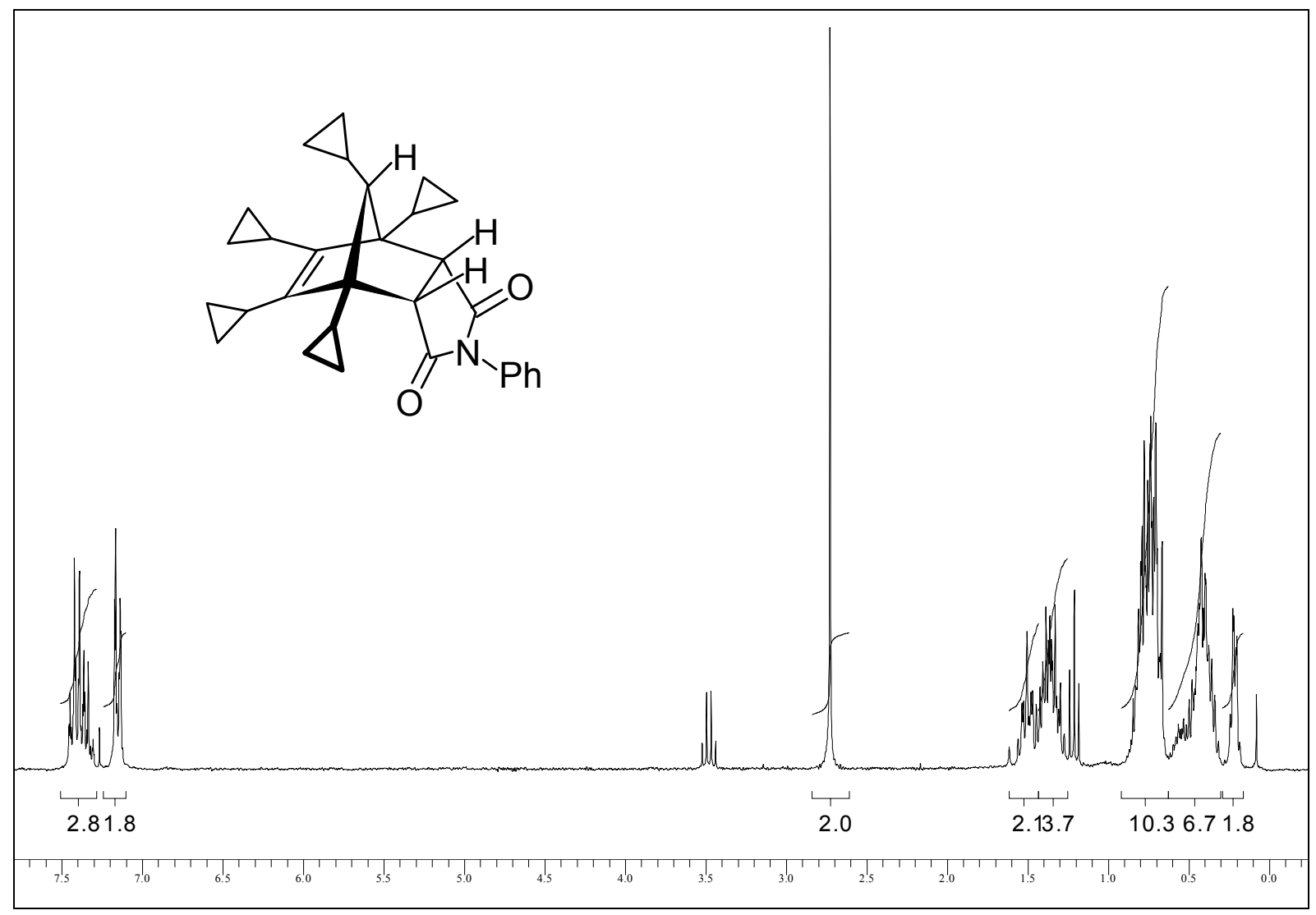

${ }^{1} \mathrm{H}-\mathrm{NMR}-$ Spektrum $\left(250 \mathrm{MHz}, \mathrm{CDCl}_{3}\right)$.

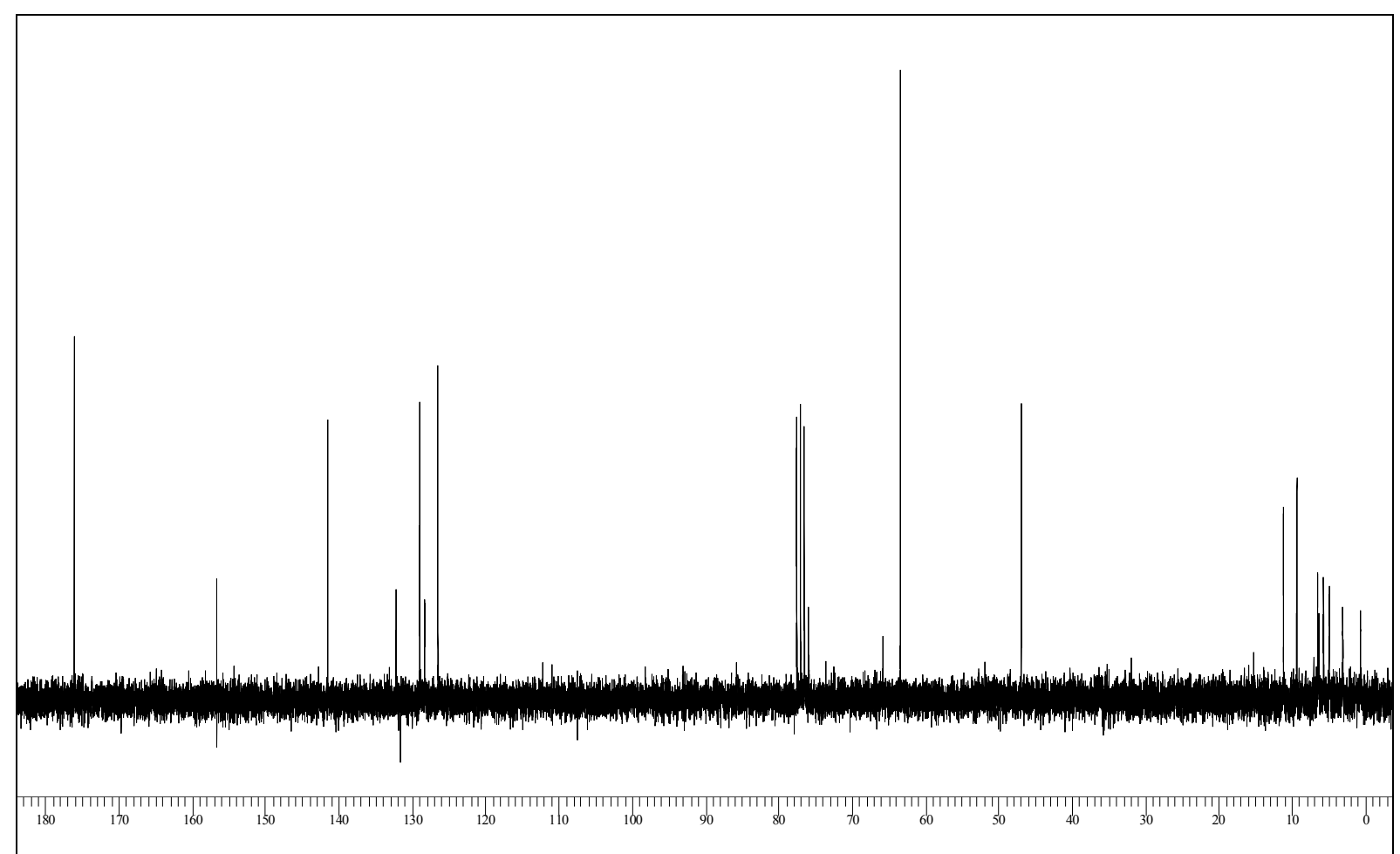

${ }^{13} \mathrm{C}-\mathrm{NMR}-$ Spektrum $\left(62.9 \mathrm{MHz}, \mathrm{CDCl}_{3}\right)$. 
3. syn-4,5,6,7-Tetracyclopropyl-2-phenyl-3a,4,7,7a-tetrahydroisoindol-1,3-dion (104)

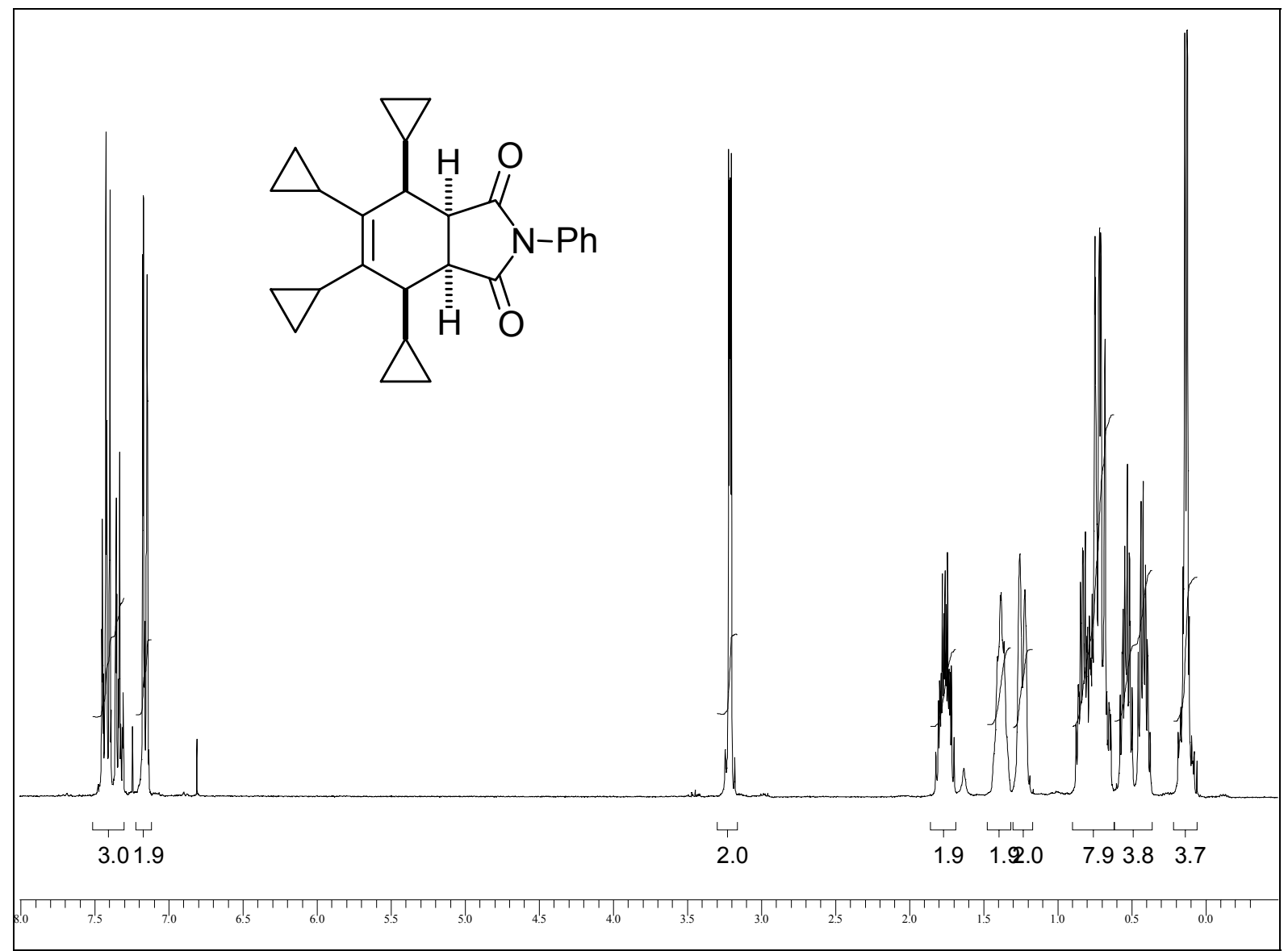

${ }^{1} \mathrm{H}-\mathrm{NMR}-$ Spektrum $\left(300 \mathrm{MHz}, \mathrm{CDCl}_{3}\right)$.

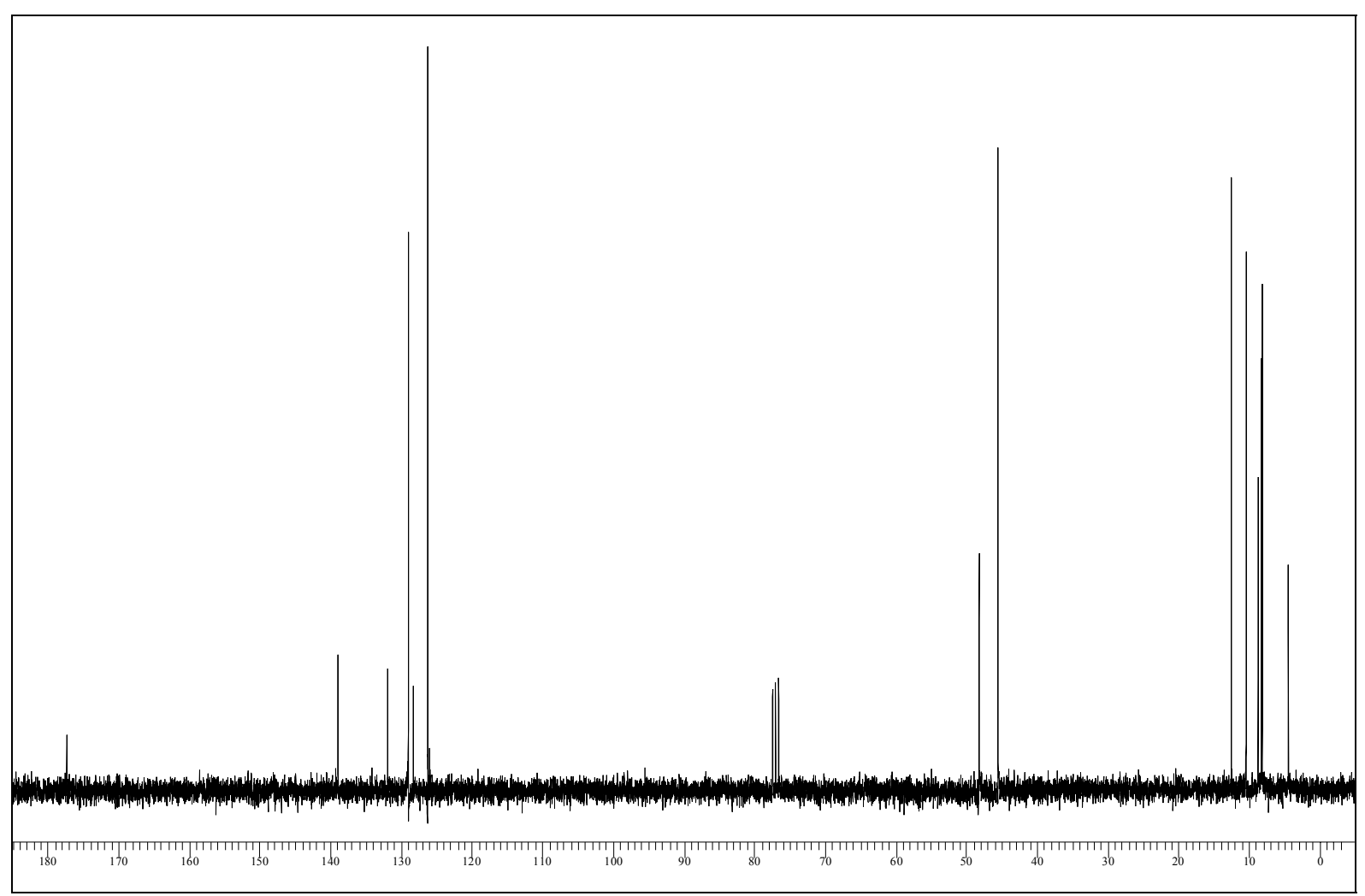

${ }^{13} \mathrm{C}$-NMR-Spektrum (75.4 MHz, $\mathrm{CDCl}_{3}$ ). 
4. 1,2,3,4,5-Pentacyclopropyl-1H-pyrrol (18b)

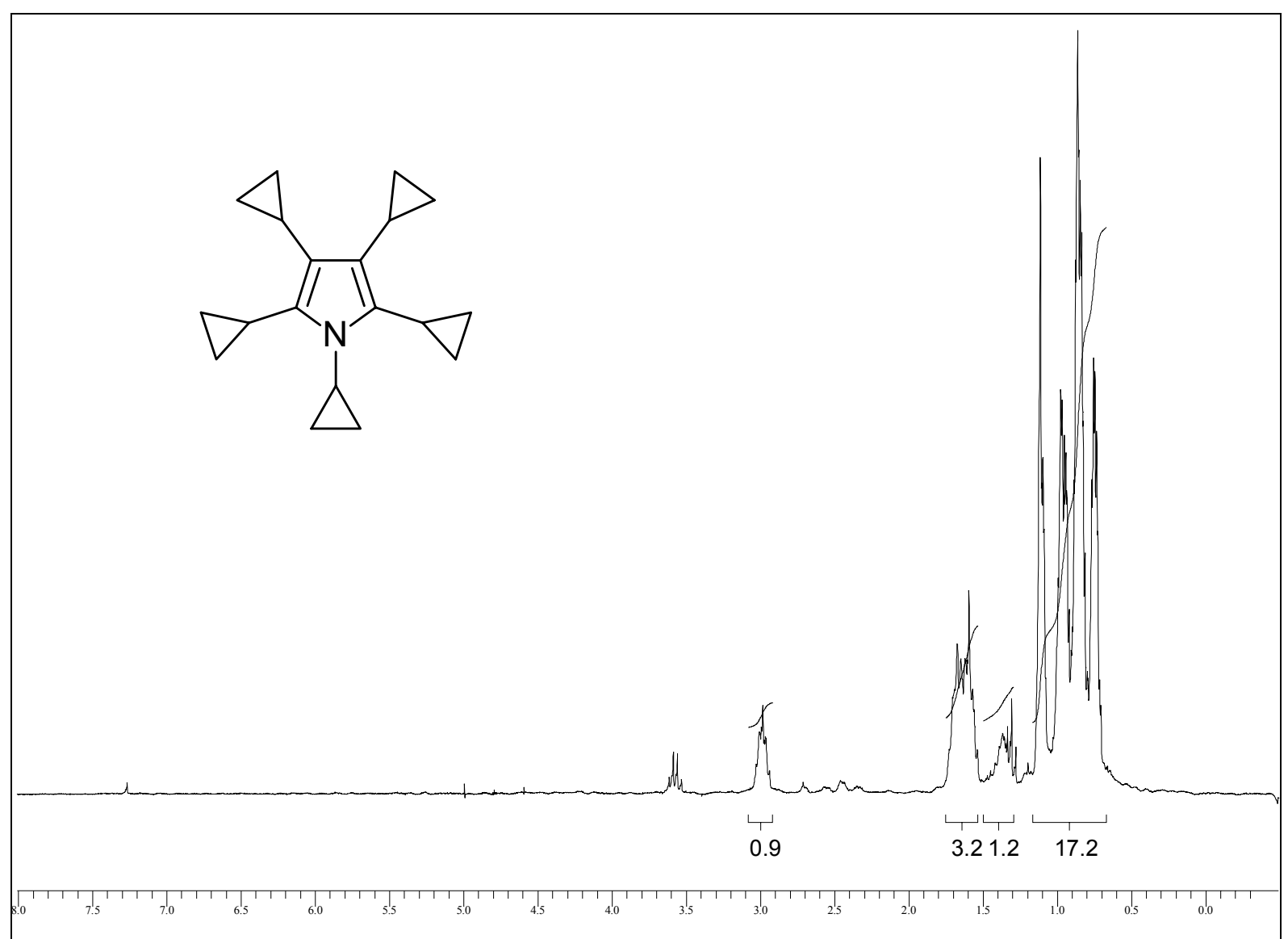

${ }^{1} \mathrm{H}-\mathrm{NMR}-$ Spektrum $\left(250 \mathrm{MHz}, \mathrm{CDCl}_{3}\right)$.

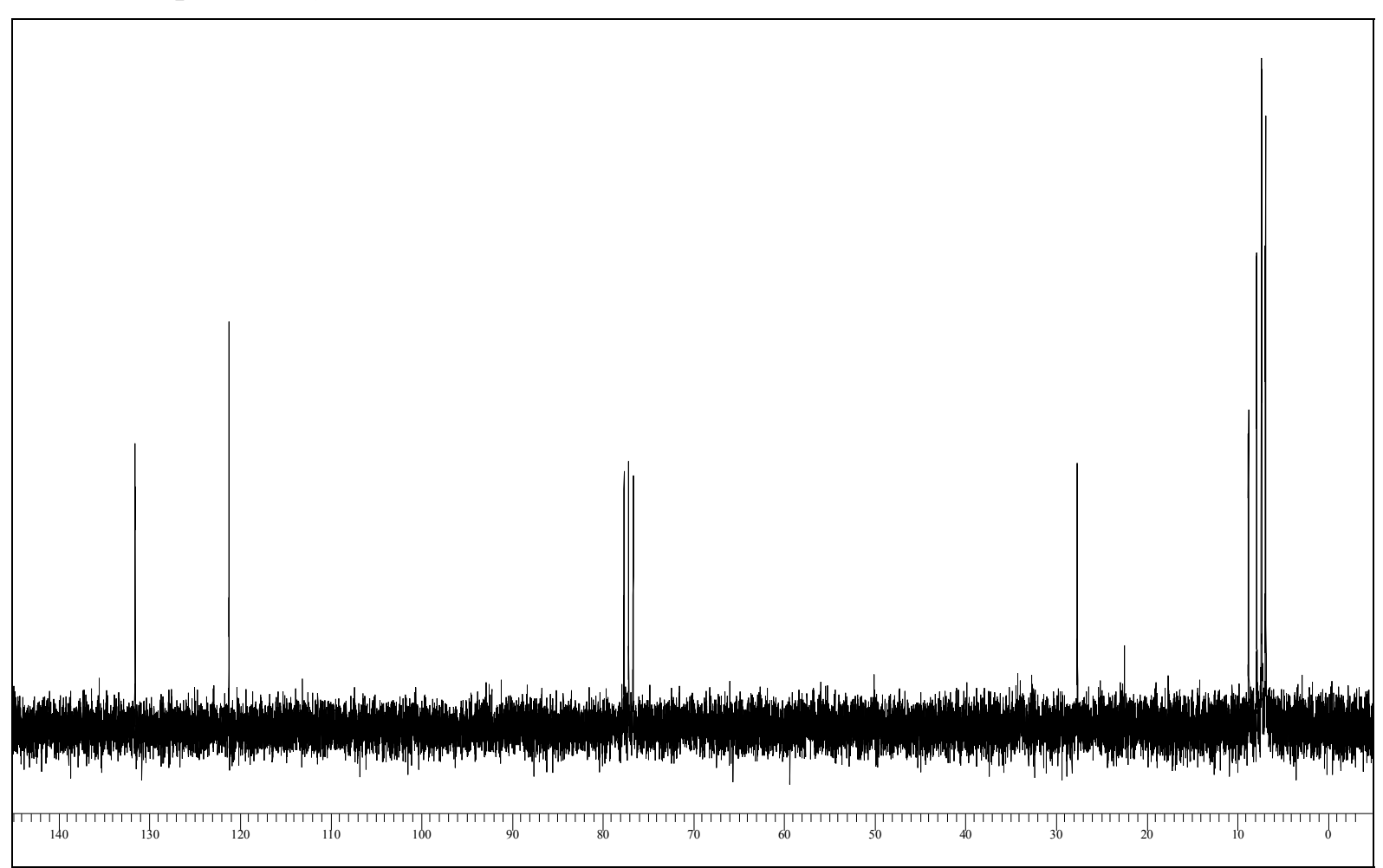

${ }^{13} \mathrm{C}$-NMR-Spektrum (62.9 MHz, $\left.\mathrm{CDCl}_{3}\right)$. 
5. 2,3,4,5-Tetracyclopropylfuran (19)

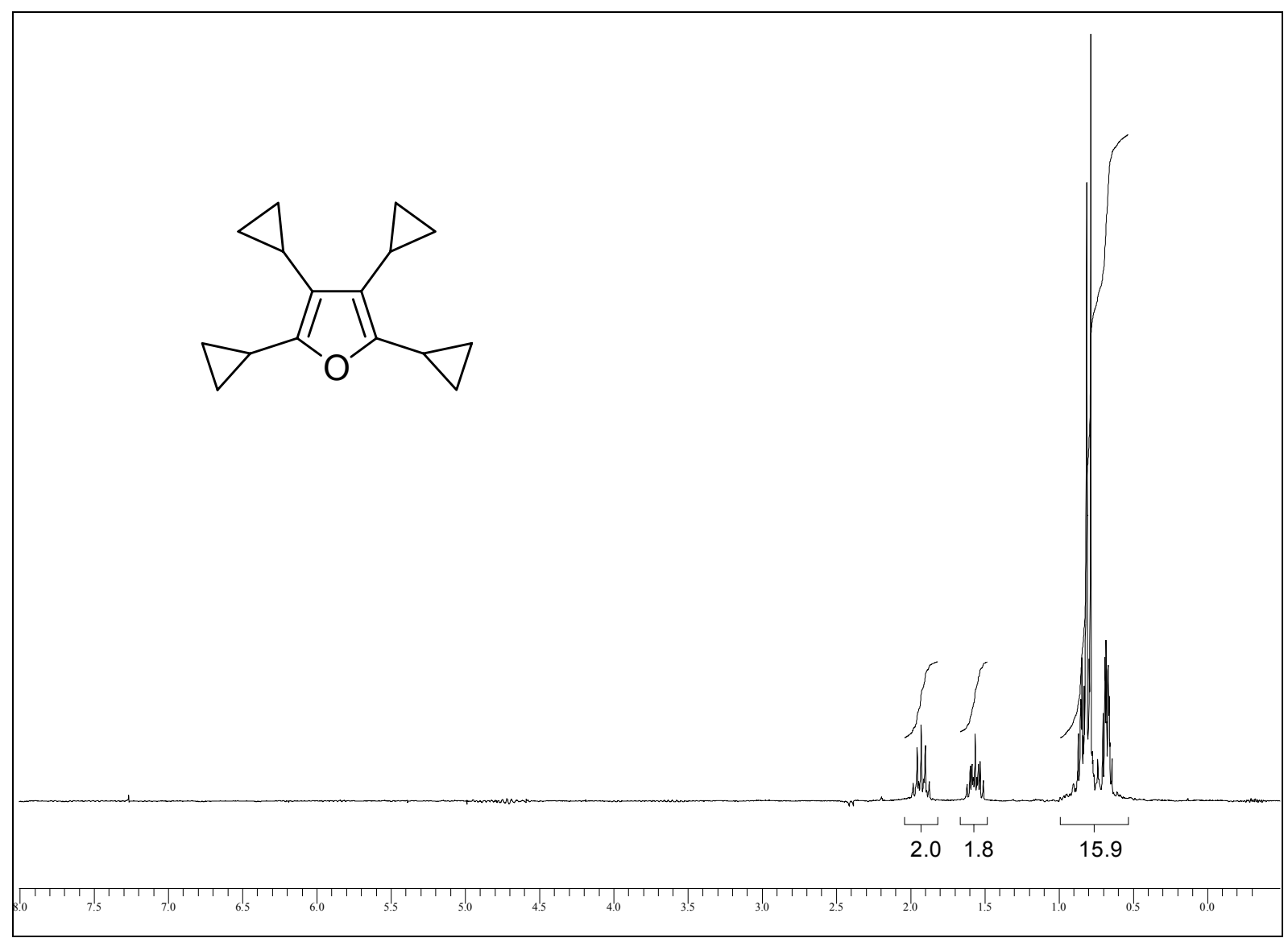

${ }^{1} \mathrm{H}-\mathrm{NMR}-$ Spektrum $\left(250 \mathrm{MHz}, \mathrm{CDCl}_{3}\right.$ ).

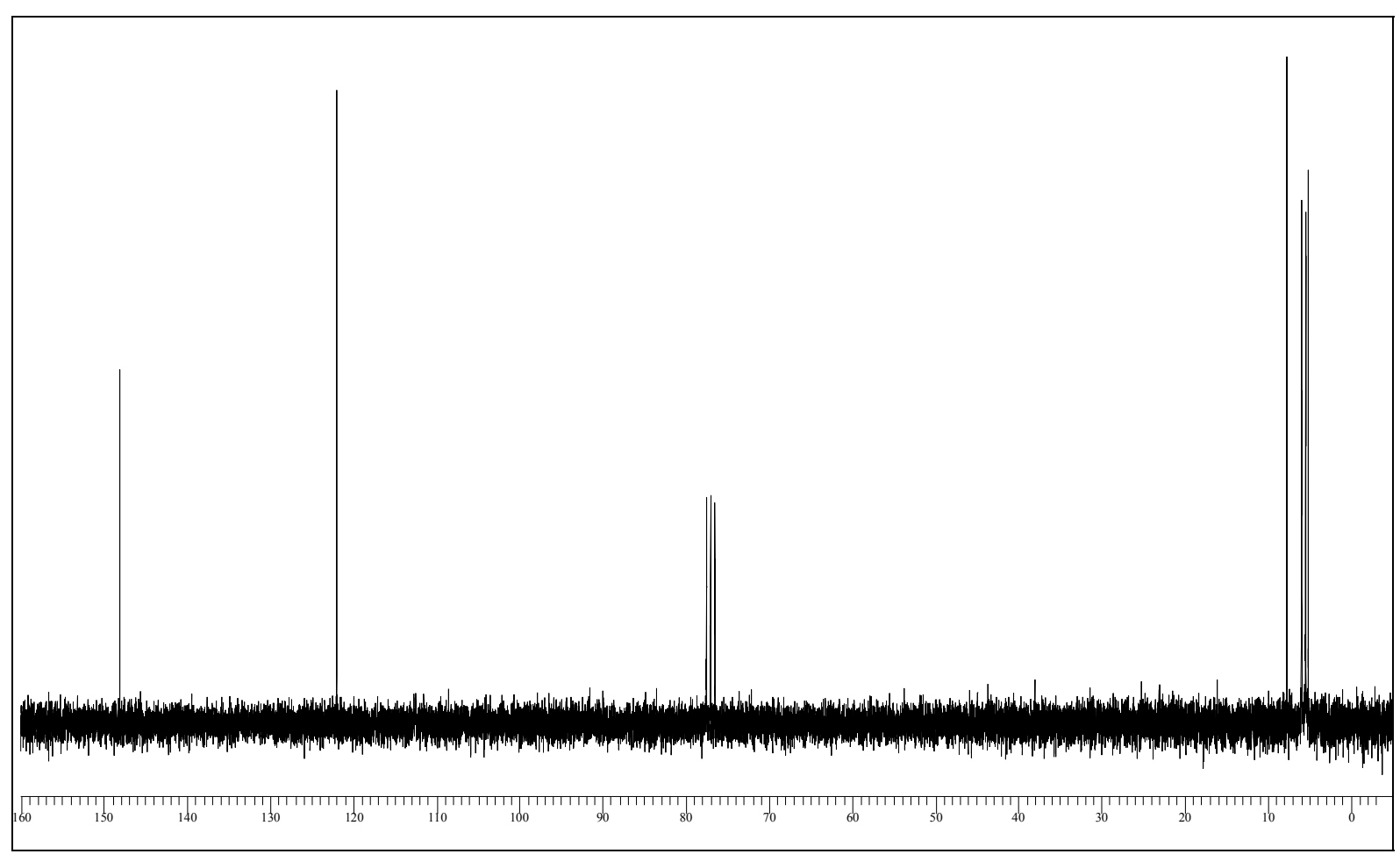

${ }^{13} \mathrm{C}$-NMR-Spektrum (62.9 MHz, $\left.\mathrm{CDCl}_{3}\right)$. 
6. 2,3,4,5-Tetracyclopropylthiophen (20)

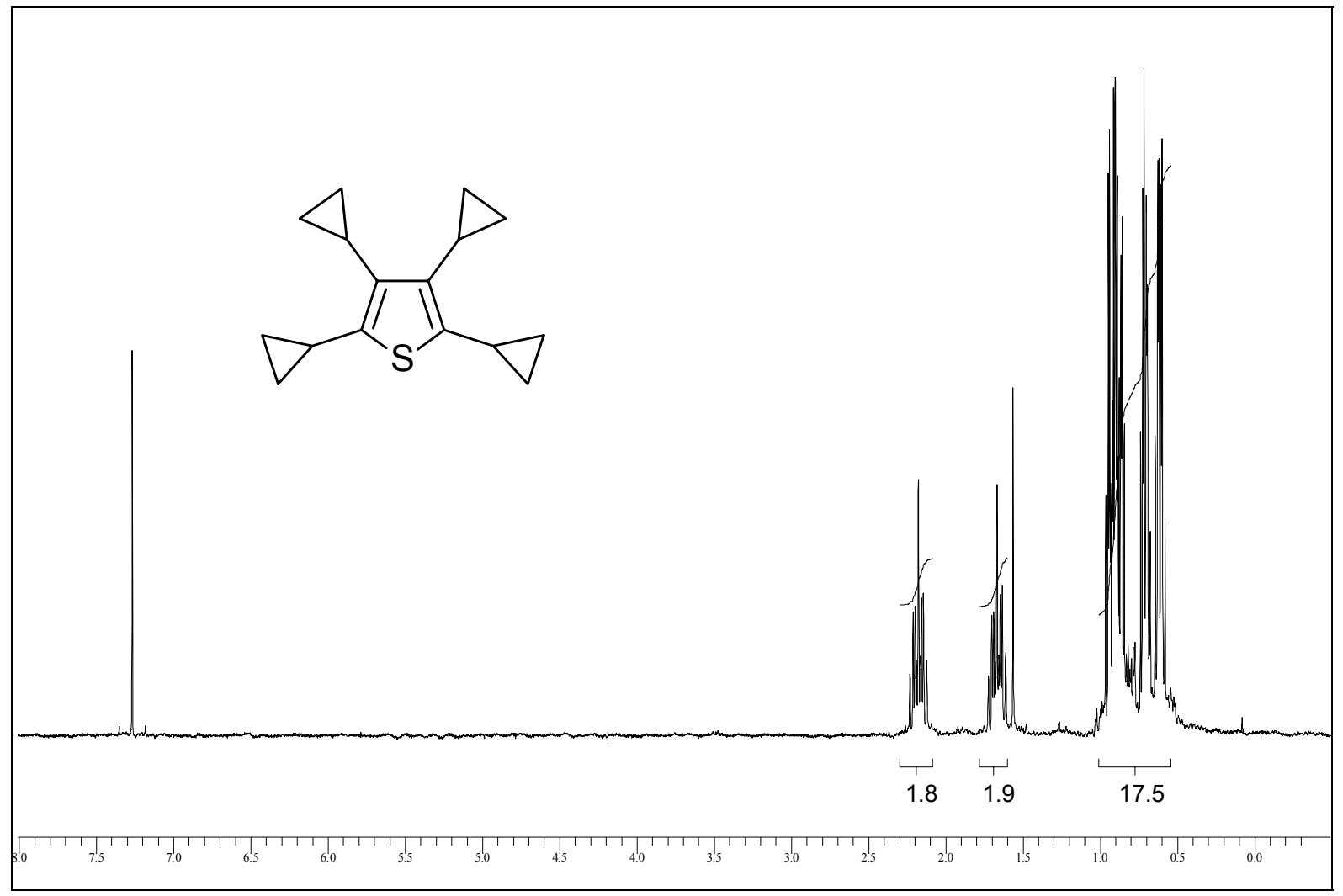

${ }^{1} \mathrm{H}-\mathrm{NMR}-$ Spektrum $\left(250 \mathrm{MHz}, \mathrm{CDCl}_{3}\right)$.

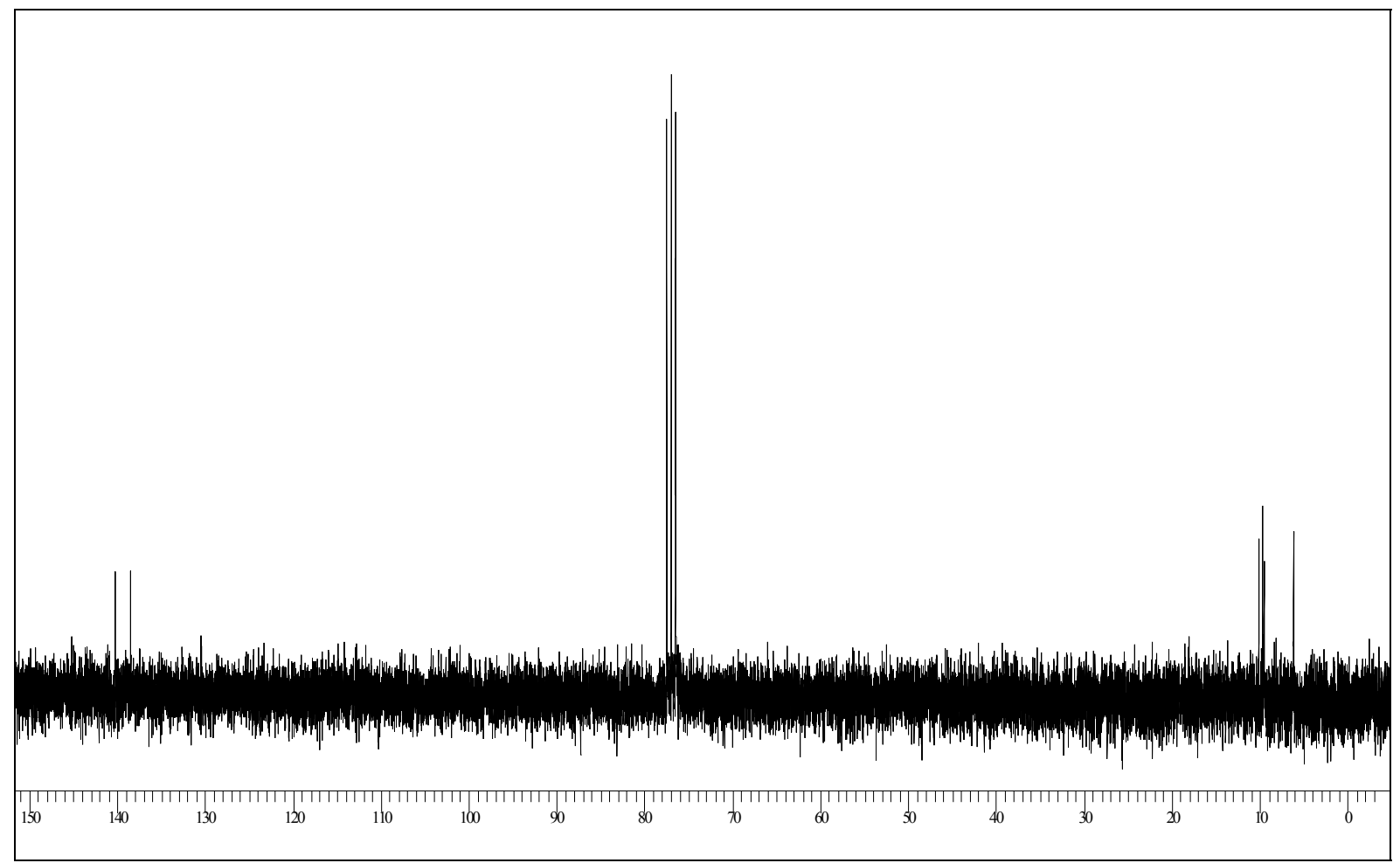

${ }^{13} \mathrm{C}$-NMR-Spektrum $\left(62.9 \mathrm{MHz}, \mathrm{CDCl}_{3}\right)$. 
7. 3-(1'-Cyclopropyl-3',3'-diphenylallyliden)-cyclobuten (200)

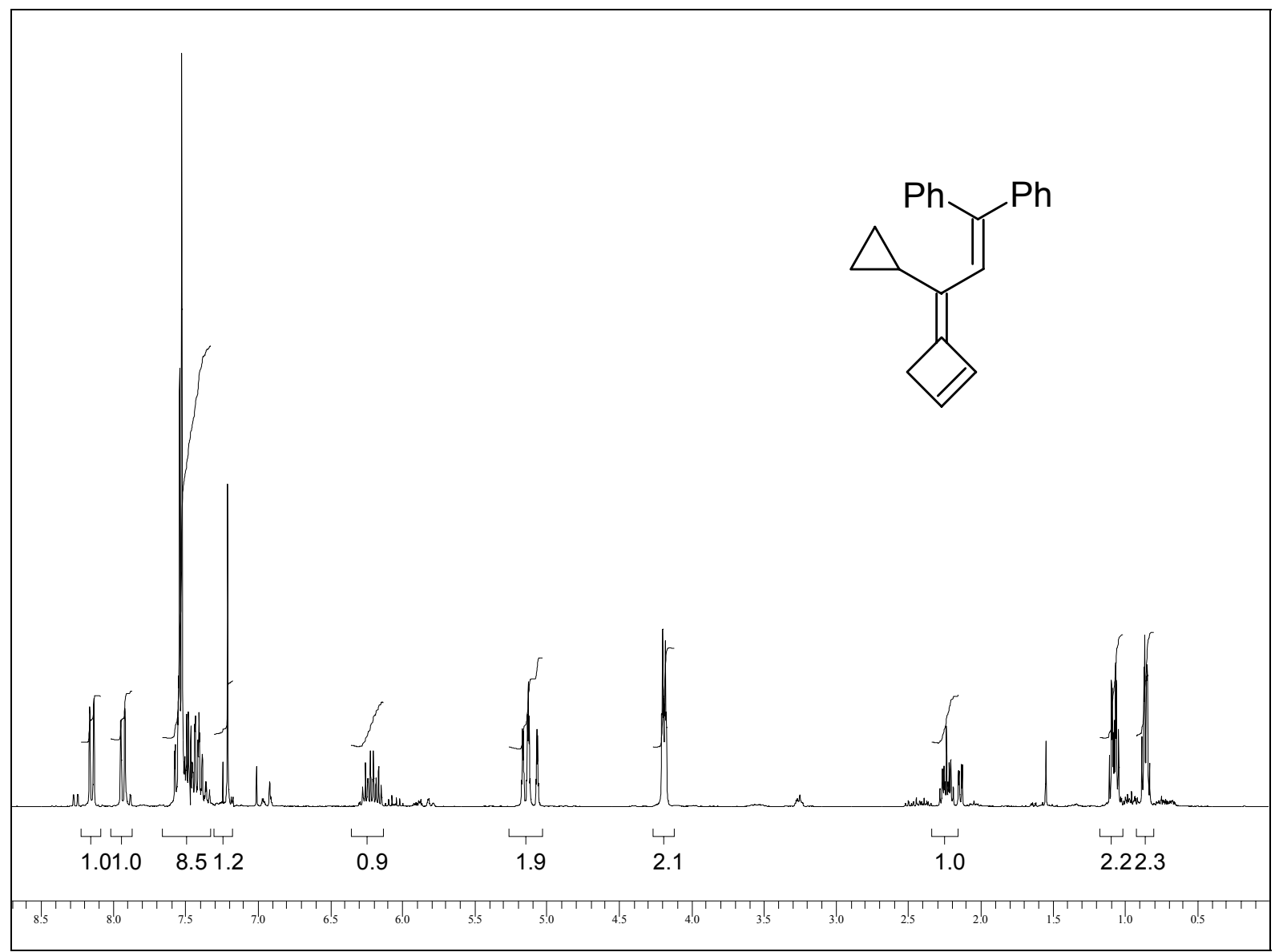

${ }^{1} \mathrm{H}-\mathrm{NMR}-$ Spektrum $\left(200 \mathrm{MHz}, \mathrm{CDCl}_{3}\right)$.

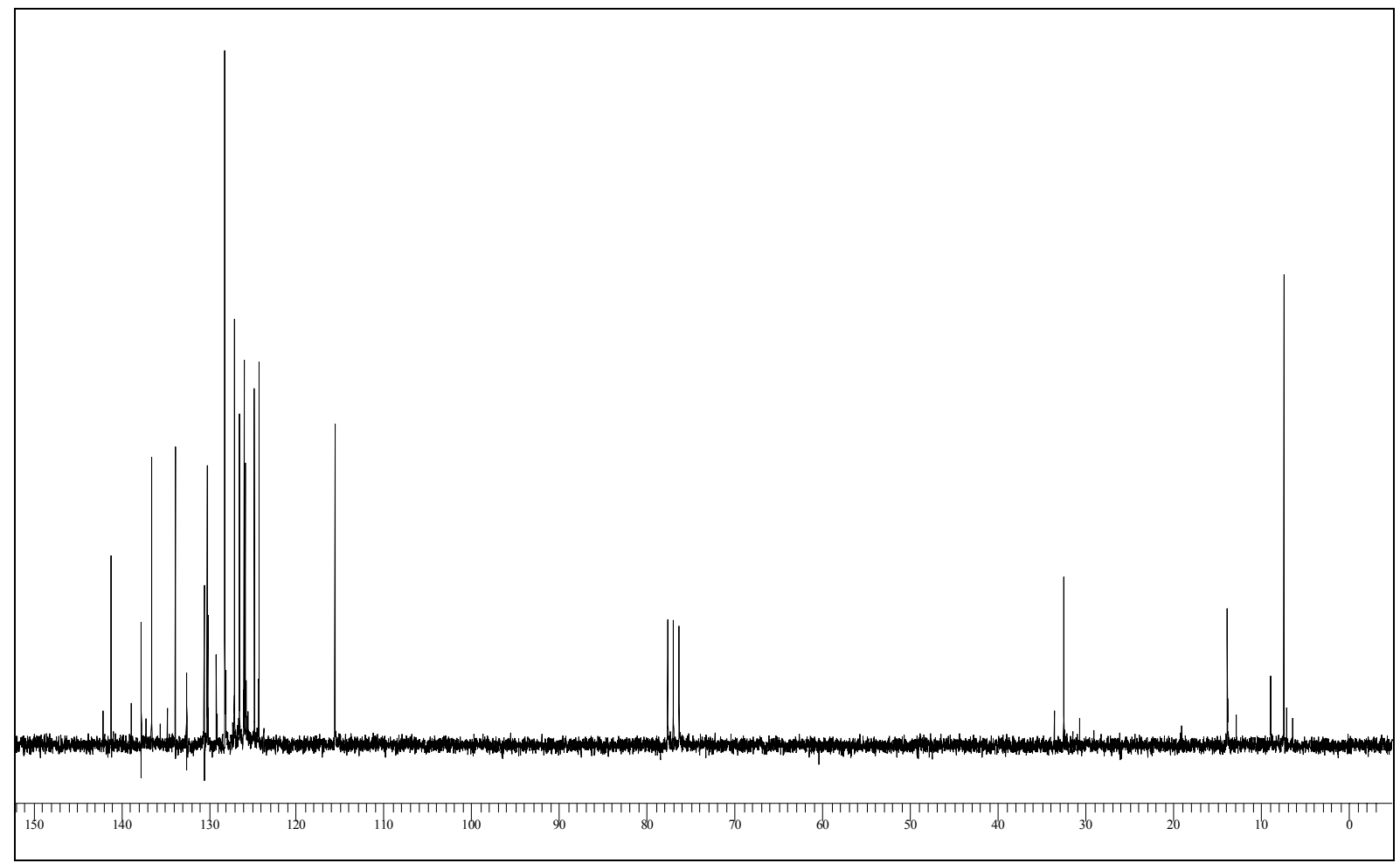

${ }^{13} \mathrm{C}$-NMR-Spektrum (50.3 MHz, $\mathrm{CDCl}_{3}$ ). 


\section{G. Röntgenographischer Teil}

1. Röntgenographische Daten von endo-1,7,8,10-Tetracyclopropyl-4-phenyl-4-aza-tricyclo[5.2.1.0 $\left.{ }^{2,6}\right]$ dec-8-en-3,5-dion (100)

Tabelle 1. Kristalldaten und Strukturverfeinerung von 100.

Strukturkennzeichen

stre 445

Summenformel

$\mathrm{C}_{27} \mathrm{H}_{29} \mathrm{NO}_{2}$

Molmasse

399.51

Temperatur

133(2) K

Wellenlänge

$0.71073 \AA$

Kristallsystem, Raumgruppe

orthorhombisch,

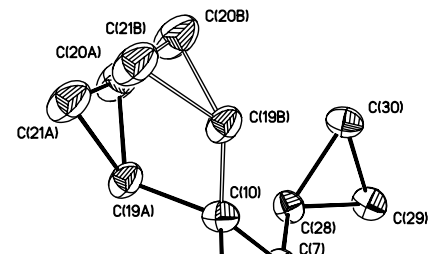

Zelldimensionen

$a=8.4108(5) \AA$

$P b c a$

$b=22.0263(13) \AA$

$\alpha=90^{\circ}$

$c=23.5459(17) \AA$

$\beta=90^{\circ}$

4362.1(5) $\AA^{3}$

Z, berechnete Dichte

$8,1.217 \mathrm{mg} / \mathrm{m}^{3}$

Absorptionskoeffizient

$0.076 \mathrm{~mm}^{-1}$

$\mathrm{F}(000)$

1712

Gemessener $\Theta$-Bereich

1.73 bis $24.83^{\circ}$

Indexgrenzen

$-9 \leq h \leq 9,-25 \leq k \leq 25,-27 \leq l \leq 27$

Anzahl der gemessenen Reflexe

38304

Unabhängige Reflexe

$3735\left[\mathrm{R}_{\text {int }}=0.0963\right]$

Observed reflections [I $>2 \sigma(\mathrm{I})] \quad 2825$

Completeness to theta $=24.83$

$99.5 \%$

Strukturverfeinerung

Full-matrix least-squares on $\mathrm{F}^{2}$

Daten / Restraints / Parameter

3735 / 0 / 281

Goodness-of-fit on $\mathrm{F}^{2}$

Endgültige R-Werte [I > 2 $\sigma(\mathrm{I})]$

$\mathrm{R} 1=0.0524, \mathrm{wR} 2=0.1042$

R-Werte (sämtliche Daten)

$\mathrm{R} 1=0.0769, \mathrm{wR} 2=0.1124$

Größtes Maximum und Minimum 137 und -188 e nm $^{-3}$ 
Tabelle 2. Atomkoordinaten und äquivalente isotrope Auslenkungsparameter $\left[\AA^{2}\right]$ von $\mathbf{1 0 0}$.

$\mathrm{U}(\mathrm{eq})$ wird bezeichnet als ein Drittel der Spur des orthogonalen $\mathrm{U}_{\mathrm{ij}}$-Tensors.

\begin{tabular}{|c|c|c|c|c|}
\hline & $\mathrm{x}$ & $\mathrm{y}$ & $\mathrm{z}$ & $\mathrm{U}(\mathrm{eq})$ \\
\hline $\mathrm{C}(1)$ & $0.3558(3)$ & $0.1878(1)$ & $0.4088(1)$ & $0.0311(5)$ \\
\hline$C(2)$ & $0.4510(3)$ & $0.1599(1)$ & $0.4602(1)$ & $0.0302(5)$ \\
\hline$C(3)$ & $0.3871(2)$ & $0.1770(1)$ & $0.5176(1)$ & $0.0307(5)$ \\
\hline $\mathrm{N}(4)$ & $0.3314(2)$ & $0.1242(1)$ & $0.5445(1)$ & $0.0307(4)$ \\
\hline$C(5)$ & $0.3454(2)$ & $0.0724(1)$ & $0.5101(1)$ & $0.0299(5)$ \\
\hline$C(6)$ & $0.4230(2)$ & $0.0908(1)$ & $0.4547(1)$ & $0.0297(5)$ \\
\hline$C(7)$ & $0.3156(2)$ & $0.0856(1)$ & $0.3999(1)$ & $0.0304(5)$ \\
\hline$C(8)$ & $0.1579(2)$ & $0.1148(1)$ & $0.4170(1)$ & $0.0298(5)$ \\
\hline$C(9)$ & $0.1826(2)$ & $0.1745(1)$ & $0.4218(1)$ & $0.0300(5)$ \\
\hline$C(10)$ & $0.3906(3)$ & $0.1375(1)$ & $0.3643(1)$ & $0.0360(5)$ \\
\hline $\mathrm{O}(11)$ & $0.3817(2)$ & $0.2272(1)$ & $0.5387(1)$ & $0.0403(4)$ \\
\hline $\mathrm{O}(12)$ & $0.3020(2)$ & $0.0221(1)$ & $0.5242(1)$ & $0.0355(4)$ \\
\hline$C(13)$ & $0.2718(3)$ & $0.1228(1)$ & $0.6016(1)$ & $0.0319(5)$ \\
\hline$C(14)$ & $0.3526(3)$ & $0.1550(1)$ & $0.6436(1)$ & $0.0386(5)$ \\
\hline$C(15)$ & $0.2984(3)$ & $0.1528(1)$ & $0.6991(1)$ & $0.0443(6)$ \\
\hline$C(16)$ & $0.1679(3)$ & $0.1179(1)$ & $0.7135(1)$ & $0.0484(6)$ \\
\hline$C(17)$ & $0.0884(3)$ & $0.0858(1)$ & $0.6717(1)$ & $0.0465(6)$ \\
\hline$C(18)$ & $0.1393(3)$ & $0.0884(1)$ & $0.6155(1)$ & $0.0384(5)$ \\
\hline$C(19 A$ & $0.3244(4)$ & $0.1454(1)$ & $0.3065(1)$ & $0.0417(8)$ \\
\hline $\mathrm{C}(20 \mathrm{~A}$ & $0.3959(5)$ & $0.1104(1)$ & $0.2576(1)$ & $0.0648(11)$ \\
\hline $\mathrm{C}(21 \mathrm{~A}$ & $0.4180(5)$ & $0.1782(1)$ & $0.2616(1)$ & $0.0603(11)$ \\
\hline$C(19 B$ & $0.524(2)$ & $0.1383(7)$ & $0.3341(7)$ & $0.0417(8)$ \\
\hline$C(20 B$ & $0.536(4)$ & $0.1110(9)$ & $0.2729(8)$ & $0.0648(11)$ \\
\hline$C(21 B$ & $0.562(3)$ & $0.1807(8)$ & $0.2832(8)$ & $0.0603(11)$ \\
\hline $\mathrm{C}(22)$ & $0.0132(2)$ & $0.0793(1)$ & $0.4296(1)$ & $0.0331(5)$ \\
\hline $\mathrm{C}(23)$ & $-0.1295(3)$ & $0.1093(1)$ & $0.4549(1)$ & $0.0425(6)$ \\
\hline $\mathrm{C}(24)$ & $-0.1343(3)$ & $0.0894(1)$ & $0.3951(1)$ & $0.0496(7)$ \\
\hline$C(25)$ & $0.4032(3)$ & $0.2517(1)$ & $0.3938(1)$ & $0.0365(5)$ \\
\hline$C(26)$ & $0.2953(3)$ & $0.2897(1)$ & $0.3571(1)$ & $0.0470(6)$ \\
\hline$C(27)$ & $0.3168(3)$ & $0.3051(1)$ & $0.4191(1)$ & $0.0419(6)$ \\
\hline$C(28)$ & $0.3056(3)$ & $0.0251(1)$ & $0.3697(1)$ & $0.0352(5)$ \\
\hline$C(29)$ & $0.3277(3)$ & $-0.0357(1)$ & $0.3979(1)$ & $0.0401(5)$ \\
\hline$C(30)$ & $0.4509(3)$ & $-0.0122(1)$ & $0.3577(1)$ & $0.0414(6)$ \\
\hline
\end{tabular}


Tabelle 3. Bindungslängen $[\mathrm{pm}]$ und -winkel $\left[{ }^{\circ}\right]$ von $\mathbf{1 0 0}$.

\begin{tabular}{|c|c|c|c|}
\hline$C(1)-C(25)$ & $150.4(3)$ & $C(5)-C(6)-C(7)$ & $116.02(17)$ \\
\hline $\mathrm{C}(1)-\mathrm{C}(9)$ & $151.7(3)$ & $\mathrm{C}(2)-\mathrm{C}(6)-\mathrm{C}(7)$ & $103.04(15)$ \\
\hline$C(1)-C(10)$ & $155.2(3)$ & $\mathrm{C}(28)-\mathrm{C}(7)-\mathrm{C}(8)$ & $116.46(17)$ \\
\hline $\mathrm{C}(1)-\mathrm{C}(2)$ & $157.5(3)$ & $\mathrm{C}(28)-\mathrm{C}(7)-\mathrm{C}(10)$ & $114.69(17)$ \\
\hline $\mathrm{C}(2)-\mathrm{C}(3)$ & $150.3(3)$ & $\mathrm{C}(8)-\mathrm{C}(7)-\mathrm{C}(10)$ & $100.65(15)$ \\
\hline $\begin{array}{l}C(2)-C(6) \\
C(3)-O(11)\end{array}$ & $\begin{array}{l}154.7(3) \\
121.2(2)\end{array}$ & $C(28)-C(7)-C(6)$ & $118.69(16)$ \\
\hline $\mathrm{C}(3)-\mathrm{N}(4)$ & $140.6(2)$ & $\mathrm{C}(8)-\mathrm{C}(7)-\mathrm{C}(6)$ & $104.52(16)$ \\
\hline$N(4)-C(5)$ & $140.4(2)$ & $C(10)-C(7)-C(6)$ & $98.98(16)$ \\
\hline $\mathrm{N}(4)-\mathrm{C}(13)$ & $143.6(3)$ & $\mathrm{C}(9)-\mathrm{C}(8)-\mathrm{C}(22)$ & $129.14(19)$ \\
\hline $\mathrm{C}(5)-\mathrm{O}(12)$ & $151.2(3)$ & $\mathrm{C}(9)-\mathrm{C}(8)-\mathrm{C}(7)$ & $107.54(18)$ \\
\hline $\mathrm{C}(6)-\mathrm{C}(7)$ & $157.9(3)$ & $\mathrm{C}(22)-\mathrm{C}(8)-\mathrm{C}(7)$ & $123.14(16)$ \\
\hline $\begin{array}{l}\mathrm{C}(7)-\mathrm{C}(28) \\
\mathrm{C}(7)-\mathrm{C}(8)\end{array}$ & $\begin{array}{l}151.4(3) \\
152.8(3)\end{array}$ & $\mathrm{C}(8)-\mathrm{C}(9)-\mathrm{C}(1)$ & $108.78(18)$ \\
\hline $\mathrm{C}(7)-\mathrm{C}(10)$ & $155.1(3)$ & $C(19 B)-C(10)-C(19 A)$ & $79.9(8)$ \\
\hline $\mathrm{C}(8)-\mathrm{C}(9)$ & $147.7(3)$ & & $1300(7)$ \\
\hline $\mathrm{C}(10)-\mathrm{C}(19 \mathrm{~B})$ & $133.0(18)$ & $C(19 B)-C(10)-C(1)$ & $130.0(7)$ \\
\hline$C(10)-C(19 A)$ & $148.0(4)$ & C(19A)-C(10)-C(7) & $115.5(2)$ \\
\hline $\mathrm{C}(13)-\mathrm{C}(18)$ & $138.6(3)$ & $C(19 B)-C(10)-C(1)$ & $120.7(7)$ \\
\hline$C(13)-C(14)$ & $139.4(3)$ & $C(19 A)-C(10)-C(1)$ & $117.67(19)$ \\
\hline$C(14)-C(15)$ & $138.5(3)$ & $C(7)-C(10)-C(1)$ & $94.91(16)$ \\
\hline$C(15)-C(16)$ & $138.3(4)$ & $C(18)-C(13)-C(14)$ & $120.2(2)$ \\
\hline C(16)-C(17) & $\begin{array}{l}138.4(4) \\
1392(3)\end{array}$ & $\mathrm{C}(18)-\mathrm{C}(13)-\mathrm{N}(4)$ & $120.86(19)$ \\
\hline $\begin{array}{l}C(17)-C(18) \\
C(19 A)-C(21 A)\end{array}$ & $\begin{array}{l}139.2(3) \\
150.3(4)\end{array}$ & $\mathrm{C}(14)-\mathrm{C}(13)-\mathrm{N}(4)$ & $118.89(19)$ \\
\hline$C(19 A)-C(20 A)$ & $151.1(4)$ & $C(15)-C(14)-C(13)$ & $119.4(2)$ \\
\hline$C(20 A)-C(21 A)$ & $150.6(4)$ & $C(16)-C(15)-C(14)$ & $120.8(2)$ \\
\hline$C(19 B)-C(21 B)$ & $155(2)$ & $C(15)-C(16)-C(17)$ & $119.6(2)$ \\
\hline C(19B)-C(20B) & $156(2)$ & $C(16)-C(17)-C(18)$ & $120.4(2)$ \\
\hline $\begin{array}{l}\mathrm{C}(20 \mathrm{~B})-\mathrm{C}(21 \mathrm{~B}) \\
\mathrm{C}(22)-\mathrm{C}(23)\end{array}$ & $\begin{array}{l}157(3) \\
1494(3)\end{array}$ & $\mathrm{C}(13)-\mathrm{C}(18)-\mathrm{C}(17)$ & $119.6(2)$ \\
\hline$C(22)-C(24)$ & $150.0(3)$ & $C(10)-C(19 A)-C(21 A)$ & $120.4(3)$ \\
\hline $\mathrm{C}(23)-\mathrm{C}(24)$ & $147.7(3)$ & $C(10)-C(19 A)-C(20 A)$ & $119.4(3)$ \\
\hline $\mathrm{C}(25)-\mathrm{C}(27)$ & $150.5(3)$ & $C(21 A)-C(19 A)-C(20 A)$ & $59.98(19)$ \\
\hline$C(25)-C(26)$ & $150.7(3)$ & $C(21 A)-C(20 A)-C(19 A)$ & $59.75(19)$ \\
\hline $\mathrm{C}(26)-\mathrm{C}(27)$ & $150.8(3)$ & $C(19 A)-C(21 A)-C(20 A)$ & $60.27(19)$ \\
\hline $\begin{array}{l}C(28)-C(30) \\
C(28)-C(29)\end{array}$ & $\begin{array}{l}149.9(3) \\
150.6(3)\end{array}$ & $C(10)-C(19 B)-C(21 B)$ & $126.3(16)$ \\
\hline $\mathrm{C}(29)-\mathrm{C}(30)$ & $149.7(3)$ & $C(10)-C(19 B)-C(20 B)$ & $122.9(16)$ \\
\hline $\mathrm{C}(25)-\mathrm{C}(1)-\mathrm{C}(9)$ & $118.80(18)$ & $C(21 B)-C(19 B)-C(20 B)$ & $60.5(11)$ \\
\hline$C(25)-C(1)-C(10)$ & $117.40(18)$ & $C(19 B)-C(20 B)-C(21 B)$ & $59.3(11)$ \\
\hline $\mathrm{C}(9)-\mathrm{C}(1)-\mathrm{C}(10)$ & $100.31(16)$ & $C(19 B)-C(21 B)-C(20 B)$ & $60.2(11)$ \\
\hline $\begin{array}{l}\mathrm{C}(25)-\mathrm{C}(1)-\mathrm{C}(2) \\
\mathrm{C}(9)-\mathrm{C}(1)-\mathrm{C}(2)\end{array}$ & $\begin{array}{l}114.23(18) \\
104.95(16)\end{array}$ & $\mathrm{C}(8)-\mathrm{C}(22)-\mathrm{C}(23)$ & $120.62(17)$ \\
\hline$C(10)-C(1)-C(2)$ & $98.24(16)$ & $\mathrm{C}(8)-\mathrm{C}(22)-\mathrm{C}(24)$ & $119.64(18)$ \\
\hline $\mathrm{C}(3)-\mathrm{C}(2)-\mathrm{C}(6)$ & $105.50(16)$ & $C(23)-C(22)-C(24)$ & $59.10(16)$ \\
\hline$C(3)-C(2)-C(1)$ & $114.27(17)$ & $C(24)-C(23)-C(22)$ & $60.64(15)$ \\
\hline$C(6)-C(2)-C(1)$ & $104.10(16)$ & $\mathrm{C}(23)-\mathrm{C}(24)-\mathrm{C}(22)$ & $60.26(15)$ \\
\hline $\mathrm{O}(11)-\mathrm{C}(3)-\mathrm{N}(4)$ & $\begin{array}{l}123.9(2) \\
12763(18)\end{array}$ & $\mathrm{C}(1)-\mathrm{C}(25)-\mathrm{C}(27)$ & $120.73(19)$ \\
\hline $\mathrm{N}(4)-\mathrm{C}(3)-\mathrm{C}(2)$ & $108.46(16)$ & $C(1)-C(25)-C(26)$ & $119.6(2)$ \\
\hline $\mathrm{C}(5)-\mathrm{N}(4)-\mathrm{C}(3)$ & $112.60(17)$ & $C(27)-C(25)-C(26)$ & $60.10(15)$ \\
\hline $\mathrm{O}(12)-\mathrm{C}(5)-\mathrm{N}(4)$ & $124.0(2)$ & $C(25)-C(26)-C(27)$ & $5989(15)$ \\
\hline $\mathrm{O}(12)-\mathrm{C}(5)-\mathrm{C}(6)$ & $127.54(18)$ & & \\
\hline $\mathrm{C}(3)-\mathrm{N}(4)-\mathrm{C}(13)$ & $123.82(16)$ & $C(25)-C(27)-C(26)$ & $60.01(15)$ \\
\hline $\mathrm{N}(4)-\mathrm{C}(5)-\mathrm{C}(6)$ & $108.49(16)$ & $C(30)-C(28)-C(29)$ & $59.73(14)$ \\
\hline$C(5)-C(6)-C(2)$ & $104.90(16)$ & $\mathrm{C}(30)-\mathrm{C}(29)-\mathrm{C}(28)$ & $59.90(14)$ \\
\hline
\end{tabular}


Tabelle 4. Anisotrope Auslenkungsparameter $\left(\AA^{2}\right)$ von 100. Der anisotrope Auslenkungsfaktor hat die Form: $-2 \pi^{2}\left[\left(\mathrm{~h} \mathrm{a}^{*}\right)^{2} \mathrm{U}_{11}+\ldots+2 \mathrm{~h} \mathrm{k} \mathrm{a*} \mathrm{b}^{*} \mathrm{U}_{12}\right]$.

\begin{tabular}{|c|c|c|c|c|c|c|}
\hline & U11 & $\mathrm{U} 22$ & U33 & $\mathrm{U} 23$ & U13 & U12 \\
\hline$C(1)$ & $0.0332(12)$ & $0.0221(9)$ & $0.0380(12)$ & $-0.0006(8)$ & $0.0043(10)$ & $-0.0016(8)$ \\
\hline $\mathrm{C}(2)$ & $0.0249(12)$ & $0.0237(10)$ & $0.0419(13)$ & $-0.0004(8)$ & $0.0005(9)$ & $-0.0012(8)$ \\
\hline$C(3)$ & $0.0278(12)$ & $0.0235(10)$ & $0.0407(12)$ & $0.0005(9)$ & $-0.0045(9)$ & $-0.0032(8)$ \\
\hline $\mathrm{N}(4)$ & $0.0332(10)$ & $0.0221(8)$ & $0.0367(10)$ & $-0.0025(7)$ & $-0.0003(8)$ & $-0.0007(7)$ \\
\hline$C(5)$ & $0.0264(12)$ & $0.0217(10)$ & $0.0416(13)$ & $-0.0032(8)$ & $-0.0055(9)$ & $0.0028(8)$ \\
\hline$C(6)$ & $0.0241(11)$ & $0.0215(9)$ & $0.0436(13)$ & $-0.0026(8)$ & $-0.0002(9)$ & $0.0015(8)$ \\
\hline$C(7)$ & $0.0313(12)$ & $0.0223(10)$ & $0.0374(12)$ & $-0.0005(8)$ & $0.0008(9)$ & $0.0001(8)$ \\
\hline $\mathrm{C}(8)$ & $0.0300(12)$ & $0.0252(10)$ & $0.0341(11)$ & $0.0000(8)$ & $-0.0010(9)$ & $0.0001(8)$ \\
\hline $\mathrm{C}(9)$ & $0.0289(12)$ & $0.0239(10)$ & $0.0372(12)$ & $0.0013(8)$ & $-0.0015(9)$ & $0.0026(8)$ \\
\hline$C(10)$ & $0.0392(14)$ & $0.0269(10)$ & $0.0421(13)$ & $-0.0025(9)$ & $0.0081(10)$ & $-0.0027(9)$ \\
\hline $\mathrm{O}(11)$ & $0.0525(11)$ & $0.0227(7)$ & $0.0458(9)$ & $-0.0051(6)$ & $-0.0023(8)$ & $-0.0033(7)$ \\
\hline $\mathrm{O}(12)$ & $0.0420(9)$ & $0.0217(7)$ & $0.0428(8)$ & $0.0011(6)$ & $-0.0031(7)$ & $-0.0015(6)$ \\
\hline $\mathrm{C}(13)$ & $0.0352(13)$ & $0.0229(9)$ & $0.0377(12)$ & $0.0009(8)$ & $-0.0012(10)$ & $0.0052(9)$ \\
\hline $\mathrm{C}(14)$ & $0.0403(14)$ & $0.0327(11)$ & $0.0428(13)$ & $-0.0047(9)$ & $-0.0016(11)$ & $-0.0008(10)$ \\
\hline$C(15)$ & $0.0548(17)$ & $0.0400(12)$ & $0.0380(13)$ & $-0.0052(10)$ & $-0.0046(11)$ & $0.0034(11)$ \\
\hline$C(16)$ & $0.0580(18)$ & $0.0472(13)$ & $0.0400(14)$ & $0.0015(11)$ & $0.0074(12)$ & $0.0092(13)$ \\
\hline $\mathrm{C}(17)$ & $0.0432(15)$ & $0.0426(13)$ & $0.0538(16)$ & $0.0054(11)$ & $0.0112(12)$ & $0.0018(11)$ \\
\hline $\mathrm{C}(18)$ & $0.0358(13)$ & $0.0327(11)$ & $0.0466(14)$ & $-0.0004(10)$ & $-0.0022(11)$ & $0.0010(9)$ \\
\hline$C(19 A)$ & $0.061(2)$ & $0.0320(13)$ & $0.0320(15)$ & $0.0019(10)$ & $0.0031(13)$ & $-0.0001(12)$ \\
\hline $\mathrm{C}(20 \mathrm{~A})$ & $0.107(3)$ & $0.0471(16)$ & $0.0403(18)$ & $-0.0031(13)$ & $0.0112(19)$ & $0.0001(19)$ \\
\hline$C(21 \mathrm{~A})$ & $0.097(3)$ & $0.0406(15)$ & $0.0433(19)$ & $0.0046(13)$ & $0.0168(18)$ & $-0.0010(17)$ \\
\hline$C(19 B)$ & $0.061(2)$ & $0.0320(13)$ & $0.0320(15)$ & $0.0019(10)$ & $0.0031(13)$ & $-0.0001(12)$ \\
\hline $\mathrm{C}(20 \mathrm{~B})$ & $0.107(3)$ & $0.0471(16)$ & $0.0403(18)$ & $-0.0031(13)$ & $0.0112(19)$ & $0.0001(19)$ \\
\hline$C(21 B)$ & $0.097(3)$ & $0.0406(15)$ & $0.0433(19)$ & $0.0046(13)$ & $0.0168(18)$ & $-0.0010(17)$ \\
\hline $\mathrm{C}(22)$ & $0.0294(12)$ & $0.0246(10)$ & $0.0453(13)$ & $0.0003(9)$ & $-0.0035(10)$ & $-0.0009(9)$ \\
\hline $\mathrm{C}(23)$ & $0.0384(15)$ & $0.0319(11)$ & $0.0573(15)$ & $-0.0037(10)$ & $0.0112(12)$ & $-0.0064(10)$ \\
\hline $\mathrm{C}(24)$ & $0.0356(15)$ & $0.0598(16)$ & $0.0533(16)$ & $0.0129(12)$ & $-0.0141(12)$ & $-0.0137(11)$ \\
\hline $\mathrm{C}(25)$ & $0.0396(13)$ & $0.0273(10)$ & $0.0426(13)$ & $0.0024(9)$ & $0.0066(11)$ & $-0.0040(9)$ \\
\hline$C(26)$ & $0.0663(18)$ & $0.0292(11)$ & $0.0455(14)$ & $0.0071(10)$ & $0.0051(13)$ & $0.0005(11)$ \\
\hline $\mathrm{C}(27)$ & $0.0528(16)$ & $0.0241(10)$ & $0.0487(14)$ & $0.0019(9)$ & $0.0100(12)$ & $-0.0021(10)$ \\
\hline $\mathrm{C}(28)$ & $0.0383(13)$ & $0.0258(10)$ & $0.0414(12)$ & $-0.0033(9)$ & $-0.0031(10)$ & $0.0009(9)$ \\
\hline C(29) & $0.0453(14)$ & $0.0246(10)$ & $0.0505(14)$ & $-0.0051(9)$ & $0.0010(11)$ & $-0.0029(9)$ \\
\hline $\mathrm{C}(30)$ & $0.0442(15)$ & $0.0302(11)$ & $0.0499(14)$ & $-0.0095(10)$ & $0.0061(11)$ & $0.0018(10)$ \\
\hline
\end{tabular}


Tabelle 5. H-Atomkoordinaten und äquivalente isotrope Auslenkungsparameter $\left(\AA^{2}\right)$ von $\mathbf{1 0 0}$.

\begin{tabular}{|c|c|c|c|c|}
\hline & $\mathrm{x}$ & $\mathrm{y}$ & $\mathrm{z}$ & $\mathrm{U}(\mathrm{eq})$ \\
\hline $\mathrm{H}(2 \mathrm{~A})$ & 0.5667 & 0.1697 & 0.4572 & 0.036 \\
\hline $\mathrm{H}(6 \mathrm{~A})$ & 0.5259 & 0.0688 & 0.4493 & 0.036 \\
\hline $\mathrm{H}(9 \mathrm{~A})$ & 0.1045 & 0.2039 & 0.4316 & 0.036 \\
\hline $\mathrm{H}(10 \mathrm{~A})$ & 0.5080 & 0.1310 & 0.3616 & 0.043 \\
\hline $\mathrm{H}(10 \mathrm{~B})$ & 0.3075 & 0.1452 & 0.3348 & 0.043 \\
\hline $\mathrm{H}(14 \mathrm{~A})$ & 0.4440 & 0.1783 & 0.6342 & 0.046 \\
\hline $\mathrm{H}(15 \mathrm{~A})$ & 0.3514 & 0.1756 & 0.7276 & 0.053 \\
\hline $\mathrm{H}(16 \mathrm{~A})$ & 0.1330 & 0.1159 & 0.7518 & 0.058 \\
\hline $\mathrm{H}(17 \mathrm{~A})$ & -0.0015 & 0.0618 & 0.6814 & 0.056 \\
\hline $\mathrm{H}(18 \mathrm{~A})$ & 0.0835 & 0.0669 & 0.5868 & 0.046 \\
\hline $\mathrm{H}(19 \mathrm{~A})$ & 0.2066 & 0.1513 & 0.3047 & 0.050 \\
\hline $\mathrm{H}(20 \mathrm{~A})$ & 0.4890 & 0.0843 & 0.2656 & 0.078 \\
\hline $\mathrm{H}(20 \mathrm{~B})$ & 0.3228 & 0.0948 & 0.2281 & 0.078 \\
\hline $\mathrm{H}(21 \mathrm{~A})$ & 0.5248 & 0.1935 & 0.2720 & 0.072 \\
\hline $\mathrm{H}(21 \mathrm{~B})$ & 0.3587 & 0.2040 & 0.2345 & 0.072 \\
\hline $\mathrm{H}(19 \mathrm{~B})$ & 0.6203 & 0.1294 & 0.3577 & 0.050 \\
\hline $\mathrm{H}(20 \mathrm{C})$ & 0.4367 & 0.0980 & 0.2542 & 0.078 \\
\hline $\mathrm{H}(20 \mathrm{D})$ & 0.6310 & 0.0862 & 0.2637 & 0.078 \\
\hline $\mathrm{H}(21 \mathrm{C})$ & 0.6709 & 0.1969 & 0.2799 & 0.072 \\
\hline$H(21 D)$ & 0.4768 & 0.2087 & 0.2704 & 0.072 \\
\hline $\mathrm{H}(22 \mathrm{~A})$ & 0.0318 & 0.0365 & 0.4419 & 0.040 \\
\hline $\mathrm{H}(23 \mathrm{~A})$ & -0.1926 & 0.0859 & 0.4829 & 0.051 \\
\hline $\mathrm{H}(23 \mathrm{~B})$ & -0.1235 & 0.1535 & 0.4625 & 0.051 \\
\hline $\mathrm{H}(24 \mathrm{~A})$ & -0.1310 & 0.1212 & 0.3653 & 0.059 \\
\hline $\mathrm{H}(24 \mathrm{~B})$ & -0.2001 & 0.0536 & 0.3856 & 0.059 \\
\hline $\mathrm{H}(25 \mathrm{~A})$ & 0.5195 & 0.2580 & 0.3873 & 0.044 \\
\hline $\mathrm{H}(26 \mathrm{~A})$ & 0.1925 & 0.2718 & 0.3453 & 0.056 \\
\hline $\mathrm{H}(26 \mathrm{~B})$ & 0.3454 & 0.3168 & 0.3287 & 0.056 \\
\hline $\mathrm{H}(27 \mathrm{~A})$ & 0.3799 & 0.3417 & 0.4286 & 0.050 \\
\hline $\mathrm{H}(27 \mathrm{~B})$ & 0.2271 & 0.2967 & 0.4451 & 0.050 \\
\hline $\mathrm{H}(28 \mathrm{~A})$ & 0.2260 & 0.0244 & 0.3381 & 0.042 \\
\hline $\mathrm{H}(29 \mathrm{~A})$ & 0.3538 & -0.0361 & 0.4389 & 0.048 \\
\hline $\mathrm{H}(29 \mathrm{~B})$ & 0.2599 & -0.0697 & 0.3850 & 0.048 \\
\hline $\mathrm{H}(30 \mathrm{~A})$ & 0.4592 & -0.0319 & 0.3200 & 0.050 \\
\hline $\mathrm{H}(30 \mathrm{~B})$ & 0.5531 & 0.0018 & 0.3739 & 0.050 \\
\hline
\end{tabular}


2. Röntgenographische Daten von endo-1,7,8,9,10-Pentacyclopropyl-4-phenyl-4-aza-tricyclo[5.2.1.0 $\left.{ }^{2,6}\right]$ dec-8-en-3,5-dion (102)

Tabelle 1. Kristalldaten und Strukturverfeinerung von 102.

Strukturkennzeichen

Summenformel

Molmasse

Temperatur

Wellenlänge

Kristallsystem, Raumgruppe

Zelldimensionen

Zellvolumen

Z, berechnete Dichte

Absorptionskoeffizient

$\mathrm{F}(000)$

Gemessener $\Theta$-Bereich

Indexgrenzen

Anzahl der gemessenen Reflexe

Unabhängige Reflexe

Observed reflections $[\mathrm{I}>2 \sigma(\mathrm{I})]$

Completeness to theta $=24.83$

Strukturverfeinerung

Daten / Restraints / Parameter

Goodness-of-fit on $\mathrm{F}^{2}$

Endgültige R-Werte [I > $2 \sigma(\mathrm{I})]$

R-Werte (sämtliche Daten)

Größtes Maximum und Minimum stre 436

$\mathrm{C}_{30} \mathrm{H}_{33} \mathrm{NO}_{2}$

439.57

133(2) K

$0.71073 \AA$

monoklin, $P 2{ }_{1} / n$

$a=10.4996(9) \AA$

$\alpha=90^{\circ}$

$b=37.910(4) \AA$

$\beta=100.577(7)^{\circ}$

$c=12.1868(12) \AA$

$\gamma=90^{\circ}$

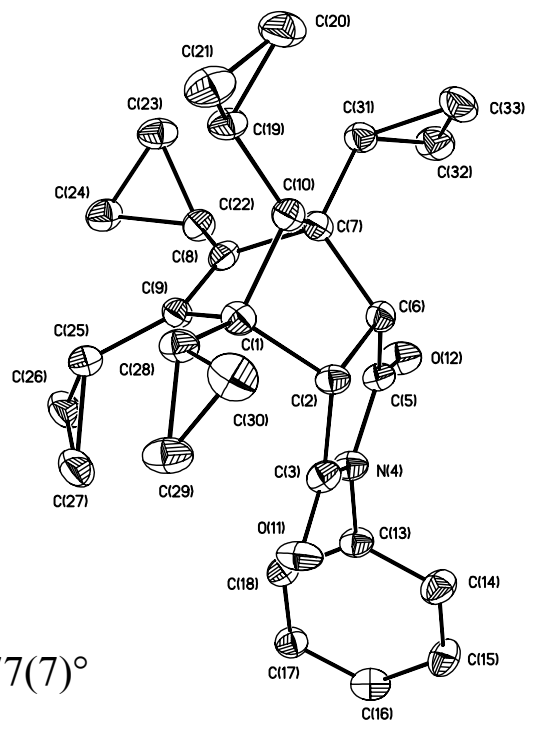

$4768.5(8) \AA^{3}$

$8,1.225 \mathrm{mg} / \mathrm{m}^{3}$

$0.076 \mathrm{~mm}^{-1}$

1888

1.78 bis $23.56^{\circ}$

$-11 \leq h \leq 10,-42 \leq k \leq 42,-12 \leq l \leq 13$

18987

$6671\left[\mathrm{R}_{\mathrm{int}}=0.1334\right]$

3274

$93.8 \%$

Full-matrix least-squares on $\mathrm{F}^{2}$

$6671 / 1 / 614$

0.977

$\mathrm{R} 1=0.0767, \mathrm{wR} 2=0.1476$

$\mathrm{R} 1=0.1656, \mathrm{wR} 2=0.1818$

425 und $-323 \mathrm{e} \mathrm{nm}^{-3}$ 
Tabelle 2. Atomkoordinaten und äquivalente isotrope Auslenkungsparameter $\left[\AA^{2}\right]$ von $\mathbf{1 0 2}$.

$\mathrm{U}(\mathrm{eq})$ wird bezeichnet als ein Drittel der Spur des orthogonalen $\mathrm{U}_{\mathrm{ij}}$-Tensors.

\begin{tabular}{|c|c|c|c|c|}
\hline & $\mathrm{x}$ & $\mathrm{y}$ & $\mathrm{z}$ & $\mathrm{U}(\mathrm{eq})$ \\
\hline$C(1)$ & $0.2038(5)$ & $0.1288(1)$ & $0.1081(5)$ & $0.0351(13)$ \\
\hline$C(2)$ & $0.2279(5)$ & $0.1171(1)$ & $0.2339(5)$ & $0.0325(13)$ \\
\hline$C(3)$ & $0.1178(6)$ & $0.1099(1)$ & $0.2923(5)$ & $0.0358(14)$ \\
\hline $\mathrm{N}(4)$ & $0.1129(5)$ & $0.0735(1)$ & $0.3120(4)$ & $0.0362(11)$ \\
\hline$C(5)$ & $0.2123(6)$ & $0.0549(1)$ & $0.2755(5)$ & $0.0344(13)$ \\
\hline$C(6)$ & $0.2998(5)$ & $0.0813(1)$ & $0.2339(5)$ & $0.0331(13)$ \\
\hline$C(7)$ & $0.3181(5)$ & $0.0774(1)$ & $0.1104(5)$ & $0.0321(13)$ \\
\hline $\mathrm{C}(8)$ & $0.1848(5)$ & $0.0708(1)$ & $0.0393(5)$ & $0.0344(13)$ \\
\hline $\mathrm{C}(9)$ & $0.1133(5)$ & $0.1005(1)$ & $0.0409(5)$ & $0.0324(13)$ \\
\hline$C(10)$ & $0.3370(5)$ & $0.1172(1)$ & $0.0830(5)$ & $0.0326(13)$ \\
\hline $\mathrm{O}(11)$ & $0.0425(4)$ & $0.1304(1)$ & $0.3242(4)$ & $0.0471(11)$ \\
\hline $\mathrm{O}(12)$ & $0.2230(4)$ & $0.0232(1)$ & $0.2838(4)$ & $0.0490(11)$ \\
\hline$C(13)$ & $0.0240(6)$ & $0.0589(1)$ & $0.3764(5)$ & $0.0369(14)$ \\
\hline$C(14)$ & $0.0641(7)$ & $0.0553(2)$ & $0.4907(5)$ & $0.0480(16)$ \\
\hline$C(15)$ & $-0.0203(7)$ & $0.0421(2)$ & $0.5553(5)$ & $0.0483(16)$ \\
\hline$C(16)$ & $-0.1454(6)$ & $0.0335(1)$ & $0.5058(5)$ & $0.0460(16)$ \\
\hline$C(17)$ & $-0.1856(6)$ & $0.0379(1)$ & $0.3917(5)$ & $0.0408(14)$ \\
\hline $\mathrm{C}(18)$ & $-0.1008(6)$ & $0.0502(1)$ & $0.3264(5)$ & $0.0392(14)$ \\
\hline$C(19)$ & $0.3671(5)$ & $0.1245(1)$ & $-0.0290(5)$ & $0.0381(14)$ \\
\hline$C(20)$ & $0.5080(6)$ & $0.1302(2)$ & $-0.0372(6)$ & $0.0546(17)$ \\
\hline $\mathrm{C}(21)$ & $0.4170(7)$ & $0.1612(2)$ & $-0.0540(6)$ & $0.0552(18)$ \\
\hline $\mathrm{C}(22)$ & $0.1508(6)$ & $0.0360(1)$ & $-0.0169(5)$ & $0.0386(14)$ \\
\hline$C(23)$ & $0.1929(6)$ & $0.0295(2)$ & $-0.1272(5)$ & $0.0471(16)$ \\
\hline $\mathrm{C}(24)$ & $0.0523(6)$ & $0.0327(2)$ & $-0.1232(5)$ & $0.0461(15)$ \\
\hline$C(25)$ & $-0.0209(6)$ & $0.1078(1)$ & $-0.0123(5)$ & $0.0416(14)$ \\
\hline$C(26)$ & $-0.1293(6)$ & $0.0821(2)$ & $-0.0067(6)$ & $0.0520(17)$ \\
\hline$C(27)$ & $-0.1252(6)$ & $0.1167(2)$ & $0.0542(6)$ & $0.0547(17)$ \\
\hline $\mathrm{C}(28)$ & $0.1656(6)$ & $0.1666(1)$ & $0.0804(5)$ & $0.0379(14)$ \\
\hline $\mathrm{C}(29)$ & $0.0849(7)$ & $0.1888(1)$ & $0.1433(6)$ & $0.0497(17)$ \\
\hline$C(30)$ & $0.2258(7)$ & $0.1970(2)$ & $0.1511(6)$ & $0.0558(18)$ \\
\hline $\mathrm{C}(31)$ & $0.4271(5)$ & $0.0536(1)$ & $0.0863(5)$ & $0.0361(14)$ \\
\hline$C(32)$ & $0.4759(6)$ & $0.0228(2)$ & $0.1588(6)$ & $0.0505(17)$ \\
\hline$C(33)$ & $0.5592(6)$ & $0.0551(2)$ & $0.1572(6)$ & $0.0518(17)$ \\
\hline$C(34)$ & $-0.0804(6)$ & $0.1725(2)$ & $-0.4190(5)$ & $0.0583(19)$ \\
\hline$C(35)$ & $-0.1305(6)$ & $0.1358(2)$ & $-0.4684(5)$ & $0.0478(16)$ \\
\hline$C(36)$ & $-0.2084(7)$ & $0.1146(2)$ & $-0.3993(6)$ & $0.0498(16)$ \\
\hline $\mathrm{N}(37)$ & $-0.3312(5)$ & $0.1088(1)$ & $-0.4634(4)$ & $0.0427(12)$ \\
\hline
\end{tabular}




$\begin{array}{lllll}\mathrm{C}(38) & -0.3498(6) & 0.1248(1) & -0.5682(5) & 0.0377(14) \\ \mathrm{C}(39) & -0.2268(6) & 0.1448(1) & -0.5756(5) & 0.0367(14) \\ \mathrm{C}(40) & -0.2337(6) & 0.1866(1) & -0.5717(5) & 0.0414(15) \\ \mathrm{C}(41) & -0.2869(7) & 0.1963(2) & -0.4668(7) & 0.0582(19) \\ \mathrm{C}(42) & -0.1967(8) & 0.1888(2) & -0.3754(6) & 0.073(2) \\ \mathrm{C}(43) & -0.0873(6) & 0.1925(2) & -0.5294(5) & 0.0466(16) \\ \mathrm{O}(44) & -0.1748(5) & 0.1029(1) & -0.3058(4) & 0.0623(13) \\ \mathrm{O}(45) & -0.4452(4) & 0.1217(1) & -0.6383(3) & 0.0430(10) \\ \mathrm{C}(46) & -0.4280(6) & 0.0875(1) & -0.4254(5) & 0.0395(14) \\ \mathrm{C}(47) & -0.4850(6) & 0.0595(2) & -0.4903(5) & 0.0427(15) \\ \mathrm{C}(48) & -0.5756(6) & 0.0386(2) & -0.4529(5) & 0.0460(16) \\ \mathrm{C}(49) & -0.6116(7) & 0.0458(2) & -0.3527(5) & 0.0499(17) \\ \mathrm{C}(50) & -0.5527(8) & 0.0735(2) & -0.2872(6) & 0.067(2) \\ \mathrm{C}(51) & -0.4628(7) & 0.0947(2) & -0.3245(6) & 0.0602(19) \\ \mathrm{C}(52 \mathrm{~A}) & -0.041(4) & 0.2247(4) & -0.513(4) & 0.042(4) \\ \mathrm{C}(53 \mathrm{~A}) & -0.016(4) & 0.2494(17) & -0.635(5) & 0.047(7) \\ \mathrm{C}(54 \mathrm{~A}) & 0.097(4) & 0.2443(6) & -0.531(3) & 0.048(5) \\ \mathrm{C}(55 \mathrm{~A}) & -0.407(3) & 0.2078(5) & -0.463(3) & 0.042(4) \\ \mathrm{C}(56 \mathrm{~A}) & -0.5360(18) & 0.1881(5) & -0.4900(14) & 0.050(3) \\ \mathrm{C}(57 \mathrm{~A}) & -0.498(3) & 0.2003(9) & -0.3694(17) & 0.062(5) \\ \mathrm{C}(52 \mathrm{~B}) & -0.042(4) & 0.2369(4) & -0.523(4) & 0.042(4) \\ \mathrm{C}(53 \mathrm{~B}) & 0.020(4) & 0.2456(17) & -0.608(5) & 0.047(7) \\ \mathrm{C}(54 \mathrm{~B}) & 0.106(4) & 0.2320(6) & -0.505(3) & 0.048(5) \\ \mathrm{C}(55 \mathrm{~B}) & -0.429(3) & 0.2195(5) & -0.491(3) & 0.042(4) \\ \mathrm{C}(56 \mathrm{~B}) & -0.5509(18) & 0.1988(5) & -0.5486(13) & 0.050(3) \\ \mathrm{C}(57 \mathrm{~B}) & -0.517(3) & 0.2065(9) & -0.4220(16) & 0.062(5) \\ \mathrm{C}(58 \mathrm{~A}) & -0.2348(12) & 0.2067(3) & -0.2747(12) & 0.048(2) \\ \mathrm{C}(58 \mathrm{~B}) & -0.1822(12) & 0.1835(3) & -0.2461(12) & 0.048(2) \\ \mathrm{C}(59 \mathrm{~A}) & -0.2418(14) & 0.2147(3) & -0.1868(12) & 0.053(3) \\ \mathrm{C}(59 \mathrm{~B}) & -0.213(3) & 0.1851(7) & -0.1571(16) & 0.130(8) \\ \mathrm{C}(60) & -0.1097(10) & 0.2137(3) & -0.1756(7) & 0.095(3) \\ \mathrm{C}(61) & 0.0484(7) & 0.1736(3) & -0.3405(7) & 0.086(3) \\ \mathrm{C}(62 \mathrm{~A}) & 0.1002(15) & 0.1516(4) & -0.2631(13) & 0.061(4) \\ \mathrm{C}(62 \mathrm{~B}) & 0.1062(12) & 0.1321(4) & -0.2749(11) & 0.046(3) \\ \mathrm{C}(63) & 0.1631(8) & 0.1522(3) & -0.3723(7) & 0.093(3) \\ \mathrm{C}(64) & -0.2868(6) & 0.2059(2) & -0.6778(6) & 0.0526(17) \\ \mathrm{C}(65) & -0.3921(7) & 0.1933(2) & -0.7662(7) & 0.071(2) \\ \mathrm{C}(66) & -0.2569(8) & 0.1948(2) & -0.7888(6) & 0.070(2)\end{array}$


Tabelle 3. Bindungslängen $[\mathrm{pm}]$ und -winkel $\left[{ }^{\circ}\right]$ von 102.

\begin{tabular}{|c|c|c|c|}
\hline $\mathrm{C}(1)-\mathrm{C}(28)$ & $151.0(7)$ & \multicolumn{2}{|l|}{$C(40)-C(43)$} \\
\hline$C(1)-C(10)$ & $154.9(8)$ & $\mathrm{C}(41)-\mathrm{C}(55 \mathrm{~A})$ & $134(3)$ \\
\hline$C(1)-C(9)$ & $156.3(8)$ & $\mathrm{C}(41)-\mathrm{C}(42)$ & $135.4(11)$ \\
\hline $\mathrm{C}(1)-\mathrm{C}(2)$ & $157.2(8)$ & $\mathrm{C}(41)-\mathrm{C}(55 \mathrm{~B})$ & $171(2)$ \\
\hline$C(2)-C(3)$ & 149.1(8) & $\mathrm{C}(42)-\mathrm{C}(58 \mathrm{~A})$ & $151.9(14)$ \\
\hline$C(2)-C(6)$ & $155.1(7)$ & $\mathrm{C}(42)-\mathrm{C}(58 \mathrm{~B})$ & $156.8(16)$ \\
\hline $\mathrm{C}(3)-\mathrm{O}(11)$ & $122.2(6)$ & $\mathrm{C}(43)-\mathrm{C}(52 \mathrm{~A})$ & $131(2)$ \\
\hline $\mathrm{C}(3)-\mathrm{N}(4)$ & $140.2(6)$ & $\mathrm{C}(43)-\mathrm{C}(52 \mathrm{~B})$ & $174.9(17)$ \\
\hline $\mathrm{N}(4)-\mathrm{C}(5)$ & $140.0(7)$ & $\mathrm{C}(46)-\mathrm{C}(51)$ & $137.2(8)$ \\
\hline $\mathrm{N}(4)-\mathrm{C}(13)$ & $143.6(7)$ & $C(46)-C(47)$ & $139.1(8)$ \\
\hline $\mathrm{C}(5)-\mathrm{O}(12)$ & $120.8(6)$ & $\mathrm{C}(47)-\mathrm{C}(48)$ & $137.8(8)$ \\
\hline$C(5)-C(6)$ & $151.1(7)$ & $\mathrm{C}(48)-\mathrm{C}(49)$ & $137.0(8)$ \\
\hline$C(6)-C(7)$ & $155.9(7)$ & $C(49)-C(50)$ & $139.4(9)$ \\
\hline $\mathrm{C}(7)-\mathrm{C}(8)$ & $152.5(8)$ & $\mathrm{C}(50)-\mathrm{C}(51)$ & $137.9(9)$ \\
\hline$C(7)-C(31)$ & $152.9(7)$ & $C(52 A)-C(54 A)$ & $168(4)$ \\
\hline$C(7)-C(10)$ & $156.6(7)$ & $\mathrm{C}(52 \mathrm{~A})-\mathrm{C}(53 \mathrm{~A})$ & $181(7)$ \\
\hline $\mathrm{C}(8)-\mathrm{C}(9)$ & $135.4(7)$ & $\mathrm{C}(53 \mathrm{~A})-\mathrm{C}(54 \mathrm{~A})$ & $158(5)$ \\
\hline $\mathrm{C}(8)-\mathrm{C}(22)$ & $150.0(7)$ & $C(55 A)-C(56 A)$ & $153(3)$ \\
\hline $\mathrm{C}(9)-\mathrm{C}(25)$ & $146.6(8)$ & $\mathrm{C}(55 \mathrm{~A})-\mathrm{C}(57 \mathrm{~A})$ & $165(4)$ \\
\hline$C(10)-C(19)$ & $148.3(8)$ & $C(56 A)-C(57 A)$ & $152(3)$ \\
\hline $\mathrm{C}(13)-\mathrm{C}(18)$ & $138.0(8)$ & $\mathrm{C}(52 \mathrm{~B})-\mathrm{C}(53 \mathrm{~B})$ & $137(7)$ \\
\hline$C(13)-C(14)$ & $138.7(8)$ & $\mathrm{C}(52 \mathrm{~B})-\mathrm{C}(54 \mathrm{~B})$ & $154(5)$ \\
\hline$C(14)-C(15)$ & $138.1(8)$ & $\mathrm{C}(53 \mathrm{~B})-\mathrm{C}(54 \mathrm{~B})$ & $150(5)$ \\
\hline$C(15)-C(16)$ & $138.0(9)$ & $\mathrm{C}(55 \mathrm{~B})-\mathrm{C}(57 \mathrm{~B})$ & $145(4)$ \\
\hline $\mathrm{C}(16)-\mathrm{C}(17)$ & $138.7(8)$ & $\mathrm{C}(55 \mathrm{~B})-\mathrm{C}(56 \mathrm{~B})$ & $156(3)$ \\
\hline $\mathrm{C}(17)-\mathrm{C}(18)$ & $138.0(8)$ & $\mathrm{C}(56 \mathrm{~B})-\mathrm{C}(57 \mathrm{~B})$ & $155(3)$ \\
\hline$C(19)-C(20)$ & $151.7(8)$ & $\mathrm{C}(58 \mathrm{~A})-\mathrm{C}(59 \mathrm{~A})$ & $112.8(17)$ \\
\hline$C(19)-C(21)$ & $153.4(8)$ & $\mathrm{C}(58 \mathrm{~A})-\mathrm{C}(60)$ & $163.5(16)$ \\
\hline $\mathrm{C}(20)-\mathrm{C}(21)$ & $150.4(9)$ & $\mathrm{C}(58 \mathrm{~B})-\mathrm{C}(59 \mathrm{~B})$ & $118.9(10)$ \\
\hline $\mathrm{C}(22)-\mathrm{C}(24)$ & $150.6(8)$ & $\mathrm{C}(58 \mathrm{~B})-\mathrm{C}(60)$ & $154.4(15)$ \\
\hline $\mathrm{C}(22)-\mathrm{C}(23)$ & $151.0(8)$ & $\mathrm{C}(59 \mathrm{~A})-\mathrm{C}(60)$ & $136.9(16)$ \\
\hline$C(23)-C(24)$ & $149.1(8)$ & $C(59 B)-C(60)$ & $158(2)$ \\
\hline $\mathrm{C}(25)-\mathrm{C}(26)$ & $150.9(8)$ & $\mathrm{C}(61)-\mathrm{C}(62 \mathrm{~A})$ & $129.9(17)$ \\
\hline $\mathrm{C}(25)-\mathrm{C}(27)$ & $151.4(8)$ & $\mathrm{C}(61)-\mathrm{C}(63)$ & $155.7(12)$ \\
\hline $\mathrm{C}(26)-\mathrm{C}(27)$ & $150.2(9)$ & $\mathrm{C}(61)-\mathrm{C}(62 \mathrm{~B})$ & $181.6(17)$ \\
\hline $\mathrm{C}(28)-\mathrm{C}(29)$ & $150.0(8)$ & $C(62 A)-C(63)$ & $159.1(17)$ \\
\hline $\mathrm{C}(28)-\mathrm{C}(30)$ & $150.5(8)$ & $\mathrm{C}(62 \mathrm{~B})-\mathrm{C}(63)$ & $161.5(14)$ \\
\hline$C(29)-C(30)$ & 149.7(9) & $\mathrm{C}(64)-\mathrm{C}(65)$ & $147.5(10)$ \\
\hline $\mathrm{C}(31)-\mathrm{C}(32)$ & $149.4(8)$ & $C(64)-C(66)$ & $150.2(10)$ \\
\hline$C(31)-C(33)$ & $149.4(8)$ & $\mathrm{C}(65)-\mathrm{C}(66)$ & $149.6(10)$ \\
\hline $\mathrm{C}(32)-\mathrm{C}(33)$ & $150.7(9)$ & $\mathrm{C}(28)-\mathrm{C}(1)-\mathrm{C}(10)$ & $116.0(4)$ \\
\hline $\mathrm{C}(34)-\mathrm{C}(61)$ & $150.7(10)$ & $\mathrm{C}(28)-\mathrm{C}(1)-\mathrm{C}(9)$ & $115.1(5)$ \\
\hline $\mathrm{C}(34)-\mathrm{C}(43)$ & $153.3(9)$ & $\mathrm{C}(10)-\mathrm{C}(1)-\mathrm{C}(9)$ & $100.7(4)$ \\
\hline$C(34)-C(42)$ & $154.7(10)$ & $\mathrm{C}(28)-\mathrm{C}(1)-\mathrm{C}(2)$ & $118.4(4)$ \\
\hline $\mathrm{C}(34)-\mathrm{C}(35)$ & $156.8(9)$ & $\mathrm{C}(10)-\mathrm{C}(1)-\mathrm{C}(2)$ & $97.0(4)$ \\
\hline$C(35)-C(36)$ & $150.9(9)$ & $\mathrm{C}(9)-\mathrm{C}(1)-\mathrm{C}(2)$ & $106.8(4)$ \\
\hline$C(35)-C(39)$ & $153.6(8)$ & $\mathrm{C}(3)-\mathrm{C}(2)-\mathrm{C}(6)$ & $105.1(4)$ \\
\hline $\mathrm{C}(36)-\mathrm{O}(44)$ & $121.2(7)$ & $\mathrm{C}(3)-\mathrm{C}(2)-\mathrm{C}(1)$ & $121.2(5)$ \\
\hline $\mathrm{C}(36)-\mathrm{N}(37)$ & $139.6(8)$ & $\mathrm{C}(6)-\mathrm{C}(2)-\mathrm{C}(1)$ & $103.8(4)$ \\
\hline $\mathrm{N}(37)-\mathrm{C}(38)$ & $139.5(8)$ & $\mathrm{O}(11)-\mathrm{C}(3)-\mathrm{N}(4)$ & $121.7(5)$ \\
\hline $\mathrm{N}(37)-\mathrm{C}(46)$ & $144.1(7)$ & $\mathrm{O}(11)-\mathrm{C}(3)-\mathrm{C}(2)$ & $129.7(5)$ \\
\hline $\mathrm{C}(38)-\mathrm{O}(45)$ & $119.7(7)$ & $\mathrm{N}(4)-\mathrm{C}(3)-\mathrm{C}(2)$ & $108.5(4)$ \\
\hline $\mathrm{C}(38)-\mathrm{C}(39)$ & $151.6(8)$ & $\mathrm{C}(5)-\mathrm{N}(4)-\mathrm{C}(3)$ & $113.0(4)$ \\
\hline$C(39)-C(40)$ & $158.4(7)$ & $\mathrm{C}(5)-\mathrm{N}(4)-\mathrm{C}(13)$ & $125.2(4)$ \\
\hline$C(40)-C(64)$ & $150.1(9)$ & $\mathrm{C}(3)-\mathrm{N}(4)-\mathrm{C}(13)$ & $121.3(4)$ \\
\hline$C(40)-C(41)$ & 153.1(9) & $\mathrm{O}(12)-\mathrm{C}(5)-\mathrm{N}(4)$ & $122.6(5)$ \\
\hline
\end{tabular}




\begin{tabular}{|c|c|}
\hline $\mathrm{O}(12)-\mathrm{C}(5)-\mathrm{C}(6)$ & $129.5(5)$ \\
\hline $\mathrm{N}(4)-\mathrm{C}(5)-\mathrm{C}(6)$ & $107.8(4)$ \\
\hline$C(5)-C(6)-C(2)$ & $104.8(4)$ \\
\hline$C(5)-C(6)-C(7)$ & $116.8(4)$ \\
\hline $\mathrm{C}(2)-\mathrm{C}(6)-\mathrm{C}(7)$ & $103.3(4)$ \\
\hline $\mathrm{C}(8)-\mathrm{C}(7)-\mathrm{C}(31)$ & $115.8(4)$ \\
\hline $\mathrm{C}(8)-\mathrm{C}(7)-\mathrm{C}(6)$ & $107.6(4)$ \\
\hline$C(31)-C(7)-C(6)$ & $118.1(5)$ \\
\hline$C(8)-C(7)-C(10)$ & $100.1(4)$ \\
\hline$C(31)-C(7)-C(10)$ & $113.6(4)$ \\
\hline $\mathrm{C}(6)-\mathrm{C}(7)-\mathrm{C}(10)$ & $99.0(4)$ \\
\hline $\mathrm{C}(9)-\mathrm{C}(8)-\mathrm{C}(22)$ & $130.7(5)$ \\
\hline$C(9)-C(8)-C(7)$ & $108.0(4)$ \\
\hline $\mathrm{C}(22)-\mathrm{C}(8)-\mathrm{C}(7)$ & $121.4(5$ \\
\hline$C(8)-C(9)-C(25)$ & $130.0(5)$ \\
\hline$C(8)-C(9)-C(1)$ & $107.1(5)$ \\
\hline $\mathrm{C}(25)-\mathrm{C}(9)-\mathrm{C}(1)$ & $122.9(4)$ \\
\hline$C(19)-C(10)-C(1)$ & $118.7(5)$ \\
\hline $\mathrm{C}(19)-\mathrm{C}(10)-\mathrm{C}(7)$ & $115.7(4)$ \\
\hline$C(1)-C(10)-C(7)$ & $94.4(4)$ \\
\hline$C(18)-C(13)-C(14)$ & $120.5(5)$ \\
\hline $\mathrm{C}(18)-\mathrm{C}(13)-\mathrm{N}(4)$ & $120.7(5)$ \\
\hline $\mathrm{C}(14)-\mathrm{C}(13)-\mathrm{N}(4)$ & $118.7(5)$ \\
\hline$C(15)-C(14)-C(13)$ & $120.0(6$ \\
\hline$C(16)-C(15)-C(14)$ & $119.7(6)$ \\
\hline$C(15)-C(16)-C(17)$ & $120.0(6)$ \\
\hline $\mathrm{C}(18)-\mathrm{C}(17)-\mathrm{C}(16)$ & $120.6(6)$ \\
\hline $\mathrm{C}(17)-\mathrm{C}(18)-\mathrm{C}(13)$ & $119.2(6)$ \\
\hline$C(10)-C(19)-C(20)$ & $117.5(5)$ \\
\hline$C(10)-C(19)-C(21)$ & $119.3(5)$ \\
\hline $\mathrm{C}(20)-\mathrm{C}(19)-\mathrm{C}(21)$ & $59.1(4)$ \\
\hline $\mathrm{C}(21)-\mathrm{C}(20)-\mathrm{C}(19)$ & $61.0(4)$ \\
\hline$C(20)-C(21)-C(19)$ & $59.9(4)$ \\
\hline$C(8)-C(22)-C(24)$ & $122.2(5)$ \\
\hline $\mathrm{C}(8)-\mathrm{C}(22)-\mathrm{C}(23)$ & $117.8(5)$ \\
\hline $\mathrm{C}(24)-\mathrm{C}(22)-\mathrm{C}(23)$ & $59.2(4)$ \\
\hline $\mathrm{C}(24)-\mathrm{C}(23)-\mathrm{C}(22)$ & $60.2(4)$ \\
\hline$C(23)-C(24)-C(22)$ & $60.5(4)$ \\
\hline$C(9)-C(25)-C(26)$ & $121.9(5)$ \\
\hline $\mathrm{C}(9)-\mathrm{C}(25)-\mathrm{C}(27)$ & $122.5(5$ \\
\hline$C(26)-C(25)-C(27)$ & $59.6(4)$ \\
\hline$C(27)-C(26)-C(25)$ & $60.4(4)$ \\
\hline $\mathrm{C}(26)-\mathrm{C}(27)-\mathrm{C}(25)$ & $60.0(4)$ \\
\hline $\mathrm{C}(29)-\mathrm{C}(28)-\mathrm{C}(30)$ & $59.7(4)$ \\
\hline$C(29)-C(28)-C(1)$ & $124.8(5$ \\
\hline $\mathrm{C}(30)-\mathrm{C}(28)-\mathrm{C}(1)$ & $122.0(5$ \\
\hline $\mathrm{C}(30)-\mathrm{C}(29)-\mathrm{C}(28)$ & $60.3(4)$ \\
\hline $\mathrm{C}(29)-\mathrm{C}(30)-\mathrm{C}(28)$ & $59.9(4)$ \\
\hline $\mathrm{C}(32)-\mathrm{C}(31)-\mathrm{C}(33)$ & $60.5(4)$ \\
\hline $\mathrm{C}(32)-\mathrm{C}(31)-\mathrm{C}(7)$ & $122.3(5)$ \\
\hline $\mathrm{C}(33)-\mathrm{C}(31)-\mathrm{C}(7)$ & $120.9(5$ \\
\hline $\mathrm{C}(31)-\mathrm{C}(32)-\mathrm{C}(33)$ & $59.7(4)$ \\
\hline $\mathrm{C}(31)-\mathrm{C}(33)-\mathrm{C}(32)$ & $59.7(4)$ \\
\hline$C(61)-C(34)-C(43)$ & $115.9(6$ \\
\hline $\mathrm{C}(61)-\mathrm{C}(34)-\mathrm{C}(42)$ & $116.3(6$ \\
\hline $\mathrm{C}(43)-\mathrm{C}(34)-\mathrm{C}(42)$ & $101.0(6$ \\
\hline$C(61)-C(34)-C(35)$ & $117.8(7)$ \\
\hline $\mathrm{C}(43)-\mathrm{C}(34)-\mathrm{C}(35)$ & $98.2(5)$ \\
\hline $\mathrm{C}(42)-\mathrm{C}(34)-\mathrm{C}(35)$ & $104.8(5$ \\
\hline$C(36)-C(35)-C(39)$ & $104.8(5$ \\
\hline
\end{tabular}

\begin{tabular}{|c|c|}
\hline $\mathrm{C}(36)-\mathrm{C}(35)-\mathrm{C}(34)$ & $115.9(5)$ \\
\hline$C(39)-C(35)-C(34)$ & $104.5(5)$ \\
\hline $\mathrm{O}(44)-\mathrm{C}(36)-\mathrm{N}(37)$ & $123.3(6)$ \\
\hline $\mathrm{O}(44)-\mathrm{C}(36)-\mathrm{C}(35)$ & $128.6(6)$ \\
\hline $\mathrm{N}(37)-\mathrm{C}(36)-\mathrm{C}(35)$ & $108.1(5)$ \\
\hline $\mathrm{C}(38)-\mathrm{N}(37)-\mathrm{C}(36)$ & $113.8(5)$ \\
\hline $\mathrm{C}(38)-\mathrm{N}(37)-\mathrm{C}(46)$ & $123.3(5)$ \\
\hline $\mathrm{C}(36)-\mathrm{N}(37)-\mathrm{C}(46)$ & $122.8(5)$ \\
\hline $\mathrm{O}(45)-\mathrm{C}(38)-\mathrm{N}(37)$ & $124.7(5)$ \\
\hline $\mathrm{O}(45)-\mathrm{C}(38)-\mathrm{C}(39)$ & $128.2(5)$ \\
\hline $\mathrm{N}(37)-\mathrm{C}(38)-\mathrm{C}(39)$ & $107.1(6)$ \\
\hline$C(38)-C(39)-C(35)$ & $105.8(5)$ \\
\hline $\mathrm{C}(38)-\mathrm{C}(39)-\mathrm{C}(40)$ & $117.1(5)$ \\
\hline $\mathrm{C}(35)-\mathrm{C}(39)-\mathrm{C}(40)$ & $103.0(5)$ \\
\hline$C(64)-C(40)-C(41)$ & $117.8(5)$ \\
\hline$C(64)-C(40)-C(43)$ & $114.0(5)$ \\
\hline $\mathrm{C}(41)-\mathrm{C}(40)-\mathrm{C}(43)$ & $100.3(5)$ \\
\hline $\mathrm{C}(64)-\mathrm{C}(40)-\mathrm{C}(39)$ & $118.2(5)$ \\
\hline$C(41)-C(40)-C(39)$ & $107.0(5)$ \\
\hline C(43)-C(40)-C(39) & $96.1(4)$ \\
\hline$C(55 A)-C(41)-C(42)$ & $124.2(14)$ \\
\hline $\mathrm{C}(55 \mathrm{~A})-\mathrm{C}(41)-\mathrm{C}(40)$ & $126.4(14)$ \\
\hline$C(42)-C(41)-C(40)$ & $109.2(6)$ \\
\hline$C(55 A)-C(41)-C(55 B)$ & $17.0(19)$ \\
\hline $\mathrm{C}(42)-\mathrm{C}(41)-\mathrm{C}(55 \mathrm{~B})$ & $135.0(12)$ \\
\hline $\mathrm{C}(40)-\mathrm{C}(41)-\mathrm{C}(55 \mathrm{~B})$ & $114.8(12)$ \\
\hline $\mathrm{C}(41)-\mathrm{C}(42)-\mathrm{C}(58 \mathrm{~A})$ & $108.8(8)$ \\
\hline C(41)-C(42)-C(34) & $106.1(6)$ \\
\hline$C(58 A)-C(42)-C(34)$ & $142.9(8)$ \\
\hline $\mathrm{C}(41)-\mathrm{C}(42)-\mathrm{C}(58 \mathrm{~B})$ & $141.1(8)$ \\
\hline $\mathrm{C}(58 \mathrm{~A})-\mathrm{C}(42)-\mathrm{C}(58 \mathrm{~B})$ & $40.2(6)$ \\
\hline $\mathrm{C}(34)-\mathrm{C}(42)-\mathrm{C}(58 \mathrm{~B})$ & $111.0(8)$ \\
\hline$C(52 A)-C(43)-C(34)$ & $112(2)$ \\
\hline$C(52 A)-C(43)-C(40)$ & $120.0(18)$ \\
\hline $\mathrm{C}(34)-\mathrm{C}(43)-\mathrm{C}(40)$ & $96.0(5)$ \\
\hline$C(52 A)-C(43)-C(52 B)$ & $8(3)$ \\
\hline $\mathrm{C}(34)-\mathrm{C}(43)-\mathrm{C}(52 \mathrm{~B})$ & $117.9(16)$ \\
\hline $\mathrm{C}(40)-\mathrm{C}(43)-\mathrm{C}(52 \mathrm{~B})$ & $113.6(15)$ \\
\hline $\mathrm{C}(51)-\mathrm{C}(46)-\mathrm{C}(47)$ & $120.3(5)$ \\
\hline $\mathrm{C}(51)-\mathrm{C}(46)-\mathrm{N}(37)$ & $119.7(5)$ \\
\hline $\mathrm{C}(47)-\mathrm{C}(46)-\mathrm{N}(37)$ & $120.0(5)$ \\
\hline $\mathrm{C}(48)-\mathrm{C}(47)-\mathrm{C}(46)$ & $120.0(6)$ \\
\hline $\mathrm{C}(49)-\mathrm{C}(48)-\mathrm{C}(47)$ & $120.1(6)$ \\
\hline $\mathrm{C}(48)-\mathrm{C}(49)-\mathrm{C}(50)$ & $119.6(6)$ \\
\hline $\mathrm{C}(51)-\mathrm{C}(50)-\mathrm{C}(49)$ & $120.6(6)$ \\
\hline $\mathrm{C}(46)-\mathrm{C}(51)-\mathrm{C}(50)$ & $119.3(6)$ \\
\hline $\mathrm{C}(43)-\mathrm{C}(52 \mathrm{~A})-\mathrm{C}(54 \mathrm{~A})$ & $134(3)$ \\
\hline C(43)-C(52A)-C(53A) & $118(4)$ \\
\hline$C(54 A)-C(52 A)-C(53 A)$ & $54(2)$ \\
\hline $\mathrm{C}(54 \mathrm{~A})-\mathrm{C}(53 \mathrm{~A})-\mathrm{C}(52 \mathrm{~A})$ & $59(2)$ \\
\hline$C(53 A)-C(54 A)-C(52 A)$ & $68(3)$ \\
\hline $\mathrm{C}(41)-\mathrm{C}(55 \mathrm{~A})-\mathrm{C}(56 \mathrm{~A})$ & $129.4(19)$ \\
\hline $\mathrm{C}(41)-\mathrm{C}(55 \mathrm{~A})-\mathrm{C}(57 \mathrm{~A})$ & $129.5(17)$ \\
\hline$C(56 A)-C(55 A)-C(57 A)$ & $57.0(16)$ \\
\hline$C(57 A)-C(56 A)-C(55 A)$ & $65.4(18)$ \\
\hline$C(56 A)-C(57 A)-C(55 A)$ & $57.6(15)$ \\
\hline $\mathrm{C}(53 \mathrm{~B})-\mathrm{C}(52 \mathrm{~B})-\mathrm{C}(54 \mathrm{~B})$ & $62(2)$ \\
\hline $\mathrm{C}(53 \mathrm{~B})-\mathrm{C}(52 \mathrm{~B})-\mathrm{C}(43)$ & $111(4)$ \\
\hline $\mathrm{C}(54 \mathrm{~B})-\mathrm{C}(52 \mathrm{~B})-\mathrm{C}(43)$ & $98.5(16)$ \\
\hline $\mathrm{C}(52 \mathrm{~B})-\mathrm{C}(53 \mathrm{~B})-\mathrm{C}(54 \mathrm{~B})$ & $65(3)$ \\
\hline
\end{tabular}

$\mathrm{C}(36)-\mathrm{C}(35)-\mathrm{C}(34) \quad 115.9(5)$

$\mathrm{O}(44)-\mathrm{C}(36)-\mathrm{C}(35) \quad 128.6(6)$

$\mathrm{N}(37)-\mathrm{C}(36)-\mathrm{C}(35) \quad 108.1(5)$

$\mathrm{C}(38)-\mathrm{N}(37)-\mathrm{C}(36) \quad 113.8(5)$

$\mathrm{C}(38)-\mathrm{N}(37)-\mathrm{C}(46) \quad 123.3(5)$

$\mathrm{O}(45)-\mathrm{C}(38)-\mathrm{N}(37) \quad 124.7(5)$

$\mathrm{O}(45)-\mathrm{C}(38)-\mathrm{C}(39) \quad 128.2(5)$

$\mathrm{N}(37)-\mathrm{C}(38)-\mathrm{C}(39) \quad 107.1(6)$

$\mathrm{C}(38)-\mathrm{C}(39)-\mathrm{C}(40) \quad 117.1(5)$

$\mathrm{C}(35)-\mathrm{C}(39)-\mathrm{C}(40) \quad 103.0(5)$

$\mathrm{C}(64)-\mathrm{C}(40)-\mathrm{C}(41) \quad 117.8(5)$

$\mathrm{C}(41)-\mathrm{C}(40)-\mathrm{C}(43) \quad 100.3(5)$

$\mathrm{C}(64)-\mathrm{C}(40)-\mathrm{C}(39) \quad 118.2(5)$

$\mathrm{C}(41)-\mathrm{C}(40)-\mathrm{C}(39) \quad 107.0(5)$

$\mathrm{C}(55 \mathrm{~A})-\mathrm{C}(41)-\mathrm{C}(42) \quad 124.2(14)$

$\mathrm{C}(55 \mathrm{~A})-\mathrm{C}(41)-\mathrm{C}(40) \quad 126.4(14)$

$\mathrm{C}(42)-\mathrm{C}(41)-\mathrm{C}(40) \quad 109.2(6)$

$\mathrm{C}(42)-\mathrm{C}(41)-\mathrm{C}(55 \mathrm{~B}) \quad 135.0(12)$

$\mathrm{C}(40)-\mathrm{C}(41)-\mathrm{C}(55 \mathrm{~B}) \quad 114.8(12)$

$\mathrm{C}(41)-\mathrm{C}(42)-\mathrm{C}(58 \mathrm{~A}) \quad 108.8(8)$

$\mathrm{C}(58 \mathrm{~A})-\mathrm{C}(42)-\mathrm{C}(34) \quad 142.9(8)$

$\mathrm{C}(41)-\mathrm{C}(42)-\mathrm{C}(58 \mathrm{~B}) \quad 141.1(8)$

$\mathrm{C}(58 \mathrm{~A})-\mathrm{C}(42)-\mathrm{C}(58 \mathrm{~B}) \quad 40.2(6)$

$\mathrm{C}(34)-\mathrm{C}(42)-\mathrm{C}(58 \mathrm{~B}) \quad 111.0(8)$

$\mathrm{C}(52 \mathrm{~A})-\mathrm{C}(43)-\mathrm{C}(40) \quad 120.0(18)$

$\mathrm{C}(34)-\mathrm{C}(43)-\mathrm{C}(40) \quad 96.0(5)$

$\mathrm{C}(52 \mathrm{~A})-\mathrm{C}(43)-\mathrm{C}(52 \mathrm{~B}) \quad 8(3)$

$\mathrm{C}(34)-\mathrm{C}(43)-\mathrm{C}(52 \mathrm{~B}) \quad 117.9(16)$

$\mathrm{C}(40)-\mathrm{C}(43)-\mathrm{C}(52 \mathrm{~B}) \quad 113.6(15)$

$\mathrm{C}(51)-\mathrm{C}(46)-\mathrm{N}(37) \quad 119.7(5)$

$\mathrm{C}(47)-\mathrm{C}(46)-\mathrm{N}(37) \quad 120.0(5)$

$\mathrm{C}(48)-\mathrm{C}(47)-\mathrm{C}(46) \quad 120.0(6)$

$\mathrm{C}(49)-\mathrm{C}(48)-\mathrm{C}(47) \quad 120.1(6)$

$\mathrm{C}(48)-\mathrm{C}(49)-\mathrm{C}(50) \quad 119.6(6)$

$\mathrm{C}(51)-\mathrm{C}(50)-\mathrm{C}(49) \quad 120.6(6)$

$\mathrm{C}(46)-\mathrm{C}(51)-\mathrm{C}(50) \quad 119.3(6)$

$\mathrm{C}(43)-\mathrm{C}(52 \mathrm{~A})-\mathrm{C}(53 \mathrm{~A}) \quad 118(4)$

$\mathrm{C}(54 \mathrm{~A})-\mathrm{C}(52 \mathrm{~A})-\mathrm{C}(53 \mathrm{~A}) \quad 54(2)$

$\mathrm{C}(54 \mathrm{~A})-\mathrm{C}(53 \mathrm{~A})-\mathrm{C}(52 \mathrm{~A}) \quad 59(2)$

$\mathrm{C}(53 \mathrm{~A})-\mathrm{C}(54 \mathrm{~A})-\mathrm{C}(52 \mathrm{~A}) \quad 68(3)$

$\mathrm{C}(41)-\mathrm{C}(55 \mathrm{~A})-\mathrm{C}(56 \mathrm{~A}) \quad 129.4(19)$

$\mathrm{C}(41)-\mathrm{C}(55 \mathrm{~A})-\mathrm{C}(57 \mathrm{~A}) \quad 129.5(17)$

$\mathrm{C}(56 \mathrm{~A})-\mathrm{C}(55 \mathrm{~A})-\mathrm{C}(57 \mathrm{~A}) \quad 57.0(16)$

$\mathrm{C}(56 \mathrm{~A})-\mathrm{C}(57 \mathrm{~A})-\mathrm{C}(55 \mathrm{~A}) \quad 57.6(15)$

$\mathrm{C}(53 \mathrm{~B})-\mathrm{C}(52 \mathrm{~B})-\mathrm{C}(54 \mathrm{~B}) \quad 62(2)$

$\mathrm{C}(53 \mathrm{~B})-\mathrm{C}(52 \mathrm{~B})-\mathrm{C}(43) \quad 111(4)$

C(52B)-C(53B)-C(54B) 65(3) 


$\begin{array}{ll}\mathrm{C}(53 \mathrm{~B})-\mathrm{C}(54 \mathrm{~B})-\mathrm{C}(52 \mathrm{~B}) & 53(3) \\ \mathrm{C}(57 \mathrm{~B})-\mathrm{C}(55 \mathrm{~B})-\mathrm{C}(56 \mathrm{~B}) & 61.9(17) \\ \mathrm{C}(57 \mathrm{~B})-\mathrm{C}(55 \mathrm{~B})-\mathrm{C}(41) & 110.8(16) \\ \mathrm{C}(56 \mathrm{~B})-\mathrm{C}(55 \mathrm{~B})-\mathrm{C}(41) & 116.2(15) \\ \mathrm{C}(57 \mathrm{~B})-\mathrm{C}(56 \mathrm{~B})-\mathrm{C}(55 \mathrm{~B}) & 55.5(17) \\ \mathrm{C}(55 \mathrm{~B})-\mathrm{C}(57 \mathrm{~B})-\mathrm{C}(56 \mathrm{~B}) & 62.6(16) \\ \mathrm{C}(59 \mathrm{~A})-\mathrm{C}(58 \mathrm{~A})-\mathrm{C}(42) & 163.5(15) \\ \mathrm{C}(59 \mathrm{~A})-\mathrm{C}(58 \mathrm{~A})-\mathrm{C}(60) & 55.8(10) \\ \mathrm{C}(42)-\mathrm{C}(58 \mathrm{~A})-\mathrm{C}(60) & 112.0(9) \\ \mathrm{C}(59 \mathrm{~B})-\mathrm{C}(58 \mathrm{~B})-\mathrm{C}(60) & 69.3(14) \\ \mathrm{C}(59 \mathrm{~B})-\mathrm{C}(58 \mathrm{~B})-\mathrm{C}(42) & 156.6(18) \\ \mathrm{C}(60)-\mathrm{C}(58 \mathrm{~B})-\mathrm{C}(42) & 114.4(9) \\ \mathrm{C}(58 \mathrm{~A})-\mathrm{C}(59 \mathrm{~A})-\mathrm{C}(60) & 81.2(11) \\ \mathrm{C}(58 \mathrm{~B})-\mathrm{C}(59 \mathrm{~B})-\mathrm{C}(60) & 66.0(12) \\ \mathrm{C}(59 \mathrm{~A})-\mathrm{C}(60)-\mathrm{C}(58 \mathrm{~B}) & 65.0(8) \\ \mathrm{C}(59 \mathrm{~A})-\mathrm{C}(60)-\mathrm{C}(59 \mathrm{~B}) & 47.3(10) \\ \mathrm{C}(58 \mathrm{~B})-\mathrm{C}(60)-\mathrm{C}(59 \mathrm{~B}) & 44.7(5) \\ \mathrm{C}(59 \mathrm{~A})-\mathrm{C}(60)-\mathrm{C}(58 \mathrm{~A}) & 43.0(7)\end{array}$

$\begin{array}{ll}\mathrm{C}(58 \mathrm{~B})-\mathrm{C}(60)-\mathrm{C}(58 \mathrm{~A}) & 38.9(6) \\ \mathrm{C}(59 \mathrm{~B})-\mathrm{C}(60)-\mathrm{C}(58 \mathrm{~A}) & 60.9(10) \\ \mathrm{C}(62 \mathrm{~A})-\mathrm{C}(61)-\mathrm{C}(34) & 130.7(10) \\ \mathrm{C}(62 \mathrm{~A})-\mathrm{C}(61)-\mathrm{C}(63) & 67.0(9) \\ \mathrm{C}(34)-\mathrm{C}(61)-\mathrm{C}(63) & 118.2(7) \\ \mathrm{C}(62 \mathrm{~A})-\mathrm{C}(61)-\mathrm{C}(62 \mathrm{~B}) & 20.8(8) \\ \mathrm{C}(34)-\mathrm{C}(61)-\mathrm{C}(62 \mathrm{~B}) & 116.4(7) \\ \mathrm{C}(63)-\mathrm{C}(61)-\mathrm{C}(62 \mathrm{~B}) & 56.6(6) \\ \mathrm{C}(61)-\mathrm{C}(62 \mathrm{~A})-\mathrm{C}(63) & 64.3(8) \\ \mathrm{C}(63)-\mathrm{C}(62 \mathrm{~B})-\mathrm{C}(61) & 53.6(6) \\ \mathrm{C}(61)-\mathrm{C}(63)-\mathrm{C}(62 \mathrm{~A}) & 48.7(7) \\ \mathrm{C}(61)-\mathrm{C}(63)-\mathrm{C}(62 \mathrm{~B}) & 69.8(7) \\ \mathrm{C}(62 \mathrm{~A})-\mathrm{C}(63)-\mathrm{C}(62 \mathrm{~B}) & 27.4(5) \\ \mathrm{C}(65)-\mathrm{C}(64)-\mathrm{C}(40) & 125.6(5) \\ \mathrm{C}(65)-\mathrm{C}(64)-\mathrm{C}(66) & 60.3(5) \\ \mathrm{C}(40)-\mathrm{C}(64)-\mathrm{C}(66) & 122.3(5) \\ \mathrm{C}(64)-\mathrm{C}(65)-\mathrm{C}(66) & 60.8(5) \\ \mathrm{C}(65)-\mathrm{C}(66)-\mathrm{C}(64) & 58.9(5)\end{array}$

Tabelle 4. Anisotrope Auslenkungsparameter $\left(\AA^{2}\right)$ von 102. Der anisotrope Auslenkungsfaktor hat die Form: $-2 \pi^{2}\left[\left(\mathrm{~h} \mathrm{a}^{*}\right)^{2} \mathrm{U}_{11}+\ldots+2 \mathrm{~h} \mathrm{k} \mathrm{a*} \mathrm{b}^{*} \mathrm{U}_{12}\right]$.

\begin{tabular}{lllllll}
\hline & $\mathrm{U} 11$ & $\mathrm{U} 22$ & $\mathrm{U} 33$ & $\mathrm{U} 23$ & $\mathrm{U} 13$ & $\mathrm{U} 12$ \\
\hline $\mathrm{C}(1)$ & $0.034(3)$ & $0.032(3)$ & $0.041(3)$ & $0.006(2)$ & $0.010(3)$ & $-0.003(2)$ \\
$\mathrm{C}(2)$ & $0.037(3)$ & $0.028(3)$ & $0.035(3)$ & $-0.002(2)$ & $0.012(3)$ & $0.002(2)$ \\
$\mathrm{C}(3)$ & $0.044(4)$ & $0.033(3)$ & $0.032(3)$ & $0.001(2)$ & $0.010(3)$ & $-0.001(3)$ \\
$\mathrm{N}(4)$ & $0.042(3)$ & $0.031(2)$ & $0.039(3)$ & $0.003(2)$ & $0.016(3)$ & $-0.001(2)$ \\
$\mathrm{C}(5)$ & $0.039(4)$ & $0.035(3)$ & $0.032(3)$ & $0.000(2)$ & $0.012(3)$ & $0.000(2)$ \\
$\mathrm{C}(6)$ & $0.032(3)$ & $0.035(3)$ & $0.031(3)$ & $-0.003(2)$ & $0.003(3)$ & $0.003(2)$ \\
$\mathrm{C}(7)$ & $0.028(3)$ & $0.033(3)$ & $0.035(3)$ & $-0.008(2)$ & $0.005(3)$ & $-0.001(2)$ \\
$\mathrm{C}(8)$ & $0.039(4)$ & $0.040(3)$ & $0.026(3)$ & $0.001(2)$ & $0.009(3)$ & $-0.002(2)$ \\
$\mathrm{C}(9)$ & $0.028(3)$ & $0.035(3)$ & $0.034(3)$ & $0.004(2)$ & $0.007(3)$ & $0.002(2)$ \\
$\mathrm{C}(10)$ & $0.033(3)$ & $0.031(3)$ & $0.033(3)$ & $-0.006(2)$ & $0.005(3)$ & $-0.002(2)$ \\
$\mathrm{O}(11)$ & $0.054(3)$ & $0.036(2)$ & $0.059(3)$ & $0.0057(19)$ & $0.034(2)$ & $0.0094(19)$ \\
$\mathrm{O}(12)$ & $0.066(3)$ & $0.029(2)$ & $0.057(3)$ & $0.0029(18)$ & $0.026(2)$ & $0.0053(19)$ \\
$\mathrm{C}(13)$ & $0.041(4)$ & $0.036(3)$ & $0.037(4)$ & $0.006(2)$ & $0.015(3)$ & $-0.003(2)$ \\
$\mathrm{C}(14)$ & $0.050(4)$ & $0.058(4)$ & $0.038(4)$ & $0.002(3)$ & $0.013(3)$ & $-0.011(3)$ \\
$\mathrm{C}(15)$ & $0.059(5)$ & $0.052(4)$ & $0.033(3)$ & $0.012(3)$ & $0.007(4)$ & $-0.003(3)$ \\
$\mathrm{C}(16)$ & $0.054(4)$ & $0.032(3)$ & $0.057(4)$ & $-0.001(3)$ & $0.022(4)$ & $-0.006(3)$ \\
$\mathrm{C}(17)$ & $0.040(4)$ & $0.042(3)$ & $0.042(4)$ & $0.001(3)$ & $0.011(3)$ & $-0.003(3)$ \\
$\mathrm{C}(18)$ & $0.042(4)$ & $0.044(3)$ & $0.033(3)$ & $0.003(3)$ & $0.010(3)$ & $0.000(3)$ \\
$\mathrm{C}(19)$ & $0.038(4)$ & $0.039(3)$ & $0.040(3)$ & $-0.002(3)$ & $0.015(3)$ & $-0.010(2)$ \\
$\mathrm{C}(20)$ & $0.041(4)$ & $0.068(4)$ & $0.058(4)$ & $-0.014(3)$ & $0.019(4)$ & $-0.012(3)$ \\
$\mathrm{C}(21)$ & $0.064(5)$ & $0.050(4)$ & $0.056(4)$ & $0.003(3)$ & $0.026(4)$ & $-0.018(3)$ \\
$\mathrm{C}(22)$ & $0.041(4)$ & $0.037(3)$ & $0.037(3)$ & $-0.011(3)$ & $0.005(3)$ & $-0.001(3)$
\end{tabular}




\begin{tabular}{|c|c|c|c|c|c|c|}
\hline$C(23)$ & $0.050(4)$ & $0.047(3)$ & $0.048(4)$ & $-0.015(3)$ & $0.019(3)$ & $-0.009(3)$ \\
\hline$C(24)$ & $0.047(4)$ & $0.052(3)$ & $0.041(4)$ & $-0.013(3)$ & $0.010(3)$ & $-0.011(3)$ \\
\hline$C(25)$ & $0.039(4)$ & $0.052(3)$ & $0.036(3)$ & $0.004(3)$ & $0.012(3)$ & $0.003(3)$ \\
\hline$C(26)$ & $0.031(4)$ & $0.065(4)$ & $0.058(4)$ & $-0.006(3)$ & $0.001(3)$ & $0.000(3)$ \\
\hline$C(27)$ & $0.034(4)$ & $0.070(4)$ & $0.057(4)$ & $-0.009(3)$ & $0.000(4)$ & $0.006(3)$ \\
\hline$C(28)$ & $0.045(4)$ & $0.029(3)$ & $0.043(3)$ & $0.002(2)$ & $0.017(3)$ & $0.005(2)$ \\
\hline$C(29)$ & $0.065(5)$ & $0.032(3)$ & $0.060(4)$ & $0.002(3)$ & $0.031(4)$ & $0.006(3)$ \\
\hline$C(30)$ & $0.061(5)$ & $0.035(3)$ & $0.073(5)$ & $-0.001(3)$ & $0.015(4)$ & $0.000(3)$ \\
\hline $\mathrm{C}(31)$ & $0.035(4)$ & $0.037(3)$ & $0.037(3)$ & $-0.006(2)$ & $0.009(3)$ & $0.003(2)$ \\
\hline$C(32)$ & $0.056(4)$ & $0.045(3)$ & $0.054(4)$ & $0.003(3)$ & $0.018(4)$ & $0.014(3)$ \\
\hline$C(33)$ & $0.038(4)$ & $0.058(4)$ & $0.059(4)$ & $-0.015(3)$ & $0.009(4)$ & $0.011(3)$ \\
\hline$C(34)$ & $0.039(4)$ & $0.108(5)$ & $0.029(4)$ & $-0.018(3)$ & $0.007(3)$ & $-0.020(4)$ \\
\hline$C(35)$ & $0.032(4)$ & $0.080(4)$ & $0.031(3)$ & $0.006(3)$ & $0.005(3)$ & $0.003(3)$ \\
\hline$C(36)$ & $0.045(4)$ & $0.069(4)$ & $0.035(4)$ & $0.006(3)$ & $0.007(4)$ & $0.003(3)$ \\
\hline N(37) & $0.039(3)$ & $0.050(3)$ & $0.039(3)$ & $0.006(2)$ & $0.007(3)$ & $-0.002(2)$ \\
\hline $\mathrm{C}(38)$ & $0.042(4)$ & $0.036(3)$ & $0.036(3)$ & $0.002(3)$ & $0.010(3)$ & $0.001(3)$ \\
\hline C(39) & $0.039(4)$ & $0.039(3)$ & $0.035(3)$ & $0.000(2)$ & $0.015(3)$ & $0.001(2)$ \\
\hline$C(40)$ & $0.037(4)$ & $0.038(3)$ & $0.052(4)$ & $-0.016(3)$ & $0.017(3)$ & $-0.004(3)$ \\
\hline$C(41)$ & $0.047(4)$ & $0.067(4)$ & $0.065(5)$ & $-0.040(4)$ & $0.022(4)$ & $-0.016(3)$ \\
\hline$C(42)$ & $0.059(5)$ & $0.116(6)$ & $0.052(5)$ & $-0.045(4)$ & $0.031(5)$ & $-0.042(4)$ \\
\hline$C(43)$ & $0.035(4)$ & $0.060(4)$ & $0.048(4)$ & $-0.015(3)$ & $0.017(3)$ & $-0.009(3)$ \\
\hline $\mathrm{O}(44)$ & $0.052(3)$ & $0.090(3)$ & $0.043(3)$ & $0.019(2)$ & $0.004(3)$ & $0.006(2)$ \\
\hline $\mathrm{O}(45)$ & $0.045(3)$ & $0.044(2)$ & $0.039(2)$ & $0.0035(18)$ & $0.005(2)$ & $-0.0059(18)$ \\
\hline$C(46)$ & $0.037(4)$ & $0.044(3)$ & $0.037(3)$ & $0.003(3)$ & $0.008(3)$ & $-0.002(3)$ \\
\hline$C(47)$ & $0.041(4)$ & $0.053(3)$ & $0.035(3)$ & $0.005(3)$ & $0.009(3)$ & $0.010(3)$ \\
\hline$C(48)$ & $0.054(4)$ & $0.038(3)$ & $0.046(4)$ & $0.002(3)$ & $0.008(4)$ & $0.002(3)$ \\
\hline C(49) & $0.058(4)$ & $0.047(4)$ & $0.050(4)$ & $0.004(3)$ & $0.024(4)$ & $-0.009(3)$ \\
\hline$C(50)$ & $0.084(6)$ & $0.066(4)$ & $0.063(5)$ & $-0.012(4)$ & $0.045(5)$ & $-0.018(4)$ \\
\hline$C(51)$ & $0.074(5)$ & $0.061(4)$ & $0.051(4)$ & $-0.010(3)$ & $0.026(4)$ & $-0.019(4)$ \\
\hline $\mathrm{C}(52 \mathrm{~A}$ & $0.048(5)$ & $0.000(11)$ & $0.085(9)$ & $-0.025(14)$ & $0.028(6)$ & $-0.020(13)$ \\
\hline $\mathrm{C}(53 \mathrm{~A}$ & $0.01(2)$ & $0.053(12)$ & $0.07(3)$ & $0.004(13)$ & $-0.003(18)$ & $0.003(14)$ \\
\hline $\mathrm{C}(54 \mathrm{~A}$ & $0.056(8)$ & $0.043(15)$ & $0.052(14)$ & $0.005(9)$ & $0.029(10)$ & $-0.026(12)$ \\
\hline $\mathrm{C}(55 \mathrm{~A}$ & $0.021(9)$ & $0.032(12)$ & $0.074(14)$ & $-0.015(9)$ & $0.007(8)$ & $0.019(7)$ \\
\hline$C(56 \mathrm{~A}$ & $0.032(5)$ & $0.064(10)$ & $0.054(11)$ & $-0.018(7)$ & $0.003(9)$ & $-0.004(6)$ \\
\hline $\mathrm{C}(57 \mathrm{~A}$ & $0.050(9)$ & $0.086(12)$ & $0.060(16)$ & $-0.006(13)$ & $0.039(14)$ & $-0.017(7)$ \\
\hline$C(52 B$ & $0.048(5)$ & $0.000(11)$ & $0.085(9)$ & $-0.025(14)$ & $0.028(6)$ & $-0.020(13)$ \\
\hline$C(53 B$ & $0.01(2)$ & $0.053(12)$ & $0.07(3)$ & $0.004(13)$ & $-0.003(18)$ & $0.003(14)$ \\
\hline $\mathrm{C}(54 \mathrm{~B}$ & $0.056(8)$ & $0.043(15)$ & $0.052(14)$ & $0.005(9)$ & $0.029(10)$ & $-0.026(12)$ \\
\hline$C(55 B$ & $0.021(9)$ & $0.032(12)$ & $0.074(14)$ & $-0.015(9)$ & $0.007(8)$ & $0.019(7)$ \\
\hline$C(56 B$ & $0.032(5)$ & $0.064(10)$ & $0.054(11)$ & $-0.018(7)$ & $0.003(9)$ & $-0.004(6)$ \\
\hline$C(57 B$ & $0.050(9)$ & $0.086(12)$ & $0.060(16)$ & $-0.006(13)$ & $0.039(14)$ & $-0.017(7)$ \\
\hline $\mathrm{C}(58 \mathrm{~A}$ & $0.031(6)$ & $0.060(7)$ & $0.053(6)$ & $-0.017(5)$ & $0.007(5)$ & $0.000(4)$ \\
\hline $\mathrm{C}(58 \mathrm{~B}$ & $0.031(6)$ & $0.060(7)$ & $0.053(6)$ & $-0.017(5)$ & $0.007(5)$ & $0.000(4)$ \\
\hline$C(60)$ & $0.109(8)$ & $0.129(7)$ & $0.044(5)$ & $-0.037(5)$ & $0.007(5)$ & $-0.020(6)$ \\
\hline$C(61)$ & $0.033(5)$ & $0.182(9)$ & $0.043(5)$ & $-0.012(5)$ & $0.006(4)$ & $-0.031(5)$ \\
\hline$C(63)$ & $0.038(5)$ & $0.178(9)$ & $0.061(5)$ & $0.028(6)$ & $0.010(5)$ & $-0.008(5)$ \\
\hline$C(64)$ & $0.040(4)$ & $0.042(3)$ & $0.074(5)$ & $0.005(3)$ & $0.004(4)$ & $0.003(3)$ \\
\hline$C(65)$ & $0.054(5)$ & $0.069(5)$ & $0.082(6)$ & $0.026(4)$ & $-0.007(5)$ & $-0.008(4)$ \\
\hline$C(66)$ & $0.078(6)$ & $0.072(5)$ & $0.060(5)$ & $0.028(4)$ & $0.015(5)$ & $0.012(4)$ \\
\hline
\end{tabular}


Tabelle 5. H-Atomkoordinaten und äquivalente isotrope Auslenkungsparameter $\left(\AA^{2}\right)$ von 102.

\begin{tabular}{|c|c|c|c|c|c|c|c|c|}
\hline & $\mathrm{x}$ & $\mathrm{y}$ & $\mathrm{z}$ & $\mathrm{U}(\mathrm{eq})$ & $\mathrm{x}$ & $\mathrm{y}$ & $\mathrm{z}$ & $\mathrm{U}(\mathrm{eq})$ \\
\hline $\mathrm{H}(2 \mathrm{~A})$ & 0.2875 & 0.1345 & 0.2788 & 0.039 & $\mathrm{H}(28 \mathrm{~A}) 0.1501$ & 0.1718 & -0.0015 & 50.045 \\
\hline $\mathrm{H}(6 \mathrm{~A})$ & 0.3858 & 0.0823 & 0.2852 & 0.040 & $\mathrm{H}(29 \mathrm{~A}) 0.0215$ & 0.2052 & 0.0998 & 0.060 \\
\hline $\mathrm{H}(10 \mathrm{~A})$ & 0.4072 & 0.1274 & 0.1411 & 0.039 & $\mathrm{H}(29 \mathrm{~B}) 0.0573$ & 0.1782 & 0.2093 & 0.060 \\
\hline $\mathrm{H}(14 \mathrm{~A})$ & 0.1496 & 0.0618 & 0.5247 & 0.058 & $\mathrm{H}(30 \mathrm{~A}) 0.2850$ & 0.1914 & 0.2220 & 0.067 \\
\hline $\mathrm{H}(15 \mathrm{~B})$ & 0.0077 & 0.0391 & 0.6334 & 0.058 & $\mathrm{H}(30 \mathrm{~B}) 0.2492$ & 0.2183 & 0.1124 & 0.067 \\
\hline $\mathrm{H}(16 \mathrm{~A})$ & -0.2039 & 0.0245 & 0.5499 & 0.055 & $\mathrm{H}(31 \mathrm{~A}) 0.4270$ & 0.0497 & 0.0051 & 0.043 \\
\hline $\mathrm{H}(17 \mathrm{~A})$ & -0.2723 & 0.0324 & 0.3583 & 0.049 & $\mathrm{H}(32 \mathrm{~A}) 0.5004$ & 0.0012 & 0.1221 & 0.061 \\
\hline $\mathrm{H}(18 \mathrm{~A})$ & -0.1280 & 0.0527 & 0.2480 & 0.047 & $\mathrm{H}(32 \mathrm{~B}) 0.4374$ & 0.0187 & 0.2261 & 0.061 \\
\hline $\mathrm{H}(19 \mathrm{~A})$ & 0.3125 & 0.1120 & -0.0932 & 0.046 & $\mathrm{H}(33 \mathrm{~A}) 0.5718$ & 0.0708 & 0.2234 & 0.062 \\
\hline $\mathrm{H}(20 \mathrm{~A})$ & 0.5729 & 0.1293 & 0.0328 & 0.066 & $\mathrm{H}(33 \mathrm{~B}) 0.6347$ & 0.0533 & 0.1195 & 0.062 \\
\hline $\mathrm{H}(20 \mathrm{~B})$ & 0.5382 & 0.1207 & -0.1036 & 0.066 & $\mathrm{H}(35 \mathrm{~A})-0.0571$ & 0.1214 & -0.4863 & 30.057 \\
\hline $\mathrm{H}(21 \mathrm{~B})$ & 0.3910 & 0.1705 & -0.1307 & 0.066 & $\mathrm{H}(39 \mathrm{~A})-0.1917$ & 0.1370 & -0.6426 & 60.044 \\
\hline $\mathrm{H}(21 \mathrm{C})$ & 0.4257 & 0.1791 & 0.0059 & 0.066 & $H(43 A)-0.0369$ & 0.1793 & -0.5783 & 30.056 \\
\hline $\mathrm{H}(22 \mathrm{~A})$ & 0.1573 & 0.0152 & 0.0342 & 0.046 & $\mathrm{H}(47 \mathrm{~A})-0.4615$ & 0.0548 & -0.5605 & 50.051 \\
\hline $\mathrm{H}(23 \mathrm{~B})$ & 0.2345 & 0.0492 & -0.1606 & 0.057 & $H(48 A)-0.6132$ & 0.0193 & -0.4966 & 60.055 \\
\hline $\mathrm{H}(23 \mathrm{C})$ & 0.2266 & 0.0057 & -0.1405 & 0.057 & $H(49 A)-0.6763$ & 0.0319 & -0.3280 & 00.060 \\
\hline $\mathrm{H}(24 \mathrm{~A})$ & -0.0009 & 0.0110 & -0.1340 & 0.055 & $\mathrm{H}(50 \mathrm{~A})-0.5747$ & 0.0778 & -0.2162 & 20.080 \\
\hline $\mathrm{H}(24 \mathrm{~B})$ & 0.0070 & 0.0544 & -0.1542 & 0.055 & $\mathrm{H}(51 \mathrm{~A})-0.4253$ & 0.1141 & -0.2809 & 90.072 \\
\hline $\mathrm{H}(25 \mathrm{~A})$ & -0.0296 & 0.1207 & -0.0849 & 0.050 & $\mathrm{H}(64 \mathrm{~A})-0.2872$ & 0.2320 & -0.6680 & 00.063 \\
\hline $\mathrm{H}(26 \mathrm{~A})$ & -0.1965 & 0.0791 & -0.0746 & 0.062 & $\mathrm{H}(65 \mathrm{~A})-0.4551$ & 0.2109 & -0.8035 & 50.085 \\
\hline $\mathrm{H}(26 \mathrm{~B})$ & -0.1081 & 0.0605 & 0.0383 & 0.062 & $\mathrm{H}(65 \mathrm{~B})-0.4292$ & 0.1697 & -0.7567 & 70.085 \\
\hline $\mathrm{H}(27 \mathrm{~A})$ & -0.1014 & 0.1162 & 0.1366 & 0.066 & $H(66 A)-0.2362$ & 0.2136 & -0.8394 & 40.084 \\
\hline $\mathrm{H}(27 \mathrm{~B})$ & -0.1898 & 0.1349 & 0.0237 & 0.066 & $\mathrm{H}(66 \mathrm{~B})-0.2103$ & 0.1723 & -0.7925 & 50.084 \\
\hline
\end{tabular}


3. Röntgenographische Daten von syn-4,5,6,7-Tetracyclopropyl-2-phenyl-3a,4,7,7a-tetrahydroisoindol-1,3-dion (104)

Tabelle 1. Kristalldaten und Strukturverfeinerung von 104.

Strukturkennzeichen

stre 443

Summenformel

$\mathrm{C}_{26} \mathrm{H}_{29} \mathrm{NO}_{2}$

Molmasse

387.50

Temperatur

133(2) K

Wellenlänge

$0.71073 \AA$

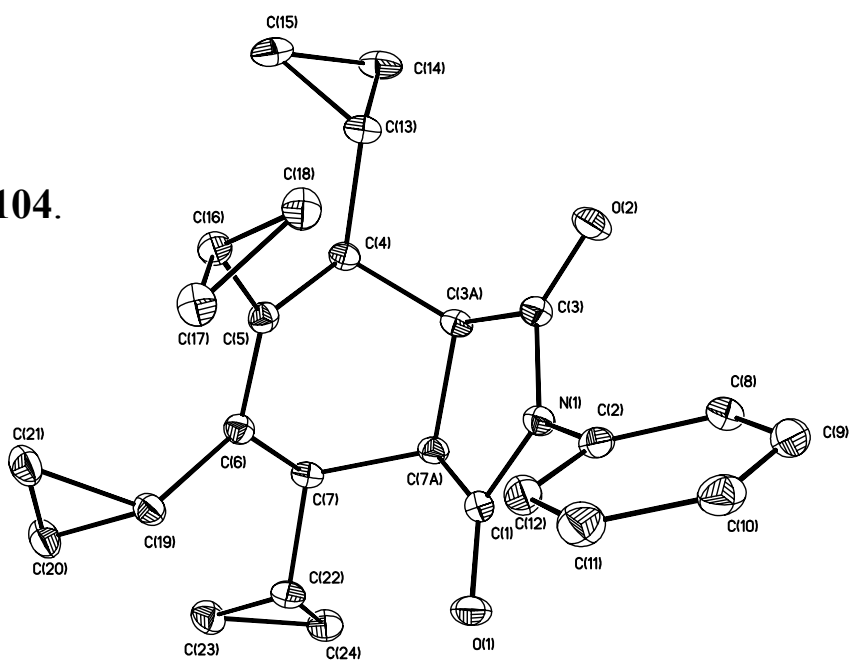

Kristallsystem, Raumgruppe

orthorombisch,

$P 22_{1} 2_{1}$

Zelldimensionen

$a=7.7020(6) \AA$

$\alpha=90^{\circ}$

$b=15.2195(11) \AA$

$\beta=90^{\circ}$

$c=17.5981(16) \AA$

$\gamma=90^{\circ}$

Zellvolumen

2062.9(3) $\AA^{3}$

Z, berechnete Dichte

$4,1.248 \mathrm{mg} / \mathrm{m}^{3}$

Absorptionskoeffizient

$0.078 \mathrm{~mm}^{-1}$

$\mathrm{F}(000)$

832

Gemessener $\Theta$-Bereich

1.77 bis $24.84^{\circ}$

Indexgrenzen

$-9 \leq h \leq 8,-17 \leq k \leq 16,-19 \leq l \leq 20$

Anzahl der gemessenen Reflexe 7185

Unabhängige Reflexe

$3533\left[\mathrm{R}_{\text {int }}=0.0567\right]$

Observed reflections $[\mathrm{I}>2 \sigma(\mathrm{I})] \quad 3043$

Completeness to theta $=24.83 \quad 99.4 \%$

Strukturverfeinerung

Full-matrix least-squares on $\mathrm{F}^{2}$

Daten / Restraints / Parameter

3533 / 0 / 262

Goodness-of-fit on $\mathrm{F}^{2}$ 1.046

Endgültige R-Werte $[\mathrm{I}>2 \sigma(\mathrm{I})]$

$\mathrm{R} 1=0.0401, \mathrm{wR} 2=0.0918$

R-Werte (sämtliche Daten)

$\mathrm{R} 1=0.0497, \mathrm{wR} 2=0.0957$

Größtes Maximum und Minimum 139 und $-179 \mathrm{e} \mathrm{nm}^{-3}$ 
Tabelle 2. Atomkoordinaten und äquivalente isotrope Auslenkungsparameter $\left[\AA^{2}\right]$ von $\mathbf{1 0 4}$.

$\mathrm{U}(\mathrm{eq})$ wird bezeichnet als ein Drittel der Spur des orthogonalen $\mathrm{U}_{\mathrm{ij}}$-Tensors.

\begin{tabular}{lcccc}
\hline & $\mathrm{x}$ & $\mathrm{y}$ & $\mathrm{z}$ & $\mathrm{U}(\mathrm{eq})$ \\
\hline $\mathrm{N}(1)$ & $0.0852(2)$ & $0.1841(1)$ & $0.6668(1)$ & $0.0233(4)$ \\
$\mathrm{O}(1)$ & $0.0978(2)$ & $0.3349(1)$ & $0.6779(1)$ & $0.0342(4)$ \\
$\mathrm{O}(2)$ & $0.1454(2)$ & $0.0362(1)$ & $0.6742(1)$ & $0.0320(4)$ \\
$\mathrm{C}(1)$ & $0.1503(3)$ & $0.2627(1)$ & $0.6967(1)$ & $0.0241(5)$ \\
$\mathrm{C}(2)$ & $-0.0564(3)$ & $0.1796(1)$ & $0.6145(1)$ & $0.0249(4)$ \\
$\mathrm{C}(3 \mathrm{~A})$ & $0.2874(3)$ & $0.1405(1)$ & $0.7606(1)$ & $0.0218(4)$ \\
$\mathrm{C}(3)$ & $0.1710(3)$ & $0.1106(1)$ & $0.6969(1)$ & $0.0235(5)$ \\
$\mathrm{C}(4)$ & $0.2125(3)$ & $0.1122(1)$ & $0.8390(1)$ & $0.0208(4)$ \\
$\mathrm{C}(5)$ & $0.0545(3)$ & $0.1684(1)$ & $0.8586(1)$ & $0.0213(4)$ \\
$\mathrm{C}(6)$ & $0.0804(3)$ & $0.2565(1)$ & $0.8591(1)$ & $0.0210(4)$ \\
$\mathrm{C}(7 \mathrm{~A})$ & $0.2913(3)$ & $0.2417(1)$ & $0.7534(1)$ & $0.0220(4)$ \\
$\mathrm{C}(7)$ & $0.2571(3)$ & $0.2878(1)$ & $0.8307(1)$ & $0.0211(4)$ \\
$\mathrm{C}(8)$ & $-0.0383(3)$ & $0.1356(1)$ & $0.5458(1)$ & $0.0279(5)$ \\
$\mathrm{C}(9)$ & $-0.1776(3)$ & $0.1330(2)$ & $0.4966(1)$ & $0.0326(5)$ \\
$\mathrm{C}(10)$ & $-0.3327(3)$ & $0.1734(2)$ & $0.5148(1)$ & $0.0336(5)$ \\
$\mathrm{C}(11)$ & $-0.3498(3)$ & $0.2167(2)$ & $0.5838(1)$ & $0.0354(6)$ \\
$\mathrm{C}(12)$ & $-0.2119(3)$ & $0.2195(2)$ & $0.6339(1)$ & $0.0303(5)$ \\
$\mathrm{C}(13)$ & $0.1890(3)$ & $0.0139(1)$ & $0.8462(1)$ & $0.0265(5)$ \\
$\mathrm{C}(14)$ & $0.3445(3)$ & $-0.0388(2)$ & $0.8692(2)$ & $0.0347(6)$ \\
$\mathrm{C}(15)$ & $0.1978(3)$ & $-0.0257(2)$ & $0.9241(1)$ & $0.0331(6)$ \\
$\mathrm{C}(16)$ & $-0.1120(3)$ & $0.1226(1)$ & $0.8750(1)$ & $0.0241(5)$ \\
$\mathrm{C}(17)$ & $-0.2839(3)$ & $0.1565(2)$ & $0.8476(1)$ & $0.0286(5)$ \\
$\mathrm{C}(18)$ & $-0.2067(3)$ & $0.0758(2)$ & $0.8114(1)$ & $0.0298(5)$ \\
$\mathrm{C}(19)$ & $-0.0473(3)$ & $0.3243(1)$ & $0.8840(1)$ & $0.0242(4)$ \\
$\mathrm{C}(20)$ & $-0.0100(3)$ & $0.3745(2)$ & $0.9563(1)$ & $0.0312(5)$ \\
$\mathrm{C}(21)$ & $-0.1603(3)$ & $0.3115(2)$ & $0.9528(1)$ & $0.0317(5)$ \\
$\mathrm{C}(22)$ & $0.2828(3)$ & $0.3864(1)$ & $0.8254(1)$ & $0.0253(5)$ \\
$\mathrm{C}(23)$ & $0.3661(3)$ & $0.4344(2)$ & $0.8903(1)$ & $0.0333(5)$ \\
$\mathrm{C}(24)$ & $0.4646(3)$ & $0.4203(2)$ & $0.8178(1)$ & $0.0316(5)$ \\
& & & & \\
\hline & & & &
\end{tabular}


Table 3. Bindungslängen $[\mathrm{pm}]$ und -winkel $\left[{ }^{\circ}\right]$ von 104.

\begin{tabular}{|c|c|c|c|}
\hline $\mathrm{N}(1)-\mathrm{C}(1)$ & $139.8(3)$ & $\mathrm{C}(3)-\mathrm{C}(3 \mathrm{~A})-\mathrm{C}(4)$ & $110.87(17)$ \\
\hline $\mathrm{N}(1)-\mathrm{C}(3)$ & $140.3(3)$ & $C(7 A)-C(3 A)-C(4)$ & $110.86(16)$ \\
\hline $\mathrm{N}(1)-\mathrm{C}(2)$ & $142.8(3)$ & $\mathrm{O}(2)-\mathrm{C}(3)-\mathrm{N}(1)$ & $122.8(2)$ \\
\hline $\mathrm{O}(1)-\mathrm{C}(1)$ & $121.7(3)$ & $\mathrm{O}(2)-\mathrm{C}(3)-\mathrm{C}(3 \mathrm{~A})$ & $128.46(19)$ \\
\hline $\mathrm{O}(2)-\mathrm{C}(3)$ & $121.7(3)$ & $\mathrm{N}(1)-\mathrm{C}(3)-\mathrm{C}(3 \mathrm{~A})$ & $108.67(17)$ \\
\hline $\mathrm{C}(1)-\mathrm{C}(7 \mathrm{~A})$ & $150.9(3)$ & $\mathrm{C}(13)-\mathrm{C}(4)-\mathrm{C}(5)$ & $116.08(17)$ \\
\hline$C(2)-C(12)$ & $138.5(3)$ & $C(13)-C(4)-C(3 A)$ & $113.11(17)$ \\
\hline$C(2)-C(8)$ & $139.0(3)$ & $\mathrm{C}(5)-\mathrm{C}(4)-\mathrm{C}(3 \mathrm{~A})$ & $109.91(16)$ \\
\hline $\mathrm{C}(3 \mathrm{~A})-\mathrm{C}(3)$ & $150.6(3)$ & $C(6)-C(5)-C(16)$ & $126.05(19)$ \\
\hline $\mathrm{C}(3 \mathrm{~A})-\mathrm{C}(7 \mathrm{~A})$ & $154.5(3)$ & $C(6)-C(5)-C(4)$ & $115.99(18)$ \\
\hline$C(3 A)-C(4)$ & $155.6(3)$ & $C(16)-C(5)-C(4)$ & $117.96(17)$ \\
\hline$C(4)-C(13)$ & $151.3(3)$ & $\mathrm{C}(5)-\mathrm{C}(6)-\mathrm{C}(19)$ & $126.13(19)$ \\
\hline $\mathrm{C}(4)-\mathrm{C}(5)$ & $152.7(3)$ & $C(5)-C(6)-C(7)$ & $116.00(18)$ \\
\hline$C(5)-C(6)$ & $135.5(3)$ & $\mathrm{C}(19)-\mathrm{C}(6)-\mathrm{C}(7)$ & $117.87(17)$ \\
\hline$C(5)-C(16)$ & $148.7(3)$ & $C(1)-C(7 A)-C(3 A)$ & $104.55(17)$ \\
\hline C(6)-C(19) & $149.2(3)$ & $\mathrm{C}(1)-\mathrm{C}(7 \mathrm{~A})-\mathrm{C}(7)$ & $111.17(16)$ \\
\hline$C(6)-C(7)$ & $152.6(3)$ & $C(3 A)-C(7 A)-C(7)$ & $112.01(16)$ \\
\hline$C(7 A)-C(7)$ & $155.5(3)$ & $C(22)-C(7)-C(6)$ & $116.49(17)$ \\
\hline$C(7)-C(22)$ & $151.6(3)$ & $\mathrm{C}(22)-\mathrm{C}(7)-\mathrm{C}(7 \mathrm{~A})$ & $111.77(17)$ \\
\hline C(8)-C(9) & $138.0(3)$ & $C(6)-C(7)-C(7 A)$ & $107.23(16)$ \\
\hline $\mathrm{C}(9)-\mathrm{C}(10)$ & $138.1(4)$ & $\mathrm{C}(9)-\mathrm{C}(8)-\mathrm{C}(2)$ & $118.8(2)$ \\
\hline $\mathrm{C}(10)-\mathrm{C}(11)$ & $138.7(3)$ & $\mathrm{C}(8)-\mathrm{C}(9)-\mathrm{C}(10)$ & $121.0(2)$ \\
\hline $\mathrm{C}(11)-\mathrm{C}(12)$ & $138.2(3)$ & $\mathrm{C}(9)-\mathrm{C}(10)-\mathrm{C}(11)$ & $119.7(2)$ \\
\hline $\mathrm{C}(13)-\mathrm{C}(14)$ & $149.7(3)$ & $\mathrm{C}(12)-\mathrm{C}(11)-\mathrm{C}(10)$ & $120.1(2)$ \\
\hline$C(13)-C(15)$ & $149.9(3)$ & $\mathrm{C}(11)-\mathrm{C}(12)-\mathrm{C}(2)$ & $119.6(2)$ \\
\hline $\mathrm{C}(14)-\mathrm{C}(15)$ & $150.0(4)$ & $\mathrm{C}(14)-\mathrm{C}(13)-\mathrm{C}(15)$ & $60.08(16)$ \\
\hline $\mathrm{C}(16)-\mathrm{C}(17)$ & $150.0(3)$ & $C(14)-C(13)-C(4)$ & $117.16(19)$ \\
\hline $\mathrm{C}(16)-\mathrm{C}(18)$ & $151.4(3)$ & $\mathrm{C}(15)-\mathrm{C}(13)-\mathrm{C}(4)$ & $118.00(19)$ \\
\hline $\mathrm{C}(17)-\mathrm{C}(18)$ & $150.6(3)$ & $\mathrm{C}(13)-\mathrm{C}(14)-\mathrm{C}(15)$ & $60.02(15)$ \\
\hline $\mathrm{C}(19)-\mathrm{C}(21)$ & $150.4(3)$ & $\mathrm{C}(13)-\mathrm{C}(15)-\mathrm{C}(14)$ & $59.91(16)$ \\
\hline$C(19)-C(20)$ & 151.1(3) & $C(5)-C(16)-C(17)$ & $122.52(18)$ \\
\hline $\mathrm{C}(20)-\mathrm{C}(21)$ & $150.5(3)$ & $C(5)-C(16)-C(18)$ & $119.55(19)$ \\
\hline $\mathrm{C}(22)-\mathrm{C}(24)$ & $149.9(3)$ & $\mathrm{C}(17)-\mathrm{C}(16)-\mathrm{C}(18)$ & $59.95(15)$ \\
\hline $\mathrm{C}(22)-\mathrm{C}(23)$ & $149.9(3)$ & $\mathrm{C}(16)-\mathrm{C}(17)-\mathrm{C}(18)$ & $60.47(14)$ \\
\hline$C(23)-C(24)$ & $150.0(3)$ & $\mathrm{C}(17)-\mathrm{C}(18)-\mathrm{C}(16)$ & $59.59(14)$ \\
\hline $\mathrm{C}(1)-\mathrm{N}(1)-\mathrm{C}(3)$ & 111.77(17) & $C(6)-C(19)-C(21)$ & $121.85(19)$ \\
\hline $\mathrm{C}(1)-\mathrm{N}(1)-\mathrm{C}(2)$ & $123.85(17)$ & C(6)-C(19)-C(20) & $118.14(19)$ \\
\hline $\mathrm{C}(3)-\mathrm{N}(1)-\mathrm{C}(2)$ & $124.32(17)$ & $\mathrm{C}(21)-\mathrm{C}(19)-\mathrm{C}(20)$ & $59.90(15)$ \\
\hline $\mathrm{O}(1)-\mathrm{C}(1)-\mathrm{N}(1)$ & 123.39(19) & $\mathrm{C}(21)-\mathrm{C}(20)-\mathrm{C}(19)$ & $59.83(15)$ \\
\hline $\mathrm{O}(1)-\mathrm{C}(1)-\mathrm{C}(7 \mathrm{~A})$ & 127.60(19) & $C(19)-C(21)-C(20)$ & $60.27(15)$ \\
\hline $\mathrm{N}(1)-\mathrm{C}(1)-\mathrm{C}(7 \mathrm{~A})$ & $109.00(17)$ & $\mathrm{C}(24)-\mathrm{C}(22)-\mathrm{C}(23)$ & $60.05(16)$ \\
\hline $\mathrm{C}(12)-\mathrm{C}(2)-\mathrm{C}(8)$ & $120.8(2)$ & $\mathrm{C}(24)-\mathrm{C}(22)-\mathrm{C}(7)$ & $117.89(19)$ \\
\hline $\mathrm{C}(12)-\mathrm{C}(2)-\mathrm{N}(1)$ & $118.73(18)$ & $\mathrm{C}(23)-\mathrm{C}(22)-\mathrm{C}(7)$ & $119.40(19)$ \\
\hline $\mathrm{C}(8)-\mathrm{C}(2)-\mathrm{N}(1)$ & $120.5(2)$ & $C(22)-C(23)-C(24)$ & $59.95(15)$ \\
\hline$C(3)-C(3 A)-C(7 A)$ & $104.56(17)$ & $C(22)-C(24)-C(23)$ & $60.00(15)$ \\
\hline
\end{tabular}


Tabelle 4. Anisotrope Auslenkungsparameter $\left(\AA^{2}\right)$ von 100. Der anisotrope Auslenkungsfaktor hat die Form: $-2 \pi^{2}\left[(\mathrm{~h} \mathrm{a})^{2} U_{11}+\ldots+2 \mathrm{~h} \mathrm{k} \mathrm{a*} \mathrm{b}^{*} \mathrm{U}_{12}\right]$.

\begin{tabular}{|c|c|c|c|c|c|c|}
\hline & U11 & $\mathrm{U} 22$ & U33 & $\mathrm{U} 23$ & U13 & U12 \\
\hline $\mathrm{N}(1)$ & $0.0295(9)$ & $0.0189(8)$ & $0.0217(8)$ & $-0.0004(7)$ & $-0.0018(8)$ & $0.0037(7)$ \\
\hline $\mathrm{O}(1)$ & $0.0525(10)$ & $0.0195(8)$ & $0.0306(8)$ & $0.0018(6)$ & $-0.0117(8)$ & $0.0029(7)$ \\
\hline $\mathrm{O}(2)$ & $0.0417(9)$ & $0.0196(8)$ & $0.0346(9)$ & $-0.0051(6)$ & $-0.0071(8)$ & $0.0027(7)$ \\
\hline$C(1)$ & $0.0314(12)$ & $0.0209(11)$ & $0.0200(10)$ & $-0.0014(8)$ & $0.0020(9)$ & $0.0011(9)$ \\
\hline $\mathrm{C}(2)$ & $0.0328(11)$ & $0.0193(10)$ & $0.0226(10)$ & $0.0025(8)$ & $-0.0021(9)$ & $-0.0017(9)$ \\
\hline$C(3 \mathrm{~A})$ & $0.0210(10)$ & $0.0171(10)$ & $0.0273(11)$ & $-0.0009(8)$ & $-0.0004(9)$ & $0.0020(8)$ \\
\hline$C(3)$ & $0.0259(11)$ & $0.0194(11)$ & $0.0252(11)$ & $0.0002(8)$ & $0.0016(9)$ & $0.0020(8)$ \\
\hline$C(4)$ & $0.0204(10)$ & $0.0184(10)$ & $0.0236(11)$ & $-0.0004(8)$ & $-0.0012(9)$ & $-0.0011(8)$ \\
\hline$C(5)$ & $0.0223(10)$ & $0.0227(11)$ & $0.0188(10)$ & $0.0007(8)$ & $-0.0024(8)$ & $-0.0004(9)$ \\
\hline$C(6)$ & $0.0214(10)$ & $0.0204(10)$ & $0.0212(10)$ & $-0.0010(8)$ & $-0.0018(9)$ & $0.0000(9)$ \\
\hline $\mathrm{C}(7 \mathrm{~A})$ & $0.0223(10)$ & $0.0172(10)$ & $0.0263(11)$ & $0.0002(8)$ & $0.0032(9)$ & $-0.0014(9)$ \\
\hline$C(7)$ & $0.0215(10)$ & $0.0164(10)$ & $0.0254(11)$ & $0.0006(8)$ & $-0.0024(9)$ & $-0.0002(8)$ \\
\hline$C(8)$ & $0.0349(12)$ & $0.0198(11)$ & $0.0289(12)$ & $-0.0010(9)$ & $0.0018(10)$ & $-0.0012(10)$ \\
\hline $\mathrm{C}(9)$ & $0.0474(14)$ & $0.0253(12)$ & $0.0249(12)$ & $-0.0005(9)$ & $-0.0062(11)$ & $-0.0063(11)$ \\
\hline$C(10)$ & $0.0400(13)$ & $0.0286(12)$ & $0.0322(12)$ & $0.0041(10)$ & $-0.0121(11)$ & $-0.0042(11)$ \\
\hline $\mathrm{C}(11)$ & $0.0358(13)$ & $0.0347(13)$ & $0.0357(13)$ & $0.0020(10)$ & $-0.0058(11)$ & $0.0050(11)$ \\
\hline$C(12)$ & $0.0354(13)$ & $0.0311(12)$ & $0.0244(11)$ & $0.0002(9)$ & $-0.0027(10)$ & $0.0090(10)$ \\
\hline$C(13)$ & $0.0284(12)$ & $0.0183(11)$ & $0.0327(12)$ & $0.0017(8)$ & $-0.0022(10)$ & $-0.0009(9)$ \\
\hline$C(14)$ & $0.0330(13)$ & $0.0203(11)$ & $0.0509(15)$ & $0.0060(10)$ & $-0.0011(12)$ & $0.0036(10)$ \\
\hline$C(15)$ & $0.0372(14)$ & $0.0236(12)$ & $0.0385(13)$ & $0.0082(9)$ & $-0.0035(11)$ & $-0.0067(10)$ \\
\hline$C(16)$ & $0.0230(11)$ & $0.0238(11)$ & $0.0255(11)$ & $-0.0004(9)$ & $0.0013(9)$ & $-0.0027(9)$ \\
\hline$C(17)$ & $0.0214(10)$ & $0.0335(12)$ & $0.0310(12)$ & $-0.0036(9)$ & $-0.0006(9)$ & $-0.0034(9)$ \\
\hline$C(18)$ & $0.0261(11)$ & $0.0332(12)$ & $0.0302(12)$ & $-0.0038(10)$ & $-0.0005(10)$ & $-0.0064(10)$ \\
\hline C(19) & $0.0254(10)$ & $0.0217(11)$ & $0.0254(11)$ & $-0.0018(8)$ & $0.0006(9)$ & $0.0037(9)$ \\
\hline $\mathrm{C}(20)$ & $0.0337(13)$ & $0.0289(12)$ & $0.0310(13)$ & $-0.0092(10)$ & $0.0047(10)$ & $0.0004(10)$ \\
\hline $\mathrm{C}(21)$ & $0.0293(12)$ & $0.0318(12)$ & $0.0341(12)$ & $-0.0068(10)$ & $0.0075(10)$ & $0.0045(10)$ \\
\hline$C(22)$ & $0.0271(11)$ & $0.0176(10)$ & $0.0311(12)$ & $0.0004(8)$ & $-0.0010(10)$ & $-0.0021(9)$ \\
\hline$C(23)$ & $0.0399(13)$ & $0.0247(11)$ & $0.0353(13)$ & $-0.0071(10)$ & $0.0020(11)$ & $-0.0067(10)$ \\
\hline$C(24)$ & $0.0330(12)$ & $0.0251(11)$ & $0.0367(13)$ & $0.0001(10)$ & $0.0019(11)$ & $-0.0087(10)$ \\
\hline
\end{tabular}


Tabelle 5. H-Atomkoordinaten und äquivalente isotrope Auslenkungsparameter $\left(\AA^{2}\right)$ von 104.

\begin{tabular}{lllll}
\hline & $\mathrm{x}$ & $\mathrm{y}$ & $\mathrm{z}$ & $\mathrm{U}(\mathrm{eq})$ \\
\hline $\mathrm{H}(3 \mathrm{AA})$ & 0.4068 & 0.1160 & 0.7537 & 0.026 \\
$\mathrm{H}(4 \mathrm{~A})$ & 0.3033 & 0.1283 & 0.8772 & 0.025 \\
$\mathrm{H}(7 \mathrm{AA})$ & 0.4063 & 0.2607 & 0.7328 & 0.026 \\
$\mathrm{H}(7 \mathrm{~A})$ & 0.3459 & 0.2652 & 0.8673 & 0.025 \\
$\mathrm{H}(8 \mathrm{~A})$ & 0.0681 & 0.1077 & 0.5329 & 0.033 \\
$\mathrm{H}(9 \mathrm{~A})$ & -0.1667 & 0.1031 & 0.4495 & 0.039 \\
$\mathrm{H}(10 \mathrm{~A})$ & -0.4274 & 0.1716 & 0.4802 & 0.040 \\
$\mathrm{H}(11 \mathrm{~A})$ & -0.4564 & 0.2444 & 0.5965 & 0.042 \\
$\mathrm{H}(12 \mathrm{~A})$ & -0.2236 & 0.2486 & 0.8094 & 0.032 \\
$\mathrm{H}(14 \mathrm{~A})$ & 0.4542 & -0.0067 & 0.8790 & 0.042 \\
$\mathrm{H}(14 \mathrm{~B})$ & 0.3590 & -0.0978 & 0.8464 & 0.042 \\
$\mathrm{H}(15 \mathrm{~A})$ & 0.1220 & -0.0767 & 0.9350 & 0.040 \\
$\mathrm{H}(15 \mathrm{~B})$ & 0.2171 & 0.0143 & 0.9676 & 0.040 \\
$\mathrm{H}(16 \mathrm{~A})$ & -0.1160 & 0.0919 & 0.9251 & 0.029 \\
$\mathrm{H}(17 \mathrm{~A})$ & -0.2843 & 0.2115 & 0.8175 & 0.034 \\
$\mathrm{H}(17 \mathrm{~B})$ & -0.3857 & 0.1486 & 0.8812 & 0.034 \\
$\mathrm{H}(18 \mathrm{~A})$ & -0.1595 & 0.0813 & 0.7593 & 0.036 \\
$\mathrm{H}(18 \mathrm{~B})$ & -0.2610 & 0.0184 & 0.8231 & 0.036 \\
$\mathrm{H}(19 \mathrm{~A})$ & -0.1005 & 0.3596 & 0.8422 & 0.029 \\
$\mathrm{H}(20 \mathrm{~A})$ & 0.0930 & 0.3570 & 0.9863 & 0.037 \\
$\mathrm{H}(20 \mathrm{~B})$ & -0.0364 & 0.4382 & 0.9570 & 0.037 \\
$\mathrm{H}(21 \mathrm{~A})$ & -0.1495 & 0.2553 & 0.9809 & 0.038 \\
$\mathrm{H}(21 \mathrm{~B})$ & -0.2789 & 0.3365 & 0.9516 & 0.038 \\
$\mathrm{H}(22 \mathrm{~A})$ & 0.1916 & 0.4195 & 0.7968 & 0.030 \\
$\mathrm{H}(23 \mathrm{~A})$ & 0.4012 & 0.3997 & 0.9353 & 0.040 \\
$\mathrm{H}(23 \mathrm{~B})$ & 0.3248 & 0.4946 & 0.9016 & 0.040 \\
$\mathrm{H}(24 \mathrm{~A})$ & 0.4839 & 0.4718 & 0.7845 & 0.038 \\
$\mathrm{H}(24 \mathrm{~B})$ & 0.5604 & 0.3769 & 0.8182 & 0.038 \\
& & & & \\
\hline
\end{tabular}




\section{Danksagungen}

Für den wissenschaftlichen Unterricht danke ich den Professoren und Privatdozenten der Georg-August-Universität Göttingen, der Universität Ulm und des University College Dublin: U. Beifuss, J. Belzner, K. Beuermann, R. Brückner, M. Buback, G. Eckold, P. J. Guiry, G. Hirsch-Ernst, G. F. Kahl, W. Knepel, H. Laatsch, J. Magull, A. de Meijere, A. Meller, U. Ritter, H. W. Roesky, Ch. Schneider, P. R. Schreiner, J. Schröder, G. M. Sheldrick, H.-U. Siehl, M. Suhm, L. F. Tietze, D. Trzeciok, K.-H. Tytko, G. Wagner, A. Zeeck.

Für die Aufnahme der Massenspektren und diesbezüglichen Problemen danke ich Herrn Dr. H. Frauendorf und Frau G. Udvarnoki. Herrn F. Hambloch danke ich für die Durchführung der Elementaranalysen und Herrn R. Machinek, sowie seinem Team, besonders Frau und Herrn Zolke für die Messung der NMR-Spektren. Frau E. Pfeil danke ich für die Aufnahme der UV-Vis-Spektren und Herrn Dr. H. P. Vögele für die schnelle Hilfe bei IR-Problemen. Herrn Prof. J. Magull und Denis Vidović danke ich für den Service bei den EinkristallRöntgenstrukturanalysen. Herrn Prof. Dr. P. R. Schreiner danke ich besonders für die Durchführung der Rechnungen und die anregenden Diskussionen.

Frau G. Keil-Knepel, Stefan Beußhausen, Michael Schelper, Heiko Schill, Karsten Rauch, Daniel Frank, Dr. Rolf Meyer und Günther Möller danke ich für ihre stete Hilfsbereitschaft bei Dienstlichem, mit Computern und Labormaterialien.

Für das sorgfältige Korrekturlesen dieser Arbeit und die anregenden Diskussionen danke ich besonders Regina, Farina, Heiko, Jasmin, Sergei und besonders Ingo und Christoph, dem ich auch herzlich für die Einführung in die Tieftemperatur-NMR-Messung und die angenehme Zeit in Ulm danke.

Für das Wohlfühl-Klima im Labor P110/112 danke ich herzlich Regina und Michael S. (Tigerbalm und Ingwer-Knoblauch-Bouillon: eine heilsame Liaison) sowie unseren Gaststudenten Abbey, Alberto, Bahaa und Swen. Für die immer frohe Runde und kulinarischen Ausflüge danke ich besonders Ingo, Sandra, Michael L., Mario, Surya, Takuya, Ligang, Baris, Daniel und Carsten. Allen weiteren Kollegen sei ebenfalls für die Zusammenarbeit gedankt. Dank gilt ebenso meinen Praktikanten aus dem OC-F-Praktikum.

Ganz besonders herzlichen Dank möchte ich meinen Eltern und meiner Schwester aussprechen, deren Verständnis und intensive Unterstützung mir dieses Studium ermöglichte. Jasmin danke ich herzlich für ihre stete Zuneigung und ihre Geduld. 


\section{Lebenslauf}

Am 28.05.1976 wurde ich als Sohn von Bärbel und Karlheinz Redlich in Göttingen geboren. Im August 1982 erfolgte die Einschulung in die Johann-Wolf-Schule (Grundschule) in Nörten-Hardenberg. Anschließend besuchte ich von August 1986 bis Juli 1988 die NovalisSchule (Orientierungsstufe) in Nörten-Hardenberg und von August 1988 bis 1995 das OttoHahn-Gymnasium in Göttingen, wo ich am 16.05.1995 die Abiturprüfung bestand. In der Zeit von Oktober 1995 bis September 1996 absolvierte ich meinen Wehrdienst beim Jagdbombergeschwader 38 "Friesland" in Schortens. Zum Wintersemester 1996/1997 nahm ich das Chemiestudium an der Georg-August-Universität in Göttingen auf und absolvierte am 22.10.1998 die Diplom-Vorprüfung.

Im Januar 2000 nahm ich an der 10th Winterschool on Organic Reactitvity in Bressanone, Italien, teil. In der Zeit von Februar bis April 2000 war ich als Praktikant am Pharmaforschungszentrum der Bayer AG im Labor von Herrn Dr. H. Meier in Wuppertal tätig (Synthese von ZNS-aktiven Cannabinoid-Derivaten). Von Mai bis Juli 2000 führte ich gefördert durch ein ERASMUS-Stipendium der Europäischen Union - einen Teil meines Hauptstudiums am University College Dublin im Arbeitskreis von Herrn Dr. P. J. Guiry (Synthese und Anwendung von axial-chiralen Liganden) in Irland durch. Im September 1999 nahm ich erfolgreich am Praktikum zur Anwendung radioaktiver Isotope teil. Während meines Hauptstudiums wurde mir als studentische Hilfskraft zeitweise die Betreuung der Studenten der Biologie und der Medizin übertragen.

Meine Diplomarbeit über die Kinetik der Heck-Reaktion unter hohem Druck und cyclopropylierte Cyclopentadienyl- und Fulvenyl-Kationen fertigte ich in der Zeit von Januar bis September 2001 unter der Leitung von Herrn Prof. Dr. A. de Meijere im Fach Organische Chemie an und erlangte am im Oktober 2001 den Akademischen Grad eines DiplomChemikers. Seit November 2001 arbeite ich als wissenschaftlicher Mitarbeiter in der Forschungsgruppe von Herrn Prof. Dr. A. de Meijere an meiner Doktorarbeit über die Erzeugung des Pentacyclopropylcyclopentadienyl-Kations und cyclopropylierte Oligoene, Carbo- und Heterocyclen und betreue Studenten der Chemie bei ihrem Organischen Fortgeschrittenen-Praktikum.

Einen Teil der experimentellen Arbeiten meiner Doktorarbeit führte ich in mehrwöchigen Forschungsaufenthalten bei Herrn Prof. Dr. H.-U. Siehl an der Universität Ulm durch. Im Juni 2003 bestand ich die Prüfung der umfassenden Sachkunde nach $\S 5$ der Chemikalienverbotsverordnung, und im Januar 2004 absolvierte ich erfolgreich einen Intensivkurs in allgemeiner Pharmakologie und Toxikologie. 